\title{
A Theory of Besov and Triebel-Lizorkin Spaces on Metric Measure Spaces Modeled on Carnot-Carathéodory Spaces
}

\author{
Yongsheng Han, ${ }^{1}$ Detlef Müller, ${ }^{2}$ and Dachun Yang $^{3}$ \\ ${ }^{1}$ Department of Mathematics, Auburn University, Auburn, AL 36849-5310, USA \\ ${ }^{2}$ Mathematisches Seminar, Christian-Albrechts-Universität Kiel, Ludewig-Meyn Strasse 4, \\ 24098 Kiel, Germany \\ ${ }^{3}$ School of Mathematical Sciences, Beijing Normal University, Laboratory of Mathematics and \\ Complex Systems, Ministry of Education, Beijing 100875, China
}

Correspondence should be addressed to Dachun Yang, dcyang@bnu.edu.cn

Received 13 February 2008; Accepted 23 May 2008

Recommended by Stephen Clark

We work on RD-spaces $\mathcal{X}$, namely, spaces of homogeneous type in the sense of Coifman and Weiss with the additional property that a reverse doubling property holds in $\mathcal{X}$. An important example is the Carnot-Carathéodory space with doubling measure. By constructing an approximation of the identity with bounded support of Coifman type, we develop a theory of Besov and TriebelLizorkin spaces on the underlying spaces. In particular, this includes a theory of Hardy spaces $H^{p}(\mathcal{X})$ and local Hardy spaces $h^{p}(\mathcal{X})$ on RD-spaces, which appears to be new in this setting. Among other things, we give frame characterization of these function spaces, study interpolation of such spaces by the real method, and determine their dual spaces when $p \geq 1$. The relations among homogeneous Besov spaces and Triebel-Lizorkin spaces, inhomogeneous Besov spaces and Triebel-Lizorkin spaces, Hardy spaces, and BMO are also presented. Moreover, we prove boundedness results on these Besov and Triebel-Lizorkin spaces for classes of singular integral operators, which include non-isotropic smoothing operators of order zero in the sense of Nagel and Stein that appear in estimates for solutions of the Kohn-Laplacian on certain classes of model domains in $\mathbb{C}^{N}$. Our theory applies in a wide range of settings.

Copyright (C) 2008 Yongsheng Han et al. This is an open access article distributed under the Creative Commons Attribution License, which permits unrestricted use, distribution, and reproduction in any medium, provided the original work is properly cited.

\section{Introduction}

The scales of Besov spaces $B_{p, q}^{s}$ and Triebel-Lizorkin spaces $F_{p, q}^{s}$ on $\mathbb{R}^{n}$, respectively, domains in $\mathbb{R}^{n}$ for the full range of parameters, $s \in \mathbb{R}$ and $0<p, q \leq \infty$, were introduced between 1959 and 1975. They cover many well-known classical concrete function spaces such as Hölder-Zygmund spaces, Sobolev spaces, fractional Sobolev spaces (also often referred to as Bessel-potential spaces), local Hardy spaces, and bmo, which have their own history. 
A comprehensive treatment of these function spaces and their history can be found in Triebel's monographs [1, 2]. For further developments, including analogous theories of function spaces on fractals, we refer to [3-6].

Metric spaces play a prominent role in many fields of mathematics. In particular, they constitute natural generalizations of manifolds admitting all kinds of singularities and still providing rich geometric structure; see [7-9]. Analysis on metric measure spaces has been studied quite intensively in recent years; see, for example, Semmes's survey [10] for a more detailed discussion and references. Of particular interest is the study of functional inequalities, like Sobolev and Poincaré inequalities, on metric measure spaces; see, for example, [11-16]. Also the theory of function spaces on metric measure spaces has seen a rapid development in recent years. Since Hajłasz in [17] introduced Sobolev spaces on any metric measure spaces, a series of papers has been devoted to the construction and investigation of Sobolev spaces of various types on metric measure spaces; see, for example, $[12,13,18-27]$.

It is well known that Calderon-Zygmund operators are in general not bounded on $L^{1}\left(\mathbb{R}^{n}\right)$, and the Hardy space $H^{1}\left(\mathbb{R}^{n}\right)$ is a good substitute for $L^{1}\left(\mathbb{R}^{n}\right)$. Coifman and Weiss [28] introduced atomic Hardy spaces $H_{\mathrm{at}}^{p}(\mathcal{X})$ for $p \in(0,1]$ when $\mathcal{X}$ is a general space of homogeneous type in the sense of Coifman and Weiss [29], that is, $\mu$ is known only to be doubling. Moreover, under the assumption that the measure of any ball in $\mathcal{X}$ is equivalent to its radius (i.e., if $\mathcal{X}$ is essentially a so-called Ahlfors 1-regular metric measure space), Coifman and Weiss [28] further established a molecular characterization of $H_{\text {at }}^{1}(\mathcal{X})$, and if $p \in(1 / 2,1]$, Macías and Segovia [30] gave a maximal function characterization of $H_{\mathrm{at}}^{p}(\mathcal{X})$. For $p$ in this range, a Lusin-area characterization for $H_{\mathrm{at}}^{p}(\mathcal{x})$ was given in [31], and Duong and Yan in [32] characterized these atomic Hardy spaces in terms of Lusin-area functions associated with certain Poisson semigroups. However, the results in [28,30,32] require that $\mathcal{X}$ is an Ahlfors 1-regular metric measure space, and the methods do not extend to arbitrary spaces of homogeneous type, even though Macías and Segovia [33] proved that any space of homogeneous type is topologically equivalent to an Ahlfors 1-regular metric measure space. On the other hand, via Littlewood-Paley theory, a theory of Besov and Triebel-Lizorkin spaces on Ahlfors $n$-regular metric measure spaces was established in [34], which was further completed in [2, 31, 35-37], and it turns out that some ideas from [31, 34, 35] still work on general metric measure spaces considered in this paper.

In this paper, we work on RD-spaces $\mathcal{X}$, that is, spaces of homogeneous type in the sense of Coifman and Weiss with the additional property that a reverse doubling property holds in $\mathcal{X}$, or equivalently, that there exists a constant $a_{0}>1$ such that for all $x \in \mathcal{X}$ and $0<r<\operatorname{diam}(\mathcal{X}) / a_{0}$, the annulus $B\left(x, a_{0} r\right) \backslash B(x, r)$ is nonempty, where and in what follows, $\operatorname{diam}(\mathcal{X})$ denotes the diameter of the metric space $(\mathcal{X}, d)$. An important class of RD-spaces is provided by Carnot-Carathéodory spaces with a doubling measure, which have been the object of intensive studies for quite a while. A Carnot-Carathéodory (or sub-Riemannian) space is a connected smooth manifold endowed with a Hörmander system of vectors $\left\{X_{1}, X_{2}, \ldots, X_{k}\right\}$, which span, together with their commutators of order $\leq m$, the tangent space at each point, and the distance function $d$ is in this case given by the Carnot-Carathéodory or control distance associated with the Hörmander system. Carnot-Carathéodory spaces arise in many places in mathematics, including control theory, the theory of hypoelliptic differential operators, and several areas of harmonic analysis and complex analysis; see [38-40] for a general discussion and detailed references. Also, this type of metric measure space plays an important role in connection with problems related to snowflaked transforms; see [13, page 99] or [2]. Examples 
of noncompact Carnot-Carathéodory spaces naturally arise as boundaries of unbounded model polynomial domains in $\mathbb{C}^{N}$ appearing in the work of Nagel and Stein; see [4143].

Our theory of function spaces on RD-spaces will use Carnot-Carathéodory spaces as a basic model, in particular well-known estimates of heat kernels on such spaces.

By constructing an approximation of the identity with bounded support of Coifman type on RD-spaces, we develop a theory of Besov and Triebel-Lizorkin spaces on the underlying spaces. In particular, this includes a theory of Hardy spaces $H^{p}(\mathcal{X})$ and local Hardy spaces $h^{p}(\mathcal{X})$ on RD-spaces, which appears to be new in this setting. Among other things, we give frame characterization of these function spaces, study interpolation of such spaces by the real method and determine their dual spaces when $p \geq 1$. The relations among homogeneous Besov spaces and Triebel-Lizorkin spaces, inhomogeneous Besov spaces and Triebel-Lizorkin spaces, Hardy spaces, and BMO are also presented. Moreover, we prove boundedness results on these Besov and Triebel-Lizorkin spaces for classes of singular integral operators in [44], which include nonisotropic smoothing operators of order zero in the sense of Nagel and Stein that appear in estimates for solutions of the Kohn-Laplacian on certain classes of model domains in $\mathbb{C}^{N}$; see [43, 45-47].

We point out that a theory of Hardy spaces on RD-spaces was also established in [48] by using spaces of test functions, the theory of distributions, and the boundedness criterion of singular integrals on spaces of test functions, which are all developed in the current paper, and by assuming that there exists a suitable Calderón reproducing formula in $L^{2}(\mathcal{X})$. These Hardy spaces are proved to coincide with some of Triebel-Lizorkin spaces in this paper. Also, some spaces of Lipschitz type on RD-spaces were recently studied in [49]. Via Calderón reproducing formulae developed in the current paper, the relations of Besov and Triebel-Lizorkin spaces introduced in the current paper with those spaces of Lipschitz type in [49] and with various known Sobolev spaces as mentioned above were established in [49]. As an application, a difference characterization of Besov and TriebelLizorkin on RD-spaces was also obtained in [49]. Moreover, it is possible to establish smooth atomic and molecular characterizations and lifting property of these spaces by using fractional integrals and derivatives, and it is also possible to develop a corresponding product theory. However, to limit the length of this paper, we will leave these topics to forthcoming papers.

Our setting of RD-spaces includes spaces with a "local" dimension strictly less than the global dimension, such as certain classes of nilpotent Lie groups. In such situations, the Lipschitz classes (in the sense of Coifman and Weiss [28]) that we consider in this paper do not compare with the usual Hölder classes (which are particular Besov spaces), which is why some of our results, for example, about duality of Besov and Triebel-Lizorkin spaces, assume a different form compared to the corresponding "classical" results on $\mathbb{R}^{n}$, respectively, on Ahlfors $n$-regular spaces.

\subsection{Underlying spaces}

We first recall the notion of a space of homogeneous type in the sense of Coifman and Weiss $[28,29]$ and then introduce the so-called RD-spaces, which are particular spaces of homogeneous type.

Definition 1.1. Let $(\mathcal{X}, d)$ be a metric space with a regular Borel measure $\mu$, which means that $\mu$ is a nonnegative countably subadditive set function defined on all subsets of $\mathcal{X}$, open sets 
are measurable, and every set is contained in a Borel set with the same measure, such that all balls defined by $d$ have finite and positive measure. For any $x \in \mathcal{X}$ and $r>0$, set

$$
B(x, r)=\{y \in X: d(x, y)<r\}
$$

(i) The triple $(\mathcal{X}, d, \mu)$ is called a space of homogeneous type if there exists a constant $C_{0} \geq 1$ such that for all $x \in \mathcal{X}$ and $r>0$,

$$
\mu(B(x, 2 r)) \leq C_{0} \mu(B(x, r)) \text { (doubling property). }
$$

(ii) Let $0<\kappa \leq n$. The triple $(\mathcal{X}, d, \mu)$ is called a $(\kappa, n)$-space if there exist constants $0<C_{1} \leq 1$ and $C_{2} \geq 1$ such that for all $x \in \mathcal{X}, 0<r<\operatorname{diam}(x) / 2$, and $1 \leq \lambda<$ $\operatorname{diam}(x) /(2 r)$,

$$
C_{1} \lambda^{\kappa} \mu(B(x, r)) \leq \mu(B(x, \lambda r)) \leq C_{2} \lambda^{n} \mu(B(x, r)),
$$

$$
\text { where } \operatorname{diam}(x)=\sup _{x, y \in x} d(x, y) \text {. }
$$

A space of homogeneous type will be called an $R D$-space if it is a $(\kappa, n)$-space for some $0<\kappa \leq n$, that is, if some "reverse" doubling condition holds.

Clearly, any Ahlfors $n$-regular metric measure space $(\mathcal{X}, d, \mu)$, which means that there exists some $n>0$ such that $\mu(B(x, r)) \sim r^{n}$ for all $x \in \mathcal{X}$ and $0<r<\operatorname{diam}(\mathcal{X}) / 2$, is an $(n, n)$-space.

Remark 1.2. (i) Obviously, any $(k, n)$-space is a space of homogeneous type with $C_{0}=C_{2} 2^{n}$. Conversely, any space of homogeneous type satisfies the second inequality of (1.3) with $C_{2}=C_{0}$ and $n=\log _{2} C_{0}$. Comparing with spaces of homogeneous type, the only additional restriction in $(k, n)$-spaces is the first inequality of (1.3).

If $\mathcal{X}$ is a $(\kappa, n)$-space, the first inequality in (1.3) implies that there exist constants $a_{0}>$ $1 / C_{1}^{1 / \kappa} \geq 1$ and $\widetilde{C}_{1}=C_{1} a_{0}^{\kappa}>1$ such that for all $x \in \mathcal{X}$ and $0<r<\operatorname{diam}(\mathcal{X}) / a_{0}$,

$$
\mu\left(B\left(x, a_{0} r\right)\right) \geq \widetilde{C}_{1} \mu(B(x, r)) \text { (reverse doubling property), }
$$

(if $a_{0}=2$, this is the classical reverse doubling condition), and therefore,

$$
B\left(x, a_{0} r\right) \backslash B(x, r) \neq \varnothing .
$$

Conversely, assume that $\mu$ satisfies the second inequality of (1.3) (i.e., $\mu$ is doubling) and (1.5) holds for some $a_{0}>1$ and for all $x \in \mathcal{X}$ and $0<r<\operatorname{diam}(\mathcal{X}) / a_{0}$. Then, $\mathcal{X}$ is a $(\kappa, n)$-space for some $\kappa>0$.

To see this, by ideas in [50, pages 11-12] (see also [51, pages 269-270]), it suffices to show that there exist constants $a_{1}>a_{0}$ and $\widetilde{C}_{1}>1$ such that for all $x \in \mathcal{X}$ and $0<r<$ $\operatorname{diam}(x) / 2 a_{0}$,

$$
\mu\left(B\left(x, a_{1} r\right)\right) \geq \tilde{C}_{1} \mu(B(x, r))
$$


which further implies that the first inequality in (1.3) also holds for some $\kappa>0$, that is, $x$ is a $(\kappa, n)$-space. To this end, fix any $\sigma \in(0,1]$. Then, if $0<r<\operatorname{diam}(\mathcal{x}) / 2 a_{0}$, we have $(1+\sigma) r<\operatorname{diam}(\mathcal{X}) / a_{0}$. Thus, by the assumption,

$$
B\left(x, a_{0}(1+\sigma) r\right) \backslash B(x,(1+\sigma) r) \neq \varnothing .
$$

Choose $y \in B\left(x, a_{0}(1+\sigma) r\right) \backslash B(x,(1+\sigma) r)$. It is easy to see that

$$
B(y, \sigma r) \cap B(x, r)=\varnothing
$$

$B(y, \sigma r) \subset B\left(x,\left[\sigma+a_{0}(1+\sigma)\right] r\right)$, and

$$
B\left(x,\left[\sigma+a_{0}(1+\sigma)\right] r\right) \subset B\left(y,\left[\sigma+2 a_{0}(1+\sigma)\right] r\right),
$$

which together with the second inequality in (1.3) imply

$$
\begin{aligned}
& \mu\left(B\left(x,\left[\sigma+a_{0}(1+\sigma)\right] r\right)\right) \\
& \quad \geq \mu(B(x, r))+\mu(B(y, \sigma r)) \\
& \quad \geq \mu(B(x, r))+C_{2}^{-1}\left(\frac{\sigma}{\sigma+2 a_{0}(1+\sigma)}\right)^{n} \mu\left(B\left(y,\left[\sigma+2 a_{0}(1+\sigma)\right] r\right)\right) \\
& \quad \geq \mu(B(x, r))+C_{2}^{-1}\left(\frac{\sigma}{\sigma+2 a_{0}(1+\sigma)}\right)^{n} \mu\left(B\left(x,\left[\sigma+a_{0}(1+\sigma)\right] r\right)\right) .
\end{aligned}
$$

This implies (1.6) with $a_{1} \equiv \sigma+a_{0}(1+\sigma)>a_{0}$ and

$$
\widetilde{C}_{1} \equiv\left[1-C_{2}^{-1}\left(\frac{\sigma}{\sigma+2 a_{0}(1+\sigma)}\right)^{n}\right]^{-1}>1
$$

Thus, $x$ is a $(\kappa, n)$-space.

Therefore, $\boldsymbol{X}$ is an RD-space if and only if $\mathcal{X}$ is a space of homogeneous type with the additional property that there exists a constant $a_{0}>1$ such that for all $x \in \mathcal{X}$ and $0<r<$ $\operatorname{diam}(\mathcal{X}) / a_{0}, B\left(x, a_{0} r\right) \backslash B(x, r) \neq \varnothing$.

(ii) From (i), it is obvious that an RD-space has no isolated points.

(iii) It is proved in [7-9] that some curvature-dimension condition on metric measure spaces implies the doubling property of the considered measure.

Remark 1.3. We recall that two metrics $d$ and $\tilde{d}$ are said to be equivalent if $d / \tilde{d}$ is uniformly bounded and uniformly bounded away from zero. In what follows, we always regard two $(\kappa, n)$-spaces or spaces of homogeneous type with equivalent metrics as the same space. 
Remark 1.4. Let $d$ be a quasimetric, which means that $d$ is a nonnegative and symmetric function on $x \times x, d(x, y)=0$ if and only if $x=y$, and there exists a constant $A_{0} \geq 1$ such that for all $x, y, z \in \mathcal{X}$,

$$
d(x, y) \leq A_{0}(d(x, z)+d(z, y))
$$

Macías and Segovia [33] proved that for any quasimetric $d$, there exists an equivalent quasimetric $\tilde{d}$ such that all balls corresponding to $\tilde{d}$ are open in the topology induced by $\tilde{d}$, and there exist constants $A_{0}^{\prime}>0$ and $\theta \in(0,1)$ such that for all $x, y, z \in \mathcal{X}$,

$$
|\tilde{d}(x, z)-\tilde{d}(y, z)| \leq A_{0}^{\prime} \tilde{d}(x, y)^{\theta}[\tilde{d}(x, z)+\tilde{d}(y, z)]^{1-\theta}
$$

which means $\tilde{d}$ has some regularity; see [33, Theorem 2].

Notice that all results below are true if $d$ is a quasimetric and has some regularity, and Remark 1.3 is also true for both such equivalent quasimetrics. From this and the above result of Macías and Segovia, it follows that all results in this paper are still true if $d$ on $x$ is only known to be a quasimetric (especially, if $\mathcal{X}$ is a so-called $d$-space of Triebel; see [2, page 189]), which is another advantage of the results in this paper compared to all known results so far.

In what follows, for the simplicity of the presentation, we always assume that $d$ is a metric, which means $A_{0}=1$ and $\theta=1$.

Remark 1.5. If $\mathcal{X}$ is a closed subset of $\mathbb{R}^{n}$ and $\mathcal{X}$ is a $(\kappa, n)$-space with the additional normalization assumption that $\mu(B(x, 1)) \sim 1$ for all $x \in \mathcal{X}$, Jonsson in [52, 53] introduced certain Besov spaces for some special indices on such sets with the aid of difference or local polynomial approximation (equivalently by atoms) and obtained some trace theorems for restrictions to $\mathcal{X}$.

Moreover, Bylund and Gudayol [54] gave out some conditions such that a compact pseudometric space becomes a $(\kappa, n)$-space with $\mu$ being a probability measure.

Remark 1.6. Under rather general circumstances, regular Borel measures have two useful properties, which are often called inner regularity and outer regularity. More precisely, if $\mu$ is a regular Borel measure, then for each Borel set $A$ of finite measure, $\mu(A)$ is the supremum of the numbers $\mu(C)$, where $C$ runs through all closed subsets of $A$; moreover, if the metric balls have finite measure, and $A$ is a Borel set, then $\mu(A)$ is the infimum of the numbers $\mu(U)$, where $U$ runs through all open supersets of $A$; see [55, Theorem 2.2.2] or [13, page 3]. These properties are used in establishing Lebesgue's differentiation theorem; see [13, pages 4-7] for the details.

We now recall the definition of Carnot-Carathéodory spaces. Let $\mathcal{X}$ be a connected smooth manifold and let $\left\{X_{1}, \ldots, X_{k}\right\}$ be $k$ given smooth real vector fields on $\chi$ satisfying Hörmander's condition of order $m$, that is, these vector fields together with their commutators of order at most $m$ span the tangent space to $\mathcal{X}$ at each point. To develop a theory of Besov and TriebelLizorkin spaces on $\mathcal{X}$, we make use of the notion of control distances associated to the vector fields. One possibility of defining a control distance is as follows: for $x, y \in \mathcal{X}$ and $\delta>0$, let $A C(x, y, \delta)$ denote the collection of absolutely continuous mappings $\varphi:[0,1] \rightarrow \mathcal{X}$ with $\varphi(0)=x$ and $\varphi(1)=y$ such that for almost every $t \in[0,1], \varphi^{\prime}(t)=\sum_{j=1}^{k} a_{j} X_{j}(\varphi(t))$, with 
$\left|a_{j}\right| \leq \delta$. Then, the control metric $d(x, y)$ from $x$ to $y$ is the infimum of the set of $\delta>0$ such that $A C(x, y, \delta) \neq \varnothing$. Hörmander's condition makes sure that $d(x, y)<\infty$ for every $x, y \in x$.

The following three specific examples of Carnot-Carathéodory spaces which are also $(\kappa, n)$-spaces naturally come from harmonic analysis and several complex variables.

(a) Compact case. If $\mathcal{X}$ is a compact $n$-dimensional Carnot-Carathéodory space and is endowed with any fixed smooth measure $\mu$ with strictly positive density, by [56, Theorem 1] (or [42, Theorem 2.2.4]), we know that $\mathcal{X}$ is an $(n, n m)$-space; see also [44].

(b) Noncompact case. Let $\Omega=\left\{(z, w) \in \mathbb{C}^{2}: \Im m[w]>P(z)\right\}$, where $P$ is a real, subharmonic, nonharmonic polynomial of degree $m$. Namely, $\Omega$ is an unbounded model domain of polynomial type in $\mathbb{C}^{2}$. Then, $\mathcal{X}=\partial \Omega$ can be identified with $\mathbb{C} \times \mathbb{R}=\{(z, t): z \in \mathbb{C}, t \in \mathbb{R}\}$. The basic $(0,1)$ Levi vector field is then $\bar{Z}=\partial / \partial \bar{z}-i(\partial P / \partial \bar{z})(\partial / \partial t)$, and we write $\bar{Z}=X_{1}+i X_{2}$. The real vector fields $\left\{X_{1}, X_{2}\right\}$ and their commutators of orders $\leq m$ span the tangent space at each point. If we endow $\mathbb{C} \times \mathbb{R}$ with the Lebesgue measure, then by [43, Proposition 3.1.1] we know that $\mathcal{X}=\mathbb{C} \times \mathbb{R}$ is a $(4, m+2)$-space; see also $[42,44,56]$.

(c) (Noncompact case) Lie groups of polynomial growth (see [56-60]). Let $G$ be a connected Lie group and fix a left invariant Haar measure $\mu$ on $G$. We assume that $G$ has polynomial volume growth, that is, if $U$ is a compact neighborhood of the identity element $e$ of $G$, then there is a constant $C>0$ such that $\mu\left(U^{n}\right) \lesssim n^{C}$ for all $n \in \mathbb{N}$ (see [56]). Then $G$ is unimodular. Furthermore, there is a nonnegative integer $n_{\infty}$ such that $\mu\left(U^{n}\right) \sim n^{n_{\infty}}$ as $n \rightarrow \infty$; see [61] and also [57, 62]. Let $X_{1}, \ldots, X_{n}$ be left invariant vector fields on $G$ that satisfy Hörmander's condition, that is, they together with their successive Lie brackets $\left[X_{i_{1}},\left[X_{i_{2}},\left[\ldots, X_{i_{k}}\right] \ldots\right]\right.$ span the tangent space of $G$ at every point of $G$. Let $d$ be the associated control metric. Then this metric is left invariant and compatible with the topology on $G$; see $[57,58]$. Moreover, by the results in $[56,57]$, we know that there is $n_{0} \in \mathbb{N}$, independent of $x$, such that $\mu(B(x, r)) \sim r^{n_{0}}$ when $0<r \leq 1$, and $\mu(B(x, r)) \sim r^{n_{\infty}}$ when $r \geq 1$. From this, it is easy to verify that $G$ is a $\left(\min \left\{n_{0}, n_{\infty}\right\}, \max \left\{n_{0}, n_{\infty}\right\}\right)$-space.

\subsection{Outline of some basic methods}

Let $\mathcal{X}$ be a Carnot-Carathéodory space as in Case (a) with $n \geq 3$ or as in Case (b), and let $\left\{X_{1}, \ldots, X_{k}\right\}$ be a family of real vector fields on $\mathcal{X}$, which are of finite-type $m$. Consider the sub-Laplacian $\mathcal{\perp}$ on $\mathcal{X}$ in self-adjoint form, given by

$$
\mathcal{L}=\sum_{j=1}^{k} X_{j}^{*} X_{j} .
$$

Here $\left(X_{j}^{*} \varphi, \psi\right)=\left(\varphi, X_{j} \psi\right)$, where

$$
(\varphi, \psi)=\int_{x} \varphi(x) \overline{\psi(x)} d \mu(x)
$$

and $\varphi, \psi \in C_{c}^{\infty}(\mathcal{X})$, the space of $C^{\infty}$ functions on $\mathcal{X}$ with compact support. In general, $X_{j}^{*}=$ $-X_{j}+a_{j}$, where $a_{j} \in \mathcal{C}^{\infty}(\mathcal{X})$. The solution of the following initial value problem for the heat 
equation,

$$
\frac{\partial u}{\partial s}(x, s)+\mathcal{L}_{x} u(x, s)=0
$$

with $u(x, 0)=f(x)$, is given by $u_{s}(x, s)=H_{s}[f](x)$, where $H_{s}$ is the operator given via the spectral theorem by $H_{s}=e^{-s \mathcal{L}}$, and an appropriate self-adjoint extension of the nonnegative operator $\mathcal{L}$ initially defined on $C_{c}^{\infty}(\mathcal{X})$. It was proved in [44] that for $f \in L^{2}(\mathcal{X})$,

$$
H_{s}[f](x)=\int_{x} H(s, x, y) f(y) d \mu(y)
$$

where $x \in \mathcal{X}$. Moreover, $H(s, x, y)$ has some nice regularity properties (see [44, Proposition 2.3.1] and [63]).

By abstracting from the properties of heat kernels $H(s, x, y)$, we will introduce notions of approximations of the identity and spaces of test functions and their dual spaces on arbitrary spaces of homogeneous type. These two spaces are, respectively, the substitutes of the space of Schwartz functions and the space of tempered distributions; see, for example, $[31,34]$. Following Coifman's idea in [64], we then construct an approximation of the identity with bounded support on metric measure spaces. We will show that our spaces of test functions are invariant under a large class of singular integral operators. The integral kernels associated to these operators satisfy a certain "second difference regularity condition," which also turns out to be necessary to this result; see [31,35]. This theorem is a main tool for establishing a Calderón reproducing formula. Let $\left\{S_{k}\right\}_{k \in \mathbb{Z}}$ be such an approximation of the identity. Set next $D_{k}=S_{k}-S_{k-1}$ for $k \in \mathbb{Z}$. Based on Coifman's ideas (see [64] for the details), on $L^{p}(\mathcal{X})$ with $p \in(1, \infty)$, we can decompose the identity operator $I$ in the strong sense as

$$
\begin{aligned}
I & =\sum_{k=-\infty}^{\infty} D_{k} \\
& =\left(\sum_{k=-\infty}^{\infty} D_{k}\right)\left(\sum_{j=-\infty}^{\infty} D_{j}\right) \\
& =\sum_{|k-j| \leq N} D_{k} D_{j}+\sum_{|k-j|>N} D_{k} D_{j} \\
& =T_{N}+R_{N} .
\end{aligned}
$$

The error term $R_{N}$ will be small of order $2^{-\delta N}$ in norm with $\delta>0$. Using the abovementioned theorem, we prove that if $N$ is large enough, then $R_{N}$ is bounded on the space of test functions with the operator norm less than 1. Therefore, if $N$ is large enough and $D_{k}^{N}=\sum_{|j| \leq N} D_{k+j}$ for $k \in \mathbb{Z}$, we then obtain the following Calderón-type reproducing formulae:

$$
f=\sum_{k=-\infty}^{\infty} T_{N}^{-1} D_{k}^{N} D_{k}(f)=\sum_{k=-\infty}^{\infty} D_{k} D_{k}^{N} T_{N}^{-1}(f)
$$


where $T_{N}^{-1}$ is the inverse of $T_{N}$ and the series converge in $L^{p}(\mathcal{X}), 1<p<\infty$, and in the space of test functions and its dual space. We also obtain a corresponding discrete version. The Calderon reproducing formula is another main tool of this paper. As soon as (1.19) is established, we can introduce Besov and Triebel-Lizorkin spaces on $\not{X}$ via approximations of the identity and prove that these spaces are independent of the choice of approximations of the identity. The Calderon reproducing formula is also employed to establish the atomic decomposition of these spaces and to prove boundedness results for operators acting on these spaces.

Remark 1.7. Let $\left\{S_{k}\right\}_{k \in \mathbb{Z}}$ be an approximation of the identity as constructed in Theorem 2.6 below. An essential difference in the theory occurs when the measure, $\mu(\mathcal{X})$, of the underlying space $\mathcal{X}$ is finite versus the case when the measure of the underlying space is infinite. When $\mu(x)<\infty$, it is not true that $\left\|S_{k}(f)\right\|_{L^{p}(x)} \rightarrow 0$ as $k \rightarrow-\infty$ for all $p \in(1, \infty)$ and $f \in$ $L^{p}(\mathcal{X})$; see the proof of Proposition 3.1(i) below. Thus, when $\mu(X)<\infty$, in the Calderon-type reproducing formulae (1.19), we should replace $D_{0}$ by $S_{0}$ and $D_{k}$ for $k \in\{-1,-2, \ldots\}$ by 0 . This means that when $\mu(x)<\infty$, we always have an inhomogeneous term corresponding to $S_{0}$, which needs some special care.

\subsection{Notation}

Finally, we introduce some notation and make some conventions. Throughout the paper, $A \sim B$ means that the ratio $A / B$ is bounded and bounded away from zero by constants that do not depend on the relevant variables in $A$ and $B ; A \lesssim B$ and $B \gtrsim A$ mean that the ratio $A / B$ is bounded by a constant independent of the relevant variables. For any $p \in[1, \infty]$, we denote by $p^{\prime}$ its conjugate index, namely, $1 / p+1 / p^{\prime}=1$. We also denote by $C$ a positive constant which is independent of the main parameters, but it may vary from line to line. Constants with subscripts, such as $C_{0}$, do not change in different occurrences. If $E$ is a subset of a metric space $(\mathcal{X}, d)$, we denote by $\chi_{E}$ the characteristic function of $E$ and define

$$
\operatorname{diam} E=\sup _{x, y \in E} d(x, y) .
$$

We also set $\mathbb{N}=\{1,2, \ldots\}$ and $\mathbb{Z}_{+}=\mathbb{N} \cup\{0\}$. For any $a, b \in \mathbb{R}$, we denote $\min \{a, b\}, \max \{a, b\}$, and $\max \{a, 0\}$ by $a \wedge b, a \vee b$, and $a_{+}$, respectively.

If $(\mathcal{X}, d, \mu)$ is a space of homogeneous type, we also introduce the volume functions $V_{\delta}(x)=\mu(B(x, \delta))$ and $V(x, y)=\mu(B(x, d(x, y)))$ for all $x, y \in X$ and $\delta>0$. By (1.2), it is easy to see that $V(x, y) \sim V(y, x)$; see also [44]. We will use this fact without further mentioning.

Throughout the whole paper, for $\epsilon \in(0,1]$ and $|s|<\epsilon$, we set

$$
p(s, \epsilon) \equiv \max \left\{\frac{n}{n+\epsilon}, \frac{n}{n+\epsilon+s}\right\} .
$$

\section{Approximations of the identity and spaces of test functions}

In this section, we will work on spaces of homogeneous type with the constant $C_{0}$ as in Definition 1.1, $(\mathcal{X}, \mu, d)$, where $\mu(x)$ can be finite or infinite. We first present some basic 
estimates which will be used throughout the whole paper. We then introduce the notion of an approximation of the identity. Following Coifman's idea in [64], we then construct an approximation of the identity with bounded support on $\mathcal{X}$. We also introduce spaces of test functions and establish the boundedness of singular integrals on these spaces, which are key tools of the whole theory.

\subsection{Approximations of the identity}

Throughout the whole paper, we denote by $M f$ the Hardy-Littlewood maximal function on $\chi$ for any $f \in L_{\text {loc }}^{1}(\mathcal{X})$. It is well known that $M$ is bounded on $L^{p}(\mathcal{X})$ with $p \in(1, \infty]$; see $[29,44]$. Some basic estimates used throughout the whole paper are stated in the following lemma, whose main part is included in [48, Lemma 2.1], and the remaining statements are obvious.

Lemma 2.1. Let $\delta>0, a>0, r>0$, and $\theta \in(0,1)$. Then the following hold.

(i) $\int_{d(x, y) \leq \delta}\left(d(x, y)^{a} / V(x, y)\right) d \mu(y) \leq C \delta^{a}$ and $\int_{d(x, y) \geq \delta}(1 / V(x, y))\left(\delta^{a} / d(x, y)^{a}\right) d \mu(y) \leq$ $C$ uniformly in $x \in \mathcal{X}$ and $\delta>0$.

(ii) $\int_{\mathcal{X}}\left(1 /\left(V_{\delta}(x)+V(x, y)\right)\right)\left(\delta^{a} /(\delta+d(x, y))^{a}\right) d(x, y)^{\eta} d \mu(y) \leq C \delta^{\eta}$ uniformly in $x \in \mathcal{X}$ and $\delta>0$, if $a>\eta \geq 0$.

(iii) If $x, x^{\prime}, x_{1} \in X$ satisfy $d\left(x, x^{\prime}\right) \leq \theta\left(r+d\left(x, x_{1}\right)\right)$, then $1 /\left(r+d\left(x^{\prime}, x_{1}\right)\right) \leq C(1 /(r+$ $\left.\left.d\left(x, x_{1}\right)\right)\right), 1 /\left(V_{r}\left(x_{1}\right)+V\left(x_{1}, x^{\prime}\right)\right) \leq C\left(1 /\left(V_{r}\left(x_{1}\right)+V\left(x_{1}, x\right)\right)\right)$, and

$$
\frac{1}{V_{r}\left(x^{\prime}\right)+V\left(x^{\prime}, x_{1}\right)} \leq C \frac{1}{V_{r}(x)+V\left(x, x_{1}\right)}
$$

uniformly in $r>0$ and $x, x^{\prime}, x_{1} \in \mathcal{X}$.

(iv) For all $f \in L_{\text {loc }}^{1}(x)$ and all $x \in \mathcal{X}, \int_{d(x, y)>\delta}(1 / V(x, y))\left(\delta^{a} / d(x, y)^{a}\right)|f(y)| d \mu(y) \leq$ $C M(f)(x)$ uniformly in $\delta>0, f \in L_{\mathrm{loc}}^{1}(x)$ and $x \in \mathcal{X}$.

(v) For any $\epsilon>0, \int_{d(y, x) \geq \delta}\left(1 /\left(V_{r}(x)+V(y, x)\right)\right)\left(1 /(r+d(y, x))^{\epsilon}\right) d \mu(y) \leq C\left(1 /(r+\delta)^{\epsilon}\right)$ uniformly in $x \in \mathcal{X}$ and $\delta, r>0$.

(vi) For any fixed $\alpha>0$, if $d(x, y) \leq \alpha r$, then $V_{r}(x) \sim V_{\alpha r}(x) \sim V_{\alpha r}(y) \sim V_{r}(y)$ uniformly in $x, y \in X$ and $r>0$.

(vii) For all $r>0$ and all $x, y \in \mathcal{X}, V_{r}(x)+V(x, y) \sim V_{r}(x)+V_{r}(y)+V(x, y) \sim V_{r}(y)+$ $V(x, y)$.

Motivated by the properties of the heat kernel defined in (1.17) in the case of CarnotCarathéodory spaces, we introduce the following notion of an approximation of the identity on $x$.

Definition 2.2. Let $\epsilon_{1} \in(0,1], \epsilon_{2}>0$, and $\epsilon_{3}>0$. A sequence $\left\{S_{k}\right\}_{k \in \mathbb{Z}}$ of bounded linear integral operators on $L^{2}(\mathcal{X})$ is said to be an approximation of the identity of order $\left(\epsilon_{1}, \epsilon_{2}, \epsilon_{3}\right)$ (for short, $\left(\epsilon_{1}, \epsilon_{2}, \epsilon_{3}\right)$-ATI) if there exists a constant $C_{3}>0$ such that for all $k \in \mathbb{Z}$ and all $x, x^{\prime}, y$, and $y^{\prime} \in \mathcal{X}, S_{k}(x, y)$, the integral kernel of $S_{k}$ is a measurable function from $\mathcal{X} \times \mathcal{X}$ into $\mathbb{C}$ satisfying

(i) $\left|S_{k}(x, y)\right| \leq C_{3}\left(1 /\left(V_{2^{-k}}(x)+V_{2^{-k}}(y)+V(x, y)\right)\right)\left(2^{-k \epsilon_{2}} /\left(2^{-k}+d(x, y)\right)^{\epsilon_{2}}\right) ;$ 
(ii) $\left|S_{k}(x, y)-S_{k}\left(x^{\prime}, y\right)\right| \leq C_{3}\left(d\left(x, x^{\prime}\right) /\left(2^{-k}+d(x, y)\right)\right)^{\epsilon_{1}}\left(1 /\left(V_{2^{-k}}(x)+V_{2^{-k}}(y)+V(x, y)\right)\right) \times$ $\left(2^{-k \epsilon_{2}} /\left(2^{-k}+d(x, y)\right)^{\epsilon_{2}}\right)$ for $d\left(x, x^{\prime}\right) \leq\left(2^{-k}+d(x, y)\right) / 2$;

(iii) property (ii) also holds with $x$ and $y$ interchanged;

(iv) $\left|\left[S_{k}(x, y)-S_{k}\left(x, y^{\prime}\right)\right]-\left[S_{k}\left(x^{\prime}, y\right)-S_{k}\left(x^{\prime}, y^{\prime}\right)\right]\right| \leq C_{3}\left(d\left(x, x^{\prime}\right) /\left(2^{-k}+d(x, y)\right)\right)^{\epsilon_{1}} \times$ $\left(d\left(y, y^{\prime}\right) /\left(2^{-k}+d(x, y)\right)\right)^{\epsilon_{1}}\left(1 /\left(V_{2^{-k}}(x)+V_{2^{-k}}(y)+V(x, y)\right)\right)\left(2^{-k \epsilon_{3}} /\left(2^{-k}+d(x, y)\right)^{\epsilon_{3}}\right)$ for $d\left(x, x^{\prime}\right) \leq\left(2^{-k}+d(x, y)\right) / 3$ and $d\left(y, y^{\prime}\right) \leq\left(2^{-k}+d(x, y)\right) / 3$;

(v) $\int_{x} S_{k}(x, y) d \mu(y)=1$;

(vi) $\int_{x} S_{k}(x, y) d \mu(x)=1$.

In case the ATI has bounded support, in the sense that $S_{k}(x, y)=0$ when $d(x, y) \gtrsim$ $2^{-k}$, then the conditions (i), (ii), (iii), and (iv) of Definition 2.2 simplify as follows (see Proposition 2.5).

Definition 2.3. Let $\epsilon_{1} \in(0,1]$. A sequence $\left\{S_{k}\right\}_{k \in \mathbb{Z}}$ of bounded linear integral operators on $L^{2}(X)$ is said to be an approximation of the identity of order $\epsilon_{1}$ with bounded support (for short, $\epsilon_{1}$-ATI with bounded support) if there exist constants $C_{4}, C_{5}>0$ such that for all $k \in \mathbb{Z}$ and all $x, x^{\prime}, y$, and $y^{\prime} \in \mathcal{X}, S_{k}(x, y)$, the integral kernel of $S_{k}$ is a measurable function from $x \times x$ into $\mathbb{C}$ satisfying (v) and (vi) of Definition 2.2 as above, and

(i) $S_{k}(x, y)=0$ if $d(x, y) \geq C_{5} 2^{-k}$ and $\left|S_{k}(x, y)\right| \leq C_{4}\left(1 /\left(V_{2^{-k}}(x)+V_{2^{-k}}(y)\right)\right)$;

(ii) $\left|S_{k}(x, y)-S_{k}\left(x^{\prime}, y\right)\right| \leq C_{4} 2^{k \epsilon_{1}} d\left(x, x^{\prime}\right)^{\epsilon_{1}}\left(1 /\left(V_{2^{-k}}(x)+V_{2^{-k}}(y)\right)\right)$ for $d\left(x, x^{\prime}\right) \leq$ $\max \left\{C_{5}, 1\right\} 2^{1-k}$;

(iii) property (ii) also holds with $x$ and $y$ interchanged;

(iv) $\left|\left[S_{k}(x, y)-S_{k}\left(x, y^{\prime}\right)\right]-\left[S_{k}\left(x^{\prime}, y\right)-S_{k}\left(x^{\prime}, y^{\prime}\right)\right]\right| \leq C_{4} 2^{2 k \epsilon_{1}} d\left(x, x^{\prime}\right)^{\epsilon_{1}} d\left(y, y^{\prime}\right)^{\epsilon_{1}} \times$ $\left(1 /\left(V_{2^{-k}}(x)+V_{2^{-k}}(y)\right)\right)$ for $d\left(x, x^{\prime}\right) \leq \max \left\{C_{5}, 1\right\} 2^{1-k}$ and $d\left(y, y^{\prime}\right) \leq \max \left\{C_{5}, 1\right\} 2^{1-k}$.

We call $\epsilon_{1}$ the regularity of $\left(\epsilon_{1}, \epsilon_{2}, \epsilon_{3}\right)$-ATI $\left\{S_{k}\right\}_{k \in \mathbb{Z}}$.

Remark 2.4. (i) Assume that $x$ is as in Case (a) with $n \geq 3$ or as in Case (b) in the introduction, and let $H(s, x, y)$ be the heat kernel defined in (1.17). Define $S_{k}(x, y)=$ $H\left(2^{-2 k}, x, y\right)$ for $k \in \mathbb{Z}$ and $x, y \in X$. Using [44, Proposition 2.3.1], we can verify that $\left\{S_{k}\right\}_{k \in \mathbb{Z}}$ is an $\left(\epsilon_{1}, \epsilon_{2}, \epsilon_{3}\right)$-ATI for any $\epsilon_{1} \in(0,1], \epsilon_{2}>0$, and $\epsilon_{3}>0$. Moreover, in this case, $S_{k}(x, y)=S_{k}(y, x)$.

(ii) If $\mathcal{X}$ is any (compact or noncompact) Ahlfors $n$-regular metric measure space or any Lie group with polynomial growth, then one can construct an $\epsilon_{1}$-ATI with bounded support for any $\epsilon_{1} \in(0,1]$ by following Coifman's idea in [64].

(iii) We remark that when we consider the existence of ATIs, the condition (iv) of Definition 2.2 is not essential, in the sense that if there exist $\left\{S_{k}\right\}_{k \in \mathbb{Z}}$ with $k \in \mathbb{Z}$ satisfying (i), (ii), (iii), (v), and (vi) of Definition 2.2, then $\left\{S_{k} \circ S_{k}\right\}_{k \in \mathbb{Z}}$ satisfy (i) through (vi) of Definition 2.2.

Proposition 2.5. Suppose $\left\{S_{k}\right\}_{k \in \mathbb{Z}}$ is a sequence of bounded linear integral operators on $L^{2}(X)$ such that $S_{k}(x, y)=0$ whenever $d(x, y) \geq C_{5} 2^{-k}$. Then $\left\{S_{k}\right\}_{k \in \mathbb{Z}}$ is an ATI if and only if it is an ATI with bounded support. 
Proof. Obviously, from the assumption that $S_{k}(x, y)=0$ whenever $d(x, y) \geq C_{5} 2^{-k}$, it easily follows that $S_{k}$ satisfies Definition 2.2(i) if and only if

$$
\left|S_{k}(x, y)\right| \lesssim \frac{1}{V_{2^{-k}}(x)+V_{2^{-k}}(y)}
$$

which appears in Definition 2.3(i).

We now establish the equivalence between Definitions 2.2(ii) and 2.3(ii), with the proof for the equivalence between (iii) of Definitions 2.2 and (iii) of Definition 2.3 being similar. Notice that $S_{k}(x, y)-S_{k}\left(x^{\prime}, y\right) \neq 0$ implies that $d(x, y)<C_{5} 2^{-k}$ or $d\left(x^{\prime}, y\right)<$ $C_{5} 2^{-k}$. Thus, $S_{k}(x, y)-S_{k}\left(x^{\prime}, y\right) \neq 0$ together with $d\left(x, x^{\prime}\right) \leq 2 \max \left\{C_{5}, 1\right\} 2^{-k}$ shows that $d(x, y)<3 \max \left\{C_{5}, 1\right\} 2^{-k}$, and hence, $2^{-k}+d(x, y) \sim 2^{-k}$ and $V_{2^{-k}}(x)+V_{2^{-k}}(y)+$ $V(x, y) \sim V_{2^{-k}}(x)+V_{2^{-k}}(y)$. From these estimates, it immediately follows that Definition 2.2(ii) implies Definition 2.3(ii), and conversely Definition 2.3(ii) implies that Definition 2.2(ii) holds whenever $d\left(x, x^{\prime}\right) \leq 2 \max \left\{C_{5}, 1\right\} 2^{-k}$. We still need to prove that Definition 2.3(ii) also implies that Definition 2.2(ii) holds when $2 \max \left\{C_{5}, 1\right\} 2^{-k}<d\left(x, x^{\prime}\right) \leq\left(2^{-k}+d(x, y)\right) / 2$. However, if $2 \max \left\{C_{5}, 1\right\} 2^{-k}<d\left(x, x^{\prime}\right) \leq\left(2^{-k}+d(x, y)\right) / 2$, we have $d(x, y)>3 \max \left\{C_{5}, 1\right\} 2^{-k}$ and $d\left(x^{\prime}, y\right) \geq d\left(x, x^{\prime}\right)-2^{-k}>C_{5} 2^{-k}$. Thus, in this case, $S_{k}(x, y)=0=S_{k}\left(x^{\prime}, y\right)$, and therefore, Definition 2.2(ii) automatically holds. This proves the equivalence between Definitions 2.2(ii) and 2.3(ii).

We now establish the equivalence between Definitions 2.2(iv) and 2.3(iv). Notice that $\left[S_{k}(x, y)-S_{k}\left(x, y^{\prime}\right)\right]-\left[S_{k}\left(x^{\prime}, y\right)-S_{k}\left(x^{\prime}, y^{\prime}\right)\right] \neq 0$ implies that $d(x, y)<C_{5} 2^{-k}$ or $d\left(x^{\prime}, y\right)<C_{5} 2^{-k}$ or $d\left(x, y^{\prime}\right)<C_{5} 2^{-k}$ or $d\left(x^{\prime}, y^{\prime}\right)<C_{5} 2^{-k}$. This together with $d\left(x, x^{\prime}\right) \leq$ $\max \left\{C_{5}, 1\right\} 2^{1-k}$ and $d\left(y, y^{\prime}\right) \leq \max \left\{C_{5}, 1\right\} 2^{1-k}$ further shows that we have $d(x, y)<$ $5 \max \left\{C_{5}, 1\right\} 2^{1-k}$, and hence, $2^{-k}+d(x, y) \sim 2^{-k}$ and $V_{2^{-k}}(x)+V_{2^{-k}}(y)+V(x, y) \sim V_{2^{-k}}(x)+$ $V_{2^{-k}}(y)$. From these estimates, we see that Definition 2.2(iv) implies Definition 2.3(iv), and conversely, Definition 2.3(iv) also implies that Definition 2.2(iv) holds when $d\left(x, x^{\prime}\right) \leq$ $2 \max \left\{C_{5}, 1\right\} 2^{-k}$ and $d\left(y, y^{\prime}\right) \leq \max \left\{C_{5}, 1\right\} 2^{1-k}$. We still need to prove that Definition 2.3(iv) also implies that Definition 2.2(iv) holds in the three cases listed below. Recall that we always assume that $d\left(x, x^{\prime}\right) \leq\left(2^{-k}+d(x, y)\right) / 3$ and $d\left(y, y^{\prime}\right) \leq\left(2^{-k}+d(x, y)\right) / 3$ in Definition 2.2(iv).

Case 1. $d\left(x, x^{\prime}\right) \leq 2 \max \left\{C_{5}, 1\right\} 2^{-k}$ and $d\left(y, y^{\prime}\right)>2 \max \left\{C_{5}, 1\right\} 2^{-k}$. In this case, $d(x, y) \geq$ $3 d\left(y, y^{\prime}\right)-2^{-k} \geq C_{5} 2^{-k}, d\left(x, y^{\prime}\right) \geq 2 d\left(y, y^{\prime}\right)-2^{-k}>C_{5} 2^{-k}, d\left(x^{\prime}, y\right)>2 d\left(y, y^{\prime}\right)-2^{-k}>C_{5} 2^{-k}$, and $d\left(x^{\prime}, y^{\prime}\right)>d\left(y, y^{\prime}\right)-2^{-k}>C_{5} 2^{-k}$. Thus $S_{k}(x, y)=S_{k}\left(x, y^{\prime}\right)=S_{k}\left(x^{\prime}, y\right)=S_{k}\left(x^{\prime}, y^{\prime}\right)=0$ and therefore Definition 2.2(iv) automatically holds in this case.

Case 2. $d\left(x, x^{\prime}\right)>2 \max \left\{C_{5}, 1\right\} 2^{-k}$ and $d\left(y, y^{\prime}\right) \leq 2 \max \left\{C_{5}, 1\right\} 2^{-k}$. This case is similar to Case 1 by symmetry.

Case 3. $d\left(x, x^{\prime}\right)>2 \max \left\{C_{5}, 1\right\} 2^{-k}$ and $d\left(y, y^{\prime}\right)>2 \max \left\{C_{5}, 1\right\} 2^{-k}$. In this case, similar to Case 1 , we have that $d(x, y) \geq 3 d\left(y, y^{\prime}\right)-2^{-k}>C_{5} 2^{-k}, d\left(x, y^{\prime}\right) \geq 2 d\left(y, y^{\prime}\right)-2^{-k}>C_{5} 2^{-k}$, $d\left(x^{\prime}, y\right) \geq 2 d\left(x, x^{\prime}\right)-2^{-k}>C_{5} 2^{-k}$, and $d\left(x^{\prime}, y^{\prime}\right) \geq d(x, y)-d\left(x, x^{\prime}\right)-d\left(y, y^{\prime}\right) \geq(d(x, y)-$ $\left.2^{1-k}\right) / 3>d\left(y, y^{\prime}\right)-2^{-k}>C_{5} 2^{-k}$. We also have $S_{k}(x, y)=S_{k}\left(x, y^{\prime}\right)=S_{k}\left(x^{\prime}, y\right)=S_{k}\left(x^{\prime}, y^{\prime}\right)=0$ and therefore (iv) automatically holds in this case. This establishes the equivalence between (iv) and (iv)', and hence completes the proof of Proposition 2.5. 
Following Coifman's idea in [64], for any $\epsilon_{1} \in(0,1]$, we can construct $\epsilon_{1}$-ATIs with bounded support on $\mathcal{X}$ (cf. also [65, Theorem (1.13)]).

Theorem 2.6. Let $(\mathcal{X}, d, \mu)$ be a space of homogeneous type as in Definition 1.1 and $\epsilon_{1} \in(0,1]$. Then there exists an approximation of the identity $\left\{S_{k}\right\}_{k \in \mathbb{Z}}$ of order $\epsilon_{1}$ with bounded support on $\mathcal{X}$, with constant $C_{5}=4$. Moreover, for all $k \in \mathbb{Z}$ and $x, y \in \mathcal{X}, S_{k}(x, y)=S_{k}(y, x)$.

Proof. Obviously, we only need to prove the theorem for $\epsilon_{1}=1$. Let $h \in C^{1}(\mathbb{R}), h(t)=1$ if $t \in[0,1], h(t)=0$ if $t \geq 2$, and $0 \leq h(t) \leq 1$ for all $t \in \mathbb{R}$. For any $k \in \mathbb{Z}, f \in L_{\text {loc }}^{1}(\mathcal{X})$ and $u \in \mathcal{X}$, we then define

$$
T_{k} f(u)=\int_{x} h\left(2^{k} d(u, w)\right) f(w) d \mu(w) .
$$

Obviously, we have $V_{2^{-k}}(u) \leq T_{k} 1(u) \leq V_{2^{1-k}}(u)$. Fix any $x \in \mathcal{X}$. By Lemma 2.1(vi), for all $u \in B\left(x, 2^{5-k}\right)$, we further have

$$
T_{k} 1(u) \sim V_{2^{-k}}(x)
$$

Thus, if $z \in B\left(x, 2^{4-k}\right)$ and $h\left(2^{k} d(z, u)\right) \neq 0$, then $u \in B\left(x, 2^{5-k}\right)$, and by (2.4), we further have

$$
T_{k}\left(\frac{1}{T_{k} 1}\right)(z)=\int_{x} h\left(2^{k} d(z, u)\right) \frac{1}{T_{k} 1(u)} d \mu(u) \sim 1
$$

For all $x, y \in \mathcal{X}$, we define

$$
S_{k}(x, y)=\frac{1}{T_{k} 1(x)}\left\{\int_{x} h\left(2^{k} d(x, z)\right) \frac{1}{T_{k}\left(1 / T_{k} 1\right)(z)} h\left(2^{k} d(z, y)\right) d \mu(z)\right\} \frac{1}{T_{k} 1(y)}
$$

We now prove that $\left\{S_{k}\right\}_{k \in \mathbb{Z}}$ is a 1-ATI with bounded support and $C_{5}=4$. It is obvious that for all $x, y \in \mathcal{X}, S_{k}(x, y)=S_{k}(y, x)$, and that if $d(x, y) \geq 2^{2-k}$, then $S_{k}(x, y)=0$. Also, it is easy to show that $\int_{x} S_{k}(x, y) d \mu(y)=1$. Moreover, if $d(x, y)<2^{2-k}$, by (2.4) and (2.5) together with Lemma 2.1(vi), we obtain

$$
0 \leq S_{k}(x, y) \lesssim \frac{1}{V_{2^{-k}}(x)} \sim \frac{1}{V_{2^{-k}}(x)+V_{2^{-k}}(y)}
$$

Thus $S_{k}(x, y)$ satisfies (i)' of Definition 2.2 with $C_{5}=4$.

We now show that $S_{k}(x, y)$ has the desired regularity in the first variable when $d\left(x, x^{\prime}\right) \leq 2^{3-k}$. Notice that in this case, $S_{k}(x, y)-S_{k}\left(x^{\prime}, y\right) \neq 0$ implies that $d(x, y)<2^{4-k}$, and hence,

$$
\frac{1}{V_{2^{-k}}(x)+V_{2^{-k}}(y)} \sim \frac{1}{V_{2^{-k}}(x)}
$$


By (2.6), we have

$$
\begin{aligned}
& S_{k}(x, y)-S_{k}\left(x^{\prime}, y\right) \\
& =\left[\frac{1}{T_{k} 1(x)}-\frac{1}{T_{k} 1\left(x^{\prime}\right)}\right]\left\{\int_{x} h\left(2^{k} d(x, z)\right) \frac{1}{T_{k}\left(1 / T_{k} 1\right)(z)} h\left(2^{k} d(z, y)\right) d \mu(z)\right\} \frac{1}{T_{k} 1(y)} \\
& \quad+\frac{1}{T_{k} 1\left(x^{\prime}\right)}\left\{\int_{x}\left[h\left(2^{k} d(x, z)\right)-h\left(2^{k} d\left(x^{\prime}, z\right)\right)\right] \frac{1}{T_{k}\left(1 / T_{k} 1\right)(z)} h\left(2^{k} d(z, y)\right) d \mu(z)\right\} \frac{1}{T_{k} 1(y)} \\
& \equiv Z_{1}+Z_{2} .
\end{aligned}
$$

To estimate $Z_{1}$, by the choice of $h$, the mean value theorem, and (2.4) together with Lemma 2.1(vi), we first have

$$
\left|\frac{1}{T_{k} 1(x)}-\frac{1}{T_{k} 1\left(x^{\prime}\right)}\right| \lesssim \frac{1}{\left[V_{2^{-k}}(x)\right]^{2}} 2^{k} d\left(x, x^{\prime}\right) \mu\left(B\left(x, 2^{4-k}\right)\right) \sim \frac{2^{k} d\left(x, x^{\prime}\right)}{V_{2^{-k}}(x)}
$$

which together with (2.4), (2.5), and (2.8) further yields

$$
\left|Z_{1}\right| \lesssim \frac{2^{k} d\left(x, x^{\prime}\right)}{V_{2^{-k}}(x)} \sim \frac{2^{k} d\left(x, x^{\prime}\right)}{V_{2^{-k}}(x)+V_{2^{-k}}(y)}
$$

To estimate $Z_{2}$, notice that $h\left(2^{k} d(x, z)\right)-h\left(2^{k} d\left(x^{\prime}, z\right)\right) \neq 0$ implies $d(x, z)<2^{4-k}$. By this observation, (2.5), the mean value theorem together with Lemma 2.1(vi), we obtain

$$
\begin{aligned}
\mid \int_{x} & {\left[h\left(2^{k} d(x, z)\right)-h\left(2^{k} d\left(x^{\prime}, z\right)\right)\right] \frac{1}{T_{k}\left(1 / T_{k} 1\right)(z)} h\left(2^{k} d(z, y)\right) d \mu(z) \mid } \\
& \lesssim 2^{k} d\left(x, x^{\prime}\right) \mu\left(B\left(x, 2^{4-k}\right)\right) \\
& \lesssim 2^{k} d\left(x, x^{\prime}\right) V_{2^{-k}}(x)
\end{aligned}
$$

which together with (2.4) and (2.8) yields

$$
\left|Z_{2}\right| \lesssim \frac{2^{k} d\left(x, x^{\prime}\right)}{V_{2^{-k}}(x)} \sim \frac{2^{k} d\left(x, x^{\prime}\right)}{V_{2^{-k}}(x)+V_{2^{-k}}(y)}
$$

In combination with the estimate for $Z_{1}$, this shows that $S_{k}(x, y)$ has the desired regularity with respect to the first variable when $d\left(x, x^{\prime}\right) \leq 2^{3-k}$. 
We finally prove that $S_{k}(x, y)$ satisfies the desired second difference condition, when $d\left(x, x^{\prime}\right) \leq 2^{3-k}$ and $d\left(y, y^{\prime}\right) \leq 2^{3-k}$. Notice that in this case, $\left[S_{k}(x, y)-S_{k}\left(x^{\prime}, y\right)\right]-\left[S_{k}\left(x, y^{\prime}\right)-\right.$ $\left.S_{k}\left(x^{\prime}, y^{\prime}\right)\right] \neq 0$ implies that $d(x, y)<2^{5-k}$. Thus, (2.8) also holds in this case. By (2.6), we have

$$
\begin{aligned}
& {\left[S_{k}(x, y)-S_{k}\left(x^{\prime}, y\right)\right]-\left[S_{k}\left(x, y^{\prime}\right)-S_{k}\left(x^{\prime}, y^{\prime}\right)\right]} \\
& =\left[\frac{1}{T_{k} 1(x)}-\frac{1}{T_{k} 1\left(x^{\prime}\right)}\right]\left\{\int_{x} h\left(2^{k} d(x, z)\right) \frac{1}{T_{k}\left(1 / T_{k} 1\right)(z)} h\left(2^{k} d(z, y)\right) d \mu(z)\right\} \\
& \quad \times\left[\frac{1}{T_{k} 1(y)}-\frac{1}{T_{k} 1\left(y^{\prime}\right)}\right]+\left[\frac{1}{T_{k} 1(x)}-\frac{1}{T_{k} 1\left(x^{\prime}\right)}\right] \frac{1}{T_{k} 1\left(y^{\prime}\right)} \\
& \quad \times\left\{\int_{x} h\left(2^{k} d(x, z)\right) \frac{1}{T_{k}\left(1 / T_{k} 1\right)(z)}\left[h\left(2^{k} d(z, y)\right)-h\left(2^{k} d\left(z, y^{\prime}\right)\right)\right] d \mu(z)\right\} \\
& \quad+\frac{1}{T_{k} 1\left(x^{\prime}\right)}\left\{\int_{x}\left[h\left(2^{k} d(x, z)\right)-h\left(2^{k} d\left(x^{\prime}, z\right)\right)\right]\right. \\
& \quad+\frac{1}{T_{k} 1\left(x^{\prime}\right)} \frac{1}{T_{k} 1\left(y^{\prime}\right)}\left\{\int_{x}\left[h\left(2^{k} d(x, z)\right)-h\left(2^{k} d\left(x^{\prime}, z\right)\right)\right]\right. \\
& \equiv
\end{aligned}
$$

The estimates (2.10), (2.5), and (2.8) together with Lemma 2.1(vi) show that

$$
\left|Z_{3}\right| \lesssim \frac{2^{k} d\left(x, x^{\prime}\right)}{V_{2^{-k}}(x)} \mu\left(B\left(x, 2^{3-k}\right)\right) \frac{2^{k} d\left(y, y^{\prime}\right)}{V_{2^{-k}}(x)} \sim 2^{2 k} d\left(x, x^{\prime}\right) d\left(y, y^{\prime}\right) \frac{1}{V_{2^{-k}}(x)+V_{2^{-k}}(y)}
$$

The estimate for $Z_{4}$ is similar to the one for $Z_{5}$, while the estimate for $Z_{5}$ can be deduced immediately from the estimates (2.12), (2.10), and (2.4) together with (2.8). Finally, to estimate $Z_{6}$, notice that $h\left(2^{k} d(x, z)\right)-h\left(2^{k} d\left(x^{\prime}, z\right)\right) \neq 0$ and $d\left(x, x^{\prime}\right) \leq 2^{3-k}$ implies that $z \in$ $B\left(x, 2^{4-k}\right)$. This observation together with the mean value theorem, (2.5), and Lemma 2.1(vi) yields

$$
\begin{aligned}
& \left|\int_{x}\left[h\left(2^{k} d(x, z)\right)-h\left(2^{k} d\left(x^{\prime}, z\right)\right)\right] \frac{1}{T_{k}\left(1 / T_{k} 1\right)(z)}\left[h\left(2^{k} d(z, y)\right)-h\left(2^{k} d\left(z, y^{\prime}\right)\right)\right] d \mu(z)\right| \\
& \quad \lesssim 2^{2 k} d\left(x, x^{\prime}\right) d\left(y, y^{\prime}\right) V_{2^{-k}}(x)
\end{aligned}
$$


which together with (2.4) and (2.8) further gives

$$
\left|Z_{6}\right| \lesssim 2^{2 k} d\left(x, x^{\prime}\right) d\left(y, y^{\prime}\right) \frac{1}{V_{2^{-k}}(x)} \sim 2^{2 k} d\left(x, x^{\prime}\right) d\left(y, y^{\prime}\right) \frac{1}{V_{2^{-k}}(x)+V_{2^{-k}}(y)}
$$

This proves that $S_{k}(x, y)$ has the desired second difference property, and hence, completes the proof of Theorem 2.6.

In the sequel, for any $f \in L^{p}(\mathcal{X})$ with $p \in[1, \infty]$ and $x \in \mathcal{X}$, we set

$$
S_{k} f(x)=\int_{x} S_{k}(x, y) f(y) d \mu(y) .
$$

We also let

$$
L_{b}^{\infty}(\mathcal{X})=\left\{f \in L^{\infty}(\mathcal{X}): f \text { has bounded support }\right\}
$$

Some basic properties of ATIs are presented in the next proposition.

Proposition 2.7. Let $\left\{S_{k}\right\}_{k \in \mathbb{Z}}$ be an $\left(\epsilon_{1}, \epsilon_{2}, \epsilon_{3}\right)$-ATI with $\epsilon_{1} \in(0,1], \epsilon_{2}>0$ and $\epsilon_{3}>0$, and let $S_{k}^{t}$ denote the adjoint operator to $S_{k}$, whose integral kernel is given by $S_{k}^{t}(x, y)=S_{k}(y, x)$. Then the following hold.

(i) For any $k \in \mathbb{Z}$ and any $x, y \in X, \int_{x}\left|S_{k}(x, y)\right| d \mu(y) \leq C$ and $\int_{x}\left|S_{k}(x, y)\right| d \mu(x) \leq C$.

(ii) For any $k \in \mathbb{Z}$ and any $f \in L_{\mathrm{loc}}^{1}(x),\left|S_{k} f(x)\right| \leq C M f(x)$.

(iii) For $1 \leq p \leq \infty$, there exists a constant $C_{p}>0$ such that for all $f \in L^{p}(\mathcal{X})$,

$$
\left\|S_{k} f\right\|_{L^{p}(x)} \leq C_{p}\|f\|_{L^{p}(x)} .
$$

(iv) For $1 \leq p<\infty$ and any $f \in L^{p}(\mathcal{X}),\left\|S_{k} f-f\right\|_{L^{p}(\mathcal{X})} \rightarrow 0$ when $k \rightarrow \infty$.

(v) Properties (ii) through (iv) also hold for $S_{k}$ replaced by $S_{k}^{t}$.

Proof. Definition 2.2(i) together with Lemma 2.1(i) shows that for any $k \in \mathbb{Z}$ and any $x \in \mathcal{X}$,

$$
\begin{aligned}
\int_{x}\left|S_{k}(x, y)\right| d \mu(y) & =\int_{d(x, y) \leq 2^{-k}}\left|S_{k}(x, y)\right| d \mu(y)+\int_{d(x, y)>2^{-k}}\left|S_{k}(x, y)\right| d \mu(y) \\
& \lesssim 1+\int_{d(x, y)>2^{-k}} \frac{1}{V(x, y)} \frac{2^{-k \epsilon_{2}}}{d(x, y)^{\epsilon_{2}}} d \mu(y) \\
& \lesssim 1
\end{aligned}
$$

A similar argument proves that $\int_{x}\left|S_{k}(x, y)\right| d \mu(x) \lesssim 1$. 
To prove (ii), by Definition 2.2(i) together with Lemma 2.1(iv), we have

$$
\begin{aligned}
\left|S_{k} f(x)\right| & \lesssim \frac{1}{V_{2^{-k}(x)}} \int_{d(x, y) \leq 2^{-k}}|f(y)| d \mu(y)+\int_{d(x, y)>2^{-k}} \frac{1}{V(x, y)} \frac{2^{-k \epsilon_{2}}}{d(x, y)^{\epsilon_{2}}}|f(y)| d \mu(y) \\
& \lesssim M f(x) .
\end{aligned}
$$

From (ii) and the $L^{p}(\mathcal{X})$-boundedness of $M$, we obtain (iii) for $p \in(1, \infty]$. For $p=1$, we obtain (iii) from (i), by Fubini's theorem.

To prove (iv), we first recall that Lebesgue's differentiation theorem holds in $x$, that is, if $f$ is locally integrable, then for almost all $x \in \mathcal{X}, x$ is a Lebesgue point which means

$$
\lim _{r \rightarrow 0} \frac{1}{\mu(B(x, r))} \int_{B(x, r)}|f(y)-f(x)| d \mu(y)=0,
$$

since the measure $\mu$ is regular; see [13, page 4] and [66, page 11].

For any given $f \in L^{p}(\mathcal{X})$ with $p \in[1, \infty]$, assume that $x_{0}$ is a Lebesgue point of $f$, then $M f\left(x_{0}\right)+\left|f\left(x_{0}\right)\right|<\infty$. By the conditions (v) and (i) of $S_{k}$ in Definition 2.2, we have

$$
\begin{aligned}
&\left|S_{k} f\left(x_{0}\right)-f\left(x_{0}\right)\right| \\
&=\left|\int_{x} S_{k}\left(x_{0}, y\right)\left[f(y)-f\left(x_{0}\right)\right] d \mu(y)\right| \\
& \leq C_{3} \int_{d\left(x_{0}, y\right)<2^{-k}} \frac{1}{V_{2^{-k}}\left(x_{0}\right)+V_{2^{-k}}(y)+V\left(x_{0}, y\right)} \frac{2^{-k \epsilon_{2}}}{\left(2^{-k}+d\left(x_{0}, y\right)\right)^{\epsilon_{2}}}\left|f(y)-f\left(x_{0}\right)\right| d \mu(y) \\
& \quad+C_{3} \sum_{l=0}^{\infty} \int_{2^{l-1} 2^{-k} \leq d\left(x_{0}, y\right)<2^{l} 2^{-k}} \ldots \\
& \leq C C_{3} \sum_{l=0}^{\infty} \frac{1}{2^{l \epsilon_{2}}} \frac{1}{V_{2^{l} 2^{-k}\left(x_{0}\right)}} \int_{d\left(x_{0}, y\right)<2^{l} 2^{-k}}\left|f(y)-f\left(x_{0}\right)\right| d \mu(y) .
\end{aligned}
$$

For any $\delta>0$, we choose $L_{0} \in \mathbb{N}$ so that

$$
C C_{3}\left[M f\left(x_{0}\right)+\left|f\left(x_{0}\right)\right|\right] \sum_{l=L_{0}+1}^{\infty} \frac{1}{2^{l \epsilon_{2}}}<\frac{\delta}{2} .
$$

Since $x_{0}$ is a Lebesgue point, by the definition, we know that there exists $K \in \mathbb{N}$ such that when $k>K$,

$$
C C_{3} \sum_{l=0}^{L_{0}} \frac{1}{2^{l \epsilon_{2}}} \frac{1}{V_{2^{l} 2^{2-k}}\left(x_{0}\right)} \int_{d\left(x_{0}, y\right)<2^{l} 2^{-k}}\left|f(y)-f\left(x_{0}\right)\right| d \mu(y)<\frac{\delta}{2}
$$


Thus $\lim _{k \rightarrow \infty} S_{k} f\left(x_{0}\right)=f\left(x_{0}\right)$, and therefore for almost everywhere $x \in \mathcal{X}$,

$$
\lim _{k \rightarrow \infty} S_{k} f(x)=f(x)
$$

This fact together with $\left|S_{k} f(x)\right| \lesssim M f(x)$ (see (ii) of this proposition), the $L^{p}(\mathcal{X})$ boundedness of $M$ with $p \in(1, \infty)$ again and Lebesgue's dominated convergence theorem, gives that $\lim _{k \rightarrow \infty}\left\|S_{k} f-f\right\|_{L^{p}(\mathcal{X})}=0$ for all $f \in L^{p}(\mathcal{X})$.

When $p=1$, we first consider $f \in L_{b}^{\infty}(\mathcal{X})$. Assume that supp $f \subset B\left(y_{0}, r_{0}\right)$ for some $y_{0} \in \mathcal{X}$ and $r_{0}>0$. For some fixed $L>2 r_{0}$, by Hölder's inequality and the conditions (i) and (v) of $S_{k}$ in Definition 2.2 together with Lemma 2.1(i), we have

$$
\begin{aligned}
\left\|S_{k} f-f\right\|_{L^{1}(x)}= & \int_{d\left(x, y_{0}\right)<L}\left|S_{k} f(x)-f(x)\right| d \mu(x)+\int_{d\left(x, y_{0}\right) \geq L}\left|S_{k} f(x)-f(x)\right| d \mu(x) \\
\leq & \mu\left(B_{L}\left(y_{0}\right)\right)^{1 / 2}\left\|S_{k} f-f\right\|_{L^{2}(x)} \\
& +C_{3} \int_{d\left(y, y_{0}\right)<r_{0}}|f(y)|\left\{\int_{d(x, y) \geq L / 2} \frac{1}{V(x, y)} \frac{2^{-k \epsilon_{2}}}{d(x, y)^{\epsilon_{2}}} d \mu(x)\right\} d \mu(y) \\
\leq & \mu\left(B_{L}\left(y_{0}\right)\right)^{1 / 2}\left\|S_{k} f-f\right\|_{L^{2}(x)}+C C_{3} 2^{-k \epsilon_{2}} L^{-\epsilon_{2}}\|f\|_{L^{1}(x)^{\prime}}
\end{aligned}
$$

which together with $L_{b}^{\infty}(\mathcal{X}) \subset L^{2}(\mathcal{X})$ and the above proved conclusion for $p \in(1, \infty)$ implies that $\lim _{k \rightarrow \infty}\left\|S_{k} f-f\right\|_{L^{1}(x)}=0$ for all $f \in L_{b}^{\infty}(\mathcal{X})$. This and the density of $L_{b}^{\infty}(\mathcal{X})$ in $L^{1}(x)$ further yield that $\lim _{k \rightarrow \infty}\left\|S_{k} f-f\right\|_{L^{1}(\mathcal{x})}=0$ for all $f \in L^{1}(\mathcal{X})$, which verify (iv).

Property (v) can be deduced from (i) through (iv) together with the symmetry, which completes the proof of Proposition 2.7.

We now introduce the space of test functions on $x$.

Definition 2.8. Let $x_{1} \in \mathcal{X}, r>0,0<\beta \leq 1$, and $\gamma>0$. A function $f$ on $\mathcal{X}$ is said to be a test function of type $\left(x_{1}, r, \beta, \gamma\right)$ if there exists a constant $C \geq 0$ such that

(i) $|f(x)| \leq C\left(1 /\left(V_{r}\left(x_{1}\right)+V\left(x_{1}, x\right)\right)\right)\left(r /\left(r+d\left(x_{1}, x\right)\right)\right)^{\gamma}$ for all $x \in \mathcal{X}$;

(ii) $|f(x)-f(y)| \leq C\left(d(x, y) /\left(r+d\left(x_{1}, x\right)\right)\right)^{\beta}\left(1 /\left(V_{r}\left(x_{1}\right)+V\left(x_{1}, x\right)\right)\right)\left(r /\left(r+d\left(x_{1}, x\right)\right)\right)^{r}$ for all $x, y \in X$ satisfying that $d(x, y) \leq\left(r+d\left(x_{1}, x\right)\right) / 2$.

Moreover, we denote by $\mathcal{G}\left(x_{1}, r, \beta, \gamma\right)$ the set of all test functions of type $\left(x_{1}, r, \beta, \gamma\right)$, and if $f \in \mathcal{G}\left(x_{1}, r, \beta, \gamma\right)$, we define its norm by

$$
\|f\|_{\mathcal{G}\left(x_{1}, r, \beta, r\right)}=\inf \{C:(\text { i) and (ii) hold }\} .
$$

The space $\mathcal{G}\left(x_{1}, r, \beta, \gamma\right)$ is called the space of test functions.

This definition of a test function of type $\left(x_{1}, r, \beta, \gamma\right)$ gives a quantified meaning to the notation of a sufficiently "smooth" function which is essentially supported in the ball of radius $r$ centered at $x_{1}$ in the sense that it decays of sufficiently high order (measured by $\gamma$ ) at infinity, and is Hölder continuous of order $\beta$ (at the right scale $r$ ). 
Obviously, $S_{k}(x, \cdot)$ for any fixed $k \in \mathbb{Z}$ and $x \in \mathcal{X}$ in Definition 2.2 is a test function of type $\left(x, 2^{-k}, \epsilon_{1}, \epsilon_{2}\right)$, and $S_{k}(\cdot, y)$ for any fixed $k \in \mathbb{Z}$ and $y \in \mathcal{X}$ in Definition 2.2 is a test function of type $\left(y, 2^{-k}, \epsilon_{1}, \epsilon_{2}\right)$. Moreover, for $\eta \in(0,1]$, let

$$
\|f\|_{\dot{C}^{\eta}(\mathcal{X})}=\sup _{\substack{x, y \in \mathcal{X} \\ x \neq y}} \frac{|f(x)-f(y)|}{d(x, y)^{\eta}}
$$

and define the homogeneous Hölder space

$$
\dot{C}^{\eta}(x)=\left\{f \in C(x):\|f\|_{\dot{C}^{\eta}(x)}<\infty\right\}
$$

we also consider the inhomogeneous Hölder space

$$
\begin{gathered}
C^{\eta}(x)=\left\{f \in C(x):\|f\|_{C^{\eta}(x)}<\infty\right\}, \\
C_{b}^{\eta}(x)=\left\{f \in C^{\eta}(x): f \text { has bounded support }\right\},
\end{gathered}
$$

where $\|f\|_{C^{\eta}(\mathcal{X})}=\|f\|_{L^{\infty}(\mathcal{X})}+\|f\|_{\dot{C}^{\eta}(\mathcal{X})}$. Then any $f \in C_{b}^{\eta}(\mathcal{X})$ is also a test function of type $\left(x_{1}, r, \eta, \gamma\right)$ for any $x_{1} \in \mathcal{X}, r>0$, and $\gamma>0$.

Now fix $x_{1} \in \mathcal{X}$ and let $\mathcal{G}(\beta, \gamma)=\mathcal{G}\left(x_{1}, 1, \beta, \gamma\right)$. It is easy to see that

$$
\mathcal{G}\left(x_{0}, r, \beta, \gamma\right)=\mathcal{G}(\beta, \gamma)
$$

with the equivalent norms for all $x_{0} \in \mathcal{X}$ and $r>0$. Furthermore, it is easy to check that $\mathcal{G}(\beta, \gamma)$ is a Banach space with respect to the norm in $\mathcal{G}(\beta, \gamma)$.

It is well known that even when $\mathcal{X}=\mathbb{R}^{n}, \mathcal{G}\left(\beta_{1}, \gamma\right)$ is not dense in $\mathcal{G}\left(\beta_{2}, \gamma\right)$ if $\beta_{1}>\beta_{2}$, which will bring us some inconvenience. To overcome this defect, in what follows, for given $\epsilon \in(0,1]$, let $\mathcal{G}_{0}^{\epsilon}(\beta, \gamma)$ be the completion of the space $\mathcal{G}(\epsilon, \epsilon)$ in $\mathcal{G}(\beta, \gamma)$ when $0<\beta, \gamma \leq \epsilon$. Obviously, $\mathcal{G}_{0}^{\epsilon}(\epsilon, \epsilon)=\mathcal{G}(\epsilon, \epsilon)$. Moreover, $f \in \mathcal{G}_{0}^{\epsilon}(\beta, \gamma)$ if and only if $f \in \mathcal{G}(\beta, \gamma)$ and there exist $\left\{f_{n}\right\}_{n \in \mathbb{N}} \subset \mathcal{G}(\epsilon, \epsilon)$ such that $\left\|f-f_{n}\right\|_{\mathcal{G}(\beta, \gamma)} \rightarrow 0$ as $n \rightarrow \infty$. If $f \in \mathcal{G}_{0}^{\epsilon}(\beta, \gamma)$, we then define $\|f\|_{\mathcal{G}_{0}^{e}(\beta, \gamma)}=\|f\|_{\mathcal{G}(\beta, \gamma)}$. Then, obviously, $\mathcal{G}_{0}^{\epsilon}(\beta, \gamma)$ is a Banach space and we also have $\|f\|_{\mathcal{G}_{0}^{e}(\beta, \gamma)}=\lim _{n \rightarrow \infty}\left\|f_{n}\right\|_{\mathcal{G}_{(\beta, \gamma)}}$ for the above chosen $\left\{f_{n}\right\}_{n \in \mathbb{N}}$.

We define the dual space $\left(\mathcal{G}_{0}^{\epsilon}(\beta, \gamma)\right)^{\prime}$ to be the set of all linear functionals $\mathcal{L}$ from $\mathcal{G}_{0}^{\epsilon}(\beta, \gamma)$ to $\mathbb{C}$ with the property that there exists $C \geq 0$ such that for all $f \in \mathcal{G}_{0}^{\epsilon}(\beta, \gamma)$,

$$
|\mathcal{L}(f)| \leq C\|f\|_{\mathcal{G}_{0}^{e}(\beta, \gamma)}
$$

We denote by $\langle h, f\rangle$ the natural pairing of elements $h \in\left(\mathcal{G}_{0}^{\epsilon}(\beta, \gamma)\right)^{\prime}$ and $f \in \mathcal{G}_{0}^{\epsilon}(\beta, \gamma)$. Clearly, for all $h \in\left(\mathcal{G}_{0}^{\epsilon}(\beta, \gamma)\right)^{\prime},\langle h, f\rangle$ is well defined for all $f \in \mathcal{G}_{0}^{\epsilon}\left(x_{1}, r, \beta, \gamma\right)$ with $x_{1} \in \mathcal{X}$ and $r>0$. 
In the sequel, we define

$$
\mathcal{G}\left(x_{1}, r, \beta, \gamma\right)=\left\{f \in \mathcal{G}\left(x_{1}, r, \beta, \gamma\right): \int_{x} f(x) d \mu(x)=0\right\}
$$

which is called the space of test functions with mean zero. The space $\mathcal{G}_{0}^{\epsilon}(\beta, \gamma)$ is defined to be the completion of the space $\mathcal{G}_{\mathcal{G}}(\epsilon, \epsilon)$ in $\mathcal{G}(\beta, \gamma)$ when $0<\beta, \gamma<\epsilon$. Moreover, if $f \in \mathcal{\mathcal { G }}_{0}^{\epsilon}(\beta, \gamma)$, we then define $\|f\|_{\mathcal{G}_{0}^{e}(\beta, \gamma)}=\|f\|_{\mathcal{G}(\beta, \gamma)}$.

By essentially following a procedure similar to that of [44, Lemma 3.5.1], we can establish some kind of decomposition for test functions with mean zero.

Proposition 2.9. Let $x_{1} \in \mathcal{X}, r>0,0<\beta \leq 1$, and $\gamma>0$, and let $f \in \mathcal{G}\left(x_{1}, r, \beta, \gamma\right)$. Then for any $\tilde{\gamma} \in(0, \gamma]$ and all $x \in \mathcal{X}$,

$$
f(x)=\sum_{l=0}^{\infty} 2^{-l \tilde{r}} \varphi_{l}(x)
$$

where $\varphi_{l}$ is an adjusted bump function associated with the ball $B\left(x_{1}, 2^{l} r\right)$, which means that there exists a constant $C>0$ independent of $r$ and $l$ such that

(i) $\operatorname{supp} \varphi_{l} \subset B\left(x_{1}, 2^{l} r\right)$;

(ii) $\left|\varphi_{l}(x)\right| \leq C\left(1 / V_{2^{l} r}\left(x_{1}\right)\right)$ for all $x \in \mathcal{X}$;

(iii) $\left\|\varphi_{l}\right\|_{\dot{C}^{\eta}(\mathcal{X})} \leq C\left(2^{l} r\right)^{-\eta}\left(1 / V_{2^{l} r}\left(x_{1}\right)\right)$ for all $0<\eta \leq \beta$;

(iv) $\int_{x} \varphi_{l}(x) d \mu(x)=0$.

Moreover, the series in (2.36) converges to $f$ pointwise, as well as in $L^{p}(\mathcal{X})$ for $p \in[1, \infty]$, and in $\mathcal{C}_{0}^{\beta \wedge \tilde{\gamma}}\left(\beta^{\prime}, \gamma^{\prime}\right)$ with $0<\beta^{\prime}, \gamma^{\prime}<(\beta \wedge \tilde{\gamma})$ and also in $\left(\mathcal{C}_{0}^{\beta \wedge} \tilde{\gamma}\left(\beta^{\prime}, \gamma^{\prime}\right)\right)^{\prime}$ with $0<\beta^{\prime}, \gamma^{\prime} \leq(\beta \wedge \gamma)$.

Proof. Fix a nonnegative function $\alpha \in C^{1}(\mathbb{R})$ such that $\alpha(t)=1$ if $t \leq 1 / 2$ and $\alpha(t)=0$ if $t \geq 1$. Let $A_{0}(x)=\alpha\left(d\left(x, x_{1}\right) / r\right) f(x)$, and for $l \in \mathbb{N}$,

$$
A_{l}(x)=\left[\alpha\left(\frac{d\left(x, x_{1}\right)}{2^{l} r}\right)-\alpha\left(\frac{d\left(x, x_{1}\right)}{2^{l-1} r}\right)\right] f(x) .
$$

Then $f(x)=\sum_{l=0}^{\infty} A_{l}(x)$, and for all $\tilde{\gamma} \in(0, \gamma]$,

$$
\left|A_{l}(x)\right| \lesssim 2^{-\widetilde{\gamma}} \frac{1}{V_{2^{l} r}\left(x_{1}\right)}
$$

Define $a_{l}=\int_{x} A_{l}(x) d \mu(x)$ and $v_{l}=\sum_{j=0}^{l} a_{j}$. Let $\tilde{\eta}_{l}(x)=\alpha\left(d\left(x, x_{1}\right) / 2^{l} r\right)$ and $\eta_{l}(x)=$ $\tilde{\eta}_{l}(x)\left[\int_{x} \tilde{\eta}_{l}(z) d \mu(z)\right]^{-1}$. Finally, if we define

$$
\tilde{A}_{l}(x)=A_{l}(x)-a_{l} \eta_{l}(x)+v_{l}\left(\eta_{l}(x)-\eta_{l+1}(x)\right)
$$


and $\varphi_{l}(x)=2^{r \widetilde{\gamma}} \widetilde{A}_{l}(x)$, then it is easy to verify that $\varphi_{l}$ satisfies (i) through (iv) of the proposition and (2.36) holds pointwise.

Obviously, $\varphi_{l} \in \mathcal{G}(\beta, \gamma)$ and therefore $\varphi_{l} \in \mathcal{G}_{0}^{\beta \wedge \tilde{\gamma}}\left(\beta^{\prime}, \gamma^{\prime}\right)$ for $0<\beta^{\prime}, \gamma^{\prime} \leq(\beta \wedge \tilde{\gamma})$. Moreover, if $l \in \mathbb{N}$ is large enough, then there exists a constant $C_{r, x_{1}}>0$, which is independent of $x$, such that for all $x \in B\left(x_{1}, 2^{l} r\right), 1+d\left(x, x_{1}\right) \leq C_{r, x_{1}} 2^{l}$ and hence

$$
\left\|\varphi_{l}\right\|_{\mathcal{G}_{0}^{\beta \wedge \tilde{\gamma}\left(\beta^{\prime}, \gamma^{\prime}\right)}} \leq C_{r, x_{1}} 2^{l \gamma^{\prime}}
$$

From this, it follows that if $\gamma^{\prime}<\tilde{\gamma}$,

$$
\left\|f-\sum_{l=0}^{L} 2^{-l \tilde{\gamma}} \varphi_{l}\right\|_{\tilde{C}_{0}^{\beta \beta \tilde{\gamma}}\left(\beta^{\prime}, \gamma^{\prime}\right)} \lesssim C_{r, x_{1}} \sum_{l=L+1}^{\infty} 2^{-l\left(\tilde{\gamma}-\gamma^{\prime}\right)} \sim C_{r, x_{1}} 2^{-L\left(\tilde{\gamma}-\gamma^{\prime}\right)} \longrightarrow 0,
$$

as $L \rightarrow \infty$. This shows that $(2.36)$ holds in $\mathcal{G}_{0}^{\beta \wedge} \tilde{\gamma}\left(\beta^{\prime}, \gamma^{\prime}\right)$ with $0<\beta^{\prime}, \gamma^{\prime}<(\beta \wedge \tilde{\gamma})$. By Fatou's lemma and Minkowski's inequality, we also obtain that for any $p \in[1, \infty]$,

$$
\begin{aligned}
\left\|f-\sum_{l=0}^{L} 2^{-\widetilde{l}} \varphi_{l}\right\|_{L^{p}(\mathcal{X})} & \leq \sum_{l=L+1}^{\infty} 2^{-l \tilde{\gamma}}\left\|\varphi_{l}\right\|_{L^{p}(x)} \\
& \lesssim \sum_{l=L+1}^{\infty} 2^{-l \tilde{\gamma}} \frac{1}{V_{2^{l} r}\left(x_{1}\right)^{1-1 / p}} \\
& \lesssim \frac{1}{V_{r}\left(x_{1}\right)^{1-1 / p}} 2^{-L \tilde{\gamma}} \\
& \longrightarrow 0,
\end{aligned}
$$

as $L \rightarrow \infty$. That is, $(2.36)$ holds in $L^{p}(\mathcal{X})$ with $p \in[1, \infty]$.

Finally, for any $\psi \in \mathcal{C}_{0}^{\beta \wedge \gamma}\left(\beta^{\prime}, \gamma^{\prime}\right)$ with $0<\beta^{\prime}, \gamma^{\prime} \leq(\beta \wedge \gamma)$, from Hölder's inequality, Fatou lemma and Minkowski's inequality, it follows that

$$
\begin{aligned}
\left|\langle f, \psi\rangle-\sum_{l=0}^{L} 2^{-l \tilde{\gamma}}\left\langle\varphi_{l}, \psi\right\rangle\right| & \leq\left\|\sum_{l=L+1}^{\infty} 2^{-l \tilde{\gamma}} \varphi_{l}\right\|_{L^{1}(\mathcal{X})}\|\psi\|_{L^{\infty}(\mathcal{X})} \\
& \lesssim\left(\sum_{l=L+1}^{\infty} 2^{-l \tilde{\gamma}}\right)\|\psi\|_{L^{\infty}(\mathcal{X})} \\
& \lesssim 2^{-L \tilde{\gamma}}\|\psi\|_{L^{\infty}(\mathcal{X})} \\
& \longrightarrow 0
\end{aligned}
$$

as $L \rightarrow \infty$. Thus, (2.36) also holds in $\left(\mathcal{C}_{0}^{\beta \wedge \gamma}\left(\beta^{\prime}, \gamma^{\prime}\right)\right)^{\prime}$ with $0<\beta^{\prime}, \gamma^{\prime} \leq(\beta \wedge \gamma)$, which completes the proof of Proposition 2.9. 
The following proposition is a slight variant of Proposition 2.9.

Proposition 2.10. Let $x_{1} \in \mathcal{X}, r>0,0<\beta \leq 1$, and $\gamma>0$, and let $f \in \mathcal{G}\left(x_{1}, r, \beta, \gamma\right)$. Then for any $\tilde{\gamma} \in(0, \gamma]$ and all $x \in \mathcal{X}$,

$$
f(x)=\sum_{l=0}^{\infty} 2^{-l \widetilde{r}} \varphi_{l}(x)
$$

where $\varphi_{l}$ is an adjusted bump function associated with the ball $B\left(x_{1}, 2^{l} r\right)$, which means that there exists a constant $C>0$ independent of $r$ and $l$ such that

(i) $\operatorname{supp} \varphi_{l} \subset B\left(x_{1}, 2^{l} r\right)$;

(ii) $\left|\varphi_{l}(x)\right| \leq C\left(1 / V_{2^{l} r}\left(x_{1}\right)\right)$ for all $x \in \mathcal{X}$;

(iii) $\left\|\varphi_{l}\right\|_{C^{n}(\mathcal{X})} \leq C\left(2^{l} r\right)^{-\eta}\left(1 / V_{2^{l} r}\left(x_{1}\right)\right)$ for all $0<\eta \leq \beta$.

Moreover, the series in (2.44) converges to $f$ pointwise, as well as in $L^{p}(\mathcal{X})$ for $p \in[1, \infty]$, and in $\mathcal{G}_{0}^{\beta \wedge \tilde{\gamma}}\left(\beta^{\prime}, \gamma^{\prime}\right)$ with $0<\beta^{\prime}, \gamma^{\prime}<(\beta \wedge \tilde{\gamma})$ and also in $\left(\mathcal{G}_{0}^{\beta \wedge \gamma}\left(\beta^{\prime}, \gamma^{\prime}\right)\right)^{\prime}$ with $0<\beta^{\prime}, \gamma^{\prime} \leq(\beta \wedge \gamma)$.

Proof. Let $\alpha \in C^{1}(\mathbb{R})$ be as in the proof of Proposition 2.9. Let $A_{0}(x)=\alpha\left(d\left(x, x_{1}\right) / r\right) f(x)$, and for $l \in \mathbb{N}$,

$$
A_{l}(x)=\left[\alpha\left(\frac{d\left(x, x_{1}\right)}{2^{l} r}\right)-\alpha\left(\frac{d\left(x, x_{1}\right)}{2^{l-1} r}\right)\right] f(x) .
$$

Then for $l \in \mathbb{Z}_{+}$, setting $\varphi_{l}(x)=2^{2 \tilde{\gamma}} A_{l}(x)$, we can verify that the $\left\{\varphi_{l}\right\}_{l \in \mathbb{Z}+}$ have all the properties stated in the proposition, which completes the proof of Proposition 2.10.

In what follows, for any $\beta \in(0,1], \gamma>0$, and $r>0$, we let

$$
\begin{aligned}
& \mathcal{G}_{b}\left(x_{1}, r, \beta, \gamma\right)=\left\{f \in \mathcal{G}\left(x_{1}, r, \beta, \gamma\right): f \text { has bounded support }\right\} \\
& \mathcal{G}_{b}\left(x_{1}, r, \beta, \gamma\right)=\left\{f \in \mathcal{G}\left(x_{1}, r, \beta, \gamma\right): f \text { has bounded support }\right\} .
\end{aligned}
$$

Also, for $\eta \in(0,1]$, let

$$
C_{b}^{\eta}(x)=\left\{f \in C_{b}^{\eta}(\mathcal{X}): \int_{x} f(x) d \mu(x)=0\right\}
$$

From Propositions 2.7, 2.9, and 2.10, it is easy to deduce the following useful result; we omit the details.

Corollary 2.11. Let $\epsilon_{1} \in(0,1]$ be as in Definition $2.2,0<\beta \leq \epsilon_{1}$ and $\gamma>0$. Then,

(i) both $\dot{C}_{b}^{\beta}(x)$ and $\AA_{b}\left(x_{1}, r, \beta, \gamma\right)$ for any fixed $x_{1} \in \mathcal{X}$ and $r>0$ are dense in $\dot{\mathcal{G}}\left(x_{1}, r, \beta, \gamma\right)$;

(ii) both $C_{b}^{\beta}(\mathcal{X})$ and $\mathcal{G}_{b}\left(x_{1}, r, \beta, \gamma\right)$ for any fixed $x_{1} \in X$ and $r>0$ are dense in both $\mathcal{G}\left(x_{1}, r, \beta, \gamma\right)$ and $L^{p}(X)$ with $p \in[1, \infty)$. 


\subsection{Boundedness of singular integrals on spaces of test functions with mean zero}

Let $\beta \in(0,1]$. In analogy with the topology of the space $\Phi\left(\mathbb{R}^{n}\right)$, in what follows, we endow the spaces $C_{b}^{\beta}(\mathcal{X})$ (resp., $C_{b}^{\beta}(\mathcal{X})$ ) with the strict inductive limit topology (see [30, page 273] or [67]) arising from the decomposition $C_{b}^{\beta}(\mathcal{X})=\bigcup_{n} C^{\beta}\left(B_{n}\right)$ (resp., $\mathcal{C}_{b}^{\beta}(\mathcal{X})=\bigcup_{n} \mathcal{C}^{\beta}\left(B_{n}\right)$ ), where $\left\{B_{n}\right\}_{n}$ is any increasing sequence of closed balls with the same center and $\mathcal{X}=\bigcup_{n} B_{n}$, and the space $C^{\beta}\left(B_{n}\right)$ (resp., $C^{\beta}\left(B_{n}\right)$ ) means the set of all functions $f \in C^{\beta}(\mathcal{X})$ (resp., $f \in C^{\beta}(\mathcal{X})$ ) with supp $f \subset B_{n}$, whose topology is given by the norm $\|\cdot\|_{C^{\beta}(\mathcal{X})}$. It is well known that the topology of $C_{b}^{\beta}(X)$ (resp., $C_{b}^{\beta}(X)$ ) is independent of the choice of closed balls $\left\{B_{n}\right\}_{n}$; see [30, page 273] or [67]. Their dual spaces $\left(C_{b}^{\beta}(x)\right)^{\prime}$ and $\left(\mathcal{C}_{b}^{\beta}(x)\right)^{\prime}$ will be endowed with the weak* topology.

We first have the following basic facts.

Proposition 2.12. Let $\beta \in(0,1]$ and let $T$ be a continuous linear operator from $C_{b}^{\beta}(\mathcal{X})$ to $\left(C_{b}^{\beta}(X)\right)^{\prime}$. Assume that $T$ has a distributional kernel $K$, which is locally integrable away from the diagonal of $\mathcal{X} \times \mathcal{X}$, in the sense that for all $\varphi, \psi \in C_{b}^{\beta}(\mathcal{X})$ with disjoint supports,

$$
\langle T \varphi, \psi\rangle=\iint_{\chi \times \chi} K(x, y) \varphi(y) \psi(x) d \mu(x) d \mu(y) .
$$

Assume also that there exists a constant $C_{T}>0$ such that for all $x, x^{\prime}, y \in \mathcal{X}$ with $d\left(x, x^{\prime}\right) \leq$ $d(x, y) / 2$ and $x \neq y$,

$$
\left|K(x, y)-K\left(x^{\prime}, y\right)\right| \leq C_{T} \frac{d\left(x, x^{\prime}\right)^{\epsilon}}{V(x, y) d(x, y)^{\epsilon}}
$$

Then $T$ can be extended to a continuous linear operator from $C^{\beta}(\mathcal{X})$ to $\left(C_{b}^{\beta}(\mathcal{X})\right)^{\prime}$.

Proof. For any $f \in C^{\beta}(\mathcal{X})$ and $g \in \mathcal{C}_{b}^{\beta}(\mathcal{X})$, suppose supp $g \subset B\left(x_{0}, r\right)$ for some $x_{0} \in \mathcal{X}$ and $r>$ 0 . Choose $\psi \in C_{b}^{\beta}(x)$ such that $\psi(x)=1$ when $x \in B\left(x_{0}, 2 r\right)$ and $\psi(x)=0$ when $x \notin B\left(x_{0}, 4 r\right)$. It is easy to see that $\psi f \in C_{b}^{\beta}(\mathcal{X})$, which shows that $\langle T(\psi f), g\rangle$ is well defined. On the other hand, we define

$$
\langle T((1-\psi) f), g\rangle=\iint_{\chi \times \mathcal{X}}\left[K(x, y)-K\left(x_{0}, y\right)\right](1-\psi(y)) f(y) g(x) d \mu(x) d \mu(y) .
$$

By (2.49) and Lemma 2.1(i), it is easy to see that the right-hand side of the above equality is finite; furthermore, if $f$ has also bounded support, this equals with

$$
\iint_{x \times \chi} K(x, y)(1-\psi(y)) f(y) g(x) d \mu(x) d \mu(y),
$$

which coincides with (2.48). Moreover, it is easy to verify that

$$
\langle T(\psi f), g\rangle+\langle T((1-\psi) f), g\rangle
$$


is independent of choice of $\psi$. Thus we can define $T f$ by letting

$$
\langle T f, g\rangle=\langle T(\psi f), g\rangle+\langle T((1-\psi) f), g\rangle \text {. }
$$

In this sense, we have $T f \in\left(\mathcal{C}_{b}^{\beta}(\mathcal{X})\right)^{\prime}$, which completes the proof of Proposition 2.12.

We also need the weak boundedness property and the strong weak boundedness property of operators; see [68] for the definition of the weak boundedness property on $\mathbb{R}^{n}$ and [34] for the definition of the strong weak boundedness property on Ahlfors 1-regular metric measure spaces.

In what follows, for $\beta \in(0,1]$, we denote by $C_{b}^{\beta}(\mathcal{X} \times \mathcal{X})$ the set of all functions $f$ on $\mathcal{X} \times \mathcal{X}$ with bounded support, which satisfy that for any $x \in \mathcal{X}$, both $f(x, \cdot)$ and $f(\cdot, x)$ are in $\dot{C}^{\beta}(x)$.

Definition 2.13. Let $\beta \in(0,1]$ and let $T$ be a continuous linear mapping from $C_{b}^{\beta}(\mathcal{X})$ to $\left(C_{b}^{\beta}(\mathcal{X})\right)^{\prime}$. The operator $T$ is said to have the weak boundedness property of order $\beta$ (for short, $T \in \mathrm{WBP}(\beta))$ if there exists a constant $C>0$ such that for all $\phi, \psi \in C_{b}^{\beta}(\mathcal{X})$ with supp $\phi$, supp $\psi \subset B(z, r)$ for some $z \in \mathcal{X}$ and $r>0,\|\phi\|_{\dot{C}^{\beta}(\mathcal{X})} \leq r^{-\beta}$ and $\|\psi\|_{\dot{C}^{\beta}(\mathcal{X})} \leq r^{-\beta}$,

$$
|\langle T \phi, \psi\rangle| \leq C \mu(B(z, r))
$$

The minimal constant $C$ as above is denoted by $\|T\|_{\mathrm{WBP}(\beta)}$.

Remark 2.14. (i) Let $\beta \in(0,1]$ and $\gamma>0$. Let us also endow the space $\mathcal{G}_{b}(\beta, \gamma)$ (resp., $\mathcal{G}_{b}(\beta, \gamma)$ ) with the strict inductive limit topology in a similar way as the space $C_{b}^{\beta}(\mathcal{X})$ (resp., $\mathcal{C}_{b}^{\beta}(\mathcal{X})$ ) and its dual space $\left(\mathcal{G}_{b}(\beta, \gamma)\right)^{\prime}$ (resp., $\left.\left(\mathcal{G}_{b}(\beta, \gamma)\right)^{\prime}\right)$ with the weak* topology. Then, as topological spaces, $C_{b}^{\beta}(\mathcal{X})=\mathcal{G}_{b}(\beta, \gamma)$ and $\left(C_{b}^{\beta}(\mathcal{X})\right)^{\prime}=\left(\mathcal{G}_{b}(\beta, \gamma)\right)^{\prime}$ (resp., $\mathcal{C}_{b}^{\beta}(\mathcal{X})=\mathcal{G}_{b}(\beta, \gamma)$ and $\left(\mathcal{C}_{b}^{\beta}(\mathcal{X})\right)^{\prime}=$ $\left.\left(\mathcal{G}_{b}(\beta, \gamma)\right)^{\prime}\right)$.

(ii) We remark that if $\mathcal{X}$ is a $(\kappa, n)$-space as in Definition 1.1, then there exists a constant $C>0$ such that for all $\phi \in \dot{C}_{b}^{\beta}(\mathcal{X})$ and all $x \in \mathcal{X}$,

$$
|\phi(x)| \leq C[\operatorname{diam}(\operatorname{supp} \phi)]^{\beta}\|\phi\|_{\dot{C}^{\beta}(\mathcal{X})} .
$$

To see this, assume that supp $\phi \subset B\left(x_{0}, r\right)$ for some $x_{0} \in \mathcal{X}$ and $r>0$. By Remark 1.2, we can find a $y_{0} \in \mathcal{X}$ such that $r \leq d\left(y_{0}, x_{0}\right) \leq 2 r$. Then $\phi\left(y_{0}\right)=0$ and for all $x \in \operatorname{supp} \phi$,

$$
|\phi(x)|=\left|\phi(x)-\phi\left(y_{0}\right)\right| \leq d\left(x, y_{0}\right)^{\beta}\|\phi\|_{\dot{C}^{\beta}(x)} \leq(3 r)^{\beta}\|\phi\|_{\dot{C}^{\beta}(x)},
$$

which is just (2.55). By this observation, we see that the functions $\phi$ and $\psi$ in Definition 2.13 also satisfy $\|\phi\|_{L^{\infty}(\mathcal{X})} \lesssim 1$ and $\|\psi\|_{L^{\infty}(\mathcal{X})} \lesssim 1$.

(iii) From Hölder's inequality, it is easy to deduce that if $T$ is bounded on $L^{p}(\mathcal{X})$ with $p \in(1, \infty)$, then $T \in \operatorname{WBP}(\beta)$ for any $\beta \in(0,1]$ and

$$
\|T\|_{\mathrm{WBP}(\beta)} \leq\|T\|_{L^{p}(\mathcal{X}) \rightarrow L^{p}(\mathcal{X})},
$$


where and in what follows, $\|T\|_{L^{p}(\mathcal{X}) \rightarrow L^{p}(\mathcal{X})}$ denotes the operator norm of $T$ from $L^{p}(\mathcal{X})$ to $L^{p}(X)$.

(iv) Following [34], we can also introduce a slightly stronger property than WBP, called strong weak boundedness property in [34] as follows: let $T$ be as in Definition 2.13. The operator $T$ is said to have the strong weak boundedness property of order $\beta$ (for short, $T \in \operatorname{SWBP}(\beta))$ if $T$ has a distributional kernel $K \in\left(C_{b}^{\beta}(\mathcal{X} \times \mathcal{X})\right)^{\prime}$ such that (2.48) holds and there exists a constant $C>0$ such that for all $f \in C_{b}^{\beta}(\mathcal{X} \times \mathcal{X})$ with supp $f \subset B\left(x_{0}, r\right) \times B\left(x_{0}, r\right)$ for some $x_{0} \in \mathcal{X}$ and $r>0,\|f(\cdot, y)\|_{\dot{C}^{\beta}(x)} \leq r^{-\beta}$ for all $y \in \mathcal{X}$ and $\|f(x, \cdot)\|_{\dot{C}^{\beta}(x)} \leq r^{-\beta}$ for all $x \in \mathcal{X}$

$$
|\langle K, f\rangle| \leq C \mu\left(B\left(x_{0}, r\right)\right)
$$

The minimal constant $C$ as above is denoted by $\|T\|_{S W B P(\beta)}$.

By the observation in (ii) of this remark, the function $f$ also satisfies that $\|f\|_{L^{\infty}(x \times x)}$ $\lesssim 1$.

(v) Let $i=1,2, \beta_{i} \in(0,1]$ and let $T$ be a continuous linear mapping from $C_{b}^{\beta_{i}}(\mathcal{X})$ to $\left(C_{b}^{\beta_{i}}(\mathcal{X})\right)^{\prime}$ with a distributional kernel $K$ as in (2.48) of Proposition 2.12. If $K$ satisfies the size condition that for all $x, y \in \mathcal{x}$ with $x \neq y$,

$$
|K(x, y)| \leq C_{T} \frac{1}{V(x, y)}
$$

then $T \in \mathrm{WBP}\left(\beta_{1}\right)$ if and only if $T \in \mathrm{WBP}\left(\beta_{2}\right)$. This fact can be proved by an argument similar to that used in the proof of Proposition 1 in [64] and we omit the details.

In what follows, standard notions from distribution theory carry over to continuous linear functionals on $C_{b}^{\beta}(\mathcal{X})$. For instance, if $\mathcal{U} \subset \mathcal{X}$ is an open subset, the restriction of $\omega \in$ $\left(C_{b}^{\beta}(\mathcal{X})\right)^{\prime}$ to $\mathcal{U}$ is defined by $\left\langle\left.\omega\right|_{\mathfrak{u}}, \varphi\right\rangle=\langle\omega, \varphi\rangle$ for all $\varphi \in C_{b}^{\beta}(\mathcal{X})$ supported in $\mathcal{U}$.

Using some ideas from Meyer in [69], we can obtain the following useful estimates which play a key role in establishing the boundedness of singular integrals on spaces of test functions with mean zero.

Lemma 2.15. Fix a bump function $\theta \in C_{b}^{1}(\mathbb{R})$ with $0 \leq \theta(x) \leq 1$ for all $x \in \operatorname{supp} \theta \subset\{x \in \mathbb{R}:|x| \leq$ $2\}$ and $\theta(x)=1$ on $\{x \in \mathcal{X}:|x| \leq 1\}$. For any fixed $z \in \mathcal{X}$ and $r>0$, let $\theta_{z, r}(y)=\theta(d(z, y) / r)$ for all $y \in \mathcal{X}$ and put $\omega_{z, r}=1-\theta_{z, r}$. Let $T$ be as in Proposition 2.12. If $T(1) \in\left(\mathcal{C}_{b}^{\beta}(\mathcal{X})\right)^{\prime}$ is 0 , then the following hold.

(i) The restriction of the linear functional $T\left(\theta_{z, r}\right) \in\left(C_{b}^{\beta}(x)\right)^{\prime}$ to the ball $B(z, r / 2)$ is a measurable function, and for a.e. $x \in B(z, r / 2)$,

$$
T\left(\theta_{z, r}\right)(x)=C_{z, r}-\int_{\chi}[K(x, y)-K(z, y)] \omega_{z, r}(y) d \mu(y),
$$

where $C_{z, r}$ is a constant independent of $x$. 
(ii) If further assuming that $T \in \operatorname{WBP}(\beta)$, then there exists a constant $C>0$ such that for a.e. $x \in B(z, r / 2)$,

$$
\left|T\left(\theta_{z, r}\right)(x)\right| \leq C\left(C_{T}+\|T\|_{\mathrm{WBP}(\beta)}\right) .
$$

Proof. Assume that $T(1)=0$ in $\left(\mathcal{C}_{b}^{\beta}(\mathcal{X})\right)^{\prime}$. For any $f \in \mathcal{C}_{b}^{\beta}(\mathcal{X})$ with supp $f \subset B(z, r / 2)$, since $\int_{x} f(x) d \mu(x)=0$, we have

$$
0=\langle T(1), f\rangle=\left\langle T\left(\theta_{z, r}\right)+\int_{\chi}[K(\cdot, y)-K(z, y)] \omega_{z, r}(y) d \mu(y), f\right\rangle .
$$

Let $\alpha \in C^{1}(\mathbb{R})$ be as in the proof of Proposition 2.9, and $\tilde{\eta}_{z, r}(y)=\alpha(d(y, z) /(r / 2))$. Set

$$
\eta_{z, r}(y)=\tilde{\eta}_{z, r}(y)\left[\int_{x} \tilde{\eta}_{z, r}(w) d \mu(w)\right]^{-1}
$$

Then $\eta_{z, r} \in C_{b}^{1}(\mathcal{X})$, supp $\eta_{z, r} \subset B(z, r / 2)$ and $\int_{\mathcal{X}} \eta_{z, r}(y) d \mu(y)=1$. For any $f \in C_{b}^{\beta}(\mathcal{X})$ with supp $f \subset B(z, r / 2)$, we set $\tilde{f}(y)=f(y)-\eta_{z, r}(y) \int_{\chi} f(y) d \mu(y)$; then $\tilde{f} \in \mathcal{C}_{b}^{\beta}(x)$ with supp $\tilde{f} \subset$ $B(z, r / 2)$. Applying (2.62) to $\tilde{f}$ and using Corollary 2.11(ii) in the case $p=1$ show that the restriction of the linear functional $T\left(\theta_{z, r}\right) \in\left(C_{b}^{\beta}(x)\right)^{\prime}$ to the ball $B(z, r / 2)$ is a measurable function, and for a.e. $x \in B(z, r / 2)$,

$$
\begin{aligned}
& \left\langle T\left(\theta_{z, r}\right)(x)+\int_{x}[K(x, y)-K(z, y)] \omega_{z, r}(y) d \mu(y), f\right\rangle \\
& \quad=\int_{x}\left\langle T\left(\theta_{z, r}\right)+\int_{x}[K(\cdot, y)-K(z, y)] \omega_{z, r}(y) d \mu(y), \eta_{z, r}\right\rangle f(x) d \mu(x),
\end{aligned}
$$

which implies (i) with

$$
C_{z, r}=\left\langle T\left(\theta_{z, r}\right)+\int_{\chi}[K(\cdot, y)-K(z, y)] \omega_{z, r}(y) d \mu(y), \eta_{z, r}\right\rangle,
$$

by noticing that $C_{z, r}$ is a constant independent of $x$ and the choice of $\eta_{z, r}$, but, it may depend on $z$ and $r$. 
To verify (ii), by (2.62) together with the definition of $T(1),(2.49)$ of Proposition 2.12 and Lemma 2.1(i), we have

$$
\begin{aligned}
\left|\left\langle T\left(\theta_{z, r}\right), f\right\rangle\right| & =\left|-\left\langle T\left(\omega_{z, r}\right), f\right\rangle\right| \\
& =\left|\iint_{\chi \times x}[K(x, y)-K(z, y)] \omega_{z, r}(y) f(x) d \mu(y) d \mu(x)\right| \\
& \leq C_{T} \int_{\chi}\left[\int_{2 d(z, x)<d(z, y)} \frac{d(x, z)^{\epsilon}}{V(z, y) d(z, y)^{\epsilon}} d \mu(y)\right]|f(x)| d \mu(x) \\
& \lesssim C_{T}\|f\|_{L^{1}(x)^{\prime}}
\end{aligned}
$$

which together with Corollary 2.11(ii) in the cases $r=1$ and $p=1$ again shows that for a.e. $x \in B(z, r / 2)$,

$$
T\left(\theta_{z, r}\right)(x)=\Omega_{z, r}(x)+C_{z, r}
$$

where $\left\|\Omega_{z, r}\right\|_{L^{\infty}(\mathcal{X})} \lesssim C_{T}$ and $C_{z, r}$ is a constant independent of $x$. We now estimate $C_{z, r}$ by using that $T \in \operatorname{WBP}(\beta)$. To this end, let $g \in C_{b}^{\beta}(\mathcal{X})$ with supp $g \subset B(z, r),\|g\|_{L^{\infty}(\mathcal{X})} \leq 1$, $\|g\|_{\dot{C}^{\beta}(x)} \leq r^{-\beta}$, and $\int_{\chi} g(x) d \mu(x) \sim \mu(B(z, r))$. From (2.67) and $T \in \operatorname{WBP}(\beta)$, it follows that

$$
\begin{aligned}
\left|C_{z, r}\right|\left|\int_{x} g(x) d \mu(x)\right| & =\left|\left\langle T\left(\theta_{z, r}\right), g\right\rangle-\int_{x} \Omega_{z, r}(x) g(x) d \mu(x)\right| \\
& \lesssim\left(\|T\|_{\mathrm{WBP}(\beta)}+C_{T}\right) \mu(B(z, r))
\end{aligned}
$$

which implies that $\left|C_{z, r}\right| \lesssim\|T\|_{\operatorname{WBP}(\beta)}+C_{T}$, and hence, completes the proof of Lemma 2.15.

We recall the notion of the space of functions with bounded mean oscillation, $\mathrm{BMO}(\mathcal{X})$, which was first introduced by John and Nirenberg in [70], and was proved to be the dual space of $H_{\mathrm{at}}^{1}(\mathcal{X})$ in [28].

Definition 2.16. Let $1 \leq q<\infty$. The space $\mathrm{BMO}_{q}(\mathcal{X})$ is defined to be the set of all $f \in L_{\text {loc }}^{q}(\mathcal{X})$ such that

$$
\|f\|_{\mathrm{BMO}_{q}(\mathcal{X})}=\sup _{x \in \mathcal{X}, r>0}\left\{\frac{1}{\mu(B(x, r))} \int_{B(x, r)}\left|f(y)-m_{B(x, r)}(f)\right|^{q} d \mu(y)\right\}^{1 / q}<\infty .
$$

When $q=1$, one denotes $\mathrm{BMO}_{1}(\mathcal{X})$ simply by $\mathrm{BMO}(\mathcal{X})$.

It was proved in [28] that for any $1 \leq q_{1}, q_{2}<\infty, \mathrm{BMO}_{q_{1}}(\mathcal{X})$ and $\mathrm{BMO}_{q_{2}}(\mathcal{X})$ are equal as vector spaces and the seminorms $\|\cdot\|_{\mathrm{BMO}_{q_{1}}(\mathcal{X})}$ and $\|\cdot\|_{\mathrm{BMO}_{q_{2}}(\mathcal{X})}$ are equivalent. Moreover, if we let $\mathcal{N} \equiv \mathbb{C}$ be the subspace of all constant functions on $\mathcal{X}$, then the quotient space $\mathrm{BMO}(\mathcal{X}) / \mathcal{N}$ becomes a Banach space in a natural way. 
Remark 2.17. Let $\beta \in(0, \epsilon]$ and $\gamma>0$. It is easy to see that $T(1)=0$ in $\left(\dot{C}_{b}^{\beta}(\mathcal{X})\right)^{\prime}$ if and only if $T(1) \in\left(\mathcal{C}_{b}^{\beta}(\mathcal{X})\right)^{\prime}$ is constant; see also [71, page 22]. Moreover, from Corollary 2.11(i), Proposition 5.21, Theorems 5.19(i) and 6.11 below, it is easy to see that $T(1) \in\left(\stackrel{C}{C}_{b}^{\beta}(\mathcal{X})\right)^{\prime}$ is constant if and only if $T(1) \in \mathrm{BMO}(\mathcal{X})$ is constant, which is also equivalent to $T(1)=0$ in $\operatorname{BMO}(x)$.

We now establish a basic boundedness result for singular integrals on spaces of test functions with mean zero, which will be a key tool for the whole paper.

Theorem 2.18. Let $\epsilon \in(0,1], \beta \in(0, \epsilon)$, and let $T$ be as in Proposition 2.12 with the distributional kernel $K$ satisfying the following additional conditions that

(i) for all $x, y \in \mathcal{X}$ with $x \neq y,|K(x, y)| \leq C_{T}(1 / V(x, y))$;

(ii) for all $x, y, y^{\prime} \in \mathcal{X}$ with $d\left(y, y^{\prime}\right) \leq d(x, y) / 2$ and $x \neq y$,

$$
\left|K(x, y)-K\left(x, y^{\prime}\right)\right| \leq C_{T} \frac{d\left(y, y^{\prime}\right)^{\epsilon}}{V(x, y) d(x, y)^{\epsilon}}
$$

(iii) for all $x, x^{\prime}, y, y^{\prime} \in x$ with $d\left(x, x^{\prime}\right) \leq d(x, y) / 3, d\left(y, y^{\prime}\right) \leq d(x, y) / 3$ and $x \neq y$,

$$
\left|\left[K(x, y)-K\left(x^{\prime}, y\right)\right]-\left[K\left(x, y^{\prime}\right)-K\left(x^{\prime}, y^{\prime}\right)\right]\right| \leq C_{T} \frac{d\left(x, x^{\prime}\right)^{\epsilon} d\left(y, y^{\prime}\right)^{\epsilon}}{V(x, y) d(x, y)^{2 \epsilon}}
$$

If $T \in \operatorname{WBP}(\beta)$ and $T(1)=0$ in $\left(\mathcal{C}_{b}^{\beta}(\mathcal{X})\right)^{\prime}$, then $T$ extends to a bounded linear operator from $\mathcal{G}\left(x_{1}, r, \beta, \gamma\right)$ to $\mathcal{G}\left(x_{1}, r, \beta, \gamma\right)$ for all $x_{1} \in \mathcal{X}, r>0$, and $\gamma \in(0, \epsilon)$. Moreover, there exists a constant $C_{\beta, \gamma, C_{0}}>0$ such that for all $f \in \mathcal{G}\left(x_{1}, r, \beta, \gamma\right)$ with any $x_{1} \in \mathcal{X}$, any $r>0$, and any $\gamma \in(0, \epsilon)$,

$$
\|T f\|_{\mathcal{G}\left(x_{1}, r, \beta, \gamma\right)} \leq C_{\beta, \gamma, C_{0}}\left(C_{T}+\|T\|_{\operatorname{WBP}(\beta)}\right)\|f\|_{\mathcal{G}\left(x_{1}, r, \beta, r\right)} .
$$

Compared with the corresponding results in [31, 35], Theorem 2.18 has three advantages. (1) Theorem 2.18 is true on spaces of homogeneous type, while the corresponding results in $[31,35]$ are proved only for $\mathbb{R}^{n}$ or for spaces of homogeneous type with $\mu(B(x, r)) \sim r$ for all $x \in \mathcal{X}$ and $0<r \leq \operatorname{diam} \mathcal{X}$, respectively. (2) We do not assume that $T^{*}(1)=0$ in Theorem 2.18. Since $T^{*}(1)=0$ was also assumed in [31, 35], by the $T(1)$-theorem in those settings, one knows that $T$ is bounded on $L^{2}(\mathcal{X})$. Thus, for any $f \in \mathcal{G}\left(x_{1}, r, \beta, \gamma\right)$ for some $x_{1} \in X, r>0, \beta \in(0,1]$ and $\gamma>0, T f \in L^{2}(\mathcal{X})$, which makes the proof much easier. (3) We only require $T \in \mathrm{WBP}(\beta)$ instead of $T \in \operatorname{SWBP}(\beta)$ in [35].

To prove Theorem 2.18, we first recall the following construction given by Christ in [72], which provides an analogue of the grid of Euclidean dyadic cubes on spaces of homogeneous type.

Lemma 2.19. Let $\chi \mathcal{X}$ be a space of homogeneous type. Then there exists a collection $\left\{Q_{\alpha}^{k} \subset \mathcal{X}: k \in\right.$ $\left.\mathbb{Z}, \alpha \in I_{k}\right\}$ of open subsets, where $I_{k}$ is some index set, and constants $\delta \in(0,1)$ and $C_{6}, C_{7}>0$ such 
that

(i) $\mu\left(\mathcal{X} \backslash \bigcup_{\alpha} Q_{\alpha}^{k}\right)=0$ for each fixed $k$ and $Q_{\alpha}^{k} \cap Q_{\beta}^{k}=\varnothing$ if $\alpha \neq \beta$;

(ii) for any $\alpha, \beta, k, l$ with $l \geq k$, either $Q_{\beta}^{l} \subset Q_{\alpha}^{k}$ or $Q_{\beta}^{l} \cap Q_{\alpha}^{k}=\varnothing$;

(iii) for each $(k, \alpha)$ and each $l<k$ there is a unique $\beta$ such that $Q_{\alpha}^{k} \subset Q_{\beta^{\prime}}^{l}$;

(iv) $\operatorname{diam}\left(Q_{\alpha}^{k}\right) \leq C_{6} \delta^{k}$;

(v) each $Q_{\alpha}^{k}$ contains some ball $B\left(z_{\alpha}^{k}, C_{7} \delta^{k}\right)$, where $z_{\alpha}^{k} \in \mathcal{X}$.

In fact, we can think of $Q_{\alpha}^{k}$ as being a dyadic cube with diameter rough $\delta^{k}$ and centered at $z_{\alpha}^{k}$. In what follows, to simplify our presentation, we always suppose $\delta=1 / 2$; otherwise, we need to replace $2^{-k}$ in the definition of ATIs by $\delta^{k}$ and some other changes are also necessary; see [34, pages 96-98] for more details.

To prove Theorem 2.18, we need another technical lemma, where we need Lemma 2.19 and $T \in \operatorname{WBP}(\beta)$; see also [69, Lemma 3] and [34, Lemma (3.12)].

In what follows, if $T f \in\left(C_{b}^{\beta}(x)\right)^{\prime}$ and $g \in C_{b}^{\beta}(x)$, we sometimes will write

$$
\int_{x} T f(x) g(x) d \mu(x)
$$

in place of $\langle T f, g\rangle$, in order to indicate more clearly the dependence on the variable $x$.

Lemma 2.20. Let $\epsilon \in(0,1], \beta \in(0, \epsilon), \gamma \in(0, \epsilon]$ and let $T$ be as in Proposition 2.12 with the distributional kernel $K$ satisfying the additional size condition (2.59). Let $\theta$ be as in Lemma 2.15 and for any fixed $x_{1} \in \mathcal{X}$ and $r>0$, one defines $\tilde{\theta} \equiv \theta_{x_{1}, 20 r}$ and $\widetilde{\omega} \equiv \omega_{x_{1}, 20 r}$ in the same way as in Lemma 2.15. If $T \in \operatorname{WBP}(\beta)$ and $T(1)=0$ in $\left(\mathcal{C}_{b}^{\beta}(\mathcal{X})\right)^{\prime}$, then for any $f \in \mathcal{G}_{b}\left(x_{1}, r, \beta, \gamma\right)$, the restriction of the linear functional $T f \in\left(C_{b}^{\beta}(x)\right)^{\prime}$ to the ball $B\left(x_{1}, 10 r\right)$ is a measurable function, and for a.e. $x \in B\left(x_{1}, 10 r\right)$,

$$
T f(x)=\int_{x} K(x, y)[f(y)-f(x)] \tilde{\theta}(y) d \mu(y)+\int_{x} K(x, y) f(y) \tilde{\omega}(y) d \mu(y)+f(x) T(\tilde{\theta})(x),
$$

where the first two integrals are absolutely convergent.

Proof. We make use of some ideas used in the proofs of Lemma 3 in [69] and Lemma (3.12) in [34]. For any fixed $f \in \mathcal{G}_{b}\left(x_{1}, r, \beta, \gamma\right)$ and any $\psi \in C_{b}^{\beta}(x)$ with supp $\psi \subset B\left(x_{1}, 10 r\right)$, we have

$$
\begin{aligned}
\langle T f, \psi\rangle= & \langle T(f \tilde{\theta}), \psi\rangle+\langle T(f \tilde{\omega}), \psi\rangle \\
= & \int_{x} T(\tilde{\theta}[f-f(x)])(x) \psi(x) d \mu(x)+\int_{x} T(\tilde{\theta})(x) f(x) \psi(x) d \mu(x) \\
& +\iint_{x \times x} K(x, y) \psi(x) f(y) \tilde{\omega}(y) d \mu(x) d \mu(y) \\
\equiv & Y_{1}+Y_{2}+Y_{3},
\end{aligned}
$$


where the first two integrals have to be interpreted in the sense of "distributions," whereas the third one is an absolutely convergent integral.

Actually, by Lemma 2.15(ii), $T(\tilde{\theta})$ is a bounded function in $B\left(x_{1}, 10 r\right)$, and we have

$$
\left|Y_{2}\right| \leq\left(\sup _{x \in B\left(x_{1}, 10 r\right)}|T(\tilde{\theta})(x)|\right) \int_{\chi}|\psi(y) f(y)| d \mu(y) \lesssim \frac{1}{V_{r}\left(x_{1}\right)}\|\psi\|_{L^{1}(x)}
$$

which is the desired estimate.

Notice that if $d\left(x, x_{1}\right)<10 r$, then $x \notin \operatorname{supp} \tilde{w}$. Moreover, if we put

$$
Y_{3,1}(x) \equiv \int_{x} K(x, y) f(y) \tilde{\omega}(y) d \mu(y)
$$

then for $x \in B\left(x_{1}, 10 r\right)$,

$$
\left|Y_{3,1}(x)\right| \lesssim \frac{1}{V_{r}\left(x_{1}\right)+V\left(x_{1}, x\right)}
$$

In fact, we first notice that $\tilde{\omega}(y) \neq 0$ implies that $d\left(y, x_{1}\right) \geq 20 r \geq 2 d\left(x, x_{1}\right)$. From this, it follows that $V\left(x_{1}, y\right) \geq \mu\left(B\left(x_{1}, 2 d\left(x, x_{1}\right)\right)\right) \geq V\left(x_{1}, x\right)$ and $d\left(y, x_{1}\right) \leq 2 d(x, y)$, which together with (1.2) shows that

$$
V\left(x_{1}, y\right) \sim V\left(y, x_{1}\right)=\mu\left(B\left(y, d\left(y, x_{1}\right)\right)\right) \leq \mu(B(y, 2 d(x, y))) \lesssim V(y, x) \sim V(x, y) .
$$

These estimates together with Lemma 2.1(i) and (2.59) imply that

$$
\begin{aligned}
\left|Y_{3,1}(x)\right| & \lesssim \int_{d\left(y, x_{1}\right) \geq 20 r} \frac{1}{V(x, y)} \frac{1}{V_{r}\left(x_{1}\right)+V\left(x_{1}, y\right)} \frac{r^{r}}{\left(r+d\left(y, x_{1}\right)\right)^{r}} d \mu(y) \\
& \lesssim \frac{1}{V_{r}\left(x_{1}\right)+V\left(x_{1}, x\right)},
\end{aligned}
$$

which is just (2.78). Noticing that $\operatorname{supp} \psi \cap \operatorname{supp}(f \tilde{\omega})=\varnothing$, by the assumption (2.48) of Proposition 2.12 and (2.78), we have

$$
\left|Y_{3}\right|=\left|\int_{x} Y_{3,1}(x) \psi(x) d \mu(x)\right| \lesssim \frac{1}{V_{r}\left(x_{1}\right)}\|\psi\|_{L^{1}(x)}
$$

which is again the desired estimate.

Let $k \in \mathbb{N}$ be large enough, which will be determined later, and let $\left\{S_{k}\right\}_{k \in \mathbb{Z}}$ be an ATI with bounded support as constructed in Theorem 2.6. Let $\eta \in C^{2}(\mathbb{R})$ be radial and $\eta(t)=1$ when $t \in\left[0,2^{3}\right], \eta(t)=0$ when $|t|>2^{4}$ and $0 \leq \eta(t) \leq 1$ for all $t \in \mathbb{R}$. For any $x, y \in \mathbb{R}$, 
we then define $\lambda_{k}(x, y)=\int_{x} S_{k}(x, z) \eta\left(2^{k} d(z, y)\right) d \mu(z)$. It is easy to see that $\lambda_{k}(x, y)=0$ if $d(x, y) \geq 2^{5-k}$, and that

$$
\lambda_{k}(x, y)=\int_{x} S_{k}(x, z) d \mu(z)=1, \quad \text { if } d(x, y)<2^{-k} .
$$

For any fixed $k \in \mathbb{N}$, choose $J \in \mathbb{N}$ so large that $C_{6} 2^{-J} \leq 2^{3-k}$. Put

$$
N_{J}=\left\{i \in I_{J}: Q_{i}^{J} \cap B\left(x, 2^{2-k}\right) \neq \varnothing \text { for some } x \in B\left(x_{1}, 10 r\right)\right\},
$$

and for all $x \in B\left(x_{1}, 10 r\right), N_{J}^{x}=\left\{i \in I_{J}: Q_{i}^{J} \cap B\left(x, 2^{2-k}\right) \neq \varnothing\right\}$, where $Q_{i}^{J}$ is the dyadic cube as in Lemma 2.19. Let $\sharp N_{J}$ and $\sharp N_{J}^{x}$ denote the cardinality of $N_{J}$ and $N_{J}^{x}$, respectively. It is easy to see that if $Q_{i}^{J} \cap B\left(x, 2^{2-k}\right) \neq \varnothing$ for some $x \in B\left(x_{1}, 10 r\right)$, then $Q_{i}^{J} \subset B\left(x_{1}, 2^{4-k}+10 r\right)$ and $B\left(x_{1}, 2^{4-k}+10 r\right) \subset B\left(z_{Q_{i}^{I}}, 2\left(2^{4-k}+10 r\right)\right)$, where $z_{Q_{i}^{I}}$ is the center of $Q_{i}^{J}$ as in Lemma 2.19. From this, and Lemma 2.19(i) and (v), it is easy to see that

$$
\sharp N_{J} \lesssim 2^{n J}\left(r+2^{-k}\right)^{n}
$$

Similarly, $\sharp N_{J}^{x} \lesssim 2^{(J-k) n}$.

We now claim that for any given $k \in \mathbb{N}$ and any given $x \in B\left(x_{1}, 10 r\right)$,

$$
\begin{aligned}
\lambda_{k}(x, \cdot) & =\lim _{J \rightarrow \infty} \sum_{i \in N_{J}} S_{k}\left(x, z_{Q_{i}^{I}}\right) \eta\left(2^{k} d\left(z_{Q_{i}^{I}}, \cdot\right)\right) \mu\left(Q_{i}^{J}\right), \\
1-\lambda_{k}(x, \cdot) & =\lim _{J \rightarrow \infty} \sum_{i \in N_{J}} S_{k}\left(x, z_{Q_{i}^{I}}\right)\left[1-\eta\left(2^{k} d\left(z_{Q_{i}^{I}}, \cdot\right)\right)\right] \mu\left(Q_{i}^{J}\right)
\end{aligned}
$$

hold in $C^{\beta}(x)$.

We only prove (2.85), the proof of (2.86) being similar. To this end, it is easy to see that

$$
\begin{aligned}
\lambda_{k}(x, y) & -\sum_{i \in N_{J}} S_{k}\left(x, z_{Q_{i}^{I}}\right) \eta\left(2^{k} d\left(z_{Q_{i}^{I}}, y\right)\right) \mu\left(Q_{i}^{J}\right) \\
= & \sum_{i \in N_{J}^{x}} \int_{Q_{i}^{J}}\left[S_{k}(x, z)-S_{k}\left(x, z_{Q_{i}^{I}}\right)\right] \eta\left(2^{k} d(z, y)\right) d \mu(z) \\
& +\sum_{i \in N_{J}^{x}} \int_{Q_{i}^{J}} S_{k}\left(x, z_{Q_{i}^{I}}\right)\left[\eta\left(2^{k} d(z, y)\right)-\eta\left(2^{k} d\left(z_{Q_{i}^{I}}, y\right)\right)\right] d \mu(z) \\
\equiv & Y_{4}(y)+Y_{5}(y) .
\end{aligned}
$$


Since $z \in Q_{i}^{J}$, by Lemma 2.19(iv), we have $d\left(z, z_{Q_{i}^{I}}\right) \leq C_{6} 2^{-J}$, which together with the regularity of $S_{k}$ yields

$$
\left|Y_{4}(y)\right| \lesssim \sum_{i \in N_{J}^{x}} \frac{2^{k-J}}{V_{2^{-k}}(x)} \mu\left(Q_{i}^{J}\right) \lesssim \frac{2^{k-J}}{V_{2^{-k}}(x)} \mu\left(B\left(x, 2^{4-k}\right)\right) \lesssim 2^{k-J} \longrightarrow 0
$$

as $J \rightarrow \infty$. Similarly, by the size condition of $S_{k}$ and the mean value theorem, we have

$$
\left|Y_{5}(y)\right| \lesssim 2^{k-J} \frac{1}{V_{2^{-k}}(x)} \sum_{i \in N_{J}^{x}} \mu\left(Q_{i}^{J}\right) \lesssim 2^{k-J} \longrightarrow 0
$$

as $J \rightarrow \infty$. Thus, as $J \rightarrow \infty$,

$$
\left\|\lambda_{k}(x, \cdot)-\sum_{i \in N_{J}} S_{k}\left(x, z_{Q_{i}^{I}}\right) \eta\left(2^{k} d\left(z_{Q_{i}^{I}}, \cdot\right)\right) \mu\left(Q_{i}^{J}\right)\right\|_{L^{\infty}(x)} \longrightarrow 0 .
$$

For any $y, y^{\prime} \in \mathcal{X}$, if $d\left(y, y^{\prime}\right) \geq 2^{k-J}$, by (2.88), we then have

$$
\left|Y_{4}(y)-Y_{4}\left(y^{\prime}\right)\right| \lesssim 2^{(k-J)(1-\beta)} d\left(y, y^{\prime}\right)^{\beta}
$$

If $d\left(y, y^{\prime}\right)<2^{k-J}$, by the regularity of $S_{k}$ and the mean value theorem, we have

$$
\begin{aligned}
\left|Y_{4}(y)-Y_{4}\left(y^{\prime}\right)\right| & =\left|\sum_{i \in N_{J}^{x}} \int_{Q_{i}^{I}}\left[S_{k}(x, z)-S_{k}\left(x, z_{Q_{i}^{I}}\right)\right]\left[\eta\left(2^{k} d(z, y)\right)-\eta\left(2^{k} d\left(z, y^{\prime}\right)\right)\right] d \mu(z)\right| \\
& \lesssim \frac{2^{2 k-J}}{V_{2^{-k}(x)}} d\left(y, y^{\prime}\right) \mu\left(B\left(x, 2^{4-k}\right)\right) \\
& \lesssim 2^{k(3-\beta)-J(2-\beta)} d\left(y, y^{\prime}\right)^{\beta}
\end{aligned}
$$

Similarly, if $d\left(y, y^{\prime}\right) \geq 2^{k-J}$, by (2.89), we then have

$$
\left|Y_{5}(y)-Y_{5}\left(y^{\prime}\right)\right| \lesssim 2^{(k-J)(1-\beta)} d\left(y, y^{\prime}\right)^{\beta}
$$

To estimate $Y_{5}(y)-Y_{5}\left(y^{\prime}\right)$ when $d\left(y, y^{\prime}\right)<2^{k-J}$, by the mean value theorem, we first see that

$$
\begin{aligned}
& \left|\left[\eta\left(2^{k} d(z, y)\right)-\eta\left(2^{k} d\left(z_{Q_{i}^{I}}, y\right)\right)\right]-\left[\eta\left(2^{k} d\left(z, y^{\prime}\right)\right)-\eta\left(2^{k} d\left(z_{Q_{i}^{J}}, y^{\prime}\right)\right)\right]\right| \lesssim 2^{k} d\left(z, z_{Q_{i}^{I}}\right), \\
& \left|\left[\eta\left(2^{k} d(z, y)\right)-\eta\left(2^{k} d\left(z_{Q_{i}^{I}}, y\right)\right)\right]-\left[\eta\left(2^{k} d\left(z, y^{\prime}\right)\right)-\eta\left(2^{k} d\left(z_{Q_{i}^{I}}, y^{\prime}\right)\right)\right]\right| \lesssim 2^{k} d\left(y, y^{\prime}\right) .
\end{aligned}
$$


Taking a suitable geometric mean of these estimates, we find that

$$
\begin{aligned}
& \left|\left[\eta\left(2^{k} d(z, y)\right)-\eta\left(2^{k} d\left(z_{Q_{i}^{I}}, y\right)\right)\right]-\left[\eta\left(2^{k} d\left(z, y^{\prime}\right)\right)-\eta\left(2^{k} d\left(z_{Q_{i}^{I}}, y^{\prime}\right)\right)\right]\right| \\
& \quad \lesssim 2^{k} d\left(y, y^{\prime}\right)^{\beta} d\left(z, z_{Q_{i}^{I}}\right)^{1-\beta} .
\end{aligned}
$$

Using this estimate and the size condition of $S_{k}$, we obtain

$$
\begin{aligned}
\left|Y_{5}(y)-Y_{5}\left(y^{\prime}\right)\right| & =\mid \sum_{i \in N_{J}^{x}} \int_{Q_{i}^{J}} S_{k}\left(x, z_{Q_{i}^{I}}\right)\left\{\left[\eta\left(2^{k} d(z, y)\right)-\eta\left(2^{k} d\left(z_{Q_{i}^{J}}, y\right)\right)\right]\right. \\
& \left.-\left[\eta\left(2^{k} d\left(z, y^{\prime}\right)\right)-\eta\left(2^{k} d\left(z_{Q_{i}^{J}}, y^{\prime}\right)\right)\right]\right\} d \mu(z) \mid \\
& \lesssim 2^{k} d\left(y, y^{\prime}\right)^{\beta} 2^{-J(1-\beta)} \frac{1}{V_{2^{-k}}(x)} \sum_{i \in N_{J}^{x}} \mu\left(Q_{i}^{J}\right) \\
& \lesssim 2^{k} d\left(y, y^{\prime}\right)^{\beta} 2^{-J(1-\beta)} .
\end{aligned}
$$

Thus,

$$
\left\|\lambda_{k}(x, \cdot)-\sum_{i \in N_{J}} S_{k}\left(x, z_{Q_{i}^{I}}\right) \eta\left(2^{k} d\left(z_{Q_{i}^{I}}, \cdot\right)\right) \mu\left(Q_{i}^{J}\right)\right\|_{\dot{C}^{\beta}(\mathcal{X})} \lesssim 2^{k(3-\beta)} 2^{-J(1-\beta)} \longrightarrow 0,
$$

as $J \rightarrow \infty$. This establishes (2.85), and hence also (2.86).

We now decompose $Y_{1}$ into

$$
\begin{aligned}
Y_{1}= & \int_{x} T\left(\tilde{\theta}[f-f(x)]\left[1-\lambda_{k}(x, \cdot)\right]\right)(x) \psi(x) d \mu(x) \\
& +\int_{x} T\left(\tilde{\theta}[f-f(x)] \lambda_{k}(x, \cdot)\right)(x) \psi(x) d \mu(x) \\
\equiv & Y_{1,1}+Y_{1,2},
\end{aligned}
$$

where both integrals have to be interpreted in the sense of "distributions."

To estimate $Y_{1,1}$, by (2.86) and (2.84), observe that

$$
\Upsilon_{1,1}=\lim _{J \rightarrow \infty} \sum_{i \in N_{J}} \int_{x} T\left(\tilde{\theta}[f-f(x)]\left[1-\eta\left(2^{k} d\left(z_{Q_{i}^{I}}, \cdot\right)\right)\right]\right)(x) S_{k}\left(x, z_{Q_{i}^{I}}\right) \psi(x) d \mu(x) \mu\left(Q_{i}^{J}\right)
$$


where the integral has to be interpreted in the sense of "distributions." However, noticing that supp $\tilde{\theta}(\cdot)\left[1-\eta\left(2^{k} d\left(z_{Q_{i}^{I}}, \cdot\right)\right)\right] \cap \operatorname{supp} S_{k}\left(\cdot, z_{Q_{i}^{I}}\right)=\varnothing$, by (2.48), we then further have

$$
\begin{aligned}
Y_{1,1}=\lim _{J \rightarrow \infty} \sum_{i \in N_{J}} \mu\left(Q_{i}^{J}\right) \iint_{\chi \times \chi} K(x, y) \tilde{\theta}(y)[f(y)-f(x)]\left[1-\eta\left(2^{k} d\left(z_{Q_{i}^{I}}, y\right)\right)\right] \\
\quad \times S_{k}\left(x, z_{Q_{i}^{I}}\right) \psi(x) d \mu(x) d \mu(y) .
\end{aligned}
$$

Notice that

$$
\begin{aligned}
& \sum_{i \in N_{J}} \mu\left(Q_{i}^{J}\right) \iint_{\chi \times \mathcal{X}}\left|K(x, y) \tilde{\theta}(y)[f(y)-f(x)]\left[1-\eta\left(2^{k} d\left(z_{Q_{i}^{I}}, y\right)\right)\right]\right|\left|S_{k}\left(x, z_{Q_{i}^{J}}\right) \psi(x)\right| d \mu(x) d \mu(y) \\
& \lesssim \mu\left(B\left(x_{1}, 2^{4-k}+10 r\right)\right) \\
& \times\left(\int _ { x } | \psi ( x ) | \left\{\int_{d(x, y) \leq\left(r+d\left(x_{1}, x\right)\right) / 2}|\tilde{\theta}(y)| \frac{1}{V(x, y)}\left(\frac{d(x, y)}{r+d\left(x_{1}, x\right)}\right)^{\beta}\right.\right. \\
& \times \frac{1}{V_{r}\left(x_{1}\right)+V\left(x, x_{1}\right)}\left(\frac{r}{r+d\left(x_{1}, x\right)}\right)^{r} d \mu(y) \\
& +\int_{\substack{d(x, y)<50 r \\
d(x, y)>\left(r+d\left(x, x_{1}\right)\right) / 2}}|\tilde{\theta}(y)|\left[\frac{1}{V_{r}\left(x_{1}\right)+V\left(x_{1}, y\right)}\left(\frac{r}{r+d\left(x_{1}, y\right)}\right)^{r}\right. \\
& \left.+\frac{1}{V_{r}\left(x_{1}\right)+V\left(x_{1}, x\right)}\left(\frac{r}{r+d\left(x_{1}, x\right)}\right)^{\gamma}\right] \\
& \left.\left.\times \frac{1}{V(x, y)} d \mu(y)\right\} d \mu(x)\right) \\
& \lesssim \mu\left(B\left(x_{1}, 2^{4-k}+10 r\right)\right) \frac{1}{V_{r}\left(x_{1}\right)}\|\psi\|_{L^{1}(x)} .
\end{aligned}
$$

Thus, by Lebesgue's dominated convergence theorem, we have

$$
\begin{aligned}
Y_{1,1} & =\iint_{x \times x} K(x, y) \tilde{\theta}(y)[f(y)-f(x)] \psi(x)\left\{\int_{x}\left[1-\eta\left(2^{k} d(z, y)\right)\right] S_{k}(x, z) d \mu(z)\right\} d \mu(x) d \mu(y) \\
& =\iint_{x_{\times} \times} K(x, y) \tilde{\theta}(y)[f(y)-f(x)] \psi(x)\left[1-\lambda_{k}(x, y)\right] d \mu(x) d \mu(y),
\end{aligned}
$$

where the last integral converges absolutely; and moreover, by (2.82) and an argument similar to $(2.101)$, we further have $\left|Y_{1,1}\right| \lesssim\left(1 / V_{r}\left(x_{1}\right)\right)\|\psi\|_{L^{1}(x)}$. 
Yongsheng Han et al.

We next prove that

$$
\lim _{k \rightarrow \infty} Y_{1,2}=0
$$

making use of our assumption that $T \in \operatorname{WBP}(\beta)$.

To this end, by (2.85) and (2.84), we have

$$
\begin{aligned}
& Y_{1,2}= \lim _{J \rightarrow \infty} \sum_{i \in N_{J}} \mu\left(Q_{i}^{J}\right) \int_{\chi} T\left(\tilde{\theta}[f-f(x)] \eta\left(2^{k} d\left(z_{Q_{i}^{I}}, \cdot\right)\right)\right)(x) S_{k}\left(x, z_{Q_{i}^{I}}\right) \psi(x) d \mu(x) \\
&=\lim _{J \rightarrow \infty} \sum_{i \in N_{J}} \mu\left(Q_{i}^{J}\right)\left\{\int_{x} T\left(\tilde{\theta}\left[f-f\left(z_{Q_{i}^{I}}\right)\right] \eta\left(2^{k} d\left(z_{Q_{i}^{I}}, \cdot\right)\right)\right)(x) S_{k}\left(x, z_{Q_{i}^{I}}\right) \psi(x) d \mu(x)\right. \\
&\left.+\int_{\chi} T\left(\tilde{\theta} \eta\left(2^{k} d\left(z_{Q_{i}^{I}} \cdot\right)\right)\right)(x)\left[f\left(z_{Q_{i}^{J}}\right)-f(x)\right] S_{k}\left(x, z_{Q_{i}^{I}}\right) \psi(x) d \mu(x)\right\} .
\end{aligned}
$$

Choose $k \in \mathbb{N}$ such that $2^{-k} \leq r / 2^{5}$. We claim that

$$
\begin{aligned}
& \left|\int_{x} T\left(\tilde{\theta}\left[f-f\left(z_{Q_{i}^{I}}\right)\right] \eta\left(2^{k} d\left(z_{Q_{i}^{I}} \cdot\right)\right)\right)(x) S_{k}\left(x, z_{Q_{i}^{I}}\right) \psi(x) d \mu(x)\right| \\
& \quad \lesssim \frac{2^{-k \beta}}{r^{\beta}} \frac{1}{V_{r}\left(x_{1}\right)}\|f\|_{\mathcal{G}\left(x_{1}, r, \beta, r\right)}\|\psi\|_{C^{\beta}(x)^{\prime}} \\
& \left|\int_{x} T\left(\tilde{\theta} \eta\left(2^{k} d\left(z_{Q_{i}^{I}}, \cdot\right)\right)\right)(x)\left[f\left(z_{Q_{i}^{I}}\right)-f(x)\right] S_{k}\left(x, z_{Q_{i}^{J}}\right) \psi(x) d \mu(x)\right| \\
& \quad \lesssim \frac{2^{-k \beta}}{r^{\beta}} \frac{1}{V_{r}\left(x_{1}\right)}\|f\|_{\mathcal{G}\left(x_{1}, r, \beta, r\right)}\|\psi\|_{C^{\beta}(x)} .
\end{aligned}
$$

We only show (2.105), the proof of (2.106) being similar.

To see (2.105), put $\phi(y)=\widetilde{\theta}(y)\left[f(y)-f\left(z_{Q_{i}^{I}}\right)\right] \eta\left(2^{k} d\left(z_{Q_{i}^{I}}, y\right)\right)$ and

$$
g(x)=S_{k}\left(x, z_{Q_{i}^{I}}\right) \psi(x)
$$

Notice that if $f, h \in C^{\beta}(\mathcal{X})$, then

$$
\|f h\|_{\dot{C}^{\beta}(\mathcal{X})} \lesssim\|f\|_{L^{\infty}(\mathcal{X})}\|h\|_{\dot{C}^{\beta}(\mathcal{X})}+\|f\|_{\dot{C}^{\beta}(\mathcal{X})}\|h\|_{L^{\infty}(\mathcal{X})} .
$$

From this, it follows that

$$
\|\phi\|_{\mathcal{C}^{\beta}(\mathcal{X})} \lesssim\|f\|_{\mathcal{G}\left(x_{1}, r, \beta, r\right)} \frac{1}{r^{\beta} V_{r}\left(x_{1}\right)}
$$


and that

$$
\|g\|_{\dot{C}^{\beta}(x)} \lesssim\|\psi\|_{C^{\beta}(x)} \frac{1}{V_{2^{-k}}\left(z_{Q_{i}^{I}}\right)} 2^{k \beta}
$$

The estimates (2.109) and (2.110) together with $T \in \mathrm{WBP}(\beta)$ imply the claim (2.105).

By (2.105) and (2.106), we know that if $2^{-k}<r / 2^{5}$, then

$$
\begin{aligned}
\left|Y_{1,2}\right| & \lesssim \frac{2^{-k \beta}}{r^{\beta}} \frac{1}{V_{r}\left(x_{1}\right)}\|f\|_{\mathcal{G}\left(x_{1}, r, \beta, r\right)}\|\psi\|_{C^{\beta}(\mathcal{X})} \lim _{J \rightarrow \infty} \sum_{i \in N_{J}} \mu\left(Q_{i}^{J}\right) \\
& \lesssim \frac{2^{-k \beta}}{r^{\beta}}\|f\|_{\mathcal{G}\left(x_{1}, r, \beta, r\right)}\|\psi\|_{C^{\beta}(\mathcal{X})^{\prime}}
\end{aligned}
$$

which implies (2.103).

Thus, we have that for any $\psi \in C_{b}^{\beta}(\mathcal{X})$ with supp $\psi \subset B\left(x_{1}, 10 r\right),|\langle T f, \psi\rangle| \lesssim\|\psi\|_{L^{1}(\mathcal{X})}$, so that $T f$ agrees with $L^{\infty}(\mathcal{X})$ function on $B\left(x_{1}, 10 r\right)$. Moreover, by $(2.102)$ and Lebesgue's dominated convergence theorem, we have

$$
\begin{aligned}
\langle T f, \psi\rangle= & \iint_{\chi \times X} K(x, y) \psi(x) \tilde{\theta}(y)[f(y)-f(x)] d \mu(y) d \mu(x) \\
& +\iint_{x \times x} K(x, y) \psi(x) \tilde{\omega}(y) f(y) d \mu(y) d \mu(x) \\
& +\int_{x} T(\tilde{\theta})(x) f(x) \psi(x) d \mu(x)
\end{aligned}
$$

as was to be proved.

Proof of Theorem 2.18. By Corollary 2.11(i), we only need to prove Theorem 2.18 for $\mathcal{G}_{b}\left(x_{1}, r\right.$, $\beta, \gamma)$.

Let $f \in \dot{\mathcal{G}}_{b}\left(x_{1}, r, \beta, \gamma\right)$. We first verify that $T f(x)$ satisfies (i) of Definition 2.8 for a.e. $x \in \mathcal{X}$. To this end, we consider two cases.

Case $1\left(d\left(x, x_{1}\right)<10 r\right)$. In this case, let $\tilde{\theta}$ and $\tilde{\omega}$ be as in Lemma 2.20. By Lemma 2.20, for a.e. $x \in B\left(x_{1}, 10 r\right)$, we have

$$
\begin{aligned}
T f(x) & =\int_{x} K(x, y)[f(y)-f(x)] \tilde{\theta}(y) d \mu(y)+\int_{x} K(x, y) f(y) \tilde{\omega}(y) d \mu(y)+f(x) T(\tilde{\theta})(x) \\
& \equiv Z_{1}+Z_{2}+Z_{3} .
\end{aligned}
$$

Lemma 2.15(ii) shows that for a.e. $x \in B\left(x_{1}, 10 r\right),\left|Z_{3}\right| \lesssim|f(x)| \lesssim 1 /\left(V_{r}\left(x_{1}\right)+V\left(x_{1}, x\right)\right)$, which gives the desired estimate. By (2.78), we have $\left|Z_{2}\right| \lesssim 1 /\left(V_{r}\left(x_{1}\right)+V\left(x_{1}, x\right)\right)$. For $Z_{1}$, the facts 
that $\tilde{\theta}(y) \neq 0$ and $d\left(x, x_{1}\right)<10 r$ imply that $d(x, y)<50 r$. The size condition on $K$ and the regularity of $f$ yield that

$$
\begin{aligned}
\left|Z_{1}\right| \leq & \int_{d(x, y) \leq\left(r+d\left(x, x_{1}\right)\right) / 2}|K(x, y)[f(y)-f(x)] \tilde{\theta}(y)| d \mu(y) \\
& +\int_{d(x, y)>\left(r+d\left(x, x_{1}\right)\right) / 2}|K(x, y) f(x) \tilde{\theta}(y)| d \mu(y) \\
& +\int_{d(x, y)>\left(r+d\left(x, x_{1}\right)\right) / 2}|K(x, y) f(y) \tilde{\theta}(y)| d \mu(y) \\
\lesssim & \left\{\frac{1}{r^{\beta}} \frac{1}{V_{r}\left(x_{1}\right)+V\left(x_{1}, x\right)} \int_{d(x, y) \leq 50 r} \frac{d(x, y)^{\beta}}{V(x, y)} d \mu(y)\right. \\
& \left.+\frac{1}{V_{r}\left(x_{1}\right)+V\left(x_{1}, x\right)} \int_{50 r \geq d(x, y)>\left(r+d\left(x, x_{1}\right)\right) / 2,} \frac{1}{d\left(x_{1}, y\right) \leq 40 r} d \mu(y)\right\} \\
& +\int_{50 r \geq d(x, y)>\left(r+d\left(x, x_{1}\right)\right) / 2} \frac{1}{d\left(x_{1}, y\right) \leq 40 r} \frac{1}{V(x, y)} \frac{1}{V_{r}\left(x_{1}\right)+V\left(x_{1}, y\right)} d \mu(y) \\
\equiv & Z_{1,1}+Z_{1,2 .} .
\end{aligned}
$$

By Lemma 2.1(i) and (1.2), we obtain

$$
\begin{aligned}
Z_{1,1} \lesssim \frac{1}{V_{r}\left(x_{1}\right)+V\left(x_{1}, x\right)}\left[1+\frac{V(x, 50 r)}{V(x, r / 2)}\right] \lesssim \frac{1}{V_{r}\left(x_{1}\right)+V\left(x_{1}, x\right)} \\
Z_{1,2} \lesssim \min \left\{\frac{1}{V_{r}\left(x_{1}\right)}, \int_{\begin{array}{c}
r / 2<d(x, y) \leq 50 r, \\
d\left(x, x_{1}\right) / 5 \leq r \leq d\left(x_{1}, y\right)
\end{array}} \frac{1}{V(x, y) V\left(x_{1}, y\right)} d \mu(y)\right. \\
\left.\quad+\frac{1}{V_{r}\left(x_{1}\right)} \int_{\substack{d(x, y)>d\left(x, x_{1}\right) / 2, r>d\left(x_{1}, y\right)}} \frac{1}{V(x, y)} d \mu(y)\right\} \\
\lesssim \frac{1}{V_{r}\left(x_{1}\right)+V\left(x_{1}, x\right)} .
\end{aligned}
$$

Combining the estimates for $Z_{1,1}$ and $Z_{1,2}$ yields the desired estimate for $Z_{1}$, which verifies that $T f(x)$ satisfies (i) of Definition 2.8 when $d\left(x, x_{1}\right)<10 r$.

Case $2\left(d\left(x, x_{1}\right) \equiv R \geq 10 r\right)$. In this case, for any $m \in \mathbb{N}$, let

$$
B_{m}=B\left(x_{1}, 10(m+1) r\right) \backslash B\left(x_{1}, 10 m r\right) .
$$

For any fixed $x_{0} \in B_{m}$, we put $I_{m}(y)=\theta\left(10 d\left(x_{0}, y\right) / 37 m r\right), J_{m}(y)=\theta\left(4 d\left(x_{1}, y\right) / 5 m r\right)$ and define $L_{m}(y)$ by $L_{m}(y)=1-I_{m}(y)-J_{m}(y)$. Notice that if $y \in \operatorname{supp} I_{m} \cap \operatorname{supp} J_{m}$, then 
$d\left(y, x_{0}\right)<37 m r / 5$ and $d\left(y, x_{1}\right)<5 m r / 2$, and hence $d\left(x_{0}, x_{1}\right) \leq d\left(x_{0}, y\right)+d\left(y, x_{1}\right)<$ $(99 / 10) m r<10 m r$, which contracts the choice of $x_{0} \in B_{m}$. Therefore, $L_{m}(y) \geq 0$. We also define $f_{1}(y)=f(y) I_{m}(y), f_{2}(y)=f(y) J_{m}(y)$ and $f_{3}(y)=f(y) L_{m}(y)$. We first establish some estimates on $f_{i}$ with $i=1,2,3$, when $x \in B_{m}$.

Obviously $f_{1}(y) \neq 0$ implies that $d\left(y, x_{0}\right)<37 m r / 5$, and therefore

$$
d\left(x_{1}, y\right) \geq d\left(x_{1}, x_{0}\right)-d\left(x_{0}, y\right)>\frac{3}{25} R
$$

which together with (1.2) and the size condition of $f$ shows that

$$
\left|f_{1}(y)\right| \lesssim \frac{1}{V_{R}\left(x_{1}\right)}\left(\frac{r}{R}\right)^{\gamma}, \quad \forall y \in X
$$

We now claim that

$$
\left|f_{1}(y)-f_{1}\left(y^{\prime}\right)\right| \lesssim \frac{1}{V_{R}\left(x_{1}\right)} \frac{d\left(y, y^{\prime}\right)^{\beta}}{R^{\beta}}\left(\frac{r}{R}\right)^{\gamma}, \quad \forall y, y^{\prime} \in \mathcal{X}
$$

To prove (2.119), we consider two cases.

Case $1\left(d\left(y, y^{\prime}\right) \leq\left(r+d\left(x_{1}, y\right)\right) / 2\right)$. In this case, we divide

$$
f_{1}(y)-f_{1}\left(y^{\prime}\right)=\left[f(y)-f\left(y^{\prime}\right)\right] I_{m}(y) \chi_{0}\left(y, y^{\prime}\right)+f\left(y^{\prime}\right)\left[I_{m}(y)-I_{m}\left(y^{\prime}\right)\right]=Z_{4}+Z_{5},
$$

where $\chi_{0}\left(y, y^{\prime}\right)=\chi_{\left\{y \in X: d\left(x_{0}, y\right)<37 m r / 5\right\}}(y)+X_{\left\{y^{\prime} \in \mathcal{X}: d\left(x_{0}, y^{\prime}\right)<37 m r / 5\right\}}\left(y^{\prime}\right)$.

The regularity of $f$ shows that

$$
\left|Z_{4}\right| \lesssim\left(\frac{d\left(y, y^{\prime}\right)}{r+d\left(x_{1}, y\right)}\right)^{\beta} \frac{1}{V_{r}\left(x_{1}\right)+V\left(x_{1}, y\right)}\left(\frac{r}{r+d\left(x_{1}, y\right)}\right)^{r} x_{0}\left(y, y^{\prime}\right) .
$$

If $x_{0}\left(y, y^{\prime}\right) \neq 0$, then $d\left(x_{0}, y\right)<37 \mathrm{mr} / 5$ or $d\left(x_{0}, y^{\prime}\right)<37 \mathrm{mr} / 5$. Notice that $d\left(y, y^{\prime}\right) \leq(r+$ $\left.d\left(x_{1}, y\right)\right) / 2$ implies that $3 d\left(x_{1}, y\right) / 2 \geq d\left(x_{1}, y^{\prime}\right)-r / 2$. If $d\left(x_{0}, y^{\prime}\right)<37 m r / 5$, we then further have $3 d\left(x_{1}, y\right) / 2 \geq d\left(x_{1}, x_{0}\right)-d\left(x_{0}, y^{\prime}\right)-r / 2>21 \mathrm{mr} / 10>R / 10$, and hence $d\left(x_{1}, y\right)>R / 15$. This together with (2.117) implies that if $x_{0}\left(y, y^{\prime}\right) \neq 0$, then we have

$$
d\left(y, x_{1}\right)>\frac{R}{15}
$$

By (2.122) and (1.2), we obtain $V_{R}\left(x_{1}\right) \lesssim V\left(x_{1}, y\right)$. These estimates prove that $\left|Z_{4}\right| \lesssim$ $\left(1 / V_{R}\left(x_{1}\right)\right)\left(d\left(y, y^{\prime}\right) / R\right)^{\beta}(r / R)^{\gamma}$.

The size condition of $f$ implies that

$$
\left|Z_{5}\right| \lesssim \frac{1}{V_{r}\left(x_{1}\right)+V\left(x_{1}, y^{\prime}\right)}\left(\frac{r}{r+d\left(x_{1}, y^{\prime}\right)}\right)^{r}\left|I_{m}(y)-I_{m}\left(y^{\prime}\right)\right| x_{0}\left(y, y^{\prime}\right)
$$


We now claim that

$$
\frac{1}{V_{r}\left(x_{1}\right)+V\left(x_{1}, y^{\prime}\right)} x_{0}\left(y, y^{\prime}\right) \lesssim \frac{1}{V_{R}\left(x_{1}\right)}
$$

To prove (2.124), we consider two subcases.

Subcase $1.1\left(r \geq d\left(x_{1}, y^{\prime}\right)\right)$. In this case, $\left(1 /\left(V_{r}\left(x_{1}\right)+V\left(x_{1}, y^{\prime}\right)\right)\right) \chi_{0}\left(y, y^{\prime}\right) \lesssim\left(1 / V_{r}\left(x_{1}\right)\right) \chi_{0}\left(y, y^{\prime}\right)$. If $d\left(x_{0}, y^{\prime}\right)<37 m r / 5$, then $d\left(x_{1}, y^{\prime}\right) \geq d\left(x_{1}, x_{0}\right)-d\left(x_{0}, y^{\prime}\right)>13 m r / 5>2 r$, which contradicts the assumption that $r \geq d\left(x_{1}, y^{\prime}\right)$. Thus, we further have

$$
\frac{1}{V_{r}\left(x_{1}\right)+V\left(x_{1}, y^{\prime}\right)} x_{0}\left(y, y^{\prime}\right) \lesssim \frac{1}{V_{r}\left(x_{1}\right)} X_{\left\{y \in X: d\left(x_{0}, y\right)<37 m r / 5\right\}}(y)
$$

Since $d\left(x_{0}, y\right)<37 m r / 5$ and $x \in B_{m}$, by (2.117), we have $d\left(y, x_{1}\right)>3 R / 25$. Moreover, from $d\left(y, x_{1}\right) \leq d\left(y, y^{\prime}\right)+d\left(y^{\prime}, x_{1}\right)$ and $d\left(y, y^{\prime}\right) \leq\left(r+d\left(x_{1}, y\right)\right) / 2$, it follows that $d\left(y, x_{1}\right) \leq r+$ $2 d\left(y^{\prime}, x_{1}\right) \leq 3 r$. Thus, in this case, $R<25 r$ and hence $V_{R}\left(x_{1}\right) \leq V_{r}\left(x_{1}\right)$. Thus, the claim (2.124) holds in this case.

Subcase $1.2\left(r<d\left(x_{1}, y^{\prime}\right)\right)$. In this case, from $r<d\left(x_{1}, y^{\prime}\right)$ and $d\left(y, y^{\prime}\right) \leq\left(r+d\left(x_{1}, y\right)\right) / 2$, it follows that $d\left(x_{1}, y\right) \leq r+2 d\left(x_{1}, y^{\prime}\right)<3 d\left(x_{1}, y^{\prime}\right)$, which together with (2.122) implies that $V_{R}\left(x_{1}\right) \lesssim V\left(x_{1}, y^{\prime}\right)$. Thus, $\left(1 /\left(V_{r}\left(x_{1}\right)+V\left(x_{1}, y^{\prime}\right)\right)\right) x_{0}\left(y, y^{\prime}\right) \lesssim 1 / V_{R}\left(x_{1}\right)$. Thus, (2.124) also holds in this case, which completes the proof of claim (2.124).

Notice that if $d\left(x_{0}, y\right)<37 m r / 5$, then $d\left(y, x_{1}\right) \leq d\left(x_{1}, x_{0}\right)+d\left(x_{0}, y\right) \lesssim R$, and if $d\left(x_{0}, y^{\prime}\right)<37 m r / 5$, since $d\left(y, y^{\prime}\right) \leq\left(r+d\left(y, x_{1}\right)\right) / 2$, we then have $d\left(y, x_{1}\right) \leq r+2 d\left(y^{\prime}, x_{1}\right) \leq$ $r+2\left[d\left(y^{\prime}, x_{0}\right)+d\left(x_{0}, x_{1}\right)\right] \lesssim R$. These estimates together with $d\left(y, y^{\prime}\right) \leq\left(r+d\left(y, x_{1}\right)\right) / 2$ again implies that if $x_{0}\left(y, y^{\prime}\right) \neq 0$, then $d\left(y, y^{\prime}\right) \lesssim R$ and $r+d\left(y, x_{1}\right) \leq 2\left(r+d\left(x_{1}, y^{\prime}\right)\right)$, which together with (2.122) further yields that $r+d\left(x_{1}, y^{\prime}\right) \gtrsim R$. All these estimates, (2.124), and the mean value theorem show that for any $\beta \in(0,1]$,

$$
\left|Z_{5}\right| \lesssim \frac{1}{V_{R}\left(x_{1}\right)}\left(\frac{r}{R}\right)^{\gamma} \frac{\left|d(y, x)-d\left(y^{\prime}, x\right)\right|}{R} \lesssim \frac{1}{V_{R}\left(x_{1}\right)}\left(\frac{d\left(y, y^{\prime}\right)}{R}\right)^{\beta}\left(\frac{r}{R}\right)^{\gamma}
$$

Case $2\left(d\left(y, y^{\prime}\right) \geq\left(r+d\left(y, x_{1}\right)\right) / 2\right)$. In this case, notice again that $f_{1}(y)-f_{1}\left(y^{\prime}\right) \neq 0$ implies that $d\left(x_{0}, y\right)<37 m r / 5$ or $d\left(x_{0}, y^{\prime}\right)<37 m r / 5$. If $d\left(x_{0}, y^{\prime}\right)<37 m r / 5$, by $d\left(y, x_{1}\right)<2 d\left(y, y^{\prime}\right)$, we have $10 m r \leq d\left(x_{0}, x_{1}\right) \leq d\left(x_{0}, y^{\prime}\right)+d\left(y^{\prime}, y\right)+d\left(y, x_{1}\right)<37 m r / 5+3 d\left(y, y^{\prime}\right)$. Thus, by $x \in B_{m}$, we further have $3 R / 25<13 m r / 5<3 d\left(y, y^{\prime}\right)$, which together with (2.117) and $d\left(y, x_{1}\right)<2 d\left(y, y^{\prime}\right)$ again implies that if $f_{1}(y)-f_{1}\left(y^{\prime}\right) \neq 0$ and $d\left(y, y^{\prime}\right) \geq\left(r+d\left(y, x_{1}\right)\right) / 2$, then $R<17 d\left(y, y^{\prime}\right)$. This estimate together with (2.118) yields that for any $\beta>0$,

$$
\left|f_{1}(y)-f_{1}\left(y^{\prime}\right)\right| \leq\left|f_{1}(y)\right|+\left|f_{1}\left(y^{\prime}\right)\right| \lesssim \frac{1}{V_{R}\left(x_{1}\right)}\left(\frac{r}{R}\right)^{\gamma} \lesssim \frac{1}{V_{R}\left(x_{1}\right)}\left(\frac{d\left(y, y^{\prime}\right)}{R}\right)^{\beta}\left(\frac{r}{R}\right)^{\gamma}
$$

Thus, (2.119) holds. 
As for $f_{3}$, first observe that obviously, for all $y \in \mathcal{X}$,

$$
\left|f_{3}(y)\right| \lesssim \frac{1}{V_{r}\left(x_{1}\right)+V\left(x_{1}, y\right)}\left(\frac{r}{r+d\left(y, x_{1}\right)}\right)^{r} X\left\{d\left(x_{1}, y\right)>R / 16\right\}(y)
$$

From (2.128) and Lemma 2.1(i), it follows that

$$
\int_{x}\left|f_{3}(y)\right| d \mu(y) \lesssim r^{\gamma} \int_{d\left(y, x_{1}\right)>R / 16} \frac{1}{V\left(x_{1}, y\right)} \frac{1}{d\left(y, x_{1}\right)^{\gamma}} d \mu(y) \lesssim\left(\frac{r}{R}\right)^{\gamma}
$$

Notice that supp $f_{1} \subset B\left(x_{1}, 137 R / 50\right)$. From this, the estimates (2.118) and (2.129) together with $\int_{x} f(x) d \mu(x)=0$ and (1.2), it follows that

$$
\begin{aligned}
\left|\int_{X} f_{2}(y) d \mu(y)\right| & =\left|\int_{X} f_{1}(y) d \mu(y)+\int_{x} f_{3}(y) d \mu(y)\right| \\
& \lesssim \frac{1}{V_{R}\left(x_{1}\right)}\left(\frac{r}{R}\right)^{\gamma} V_{137 R / 50}\left(x_{1}\right)+\left(\frac{r}{R}\right)^{\gamma} \\
& \lesssim\left(\frac{r}{R}\right)^{\gamma} .
\end{aligned}
$$

Since $x \in B_{m}$, we have $1 /\left(V_{r}\left(x_{1}\right)+V\left(x_{1}, x\right)\right) \sim 1 / V_{R}\left(x_{1}\right)$. Now, for any $m \in \mathbb{N}$ and $y \in \mathcal{X}$, put $U_{m, r}(y)=\theta\left(d\left(x_{1}, y\right) / 42(m+1) r\right)$. Notice that supp $f_{1} \cap \operatorname{supp}\left(1-U_{m, r}\right)=\varnothing$. By Lemma 2.20, for a.e. $x \in B_{m}$, we have

$$
\begin{aligned}
T f_{1}(x) & =\int_{x} K(x, y)\left[f_{1}(y)-f_{1}(x)\right] U_{m, r}(y) d \mu(y)+f_{1}(x) T\left(U_{m, r}\right)(x) \\
& \equiv Z_{6}(x)+Z_{7}(x) .
\end{aligned}
$$

From Lemma 2.15(ii) and (2.118), it follows that for a.e. $x \in B_{m}$,

$$
\left|Z_{7}(x)\right| \lesssim\left|f_{1}(x)\right| \lesssim \frac{1}{V_{R}\left(x_{1}\right)}\left(\frac{r}{R}\right)^{\gamma}
$$

As for $Z_{6}(x)$, notice that $x \in B_{m}$ together with $U_{m, r}(y) \neq 0$ implies that $d(x, y)<18 R$; hence, by (2.119), the size condition on $K$ and Lemma 2.1(i), we obtain

$$
\left|Z_{6}(x)\right| \lesssim \int_{d(x, y)<18 R} \frac{1}{V(x, y)} \frac{1}{V_{R}\left(x_{1}\right)} \frac{d(x, y)^{\beta}}{R^{\beta}}\left(\frac{r}{R}\right)^{\gamma} d \mu(y) \lesssim \frac{1}{V_{R}\left(x_{1}\right)}\left(\frac{r}{R}\right)^{\gamma}
$$

Combining the estimate for $Z_{6}(x)$ with $Z_{7}(x)$ gives us the desired estimate for $T f_{1}(x)$ for a.e. $x \in B_{m}$. 
Since $x \notin \operatorname{supp} f_{2}$, we can write

$$
\begin{aligned}
T f_{2}(x) & =\int_{x}\left[K(x, y)-K\left(x, x_{1}\right)\right] f_{2}(y) d \mu(y)+K\left(x, x_{1}\right) \int_{x} f_{2}(y) d \mu(y) \\
& \equiv Z_{8}(x)+Z_{9}(x) .
\end{aligned}
$$

The assumption (ii) of the theorem, the support condition of $f_{2}$, and the fact that $\gamma<\epsilon$ together with Lemma 2.1(i) yield that

$$
\begin{aligned}
\left|Z_{8}(x)\right| & \leq \int_{d\left(x_{1}, y\right) \leq R / 2}\left|K(x, y)-K\left(x, x_{1}\right)\right|\left|f_{2}(y)\right| d \mu(y) \\
& \lesssim \frac{1}{V\left(x, x_{1}\right) d\left(x, x_{1}\right)^{\epsilon}} \int_{d\left(x_{1}, y\right) \leq R / 2} d\left(x_{1}, y\right)^{\epsilon} \frac{1}{V\left(x_{1}, y\right)} \frac{r^{\gamma}}{d\left(x_{1}, y\right)^{\gamma}} d \mu(y) \\
& \lesssim \frac{1}{V_{R}\left(x_{1}\right)}\left(\frac{r}{R}\right)^{\gamma} .
\end{aligned}
$$

From the size condition on $K$, and (2.130), it also follows that

$$
\left|Z_{9}(x)\right| \lesssim \frac{1}{V\left(x, x_{1}\right)}\left(\frac{r}{R}\right)^{r} \lesssim \frac{1}{V_{R}\left(x_{1}\right)}\left(\frac{r}{R}\right)^{\gamma}
$$

which together with the estimate for $Z_{8}(x)$ gives the desired estimate for $T f_{2}(x)$ for a.e. $x \in$ $B_{m}$.

Notice that $f_{3}(x) \neq 0$ implies that $d\left(x_{1}, y\right)>R / 16$ and $d\left(x_{0}, y\right)>37 m r / 10$. If we now further assume that $x \in B_{m} \cap B\left(x_{0}, m r / 10\right)$, then $d(x, y) \geq d\left(y, x_{0}\right)-d\left(x_{0}, x\right)>18 m r / 5>$ $9 R / 50$. From this, (1.17), and (1.2), it follows that $V_{R}\left(x_{1}\right) \sim V_{R}(x) \lesssim V(x, y)$. This together with (2.128), the size condition on $K$ and Lemma 2.1(i) yields that

$$
\begin{aligned}
\left|T f_{3}(x)\right| & =\left|\int_{\substack{d(x, y)>9 R / 50, d\left(x_{1}, y\right)>R / 16}} K(x, y) f_{3}(y) d \mu(y)\right| \\
& \lesssim \int_{\substack{d(x, y)>9 R / 50, d\left(x_{1}, y\right)>R / 16}} \frac{1}{V(x, y)} \frac{1}{V\left(x_{1}, y\right)} \frac{r^{\gamma}}{d\left(y, x_{1}\right)^{\gamma}} d \mu(y) \\
& \lesssim \frac{1}{V_{R}\left(x_{1}\right)}\left(\frac{r}{R}\right)^{\gamma},
\end{aligned}
$$

which is the desired estimate.

Thus, we have verified that $T f(x)$ for a.e. $x \in B_{m} \cap B\left(x_{0}, m r / 10\right)$ satisfies the size condition (i) of Definition 2.8, with constants independent of $m, r$, and $x_{1}$. By the Besicovitch covering lemma (see, e.g., [13, Theorem 1.16, pages 8-9]), this implies that for a.e. $x \in B_{m}$, $T f(x)$ satisfies the size condition (i) of Definition 2.8, with constants independent of $m, r$, and $x_{1}$. In combination with Case 1 , we thus see that for a.e. $x \in \mathcal{X}, T f(x)$ satisfies the size condition (i) of Definition 2.8. 
We now turn to verify that $T f(x)$ for a.e. $x \in \mathcal{X}$ satisfies the regularity condition (ii) of Definition 2.8. In what follows, we fix $x^{\prime}$ near $x$ and put $\delta \equiv d\left(x, x^{\prime}\right)$. We first remark that for a.e. $x, x^{\prime} \in \mathcal{X}$ such that $(1 / 160)(r+R)<\delta \leq(1 / 2)(r+R)$, by the size condition of $T f(x)$ and Lemma 2.1(iii), we obtain

$$
\begin{aligned}
\left|T f(x)-T f\left(x^{\prime}\right)\right| & \lesssim \frac{1}{V_{r}\left(x_{1}\right)+V\left(x_{1}, x\right)}\left(\frac{r}{r+d\left(x, x_{1}\right)}\right)^{r}+\frac{1}{V_{r}\left(x_{1}\right)+V\left(x_{1}, x^{\prime}\right)}\left(\frac{r}{r+d\left(x^{\prime}, x_{1}\right)}\right)^{r} \\
& \lesssim\left(\frac{d\left(x, x^{\prime}\right)}{r+d\left(x, x_{1}\right)}\right)^{\beta} \frac{1}{V_{r}\left(x_{1}\right)+V\left(x_{1}, x\right)}\left(\frac{r}{r+d\left(x, x_{1}\right)}\right)^{r}
\end{aligned}
$$

which is the desired estimate. In what follows, we only need to estimate $\left|T f(x)-T f\left(x^{\prime}\right)\right|$ for a.e. $x, x^{\prime} \in \mathcal{X}$ such that $\delta \leq(1 / 160)(r+R)$ by considering two cases. Recall that $R \equiv d\left(x, x_{1}\right)$.

Case $1(R<10 r)$. In this case, we divide the ball $B\left(x_{1}, 10 r\right)$ into the union of annuli $B_{m_{1}}=\{x \in$ $\left.x: 10 r /\left(m_{1}+1\right) \leq d\left(x, x_{1}\right)<10 r / m_{1}\right\}$, where $m_{1} \in \mathbb{N}$. For any fixed $x_{0} \in B_{m_{1}}$ and $m_{2} \in \mathbb{N}$, we put $u_{m_{1}, m_{2}}(y)=\theta\left(20 m_{1} m_{2} d\left(x_{0}, y\right) /\left(m_{1}+10\right) r\right)$ and let $w_{m_{1}, m_{2}}(y)=1-\theta_{m_{1}, m_{2}}(y)$ for all $y \in \mathcal{X}$. By Lemmas 2.15 and 2.20, for a.e. $x \in B\left(x_{0},\left(m_{1}+10\right) r / 40 m_{1} m_{2}\right)$,

$$
\begin{aligned}
T f(x)= & \int_{x} K(x, y)[f(y)-f(x)] u_{m_{1}, m_{2}}(y) d \mu(y) \\
& +\left[\int_{x} K(x, y) f(y) w_{m_{1}, m_{2}}(y) d \mu(y)+f(x) T\left(u_{m_{1}, m_{2}}\right)(x)\right] \\
\equiv & \Gamma_{1}(x)+\Gamma_{2}(x), \\
& \left|T\left(u_{m_{1}, m_{2}}\right)(x)\right| \lesssim C_{T}+\|T\|_{\operatorname{WBP}(\beta),} \\
T\left(u_{m_{1}, m_{2}}\right)(x)= & C_{m_{1}, m_{2}, r}-\int_{x}\left[K(x, y)-K\left(x_{0}, y\right)\right] w_{m_{1}, m_{2}}(y) d \mu(y),
\end{aligned}
$$

where $C_{m_{1}, m_{2}, r}$ is a constant independent of $x$. For any

$$
x \in B\left(x_{0}, \frac{\left(m_{1}+11\right) r}{160\left(m_{1}+1\right)\left(m_{2}+1\right)}\right)
$$

we then consider $x^{\prime}$ in the annulus $(r+R) / 160\left(m_{2}+1\right)<d\left(x, x^{\prime}\right) \leq(r+R) / 160 m_{2}$. It is easy to check $x^{\prime} \in B\left(x_{0},\left(m_{1}+10\right) r / 40 m_{1} m_{2}\right)$. Assume that $x, x^{\prime}$ both satisfy (2.139), (2.140), and (2.141). Notice that $u_{m_{1}, m_{2}}(y) \neq 0$ implies that $d\left(y, x_{0}\right) \leq\left(m_{1}+10\right) r / 10 m_{1} m_{2}$, and hence $d(x, y) \leq d\left(x, x_{0}\right)+d\left(x_{0}, y\right)<17\left(m_{1}+11\right) r / 160 m_{1} m_{2}<68 d\left(x, x^{\prime}\right) \equiv 68 \delta$ and $d\left(x^{\prime}, y\right) \leq 69 \delta$. 
Therefore, the size condition on $K$, the regularity of $f$, and Lemma 2.1(i) show that

$$
\begin{aligned}
\left|\Gamma_{1}(x)\right| & =\left|\int_{x} K(x, y)[f(y)-f(x)] u_{m_{1}, m_{2}}(y) d \mu(y)\right| \\
& \leq \int_{d(x, y) \leq 68 \delta}|K(x, y)||f(y)-f(x)| d \mu(y) \\
& \lesssim\left(\frac{d\left(x, x^{\prime}\right)}{r+d\left(x, x_{1}\right)}\right)^{\beta} \frac{1}{V_{r}\left(x_{1}\right) V\left(x_{1}, x\right)}\left(\frac{r}{r+d\left(x_{1}, x\right)}\right)^{r}
\end{aligned}
$$

and a similar estimate also holds for $\Gamma_{1}\left(x^{\prime}\right)$. Thus, we clearly obtain the desired estimate for $\Gamma_{1}(x)-\Gamma_{1}\left(x^{\prime}\right)$.

Now by (2.141), we have

$$
\begin{aligned}
\Gamma_{2}(x) & -\Gamma_{2}\left(x^{\prime}\right) \\
= & \int_{x}\left[K(x, y)-K\left(x^{\prime}, y\right)\right][f(y)-f(x)] w_{m_{1}, m_{2}}(y) d \mu(y) \\
& +f(x)\left\{T\left(u_{m_{1}, m_{2}}\right)(x)+\int_{x}\left[K(x, y)-K\left(x^{\prime}, y\right)\right] w_{m_{1}, m_{2}}(y) d \mu(y)\right\}-f\left(x^{\prime}\right) T\left(u_{m_{1}, m_{2}}\right)\left(x^{\prime}\right) \\
= & \int_{x}\left[K(x, y)-K\left(x^{\prime}, y\right)\right][f(y)-f(x)] w_{m_{1}, m_{2}}(y) d \mu(y)+\left[f(x)-f\left(x^{\prime}\right)\right] T\left(u_{m_{1}, m_{2}}\right)\left(x^{\prime}\right) \\
\equiv & \Gamma_{2,1}+\Gamma_{2,2} .
\end{aligned}
$$

The estimate (2.140) and the regularity of $f$ then show that

$$
\left|\Gamma_{2,2}\right| \lesssim\left|f(x)-f\left(x^{\prime}\right)\right| \lesssim\left(\frac{d\left(x, x^{\prime}\right)}{r+d\left(x, x_{1}\right)}\right)^{\beta} \frac{1}{V_{r}\left(x_{1}\right)+V\left(x_{1}, x\right)}\left(\frac{r}{r+d\left(x_{1}, x\right)}\right)^{r}
$$

Notice that $w_{m_{1}, m_{2}}(y) \neq 0$ implies that $d(x, y)>6 \delta$. The regularity (2.49) on $K$, the regularity and the size condition of $f$, and Lemma 2.1(i) together with the fact that $\beta<\epsilon$ give

$$
\begin{aligned}
\left|\Gamma_{2,1}\right| \lesssim \int_{\substack{d(x, y)>6 \delta, d(x, y) \leq\left(r+d\left(x, x_{1}\right)\right) / 2}} \frac{d\left(x, x^{\prime}\right)^{\epsilon}}{V(x, y) d(x, y)^{\epsilon}}\left(\frac{d(x, y)}{r+d\left(x, x_{1}\right)}\right)^{\beta} \frac{1}{V_{r}\left(x_{1}\right)+V\left(x_{1}, x\right)}\left(\frac{r}{r+d\left(x_{1}, x\right)}\right)^{r} d \mu(y) \\
+\int_{\substack{d(x, y)>6 \delta, d(x, y)>\left(r+d\left(x, x_{1}\right)\right) / 2}} \frac{d\left(x, x^{\prime}\right)^{\epsilon}}{V(x, y) d(x, y)^{\epsilon}} \\
\quad \times\left[\frac{1}{V_{r}\left(x_{1}\right)+V\left(x_{1}, x\right)}\left(\frac{r}{r+d\left(x_{1}, x\right)}\right)^{r}+\frac{1}{V_{r}\left(x_{1}\right)+V\left(x_{1}, y\right)}\left(\frac{r}{r+d\left(x_{1}, y\right)}\right)^{r}\right] d \mu(y) \\
\lesssim\left(\frac{d\left(x, x^{\prime}\right)}{r+d\left(x, x_{1}\right)}\right)^{\beta} \frac{1}{V_{r}\left(x_{1}\right)+V\left(x_{1}, x\right)}\left(\frac{r}{r+d\left(x_{1}, x\right)}\right)^{r},
\end{aligned}
$$


where, in the last inequality, we used that $r+d\left(x_{1}, x\right) \leq 11 r \lesssim r+d\left(x_{1}, y\right)$ and

$$
\frac{1}{V_{r}\left(x_{1}\right)+V\left(x_{1}, y\right)} \leq \frac{1}{V_{r}\left(x_{1}\right)} \lesssim \frac{1}{V_{r}\left(x_{1}\right)+V\left(x_{1}, x\right)}
$$

which follows from $V\left(x_{1}, x\right) \leq \mu\left(B\left(x_{1}, 10 r\right)\right) \lesssim V_{r}\left(x_{1}\right)$, by (1.2). Thus, for any $x_{0} \in B_{m_{1}}$, and a.e. $x \in B\left(x_{0},\left(m_{1}+11\right) r / 160\left(m_{1}+1\right)\left(m_{2}+1\right)\right)$ and a.e. $x^{\prime} \in \mathcal{X}$ satisfying $(r+R) / 160\left(m_{2}+1\right)<$ $d\left(x, x^{\prime}\right) \leq(r+R) / 160 m_{2}, T f(x)-T f\left(x^{\prime}\right)$ has the desired regularity with constants independent of $m_{1}$ and $m_{2}$. Again, by the Besicovitch covering lemma, we further see that for a.e. $x \in B_{m_{1}}$ and a.e. $x^{\prime} \in \mathcal{X}$ satisfying $(r+R) / 160\left(m_{2}+1\right)<d\left(x, x^{\prime}\right) \leq(r+R) / 160 m_{2}, T f(x)-T f\left(x^{\prime}\right)$ has the desired regularity with constants independent of $m_{1}$ and $m_{2}$, which implies that there exists a measurable set $x_{1}$ such that $\mu\left(x_{1}\right)=0$ and for all $x \in B\left(x_{1}, 10 r\right) \backslash X_{1}$ and all $x^{\prime} \in \mathcal{X} \backslash X_{1}$ with $d\left(x, x^{\prime}\right) \leq(1 / 160)(r+R), T f(x)-T f\left(x^{\prime}\right)$ has the desired regularity.

Case $2(R \geq 10 r)$. In this case, we rename $B_{m}$ in Case 2 of the proof for the size condition of $T f$ by $\widetilde{B}_{m_{1}}$. Namely, for $m_{1} \in \mathbb{N}$, we consider annuli

$$
\widetilde{B}_{m_{1}}=B\left(x_{1}, 10\left(m_{1}+1\right) r\right) \backslash B\left(x_{1}, 10 m_{1} r\right) .
$$

For any fixed $\widetilde{x}_{0} \in \widetilde{B}_{m_{1}}$, we define $I_{m_{1}}, J_{m_{1}}$, and $L_{m_{1}}$ in the same way as in Case 1 with $m$ and $x_{0}$ replaced, respectively, by $m_{1}$ and $\tilde{x}_{0}$ here. Let $f_{1}(y)=f(y) I_{m_{1}}(y), f_{2}(y)=f(y) J_{m_{1}}(y)$ and $f_{3}(y)=f(y) L_{m_{1}}(y)$ for all $y \in \mathcal{X}$. Then the estimates (2.119) and (2.130) still hold for $f_{1}, f_{2}$, and $f_{3}$ here, when $x \in \widetilde{B}_{m_{1}}$.

Now, for $m_{2} \in \mathbb{N}$, we put $\tilde{u}_{m_{1}, m_{2}}(y)=\theta\left(20 m_{2} d\left(\tilde{x}_{0}, y\right) /\left(10 m_{1}+11\right) r\right)$ and let $\tilde{w}_{m_{1}, m_{2}}(y)=$ $1-\tilde{\theta}_{m_{1}, m_{2}}(y)$ for all $y \in \mathcal{X}$. By Lemmas 2.15 and 2.20 , for a.e. $x \in B\left(\tilde{x}_{0},\left(10 m_{1}+11\right) r / 40 m_{2}\right)$,

$$
\begin{aligned}
& T f_{1}(x)= \int_{x} K(x, y)\left[f_{1}(y)-f_{1}(x)\right] \tilde{u}_{m_{1}, m_{2}}(y) d \mu(y) \\
&+\left[\int_{x} K(x, y) f_{1}(y) \tilde{w}_{m_{1}, m_{2}}(y) d \mu(y)+f_{1}(x) T\left(\tilde{u}_{m_{1}, m_{2}}\right)(x)\right] \\
& \equiv \Gamma_{3}(x)+\Gamma_{4}(x), \\
&\left|T\left(\tilde{u}_{m_{1}, m_{2}}\right)(x)\right| \lesssim C_{T}+\|T\|_{\operatorname{WBP}(\beta),} \\
& T\left(\tilde{u}_{m_{1}, m_{2}}\right)(x)=\tilde{C}_{m_{1}, m_{2}, r}-\int_{x}\left[K(x, y)-K\left(\tilde{x}_{0}, y\right)\right] \tilde{w}_{m_{1}, m_{2}}(y) d \mu(y),
\end{aligned}
$$

where $\widetilde{C}_{m_{1}, m_{2}, r}$ is a constant independent of $x$. For any $x \in B\left(\tilde{x}_{0},\left(10 m_{1}+1\right) r / 160\left(m_{2}+1\right)\right)$, we then consider $x^{\prime}$ in the annulus $(r+R) / 160\left(m_{2}+1\right)<d\left(x, x^{\prime}\right) \leq(r+R) / 160 m_{2}$. It is easy to check $x^{\prime} \in B\left(\widetilde{x}_{0},\left(10 m_{1}+11\right) r / 40 m_{2}\right)$. We also restrict $x \in \widetilde{B}_{m_{1}}$. Assume that $x, x^{\prime}$ both satisfy (2.149), (2.150), and (2.151). Notice that $\tilde{u}_{m_{1}, m_{2}}(y) \neq 0$ implies that $d\left(y, \tilde{x}_{0}\right) \leq\left(10 m_{1}+\right.$ 11) $r / 10 m_{2}$, which implies $d(x, y) \leq d\left(x, \tilde{x}_{0}\right)+d\left(\tilde{x}_{0}, y\right)<177\left(10 m_{1}+1\right) r / 160 m_{2}<354 d\left(x, x^{\prime}\right) \equiv$ $354 \delta$ and $d\left(x^{\prime}, y\right) \leq 355 \delta$. Therefore, the size condition on $K,(2.119)$, and Lemma 2.1(i) show 
that

$$
\begin{aligned}
\left|\Gamma_{3}(x)\right| & \lesssim \int_{d(x, y) \leq 354 \delta} \frac{1}{V(x, y)} \frac{1}{V_{R}\left(x_{1}\right)} \frac{d(x, y)^{\beta}}{R^{\beta}}\left(\frac{r}{R}\right)^{\gamma} d \mu(y) \\
& \lesssim \frac{1}{V_{R}\left(x_{1}\right)}\left(\frac{d\left(x, x^{\prime}\right)}{R}\right)^{\beta}\left(\frac{r}{R}\right)^{\gamma}
\end{aligned}
$$

and a similar estimate also holds for $\Gamma_{4}\left(x^{\prime}\right)$. Thus, we obtain the desired estimate for $\Gamma_{3}(x)-$ $\Gamma_{4}\left(x^{\prime}\right)$.

Now by (2.151) and some computations similar to $\Gamma_{2}(x)-\Gamma_{2}\left(x^{\prime}\right)$, we have

$$
\begin{aligned}
\Gamma_{4}(x)-\Gamma_{4}\left(x^{\prime}\right)= & \int_{x}\left[K(x, y)-K\left(x^{\prime}, y\right)\right]\left[f_{1}(y)-f_{1}(x)\right] \tilde{w}_{m_{1}, m_{2}}(y) d \mu(y) \\
& +\left[f_{1}(x)-f_{1}\left(x^{\prime}\right)\right] T\left(\tilde{u}_{m_{1}, m_{2}}\right)\left(x^{\prime}\right) \\
\equiv & \Gamma_{4,1}+\Gamma_{4,2} .
\end{aligned}
$$

The estimate (2.150) together with (2.119) then yields

$$
\left|\Gamma_{4,2}\right| \lesssim\left|f_{1}(x)-f_{1}\left(x^{\prime}\right)\right| \lesssim \frac{1}{V_{R}\left(x_{1}\right)}\left(\frac{d\left(x, x^{\prime}\right)}{R}\right)^{\beta}\left(\frac{r}{R}\right)^{\gamma}
$$

Notice that $\widetilde{w}_{m_{1}, m_{2}}(y) \neq 0$ implies $d(x, y)>7 \delta$. Then by the regularity (2.49) on $K,(2.119)$, and Lemma 2.1(i) together with $\beta<\epsilon$, we further have

$$
\begin{aligned}
\left|\Gamma_{4,1}\right| & \lesssim \int_{d(x, y)>7 \delta} \frac{d\left(x, x^{\prime}\right)^{\epsilon}}{V(x, y) d(x, y)^{\epsilon}} \frac{1}{V_{R}\left(x_{1}\right)}\left(\frac{d(x, y)}{R}\right)^{\beta}\left(\frac{r}{R}\right)^{\gamma} d \mu(y) \\
& \lesssim \frac{1}{V_{R}\left(x_{1}\right)}\left(\frac{d\left(x, x^{\prime}\right)}{R}\right)^{\beta}\left(\frac{r}{R}\right)^{\gamma} .
\end{aligned}
$$

Thus, for any $\tilde{x}_{0} \in \widetilde{B}_{m_{1}}$, and a.e. $x \in B\left(\tilde{x}_{0},\left(10 m_{1}+1\right) r / 160\left(m_{2}+1\right)\right) \cap \widetilde{B}_{m_{1}}$ and a.e. $x^{\prime} \in \mathcal{X}$ satisfying $(r+R) / 160\left(m_{2}+1\right)<d\left(x, x^{\prime}\right) \leq(r+R) / 160 m_{2}, T f_{1}(x)-T f_{1}\left(x^{\prime}\right)$ has the desired regularity with constants independent of $m_{1}$ and $m_{2}$.

Notice that $f_{2}(x)=0=f_{2}\left(x^{\prime}\right)$. We write

$$
\begin{aligned}
\left|T f_{2}(x)-T f_{2}\left(x^{\prime}\right)\right|= & \left|\int_{x}\left[K(x, y)-K\left(x^{\prime}, y\right)\right] f_{2}(y) d \mu(y)\right| \\
\leq & \left|\int_{x}\left\{\left[K(x, y)-K\left(x^{\prime}, y\right)\right]-\left[K\left(x, x_{1}\right)-K\left(x^{\prime}, x_{1}\right)\right]\right\} f_{2}(y) d \mu(y)\right| \\
& +\left|K\left(x, x_{1}\right)-K\left(x^{\prime}, x_{1}\right)\right|\left|\int_{x} f_{2}(y) d \mu(y)\right| \\
\equiv & \Gamma_{5}+\Gamma_{6} .
\end{aligned}
$$


Since $d\left(x, x^{\prime}\right) \leq(11 / 1600) R$, the regularity (2.49) on $K,(2.130)$, and $\beta<\epsilon$ prove

$$
\Gamma_{6} \lesssim \frac{d\left(x, x^{\prime}\right)^{\epsilon}}{V\left(x_{1}, x\right) d\left(x_{1}, x\right)^{\epsilon}}\left(\frac{r}{R}\right)^{\gamma} \lesssim \frac{1}{V_{R}\left(x_{1}\right)}\left(\frac{d\left(x, x^{\prime}\right)}{R}\right)^{\beta}\left(\frac{r}{R}\right)^{\gamma}
$$

Notice that $f_{2}(y) \neq 0$ implies that $d\left(x_{1}, y\right) \leq R / 4$. Then the regularity (iii) on $K$ together with the size condition of $f$, the fact that $\gamma<\epsilon$ and Lemma 2.1(i) yields that

$$
\begin{aligned}
\Gamma_{5} & \lesssim \frac{1}{V_{R}\left(x_{1}\right)} \int_{d\left(x_{1}, y\right) \leq R / 4} \frac{d\left(x, x^{\prime}\right)^{\epsilon} d\left(x_{1}, y\right)^{\epsilon}}{d\left(x_{1}, x\right)^{2 \epsilon}} \frac{1}{V_{r}\left(x_{1}\right)+V\left(x_{1}, y\right)}\left(\frac{r}{r+d\left(x_{1}, y\right)}\right)^{\gamma} d \mu(y) \\
& \lesssim \frac{1}{V_{R}\left(x_{1}\right)}\left(\frac{d\left(x, x^{\prime}\right)}{R}\right)^{\beta}\left(\frac{r}{R}\right)^{\gamma} .
\end{aligned}
$$

Thus, we have also obtained the desired regularity for $T f_{2}(x)-T f_{2}\left(x^{\prime}\right)$ for all $x \in \widetilde{B}_{m_{1}}$ and $d\left(x, x^{\prime}\right) \leq(1 / 160)(r+R)$.

Notice that $f_{3}(y) \neq 0$ implies that $d\left(x_{1}, y\right)>R / 16$. Moreover, if

$$
x \in B\left(\tilde{x}_{0}, \frac{\left(10 m_{1}+1\right) r}{160\left(m_{2}+1\right)}\right) \cap \widetilde{B}_{m_{1}}
$$

then $f_{3}(y) \neq 0$ also implies that $d(x, y)>7 R / 40$. Since $\delta \leq(11 / 1600) R$, we then have $\delta \leq$ $(11 / 280) d(x, y)$. Then the regularity (2.49) on $K$, the size condition of $f$, and Lemma 2.1(i) show that

$$
\begin{aligned}
\left|T f_{3}(x)-T f_{3}\left(x^{\prime}\right)\right| & \lesssim \int_{\substack{d\left(x_{1}, y\right)>R / 16 \\
d(x, y)>7 R / 40}}\left|K(x, y)-K\left(x^{\prime}, y\right)\right|\left|f_{3}(y)\right| d \mu(y) \\
& \lesssim \int_{\substack{d\left(x_{1}, y\right)>R / 16, d(x, y)>7 R / 40}} \frac{d\left(x, x^{\prime}\right)^{\epsilon}}{V(x, y) d(x, y)^{\epsilon}} \frac{1}{V_{r}\left(x_{1}\right)+V\left(x_{1}, y\right)} \frac{r^{\gamma}}{d\left(x_{1}, y\right)^{\gamma}} d \mu(y) \\
& \lesssim \frac{1}{V_{R}\left(x_{1}\right)}\left(\frac{d\left(x, x^{\prime}\right)}{R}\right)^{\beta}\left(\frac{r}{R}\right)^{\gamma},
\end{aligned}
$$

where in the last step, we used the fact that $\beta<\epsilon$ and $d\left(x, x^{\prime}\right) \leq(11 / 1600) R$, and in the third-to-last inequality, we used the fact that

$$
V_{R}\left(x_{1}\right) \sim V\left(x, x_{1}\right) \leq \mu\left(B\left(x, \frac{40 d(x, y)}{7}\right)\right) \lesssim V(x, y)
$$

Thus, for all $x \in B\left(\widetilde{x}_{0},\left(10 m_{1}+1\right) r / 160\left(m_{2}+1\right)\right) \cap \widetilde{B}_{m_{1}}$ and $d\left(x, x^{\prime}\right) \leq(1 / 160)(r+R)$, we obtain the desired regularity for $T f_{3}(x)-T f_{3}\left(x^{\prime}\right)$. 
Summarizing the above estimates, we see that for any $\widetilde{x}_{0} \in \widetilde{B}_{m_{1}}$, and a.e. $x \in \widetilde{B}_{m_{1}} \cap$ $B\left(\tilde{x}_{0},\left(10 m_{1}+1\right) r / 160\left(m_{2}+1\right)\right)$ and a.e. $x^{\prime} \in X$ satisfying $(r+R) / 160\left(m_{2}+1\right)<d\left(x, x^{\prime}\right) \leq$ $(r+R) / 160 m_{2}, T f(x)-T f\left(x^{\prime}\right)$ has the desired regularity with constants independent of $m_{1}$ and $m_{2}$. By the Besicovitch covering lemma again, similarly to Case 1, we obtain that there exists a measurable set $\mathcal{X}_{2}$ such that $\mu\left(\mathcal{X}_{2}\right)=0$, and for all $x \in \mathcal{X} \backslash\left(B\left(x_{1}, 10 r\right) \cup \mathcal{X}_{2}\right)$ and all $x^{\prime} \in \mathcal{X} \backslash \mathcal{X}_{2}$ with $d\left(x, x^{\prime}\right) \leq(1 / 160)(r+R), T f(x)-T f\left(x^{\prime}\right)$ has the desired regularity.

Thus, there exists a measurable set $x_{3}$ such that for all $x, x^{\prime} \in \mathcal{X} \backslash \mathcal{X}_{3}$ with $d\left(x, x^{\prime}\right) \leq$ $(1 / 2)\left(r+d\left(x, x_{1}\right)\right), T f(x)-T f\left(x^{\prime}\right)$ satisfies the regularity condition (ii) of Definition 2.8, which implies that for all $x, x^{\prime} \in X \backslash x_{3}$ with $d\left(x, x^{\prime}\right) \leq r / 2$,

$$
\left|T f(x)-T f\left(x^{\prime}\right)\right| \lesssim \frac{1}{V_{r}\left(x_{1}\right) r^{\beta}} d\left(x, x^{\prime}\right)^{\beta}
$$

Now, for any $x \in \mathcal{X}$, we choose $\left\{x_{n}\right\}_{n \in \mathbb{N}} \subset\left(\mathcal{X} \backslash \mathcal{X}_{3}\right)$ such that $d\left(x_{n}, x\right) \rightarrow 0$ as $n \rightarrow \infty$. Then $\left\{T f\left(x_{n}\right)\right\}_{n \in \mathbb{N}}$ is a Cauchy sequence in $\mathbb{C}$. We then define $g(x)=\lim _{n \rightarrow \infty} T f\left(x_{n}\right)$. It is easy to show that $g$ is a well-defined continuous function lying in $\mathcal{G}\left(x_{1}, r, \beta, \gamma\right)$, which agrees with $T f$ almost everywhere. We may thus choose $g$ as a representative of $T f$, for which we then have

$$
\|T f\|_{\mathcal{G}\left(x_{1}, r, \beta, \gamma\right)} \lesssim\left(C_{T}+\|T\|_{\mathrm{WBP}(\beta)}\right)\|f\|_{\mathcal{G}\left(x_{1}, r, \beta, \gamma\right)} .
$$

This completes the proof of Theorem 2.18.

Remark 2.21. It was proved in [35] that the condition (iii) of Theorem 2.18 is also necessary for an operator $T$ with distributional kernel $K$ to be bounded from $\mathcal{G}\left(x_{1}, r, \beta, \gamma\right)$ to $\mathcal{G}\left(x_{1}, r, \beta, \gamma\right)$ for all $x_{1} \in \mathcal{X}$ and $r>0$; see [35, Theorem 4] or [31].

By Remark 2.14(iii), we immediately obtain the following conclusion, which is convenient in applications.

Corollary 2.22. Let $\epsilon \in(0,1], \beta \in(0, \epsilon)$, and let $T$ be as in Proposition 2.12, with the distributional kernel $K$ satisfying (i), (ii), and (iii) of Theorem 2.18. If $T$ is bounded on $L^{p}(X)$ for a certain $p \in$ $(1, \infty)$ and $T(1)=0$ in $\left(\mathcal{C}_{b}^{\beta}(\mathcal{X})\right)^{\prime}$, then $T$ extends to a bounded linear operator from $\mathcal{G}_{(}\left(x_{1}, r, \beta, \gamma\right)$ to $\mathcal{G}\left(x_{1}, r, \beta, \gamma\right)$ for all $x_{1} \in \mathcal{X}, r>0$ and $\gamma \in(0, \epsilon)$. Moreover, there exists a constant $C_{\beta, \gamma}, C_{0}>0$ such that for all $f \in \mathcal{G}\left(x_{1}, r, \beta, \gamma\right)$ with any $x_{1} \in \mathcal{X}$, any $r>0$, and any $\gamma \in(0, \epsilon)$,

$$
\|T f\|_{\mathcal{G}_{(}\left(x_{1}, r, \beta, \gamma\right)} \leq C_{\beta, \gamma, C_{0}}\left(C_{T}+\|T\|_{L^{p}(\mathcal{X}) \rightarrow L^{p}(\mathcal{X})}\right)\|f\|_{\mathcal{G}\left(x_{1}, r, \beta, \gamma\right)} .
$$

By an argument similar to the proof of Lemma 2.20, we can easily obtain the following result, which is of independent interest; see also [69, Lemma 2], [71, Lemma 1, page 119] for $\mathbb{R}^{n}$, and [34, Lemma (3.12)] for Ahlfors 1-regular metric measure spaces. Moreover, instead of $T \in \operatorname{SWBP}(\beta)$ in [34, Lemma (3.12)], we only need that $T \in \mathrm{WBP}(\beta)$.

Corollary 2.23. Let $T$ be as in Lemma 2.20. Then there exists a constant $C>0$ such that for all $f \in \dot{C}_{b}^{\beta}(x)$,

$$
\|T f\|_{L^{\infty}(\mathcal{X})} \leq C\left(C_{T}+\|T\|_{\mathrm{WBP}(\beta)}\right)[\operatorname{diam}(\operatorname{supp} f)]^{\beta}\|f\|_{\dot{C}^{\beta}(\mathcal{X})} .
$$


Proof. Assume that supp $f \subset B\left(x_{0}, r\right)$ for some $x_{0} \in \mathcal{X}$ and $r>0$. Let $\theta_{x_{0}, 10 r}$ be defined in the same way as in Lemma 2.15. An argument similar to the proof of Lemma 2.20 yields that when $d\left(x, x_{0}\right)<5 r$,

$$
T f(x)=\int_{x} K(x, y)[f(y)-f(x)] \theta_{x_{0}, 10 r}(y) d \mu(y)+f(x) \int_{x} K(x, y) \theta_{x_{0}, 10 r}(y) d \mu(y) .
$$

From this, Lemma 2.1(i), and Lemma 2.15(ii) together with (2.55), it follows that

$$
\begin{aligned}
|T f(x)| & \lesssim C_{T}\|f\|_{\dot{C}^{\beta}(x)} \int_{d(x, y)<25 r} \frac{1}{V(x, y)} d(x, y)^{\beta} d \mu(y)+\|f\|_{L^{\infty}(x)}\left(C_{T}+\|T\|_{\mathrm{WBP}(\beta)}\right) \\
& \lesssim\left(C_{T}+\|T\|_{\mathrm{WBP}(\beta)}\right) r^{\beta}\|f\|_{\dot{C}^{\beta}(x)} .
\end{aligned}
$$

If $d\left(x, x_{0}\right) \geq 5 r$ and $d\left(y, x_{0}\right)<r$, then $d(y, x)>4 d\left(x, x_{0}\right) / 5$, and by Lemma 2.1(i) and (2.55), we also have

$$
\begin{aligned}
|T f(x)| & \leq C_{T} \int_{d(x, y)>4 d\left(x, x_{0}\right) / 5} \frac{1}{V(x, y)}|f(y)| d \mu(y) \\
& \lesssim C_{T} \frac{1}{V\left(x_{0}, x\right)}\|f\|_{L^{\infty}(x)} V_{r}\left(x_{0}\right) \\
& \lesssim C_{T}[\operatorname{diam}(\operatorname{supp} f)]^{\beta}\|f\|_{\dot{C}^{\beta}(x)^{\prime}}
\end{aligned}
$$

which completes the proof of Corollary 2.23.

From Theorem 2.18, it is easy to deduce the following result which is convenient in applications.

Corollary 2.24. Let $T$ be as in Theorem 2.18 or Corollary 2.22. If $\widetilde{\epsilon} \in(0, \epsilon)$, then $T$ is bounded from $\mathcal{G}_{0}^{\tilde{e}}\left(x_{1}, r, \beta, \gamma\right)$ to $\mathcal{G}_{0}^{\tilde{\epsilon}}\left(x_{1}, r, \beta, \gamma\right)$ for all $x_{1} \in \mathcal{X}, r>0$, and $0<\beta, \gamma<\tilde{e}$. Moreover, there exists a constant $C>0$ such that for all $f \in \mathcal{G}_{0}^{\tilde{e}}\left(x_{1}, r, \beta, \gamma\right)$ with any $x_{1} \in \mathcal{X}$ and any $r>0$,

$$
\|T f\|_{\mathcal{G}_{0}^{\tilde{e}}\left(x_{1}, r, \beta, \gamma\right)} \leq C_{\beta, \gamma, C_{0}}\left(C_{T}+\|T\|_{\mathrm{WBP}(\beta)}\right)\|f\|_{\mathcal{G}_{0}^{\tilde{e}}\left(x_{1}, r, \beta, \gamma\right)}
$$

(or resp., $\left.\|T f\|_{\mathcal{G}_{0}^{\tilde{e}}\left(x_{1}, r, \beta, r\right)} \leq C_{\beta, r, C_{0}}\left(C_{T}+\|T\|_{L^{p}(\mathcal{X}) \rightarrow L^{p}(\mathcal{X})}\right)\|f\|_{\mathcal{G}_{0}^{\tilde{\varepsilon}}\left(x_{1}, r, \beta, r\right)}\right)$.

Proof. Let $f \in \mathcal{\mathcal { G }}_{0}^{\tilde{\varepsilon}}\left(x_{1}, r, \beta, \gamma\right)$. By the definition of $\mathcal{\mathcal { C }}_{0}^{\tilde{\varepsilon}}\left(x_{1}, r, \beta, \gamma\right)$, there exists

$$
\left\{f_{n}\right\}_{n=1}^{\infty} \subset \grave{G}\left(x_{1}, r, \widetilde{\epsilon}, \widetilde{\epsilon}\right)
$$

such that

$$
\lim _{n \rightarrow \infty}\left\|f_{n}-f\right\|_{\mathcal{G}\left(x_{1}, r, \beta, r\right)}=0 .
$$


By Theorem 2.18 or Corollary 2.22, we have $T f_{n} \in \mathcal{G}\left(x_{1}, r, \widetilde{\epsilon}, \widetilde{\epsilon}\right)$ and

$$
\left\|T f_{n}-T f\right\|_{\mathcal{G}\left(x_{1}, r, \beta, r\right)} \lesssim\left\|f-f_{n}\right\|_{\mathcal{G}\left(x_{1}, r, \beta, r\right)} \longrightarrow 0,
$$

as $n \rightarrow \infty$. Thus, $T f \in \mathcal{G}_{0}^{\tilde{e}}\left(x_{1}, r, \beta, \gamma\right)$ and

$$
\|T f\|_{\mathcal{G}_{0}^{\tilde{\epsilon}}\left(x_{1}, r, \beta, \gamma\right)}=\|T f\|_{\mathcal{G}_{\left(x_{1}, r, \beta, \gamma\right)}} \lesssim\|f\|_{\mathcal{G}\left(x_{1}, r, \beta, \gamma\right)}=\|f\|_{\mathcal{G}_{0}^{\tilde{f}}\left(x_{1}, r, \beta, \gamma\right)},
$$

which completes the proof of Corollary 2.24.

\subsection{Boundedness of singular integrals on spaces of test functions}

In this subsection, we establish the boundedness on $\mathcal{G}\left(x_{1}, r, \beta, \gamma\right)$ of singular integrals. Since the functions in $\mathcal{G}\left(x_{1}, r, \beta, \gamma\right)$ may have nonvanishing integral, this requires, as usual, some extra size decay conditions on the integral kernels; see, for example, [71, page 123].

The following result is an inhomogeneous variant of Proposition 2.12.

Proposition 2.25. Let $\beta \in(0,1], \sigma>0, r_{0}>0$, and let $T$ be as in Proposition 2.12 with the distributional kernel $K$ satisfying the following extra size condition that for all $x, y \in \mathcal{X}$ with $d(x, y) \geq r_{0}$,

$$
|K(x, y)| \leq C_{T} \frac{1}{V(x, y)} \frac{r_{0}^{\sigma}}{d(x, y)^{\sigma}}
$$

Then $T$ can be extended to a continuous linear operator from $C^{\beta}(\mathcal{X})$ to $\left(C_{b}^{\beta}(\mathcal{X})\right)^{\prime}$.

Proof. Let $f \in C^{\beta}(\mathcal{X})$ and $g \in C_{b}^{\beta}(\mathcal{X})$, and suppose supp $g \subset B\left(x_{0}, r\right)$ for some $x_{0} \in \mathcal{X}$ and $r>0$. Choose $\psi \in C_{b}^{\beta}(x)$ such that $\psi(x)=1$ when $x \in B\left(x_{0}, 2 \max \left\{r, r_{0}\right\}\right)$ and $\psi(x)=0$ when $x \notin B\left(x_{0}, 4 \max \left\{r, r_{0}\right\}\right)$. It is easy to see that $\psi f \in C_{b}^{\beta}(\mathcal{X})$, which implies that $\langle T(\psi f), g\rangle$ is well defined. On the other hand, we define

$$
\langle T((1-\psi) f), g\rangle=\iint_{\chi \times \mathcal{X}} K(x, y)(1-\psi(y)) f(y) g(x) d \mu(y) d \mu(x) .
$$

By the size condition (2.174) and Lemma 2.1(i), it is easy to check that the right-hand side of the above equality is finite; furthermore, if $f$ has also bounded support, this coincides with (2.48). Moreover, it is easy to verify that $\langle T(\psi f), g\rangle+\langle T((1-\psi) f), g\rangle$ is independent of choice of $\psi$. Thus we can define $T f$ by

$$
\langle T f, g\rangle=\langle T(\psi f), g\rangle+\langle T((1-\psi) f), g\rangle,
$$

so that $T f \in\left(C_{b}^{\beta}(\mathcal{X})\right)^{\prime}$. It is clear that then $T$ is continuous, which completes the proof of Proposition 2.25. 
We now establish an inhomogeneous variant of Lemma 2.15. Here, in contrast to Lemma 2.15, we do not need $T \in \mathrm{WBP}(\beta)$.

Lemma 2.26. Let $\theta$ be as in Lemma 2.15. For any fixed $z \in X$ and $r>0$, let $\theta_{z, \max \left\{r, r_{0}\right\}}$ and $\omega_{z, \max \left\{r, r_{0}\right\}}$ be defined as in Lemma 2.15. Let $T$ be as in Proposition 2.25. If, for a certain $\beta \in(0, \epsilon]$, $T(1) \in\left(C_{b}^{\beta}(x)\right)^{\prime}$ is a constant $C_{T(1)}$, then there exists a constant $C>0$ such that

(i) for all $x \in B\left(z, \max \left\{r, r_{0}\right\} / 2\right),\left|T\left(\omega_{z, \max \left\{r, r_{0}\right\}}\right)(x)\right| \leq C C_{T}$;

(ii) the restriction of the linear functional $T\left(\theta_{z, \max \left\{r, r_{0}\right\}}\right)+T\left(\omega_{z, \max \left\{r, r_{0}\right\}}\right) \in\left(C_{b}^{\beta}(x)\right)^{\prime}$ to the ball $B\left(z, \max \left\{r, r_{0}\right\} / 2\right)$ is a constant; namely, for all $x \in B\left(z, \max \left\{r, r_{0}\right\} / 2\right)$,

$$
T\left(\theta_{z, \max \left\{r, r_{0}\right\}}\right)(x)+T\left(\omega_{z, \max \left\{r, r_{0}\right\}}\right)(x)=C_{T(1)} ;
$$

(iii) for all $x \in B\left(z, \max \left\{r, r_{0}\right\} / 2\right),\left|T\left(\theta_{z, \max \left\{r, r_{0}\right\}}\right)(x)\right| \leq C\left(C_{T}+\left|C_{T(1)}\right|\right)$.

Proof. By the definitions of $\theta_{z, \max \left\{r, r_{0}\right\}}$ and $\omega_{z, \max \left\{r, r_{0}\right\}}$, we know that

$$
\omega_{z, \max \left\{r, r_{0}\right\}}(y) \neq 0
$$

implies that $d(y, z)>\max \left\{r, r_{0}\right\}$, which together with $d(x, z)<\max \left\{r, r_{0}\right\} / 2$ shows that $d(x, y)>\max \left\{r, r_{0}\right\} / 2$. Therefore, by (2.174) and Lemma 2.1(i), for all $x \in \mathcal{X}$ with $d(x, z)<$ $\max \left\{r, r_{0}\right\} / 2$, we have

$$
\begin{aligned}
\left|T\left(\omega_{z, \max \left\{r, r_{0}\right\}}\right)(x)\right| & =\left|\int_{d(x, y)>\max \left\{r, r_{0}\right\} / 2} K(x, y) \omega_{z, \max \left\{r, r_{0}\right\}}(y) d \mu(y)\right| \\
& \leq C_{T} \int_{d(x, y)>\max \left\{r, r_{0}\right\} / 2} \frac{1}{V(x, y)} \frac{r_{0}^{\sigma}}{d(x, y)^{\sigma}} d \mu(y) \\
& \leq C C_{T},
\end{aligned}
$$

which is (i).

To see (ii), for any $f \in \mathcal{G}_{b}(\beta, \gamma)$ with supp $f \subset B\left(z, \max \left\{r, r_{0}\right\} / 2\right)$, by the definition of $T(1)=C_{T(1)}$ in $\left(C_{b}^{\beta}(x)\right)^{\prime}$, we have

$$
C_{T(1)} \int_{x} f(x) d \mu(x)=\left\langle T\left(\theta_{z, \max \left\{r, r_{0}\right\}}\right), f\right\rangle+\left\langle T\left(\omega_{z, \max \left\{r, r_{0}\right\}}\right), f\right\rangle
$$

which together with Corollary 2.11(ii) gives the conclusion (ii) of this lemma.

The conclusion (iii) can be deduced from the conclusion (i) and the conclusion (ii), which completes the proof of Lemma 2.26.

We recall the notion of the space $\operatorname{bmo}(\mathcal{X})$, which, when $\mathcal{X}=\mathbb{R}^{n}$ and $\mu$ is the $n$ dimensional Lebesgue measure, was first introduced by Goldberg [73]. A variant in the setting of spaces of homogeneous type can be found in [74]. 
Definition 2.27. Let $1 \leq q<\infty$. The space $\operatorname{bmo}_{q}(x)$ is defined to be the set of all $f \in L_{\mathrm{loc}}^{q}(x)$ such that

$$
\begin{aligned}
\|f\|_{\mathrm{bmo}_{q}(\mathcal{X})}= & \sup _{x \in \mathcal{X}, 0<r<1}\left\{\frac{1}{\mu(B(x, r))} \int_{B(x, r)}\left|f(y)-m_{B(x, r)}(f)\right|^{q} d \mu(y)\right\}^{1 / q} \\
& +\sup _{x \in \mathcal{X}, r \geq 1}\left\{\frac{1}{\mu(B(x, r))} \int_{B(x, r)}|f(y)|^{q} d \mu(y)\right\}^{1 / q}
\end{aligned}
$$

is finite. When $q=1$, one denotes bmo $_{1}(\mathcal{X})$ simply by bmo $(\mathcal{X})$.

It was proved in [74] that for any $1 \leq q_{1}, q_{2}<\infty, \operatorname{bmo}_{q_{1}}(\mathcal{X})=\operatorname{bmo}_{q_{2}}(\mathcal{X})$ with equivalent norms.

Remark 2.28. From Corollary 2.11(ii), Proposition 5.46, Theorems 5.44(i) and 6.28 below, it is easy to see that $T(1)=C_{T(1)}$ in $\left(C_{b}^{\beta}(\mathcal{X})\right)^{\prime}$ if and only if $T(1)=C_{T(1)}$ in $\operatorname{bmo}(\mathcal{X})$.

We can now present the inhomogeneous variant of Theorem 2.18 as follows.

Theorem 2.29. Let $\epsilon \in(0,1], \sigma>0, r_{0}>0$, and let $T$ be as in Proposition 2.25 with the distributional kernel $K$ also satisfying the conditions (i) through (iii) in Theorem 2.18 and the following additional regularity condition: for all $x, x^{\prime}, y \in X$ with $d\left(x, x^{\prime}\right) \leq d(x, y) / 2$ and $d(x, y) \geq r_{0}$,

$$
\left|K(x, y)-K\left(x^{\prime}, y\right)\right| \leq C_{T} \frac{1}{V(x, y)}\left(\frac{d\left(x, x^{\prime}\right)}{d(x, y)}\right)^{\epsilon}\left(\frac{r_{0}}{d(x, y)}\right)^{\sigma}
$$

If $T \in \operatorname{WBP}(\beta)$ for a certain $\beta \in(0, \epsilon)$ and $T(1)=C_{T(1)}$ in $\left(C_{b}^{\beta}(x)\right)^{\prime}$, then $T$ extends to a bounded linear operator from $\mathcal{G}_{1}\left(x_{1}, r_{0}, \beta, \gamma\right)$ to itself for any $x_{1} \in \mathcal{X}$ and any $\gamma \in(0, \sigma] \cap(0, \epsilon)$. Moreover, there is a constant $C_{\beta, \gamma, \sigma, C_{0}}>0$ such that for all $f \in \mathcal{G}\left(x_{1}, r_{0}, \beta, \gamma\right)$ with any $x_{1} \in \mathcal{X}$ and any $\gamma \in(0, \sigma] \cap(0, \epsilon)$,

$$
\|T f\|_{\mathcal{G}\left(x_{1}, r_{0}, \beta, \gamma\right)} \leq C_{\beta, \gamma, \sigma, C_{0}}\left(C_{T}+\left|C_{T(1)}\right|\right)\|f\|_{\left.\mathcal{G}_{\left(x_{1}, r_{0}, \beta, \gamma\right.}\right)} .
$$

Proof. We prove Theorem 2.29 by essentially following the same argument as the proof of Theorem 2.18, and we only give an outline. We use the notations as in the proof of Theorem 2.18. By Corollary 2.11(ii), we only need to prove Theorem 2.29 for $\mathcal{G}_{b}\left(x_{1}, r_{0}, \beta, \gamma\right)$.

Let $f \in \mathcal{G}_{b}\left(x_{1}, r_{0}, \beta, \gamma\right)$. We first verify that $T f(x)$ for a.e. $x \in \mathcal{X}$ satisfies Definition 2.8(i) by considering two cases.

Case $1\left(d\left(x, x_{1}\right)<10 r_{0}\right)$. In this case, let $\theta_{x_{1}, 20 r_{0}}$ and $\omega_{x_{1}, 20 r_{0}}$ be defined in the same way as in Lemma 2.15. Instead of Lemma 2.15(ii) by Lemma 2.26(iii), following the procedure of Lemma 2.20 (here we need $T \in \mathrm{WBP}(\beta)$ ), for a.e. $x_{0} \in B\left(x_{1} 10 r_{0}\right)$, we have

$$
\begin{aligned}
T f(x)= & \int_{x} K(x, y)[f(y)-f(x)] \theta_{x_{1}, 20 r_{0}}(y) d \mu(y) \\
& +\int_{x} K(x, y) f(y) \omega_{x_{1}, 20 r_{0}}(y) d \mu(y)+f(x) T\left(\theta_{x_{1}, 20 r_{0}}\right)(x) .
\end{aligned}
$$


Using Lemma 2.26(iii) to replace Lemma 2.15(ii) and following the same proof as that of Theorem 2.18 give the desired estimate of this case.

Case $2\left(d\left(x, x_{1}\right) \equiv R \geq 10 r_{0}\right)$. In this case, we use all the notation as in the proof of Theorem 2.18, but with $r$ replaced by $r_{0}$. The estimates (2.118), (2.119), (2.128), and (2.129) with $r$ replaced by $r_{0}$ hold in the current setting. Instead of (2.130), by Lemma 2.1(ii), we now have

$$
\left|\int_{x} f_{2}(y) d \mu(y)\right| \lesssim 1
$$

Replacing Lemma 2.15(ii) by Lemma 2.26(iii) and using $T \in \mathrm{WBP}(\beta)$, by following the proof of Lemma 2.20, for a.e. $x \in B_{m}$, we have

$$
T f_{1}(x)=\int_{x} K(x, y)\left[f_{1}(y)-f_{1}(x)\right] U_{m, r_{0}}(y) d \mu(y)+f_{1}(x) T\left(U_{m, r_{0}}\right)(x) .
$$

Then Lemma 2.26(iii) together with an argument similar to the proof of Theorem 2.18 gives the desired estimate for $T f_{1}(x)$ in this case.

Since $x \notin \operatorname{supp} f_{2}$, we can write

$$
\begin{aligned}
T f_{2}(x) & =\int_{x}\left[K(x, y)-K\left(x, x_{1}\right)\right] f_{2}(y) d \mu(y)+K\left(x, x_{1}\right) \int_{x} f_{2}(y) d \mu(y) \\
& \equiv Z_{8}(x)+Z_{9}(x) .
\end{aligned}
$$

The estimate for $Z_{8}(x)$ is as in the proof of Theorem 2.18. Since $R \geq 10 r_{0}$ and $\gamma \leq \sigma$, by (2.174) and (2.185), we have

$$
\left|Z_{9}(x)\right| \lesssim \frac{1}{V_{R}\left(x_{1}\right)}\left(\frac{r_{0}}{R}\right)^{\sigma} \lesssim \frac{1}{V_{R}\left(x_{1}\right)}\left(\frac{r_{0}}{R}\right)^{\gamma}
$$

which is the desired estimate.

The estimate for $T f_{3}(x)$ in this case is also similar to the proof of Theorem 2.18. Thus, $T(f)(x)$ for a.e. $x \in \mathcal{X}$ satisfies Definition 2.2(i).

To verify that $T(f)(x)$ for a.e. $x \in \mathcal{X}$ satisfies Definition 2.2(ii), similarly to the proof of Theorem 2.18, we can always assume that $d\left(x, x^{\prime}\right) \leq(1 / 160)\left(r_{0}+d\left(x, x_{1}\right)\right)$, and we only need to consider the following two cases.

Case $1\left(R<10 r_{0}\right)$. In this case, if we replace Lemma 2.15(i) and (ii), respectively, by Lemma 2.26(ii) and (iii), the same proof as in the proof of Theorem 2.18 gives the desired estimates. 
Case $2\left(R \geq 10 r_{0}\right)$. In this case, the same argument as in the proof of Theorem 2.18 together with Lemma 2.15 replaced by Lemma 2.26 yields the desired estimates for $T f_{1}(x)$ and $T f_{3}(x)$. Noticing that $f_{2}(x)=0=f_{2}\left(x^{\prime}\right)$, we now estimate $T f_{2}$ by writing

$$
\begin{aligned}
\left|T f_{2}(x)-T f_{2}\left(x^{\prime}\right)\right|= & \left|\int_{x}\left[K(x, y)-K\left(x^{\prime}, y\right)\right] f_{2}(y) d \mu(y)\right| \\
\leq & \left|\int_{x}\left\{\left[K(x, y)-K\left(x^{\prime}, y\right)\right]-\left[K\left(x, x_{1}\right)-K\left(x^{\prime}, x_{1}\right)\right]\right\} f_{2}(y) d \mu(y)\right| \\
& +\left|K\left(x, x_{1}\right)-K\left(x^{\prime}, x_{1}\right)\right|\left|\int_{x} f_{2}(y) d \mu(y)\right| \\
\equiv & \Gamma_{5}+\Gamma_{6} .
\end{aligned}
$$

The estimate for $\Gamma_{5}$ is as in the proof of Theorem 2.18. To estimate $\Gamma_{6}$, by (2.182) and (2.185) together with $\gamma \leq \sigma$, we have

$$
\Gamma_{6} \lesssim \frac{1}{V\left(x, x_{1}\right)}\left(\frac{d\left(x, x^{\prime}\right)}{d\left(x, x_{1}\right)}\right)^{\epsilon}\left(\frac{r_{0}}{d\left(x, x_{1}\right)}\right)^{\sigma} \lesssim \frac{1}{V_{R}\left(x_{1}\right)}\left(\frac{d\left(x, x^{\prime}\right)}{R}\right)^{\beta}\left(\frac{r_{0}}{R}\right)^{\gamma}
$$

which is the desired estimate. Then an extension via the Besicovitch covering lemma as in the proof of Theorem 2.18 completes the proof of Theorem 2.29.

Remark 2.30. A regularity condition similar to (2.182) also appears in [71, page 123]. This additional regularity assumption is used only in the estimate of $\Gamma_{6}$. Instead of (2.182) by requiring $\gamma \in(0, \sigma(\epsilon-\beta) / \epsilon]$, we can obtain a similar conclusion. In fact, since $d\left(x^{\prime}, x_{1}\right) \geq$ $d\left(x, x_{1}\right)-d\left(x, x^{\prime}\right) \geq 189 r_{0} / 20 \geq r_{0}$ and $d\left(x^{\prime}, x_{1}\right) \geq 189 R / 200 \geq R / 2$, by (2.174) and (2.185), we have

$$
\Gamma_{6} \lesssim \frac{1}{V\left(x, x_{1}\right)}\left(\frac{r_{0}}{d\left(x, x_{1}\right)}\right)^{\sigma}+\frac{1}{V\left(x^{\prime}, x_{1}\right)}\left(\frac{r_{0}}{d\left(x^{\prime}, x_{1}\right)}\right)^{\sigma} \lesssim \frac{1}{V\left(x, x_{1}\right)}\left(\frac{r_{0}}{d\left(x, x_{1}\right)}\right)^{\sigma}
$$

On the other hand, by (2.49) and (2.185), we also have $\Gamma_{6} \lesssim\left(1 / V\left(x, x_{1}\right)\right)\left(d\left(x, x^{\prime}\right) / d\left(x, x_{1}\right)\right)^{\epsilon}$. Combining both estimates yields that

$$
\Gamma_{6} \lesssim \frac{1}{V\left(x, x_{1}\right)}\left(\frac{d\left(x, x^{\prime}\right)}{d\left(x, x_{1}\right)}\right)^{\beta}\left(\frac{r_{0}}{d\left(x, x_{1}\right)}\right)^{\sigma(1-\beta / \epsilon)} \lesssim \frac{1}{V_{R}\left(x_{1}\right)}\left(\frac{d\left(x, x^{\prime}\right)}{R}\right)^{\beta}\left(\frac{r_{0}}{R}\right)^{\gamma}
$$

which is the desired estimate.

By Remark 2.14(iii) again, we can obtain the following conclusion which is convenient in applications. 
Corollary 2.31. Let $\epsilon \in(0,1], \sigma>0, r_{0}>0$, and let $T$ be as in Proposition 2.25 with the distributional kernel $K$ also satisfying Theorem 2.18(i) through (iii) and (2.182). If $T$ is bounded on $L^{p}(X)$ for a certain $p \in(1, \infty)$ and $T(1)=C_{T(1)}$ in $\left(C_{b}^{\beta}(X)\right)^{\prime}$, then $T$ extends to a bounded linear operator from $\mathcal{G}\left(x_{1}, r_{0}, \beta, \gamma\right)$ to itself for any $x_{1} \in \mathcal{X}$, any $\beta \in(0, \epsilon)$, and any $\gamma \in(0, \sigma] \cap(0, \epsilon)$. Moreover, there is a constant $C_{\beta, \gamma, \sigma, C_{0}}>0$ such that for all $f \in \mathcal{G}\left(x_{1}, r_{0}, \beta, \gamma\right)$ with any $x_{1} \in \mathcal{X}$ and any $\gamma \in(0, \sigma] \cap(0, \epsilon)$,

$$
\|T f\|_{\mathcal{G}\left(x_{1}, r_{0}, \beta, \gamma\right)} \leq C_{\beta, \gamma, \sigma, C_{0}}\left(C_{T}+\left|C_{T(1)}\right|\right)\|f\|_{\mathcal{G}_{\left(x_{1}, r_{0}, \beta, \gamma\right)}} .
$$
details.

The proof of the following corollary is similar to that of Corollary 2.24. We omit the

Corollary 2.32. Let $T$ be as in Theorem 2.29 or Corollary 2.31. If $\widetilde{\epsilon} \in(0, \epsilon)$, then $T$ is bounded from $\mathcal{G}_{0}^{\tilde{e}}\left(x_{1}, r_{0}, \beta, \gamma\right)$ to $\mathcal{G}_{0}^{\tilde{e}}\left(x_{1}, r_{0}, \beta, \gamma\right)$ for all $x_{1}, \beta, \gamma$ as in Theorem 2.29. Moreover, there exists a constant $C_{\beta, \gamma, \sigma, C_{0}}>0$ such that for all $f \in \mathcal{G}_{0}^{\tilde{E}}\left(x_{1}, r_{0}, \beta, \gamma\right)$ with any $x_{1} \in \mathcal{X}$,

$$
\|T f\|_{\mathcal{G}\left(x_{1}, r_{0}, \beta, \gamma\right)} \leq C_{\beta, \gamma, \sigma, C_{0}}\left(C_{T}+\left|C_{T(1)}\right|\right)\|f\|_{\mathcal{G}\left(x_{1}, r_{0}, \beta, \gamma\right)} .
$$

\section{Continuous Calderón reproducing formulae}

From now on till the end of this paper, we will always assume that $\mathcal{X}$ is an RD-space. In this section, using Corollaries 2.22 and 2.31, we establish homogeneous and inhomogeneous Calderón reproducing formulae.

\subsection{Homogeneous continuous Calderón reproducing formulae}

In this subsection, we always assume that $\operatorname{diam}(\mathcal{X})=\infty$.

Proposition 3.1. Let $\left\{S_{k}\right\}_{k \in \mathbb{Z}}$ be an $\left(\epsilon_{1}, \epsilon_{2}, \epsilon_{3}\right)$-ATI with $\epsilon_{1} \in(0,1], \epsilon_{2}>0$, and $\epsilon_{3}>0$ and let $S_{k}^{t}$ be the adjoint operator of $S_{k}$ for any $k \in \mathbb{Z}$. Then the following hold.

(i) For $p \in(1, \infty)$ and any $f \in L^{p}(\mathcal{X}),\left\|S_{k} f\right\|_{L^{p}(x)} \rightarrow 0$ when $k \rightarrow-\infty$.

(ii) Let $D_{k}=S_{k}-S_{k-1}$ for $k \in \mathbb{Z}$. Then $\sum_{k=-\infty}^{\infty} D_{k}=I$ in $L^{p}(\mathcal{X})$ for $p \in(1, \infty)$, where $I$ is the identity on $L^{p}(X)$.

(iii) Properties (i) and (ii) also hold when $S_{k}$ is replaced by $S_{k}^{t}$.

Proof. Let $1 / p+1 / q=1$. Then Definition 2.2(i), Hölder's inequality, (1.2) and (1.3) together with $\mu(X)=\infty$ yield that

$$
\begin{aligned}
\left|S_{k} f(x)\right| & \lesssim \frac{1}{V_{2^{-k}}(x)} \int_{d(x, y)<2^{-k}}|f(y)| d \mu(y)+\int_{d(x, y) \geq 2^{-k}} \frac{1}{V(x, y)}|f(y)| d \mu(y) \\
& \lesssim\left\{\frac{1}{V_{2^{-k}}(x)^{1 / p}}+\left[\sum_{l=0}^{\infty} \int_{2^{l} 2^{-k} \leq d(x, y)<2^{l+1} 2^{-k}} \frac{1}{V(x, y)^{q}} d \mu(y)\right]^{1 / q}\right\}\|f\|_{L^{p}(x)} \\
& \lesssim \frac{1}{V_{2^{-k}}(x)^{1 / p}}\|f\|_{L^{p}(x)} \\
& \longrightarrow 0
\end{aligned}
$$


as $k \rightarrow-\infty$. This together with Proposition 2.7(ii) and Lebesgue's dominated convergence theorem implies that $\left\|S_{k} f\right\|_{L^{p}(\mathcal{X})} \rightarrow 0$ when $k \rightarrow-\infty$.

Property (ii) is a simple corollary of Property (i) together with Proposition 2.7(iv). Property (iii) follows by symmetry, which completes the proof of Proposition 3.1.

Before we establish the continuous Calderón reproducing formulae, we need a technical lemma. We recall that we denote $\min \{a, b\}$ by $a \wedge b$ for any $a, b \in \mathbb{R}$.

Lemma 3.2. Let $\epsilon_{1} \in(0,1], \epsilon_{2}>0, \epsilon_{3}>0,\left\{S_{k}\right\}_{k \in \mathbb{Z}}$, and let $\left\{E_{k}\right\}_{k \in \mathbb{Z}}$ be two $\left(\epsilon_{1}, \epsilon_{2}, \epsilon_{3}\right)$-ATIs. Let $P_{k}=S_{k}-S_{k-1}$ and $Q_{k}=E_{k}-E_{k-1}$ for $k \in \mathbb{Z}$. Then for any $\epsilon_{1}^{\prime} \in\left(0, \epsilon_{1} \wedge \epsilon_{2}\right)$, there exist positive constants $C$ (depending on $\epsilon_{1}^{\prime}, \epsilon_{1}, \epsilon_{2}$, and $\epsilon_{3}$ ), $\delta$ (depending on $\epsilon_{1}^{\prime}, \epsilon_{1}$, and $\epsilon_{2}$ ), and $\sigma$ (depending on $\epsilon_{1}^{\prime}, \epsilon_{1}, \epsilon_{2}$, and $\left.\epsilon_{3}\right)$ such that $P_{l} Q_{k}(x, y)$, the kernel of $P_{l} Q_{k}$, satisfies the following estimates that for all $x, y \in X$ and all $k, l \in \mathbb{Z}$,

$$
\left|P_{l} Q_{k}(x, y)\right| \leq C 2^{-|k-l| \epsilon_{1}^{\prime}} \frac{1}{V_{2^{-(k \wedge l)}}(x)+V_{2^{-(k \wedge l)}}(y)+V(x, y)} \frac{2^{-(k \wedge l) \epsilon_{2}}}{\left(2^{-(k \wedge l)}+d(x, y)\right)^{\epsilon_{2}}}
$$

for $d\left(y, y^{\prime}\right) \leq(1 / 4) d(x, y)$ and all $k, l \in \mathbb{Z}$,

$$
\begin{aligned}
& \left|P_{l} Q_{k}(x, y)-P_{l} Q_{k}\left(x, y^{\prime}\right)\right| \\
& \quad \leq C 2^{-|k-l| \delta}\left(\frac{d\left(y, y^{\prime}\right)}{2^{-(l \wedge k)}+d(x, y)}\right)^{\epsilon_{1}^{\prime}} \frac{1}{V_{2^{-(k \wedge l)}}(x)+V_{2^{-(k \wedge l)}}(y)+V(x, y)} \frac{2^{-(k \wedge l) \epsilon_{2}}}{\left(2^{-(k \wedge l)}+d(x, y)\right)^{\epsilon_{2}}}
\end{aligned}
$$

for $d\left(x, x^{\prime}\right) \leq(1 / 4) d(x, y)$ and all $k, l \in \mathbb{Z}$,

$$
\begin{aligned}
& \left|P_{l} Q_{k}(x, y)-P_{l} Q_{k}\left(x^{\prime}, y\right)\right| \\
& \quad \leq C 2^{-|k-l| \delta}\left(\frac{d\left(x, x^{\prime}\right)}{2^{-(l \wedge k)}+d(x, y)}\right)^{\epsilon_{1}^{\prime}} \frac{1}{V_{2^{-(k \wedge l)}}(x)+V_{2^{-(k \wedge l)}}(y)+V(x, y)} \frac{2^{-(k \wedge l) \epsilon_{2}}}{\left(2^{-(k \wedge l)}+d(x, y)\right)^{\epsilon_{2}}}
\end{aligned}
$$

and for $d\left(x, x^{\prime}\right) \leq(1 / 8) d(x, y), d\left(y, y^{\prime}\right) \leq(1 / 8) d(x, y)$, and all $k, l \in \mathbb{Z}$,

$$
\begin{aligned}
& \left|\left[P_{l} Q_{k}(x, y)-P_{l} Q_{k}\left(x^{\prime}, y\right)\right]-\left[P_{l} Q_{k}\left(x, y^{\prime}\right)-P_{l} Q_{k}\left(x^{\prime}, y^{\prime}\right)\right]\right| \\
& \leq C 2^{-|k-l| \delta}\left(\frac{d\left(x, x^{\prime}\right)}{2^{-(l \wedge k)}+d(x, y)}\right)^{\epsilon_{1}^{\prime}}\left(\frac{d\left(y, y^{\prime}\right)}{2^{-(l \wedge k)}+d(x, y)}\right)^{\epsilon_{1}^{\prime}} \\
& \quad \times \frac{1}{V_{2^{-(k \wedge l)}}(x)+V_{2^{-(k \wedge l)}}(y)+V(x, y)} \frac{2^{-(k \wedge l) \sigma}}{\left(2^{-(k \wedge l)}+d(x, y)\right)^{\sigma}} .
\end{aligned}
$$

Proof. By symmetry, we may assume that $k \geq l$.

Noticing that

$$
\int_{x} Q_{k}(x, y) d \mu(x)=0,
$$


we can write

$$
\begin{aligned}
\left|P_{l} Q_{k}(x, y)\right|= & \left|\int_{x}\left[P_{l}(x, z)-P_{l}(x, y)\right] Q_{k}(z, y) d \mu(z)\right| \\
\leq & \int_{d(z, y) \leq\left(2^{-l}+d(x, y)\right) / 2}\left|P_{l}(x, z)-P_{l}(x, y)\right|\left|Q_{k}(z, y)\right| d \mu(z) \\
& +\int_{d(z, y)>\left(2^{-l}+d(x, y)\right) / 2}\left|P_{l}(x, z)\right|\left|Q_{k}(z, y)\right| d \mu(z) \\
& +\left|P_{l}(x, y)\right| \int_{d(z, y)>\left(2^{-l}+d(x, y)\right) / 2}\left|Q_{k}(z, y)\right| d \mu(z) \\
\equiv & Z_{1}+Z_{2}+Z_{3} .
\end{aligned}
$$

The regularity of $P_{l}$ and the size condition of $Q_{k}$ together with Lemma 2.1(i) show that for any $\epsilon_{1}^{\prime} \in\left(0, \epsilon_{1} \wedge \epsilon_{2}\right)$,

$$
\begin{aligned}
Z_{1} \lesssim & 2^{-k \epsilon_{1}^{\prime}} \frac{1}{V_{2^{-l}}(x)+V_{2^{-l}}(y)+V(x, y)} \frac{2^{-l \epsilon_{2}}}{\left(2^{-l}+d(x, y)\right)^{\epsilon_{1}+\epsilon_{2}}} \\
& \times \int_{d(z, y) \leq\left(2^{-l}+d(x, y)\right) / 2} d(z, y)^{\epsilon_{1}-\epsilon_{1}^{\prime}} \frac{1}{V(z, y)} d \mu(y) \\
\lesssim & 2^{-(k-l) \epsilon_{1}^{\prime}} \frac{1}{V_{2^{-l}}(x)+V_{2^{-l}}(y)+V(x, y)} \frac{2^{-l \epsilon_{2}}}{\left(2^{-l}+d(x, y)\right)^{\epsilon_{2}}} .
\end{aligned}
$$

The size conditions for $P_{l}$ and $Q_{k}$ together with Lemma 2.1(i) prove that

$$
\begin{aligned}
Z_{2} & \lesssim \frac{1}{V_{2^{-l}}(x)} \frac{2^{-k \epsilon_{2}}}{\left(2^{-l}+d(x, y)\right)^{\epsilon_{1}^{\prime}}} \int_{d(z, y)>\left(2^{-l}+d(x, y)\right) / 2} \frac{1}{V(z, y)} \frac{1}{d(z, y)^{\epsilon_{2}-\epsilon_{1}^{\prime}}} d \mu(z) \\
& \lesssim 2^{-(k-l) \epsilon_{2}} \frac{1}{V_{2^{-l}}(x)} \frac{2^{-l \epsilon_{2}}}{\left(2^{-l}+d(x, y)\right)^{\epsilon_{2}}}, \\
Z_{2} & \lesssim \frac{2^{-k \epsilon_{2}}}{\left(2^{-l}+d(x, y)\right)^{\epsilon_{2}}} \int_{d(z, y)>\left(2^{-l}+d(x, y)\right) / 2}\left|P_{l}(x, z)\right| \frac{1}{V(z, y)} d \mu(z) \\
& \lesssim 2^{-(k-l) \epsilon_{2}} \frac{1}{V_{2^{-l}}(y)+V(x, y)} \frac{2^{-l \epsilon_{2}}}{\left(2^{-l}+d(x, y)\right)^{\epsilon_{2}}},
\end{aligned}
$$

where in the last step, we used Proposition 2.7(i) and the fact that $1 / V(z, y) \lesssim 1 /\left(V_{2^{-l}}(y)+\right.$ $V(x, y))$, by $d(z, y)>\left(2^{-l}+d(x, y)\right) / 2$ together with (1.2). Thus,

$$
Z_{2} \lesssim 2^{-(k-l) \epsilon_{1}^{\prime}} \frac{1}{V_{2^{-l}}(x)+V_{2^{-l}}(y)+V(x, y)} \frac{2^{-l \epsilon_{2}}}{\left(2^{-l}+d(x, y)\right)^{\epsilon_{2}}}
$$


Similarly, by Lemma 2.1(v),

$$
\begin{aligned}
Z_{3} & \lesssim \frac{1}{V_{2^{-l}}(x)+V_{2^{-l}}(y)+V(x, y)} \int_{d(z, y)>\left(2^{-l}+d(x, y)\right) / 2} \frac{1}{V_{2^{-k}}(y)+V(z, y)} \frac{2^{-k \epsilon_{2}}}{\left(2^{-k}+d(z, y)\right)^{\epsilon_{2}}} d \mu(z) \\
& \lesssim 2^{-(k-l) \epsilon_{2}} \frac{1}{V_{2^{-l}}(x)+V_{2^{-l}}(y)+V(x, y)} \frac{2^{-l \epsilon_{2}}}{\left(2^{-l}+d(x, y)\right)^{\epsilon_{2}}},
\end{aligned}
$$

which completes the proof of (3.2).

The proofs for (3.3) and (3.4) are similar and we only verify (3.3). To this end, it suffices to prove

$$
\left|P_{l} Q_{k}(x, y)-P_{l} Q_{k}\left(x, y^{\prime}\right)\right| \lesssim\left(\frac{d\left(y, y^{\prime}\right)}{2^{-l}+d(x, y)}\right)^{\epsilon_{1}} \frac{1}{V_{2^{-l}}(x)+V_{2^{-l}}(y)+V(x, y)} \frac{2^{-l \epsilon_{2}}}{\left(2^{-l}+d(x, y)\right)^{\epsilon_{2}}}
$$

for $d\left(y, y^{\prime}\right) \leq(1 / 4) d(x, y)$. To see this, by (3.2), for any $\epsilon_{1}^{\prime} \in\left(0, \epsilon_{1} \wedge \epsilon_{2}\right)$,

$$
\begin{aligned}
\left|P_{l} Q_{k}(x, y)-P_{l} Q_{k}\left(x, y^{\prime}\right)\right| \lesssim 2^{-(k-l) \epsilon_{1}^{\prime}} & {\left[\frac{1}{V_{2^{-l}}(x)+V_{2^{-l}}(y)+V(x, y)} \frac{2^{-l \epsilon_{2}}}{\left(2^{-l}+d(x, y)\right)^{\epsilon_{2}}}\right.} \\
& \left.+\frac{1}{V_{2^{-l}}(x)+V_{2^{-l}}\left(y^{\prime}\right)+V\left(x, y^{\prime}\right)} \frac{2^{-l \epsilon_{2}}}{\left(2^{-l}+d\left(x, y^{\prime}\right)\right)^{\epsilon_{2}}}\right]
\end{aligned}
$$

The assumption that $d\left(y, y^{\prime}\right) \leq(1 / 4) d(x, y)$ together with Lemma 2.1(iii) further yields that

$$
\left|P_{l} Q_{k}(x, y)-P_{l} Q_{k}\left(x, y^{\prime}\right)\right| \lesssim 2^{-(k-l) \epsilon_{1}^{\prime}} \frac{1}{V_{2^{-l}}(x)+V_{2^{-l}}(y)+V(x, y)} \frac{2^{-l \epsilon_{2}}}{\left(2^{-l}+d(x, y)\right)^{\epsilon_{2}}}
$$

Let $\sigma \in(0,1)$. Then the geometric mean between (3.12) and (3.14) gives

$$
\begin{aligned}
& \left|P_{l} Q_{k}(x, y)-P_{l} Q_{k}\left(x, y^{\prime}\right)\right| \\
& \quad=\left|P_{l} Q_{k}(x, y)-P_{l} Q_{k}\left(x, y^{\prime}\right)\right|^{\sigma}\left|P_{l} Q_{k}(x, y)-P_{l} Q_{k}\left(x, y^{\prime}\right)\right|^{1-\sigma} \\
& \quad \lesssim 2^{-(k-l) \epsilon_{1}^{\prime}(1-\sigma)}\left(\frac{d\left(y, y^{\prime}\right)}{2^{-l}+d(x, y)}\right)^{\sigma \epsilon_{1}} \frac{1}{V_{2^{-l}}(x)+V_{2^{-l}}(y)+V(x, y)} \frac{2^{-l \epsilon_{2}}}{\left(2^{-l}+d(x, y)\right)^{\epsilon_{2}}}
\end{aligned}
$$

which is (3.3). 
We now verify (3.12). By (3.6), we can write

$$
\begin{aligned}
\left|P_{l} Q_{k}(x, y)-P_{l} Q_{k}\left(x, y^{\prime}\right)\right| & =\left|\int_{x}\left[P_{l}(x, z)-P_{l}(x, y)\right]\left[Q_{k}(z, y)-Q_{k}\left(z, y^{\prime}\right)\right] d \mu(z)\right| \\
& \leq \sum_{i=1}^{3} \int_{W_{i}}\left|P_{l}(x, z)-P_{l}(x, y)\right|\left|Q_{k}(z, y)-Q_{k}\left(z, y^{\prime}\right)\right| d \mu(z) \\
& \equiv \sum_{i=4}^{6} Z_{i}
\end{aligned}
$$

where

$$
\begin{aligned}
& W_{1}=\left\{z \in X: d\left(y, y^{\prime}\right) \leq \frac{2^{-l}+d(x, y)}{4} \leq \frac{2^{-k}+d(z, y)}{2}\right\} \\
& W_{2}=\left\{z \in X: d\left(y, y^{\prime}\right) \leq \frac{2^{-k}+d(z, y)}{2} \leq \frac{2^{-l}+d(x, y)}{4}\right\} \\
& W_{3}=\left\{z \in X: d\left(y, y^{\prime}\right) \geq \frac{2^{-k}+d(z, y)}{2}\right\}
\end{aligned}
$$

The regularity for $Q_{k}$ and the size condition for $P_{l}$ together with the assumption $k \geq l$ implies that

$$
\begin{aligned}
& Z_{4} \lesssim \int_{W_{1}} {\left[\left|P_{l}(x, z)\right|+\frac{1}{V_{2^{-l}}(x)+V_{2^{-l}}(y)+V(x, y)} \frac{2^{-l \epsilon_{2}}}{\left(2^{-l}+d(x, y)\right)^{\epsilon_{2}}}\right] } \\
& \times \frac{d\left(y, y^{\prime}\right)^{\epsilon_{1}}}{\left(2^{-k}+d(z, y)\right)^{\epsilon_{1}}} \frac{1}{V_{2^{-k}}(z)+V_{2^{-k}}(y)+V(z, y)} \frac{2^{-k \epsilon_{2}}}{\left(2^{-k}+d(z, y)\right)^{\epsilon_{2}}} d \mu(z) \\
& \equiv Z_{4,1}+Z_{4,2} .
\end{aligned}
$$

We now claim that if $k \geq l$ and $2^{-l}+d(x, y) \lesssim 2^{-k}+d(z, y)$, then for all $x, y, z \in x$,

$$
\frac{1}{V_{2^{-k}}(y)+V(z, y)} \lesssim \frac{1}{V_{2^{-l}}(y)+V(x, y)}
$$

In fact, if $2^{-k} \geq d(z, y)$, then $2^{-l}+d(x, y) \lesssim 2^{-k}+d(z, y) \lesssim 2^{-k}$, and therefore $V_{2^{-l}}(y) \lesssim$ $V_{2^{-k}}(y)$ and $V(x, y) \sim V(y, x) \lesssim V_{2^{-k}}(y)$. Thus, (3.19) holds in this case. If $2^{-k}<d(z, y)$, then $2^{-l}+d(x, y) \lesssim d(z, y)$ and hence, $V_{2^{-l}}(y) \lesssim V(y, z) \sim V(z, y)$ and $V(x, y) \sim V(y, x) \lesssim V(z, y)$, which verifies (3.19). Proposition 2.7(i) and the estimate (3.19) together with $2^{-l}+d(x, y) \leq$ $2\left(2^{-k}+d(z, y)\right)$ and $k \geq l$ imply that

$$
\begin{aligned}
Z_{4,1} & \lesssim \frac{d\left(y, y^{\prime}\right)^{\epsilon_{1}}}{\left(2^{-l}+d(x, y)\right)^{\epsilon_{1}}} \frac{1}{V_{2^{-l}}(y)+V(x, y)} \frac{2^{-l \epsilon_{2}}}{\left(2^{-l}+d(x, y)\right)^{\epsilon_{2}}} \int_{x}\left|P_{l}(x, z)\right| d \mu(z) \\
& \lesssim \frac{d\left(y, y^{\prime}\right)^{\epsilon_{1}}}{\left(2^{-l}+d(x, y)\right)^{\epsilon_{1}}} \frac{1}{V_{2^{-l}}(y)+V(x, y)} \frac{2^{-l \epsilon_{2}}}{\left(2^{-l}+d(x, y)\right)^{\epsilon_{2}}}
\end{aligned}
$$


and the size condition of $P_{l}$ together with Lemma 2.1(i) yields that

$$
\begin{aligned}
Z_{4,1} & \lesssim \frac{d\left(y, y^{\prime}\right)^{\epsilon_{1}}}{\left(2^{-l}+d(x, y)\right)^{\epsilon_{1}}} \frac{1}{V_{2^{-l}}(x)}\left\{\int_{d(z, y) \leq 2^{-k}}+\int_{d(z, y)>2^{-k}} W_{1^{\prime}}\right\} \frac{1}{V_{2^{-k}}(y)+V(z, y)} \frac{2^{-k \epsilon_{2}}}{\left(2^{-k}+d(z, y)\right)^{\epsilon_{2}}} d \mu(z) \\
& \lesssim \frac{d\left(y, y^{\prime}\right)^{\epsilon_{1}}}{\left(2^{-l}+d(x, y)\right)^{\epsilon_{1}}} \frac{1}{V_{2^{-l}}(x)} \frac{2^{-l \epsilon_{2}}}{\left(2^{-l}+d(x, y)\right)^{\epsilon_{2}}} .
\end{aligned}
$$

Similarly, using Lemma 2.1(ii), we have

$$
\begin{aligned}
Z_{4,2} \lesssim & \frac{d\left(y, y^{\prime}\right)^{\epsilon_{1}}}{\left(2^{-l}+d(x, y)\right)^{\epsilon_{1}}} \frac{1}{V_{2^{-l}}(x)+V_{2^{-l}}(y)+V(x, y)} \frac{2^{-l \epsilon_{2}}}{\left(2^{-l}+d(x, y)\right)^{\epsilon_{2}}} \\
& \times \int_{W_{1}} \frac{1}{V_{2^{-k}}(y)+V(z, y)} \frac{2^{-k \epsilon_{2}}}{\left(2^{-k}+d(z, y)\right)^{\epsilon_{2}}} d \mu(z) \\
\lesssim & \frac{d\left(y, y^{\prime}\right)^{\epsilon_{1}}}{\left(2^{-l}+d(x, y)\right)^{\epsilon_{1}}} \frac{1}{V_{2^{-l}}(x)+V_{2^{-l}}(y)+V(x, y)} \frac{2^{-l \epsilon_{2}}}{\left(2^{-l}+d(x, y)\right)^{\epsilon_{2}}}
\end{aligned}
$$

which completes the estimate for $Z_{4}$.

The regularities of $P_{l}$ and $Q_{k}$ and Lemma 2.1(ii) show that

$$
\begin{aligned}
Z_{5} \lesssim \int_{W_{2}} \frac{d(y, z)^{\epsilon_{1}}}{\left(2^{-l}+d(x, y)\right)^{\epsilon_{1}}} \frac{1}{V_{2^{-l}}(x)+V_{2^{-l}}(y)+V(x, y)} \frac{2^{-l \epsilon_{2}}}{\left(2^{-l}+d(x, y)\right)^{\epsilon_{2}}} \\
\quad \times \frac{d\left(y, y^{\prime}\right)^{\epsilon_{1}}}{\left(2^{-k}+d(y, z)\right)^{\epsilon_{1}}} \frac{1}{V_{2^{-k}}(y)+V_{2^{-k}}(z)+V(z, y)} \frac{2^{-k \epsilon_{2}}}{\left(2^{-k}+d(z, y)\right)^{\epsilon_{2}}} d \mu(z) \\
\lesssim \frac{d\left(y, y^{\prime}\right)^{\epsilon_{1}}}{\left(2^{-l}+d(x, y)\right)^{\epsilon_{1}}} \frac{1}{V_{2^{-l}}(x)+V_{2^{-l}}(y)+V(x, y)} \frac{2^{-l \epsilon_{2}}}{\left(2^{-l}+d(x, y)\right)^{\epsilon_{2}}} .
\end{aligned}
$$

From $(1 / 4) d(x, y) \geq d\left(y, y^{\prime}\right) \geq(1 / 2)\left(2^{-k}+d(z, y)\right)$, it follows that $d(z, y) \leq(1 / 2) d(x, y) \leq$ $(1 / 2)\left(2^{-l}+d(x, y)\right)$. Then the regularity of $P_{l}$ and the size condition of $Q_{k}$ together with Proposition 2.7(i) and $d(y, z) \leq 2 d\left(y, y^{\prime}\right)$ prove that

$$
\begin{aligned}
Z_{6} \lesssim & \frac{1}{V_{2^{-l}}(x)+V_{2^{-l}}(y)+V(x, y)} \frac{2^{-l \epsilon_{2}}}{\left(2^{-l}+d(x, y)\right)^{\epsilon_{2}}} \\
& \times \int_{W_{3}} \frac{d(y, z)^{\epsilon_{1}}}{\left(2^{-l}+d(x, y)\right)^{\epsilon_{1}}}\left[\left|Q_{k}(z, y)\right|+\left|Q_{k}\left(z, y^{\prime}\right)\right|\right] d \mu(z) \\
& \lesssim \frac{d\left(y, y^{\prime}\right)^{\epsilon_{1}}}{\left(2^{-l}+d(x, y)\right)^{\epsilon_{1}}} \frac{1}{V_{2^{-l}}(x)+V_{2^{-l}}(y)+V(x, y)} \frac{2^{-l \epsilon_{2}}}{\left(2^{-l}+d(x, y)\right)^{\epsilon_{2}}}
\end{aligned}
$$

Thus, (3.12) holds and this finishes the proof of (3.3). 
We finally prove (3.5) in the case $k \geq l$. To this end, it suffices to verify that for any $\epsilon_{1}^{\prime} \in\left(0, \epsilon_{1} \wedge \epsilon_{2}\right), d\left(x, x^{\prime}\right) \leq(1 / 8) d(x, y)$ and $d\left(y, y^{\prime}\right) \leq(1 / 8) d(x, y)$,

$$
\begin{aligned}
& \left|\left[P_{l} Q_{k}(x, y)-P_{l} Q_{k}\left(x^{\prime}, y\right)\right]-\left[P_{l} Q_{k}\left(x, y^{\prime}\right)-P_{l} Q_{k}\left(x^{\prime}, y^{\prime}\right)\right]\right| \\
& \quad \lesssim \frac{d\left(x, x^{\prime}\right)^{\epsilon_{1}^{\prime}}}{\left(2^{-l}+d(x, y)\right)^{\epsilon_{1}^{\prime}}} \frac{d\left(y, y^{\prime}\right)^{\epsilon_{1}^{\prime}}}{\left(2^{-l}+d(x, y)\right)^{\epsilon_{1}^{\prime}}} \frac{1}{V_{2^{-l}}(x)+V_{2^{-l}}(y)+V(x, y)} \frac{2^{-l \sigma}}{\left(2^{-l}+d(x, y)\right)^{\sigma}},
\end{aligned}
$$

where $\sigma=\left(\epsilon_{2}-\epsilon_{1}^{\prime}\right) \wedge \epsilon_{3}>0$. To see this, the estimate (3.4) implies that for any $\epsilon_{1}^{\prime} \in\left(0, \epsilon_{1} \wedge \epsilon_{2}\right)$, $d\left(x, x^{\prime}\right) \leq(1 / 8) d(x, y)$, and $d\left(y, y^{\prime}\right) \leq(1 / 8) d(x, y)$,

$$
\begin{aligned}
& \left|\left[P_{l} Q_{k}(x, y)-P_{l} Q_{k}\left(x^{\prime}, y\right)\right]-\left[P_{l} Q_{k}\left(x, y^{\prime}\right)-P_{l} Q_{k}\left(x^{\prime}, y^{\prime}\right)\right]\right| \\
& \lesssim 2^{-(k-l) \delta} \frac{d\left(x, x^{\prime}\right)^{\epsilon_{1}^{\prime}}}{\left(2^{-l}+d(x, y)\right)^{\epsilon_{1}^{\prime}}} \frac{1}{V_{2^{-l}}(x)+V_{2^{-l}}(y)+V(x, y)} \frac{2^{-l \epsilon_{2}}}{\left(2^{-l}+d(x, y)\right)^{\epsilon_{2}}} \\
& \quad+2^{-(k-l) \delta} \frac{d\left(x, x^{\prime}\right)^{\epsilon_{1}^{\prime}}}{\left(2^{-l}+d\left(x, y^{\prime}\right)\right)^{\epsilon_{1}^{\prime}}} \frac{1}{V_{2^{-l}}(x)+V_{2^{-l}}\left(y^{\prime}\right)+V\left(x, y^{\prime}\right)} \frac{2^{-l \epsilon_{2}}}{\left(2^{-l}+d\left(x, y^{\prime}\right)\right)^{\epsilon_{2}}},
\end{aligned}
$$

where $\delta>0$ depends only on $\epsilon_{1}^{\prime}, \epsilon_{1}$, and $\epsilon_{2}$. The assumption $d\left(y, y^{\prime}\right) \leq(1 / 8) d(x, y)$ together with Lemma 2.1(iii) further shows that for any $\epsilon_{1}^{\prime} \in\left(0, \epsilon_{1} \wedge \epsilon_{2}\right), d\left(x, x^{\prime}\right) \leq(1 / 8) d(x, y)$ and $d\left(y, y^{\prime}\right) \leq(1 / 8) d(x, y)$,

$$
\begin{aligned}
& \left|\left[P_{l} Q_{k}(x, y)-P_{l} Q_{k}\left(x^{\prime}, y\right)\right]-\left[P_{l} Q_{k}\left(x, y^{\prime}\right)-P_{l} Q_{k}\left(x^{\prime}, y^{\prime}\right)\right]\right| \\
& \quad \lesssim 2^{-(k-l) \delta} \frac{d\left(x, x^{\prime}\right)^{\epsilon_{1}^{\prime}}}{\left(2^{-l}+d(x, y)\right)^{\epsilon_{1}^{\prime}}} \frac{1}{V_{2^{-l}}(x)+V_{2^{-l}}(y)+V(x, y)} \frac{2^{-l \epsilon_{2}}}{\left(2^{-l}+d(x, y)\right)^{\epsilon_{2}}}
\end{aligned}
$$

By the estimate (3.3) and symmetry, we further obtain that for any $\epsilon_{1}^{\prime} \in\left(0, \epsilon_{1} \wedge \epsilon_{2}\right), d\left(x, x^{\prime}\right) \leq$ $(1 / 8) d(x, y)$ and $d\left(y, y^{\prime}\right) \leq(1 / 8) d(x, y)$,

$$
\begin{aligned}
& \left|\left[P_{l} Q_{k}(x, y)-P_{l} Q_{k}\left(x^{\prime}, y\right)\right]-\left[P_{l} Q_{k}\left(x, y^{\prime}\right)-P_{l} Q_{k}\left(x^{\prime}, y^{\prime}\right)\right]\right| \\
& \quad \lesssim 2^{-(k-l) \delta} \frac{d\left(y, y^{\prime}\right)^{\epsilon_{1}^{\prime}}}{\left(2^{-l}+d(x, y)\right)^{\epsilon_{1}^{\prime}}} \frac{1}{V_{2^{-l}}(x)+V_{2^{-l}}(y)+V(x, y)} \frac{2^{-l \epsilon_{2}}}{\left(2^{-l}+d(x, y)\right)^{\epsilon_{2}}},
\end{aligned}
$$

where $\delta>0$ depends only on $\epsilon_{1}^{\prime}, \epsilon_{1}$, and $\epsilon_{2}$. Then the geometric mean among (3.25), (3.27), and (3.28) gives the desired estimate (3.5). 
Using (3.6), we now prove (3.25) by writing that

$$
\begin{aligned}
& \left|\left[P_{l} Q_{k}(x, y)-P_{l} Q_{k}\left(x^{\prime}, y\right)\right]-\left[P_{l} Q_{k}\left(x, y^{\prime}\right)-P_{l} Q_{k}\left(x^{\prime}, y^{\prime}\right)\right]\right| \\
& \quad=\left|\int_{x}\left\{\left[P_{l}(x, z)-P_{l}\left(x^{\prime}, z\right)\right]-\left[P_{l}(x, y)-P_{l}\left(x^{\prime}, y\right)\right]\right\}\left[Q_{k}(z, y)-Q_{k}\left(z, y^{\prime}\right)\right] d \mu(z)\right| \\
& \quad \leq \sum_{i=1}^{4} \int_{W_{i}}\left|\left[P_{l}(x, z)-P_{l}\left(x^{\prime}, z\right)\right]-\left[P_{l}(x, y)-P_{l}\left(x^{\prime}, y\right)\right]\right|\left|Q_{k}(z, y)-Q_{k}\left(z, y^{\prime}\right)\right| d \mu(z) \\
& \quad \equiv \sum_{i=1}^{4} Y_{i}
\end{aligned}
$$

where

$$
\begin{aligned}
& W_{1}=\left\{z \in x: d\left(y, y^{\prime}\right) \leq \frac{2^{-k}+d(z, y)}{2} \leq \frac{2^{-l}+d(x, y)}{8}\right\}, \\
& W_{2}=\left\{z \in x: d\left(y, y^{\prime}\right) \leq \frac{2^{-l}+d(x, y)}{8} \leq \frac{2^{-k}+d(z, y)}{2}, \text { and } d\left(x, x^{\prime}\right) \leq \frac{2^{-l}+d(z, x)}{2}\right\}, \\
& W_{3}=\left\{z \in x: d\left(y, y^{\prime}\right) \leq \frac{2^{-l}+d(x, y)}{8} \leq \frac{2^{-k}+d(z, y)}{2}, \text { and } d\left(x, x^{\prime}\right)>\frac{2^{-l}+d(z, x)}{2}\right\}, \\
& W_{4}=\left\{z \in x: d\left(y, y^{\prime}\right)>\frac{2^{-k}+d(z, y)}{2}\right\} .
\end{aligned}
$$

If $z \in W_{1}$, then $d(z, y) \leq(1 / 4)\left(2^{-l}+d(x, y)\right), d\left(x, x^{\prime}\right) \leq(1 / 8) d(x, y) \leq(1 / 8)\left(2^{-l}+\right.$ $d(x, y))$, and $d\left(y, y^{\prime}\right) \leq(1 / 2)\left(2^{-k}+d(z, y)\right)$, which together with the second difference condition of $P_{l}$, the regularity of $Q_{k}$ and Lemma 2.1(ii) shows

$$
\begin{aligned}
Y_{1} \lesssim & \frac{d\left(x, x^{\prime}\right)^{\epsilon_{1}}}{\left(2^{-l}+d(x, y)\right)^{\epsilon_{1}}} \frac{1}{V_{2^{-l}}(x)+V_{2^{-l}}(y)+V(x, y)} \frac{2^{-l \epsilon_{3}}}{\left(2^{-l}+d(x, y)\right)^{\epsilon_{3}}} \\
& \times \int_{W_{1}} \frac{d(y, z)^{\epsilon_{1}}}{\left(2^{-l}+d(x, y)\right)^{\epsilon_{1}}} \frac{d\left(y, y^{\prime}\right)^{\epsilon_{1}}}{\left(2^{-k}+d(z, y)\right)^{\epsilon_{1}}} \\
& \quad \times \frac{2^{-k \epsilon_{2}}}{V_{2^{-k}}(z)+V_{2^{-k}}(y)+V(z, y)} \frac{1}{\left(2^{-k}+d(z, y)\right)^{\epsilon_{2}}} d \mu(z) \\
\lesssim & \frac{d\left(x, x^{\prime}\right)^{\epsilon_{1}}}{\left(2^{-l}+d(x, y)\right)^{\epsilon_{1}}} \frac{d\left(y, y^{\prime}\right)^{\epsilon_{1}}}{\left(2^{-l}+d(x, y)\right)^{\epsilon_{1}}} \frac{2^{-l \epsilon_{3}}}{V_{2^{-l}}(x)+V_{2^{-l}}(y)+V(x, y)} \frac{1}{\left(2^{-l}+d(x, y)\right)^{\epsilon_{3}}} .
\end{aligned}
$$


If $z \in W_{4}$, then $(1 / 2)\left(2^{-k}+d(z, y)\right)<d\left(y, y^{\prime}\right) \leq(1 / 8) d(x, y)$, and therefore, $d(z, y) \leq$ $2 d\left(y, y^{\prime}\right) \leq(1 / 4) d(x, y) \leq(1 / 4)\left(2^{-l}+d(x, y)\right)$ and $d\left(x, x^{\prime}\right) \leq(1 / 8) d(x, y) \leq(1 / 8)\left(2^{-l}+\right.$ $d(x, y))$. From this, the regularity of $P_{l}$ and Proposition 2.7(i), it follows that

$$
\begin{aligned}
Y_{4} \lesssim & \frac{d\left(x, x^{\prime}\right)^{\epsilon_{1}}}{\left(2^{-l}+d(x, y)\right)^{\epsilon_{1}}} \frac{d\left(y, y^{\prime}\right)^{\epsilon_{1}}}{\left(2^{-l}+d(x, y)\right)^{\epsilon_{1}}} \frac{1}{V_{2^{-l}}(x)+V_{2^{-l}}(y)+V(x, y)} \\
& \times \frac{2^{-l \epsilon_{3}}}{\left(2^{-l}+d(x, y)\right)^{\epsilon_{3}}} \int_{x}\left[\left|Q_{k}(z, y)\right|+\left|Q_{k}\left(z, y^{\prime}\right)\right|\right] d \mu(z) \\
& \lesssim \frac{d\left(x, x^{\prime}\right)^{\epsilon_{1}}}{\left(2^{-l}+d(x, y)\right)^{\epsilon_{1}}} \frac{d\left(y, y^{\prime}\right)^{\epsilon_{1}}}{\left(2^{-l}+d(x, y)\right)^{\epsilon_{1}}} \frac{2^{-l \epsilon_{3}}}{V_{2^{-l}}(x)+V_{2^{-l}}(y)+V(x, y)} \frac{1}{\left(2^{-l}+d(x, y)\right)^{\epsilon_{3}}} .
\end{aligned}
$$

If $z \in W_{2}$, then $d\left(x, x^{\prime}\right) \leq(1 / 2)\left(2^{-l}+d(z, x)\right), d\left(y, y^{\prime}\right) \leq(1 / 2)\left(2^{-k}+d(z, y)\right)$, and $d\left(x, x^{\prime}\right) \leq(1 / 8) d(x, y) \leq(1 / 8)\left(2^{-l}+d(x, y)\right)$, which together with the regularity of $P_{l}$ and $Q_{k}$ implies that

$$
\begin{aligned}
Y_{2} \lesssim \int_{W_{2}} & {\left[\frac{d\left(x, x^{\prime}\right)^{\epsilon_{1}}}{\left(2^{-l}+d(x, z)\right)^{\epsilon_{1}}} \frac{1}{V_{2^{-l}}(x)+V_{2^{-l}}(z)+V(x, z)} \frac{2^{-l \epsilon_{2}}}{\left(2^{-l}+d(x, z)\right)^{\epsilon_{2}}}\right.} \\
& \left.+\frac{d\left(x, x^{\prime}\right)^{\epsilon_{1}}}{\left(2^{-l}+d(x, y)\right)^{\epsilon_{1}}} \frac{1}{V_{2^{-l}}(x)+V_{2^{-l}}(y)+V(x, y)} \frac{2^{-l \epsilon_{2}}}{\left(2^{-l}+d(x, y)\right)^{\epsilon_{2}}}\right] \\
& \times \frac{d\left(y, y^{\prime}\right)^{\epsilon_{1}}}{\left(2^{-k}+d(y, z)\right)^{\epsilon_{1}}} \frac{1}{V_{2^{-k}}(z)+V_{2^{-k}}(y)+V(z, y)} \frac{2^{-k \epsilon_{2}}}{\left(2^{-k}+d(z, y)\right)^{\epsilon_{2}}} d \mu(z) \\
\equiv Y_{2,1} & +Y_{2,2} .
\end{aligned}
$$

The fact that $z \in W_{2}$, which implies that $2^{-k}+d(z, y) \geq(1 / 4)\left(2^{-l}+d(x, y)\right)$, together with Lemma 2.1(ii) yields that

$$
\begin{aligned}
Y_{2,2} \lesssim & \frac{d\left(x, x^{\prime}\right)^{\epsilon_{1}}}{\left(2^{-l}+d(x, y)\right)^{\epsilon_{1}}} \frac{d\left(y, y^{\prime}\right)^{\epsilon_{1}}}{\left(2^{-l}+d(x, y)\right)^{\epsilon_{1}}} \frac{1}{V_{2^{-l}}(x)+V_{2^{-l}}(y)+V(x, y)} \\
& \times \frac{2^{-l \epsilon_{2}}}{\left(2^{-l}+d(x, y)\right)^{\epsilon_{2}}} \int_{x} \frac{1}{V_{2^{-k}}(y)+V(z, y)} \frac{2^{-k \epsilon_{2}}}{\left(2^{-k}+d(z, y)\right)^{\epsilon_{2}}} d \mu(z) \\
& \lesssim \frac{d\left(x, x^{\prime}\right)^{\epsilon_{1}}}{\left(2^{-l}+d(x, y)\right)^{\epsilon_{1}}} \frac{d\left(y, y^{\prime}\right)^{\epsilon_{1}}}{\left(2^{-l}+d(x, y)\right)^{\epsilon_{1}}} \frac{2^{-l \epsilon_{2}}}{V_{2^{-l}}(x)+V_{2^{-l}}(y)+V(x, y)} \frac{1}{\left(2^{-l}+d(x, y)\right)^{\epsilon_{2}}} .
\end{aligned}
$$


Similarly, the fact that $2^{-k}+d(z, y) \geq(1 / 4)\left(2^{-l}+d(x, y)\right)$ implies that

$$
\begin{aligned}
Y_{2,1} \lesssim \frac{d\left(y, y^{\prime}\right)^{\epsilon_{1}}}{\left(2^{-l}+d(x, y)\right)^{\epsilon_{1}}} \int_{W_{2}} \frac{d\left(x, x^{\prime}\right)^{\epsilon_{1}}}{\left(2^{-l}+d(x, z)\right)^{\epsilon_{1}}} \frac{1}{V_{2^{-l}}(x)+V_{2^{-l}}(z)+V(x, z)} \\
\quad \times \frac{2^{-l \epsilon_{2}}}{\left(2^{-l}+d(x, z)\right)^{\epsilon_{2}}} \frac{2^{-k \epsilon_{2}}}{V_{2^{-k}}(z)+V_{2^{-k}}(y)+V(z, y)} \frac{1}{\left(2^{-k}+d(z, y)\right)^{\epsilon_{2}}} d \mu(z) .
\end{aligned}
$$

The estimate (3.19) together with the facts that $k \geq l$ and $2^{-k}+d(z, y) \geq(1 / 4)\left(2^{-l}+d(x, y)\right)$ and Lemma 2.1(ii) yields that for any $\epsilon_{1}^{\prime} \in\left(0, \epsilon_{1} \wedge \epsilon_{2}\right)$,

$$
\begin{aligned}
Y_{2,1} \lesssim & \frac{d\left(y, y^{\prime}\right)^{\epsilon_{1}}}{\left(2^{-l}+d(x, y)\right)^{\epsilon_{1}}} \frac{1}{V_{2^{-l}}(y)+V(x, y)} d\left(x, x^{\prime}\right)^{\epsilon_{1}^{\prime}} \frac{2^{-l \epsilon_{2}}}{\left(2^{-l}+d(x, y)\right)^{\epsilon_{2}}} \\
& \times \int_{x} \frac{1}{V_{2^{-l}}(x)+V(x, z)} \frac{2^{-l \epsilon_{2}}}{\left(2^{-l}+d(x, z)\right)^{\epsilon_{2}+\epsilon_{1}^{\prime}}} d \mu(z) \\
\lesssim & \frac{d\left(y, y^{\prime}\right)^{\epsilon_{1}}}{\left(2^{-l}+d(x, y)\right)^{\epsilon_{1}}} \frac{1}{V_{2^{-l}}(y)+V(x, y)} \frac{d\left(x, x^{\prime}\right)^{\epsilon_{1}^{\prime}}}{\left(2^{-l}+d(x, y)\right)^{\epsilon_{1}^{\prime}}} \frac{2^{-l\left(\epsilon_{2}-\epsilon_{1}^{\prime}\right)}}{\left(2^{-l}+d(x, y)\right)^{\epsilon_{2}-\epsilon_{1}^{\prime}}}
\end{aligned}
$$

and similarly, Lemma 2.1(i) implies that

$$
\begin{aligned}
Y_{2,1} \lesssim & \frac{d\left(y, y^{\prime}\right)^{\epsilon_{1}}}{\left(2^{-l}+d(x, y)\right)^{\epsilon_{1}}} \frac{1}{V_{2^{-l}}(x)} d\left(x, x^{\prime}\right)^{\epsilon_{1}^{\prime}} 2^{l \epsilon_{1}^{\prime}} \int_{W_{2}} \frac{1}{V_{2^{-k}}(y)+V(z, y)} \frac{2^{-k \epsilon_{2}}}{\left(2^{-k}+d(z, y)\right)^{\epsilon_{2}}} d \mu(z) \\
\lesssim & \frac{d\left(y, y^{\prime}\right)^{\epsilon_{1}}}{\left(2^{-l}+d(x, y)\right)^{\epsilon_{1}}} \frac{1}{V_{2^{-l}}(x)} d\left(x, x^{\prime}\right)^{\epsilon_{1}^{\prime}} 2^{l \epsilon_{1}^{\prime}} \\
& \times\left\{\frac{2^{-l \epsilon_{2}}}{\left(2^{-l}+d(x, y)\right)^{\epsilon_{2}}}+2^{-l \epsilon_{2}} \int_{d(z, y) \geq(1 / 8)\left(2^{-l}+d(x, y)\right),} \frac{1}{d(z, y) \geq 2^{-k}} \frac{1}{d(z, y)^{\epsilon_{2}}} d \mu(z)\right\} \\
\lesssim & \frac{d\left(y, y^{\prime}\right)^{\epsilon_{1}}}{\left(2^{-l}+d(x, y)\right)^{\epsilon_{1}}} \frac{1}{V_{2^{-l}}(x)} \frac{d\left(x, x^{\prime}\right)^{\epsilon_{1}^{\prime}}}{\left(2^{-l}+d(x, y)\right)^{\epsilon_{1}^{\prime}}} \frac{2^{-l\left(\epsilon_{2}-\epsilon_{1}^{\prime}\right)}}{\left(2^{-l}+d(x, y)\right)^{\epsilon_{2}-\epsilon_{1}^{\prime}}},
\end{aligned}
$$

which completes the estimate for $Y_{2}$.

Finally, to estimate $Y_{3}$, since $d\left(y, y^{\prime}\right) \leq(1 / 2)\left(2^{-k}+d(z, y)\right)$ and

$$
d\left(x, x^{\prime}\right) \leq \frac{d(x, y)}{8} \leq \frac{2^{-l}+d(x, y)}{8}
$$


by the regularities of $P_{l}$ and $Q_{k}$, we then have

$$
\begin{aligned}
Y_{3} \lesssim & \int_{W_{3}}\left[\left|P_{l}(x, z)\right|+\left|P_{l}\left(x^{\prime}, z\right)\right|+\frac{d\left(x, x^{\prime}\right)^{\epsilon_{1}}}{\left(2^{-l}+d(x, y)\right)^{\epsilon_{1}}} \frac{1}{V_{2^{-l}}(x)+V_{2^{-l}}(y)+V(x, y)} \frac{2^{-l \epsilon_{2}}}{\left(2^{-l}+d(x, y)\right)^{\epsilon_{2}}}\right] \\
& \times \frac{d\left(y, y^{\prime}\right)^{\epsilon_{1}}}{\left(2^{-k}+d(z, y)\right)^{\epsilon_{1}}} \frac{1}{V_{2^{-k}}(z)+V_{2^{-k}}(y)+V(z, y)} \frac{2^{-k \epsilon_{2}}}{\left(2^{-k}+d(z, y)\right)^{\epsilon_{2}}} d \mu(z) \\
\equiv & \sum_{i=1}^{3} Y_{3, i} .
\end{aligned}
$$

The fact that $2^{-k}+d(z, y) \geq(1 / 4)\left(2^{-l}+d(x, y)\right)$ and Lemma 2.1(ii) imply that

$$
\begin{aligned}
Y_{3,3} \lesssim & \frac{d\left(x, x^{\prime}\right)^{\epsilon_{1}}}{\left(2^{-l}+d(x, y)\right)^{\epsilon_{1}}} \frac{d\left(y, y^{\prime}\right)^{\epsilon_{1}}}{\left(2^{-l}+d(x, y)\right)^{\epsilon_{1}}} \frac{1}{V_{2^{-l}}(x)+V_{2^{-l}}(y)+V(x, y)} \\
& \times \frac{2^{-l \epsilon_{2}}}{\left(2^{-l}+d(x, y)\right)^{\epsilon_{2}}} \int_{x} \frac{1}{V_{2^{-k}}(y)+V(z, y)} \frac{2^{-k \epsilon_{2}}}{\left(2^{-k}+d(z, y)\right)^{\epsilon_{2}}} d \mu(z) \\
& \lesssim \frac{d\left(x, x^{\prime}\right)^{\epsilon_{1}}}{\left(2^{-l}+d(x, y)\right)^{\epsilon_{1}}} \frac{d\left(y, y^{\prime}\right)^{\epsilon_{1}}}{\left(2^{-l}+d(x, y)\right)^{\epsilon_{1}}} \frac{2^{-l \epsilon_{2}}}{V_{2^{-l}}(x)+V_{2^{-l}}(y)+V(x, y)}
\end{aligned}
$$

The facts that $k \geq l, 2^{-k}+d(z, y) \geq(1 / 4)\left(2^{-l}+d(x, y)\right),(3.19)$, and Proposition 2.7(i) yield that for any $\epsilon_{1}^{\prime} \in\left(0, \epsilon_{1} \wedge \epsilon_{2}\right)$,

$$
\begin{aligned}
Y_{3,1} & \lesssim \frac{d\left(y, y^{\prime}\right)^{\epsilon_{1}}}{\left(2^{-l}+d(x, y)\right)^{\epsilon_{1}}} \int_{W_{3}}\left|P_{l}(x, z)\right| \frac{1}{V_{2^{-k}}(z)+V_{2^{-k}}(y)+V(z, y)} \frac{2^{-k \epsilon_{2}}}{\left(2^{-k}+d(z, y)\right)^{\epsilon_{2}}} d \mu(z) \\
& \lesssim \frac{d\left(x, x^{\prime}\right)^{\epsilon_{1}^{\prime}}}{\left(2^{-l}+d(x, y)\right)^{\epsilon_{1}^{\prime}}} \frac{d\left(y, y^{\prime}\right)^{\epsilon_{1}}}{\left(2^{-l}+d(x, y)\right)^{\epsilon_{1}}} \frac{1}{V_{2^{-l}}(y)+V(x, y)} \frac{2^{-l\left(\epsilon_{2}-\epsilon_{1}^{\prime}\right)}}{\left(2^{-l}+d(x, y)\right)^{\epsilon_{2}-\epsilon_{1}^{\prime}}}
\end{aligned}
$$

where, in the last step, we used the fact that $2^{-l}<2 d\left(x, x^{\prime}\right)$ in this case. Similarly, by the size condition of $P_{l}$ and Lemma 2.1(i), we further have

$$
\begin{aligned}
Y_{3,1} \lesssim & \frac{d\left(y, y^{\prime}\right)^{\epsilon_{1}}}{\left(2^{-l}+d(x, y)\right)^{\epsilon_{1}}} \frac{1}{V_{2^{-l}}(x)} \int_{W_{3}} \frac{1}{V_{2^{-k}}(y)+V(z, y)} \frac{2^{-k \epsilon_{2}}}{\left(2^{-k}+d(z, y)\right)^{\epsilon_{2}}} d \mu(z) \\
\lesssim & \frac{d\left(y, y^{\prime}\right)^{\epsilon_{1}}}{\left(2^{-l}+d(x, y)\right)^{\epsilon_{1}}} \frac{1}{V_{2^{-l}}(x)} \\
& \times\left\{\frac{2^{-l \epsilon_{2}}}{\left(2^{-l}+d(x, y)\right)^{\epsilon_{2}}}+2^{-l \epsilon_{2}} \int_{d(z, y) \geq(1 / 8)\left(2^{-l}+d(x, y)\right),} \frac{1}{d(z, y) \geq 2^{-k}} \frac{1}{d(z, y)^{\epsilon_{2}}} d \mu(z)\right\} \\
\lesssim & \frac{d\left(x, x^{\prime}\right)^{\epsilon_{1}^{\prime}}}{\left(2^{-l}+d(x, y)\right)^{\epsilon_{1}^{\prime}}} \frac{d\left(y, y^{\prime}\right)^{\epsilon_{1}}}{\left(2^{-l}+d(x, y)\right)^{\epsilon_{1}}} \frac{1}{V_{2^{-l}(x)}} \frac{2^{-l\left(\epsilon_{2}-\epsilon_{1}^{\prime}\right)}}{\left(2^{-l}+d(x, y)\right)^{\epsilon_{2}-\epsilon_{1}^{\prime}} .}
\end{aligned}
$$


Notice that in this case, $2^{-l} \leq 2 d\left(x, x^{\prime}\right) \leq(1 / 4) d(x, y)$ and therefore $V_{2^{-l}}(x) \leq V(x, y)$. Thus, by (3.19), we have that for $z \in W_{3}, 1 /\left(V_{2^{-k}}(y)+V(z, y)\right) \lesssim 1 /\left(V_{2^{-l}}(y)+V(x, y)\right) \lesssim 1 /\left(V_{2^{-l}}(x)+\right.$ $\left.V_{2^{-l}}(y)+V(x, y)\right)$, which together with Proposition 2.7(i) shows

$$
\begin{aligned}
Y_{3,2} & \lesssim \frac{d\left(y, y^{\prime}\right)^{\epsilon_{1}}}{\left(2^{-l}+d(x, y)\right)^{\epsilon_{1}}} \frac{2^{-l \epsilon_{2}}}{\left(2^{-l}+d(x, y)\right)^{\epsilon_{2}}} \int_{W_{3}}\left|P_{l}\left(x^{\prime}, z\right)\right| \frac{1}{V_{2^{-k}}(z)+V_{2^{-k}}(y)+V(z, y)} d \mu(z) \\
& \lesssim \frac{d\left(x, x^{\prime}\right)^{\epsilon_{1}^{\prime}}}{\left(2^{-l}+d(x, y)\right)^{\epsilon_{1}^{\prime}}} \frac{d\left(y, y^{\prime}\right)^{\epsilon_{1}}}{\left(2^{-l}+d(x, y)\right)^{\epsilon_{1}}} \frac{1}{V_{2^{-l}}(x)+V_{2^{-l}}(y)+V(x, y)} \frac{2^{-l\left(\epsilon_{2}-\epsilon_{1}^{\prime}\right)}}{\left(2^{-l}+d(x, y)\right)^{\epsilon_{2}-\epsilon_{1}^{\prime}}} .
\end{aligned}
$$

This completes the proof of (3.25), and hence, the proof of Lemma 3.2.

Remark 3.3. From the proof of (3.2) in Lemma 3.2, it is easy to see that (3.2) still holds if $P_{l}$ has the required regularity only in the second variable, and $Q_{k}$ the first variable. This observation is useful in applications.

In what follows, let $\left\{S_{k}\right\}_{k \in \mathbb{Z}}$ be an $\left(\epsilon_{1}, \epsilon_{2}, \epsilon_{3}\right)$-ATI with $\epsilon_{1} \in(0,1], \epsilon_{2}>0$, and $\epsilon_{3}>0$ as in Definition 2.2. Set $D_{k}=S_{k}-S_{k-1}$ for $k \in \mathbb{Z}$. To establish continuous Calderón reproducing formulae, by following Coifman's idea (see [64]) and Proposition 3.1(ii), we write for any $N \in \mathbb{N}$,

$$
\begin{aligned}
I & =\left(\sum_{k=-\infty}^{\infty} D_{k}\right)\left(\sum_{j=-\infty}^{\infty} D_{j}\right) \\
& =\sum_{|l|>N} \sum_{k=-\infty}^{\infty} D_{k+l} D_{k}+\sum_{k=-\infty}^{\infty} D_{k}^{N} D_{k} \\
& =R_{N}+T_{N}
\end{aligned}
$$

in $L^{p}(\mathcal{X})$ with $p \in(1, \infty)$, where $D_{k}^{N}=\sum_{|l| \leq N} D_{k+l}$. To verify that $T_{N}^{-1}$ exists and is bounded on any space of test functions, we first prove that $R_{N}$ is bounded on $L^{2}(\mathcal{X})$ with a small operator norm.

Lemma 3.4. Let $N \in \mathbb{N}$ and let $R_{N}$ be as in (3.44). Then there exist constants $C>0$ and $\delta>0$, independent of $N$, such that for all $f \in L^{2}(X)$,

$$
\left\|R_{N}(f)\right\|_{L^{2}(x)} \leq C 2^{-N \delta}\|f\|_{L^{2}(x)} .
$$

Proof. To prove the lemma, by applying the Cotlar-Stein lemma (see [75, page 280] or [64]), we see that it suffices to verify that for any $\sigma \in(0,1), \epsilon_{1}^{\prime} \in\left(0, \epsilon_{1} \wedge \epsilon_{2}\right)$ with $i=1,2,3$, and all $k_{1}, l_{1}, k_{2}, l_{2} \in \mathbb{Z}$,

$$
\begin{aligned}
&\left\|D_{k_{1}+l_{1}} D_{k_{1}}\left(D_{k_{2}+l_{2}} D_{k_{2}}\right)^{t}\right\|_{L^{2}(\mathcal{X}) \rightarrow L^{2}(x)} \lesssim 2^{-\left|l_{1}\right| \epsilon_{1}^{\prime} \sigma} 2^{-\left|l_{2}\right| \epsilon_{2}^{\prime} \sigma} 2^{-\left|k_{1}-k_{2}\right| \epsilon_{3}^{\prime}(1-\sigma)}, \\
&\left\|\left(D_{k_{2}+l_{2}} D_{k_{2}}\right)^{t} D_{k_{1}+l_{1}} D_{k_{1}}\right\|_{L^{2}(\mathcal{X}) \rightarrow L^{2}(\mathcal{X})} \lesssim 2^{-\left|l_{1}\right| \epsilon_{1}^{\prime} \sigma} 2^{-\left|l_{2}\right| \epsilon_{2}^{\prime} \sigma} 2^{-\left|k_{1}-k_{2}\right| \epsilon_{3}^{\prime}(1-\sigma)} .
\end{aligned}
$$


We only prove (3.46), the proof for the last inequality being similar. In fact, Lemma 3.2 and Proposition 2.7 yield that

$$
\begin{aligned}
\left\|D_{k_{1}+l_{1}} D_{k_{1}}\left(D_{k_{2}+l_{2}} D_{k_{2}}\right)^{t}\right\|_{L^{2}(\mathcal{X}) \rightarrow L^{2}(\mathcal{X})} & \lesssim 2^{-\left|l_{1}\right| \epsilon_{1}^{\prime}} 2^{-\left|l_{2}\right| \epsilon_{2}^{\prime}}, \\
\left\|D_{k_{1}+l_{1}} D_{k_{1}} D_{k_{2}}^{t} D_{k_{2}+l_{2}}^{t}\right\|_{L^{2}(\mathcal{X}) \rightarrow L^{2}(\mathcal{X})} & \lesssim 2^{-\left|k_{1}-k_{2}\right| \epsilon_{3}^{\prime}} .
\end{aligned}
$$

Then the geometric mean of the above two estimates gives the estimate (3.46), which completes the proof of Lemma 3.4.

We now establish some estimates for the kernel, $R_{N}(x, y)$, of the operator $R_{N}$. To this end, we first give a technical lemma.

Lemma 3.5. For any $\sigma \geq s>0$ and $x, y \in \mathcal{X}$ with $x \neq y$,

$$
\sum_{k=-\infty}^{\infty} \frac{1}{V_{2^{-k}}(x)+V(x, y)} \frac{2^{-k s}}{\left(2^{-k}+d(x, y)\right)^{\sigma}} \lesssim \frac{1}{V(x, y)} \frac{1}{d(x, y)^{\sigma-s}}
$$

Proof. For any $x, y \in \mathcal{X}$, choose $k_{0} \in \mathbb{Z}$ such that $2^{-k_{0}}<d(x, y) \leq 2^{-k_{0}+1}$. Then if $k \leq k_{0}$, by (1.3), we have

$$
\begin{aligned}
V_{2^{-k}}(x) & =\mu\left(B\left(x, 2^{-\left(k-k_{0}\right)} 2^{-k_{0}}\right)\right) \\
& \geq 2^{-\kappa\left(k-k_{0}\right)} \mu\left(B\left(x, 2^{-k_{0}}\right)\right) \\
& \gtrsim 2^{-\kappa\left(k-k_{0}\right)} V(x, y) .
\end{aligned}
$$

Therefore,

$$
\begin{aligned}
\sum_{k=-\infty}^{\infty} & \frac{1}{V_{2^{-k}}(x)+V(x, y)} \frac{2^{-k s}}{\left(2^{-k}+d(x, y)\right)^{\sigma}} \\
& \leq \frac{1}{d(x, y)^{\sigma-s}} \sum_{k=-\infty}^{k_{0}} \frac{1}{V_{2^{-k}}(x)}+\frac{1}{V(x, y) d(x, y)^{\sigma}} \sum_{k=k_{0}+1}^{\infty} 2^{-k s} \\
& \lesssim \frac{1}{V(x, y)} \frac{1}{d(x, y)^{\sigma-s}}\left\{\sum_{k=-\infty}^{k_{0}} 2^{-\kappa\left(k-k_{0}\right)}+1\right\} \\
& \lesssim \frac{1}{V(x, y)} \frac{1}{d(x, y)^{\sigma-s}}
\end{aligned}
$$

which completes the proof of Lemma 3.5.

Lemma 3.6. Let $N \in \mathbb{N}$, let $R_{N}$ be as in (3.44), and let $R_{N}(x, y)$ be its kernel. Then for any $\epsilon_{1}^{\prime} \in$ $\left(0, \epsilon_{1} \wedge \epsilon_{2}\right)$, there exists a constant $\delta>0$, independent of $N$, such that $R_{N}$ satisfies all the conditions of Corollary 2.22 with $\epsilon$ replaced by $\epsilon_{1}^{\prime}$ and $C_{R_{N}} \lesssim 2^{-\delta N}$. Moreover, $R_{N}^{*}(1)=0$. 
Proof. Let $\epsilon_{1}^{\prime} \in\left(0, \epsilon_{1} \wedge \epsilon_{2}\right)$. Write

$$
\begin{aligned}
R_{N}(x, y) & =\sum_{l=N+1}^{\infty} \sum_{k=-\infty}^{\infty}\left(D_{k+l} D_{k}\right)(x, y)+\sum_{l=-\infty}^{-N-1} \sum_{k=-\infty}^{\infty}\left(D_{k+l} D_{k}\right)(x, y) \\
& =R_{N}^{1}(x, y)+R_{N}^{2}(x, y) .
\end{aligned}
$$

By (3.2) and Lemma 3.5, we have that for any $x, y \in \mathcal{X}$ with $x \neq y$,

$$
\begin{aligned}
\left|R_{N}(x, y)\right| \leq & \left|R_{N}^{1}(x, y)\right|+\left|R_{N}^{2}(x, y)\right| \\
\lesssim & \sum_{l=N+1}^{\infty} 2^{-l \epsilon_{1}^{\prime}} \sum_{k=-\infty}^{\infty} \frac{1}{V_{2^{-k}}(x)+V(x, y)} \frac{2^{-k \epsilon_{2}}}{\left(2^{-k}+d(x, y)\right)^{\epsilon_{2}}} \\
& +\sum_{l=-\infty}^{-N-1} 2^{l \epsilon_{1}^{\prime}} \sum_{k=-\infty}^{\infty} \frac{1}{V_{2^{-(k+l)}}(x)+V(x, y)} \frac{2^{-(k+l) \epsilon_{2}}}{\left(2^{-(k+l)}+d(x, y)\right)^{\epsilon_{2}}} \\
\lesssim & 2^{-N \epsilon_{1}^{\prime}} \frac{1}{V(x, y)} .
\end{aligned}
$$

Thus, $R_{N}$ satisfies (i) of Theorem 2.18.

The estimate (3.3) and Lemma 3.5 show that for any $x, y, y^{\prime} \in \mathcal{x}$ with $x \neq y$ and $d\left(y, y^{\prime}\right) \leq(1 / 4) d(x, y)$,

$$
\begin{aligned}
& \left|R_{N}(x, y)-R_{N}\left(x, y^{\prime}\right)\right| \\
& \leq\left|R_{N}^{1}(x, y)-R_{N}^{1}\left(x, y^{\prime}\right)\right|+\left|R_{N}^{2}(x, y)-R_{N}^{2}\left(x, y^{\prime}\right)\right| \\
& \lesssim \frac{d\left(y, y^{\prime}\right)^{\epsilon_{1}^{\prime}}}{d(x, y)^{\epsilon_{1}^{\prime}}}\left\{\sum_{l=N+1}^{\infty} 2^{-l \delta} \sum_{k=-\infty}^{\infty} \frac{1}{V_{2^{-k}}(x)+V(x, y)} \frac{2^{-k \epsilon_{2}}}{\left(2^{-k}+d(x, y)\right)^{\epsilon_{2}}}\right. \\
& \left.+\sum_{l=-\infty}^{-N-1} 2^{l \delta} \sum_{k=-\infty}^{\infty} \frac{1}{V_{2^{-(k+l)}}(x)+V(x, y)} \frac{2^{-(k+l) \epsilon_{2}}}{\left(2^{-(k+l)}+d(x, y)\right)^{\epsilon_{2}}}\right\} \\
& \lesssim 2^{-N \delta} \frac{d\left(y, y^{\prime}\right)^{\epsilon_{1}^{\prime}}}{d(x, y)^{\epsilon_{1}^{\prime}}} \frac{1}{V(x, y)}
\end{aligned}
$$

and the estimate (3.4) and the symmetry also yield that

$$
\left|R_{N}(y, x)-R_{N}\left(y^{\prime}, x\right)\right| \lesssim 2^{-N \delta} \frac{d\left(y, y^{\prime}\right)^{\epsilon_{1}^{\prime}}}{d(x, y)^{\epsilon_{1}^{\prime}}} \frac{1}{V(x, y)}
$$

which shows that $R_{N}$ satisfies (2.49) and (ii) of Theorem 2.18. 
Using (3.5) and Lemma 3.5 then gives that for $x, x^{\prime}, y, y^{\prime} \in \mathcal{X}$ with $x \neq y$ and $d\left(x, x^{\prime}\right) \leq$ $(1 / 8) d(x, y)$ and $d\left(y, y^{\prime}\right) \leq(1 / 8) d(x, y)$,

$$
\begin{aligned}
& \left|\left[R_{N}(x, y)-R_{N}\left(x^{\prime}, y\right)\right]-\left[R_{N}\left(x, y^{\prime}\right)-R_{N}\left(x^{\prime}, y^{\prime}\right)\right]\right| \\
& \leq||\left[R_{N}^{1}(x, y)-R_{N}^{1}\left(x^{\prime}, y\right)\right]-\left[R_{N}^{1}\left(x, y^{\prime}\right)-R_{N}^{1}\left(x^{\prime}, y^{\prime}\right)\right] \mid \\
& \quad+\left|\left[R_{N}^{2}(x, y)-R_{N}^{2}\left(x^{\prime}, y\right)\right]-\left[R_{N}^{2}\left(x, y^{\prime}\right)-R_{N}^{2}\left(x^{\prime}, y^{\prime}\right)\right]\right| \\
& \lesssim \frac{d\left(x, x^{\prime}\right)^{\epsilon_{1}^{\prime}}}{d(x, y)^{\epsilon_{1}^{\prime}}} \frac{d\left(y, y^{\prime}\right)^{\epsilon_{1}^{\prime}}}{d(x, y)^{\epsilon_{1}^{\prime}}}\left\{\sum_{l=N+1}^{\infty} 2^{-l \delta} \sum_{k=-\infty}^{\infty} \frac{1}{V_{2^{-k}}(x)+V(x, y)} \frac{2^{-k \sigma}}{\left(2^{-k}+d(x, y)\right)^{\sigma}}\right. \\
& \left.\quad+\sum_{l=-\infty}^{-N-1} 2^{l \delta} \sum_{k=-\infty}^{\infty} \frac{1}{V_{2^{-(k+l)}}(x)+V(x, y)} \frac{2^{-(k+l) \sigma}}{\left(2^{-(k+l)}+d(x, y)\right)^{\sigma}}\right\} \\
& \lesssim 2^{-N \delta} \frac{d\left(x, x^{\prime}\right)^{\epsilon_{1}^{\prime}}}{d(x, y)^{\epsilon_{1}^{\prime}}} \frac{d\left(y, y^{\prime}\right)^{\epsilon_{1}^{\prime}}}{d(x, y)^{\epsilon_{1}^{\prime}}} \frac{1}{V(x, y)^{\prime}},
\end{aligned}
$$

which shows that $R_{N}$ satisfies (iii) of Theorem 2.18.

Moreover, by the vanishing moments of $D_{k}$, we obviously have $R_{N}(1)=0=R_{N}^{*}(1)$. Thus, $R_{N}$ satisfies all the conditions of Corollary 2.22 with $\epsilon$ replaced by $\epsilon_{1}^{\prime}$ and $C_{R_{N}} \lesssim 2^{-\delta N}$, which completes the proof of Lemma 3.6.

From Lemma 3.6 and Corollary 2.22, it is easy to deduce the boundedness of $T_{N}$ on spaces of test functions when $N$ is large enough.

Proposition 3.7. Let $\epsilon_{1} \in(0,1], \epsilon_{2}>0, \epsilon_{3}>0$, and let $\left\{S_{k}\right\}_{k \in \mathbb{Z}}$ be an $\left(\epsilon_{1}, \epsilon_{2}, \epsilon_{3}\right)$-ATI. For $N \in \mathbb{N}$, let $R_{N}$ and $T_{N}$ be as in (3.44). Then there exist constants $C_{8}>0$ and $\delta>0$, which are independent of $N$, such that for all $f \in \mathcal{G}\left(x_{1}, r, \beta, \gamma\right)$ with $x_{1} \in \mathcal{X}, r>0$ and $0<\beta, \gamma<\left(\epsilon_{1} \wedge \epsilon_{2}\right)$,

$$
\left\|R_{N}(f)\right\|_{\mathcal{G}\left(x_{1}, r, \beta, r\right)} \leq C_{8} 2^{-N \delta}\|f\|_{\mathcal{G}\left(x_{1}, r, \beta, r\right)} .
$$

Moreover, if $\mathrm{N}$ is so large that

$$
C_{8} 2^{-N \delta}<1
$$

then $T_{N}^{-1}$ exists and maps any space of test functions to itself. More precisely, there exists a constant $C>0$ such that for all $f \in \dot{\mathcal{G}}\left(x_{1}, r, \beta, \gamma\right)$ with $x_{1} \in \mathcal{X}, r>0$, and $0<\beta, \gamma<\left(\epsilon_{1} \wedge \epsilon_{2}\right)$,

$$
\left\|T_{N}^{-1}(f)\right\|_{\mathcal{G}\left(x_{1}, r, \beta, r\right)} \leq C\|f\|_{\mathcal{G}\left(x_{1}, r, \beta, r\right)} .
$$


Proof. Applying Corollary 2.22 together with Lemmas 3.4 and 3.6 gives (3.56). Moreover, if we choose $N \in \mathbb{N}$ such that (3.57) holds, then by (3.56), we have that for all $f \in \mathcal{G}\left(x_{1}, r, \beta, \gamma\right)$,

$$
\begin{aligned}
\left\|T_{N}^{-1}(f)\right\|_{\mathcal{G}\left(x_{1}, r, \beta, r\right)} & =\left\|\left(I-R_{N}\right)^{-1}(f)\right\|_{\mathcal{G}\left(x_{1}, r, \beta, r\right)} \\
& =\left\|\sum_{l=0}^{\infty}\left(R_{N}\right)^{l}(f)\right\|_{\mathcal{G}\left(x_{1}, r, \beta, r\right)} \\
& \leq \sum_{l=0}^{\infty}\left(C_{8} 2^{-N \delta}\right)^{l}\|f\|_{\mathcal{G}\left(x_{1}, r, \beta, \gamma\right)} \\
& \lesssim\|f\|_{\mathcal{G}\left(x_{1}, r, \beta, r\right)^{\prime}}
\end{aligned}
$$

which completes the proof of Proposition 3.7.

Proposition 3.8. Let $p \in(1, \infty), \epsilon_{1} \in(0,1], \epsilon_{2}>0, \epsilon_{3}>0$ and let $\left\{S_{k}\right\}_{k \in \mathbb{Z}}$ be an $\left(\epsilon_{1}, \epsilon_{2}, \epsilon_{3}\right)$-ATI. For $N \in \mathbb{N}$, let $R_{N}$ and $T_{N}$ be as in (3.44). Then there exist constants $C_{9}>0$ and $\delta>0$, which are independent of $N$, such that for all $f \in L^{p}(\mathcal{X})$

$$
\left\|R_{N}(f)\right\|_{L^{p}(\mathcal{X})} \leq C_{9} 2^{-N \delta}\|f\|_{L^{p}(\mathcal{X})} .
$$

Moreover, if $N$ is so large that

$$
\mathrm{C}_{9} 2^{-\mathrm{N} \delta}<1
$$

then $T_{N}^{-1}$ exists and is bounded on $L^{p}(\mathcal{X})$, and there exists a constant $C>0$ such that for all $f \in$ $L^{p}(\mathcal{X})$

$$
\left\|T_{N}^{-1}(f)\right\|_{L^{p}(\mathcal{X})} \leq C\|f\|_{L^{p}(\mathcal{X})} .
$$

Proof. We use the same notation as in Lemma 3.6. From Lemma 3.6 together with the proposition in [75, page 29], it is easy to see that $R_{N}$ is a singular integral satisfying the condition (10) in [75, page 19] with $A \lesssim 2^{-N \delta}$. This fact and the corollary in [75, page 19] together with a duality argument and Lemma 3.4 prove that $R_{N}$ is also bounded on $L^{p}(\mathcal{X})$ for $p \in(1, \infty)$ and $\left\|R_{N}\right\|_{L^{p}(x) \rightarrow L^{p}(\mathcal{X})} \lesssim 2^{-N \delta}$. That is, (3.60) holds. If we choose $N \in \mathbb{N}$ so large that (3.61) holds, by an argument similar to the proof of Proposition 3.7, we can prove that $T_{N}^{-1}$ is bounded on $L^{p}(\mathcal{X})$ for $p \in(1, \infty)$.

Let $\epsilon_{1} \in(0,1], \epsilon_{2}>0, \epsilon_{3}>0$, and let $\left\{S_{k}\right\}_{k=0}^{\infty}$ be an $\left(\epsilon_{1}, \epsilon_{2}, \epsilon_{3}\right)$-ATI. Set $D_{k}=S_{k}-S_{k-1}$ for $k \in \mathbb{Z}$. For any $f \in L^{p}(\mathcal{X})$ with $p \in(1, \infty)$ and $x \in \mathcal{X}$, the Littlewood-Paley g-function $\dot{g}(f)$ is defined by

$$
\dot{g}(f)(x)=\left\{\sum_{k=-\infty}^{\infty}\left|D_{k}(f)(x)\right|^{2}\right\}^{1 / 2} .
$$


Lemma 3.9. Let $\epsilon_{1} \in(0,1], \epsilon_{2}>0, \epsilon_{3}>0$, and let $\left\{S_{k}\right\}_{k \in \mathbb{Z}}$ be an $\left(\epsilon_{1}, \epsilon_{2}, \epsilon_{3}\right)$-ATI. Let $D_{k}=S_{k}-S_{k-1}$ for $k \in \mathbb{Z}$ and let $\dot{g}(f)$ for $f \in L^{p}(\mathcal{X})$ with $p \in(1, \infty)$ be as in (3.63). Then there exists a constant $C_{p}>0$ such that for all $f \in L^{p}(\mathcal{X})$,

$$
\|\dot{g}(f)\|_{L^{p}(x)} \leq C_{p}\|f\|_{L^{p}(x)}
$$

Proof. By Khinchin's inequality (see [76, page 165]) and Minkowski's inequality, we first have that for any $N \in \mathbb{N}$,

$$
\begin{aligned}
\left\|\left\{\sum_{k=-N}^{N}\left|D_{k}(f)\right|^{2}\right\}^{1 / 2}\right\|_{L^{p}(\mathcal{x})} & \lesssim\left\|\frac{1}{2^{2 N}} \sum_{\sigma_{-N}} \cdots \sum_{\sigma_{N}} \sum_{k=-N}^{N} \sigma_{k} D_{k}(f)\right\|_{L^{p}(\mathcal{x})} \\
& \lesssim \frac{1}{2^{2 N}} \sum_{\sigma_{-N}} \cdots \sum_{\sigma_{N}}\left\|\sum_{k=-N}^{N} \sigma_{k} D_{k}(f)\right\|_{L^{p}(\mathcal{X})},
\end{aligned}
$$

where $\sigma_{k}=1$ or -1 for $k \in\{-N, \ldots, N\}$. For any fixed $\sigma=\left\{\sigma_{k}\right\}_{k=-N}^{N}$, we set $T_{N}^{\sigma}=\sum_{k=-N}^{N} \sigma_{k} D_{k}$ and denote its kernel by $K_{N}^{\sigma}$. Similarly to the proof of Lemma 3.6, it is easy to verify that $K_{N}^{\sigma}$ and $\left(K_{N}^{\sigma}\right)^{*}$ are standard Calderon-Zygmund kernels, with constants independent of $N$ and $\sigma$. Then if we can verify that $T_{N}^{\sigma}$ is bounded on $L^{2}(\mathcal{X})$ with $\left\|T_{N}^{\sigma}\right\|_{L^{2}(x) \rightarrow L^{2}(\mathcal{X})} \lesssim 1$, then by the corollary in [75, page 22] together with a duality argument, we obtain that for $p \in(1, \infty)$, $\left\|T_{N}^{\sigma}\right\|_{L^{p}(\mathcal{X}) \rightarrow L^{p}(\mathcal{X})} \lesssim 1$. Therefore, for all $N \in \mathbb{N}$ and all $f \in L^{p}(\mathcal{X})$,

$$
\left\|\left\{\sum_{k=-N}^{N}\left|D_{k}(f)\right|^{2}\right\}^{1 / 2}\right\|_{L^{p}(x)} \lesssim\|f\|_{L^{p}(x)} .
$$

Then Fatou's lemma further shows that $\|\dot{g}(f)\|_{L^{p}(\mathcal{X})} \lesssim\|f\|_{L^{p}(x)}$. To finish the proof of Lemma 3.9, it remains to verify that $T_{N}^{\sigma}$ is bounded on $L^{2}(\mathcal{X})$. By the Cotlar-Stein lemma, it suffices to verify that for any $\epsilon_{1}^{\prime} \in\left(0, \epsilon_{1} \wedge \epsilon_{2}\right)$ and all $j, k \in \mathbb{Z}$,

$$
\begin{aligned}
&\left\|\sigma_{k} D_{k}\left(\sigma_{j} D_{j}\right)^{t}\right\|_{L^{2}(\mathcal{X}) \rightarrow L^{2}(\mathcal{X})} \lesssim 2^{-|k-j| \epsilon_{1}^{\prime},} \\
&\left\|\left(\sigma_{j} D_{j}\right)^{t} \sigma_{k} D_{k}\right\|_{L^{2}(\mathcal{X}) \rightarrow L^{2}(\mathcal{X})} \lesssim 2^{-|k-j| \epsilon_{1}^{\prime}} .
\end{aligned}
$$

However, these two estimates are a simple corollary of (3.2) in Lemma 3.2. This completes the proof of Lemma 3.9.

We can now establish a continuous Calderón reproducing formula.

Theorem 3.10. Let $\epsilon_{1} \in(0,1], \epsilon_{2}>0, \epsilon_{3}>0, \epsilon \in\left(0, \epsilon_{1} \wedge \epsilon_{2}\right)$ and let $\left\{S_{k}\right\}_{k \in \mathbb{Z}}$ be an $\left(\epsilon_{1}, \epsilon_{2}, \epsilon_{3}\right)$-ATI. Set $D_{k}=S_{k}-S_{k-1}$ for $k \in \mathbb{Z}$. Then there exists a family of linear operators $\left\{\tilde{D}_{k}\right\}_{k \in \mathbb{Z}}$ such that for all $f \in \mathcal{G}_{0}^{\epsilon}(\beta, \gamma)$ with $0<\beta, \gamma<\epsilon$,

$$
f=\sum_{k=-\infty}^{\infty} \tilde{D}_{k} D_{k}(f)
$$


where the series converges in both the norm of $\mathcal{G}_{0}^{e}(\beta, \gamma)$ and the norm of $L^{p}(x)$ for $p \in(1, \infty)$. Moreover, the kernels of the operators $\widetilde{D}_{k}$ satisfy the conditions (i) and (ii) of Definition 2.2 with $\epsilon_{1}$ and $\epsilon_{2}$ replaced by $\epsilon^{\prime} \in\left(\epsilon, \epsilon_{1} \wedge \epsilon_{2}\right)$, and $\int_{x} \widetilde{D}_{k}(x, y) d \mu(y)=\int_{x} \widetilde{D}_{k}(x, y) d \mu(x)=0$.

Proof. Fix a large integer $N$ such that (3.57) and (3.61) hold and, therefore, Propositions 3.7 and 3.8 hold. Let $D_{k}^{N}$ for $k \in \mathbb{Z}$ be as in (3.44). It is easy to check that $D_{k}^{N}(\cdot, y) \in$ $\mathcal{G}\left(y, 2^{-j}, \epsilon_{1}, \epsilon_{2}\right)$ for all $k \in \mathbb{Z}$. Define $\widetilde{D}_{k}(x, y)=T_{N}^{-1}\left(D_{k}^{N}(\cdot, y)\right)(x)$ for $k \in \mathbb{Z}$, where $T_{N}^{-1}$ is defined as in Proposition 3.7. Then Proposition 3.7 shows that $\widetilde{D}_{k}$ for $k \in \mathbb{Z}$ satisfies all the conclusions of the theorem, and formally, we also have (3.68). We still need to verify that the series in (3.68) converges in both the norm of $\mathcal{C}_{0}^{\epsilon}(\beta, \gamma)$ and the norm of $L^{p}(\mathcal{X})$ with $p \in(1, \infty)$.

Let $\widetilde{\epsilon}=\epsilon_{1} \wedge \epsilon_{2}$. We first prove that the series in (3.68) converges in the norm of $\mathcal{G}_{0}^{\epsilon}(\beta, \gamma)$ with $0<\beta, \gamma<\epsilon$. Let $f \in \mathcal{G}^{\prime}\left(\beta^{\prime}, \gamma^{\prime}\right)$ with $\beta<\beta^{\prime}<\tilde{\epsilon}$ and $\gamma<\gamma^{\prime}<\widetilde{\epsilon}$. Then, for $L \in \mathbb{N}$, we write

$$
\begin{aligned}
\sum_{|k| \leq L} \tilde{D}_{k} D_{k}(f) & =T_{N}^{-1}\left(\sum_{|k| \leq L} D_{k}^{N} D_{k}\right)(f) \\
& =T_{N}^{-1}\left(T_{N}-\sum_{|k| \geq L+1} D_{k}^{N} D_{k}\right)(f) \\
& =T_{N}^{-1} T_{N}(f)-T_{N}^{-1}\left(\sum_{|k| \geq L+1} D_{k}^{N} D_{k}\right)(f) \\
& =f-\lim _{j \rightarrow \infty}\left(R_{N}\right)^{j}(f)-T_{N}^{-1}\left(\sum_{|k| \geq L+1} D_{k}^{N} D_{k}\right)(f)
\end{aligned}
$$

We now verify that

$$
\lim _{L \rightarrow \infty}\left\|f-\sum_{|k| \leq L} \widetilde{D}_{k} D_{k}(f)\right\|_{\mathcal{G}(\beta, \gamma)}=0
$$

To see this, we write

$$
\begin{aligned}
& \left\|\sum_{|k| \leq L} \widetilde{D}_{k} D_{k}(f)-f\right\|_{\mathcal{G}(\beta, \gamma)} \\
& \quad \leq \lim _{j \rightarrow \infty}\left\|\left(R_{N}\right)^{j}(f)\right\|_{\mathcal{G}(\beta, \gamma)}+\left\|T_{N}^{-1}\left(\sum_{|k| \geq L+1} D_{k}^{N} D_{k}\right)(f)\right\|_{\mathcal{G}(\beta, \gamma)} .
\end{aligned}
$$

Notice that $\mathcal{G}^{\prime}\left(\beta^{\prime}, \gamma^{\prime}\right) \subset \mathcal{G}(\beta, \gamma)$. By (3.56) and (3.57), we have

$$
\lim _{j \rightarrow \infty}\left\|\left(R_{N}\right)^{j}(f)\right\|_{\mathcal{G}(\beta, \gamma)} \leq \lim _{j \rightarrow \infty}\left(C_{8} 2^{-N \delta}\right)^{j}\|f\|_{\mathcal{G}(\beta, r)}=0
$$


we remark that this is also true even when $\beta=\beta^{\prime}$ and $\gamma=\gamma^{\prime}$. We now prove that

$$
\lim _{L \rightarrow \infty}\left\|T_{N}^{-1}\left(\sum_{|k| \geq L+1} D_{k}^{N} D_{k}\right)(f)\right\|_{\mathcal{G}(\beta, \gamma)}=0
$$

To this end, by Proposition 3.7, it suffices to verify that there exists some $\sigma>0$ such that for all $0<\beta<\beta^{\prime}<\tilde{\epsilon}$ and $0<\gamma<\gamma^{\prime}<\tilde{\epsilon}$ and all $f \in \mathcal{G}_{(}\left(\beta^{\prime}, \gamma^{\prime}\right)$,

$$
\left\|\sum_{|k| \geq L+1} D_{k}^{N} D_{k}(f)\right\|_{\mathcal{G}(\beta, \gamma)} \lesssim 2^{-\sigma L}\|f\|_{\mathcal{G}\left(\beta^{\prime}, \gamma^{\prime}\right)}
$$

Similarly to the proof of (3.3), by Lemma 2.1(iii) and the geometric mean, we can reduce the proof of (3.74) to verifying that there exists some $\sigma>0$ such that for all $f \in \dot{\mathcal{G}}\left(\beta^{\prime}, \gamma^{\prime}\right)$ and all $x \in \mathcal{X}$

$$
\left|\sum_{|k| \geq L+1} D_{k}^{N} D_{k}(f)(x)\right| \lesssim 2^{-\sigma L}\|f\|_{\mathcal{G}\left(\beta^{\prime}, \gamma^{\prime}\right)} \frac{1}{V_{1}\left(x_{1}\right)+V\left(x_{1}, x\right)} \frac{1}{\left(1+d\left(x, x_{1}\right)\right)^{\gamma}}
$$

and for all $x, x^{\prime} \in \mathcal{X}$ with $d\left(x, x^{\prime}\right) \leq(1 / 2)\left(1+d\left(x, x_{1}\right)\right)$,

$$
\begin{aligned}
& \left|\sum_{|k| \geq L+1} D_{k}^{N} D_{k}(f)(x)-\sum_{|k| \geq L+1} D_{k}^{N} D_{k}(f)\left(x^{\prime}\right)\right| \\
& \quad \lesssim\|f\|_{\mathcal{G}\left(\beta^{\prime}, \gamma^{\prime}\right)} \frac{d\left(x, x^{\prime}\right)^{\beta^{\prime}}}{\left(1+d\left(x, x_{1}\right)\right)^{\beta^{\prime}}} \frac{1}{V_{1}\left(x_{1}\right)+V\left(x_{1}, x\right)} \frac{1}{\left(1+d\left(x, x_{1}\right)\right)^{\gamma^{\prime}}} .
\end{aligned}
$$

Similarly to the proofs of Lemmas 3.4 and 3.6 and using some estimates similar to (3.2) and (3.12), we easily obtain that for any $L \in \mathbb{N}$, the operator $T_{L}=\sum_{|k| \geq L+1} D_{k}^{N} D_{k}$ satisfies all the conditions of Corollary 2.22 with $\epsilon$ replaced by $\widetilde{\epsilon}, C_{T_{L}} \lesssim 1$ and

$$
\left\|T_{L}\right\|_{L^{2}(x) \rightarrow L^{2}(x)} \lesssim 1
$$

Corollary 2.22 then shows that $T_{L}$ is bounded on $\mathcal{G}_{1}\left(\beta^{\prime}, \gamma^{\prime}\right)$ for any $0<\beta^{\prime}, \gamma^{\prime}<\widetilde{\epsilon}$. In particular, we see that (3.76) holds. 
To verify (3.75), we simply denote $D_{k}^{N} D_{k}$ by $E_{k}$. By Lemma 3.2 and its proof, it is easy to see that $E_{k}(x, y)$, the kernel of $E_{k}$, still satisfies (i) to (iv) of Definition 2.2 with a constant depending on $N$; see (3.2) and (3.12). Moreover, $E_{k}(1)=0$. For $f \in$ $\stackrel{\mathcal{G}}{(}\left(\beta^{\prime}, \gamma^{\prime}\right)$,

$$
\begin{aligned}
\left|\sum_{k=L+1}^{\infty} D_{k}^{N} D_{k}(f)(x)\right|= & \left|\sum_{k=L+1}^{\infty} \int_{x} E_{k}(x, y)[f(y)-f(x)] d \mu(y)\right| \\
\leq & \sum_{k=L+1}^{\infty} \int_{d(x, y) \leq\left(1+d\left(x_{1}, x\right)\right) / 2}\left|E_{k}(x, y)\right||f(y)-f(x)| d \mu(y) \\
& +\sum_{k=L+1}^{\infty} \int_{d(x, y)>\left(1+d\left(x_{1}, x\right)\right) / 2}\left|E_{k}(x, y)\right||f(y)| d \mu(y) \\
& +\sum_{k=L+1}^{\infty} \int_{d(x, y)>\left(1+d\left(x_{1}, x\right)\right) / 2}\left|E_{k}(x, y)\right||f(x)| d \mu(y) \\
\equiv & \sum_{i=1}^{3} Z_{i} .
\end{aligned}
$$

The size estimates for $E_{k}$ and the regularity of $f$ imply together with Lemma 2.1(ii) that

$$
\begin{aligned}
Z_{1} \lesssim \sum_{k=L+1}^{\infty} \int_{d(x, y) \leq\left(1+d\left(x_{1}, x\right)\right) / 2} & \frac{1}{V_{2^{-k}}(x)+V_{2^{-k}}(y)+V(x, y)} \frac{2^{-k \epsilon_{2}}}{\left(2^{-k}+d(x, y)\right)^{\epsilon_{2}}} \\
& \times \frac{d(x, y)^{\beta^{\prime}}}{\left(1+d\left(x_{1}, x\right)\right)^{\beta^{\prime}}} \frac{1}{V_{1}\left(x_{1}\right)+V\left(x_{1}, x\right)} \frac{1}{\left(1+d\left(x_{1}, x\right)\right)^{\gamma^{\prime}}} d \mu(y) \\
& \lesssim 2^{-L \beta^{\prime}} \frac{1}{V_{1}\left(x_{1}\right)+V\left(x_{1}, x\right)} \frac{1}{\left(1+d\left(x_{1}, x\right)\right)^{\gamma^{\prime}}} .
\end{aligned}
$$

Similarly, Lemma 2.1(i) yields that

$$
\begin{aligned}
Z_{3} \lesssim \sum_{k=L+1}^{\infty} \int_{d(x, y)>\left(1+d\left(x_{1}, x\right)\right) / 2} & \frac{1}{V_{2^{-k}}(x)+V(x, y)} \frac{2^{-k \epsilon_{2}}}{\left(2^{-k}+d(x, y)\right)^{\epsilon_{2}}} d \mu(y) \\
& \times \frac{1}{V_{1}\left(x_{1}\right)+V\left(x_{1}, x\right)} \frac{1}{\left(1+d\left(x_{1}, x\right)\right)^{\gamma^{\prime}}} \\
& \lesssim 2^{-L \epsilon_{2}} \frac{1}{V_{1}\left(x_{1}\right)+V\left(x_{1}, x\right)} \frac{1}{\left(1+d\left(x_{1}, x\right)\right)^{\gamma^{\prime}}} .
\end{aligned}
$$


Since $d(x, y)>\left(1+d\left(x_{1}, x\right)\right) / 2$, we have $V\left(x, x_{1}\right) \lesssim V(x, y)$. This estimate together with Lemma 2.1 shows that

$$
\begin{aligned}
Z_{2} \lesssim \sum_{k=L+1}^{\infty} \int_{d(x, y)>\left(1+d\left(x_{1}, x\right)\right) / 2} & \frac{1}{V_{2^{-k}}(x)+V_{2-k}(y)+V(x, y)} \frac{2^{-k \epsilon_{2}}}{\left(2^{-k}+d(x, y)\right)^{\epsilon_{2}}} \\
& \times \frac{1}{V_{1}\left(x_{1}\right)+V\left(x_{1}, y\right)} \frac{1}{\left(1+d\left(x_{1}, y\right)\right)^{\gamma^{\prime}}} d \mu(y) \\
& \lesssim 2^{-L \epsilon_{2}} \min \left\{\frac{1}{V_{1}\left(x_{1}\right)}, \frac{1}{V\left(x, x_{1}\right)}\right\} \frac{1}{\left(1+d\left(x_{1}, x\right)\right)^{\epsilon_{2}}} \\
& \lesssim 2^{-L \epsilon_{2}} \frac{1}{V_{1}\left(x_{1}\right)+V\left(x_{1}, x\right)} \frac{1}{\left(1+d\left(x_{1}, x\right)\right)^{\gamma^{\prime}}},
\end{aligned}
$$

which completes the proof of (3.75) for the operator $\sum_{k=L+1}^{\infty} D_{k}^{N} D_{k}$.

Since $\int_{x} f(y) d \mu(y)=0$, we can write

$$
\begin{aligned}
\left|\sum_{k=-\infty}^{-L-1} D_{k}^{N} D_{k}(f)(x)\right| & =\left|\sum_{k=-\infty}^{-L-1} \int_{x}\left[E_{k}(x, y)-E_{k}\left(x, x_{1}\right)\right] f(y) d \mu(y)\right| \\
& \leq \sum_{k=-\infty}^{-L-1} \int_{d\left(x_{1}, y\right) \leq\left(2^{-k}+d\left(x_{1}, x\right)\right) / 2}\left|E_{k}(x, y)-E_{k}\left(x, x_{1}\right)\right||f(y)| d \mu(y) \\
& +\sum_{k=-\infty}^{-L-1} \int_{d\left(x_{1}, y\right)>\left(2^{-k}+d\left(x_{1}, x\right)\right) / 2}\left|E_{k}(x, y)\right||f(y)| d \mu(y) \\
& +\sum_{k=-\infty}^{-L-1} \int_{d\left(x_{1}, y\right)>\left(2^{-k}+d\left(x_{1}, x\right)\right) / 2}\left|E_{k}\left(x, x_{1}\right)\right||f(y)| d \mu(y) \\
& \equiv \sum_{i=1}^{3} Y_{i} .
\end{aligned}
$$

The regularity of $E_{k}$ in the second variable and the size condition of $f$ together with Lemma 2.1(i) yield that

$$
\begin{aligned}
Y_{1} \lesssim \sum_{k=-\infty}^{-L-1} \int_{d\left(x_{1}, y\right) \leq\left(2^{-k}+d\left(x_{1}, x\right)\right) / 2} & \frac{d\left(y, x_{1}\right)^{\epsilon_{1}}}{\left(2^{-k}+d\left(x, x_{1}\right)\right)^{\epsilon_{1}}} \frac{2^{-k \epsilon_{2}}}{\left(2^{-k}+d\left(x, x_{1}\right)\right)^{\epsilon_{2}}} \frac{1}{V_{2^{-k}}(x)+V_{2^{-k}}\left(x_{1}\right)+V\left(x, x_{1}\right)} \\
& \times \frac{1}{V_{1}\left(x_{1}\right)+V\left(x_{1}, y\right)} \frac{1}{\left(1+d\left(x_{1}, y\right)\right)^{\gamma^{\prime}}} d \mu(y) \\
& \lesssim 2^{-L\left(\gamma^{\prime}-\gamma\right)} \frac{1}{V_{1}\left(x_{1}\right)+V\left(x, x_{1}\right)} \frac{1}{\left(1+d\left(x, x_{1}\right)\right)^{\gamma}} .
\end{aligned}
$$


Similarly, Lemma 2.1(ii) shows that

$$
\begin{aligned}
Y_{3} \lesssim \sum_{k=-\infty}^{-L-1} \int_{d\left(x_{1}, y\right)>\left(2^{-k}+d\left(x_{1}, x\right)\right) / 2} & \frac{1}{V_{2^{-k}}(x)+V_{2^{-k}}\left(x_{1}\right)+V\left(x, x_{1}\right)} \\
& \times \frac{2^{-k \epsilon_{2}}}{\left(2^{-k}+d\left(x, x_{1}\right)\right)^{\epsilon_{2}}} \frac{1}{V_{1}\left(x_{1}\right)+V\left(x_{1}, y\right)} \frac{1}{\left(1+d\left(x_{1}, y\right)\right)^{\gamma^{\prime}}} d \mu(y) \\
& \lesssim 2^{-L\left(\gamma^{\prime}-\gamma\right) / 2} \frac{1}{V_{1}\left(x_{1}\right)+V\left(x, x_{1}\right)} \frac{1}{\left(1+d\left(x_{1}, x\right)\right)^{\gamma}} .
\end{aligned}
$$

Since $k<0$ and $d\left(x_{1}, y\right)>\left(2^{-k}+d\left(x_{1}, x\right)\right) / 2>\left(1+d\left(x_{1}, x\right)\right) / 2$, then $V\left(x_{1}, x\right) \lesssim V\left(x_{1}, y\right)$. From this and Lemma 2.1(ii), it follows that

$$
\begin{aligned}
Y_{2} \lesssim \sum_{k=-\infty}^{-L-1} \int_{d\left(x_{1}, y\right)>\left(2^{-k}+d\left(x_{1}, x\right)\right) / 2} & \frac{1}{V_{2^{-k}}(x)+V_{2^{-k}}(y)+V(x, y)} \\
& \times \frac{2^{-k \epsilon_{2}}}{\left(2^{-k}+d(x, y)\right)^{\epsilon_{2}}} \frac{1}{V_{1}\left(x_{1}\right)+V\left(x_{1}, y\right)} \frac{1}{\left(1+d\left(x_{1}, y\right)\right)^{\gamma^{\prime}}} d \mu(y) \\
& \lesssim 2^{-L\left(\gamma^{\prime}-\gamma\right)} \frac{1}{V_{1}\left(x_{1}\right)+V\left(x, x_{1}\right)} \frac{1}{\left(1+d\left(x_{1}, x\right)\right)^{\gamma}}
\end{aligned}
$$

which completes the proof of (3.75). Hence, we obtain (3.74). Therefore for $f \in \mathcal{G}\left(\beta^{\prime}, \gamma^{\prime}\right)$ with $\beta<\beta^{\prime}<\widetilde{\epsilon}$ and $\gamma<\gamma^{\prime}<\widetilde{\epsilon}$, (3.73) holds. Combining the estimate (3.73) with (3.72) shows (3.70).

Let now $f \in \mathcal{G}_{0}^{\epsilon}(\beta, \gamma)$ with $0<\beta, \gamma<\epsilon$. By definition, there exists a sequence $\left\{f_{n}\right\}_{n=1}^{\infty} \subset \mathcal{G}(\epsilon, \epsilon)$ such that $\lim _{n \rightarrow \infty}\left\|f-f_{n}\right\|_{\mathcal{G}(\beta, \gamma)}=0$. For any given $L \in \mathbb{N}$, using some estimates similar to (3.2) and (3.12) (see also Remark 3.16), similarly to the proof of Lemma 3.6, we can easily verify that for any $L \in \mathbb{N}$, the operator $\widetilde{T}_{L}=\sum_{|k| \leq L} D_{k}^{N} D_{k}$ satisfies all the conditions of Corollary 2.22 with $\epsilon$ replaced by $\widetilde{\epsilon}, C_{\widetilde{T}_{L}} \lesssim 1$, and $\left\|\widetilde{T}_{L}\right\|_{L^{2}(\chi) \rightarrow L^{2}(\chi)} \lesssim 1$. Thus $\widetilde{T}_{L}$ is bounded on $\mathcal{G}(\beta, \gamma)$ and $\tilde{T}_{L}^{*}(1)=0$ by the vanishing moment of $D_{k}^{N}$, which together with the boundedness of $T_{N}^{-1}$ on $\mathcal{G}(\beta, \gamma)$ yields

$$
\begin{aligned}
\| f- & \sum_{|k| \leq L} \tilde{D}_{k} D_{k}(f) \|_{\mathcal{G}(\beta, \gamma)} \\
& \lesssim\left\|f-f_{n}\right\|_{\mathcal{G}(\beta, \gamma)}+\left\|f_{n}-T_{N}^{-1}\left(\tilde{T}_{L}\left(f_{n}\right)\right)\right\|_{\mathcal{G}(\beta, r)}+\left\|T_{N}^{-1}\left(\tilde{T}_{L}\left(f_{n}\right)\right)-T_{N}^{-1}\left(\widetilde{T}_{L}(f)\right)\right\|_{\mathcal{G}(\beta, \gamma)} \\
& \lesssim\left\|f-f_{n}\right\|_{\mathcal{G}(\beta, \gamma)}+\left\|f_{n}-T_{N}^{-1}\left(\tilde{T}_{L}\left(f_{n}\right)\right)\right\|_{\mathcal{G}(\beta, r)} .
\end{aligned}
$$


For any given $\delta>0$, fix some $n \in \mathbb{N}$ such that $\left\|f-f_{n}\right\|_{\mathcal{G}(\beta, \gamma)} \leq \delta / 4$. By (3.70), for this chosen $n$, there exists some $L_{0} \in \mathbb{N}$ such that if $L>L_{0}$, then

$$
\left\|f_{n}-T_{N}^{-1}\left(\widetilde{T}_{L}\left(f_{n}\right)\right)\right\|_{\mathcal{G}(\beta, \gamma)}<\frac{\delta}{2}
$$

Thus, when $L>L_{0}$,

$$
\left\|f-\sum_{|k| \leq L} \widetilde{D}_{k} D_{k}(f)\right\|_{\mathcal{G}(\beta, \gamma)}<\delta .
$$

Since $\widetilde{T}_{L}^{*}(1)=0$ and $R_{N}^{*}(1)=0$ and since $\widetilde{T}_{L}$ and $T_{N}^{-1}$ are bounded from $\mathcal{G}(x, r, \beta, \gamma)$ to $\mathcal{G}(x, r, \beta, \gamma)$ for any $x \in \mathcal{X}, r>0$, and $0<\beta, \gamma<\tilde{\epsilon}$, it follows that for $\epsilon \in(0, \tilde{\epsilon})$, $\sum_{|k| \leq L} \widetilde{D}_{k} D_{k}\left(f_{n}\right)=T_{N}^{-1} \widetilde{T}_{L}\left(f_{n}\right) \in \mathscr{\mathcal { G }}(\epsilon, \epsilon)$. Moreover,

$$
\begin{aligned}
\left\|\left\{f-\sum_{|k| \leq L} \tilde{D}_{k} D_{k}(f)\right\}-\left\{f_{n}-\sum_{|k| \leq L} \tilde{D}_{k} D_{k}\left(f_{n}\right)\right\}\right\|_{\mathcal{G}(\beta, \gamma)} & \leq\left\|f-f_{n}\right\|_{\mathcal{G}(\beta, \gamma)}+\left\|T_{N}^{-1} \widetilde{T}_{L}\left(f-f_{n}\right)\right\|_{\mathcal{G}(\beta, \gamma)} \\
& \lesssim\left\|f-f_{n}\right\|_{\mathcal{G}(\beta, \gamma)} \\
& \longrightarrow 0,
\end{aligned}
$$

as $n \rightarrow \infty$. Thus, $f-\sum_{|k| \leq L} \widetilde{D}_{k} D_{k}(f) \in \mathcal{G}_{0}^{\epsilon}(\beta, \gamma)$. By (3.88), we further have that when $L>L_{0}$,

$$
\left\|f-\sum_{|k| \leq L} \tilde{D}_{k} D_{k}(f)\right\|_{\tilde{\mathcal{G}}_{0}^{e}(\beta, \gamma)}<\delta,
$$

which implies that (3.68) holds in the norm of $\dot{\mathcal{G}}_{0}^{\epsilon}(\beta, \gamma)$.

We now verify that (3.68) also holds in $L^{p}(\mathcal{X})$ for $p \in(1, \infty)$. By Proposition 3.8 and the choice of $N$, we have

$$
\begin{gathered}
\lim _{j \rightarrow \infty}\left\|\left(R_{N}\right)^{j}(f)\right\|_{L^{p}(\mathcal{X})} \leq \lim _{j \rightarrow \infty}\left(C_{9} 2^{-N \delta}\right)^{j}\|f\|_{L^{p}(\mathcal{X})}=0, \\
\left\|T_{N}^{-1}\left(\sum_{|k| \geq L+1} D_{k}^{N} D_{k}\right)(f)\right\|_{L^{p}(\mathcal{X})} \lesssim\left\|\left(\sum_{|k| \geq L+1} D_{k}^{N} D_{k}\right)(f)\right\|_{L^{p}(\mathcal{x})} .
\end{gathered}
$$


Moreover, by Lemma 3.9 and Hölder's inequality, we obtain

$$
\begin{aligned}
& \left\|\left(\sum_{|k| \geq L+1} D_{k}^{N} D_{k}\right)(f)\right\|_{L^{p}(\mathcal{X})} \\
& =\sup _{\|h\|_{L^{\prime}(x)} \leq 1}\left|\left\langle\sum_{|k| \geq L+1} D_{k}^{N} D_{k}(f), h\right\rangle\right| \\
& \leq \sup _{\|h\|_{L^{p^{\prime}}(x)} \leq 1}\left\|\left(\sum_{|k| \geq L+1}\left|D_{k}(f)\right|^{2}\right)^{1 / 2}\right\|\left\|_{L^{p}(\mathcal{X})}\right\|\left(\sum_{|k| \geq L+1}\left|\left(D_{k}^{N}\right)^{t}(h)\right|^{2}\right)^{1 / 2} \|_{L^{p^{\prime}}(\mathcal{X})} \\
& \lesssim\left\|\left(\sum_{|k| \geq L+1}\left|D_{k}(f)\right|^{2}\right)^{1 / 2}\right\|_{L^{p}(x)} \\
& \lesssim\|f\|_{L^{p}(\mathcal{X})} \text {. }
\end{aligned}
$$

Thus,

$$
\lim _{L \rightarrow \infty}\left\|\left(\sum_{|k| \geq L+1} D_{k}^{N} D_{k}\right)(f)\right\|_{L^{p}(\mathcal{X})} \lesssim \lim _{L \rightarrow \infty}\left\|\left(\sum_{|k| \geq L+1}\left|D_{k}(f)\right|^{2}\right)^{1 / 2}\right\|_{L^{p}(\mathcal{X})}=0 .
$$

From these estimates, we finally deduce that (3.68) holds in $L^{p}(\mathcal{X})$ with $p \in(1, \infty)$, which completes the proof of Theorem 3.10.

By an argument similar to the proof of Theorem 3.10, we obtain another continuous Calderón reproducing formula (we omit the details).

Theorem 3.11. Let $\epsilon_{1} \in(0,1], \epsilon_{2}>0, \epsilon_{3}>0, \epsilon \in\left(0, \epsilon_{1} \wedge \epsilon_{2}\right)$, and let $\left\{S_{k}\right\}_{k \in \mathbb{Z}}$ be an $\left(\epsilon_{1}, \epsilon_{2}, \epsilon_{3}\right)$-ATI. Set $D_{k}=S_{k}-S_{k-1}$ for $k \in \mathbb{Z}$. Then there exists a family of linear operators $\left\{\bar{D}_{k}\right\}_{k \in \mathbb{Z}}$ such that for all $f \in \mathcal{G}_{0}^{\epsilon}(\beta, \gamma)$ with $0<\beta, \gamma<\epsilon$,

$$
f=\sum_{k=-\infty}^{\infty} D_{k} \bar{D}_{k}(f)
$$

where the series converges in both the norm of $\dot{\mathcal{C}}_{0}^{\epsilon}(\beta, \gamma)$ and the norm of $L^{p}(\mathcal{X})$ for $p \in(1, \infty)$. Moreover, the kernels of the operators $\bar{D}_{k}$ satisfy the conditions (i) and (iii) of Definition 2.2 with $\epsilon_{1}$ and $\epsilon_{2}$ replaced by $\epsilon^{\prime} \in\left(\epsilon, \epsilon_{1} \wedge \epsilon_{2}\right)$, and $\int_{x} \bar{D}_{k}(x, y) d \mu(y)=\int_{x} \bar{D}_{k}(x, y) d \mu(x)=0$.

To establish some Calderón reproducing formulae in spaces of distributions, we first need to understand the action of the operators $D_{k}$ on spaces of distributions.

To this end, for all $x, y \in \mathcal{X}$, let $\varphi(x, y)$ satisfy the conditions (i) through (iii) with $k=0$ of Definition 2.2, and $\int_{x} \varphi(x, y) d \mu(y)=0=\int_{\chi} \varphi(x, y) d \mu(x)$. Let $\epsilon$ be as in Theorem 3.10, 
$0<\beta, \gamma<\epsilon$, and $f \in \mathcal{G}_{0}^{\epsilon}(\beta, \gamma)$. We then define

$$
\Psi(f)(x)=\int_{x} \varphi(x, y) f(y) d \mu(y)
$$

Let $u \in\left(\mathcal{C}_{0}^{\epsilon}(\beta, \gamma)\right)^{\prime}$. In analogy with the theory of distributions on $\mathbb{R}^{n}$, there exist two ways to define $\Psi(u) \in\left(\mathcal{C}_{0}^{e}(\beta, \gamma)\right)^{\prime}$. One way is to define $\Psi(u) \in\left(\mathcal{G}_{0}^{\epsilon}(\beta, \gamma)\right)^{\prime}$ by duality, that is, for all $f \in \dot{\mathcal{G}}_{0}^{\epsilon}(\beta, \gamma)$, we put

$$
\langle\Psi(u), f\rangle=\left\langle u, \Psi^{t}(f)\right\rangle
$$

where $\Psi^{t}$ denotes the integral operator with the kernel $\varphi^{t}(x, y)=\varphi(y, x)$ for all $x, y \in X$. Alternatively, we define pointwise

$$
\tilde{\Psi}(u)(x)=\langle u, \varphi(x, \cdot)\rangle .
$$

We now show that both definitions actually coincide.

Lemma 3.12. Let $\epsilon$ be as in Theorem 3.10 and let $0<\beta, \gamma<\epsilon$. Let $u \in\left(\mathcal{C}_{0}^{\epsilon}(\beta, \gamma)\right)^{\prime}$ and let $\Psi(u)$ and $\widetilde{\Psi}(u)$ be defined, respectively, as in (3.96) and (3.97). Then $\Psi(u)=\widetilde{\Psi}(u)$ in $\left(\mathcal{G}_{0}^{\epsilon}(\beta, \gamma)\right)^{\prime}$.

Proof. To establish this lemma, it suffices to show that for all $f \in \mathcal{G}(\epsilon, \epsilon)$,

$$
\int_{x} \tilde{\Psi}(u)(x) f(x) d \mu(x)=\left\langle u, \Psi^{t}(f)\right\rangle
$$

To this end, for $L \in \mathbb{N}$ large enough, we define

$$
T_{L}(f)(x)=\int_{B\left(x_{1}, L\right)} \varphi(y, x) f(y) d \mu(y)
$$

Let $\beta, \gamma$ be as in the lemma. Using Theorem 2.18 and some routine computations, we have $T_{L}(f) \in \dot{\mathcal{G}}(\epsilon, \epsilon)$ and $\lim _{L \rightarrow \infty}\left\|\Psi^{t}(f)-T_{L}(f)\right\|_{\mathcal{G}(\beta, \gamma)}=0$. Thus, $\left\langle u, \Psi^{t}(f)\right\rangle=\lim _{L \rightarrow \infty}\left\langle u, T_{L}(f)\right\rangle$.

Now for fixed $L \in \mathbb{N}$ large enough, and for any $J \in \mathbb{N}$, let $N_{J}=\left\{i \in I_{J}: Q_{i}^{J} \cap\right.$ $\left.B\left(x_{1}, L\right) \neq \varnothing\right\}$, where $\left\{Q_{i}^{J}\right\}_{J \in \mathbb{N}, i \in I_{J}}$ are dyadic cubes of $\mathcal{X}$ as in Lemma 2.19. If $Q_{i}^{J} \cap B\left(x_{1}, L\right) \neq \varnothing$ and $L$ is large enough, then $B\left(x_{1}, L\right) \subset B\left(z_{i}^{J}, 3 L\right)$ and $Q_{i}^{J} \subset B\left(x_{1}, 2 L\right)$, where $z_{i}^{J}$ is the center of $Q_{i}^{J}$ as in Lemma 2.19. These facts imply that $\sharp N_{J} \lesssim\left(L 2^{j}\right)^{n}$. By Lemma 2.19 , we write

$$
\begin{aligned}
T_{L}(f)(x) & =\sum_{i \in N_{J}} \int_{Q_{i}^{J} \cap B\left(x_{1}, L\right)}\left[\varphi(y, x)-\varphi\left(y_{Q_{i}^{I}}, x\right)\right] f(y) d \mu(y)+\sum_{i \in N_{J}} \varphi\left(y_{Q_{i}^{I}}, x\right) \int_{Q_{i}^{J} \cap B\left(x_{1}, L\right)} f(y) d \mu(y) \\
& \equiv T_{L, J}^{1}(f)(x)+T_{L, J}^{2}(f)(x),
\end{aligned}
$$


where $y_{Q_{i}^{I}}$ is any point in $Q_{i}^{J} \cap B\left(x_{1}, L\right)$. For fixed $L \in \mathbb{N}$ large enough, using Theorem 2.18 and some routine computations again, we have $T_{L, J}^{1}(f) \in \mathcal{G}(\epsilon, \epsilon)$ and

$$
\lim _{J \rightarrow \infty}\left\|T_{L, J}^{1}(f)\right\|_{\mathcal{G}(\beta, \gamma)}=0
$$

Thus,

$$
\begin{aligned}
\left\langle u, \Psi^{t}(f)\right\rangle & =\lim _{L \rightarrow \infty} \lim _{J \rightarrow \infty}\left\langle u, T_{L, J}^{2}(f)\right\rangle \\
& =\lim _{L \rightarrow \infty} \lim _{J \rightarrow \infty} \sum_{i \in N_{J}} \widetilde{\Psi}(u)\left(y_{Q_{i}^{I}}\right) \int_{Q_{i}^{J} \cap B\left(x_{1}, L\right)} f(y) d \mu(y) \\
& =\int_{\chi} \widetilde{\Psi}(u)(y) f(y) d \mu(y)+\lim _{L \rightarrow \infty} \lim _{J \rightarrow \infty} \sum_{i \in N_{J}} \int_{Q_{i}^{J} \cap B\left(x_{1}, L\right)}\left[\widetilde{\Psi}(u)\left(y_{Q_{i}^{I}}\right)-\widetilde{\Psi}(u)(y)\right] f(y) d \mu(y) .
\end{aligned}
$$

It is not so difficult to verify that

$$
\begin{aligned}
& \sum_{i \in N_{J}}\left[\varphi\left(\cdot, y_{Q_{i}^{I}}\right)-\varphi(\cdot, y)\right] \chi_{Q_{i}^{I} \cap B\left(x_{1}, L\right)}(y) \in \mathcal{G}(\epsilon, \epsilon), \\
& \left\|\sum_{i \in N_{J}}\left[\varphi\left(\cdot, y_{Q_{i}^{I}}\right)-\varphi(\cdot, y)\right] \chi_{Q_{i}^{J} \cap B\left(x_{1}, L\right)}(y)\right\|_{\mathcal{G}(\beta, \gamma)} \leq C 2^{-J \epsilon_{1}}
\end{aligned}
$$

uniformly in $y \in \mathcal{X}$. From this, it follows that $\left|\widetilde{\Psi}(u)\left(y_{Q_{i}^{J}}\right)-\tilde{\Psi}(u)(y)\right| \leq C_{u} 2^{-J \epsilon_{1}}$ uniformly in $y \in \mathcal{X}$, which along with Lebesgue's dominated convergence theorem shows that

$$
\lim _{J \rightarrow \infty} \sum_{i \in N_{J}} \int_{Q_{i}^{I} \cap B\left(x_{1}, L\right)}\left[\tilde{\Psi}(u)\left(y_{Q_{i}^{J}}\right)-\widetilde{\Psi}(u)(y)\right] f(y) d \mu(y)=0 .
$$

Thus, (3.98) is true and this completes the proof of Lemma 3.12.

Theorems 3.10 and 3.11 in combination with a duality argument and Lemma 3.12 show that continuous Calderón reproducing formulae also hold in spaces of distributions.

Theorem 3.13. Let all the notation be as in Theorems 3.10 and 3.11. Then for all $f \in\left(\mathcal{G}_{0}^{\epsilon}(\beta, \gamma)\right)^{\prime}$ with $0<\beta, \gamma<\epsilon,(3.68)$ and (3.94) hold in $\left(\mathcal{G}_{0}^{\epsilon}(\beta, \gamma)\right)^{\prime}$.

Finally, let us end this subsection by establishing a Littlewood-Paley theorem associated to ATIs via Theorem 3.10, which is a generalization of [44, Proposition 2.5.1]. However, the method used here is different from that in [44].

To this end, we need the following Fefferman-Stein vector-valued maximal function inequality in [77]; see also [44, Equation (2.11)] and [75, Chapter II, Section 1]. 
Lemma 3.14. Let $1<p<\infty, 1<q \leq \infty$, and let $M$ be the Hardy-Littlewood maximal operator on $x$. Let $\left\{f_{k}\right\}_{k \in \mathbb{Z}} \subset L^{p}(\mathcal{X})$ be a sequence of measurable functions on $\mathcal{X}$. Then

$$
\left\|\left\{\sum_{k=-\infty}^{\infty}\left|M\left(f_{k}\right)\right|^{q}\right\}^{1 / q}\right\|_{L^{p}(\mathcal{X})} \leq C\left\|\left\{\sum_{k=-\infty}^{\infty}\left|f_{k}\right|^{q}\right\}^{1 / q}\right\|_{L^{p}(\mathcal{X})},
$$

where $C$ is independent of $\left\{f_{k}\right\}_{k \in \mathbb{Z}}$.

Proposition 3.15. Let $\epsilon_{1} \in(0,1], \epsilon_{2}>0, \epsilon_{3}>0$, and let $\left\{S_{k}\right\}_{k \in \mathbb{Z}}$ be an $\left(\epsilon_{1}, \epsilon_{2}, \epsilon_{3}\right)$-ATI. Let $D_{k}=$ $S_{k}-S_{k-1}$ for $k \in \mathbb{Z}$ and let $\dot{g}(f)$ for $f \in L^{p}(X)$ with $p \in(1, \infty)$ be as in (3.63). Then there exists a constant $C_{p}>0$ such that for all $f \in L^{p}(\mathcal{X})$,

$$
C_{p}^{-1}\|f\|_{L^{p}(\mathcal{X})} \leq\|\dot{g}(f)\|_{L^{p}(\mathcal{X})} \leq C_{p}\|f\|_{L^{p}(\mathcal{X})}
$$

Proof. By Lemma 3.9, we only need to verify the first inequality. To this end, for any $f \in$ $L^{p}(\mathcal{X})$, Theorem 3.10 shows that there exist operators $\left\{\widetilde{D}_{k}\right\}_{k \in \mathbb{Z}}$ as in Theorem 3.10 such that

$$
f=\sum_{k=-\infty}^{\infty} \tilde{D}_{k} D_{k}(f)
$$

in $L^{p}(\mathcal{X})$. For $1<p<\infty$, let $1 / p+1 / p^{\prime}=1$. We first claim that for any $h \in L^{p^{\prime}}(\mathcal{X})$,

$$
\left\|\left\{\sum_{k=-\infty}^{\infty}\left|\tilde{D}_{k}^{t}(h)\right|^{2}\right\}^{1 / 2}\right\|_{L^{p^{\prime}}(x)} \lesssim\|h\|_{L^{p^{\prime}}(x)}
$$

Let $\epsilon \in\left(0, \epsilon_{1} \wedge \epsilon_{2}\right)$. In fact, by (3.2) of Lemma 3.2 and Remark 3.3, we have that for any $\epsilon_{1}^{\prime} \in\left(0, \epsilon_{1} \wedge \epsilon_{2}\right)$,

$$
\left|\tilde{D}_{k}^{t} \widetilde{D}_{l}(x, y)\right| \lesssim 2^{-|k-l| \epsilon^{\prime}} \frac{1}{V_{2^{-(k \wedge l)}}(x)+V_{2^{-(k \wedge l)}}(y)+V(x, y)} \frac{2^{-(k \wedge l) \epsilon}}{\left(2^{-(k \wedge l)}+d(x, y)\right)^{\epsilon}}
$$

From this and Lemma 2.1(iv), it follows that for $x \in \mathcal{X}$,

$$
\begin{aligned}
\left|D_{k}^{t} \tilde{D}_{l}(h)(x)\right| & \lesssim 2^{-|k-l| \epsilon^{\prime}} \int_{x} \frac{1}{V_{2^{-(k \wedge l)}}(x)+V_{2^{-(k \wedge l)}}(y)+V(x, y)} \frac{2^{-(k \wedge l) \epsilon}}{\left(2^{-(k \wedge l)}+d(x, y)\right)^{\epsilon}}|h(y)| d \mu(y) \\
& \lesssim 2^{-|k-l| \epsilon^{\prime}} M(h)(x)
\end{aligned}
$$


which together with (3.107), Hölder's inequality, Lemma 3.14, and Lemma 3.9 yields that

$$
\begin{aligned}
\left\|\left\{\sum_{k=-\infty}^{\infty}\left|\tilde{D}_{k}^{t}(h)\right|^{2}\right\}^{1 / 2}\right\|_{L^{p^{\prime}}(\mathcal{X})} & \lesssim\left\|\left\{\sum_{k=-\infty}^{\infty}\left[\sum_{l=-\infty}^{\infty} 2^{-|k-l| \epsilon^{\prime}} M\left(D_{l}(h)\right)\right]^{2}\right\}^{1 / 2}\right\|_{L^{p^{\prime}}(\mathcal{X})} \\
& \lesssim\left\|\left\{\sum_{l=-\infty}^{\infty}\left[M\left(D_{l}(h)\right)\right]^{2}\right\}^{1 / 2}\right\|_{L^{p^{\prime}}(\mathcal{X})} \\
& \lesssim\|h\|_{L^{p^{\prime}}(\mathcal{X})} .
\end{aligned}
$$

Thus, (3.108) holds.

Using (3.108) and (3.107) together with a duality argument and Hölder's inequality gives

$$
\begin{aligned}
\|f\|_{L^{p}(x)} & =\sup _{\|h\|_{L^{p^{\prime}}(\mathcal{x})} \leq 1}\left|\left\langle\sum_{k=-\infty}^{\infty} \widetilde{D}_{k} D_{k}(f), h\right\rangle\right| \\
& \leq \sup _{\|h\|_{L^{\prime}(x)} \leq 1}\|\dot{g}(f)\|_{L^{p}(x)}\left\|\left\{\sum_{k=-\infty}^{\infty}\left|\tilde{D}_{k}^{t}(h)\right|^{2}\right\}^{1 / 2}\right\|_{L^{p^{\prime}}(\mathcal{x})} \\
& \lesssim\|\dot{g}(f)\|_{L^{p}(\mathcal{x})^{\prime}}
\end{aligned}
$$

which completes the proof of Proposition 3.15.

Remark 3.16. From the proof of Proposition 3.15, it is easy to see that Lemma 3.9 is still true if $D_{k}$ there is replaced by $\widetilde{D}_{k}^{t}$ for $k \in \mathbb{Z}$, which has regularity only in the second variable; see (3.108).

\subsection{Inhomogeneous continuous Calderón reproducing formulae}

In this subsection, we have no restriction on $\operatorname{diam}(\mathcal{X})$, which means $\operatorname{diam}(\mathcal{X})<\infty$ or $\operatorname{diam}(\mathcal{X})=\infty$. We first introduce the following inhomogeneous approximation of the identity on $x$.

Definition 3.17. Let $\epsilon_{1} \in(0,1], \epsilon_{2}>0$, and $\epsilon_{3}>0$. A sequence $\left\{S_{k}\right\}_{k \in \mathbb{Z}_{+}}$of linear operators is said to be an inhomogeneous approximation of the identity of order $\left(\epsilon_{1}, \epsilon_{2}, \epsilon_{3}\right)$ (for short, $\left(\epsilon_{1}, \epsilon_{2}, \epsilon_{3}\right)$ IATI) if $S_{k}$ for $k \in \mathbb{Z}_{+}$satisfies Definition 2.2.

A sequence $\left\{S_{k}\right\}_{k \in \mathbb{Z}_{+}}$of linear operators is said to be an inhomogeneous approximation of the identity of order $\epsilon_{1}$ with bounded support (for short, $\epsilon_{1}$-IATI with bounded support) if $S_{k}$ for $k \in \mathbb{Z}_{+}$satisfies Definition 2.3.

The following proposition is a simple corollary of Proposition 2.7(iv) and (v).

Proposition 3.18. Let $\epsilon_{1} \in(0,1], \epsilon_{2}>0, \epsilon_{3}>0$, let $\left\{S_{k}\right\}_{k \in \mathbb{Z}_{+}}$be an $\left(\epsilon_{1}, \epsilon_{2}, \epsilon_{3}\right)$-IATI, and let $S_{k}^{t}$ be the adjoint operator of $S_{k}$ for any $k \in \mathbb{Z}_{+}$. Let $D_{k}=S_{k}-S_{k-1}$ for $k \in \mathbb{N}$ and $D_{0}=S_{0}$. Then $I=\sum_{k=0}^{\infty} D_{k}$ in $L^{p}(\mathcal{X})$ for $p \in[1, \infty)$. The same is true for $\left\{S_{k}^{t}\right\}_{k \in \mathbb{Z}_{+}}$. 
To establish the continuous inhomogeneous Calderón reproducing formulae, we need a technical lemma, which is a variant of Lemma 3.2.

Lemma 3.19. Let $\epsilon_{1} \in(0,1], \epsilon_{2}>0$, and $\epsilon_{3}>0,\left\{S_{k}\right\}_{k \in \mathbb{Z}_{+}}$and let $\left\{E_{k}\right\}_{k \in \mathbb{Z}_{+}}$be two $\left(\epsilon_{1}, \epsilon_{2}, \epsilon_{3}\right)$ IATIs. Let $P_{k}=S_{k}-S_{k-1}$ and $Q_{k}=E_{k}-E_{k-1}$ for $k \in \mathbb{N}, P_{0}=S_{0}$, and $Q_{0}=E_{0}$. Then for any $\epsilon_{1}^{\prime} \in\left(0, \epsilon_{1} \wedge \epsilon_{2}\right)$, there exist constants $C>0, \delta>0$, and $\sigma>0$ as in Lemma 3.2 such that the estimates (3.2) to (3.5) are still true for these $\left\{P_{l}\right\}_{k \in \mathbb{Z}_{+}}$and $\left\{Q_{k}\right\}_{k \in \mathbb{Z}_{+}}$.

Proof. The proof of Lemma 3.19 is essentially as in that of Lemma 3.2. The only different situations are the cases when $l=0$ or $k=0$. Let us prove (3.2) for $l=0=k$ to show the difference. In this case, by the size condition of $P_{0}$ and $Q_{0}$, we have

$$
\begin{aligned}
\left|P_{0} Q_{0}(x, y)\right|= & \left|\int_{x} S_{0}(x, z) Q_{0}(z, y) d \mu(z)\right| \\
\lesssim & \quad \int_{d(x, z) \leq(1 / 2) d(x, y)} \frac{1}{V_{1}(x)+V_{1}(z)+V(x, z)} \frac{1}{(1+d(x, z))^{\epsilon_{2}}} \\
& +\int_{d(x, z)>(1 / 2) d(x, y)} \cdots
\end{aligned}
$$

Since $d(x, z)>(1 / 2) d(x, y)$ implies that $d(y, z) \leq d(x, y) / 2$, by symmetry, the estimates of the first and the second terms are similar and we only estimate the first term. To this end, since $d(x, z) \leq d(x, y) / 2 \leq(1+d(x, y)) / 2$, by Lemma 2.1(iii), we have $1 /(1+d(z, y)) \lesssim$ $1 /(1+d(x, y))$ and $1 /\left(V_{1}(z)+V(z, y)\right) \lesssim 1 /\left(V_{1}(x)+V(x, y)\right)$, which further implies that $1 /\left(V_{1}(z)+V_{1}(y)+V(z, y)\right) \lesssim 1 /\left(V_{1}(x)+V_{1}(y)+V(x, y)\right)$. These estimates together with Lemma 2.1(ii) yield that

$$
\left|P_{0} Q_{0}(x, y)\right| \lesssim \frac{1}{V_{1}(x)+V_{1}(y)+V(x, y)} \frac{1}{(1+d(x, y))^{\epsilon_{2}}}
$$

which is the desired estimate and hence completes the proof of Lemma 3.19.

Now, let $\epsilon_{1} \in(0,1], \epsilon_{2}>0, \epsilon_{3}>0$, and $\left\{S_{k}\right\}_{k \in \mathbb{Z}_{+}}$be an $\left(\epsilon_{1}, \epsilon_{2}, \epsilon_{3}\right)$-IATI as in Definition 3.17. Throughout this subsection, we always assume that $D_{k}=S_{k}-S_{k-1}$ for $k \in \mathbb{N}$, $D_{0}=S_{0}$ and $D_{k}=0$ if $k \in\{-1,-2, \ldots\}$. Similar to (3.44), by Proposition 3.18, for any $N \in \mathbb{N}$, we write

$$
I=\left(\sum_{k=0}^{\infty} D_{k}\right)\left(\sum_{j=0}^{\infty} D_{j}\right)=\sum_{|l|>N} \sum_{k=0}^{\infty} D_{k+l} D_{k}+\sum_{k=0}^{\infty} D_{k}^{N} D_{k}=R_{N}+T_{N}
$$

in $L^{p}(\mathcal{X})$ with $p \in(1, \infty)$, where $D_{k}^{N}=\sum_{|l| \leq N} D_{k+l}$. 
Repeating the proof of Lemma 3.4, we obtain the following.

Lemma 3.20. Let $N \in \mathbb{N}$ and let $R_{N}$ be as in (3.115). Then there exist constants $C>0$ and $\delta>0$, independent of $N$, such that for all $f \in L^{2}(\mathcal{X})$,

$$
\left\|R_{N}(f)\right\|_{L^{2}(\mathcal{X})} \leq C 2^{-N \delta}\|f\|_{L^{2}(\chi)} .
$$

We now establish some estimates for the kernel, $R_{N}(x, y)$, of the operator $R_{N}$. To this end, we first give a technical lemma, which is a simple corollary of Lemma 3.5.

Lemma 3.21. For any $\sigma \geq s>0$ and $x, y \in X$ with $x \neq y$,

$$
\sum_{k=0}^{\infty} \frac{1}{V_{2^{-k}}(x)+V(x, y)} \frac{2^{-k s}}{\left(2^{-k}+d(x, y)\right)^{\sigma}} \lesssim \frac{1}{V(x, y)} \frac{1}{d(x, y)^{\sigma-s}}
$$

Lemma 3.22. Let $N \in \mathbb{N}$, let $R_{N}$ be as in (3.115), and let $R_{N}(x, y)$ be its kernel. Then for any $\epsilon \in\left(0, \epsilon_{1} \wedge \epsilon_{2}\right)$, there exists a constant $\delta>0$, independent of $N$, such that $R_{N}$ satisfies all the conditions of Corollary 2.22 and $C_{R_{N}} \lesssim 2^{-\delta N}$. Moreover, $R_{N}^{*}(1)=0$.

Replacing Lemmas 3.2 and 3.5, respectively, by Lemmas 3.19 and 3.21, and repeating the proof of Lemma 3.6, we obtain Lemma 3.22. In combination with Lemma 3.20, this leads to the following variant of Proposition 3.7. We omit the details.

Proposition 3.23. Let $\epsilon_{1} \in(0,1], \epsilon_{2}>0, \epsilon_{3}>0$, and let $\left\{S_{k}\right\}_{k \in \mathbb{Z}_{+}}$be an $\left(\epsilon_{1}, \epsilon_{2}, \epsilon_{3}\right)$-IATI. For $N \in \mathbb{N}$, let $R_{N}$ and $T_{N}$ be as in (3.115). Then there exist constants $C_{8}>0$ and $\delta>0$, which are independent of $N$, such that $R_{N}$ is bounded on any space of test functions, $\mathcal{G}\left(x_{1}, r, \beta, \gamma\right)$ with $x_{1} \in \mathcal{X}$, $r>0$, and $0<\beta, \gamma<\left(\epsilon_{1} \wedge \epsilon_{2}\right)$, and its operator norm is bounded by $C_{8} 2^{-N \delta}$. Moreover, if $N$ is so large that (3.57) holds, then $T_{N}^{-1}$ exists and is bounded on $\mathcal{G}\left(x_{1}, r, \beta, \gamma\right)$, if $x_{1} \in \mathcal{X}, r>0$ and $0<\beta, \gamma<\left(\epsilon_{1} \wedge \epsilon_{2}\right)$.

Via Lemmas 3.20 and 3.22, similar to the proof of Proposition 3.8, we can obtain the following version of Proposition 3.8.

Proposition 3.24. Let $p \in(1, \infty), \epsilon_{1} \in(0,1], \epsilon_{2}>0, \epsilon_{3}>0$, and let $\left\{S_{k}\right\}_{k \in \mathbb{Z}_{+}}$be an $\left(\epsilon_{1}, \epsilon_{2}, \epsilon_{3}\right)$ IATI. For $N \in \mathbb{N}$, let $R_{N}$ and $T_{N}$ be as in (3.115). Then there exist constants $C_{9}>0$ and $\delta>0$, which are independent of $N$, such that $R_{N}$ is bounded on $L^{p}(\mathcal{X})$ with the operator norm bounded by $C_{9} 2^{-N \delta}$. Moreover, if $N$ is so large that (3.61) holds, then $T_{N}^{-1}$ exists and is bounded on $L^{p}(x)$.

Let $\epsilon_{1} \in(0,1], \epsilon_{2}>0, \epsilon_{3}>0$, and let $\left\{S_{k}\right\}_{k \in \mathbb{Z}_{+}}$be an $\left(\epsilon_{1}, \epsilon_{2}, \epsilon_{3}\right)$-IATI. Set $D_{k}=S_{k}-S_{k-1}$ for $k \in \mathbb{N}$ and $D_{0}=S_{0}$. For any $f \in L^{p}(\mathcal{X})$ with $p \in(1, \infty)$ and $x \in \mathcal{X}$, the inhomogeneous Littlewood-Paley $g$-function $g(f)$ is defined by

$$
g(f)(x)=\left\{\sum_{k=0}^{\infty}\left|D_{k}(f)(x)\right|^{2}\right\}^{1 / 2} .
$$

Applying the Cotlar-Stein lemma and using a procedure similar to the proof of Lemma 3.9, we obtain the boundedness on $L^{p}(\mathcal{X})$ with $p \in(1, \infty)$ for the Littlewood-Paley $g$-function as below. 
Lemma 3.25. Let $\epsilon_{1} \in(0,1], \epsilon_{2}>0, \epsilon_{3}>0$, and let $\left\{S_{k}\right\}_{k \in \mathbb{Z}_{+}}$be an $\left(\epsilon_{1}, \epsilon_{2}, \epsilon_{3}\right)$-IATI. Let $D_{k}=$ $S_{k}-S_{k-1}$ for $k \in \mathbb{N}, D_{0}=S_{0}$, and $g(f)$ for $f \in L^{p}(X)$ with $p \in(1, \infty)$ being as in (3.118). Then there exists a constant $C_{p}>0$ such that for all $f \in L^{p}(\mathcal{X})$,

$$
\|g(f)\|_{L^{p}(x)} \leq C_{p}\|f\|_{L^{p}(x)}
$$

We can now establish an inhomogeneous continuous Calderón reproducing formula.

Theorem 3.26. Let $\epsilon_{1} \in(0,1], \epsilon_{2}>0, \epsilon_{3}>0, \epsilon \in\left(0, \epsilon_{1} \wedge \epsilon_{2}\right)$, and let $\left\{S_{k}\right\}_{k \in \mathbb{Z}_{+}}$be an $\left(\epsilon_{1}, \epsilon_{2}, \epsilon_{3}\right)$ IATI. Set $D_{k}=S_{k}-S_{k-1}$ for $k \in \mathbb{Z}$ and $D_{0}=S_{0}$. Then there exist $N \in \mathbb{N}$ and a family of linear operators $\left\{\widetilde{D}_{k}\right\}_{k \in \mathbb{Z}_{+}}$such that for all $f \in \mathcal{G}_{0}^{\epsilon}(\beta, \gamma)$ with $0<\beta, \gamma<\epsilon$,

$$
f=\sum_{k=0}^{\infty} \widetilde{D}_{k} D_{k}(f)
$$

where the series converges both in the norm of $\mathcal{G}_{0}^{\epsilon}(\beta, \gamma)$ and the norm of $L^{p}(\mathcal{X})$ for $p \in(1, \infty)$. Moreover, the kernels of the operators $\widetilde{D}_{k}$ satisfy the conditions (i) and (ii) of Definition 2.2 with $\epsilon_{1}$ and $\epsilon_{2}$ replaced by $\epsilon^{\prime} \in\left(\epsilon, \epsilon_{1} \wedge \epsilon_{2}\right)$, and $\int_{x} \widetilde{D}_{k}(x, y) d \mu(y)=\int_{x} \widetilde{D}_{k}(x, y) d \mu(x)=1$ when $0 \leq k \leq N$; $=0$ when $k>N$.

Proof. We prove this theorem by an argument similar to that of Theorem 3.10. Fix a large integer $N \in \mathbb{N}$ such that (3.57) and (3.61) hold and, therefore, Propositions 3.23 and 3.24 hold. Let $D_{k}^{N}$ be as in (3.115). Then,

$$
D_{k}^{N}=\sum_{|j| \leq N} D_{k+j}= \begin{cases}\sum_{j=0}^{k+N} D_{j}, & \text { for } 0 \leq k \leq N \\ \sum_{j=k-N}^{k+N} D_{j}, & \text { for } k>N .\end{cases}
$$

Thus, for $k>N, D_{k}^{N}(\cdot, y) \in \mathcal{G}\left(y, 2^{-k}, \epsilon_{1}, \epsilon_{2}\right)$, and we then define

$$
\widetilde{D}_{k}(x, y)=T_{N}^{-1}\left(D_{k}^{N}(\cdot, y)\right)(x) .
$$

By Proposition 3.23, we know that for $k>N$ and any $0<\beta, \gamma<\left(\epsilon_{1} \wedge \epsilon_{2}\right), \widetilde{D}_{k}(\cdot, y) \in$ $\mathcal{G}\left(y, 2^{-k}, \beta, \gamma\right)$. Moreover, $\left(T_{N}^{-1}\right)^{*}(1)=\sum_{i=0}^{\infty}\left(R_{N}^{*}\right)^{i}(1)=1$, which implies that

$$
\int_{x} \tilde{D}_{k}(x, y) d \mu(x)=\int_{x} D_{k}^{N}(x, y) d \mu(x)=0
$$

Obviously, we have

$$
\int_{x} \tilde{D}_{k}(x, y) d \mu(y)=T_{N}^{-1}\left\{\int_{x} D_{k}^{N}(\cdot, y) d \mu(y)\right\}(x)=0 .
$$


Thus, $\widetilde{D}_{k}$ for $k>N$ satisfies all the conditions of Theorem 3.26. If $k \in\{0, \ldots, N\}$, then

$$
\int_{x} D_{k}^{N}(x, y) d \mu(x)=1=\int_{x} D_{k}^{N}(x, y) d \mu(y)
$$

Thus, in this case, we cannot directly apply Proposition 3.23. Notice that

$$
T_{N}^{-1}=\sum_{i=0}^{\infty}\left(R_{N}\right)^{i}
$$

Using Lemma 3.19, we easily show that for $\epsilon_{1}^{\prime} \in\left(0, \epsilon_{1} \wedge \epsilon_{2}\right)$, there exist positive constants $\widetilde{C}$ and $\delta$ depending on $\epsilon_{1}^{\prime}$ such that $R_{N}(x, y)$ satisfies (2.174) and (2.185) with $r_{0}=1$ and $C_{T} \lesssim \widetilde{C} 2^{-N \delta}$. Thus, by Corollary 2.31 , we know that $R_{N}\left(S_{0}(\cdot, y)\right) \in \mathcal{G}\left(y, 1, \epsilon_{1}^{\prime}, \epsilon_{2}\right)$ and

$$
\left\|R_{N}\left(S_{0}(\cdot, y)\right)\right\|_{\mathcal{G}\left(y, 1, \epsilon_{1}^{\prime}, \epsilon_{2}\right)} \leq \widetilde{C} 2^{-N \delta}
$$

Notice that $R_{N}^{*}(1)=0$. Applying Proposition 3.23 yields that there exist two positive constants $C_{8}$ and $\delta$, which are independent of $N$, such that for all $j \in \mathbb{N}$ and all $y \in \mathcal{X}$, $R_{N}^{j}\left(S_{0}(\cdot, y)\right) \in \dot{\mathcal{G}}(y, 1, \beta, \gamma)$ and

$$
\left\|R_{N}^{j}\left(S_{0}(\cdot, y)\right)\right\|_{\mathcal{G}(y, 1, \beta, \gamma)} \leq\left(C_{8} 2^{-N \delta}\right)^{j},
$$

where $\beta, \gamma \in\left(0, \epsilon_{1} \wedge \epsilon_{2}\right)$. Thus, if we choose $N$ so large that (3.57) holds, then by (3.126), we know that $\widetilde{D}_{k}$ for $k \in\{0,1, \ldots, N\}$ satisfies the conditions (i) and (ii) of Definition 2.2 with $\epsilon_{1}$ and $\epsilon_{2}$ replaced by $\epsilon \in\left(0, \epsilon_{1} \wedge \epsilon_{2}\right)$. Moreover, from (3.126) together with $R_{N}(1)=$ $0=R_{N}^{*}(1), \int_{\chi} S_{0}(x, y) d \mu(y)=1=\int_{\chi} S_{0}(x, y) d \mu(x)$ and for $k \in \mathbb{N}, \int_{x} D_{k}(x, y) d \mu(y)=$ $0=\int_{x} D_{k}(x, y) d \mu(x)$, it follows that for $k \in\{0,1, \ldots, N\}, \int_{x} \widetilde{D}_{k}(x, y) d \mu(y)=1=$ $\int_{x} \widetilde{D}_{k}(x, y) d \mu(x)$.

Now it remains to prove that the series in (3.120) converges in both the norm of $\mathcal{G}_{0}^{\epsilon}(\beta, \gamma)$ with $0<\beta, \gamma<\epsilon$ and the norm of $L^{p}(\mathcal{X})$ with $p \in(1, \gamma)$.

To verify (3.120) converges in $\mathcal{G}_{0}^{\epsilon}(\beta, \gamma)$ with $\beta, \gamma \in(0, \epsilon)$, similarly to (3.69), for $f \in$ $\mathcal{G}_{0}^{\epsilon}\left(\beta^{\prime}, \gamma^{\prime}\right)$ with $\beta^{\prime} \in(\beta, \widetilde{\epsilon})$ and $\gamma^{\prime} \in(\gamma, \widetilde{\epsilon})$, and $L \in \mathbb{N}$ with $L>N+1$, we have

$$
\sum_{k=0}^{L} \tilde{D}_{k} D_{k}(f)=f-\lim _{j \rightarrow \infty}\left(R_{N}\right)^{j}(f)-T_{N}^{-1}\left(\sum_{k=L+1}^{\infty} D_{k}^{N} D_{k}\right)(f) .
$$

Thus,

$$
\left\|\sum_{k=0}^{L} \widetilde{D}_{k} D_{k}(f)-f\right\|_{\mathcal{G}(\beta, r)} \leq \lim _{j \rightarrow \infty}\left\|\left(R_{N}\right)^{j}(f)\right\|_{\mathcal{G}(\beta, r)}+\left\|T_{N}^{-1}\left(\sum_{k=L+1}^{\infty} D_{k}^{N} D_{k}\right)(f)\right\|_{\mathcal{G}(\beta, r)} .
$$


Applying Corollary 2.31 again, we obtain that there exists $\delta>0$ such that for all $x \in \mathcal{X}$,

$$
\left|R_{N}(f)(x)\right| \lesssim 2^{-N \delta} \frac{1}{V_{1}\left(x_{1}\right)+V\left(x_{1}, x\right)} \frac{1}{\left(1+d\left(x_{1}, x\right)\right)^{\gamma^{\prime}}}
$$

and for any $\beta \in\left(0, \beta^{\prime}\right)$, and $x, x^{\prime} \in \mathcal{X}$ with $d\left(x, x^{\prime}\right) \leq\left(1+d\left(x_{1}, x\right)\right) / 2$,

$$
\left|R_{N}(f)(x)-R_{N}(f)\left(x^{\prime}\right)\right| \lesssim 2^{-N \delta}\left(\frac{d\left(x, x^{\prime}\right)}{1+d\left(x_{1}, x\right)}\right)^{\beta} \frac{1}{V_{1}\left(x_{1}\right)+V\left(x_{1}, x\right)} \frac{1}{\left(1+d\left(x_{1}, x\right)\right)^{\gamma^{\prime}}}
$$

Notice that $R_{N}^{*}(1)=0$, which together with (3.131) and (3.132) shows that $R_{N}(f) \in \dot{\mathcal{G}}(\beta, \gamma)$. From this and Proposition 3.23, it follows that

$$
\lim _{j \rightarrow \infty}\left\|\left(R_{N}\right)^{j}(f)\right\|_{\mathcal{G}(\beta, \gamma)} \leq \lim _{j \rightarrow \infty}\left(C_{8} 2^{-N \delta}\right)^{j}\|f\|_{\mathcal{G}(\beta, \gamma)}=0
$$

To prove that the second term on the right-hand side of (3.130) tends to 0 as $L \rightarrow \infty$, by $\left(D_{k}^{N}\right)^{*}(1)=0$ when $L \geq N$ (see (3.121)), and Proposition 3.24, we only need to verify that for a certain $\sigma>0$,

$$
\left\|\sum_{k=L+1}^{\infty} D_{k}^{N} D_{k}(f)\right\|_{\mathcal{G}(\beta, \gamma)} \lesssim 2^{-\sigma L}\|f\|_{\mathcal{G}\left(\beta^{\prime}, \gamma^{\prime}\right)} .
$$

In fact, (3.134) can be deduced from the following two estimates that for all $x \in \mathcal{X}$,

$$
\left|\sum_{k=L+1}^{\infty} D_{k}^{N} D_{k}(f)(x)\right| \lesssim 2^{-\left(\beta^{\prime} \wedge \gamma^{\prime}\right) L}\|f\|_{\mathcal{G}\left(\beta^{\prime}, \gamma^{\prime}\right)} \frac{1}{V_{1}\left(x_{1}\right)+V\left(x_{1}, x\right)} \frac{1}{\left(1+d\left(x_{1}, x\right)\right)^{\gamma^{\prime}}}
$$

and for $x, x^{\prime} \in \mathcal{X}$ with $d\left(x, x^{\prime}\right) \leq\left(1+d\left(x_{1}, x\right)\right) / 2$,

$$
\begin{aligned}
& \left|\sum_{k=L+1}^{\infty} D_{k}^{N} D_{k}(f)(x)-\sum_{k=L+1}^{\infty} D_{k}^{N} D_{k}(f)\left(x^{\prime}\right)\right| \\
& \quad \lesssim 2^{-(1-\theta)\left(\beta^{\prime} \wedge \gamma^{\prime}\right) L}\|f\|_{\mathcal{G}\left(\beta^{\prime}, \gamma^{\prime}\right)}\left(\frac{d\left(x, x^{\prime}\right)}{1+d\left(x_{1}, x\right)}\right)^{\theta \beta^{\prime}} \frac{1}{V_{1}\left(x_{1}\right)+V\left(x_{1}, x\right)} \frac{1}{\left(1+d\left(x_{1}, x\right)\right)^{\gamma^{\prime}}}
\end{aligned}
$$

where $\theta \in(0,1)$. 
Let $E_{k}=D_{k}^{N} D_{k}$. It is easy to check that $E_{k}$ for $k \in \mathbb{N}$ satisfies all estimates of Definition 2.2 with $\epsilon$ replaced by $\widetilde{\epsilon}$ and a coefficient depending on $N$. Moreover, for $k \in \mathbb{N}$, $E_{k}(1)=0=E_{k}^{*}(1)$. Thus, by Lemma 2.1(ii),

$$
\begin{aligned}
& \left|D_{k}^{N} D_{k}(f)(x)\right| \\
& \leq \int_{d(x, y) \leq\left(1+d\left(x_{1}, x\right)\right) / 2}\left|E_{k}(x, y)\right||f(y)-f(x)| d \mu(y) \\
& \quad+\int_{d(x, y)>\left(1+d\left(x_{1}, x\right)\right) / 2}\left|E_{k}(x, y)\right|[|f(y)|+|f(x)|] d \mu(y) \\
& \leq\|f\|_{\mathcal{G}\left(\beta^{\prime}, \gamma^{\prime}\right)}\left\{\int_{d(x, y) \leq\left(1+d\left(x_{1}, x\right)\right) / 2}\left|E_{k}(x, y)\right|\left(\frac{d(x, y)}{1+d\left(x_{1}, x\right)}\right)^{\beta^{\prime}}\right. \\
& \quad \times \frac{1}{V_{1}\left(x_{1}\right)+V\left(x_{1}, x\right)} \frac{1}{\left(1+d\left(x_{1}, x\right)\right)^{\gamma^{\prime}}} d \mu(y) \\
& \quad \int_{d(x, y)>\left(1+d\left(x_{1}, x\right)\right) / 2}\left|E_{k}(x, y)\right|\left[\frac{1}{V_{1}\left(x_{1}\right)+V\left(x_{1}, y\right)} \frac{1}{\left(1+d\left(x_{1}, y\right)\right)^{\gamma^{\prime}}}\right. \\
& \left.\leq\|f\|_{\mathcal{G}\left(\beta^{\prime}, \gamma^{\prime}\right)}\left\{2^{-k \beta^{\prime}} \frac{1}{V_{1}\left(x_{1}\right)+V\left(x_{1}, x\right)} \frac{1}{V_{1}\left(x_{1}\right)+V\left(x_{1}, x\right)} \frac{1}{\left(1+d\left(x_{1}, x\right)\right)^{\gamma^{\prime}}}+Z\right\}, d \mu(y)\right\}
\end{aligned}
$$

and by Lemma 2.1(i) and (ii), we further have

$$
\begin{aligned}
Z \lesssim \int_{d(x, y)>\left(1+d\left(x_{1}, x\right)\right) / 2} \frac{1}{V_{2^{-k}(x)+V(x, y)}} \frac{2^{-k \epsilon_{2}}}{\left(2^{-k}+d(x, y)\right)^{\epsilon_{2}}} \frac{1}{V_{1}\left(x_{1}\right)+V\left(x_{1}, y\right)} \frac{1}{\left(1+d\left(x_{1}, y\right)\right)^{\gamma^{\prime}}} d \mu(y) \\
\lesssim \min \left\{\frac{2^{-k \gamma^{\prime}}}{V_{1}\left(x_{1}\right)} \int_{d(x, y)>\left(1+d\left(x_{1}, x\right)\right) / 2} \frac{1}{V(x, y)} \frac{1}{d(x, y)^{\gamma^{\prime}}} d \mu(y),\right. \\
\\
\left.\quad \frac{2^{-k \gamma^{\prime}}}{V\left(x_{1}, x\right)} \frac{1}{\left(1+d\left(x_{1}, x\right)\right)^{\gamma^{\prime}}} \int_{\chi} \frac{1}{V_{1}\left(x_{1}\right)+V\left(x_{1}, y\right)} \frac{1}{\left(1+d\left(x_{1}, y\right)\right)^{\gamma^{\prime}}} d \mu(y)\right\} \\
2^{-k \gamma^{\prime}} \frac{1}{V_{1}\left(x_{1}\right)+V\left(x_{1}, x\right)} \frac{1}{\left(1+d\left(x_{1}, x\right)\right)^{\gamma^{\prime}}} .
\end{aligned}
$$

From this, it is easy to deduce that (3.135) holds. 
On the other hand, similarly to the proof of (3.12), we can verify that for $x, x^{\prime} \in \mathcal{X}$ with $d\left(x, x^{\prime}\right) \leq\left(1+d\left(x, x_{1}\right)\right) / 4$,

$$
\begin{aligned}
& \left|D_{k}^{N} D_{k}(f)(x)-D_{k}^{N} D_{k}(f)\left(x^{\prime}\right)\right| \\
& \quad \lesssim\|f\|_{\mathcal{G}\left(\beta^{\prime}, \gamma^{\prime}\right)}\left(\frac{d\left(x, x^{\prime}\right)}{1+d\left(x_{1}, x\right)}\right)^{\beta^{\prime}} \frac{1}{V_{1}\left(x_{1}\right)+V\left(x_{1}, x\right)} \frac{1}{\left(1+d\left(x_{1}, x\right)\right)^{\gamma^{\prime}}},
\end{aligned}
$$

and by (3.137) together with Lemma 2.1(iii),

$$
\left|D_{k}^{N} D_{k}(f)(x)-D_{k}^{N} D_{k}(f)\left(x^{\prime}\right)\right| \lesssim 2^{-k\left(\beta^{\prime} \wedge \gamma^{\prime}\right)}\|f\|_{\mathcal{G}\left(\beta^{\prime}, \gamma^{\prime}\right)} \frac{1}{V_{1}\left(x_{1}\right)+V\left(x_{1}, x\right)} \frac{1}{\left(1+d\left(x_{1}, x\right)\right)^{\gamma^{\prime}}} .
$$

These estimates together with the geometric means yield (3.136). Thus,

$$
\lim _{L \rightarrow \infty}\left\|\sum_{k=0}^{L} \widetilde{D}_{k} D_{k}(f)-f\right\|_{\mathcal{G}(\beta, \gamma)}=0 .
$$

Then repeating the proof of Theorem 3.10 further shows that (3.120) holds in the norm of $\mathcal{G}_{0}^{\epsilon}(\beta, \gamma)$. Its convergence in $L^{p}(\mathcal{X})$ for $p \in(1, \infty)$ can be proved in a way similar to Theorem 3.10 if instead of Lemma 3.9 by Lemma 3.25, which completes the proof of Theorem 3.26.

Remark 3.27. From the proof of Theorem 3.26, it is easy to see that $N$ in Theorem 3.26 can be chosen so that both (3.57) and (3.61) are satisfied. In order to defray our notation and simplify our presentation, we will assume in the sequel that $N=0$.

A similar argument as for the proof of Theorem 3.26 leads to the following variant of the inhomogeneous continuous Calderón reproducing formula (we omit the details).

Theorem 3.28. Let $\epsilon_{1} \in(0,1], \epsilon_{2}>0, \epsilon_{3}>0, \epsilon \in\left(0, \epsilon_{1} \wedge \epsilon_{2}\right)$, and let $\left\{S_{k}\right\}_{k \in \mathbb{Z}_{+}}$be an $\left(\epsilon_{1}, \epsilon_{2}, \epsilon_{3}\right)$ IATI. Set $D_{k}=S_{k}-S_{k-1}$ for $k \in \mathbb{Z}$ and $D_{0}=S_{0}$. Then there exists a family of linear operators $\left\{\bar{D}_{k}\right\}_{k \in \mathbb{Z}_{+}}$such that for all $f \in \mathcal{G}_{0}^{\epsilon}(\beta, \gamma)$ with $0<\beta, \gamma<\epsilon$,

$$
f=\sum_{k=0}^{\infty} D_{k} \bar{D}_{k}(f)
$$

where the series converges in both the norm of $\mathcal{G}_{0}^{\epsilon}(\beta, \gamma)$ and the norm of $L^{p}(\mathcal{X})$ for $p \in(1, \infty)$. Moreover, the kernels of the operators $\bar{D}_{k}$ satisfy the conditions (i) and (iii) of Definition 2.2 with $\epsilon_{1}$ and $\epsilon_{2}$ replaced by $\epsilon^{\prime} \in\left(\epsilon, \epsilon_{1} \wedge \epsilon_{2}\right)$, and $\int_{x} \bar{D}_{k}(x, y) d \mu(y)=\int_{x} \bar{D}_{k}(x, y) d \mu(x)=1$ when $k=0 ;=0$ when $k \in \mathbb{N}$.

Theorems 3.26 and 3.28 in combination with a duality argument and Lemma 3.12 show that the inhomogeneous Calderón reproducing formulae also hold in spaces of distributions. 
Theorem 3.29. Let all the notation be as in Theorems 3.26 and 3.28. Then for all $f \in\left(\mathcal{G}_{0}^{\epsilon}(\beta, \gamma)\right)^{\prime}$ with $0<\beta, \gamma<\epsilon,(3.120)$ and (3.142) hold in $\left(\mathcal{G}_{0}^{\epsilon}(\beta, \gamma)\right)^{\prime}$.

Finally, we have the following analogue of Proposition 3.15 for the inhomogeneous Littlewood-Paley g-function, based on Theorem 3.26 (we omit the proof).

Proposition 3.30. Let $\epsilon_{1} \in(0,1], \epsilon_{2}>0, \epsilon_{3}>0$, and let $\left\{S_{k}\right\}_{k \in \mathbb{Z}_{+}}$be an $\left(\epsilon_{1}, \epsilon_{2}, \epsilon_{3}\right)$-IATI. Set $D_{k}=S_{k}-S_{k-1}$ for $k \in \mathbb{Z}, D_{0}=S_{0}$ and $g(f)$ for $f \in L^{p}(\mathcal{X})$ with $p \in(1, \infty)$ be as in (3.118). Then there exists a constant $C_{p}>0$ such that for all $f \in L^{p}(\mathcal{X})$,

$$
C_{p}\|f\|_{L^{p}(\chi)} \leq\|g(f)\|_{L^{p}(\chi)} \leq C_{p}\|f\|_{L^{p}(\chi)} .
$$

Remark 3.31. Similarly as in Remark 3.16, it is easy to see that Lemma 3.25 is still true if $D_{k}$ therein is replaced by the kernel $\widetilde{D}_{k}^{t}$ for $k \in \mathbb{Z}_{+}$, which has regularity only in the second variable.

\section{Discrete Calderón reproducing formulae}

In this section, we will establish some discrete Calderón reproducing formulae which play a key role in the theory of function spaces, especially in obtaining a frame characterization. To obtain these discrete Calderón reproducing formulae, we mainly use Corollaries 2.22 and 2.31 again.

In the following, for $k \in \mathbb{Z}$ and $\tau \in I_{k}$, we denote by $Q_{\tau}^{k, v}, v=1,2, \ldots, N(k, \tau)$, the set of all cubes $Q_{\tau^{\prime}}^{k+j} \subset Q_{\tau}^{k}$, where $Q_{\tau}^{k}$ is the dyadic cube as in Lemma 2.19 and $j$ is a positive large integer such that

$$
2^{-j} C_{6}<\frac{1}{3}
$$

Denote by $z_{\tau}^{k, v}$ the "center" of $Q_{\tau}^{k, v}$ as in Lemma 2.19 and by $y_{\tau}^{k, v}$ a point in $Q_{\tau}^{k, v}$.

\subsection{Homogeneous discrete Calderón reproducing formulae}

In this subsection, we always assume that $\operatorname{diam}(X)=\infty, \epsilon_{1} \in(0,1], \epsilon_{2}>0, \epsilon_{3}>0$, and $\left\{S_{k}\right\}_{k \in \mathbb{Z}}$ is an $\left(\epsilon_{1}, \epsilon_{2}, \epsilon_{3}\right)$-ATI. Set $D_{k}=S_{k}-S_{k-1}$ for $k \in \mathbb{Z}$.

Let all the notation be as in (3.44). We now introduce the following discrete Riemann sum operator on $x$,

$$
S(f)(x)=\sum_{k=-\infty}^{\infty} \sum_{\tau \in I_{k}} \sum_{\nu=1}^{N(k, \tau)} \int_{Q_{\tau}^{k, \nu}} D_{k}^{N}(x, y) d \mu(y) D_{k}(f)\left(y_{\tau}^{k, v}\right) .
$$

We first verify that $S$ is well defined and bounded on $L^{2}(\mathcal{X})$ via the Littlewood-Paley theorem for the homogeneous $g$-function as in (3.63), Proposition 3.15. To do so, let us first establish the following estimate by using Proposition 3.15. 
Lemma 4.1. There exists a constant $C>0$ such that for all $y_{\tau}^{k, v} \in Q_{\tau}^{k, v}$ and all $f \in L^{2}(\mathcal{X})$,

$$
\sum_{k=-\infty}^{\infty} \sum_{\tau \in I_{k}} \sum_{\nu=1}^{N(k, \tau)} \mu\left(Q_{\tau}^{k, v}\right)\left|D_{k}(f)\left(y_{\tau}^{k, v}\right)\right|^{2} \leq C\|f\|_{L^{2}(x)}^{2} .
$$

Proof. By Theorem 3.10, there exists a family of linear operators $\left\{\widetilde{D}_{k}\right\}_{k=-\infty}^{\infty}$ as in Theorem 3.10 such that for all $f \in L^{2}(\mathcal{X}), f=\sum_{l=-\infty}^{\infty} \widetilde{D}_{l} D_{l}(f)$. By Lemma 3.2 together with Remark 3.3, we have that for any $\epsilon_{1}^{\prime} \in\left(0, \epsilon_{1} \wedge \epsilon_{2}\right)$ and any $\epsilon_{1}^{\prime \prime} \in\left(0, \epsilon_{1}^{\prime}\right)$, all $x, z \in \mathcal{X}$ and all $k, l \in \mathbb{Z}$,

$$
\left|D_{k} \widetilde{D}_{l}(z, x)\right| \lesssim 2^{-|k-l| \epsilon_{1}^{\prime \prime}} \frac{1}{V_{2^{-(k \wedge l)}}(x)+V_{2^{-(k \wedge l)}}(z)+V(z, x)} \frac{2^{-(k \wedge l) \epsilon_{1}^{\prime}}}{\left(2^{-(k \wedge l)}+d(z, x)\right)^{\epsilon_{1}^{\prime}}} .
$$

Notice that for all $x \in \mathcal{X}$ and any $z, y \in Q_{\tau}^{k, v}$, by Lemma 2.19(iv), we have that $d(y, z) \leq$ $C_{6} 2^{-j} 2^{-k} \leq C_{6} 2^{-j} 2^{-(k \wedge l)} \leq C_{6} 2^{-j}\left(2^{-(k \wedge l)}+d(x, y)\right)$, where $j \in \mathbb{N}$ satisfies (4.1). Thus, for all $x \in \mathcal{X}$, any $y, z \in Q_{\tau}^{k, v}$ and all $k, l \in \mathbb{Z}$, Lemma 2.1(iii) shows that

$$
\begin{aligned}
& \left|D_{k} \tilde{D}_{l}(z, x)\right| x_{Q_{\tau}^{k, v}}(z) \\
& \quad \lesssim 2^{-|k-l| \epsilon_{1}^{\prime \prime}} \frac{1}{V_{2^{-(k \wedge l)}}(x)+V_{2^{-(k \wedge l)}}(y)+V(y, x)} \frac{2^{-(k \wedge l) \epsilon_{1}^{\prime}}}{\left(2^{-(k \wedge l)}+d(y, x)\right)^{\epsilon_{1}^{\prime}}} x_{Q_{\tau}^{k, v}}(y) .
\end{aligned}
$$

From this and Lemma 2.1(iv), it follows that for $k \in \mathbb{Z}$,

$$
\begin{aligned}
\left|D_{k}(f)\left(y_{\tau}^{k, v}\right)\right| & \leq \sum_{l=-\infty}^{\infty} \int_{x}\left|\left(D_{k} \widetilde{D}_{l}\right)\left(y_{\tau}^{k, v}, x\right)\right|\left|D_{l}(f)(x)\right| d \mu(x) \\
& \lesssim \sum_{l=-\infty}^{\infty} 2^{-|k-l| \epsilon_{1}^{\prime \prime}} M\left(D_{l}(f)\right)(y) \chi_{Q_{\tau}^{k, v}}(y),
\end{aligned}
$$

where $M$ is the Hardy-Littlewood maximal function on $\mathcal{X}$. By (4.6), the construction of $Q_{\tau}^{k, v}$ (see Lemma 2.19), Lemmas 3.14 and 3.9, we obtain

$$
\begin{aligned}
\sum_{k=-\infty}^{\infty} \sum_{\tau \in I_{k}} \sum_{v=1}^{N(k, \tau)} \mu\left(Q_{\tau}^{k, v}\right)\left|D_{k}(f)\left(y_{\tau}^{k, \nu}\right)\right|^{2} & \lesssim \sum_{k=-\infty}^{\infty} \int_{x}\left[\sum_{l=-\infty}^{\infty} 2^{-|k-l| \epsilon_{1}^{\prime \prime}} M\left(D_{l}(f)\right)(y)\right]^{2} d \mu(y) \\
& \lesssim \sum_{l=-\infty}^{\infty}\left\|M\left(D_{l}(f)\right)\right\|_{L^{2}(x)}^{2} \\
& \lesssim\|f\|_{L^{2}(\mathcal{X})^{\prime}}^{2}
\end{aligned}
$$

which proves Lemma 4.1.

The next lemma can be proved in a way similar as in the proof of Theorem (1.14) in [78, page 12]. The main idea is to combine Theorem 3.10, Lemma 2.19, and Hölder's inequality with a duality argument. We omit the details here; see also [36]. 
Lemma 4.2. Suppose that a sequence $\left\{a_{\tau}^{k, v}: k \in \mathbb{Z}, \tau \in I_{k}, v=1, \ldots, N(k, \tau)\right\}$ of numbers satisfies $\sum_{k=-\infty}^{\infty} \sum_{\tau \in I_{k}} \sum_{v=1}^{N(k, \tau)}\left|a_{\tau}^{k, v}\right|^{2}<\infty$. Then the function defined by

$$
f(x)=\sum_{k=-\infty}^{\infty} \sum_{\tau \in I_{k}} \sum_{\nu=1}^{N(k, \tau)}\left[\mu\left(Q_{\tau}^{k, v}\right)\right]^{-1 / 2} a_{\tau}^{k, v} \int_{Q_{\tau}^{k, \nu}} D_{k}^{N}(x, y) d \mu(y)
$$

is in $L^{2}(x)$. Moreover,

$$
\|f\|_{L^{2}(\mathcal{X})}^{2} \leq C \sum_{k=-\infty}^{\infty} \sum_{\tau \in I_{k}} \sum_{\nu=1}^{N(k, \tau)}\left|a_{\tau}^{k, v}\right|^{2}
$$

Lemmas 4.1 and 4.2 yield the boundedness of the discrete Riemann sum operator $S$ on $L^{2}(x)$

Proposition 4.3. Let the notation be the same as above with $j$ satisfying (4.1). Then the discrete Riemann sum operator $S$ in (4.2) is bounded on $L^{2}(\mathcal{X})$. That is, there is a constant $C>0$, only depending on $N$, such that for all $f \in L^{2}(\mathcal{X})$,

$$
\|S(f)\|_{L^{2}(x)} \leq C\|f\|_{L^{2}(x)}
$$

Next we prove that the discrete Riemann sum operator $S$ is invertible and $S^{-1}$ maps $\mathcal{G}\left(x_{1}, r, \beta, \gamma\right)$ into itself. To do this, we define $R=I-S$ and first establish some estimates on the kernel, $R(x, y)$, of the operator $R$. To this end, by (3.44), we write

$$
\begin{aligned}
R(f)(x) & =(I-S)(f)(x) \\
& =\sum_{k=-\infty}^{\infty} \sum_{\tau \in I_{k}} \sum_{v=1}^{N(k, \tau)} \int_{Q_{\tau}^{k, v}} D_{k}^{N}(x, y)\left[D_{k}(f)(y)-D_{k}(f)\left(y_{\tau}^{k, v}\right)\right] d \mu(y)+\sum_{|l|>N} \sum_{k=-\infty}^{\infty} D_{k+l} D_{k}(f)(x) \\
& \equiv \sum_{k=-\infty}^{\infty} G_{k}(f)(x)+R_{N}(f)(x) \\
& \equiv G(f)(x)+R_{N}(f)(x) .
\end{aligned}
$$

Let $G_{k}(x, y)$ be the kernel of $G_{k}$ for $k \in \mathbb{Z}$. We now verify that $G_{k}(x, y)$, and hence $G(x, y)$, satisfies all the desired estimates. Clearly,

$$
\begin{aligned}
G(x, y) & =\sum_{k=-\infty}^{\infty} \sum_{\tau \in I_{k}} \sum_{\nu=1}^{N(k, \tau)} \int_{Q_{\tau}^{k, \nu}} D_{k}^{N}(x, z)\left[D_{k}(z, y)-D_{k}\left(y_{\tau}^{k, v}, y\right)\right] d \mu(z) \\
& =\sum_{k=-\infty}^{\infty} G_{k}(x, y) .
\end{aligned}
$$

We need the following two technical lemmas. 
Lemma 4.4. Let $0 \leq \lambda, \mu \leq 1, \epsilon_{1} \in(0,1], \mu \epsilon_{1}<v_{1}, \lambda \epsilon_{1}<v_{2}$, and $k \in \mathbb{Z}$. Then there exists a constant $C>0$ such that for all $x, y \in \mathcal{X}$ and all $k \in \mathbb{Z}$,

$$
\begin{aligned}
& \int_{x} \frac{d\left(x, x^{\prime}\right)^{\lambda \epsilon_{1}}}{\left(2^{-k}+d(x, z)\right)^{\lambda \epsilon_{1}}} \frac{1}{V_{2^{-k}}(x)+V_{2^{-k}}(z)+V(x, z)} \frac{2^{-k v_{1}}}{\left(2^{-k}+d(x, z)\right)^{v_{1}}} \\
& \quad \times \frac{d\left(y, y^{\prime}\right)^{\mu \epsilon_{1}}}{\left(2^{-k}+d(z, y)\right)^{\mu \epsilon_{1}}} \frac{1}{V_{2^{-k}}(z)+V_{2^{-k}}(y)+V(z, y)} \frac{2^{-k v_{2}}}{\left(2^{-k}+d(z, y)\right)^{v_{2}}} d \mu(z) \\
& \leq C \frac{d\left(x, x^{\prime}\right)^{\lambda \epsilon_{1}}}{\left(2^{-k}+d(x, y)\right)^{\lambda \epsilon_{1}}} \frac{d\left(y, y^{\prime}\right)^{\mu \epsilon_{1}}}{\left(2^{-k}+d(x, y)\right)^{\mu \epsilon_{1}}} \frac{1}{V_{2^{-k}}(x)+V_{2^{-k}}(y)+V(x, y)} \\
& \quad \times\left\{\frac{2^{-k\left(v_{2}-\lambda \epsilon_{1}\right)}}{\left(2^{-k}+d(x, y)\right)^{v_{2}-\lambda \epsilon_{1}}}+\frac{2^{-k\left(v_{1}-\mu \epsilon_{1}\right)}}{\left(2^{-k}+d(x, y)\right)^{v_{1}-\mu \epsilon_{1}}}\right\} .
\end{aligned}
$$

Proof. Write

$$
\begin{aligned}
& \int_{x} \frac{d\left(x, x^{\prime}\right)^{\lambda \epsilon_{1}}}{\left(2^{-k}+d(x, z)\right)^{\lambda \epsilon_{1}}} \frac{1}{V_{2^{-k}}(x)+V_{2^{-k}}(z)+V(x, z)} \frac{2^{-k v_{1}}}{\left(2^{-k}+d(x, z)\right)^{v_{1}}} \\
& \times \frac{d\left(y, y^{\prime}\right)^{\mu \epsilon_{1}}}{\left(2^{-k}+d(z, y)\right)^{\mu \epsilon_{1}}} \frac{1}{V_{2^{-k}}(z)+V_{2^{-k}}(y)+V(z, y)} \frac{2^{-k v_{2}}}{\left(2^{-k}+d(z, y)\right)^{v_{2}}} d \mu(z) \\
& =\int_{d(x, z) \geq d(x, y) / 2} \frac{d\left(x, x^{\prime}\right)^{\lambda \epsilon_{1}}}{\left(2^{-k}+d(x, z)\right)^{\lambda \epsilon_{1}}} \frac{1}{V_{2^{-k}}(x)+V_{2^{-k}}(z)+V(x, z)} \\
& \times \frac{2^{-k v_{1}}}{\left(2^{-k}+d(x, z)\right)^{v_{1}}} \frac{d\left(y, y^{\prime}\right)^{\mu \epsilon_{1}}}{\left(2^{-k}+d(z, y)\right)^{\mu \epsilon_{1}}} \frac{2^{-k v_{2}}}{\left(2^{-k}+d(z, y)\right)^{v_{2}}} \\
& \times \frac{1}{V_{2^{-k}}(z)+V_{2^{-k}}(y)+V(z, y)} d \mu(z)+\int_{d(x, z)<d(x, y) / 2} \ldots \\
& \equiv Z_{1}+Z_{2} \text {. }
\end{aligned}
$$

For $Z_{1}$, the fact $d(x, z) \geq d(x, y) / 2$ implies that $V(x, y) \leq|B(x, 2 d(x, z))| \lesssim V(x, z)$, which together with Lemma 2.1(ii) shows that

$$
\begin{aligned}
Z_{1} \lesssim & \frac{d\left(x, x^{\prime}\right)^{\lambda \epsilon_{1}}}{\left(2^{-k}+d(x, y)\right)^{\lambda \epsilon_{1}}} \frac{1}{V_{2^{-k}}(x)+V(x, y)} \frac{2^{-k v_{1}}}{\left(2^{-k}+d(x, y)\right)^{v_{1}}} \\
& \times d\left(y, y^{\prime}\right)^{\mu \epsilon_{1}} \int_{x} \frac{1}{V_{2^{-k}}(y)+V(z, y)} \frac{2^{-k v_{2}}}{\left(2^{-k}+d(z, y)\right)^{\nu_{2}+\mu \epsilon_{1}}} d \mu(z) \\
& \lesssim \frac{d\left(x, x^{\prime}\right)^{\lambda \epsilon_{1}}}{\left(2^{-k}+d(x, y)\right)^{\lambda \epsilon_{1}}} \frac{d\left(y, y^{\prime}\right)^{\mu \epsilon_{1}}}{\left(2^{-k}+d(x, y)\right)^{\mu \epsilon_{1}}} \frac{1}{V_{2^{-k}}(x)+V(x, y)} \frac{2^{-k\left(v_{1}-\mu \epsilon_{1}\right)}}{\left(2^{-k}+d(x, y)\right)^{\nu_{1}-\mu \epsilon_{1}}}
\end{aligned}
$$


For $Z_{2}$, the fact $d(x, z)<d(x, y) / 2$ implies that $d(z, y)>d(x, y) / 2$ and therefore, $V(x, y) \sim V(y, x) \lesssim V(y, z) \sim V(z, y)$. From these facts and Lemma 2.1(ii), it follows that

$$
\begin{aligned}
Z_{2} \lesssim d\left(x, x^{\prime}\right)^{\lambda \epsilon_{1}} \frac{d\left(y, y^{\prime}\right)^{\mu \epsilon_{1}}}{\left(2^{-k}+d(x, y)\right)^{\mu \epsilon_{1}}} \frac{2^{-k v_{2}}}{\left(2^{-k}+d(x, y)\right)^{v_{2}}} \\
\quad \times \min \left\{\frac{1}{V(x, y)} \int_{x} \frac{1}{V_{2^{-k}}(x)+V(x, z)} \frac{2^{-k v_{1}}}{\left(2^{-k}+d(x, z)\right)^{\nu_{1}+\lambda \epsilon_{1}}} d \mu(z)\right. \\
\left.\quad \frac{1}{V_{2^{-k}(x)}} \frac{1}{V(x, y)} 2^{k \lambda \epsilon_{1}} \mu\left(B\left(x, \frac{d(x, y)}{2}\right)\right)\right\} \\
\lesssim \frac{d\left(x, x^{\prime}\right)^{\lambda \epsilon_{1}}}{\left(2^{-k}+d(x, y)\right)^{\lambda \epsilon_{1}}} \frac{d\left(y, y^{\prime}\right)^{\mu \epsilon_{1}}}{\left(2^{-k}+d(x, y)\right)^{\mu \epsilon_{1}}} \frac{1}{V_{2^{-k}}(x)+V(x, y)} \frac{2^{-k\left(v_{2}-\lambda \epsilon_{1}\right)}}{\left(2^{-k}+d(x, y)\right)^{\nu_{2}-\lambda \epsilon_{1}}} .
\end{aligned}
$$

Moreover, by symmetry, we also have

$$
\begin{aligned}
& \int \frac{d\left(x, x^{\prime}\right)^{\lambda \epsilon_{1}}}{x\left(2^{-k}+d(x, z)\right)^{\lambda \epsilon_{1}}} \frac{1}{V_{2^{-k}}(x)+V_{2^{-k}}(z)+V(x, z)} \frac{2^{-k v_{1}}}{\left(2^{-k}+d(x, z)\right)^{v_{1}}} \\
& \quad \times \frac{d\left(y, y^{\prime}\right)^{\mu \epsilon_{1}}}{\left(2^{-k}+d(z, y)\right)^{\mu \epsilon_{1}}} \frac{1}{V_{2^{-k}}(z)+V_{2^{-k}}(y)+V(z, y)} \frac{2^{-k v_{2}}}{\left(2^{-k}+d(z, y)\right)^{\nu_{2}}} d \mu(z) \\
& \lesssim \frac{d\left(x, x^{\prime}\right)^{\lambda \epsilon_{1}}}{\left(2^{-k}+d(x, y)\right)^{\lambda \epsilon_{1}}} \frac{d\left(y, y^{\prime}\right)^{\mu \epsilon_{1}}}{\left(2^{-k}+d(x, y)\right)^{\mu \epsilon_{1}}} \frac{1}{V_{2^{-k}}(y)+V(x, y)} \\
& \quad \times\left\{\frac{2^{-k\left(v_{2}-\lambda \epsilon_{1}\right)}}{\left(2^{-k}+d(x, y)\right)^{\nu_{2}-\lambda \epsilon_{1}}}+\frac{2^{-k\left(v_{1}-\mu \epsilon_{1}\right)}}{\left(2^{-k}+d(x, y)\right)^{\nu_{1}-\mu \epsilon_{1}}}\right\} .
\end{aligned}
$$

Combining these estimates completes the proof of Lemma 4.4.

Lemma 4.5. Let $G_{k}(x, y)$ for $k \in \mathbb{Z}$ be as above. Then for any $\lambda, \mu \in(0,1)$ satisfying that $\lambda \epsilon_{1}<\epsilon_{2}$, there exists a constant $C_{N}>0$, independent of $j$, such that

(i) $\left|G_{k}(x, y)\right| \leq C_{N} 2^{-j \epsilon_{1}}\left(1 /\left(V_{2^{-k}}(x)+V_{2^{-k}}(y)+V(x, y)\right)\right)\left(2^{-k \epsilon_{2}} /\left(2^{-k}+d(x, y)\right)^{\epsilon_{2}}\right)$;

(ii) for $y, y^{\prime} \in x$ with $d\left(y, y^{\prime}\right) \leq\left(2^{-k}+d(x, y)\right) / 2$,

$$
\begin{aligned}
\left|G_{k}(x, y)-G_{k}\left(x, y^{\prime}\right)\right| \leq & C_{N} 2^{-j \epsilon_{1}} \frac{d\left(y, y^{\prime}\right)^{\mu \epsilon_{1}}}{\left(2^{-k}+d(x, y)\right)^{\mu \epsilon_{1}}} \frac{1}{V_{2^{-k}}(x)+V_{2^{-k}}(y)+V(x, y)} \\
& \times\left\{\frac{2^{-k \epsilon_{3}}}{\left(2^{-k}+d(x, y)\right)^{\epsilon_{3}}}+\frac{2^{-k\left(\epsilon_{2}-\mu \epsilon_{1}\right)}}{\left(2^{-k}+d(x, y)\right)^{\epsilon_{2}-\mu \epsilon_{1}}}\right\} ;
\end{aligned}
$$


(iii) $\left|G_{k}(x, y)-G_{k}\left(x^{\prime}, y\right)\right| \leq C_{N} 2^{-j \epsilon_{1}}\left(d\left(x, x^{\prime}\right)^{\lambda \epsilon_{1}} /\left(2^{-k}+d(x, y)\right)^{\lambda \epsilon_{1}}\right)\left(1 /\left(V_{2^{-k}}(x)+V_{2^{-k}}(y)+\right.\right.$ $V(x, y)))\left(2^{-k\left(\epsilon_{2}-\lambda \epsilon_{1}\right)} /\left(2^{-k}+d(x, y)\right)^{\epsilon_{2}-\lambda \epsilon_{1}}\right)$ for $x, x^{\prime} \in x$ with $d\left(x, x^{\prime}\right) \leq\left(2^{-k}+\right.$ $d(x, y)) / 2$;

(iv) $\int_{x} G_{k}(x, y) d \mu(y)=0=\int_{x} G_{k}(x, y) d \mu(x)$.

Proof. Since $\int_{x} D_{k}(x, y) d \mu(y)=0=\int_{x} D_{k}^{N}(x, y) d \mu(x)$, by the definition of $G_{k}$ in (4.12), we easily see that (iv) holds. $z \in Q_{\tau}^{k, v}$

By the construction of dyadic cubes in Lemma 2.19, we also easily see that for any

$$
d\left(z, y_{\tau}^{k, v}\right) \leq C_{6} 2^{-(k+j)}=C_{6} 2^{-j} 2^{-k} \leq C_{6} 2^{-j}\left(2^{-k}+d(y, z)\right)
$$

We recall that $j$ always satisfies (4.1). Then, the regularity of $D_{k}$ and the size estimates of $D_{k}^{N}$ together with Lemma 4.4 in the case $\lambda=\mu=0$ yield that

$$
\begin{aligned}
\left|G_{k}(x, y)\right| & =\left|\sum_{\tau \in I_{k}} \sum_{v=1}^{N(k, \tau)} \int_{Q_{\tau}^{k, v}} D_{k}^{N}(x, z)\left[D_{k}(z, y)-D_{k}\left(y_{\tau}^{k, v}, y\right)\right] d \mu(z)\right| \\
& \leq C_{N} 2^{-j \epsilon_{1}} \frac{1}{V_{2^{-k}}(x)+V_{2^{-k}}(y)+V(x, y)} \frac{2^{-k \epsilon_{2}}}{\left(2^{-k}+d(x, y)\right)^{\epsilon_{2}}}
\end{aligned}
$$

which shows $G_{k}(x, y)$ satisfies (i).

Write

$$
\begin{aligned}
& G_{k}(x, y)-G_{k}\left(x, y^{\prime}\right) \\
& \quad=\sum_{\tau \in I_{k}} \sum_{\nu=1}^{N(k, \tau)} \int_{Q_{\tau}^{k, v}} D_{k}^{N}(x, z)\left\{\left[D_{k}(z, y)-D_{k}\left(y_{\tau}^{k, v}, y\right)\right]-\left[D_{k}\left(z, y^{\prime}\right)-D_{k}\left(y_{\tau}^{k, v}, y^{\prime}\right)\right]\right\} d \mu(z) .
\end{aligned}
$$

We now verify that $G_{k}(x, y)$ satisfies (ii) by considering the following two cases.

Case $1\left(d\left(y, y^{\prime}\right) \leq\left(2^{-k}+d(z, y)\right) / 3\right)$. In this case, from (4.19) with $j$ satisfying (4.1), the size estimates of $D_{k}^{N}$ and the second difference regularity of $D_{k}$ and Lemma 4.4 with $\lambda=0, \mu \in$ $(0,1), v_{1}=\epsilon_{2}$, and $v_{2}=\epsilon_{3}$, it follows that

$$
\begin{aligned}
& \left|G_{k}(x, y)-G_{k}\left(x, y^{\prime}\right)\right| \\
& \lesssim 2^{-j \epsilon_{1}} \int_{x}\left|D_{k}^{N}(x, z)\right| \frac{d\left(y, y^{\prime}\right)^{\mu \epsilon_{1}}}{\left(2^{-k}+d(z, y)\right)^{\mu \epsilon_{1}}} \frac{1}{V_{2^{-k}}(z)+V_{2-k}(y)+V(z, y)} \frac{2^{-k \epsilon_{3}}}{\left(2^{-k}+d(z, y)\right)^{\epsilon_{3}}} d \mu(z) \\
& \leq C_{N} 2^{-j \epsilon_{1}} \frac{d\left(y, y^{\prime}\right)^{\mu \epsilon_{1}}}{\left(2^{-k}+d(x, y)\right)^{\mu \epsilon_{1}}} \frac{1}{V_{2^{-k}}(x)+V_{2^{-k}}(y)+V(x, y)}\left\{\frac{2^{-k \epsilon_{3}}}{\left(2^{-k}+d(x, y)\right)^{\epsilon_{3}}}+\frac{2^{-k\left(\epsilon_{2}-\mu \epsilon_{1}\right)}}{\left(2^{-k}+d(x, y)\right)^{\epsilon_{2}-\mu \epsilon_{1}}}\right\} .
\end{aligned}
$$


Case $2\left(\left(2^{-k}+d(z, y)\right) / 3<d\left(y, y^{\prime}\right) \leq\left(2^{-k}+d(x, y)\right) / 2\right)$. In this case, the estimate (i), Lemma 2.1(iii) together with the fact that $2^{-k}<3 d\left(y, y^{\prime}\right)$ show that for any $\mu \in$ $(0,1)$,

$$
\begin{aligned}
& \left|G_{k}(x, y)-G_{k}\left(x, y^{\prime}\right)\right| \\
& \leq\left|G_{k}(x, y)\right|+\left|G_{k}\left(x, y^{\prime}\right)\right| \\
& \leq C_{N} 2^{-j \epsilon_{1}}\left\{\frac{1}{V_{2^{-k}}(x)+V_{2^{-k}}(y)+V(x, y)} \frac{2^{-k \epsilon_{2}}}{\left(2^{-k}+d(x, y)\right)^{\epsilon_{2}}}\right. \\
& \left.\quad+\frac{1}{V_{2^{-k}}(x)+V_{2^{-k}}\left(y^{\prime}\right)+V\left(x, y^{\prime}\right)} \frac{2^{-k \epsilon_{2}}}{\left(2^{-k}+d\left(x, y^{\prime}\right)\right)^{\epsilon_{2}}}\right\} \\
& \leq C_{N} 2^{-j \epsilon_{1}} \frac{d\left(y, y^{\prime}\right)^{\mu \epsilon_{1}}}{\left(2^{-k}+d(x, y)\right)^{\mu \epsilon_{1}}} \frac{1}{V_{2^{-k}}(x)+V_{2^{-k}}(y)+V(x, y)} \frac{2^{-k\left(\epsilon_{2}-\mu \epsilon_{1}\right)}}{\left(2^{-k}+d(x, y)\right)^{\epsilon_{2}-\mu \epsilon_{1}}},
\end{aligned}
$$

which completes the proof of (ii).

We verify (iii) by writing

$$
\begin{aligned}
G_{k}(x, y) & -G_{k}\left(x^{\prime}, y\right) \\
= & \sum_{\tau \in I_{k}} \sum_{v=1}^{N(k, \tau)} \int_{Q_{\tau}^{k, v}}\left[D_{k}^{N}(x, z)-D_{k}^{N}\left(x^{\prime}, z\right)\right]\left[D_{k}(z, y)-D_{k}\left(y_{\tau}^{k, v}, y\right)\right] d \mu(z) .
\end{aligned}
$$

If $d\left(x, x^{\prime}\right) \leq\left(2^{-k}+d(x, z)\right) / 2$, the estimate (4.19) with $j$ satisfying (4.1), the regularity of $D_{k}^{N}$ and $D_{k}$, Lemma 4.4 with $\lambda \in(0,1), \mu=0$, and $\nu_{1}=v_{2}=\epsilon_{2}$ show that

$$
\begin{aligned}
& \left|G_{k}(x, y)-G_{k}\left(x^{\prime}, y\right)\right| \\
& \leq C_{N} 2^{-j \epsilon_{1}} \int_{x} \frac{d\left(x, x^{\prime}\right)^{\lambda \epsilon_{1}}}{\left(2^{-k}+d(x, z)\right)^{\lambda \epsilon_{1}}} \frac{1}{V_{2^{-k}}(x)+V_{2^{-k}}(z)+V(x, z)} \\
& \quad \times \frac{2^{-k \epsilon_{2}}}{\left(2^{-k}+d(x, z)\right)^{\epsilon_{2}}} \frac{1}{V_{2^{-k}}(z)+V_{2^{-k}}(y)+V(z, y)} \frac{2^{-k \epsilon_{2}}}{\left(2^{-k}+d(z, y)\right)^{\epsilon_{2}}} d \mu(z) \\
& \leq C_{N} 2^{-j \epsilon_{1}} \frac{d\left(x, x^{\prime}\right)^{\lambda \epsilon_{1}}}{\left(2^{-k}+d(x, y)\right)^{\lambda \epsilon_{1}}} \frac{1}{V_{2^{-k}}(x)+V_{2^{-k}}(y)+V(x, y)} \frac{2^{-k\left(\epsilon_{2}-\lambda \epsilon_{1}\right)}}{\left(2^{-k}+d(x, y)\right)^{\epsilon_{2}-\lambda \epsilon_{1}}} .
\end{aligned}
$$


If $\left(2^{-k}+d(x, z)\right) / 2<d\left(x, x^{\prime}\right) \leq\left(2^{-k}+d(x, y)\right) / 2$, the estimate (i), Lemma 2.1(iii) together with the fact $2^{-k}<2 d\left(x, x^{\prime}\right)$ yield that

$$
\begin{aligned}
&\left|G_{k}(x, y)-G_{k}\left(x^{\prime}, y\right)\right| \leq C_{N} 2^{-j \epsilon_{1}}\{ \frac{1}{V_{2^{-k}}(x)+V_{2^{-k}}(y)+V(x, y)} \frac{2^{-k \epsilon_{2}}}{\left(2^{-k}+d(x, y)\right)^{\epsilon_{2}}} \\
&\left.+\frac{1}{V_{2^{-k}}\left(x^{\prime}\right)+V_{2^{-k}}(y)+V\left(x^{\prime}, y\right)} \frac{2^{-k \epsilon_{2}}}{\left(2^{-k}+d\left(x^{\prime}, y\right)\right)^{\epsilon_{2}}}\right\} \\
& \leq C_{N} 2^{-j \epsilon_{1}} \frac{d\left(x, x^{\prime}\right)^{\lambda \epsilon_{1}}}{\left(2^{-k}+d(x, y)\right)^{\lambda \epsilon_{1}}} \frac{1}{V_{2^{-k}}(x)+V_{2^{-k}}(y)+V(x, y)} \frac{2^{-k\left(\epsilon_{2}-\lambda \epsilon_{1}\right)}}{\left(2^{-k}+d(x, y)\right)^{\epsilon_{2}-\lambda \epsilon_{1}}},
\end{aligned}
$$

which completes the proof of (iii), and hence the proof of Lemma 4.5.

Lemma 4.6. Let $N \in \mathbb{N}$ and $G$ be as in (4.12). Then there exist a constant $C_{N}>0$ independent of $j$ such that

$$
\|G\|_{L^{2}(\chi) \rightarrow L^{2}(\mathcal{X})} \leq C_{N} 2^{-j \epsilon_{1}}
$$

Proof. Using Lemma 4.5 and repeating the proof of (3.2) in Lemma 3.2 yield that for any $\epsilon_{1}^{\prime} \in$ $\left(0, \epsilon_{1} \wedge \epsilon_{2}\right)$ and all $k, j \in \mathbb{Z}$,

$$
\begin{aligned}
& \left\|G_{k} G_{j}^{t}\right\|_{L^{2}(\mathcal{X}) \rightarrow L^{2}(\mathcal{X})} \leq C_{N} 2^{-j \epsilon_{1}} 2^{-|k-j| \epsilon_{1}^{\prime}}, \\
& \left\|G_{j}^{t} G_{k}\right\|_{L^{2}(\mathcal{X}) \rightarrow L^{2}(\mathcal{X})} \leq C_{N} 2^{-j \epsilon_{1}} 2^{-|k-j| \epsilon_{1}^{\prime},}
\end{aligned}
$$

where $C_{N}>0$ is a constant depending on $\epsilon_{1}^{\prime}$, but, independent of $j$ and $k$. These estimates together with the Cotlar-Stein lemma and $G=\sum_{k=-\infty}^{\infty} G_{k}$ yields the conclusion of Lemma 4.6. formula.

The following lemma is a key lemma to establish the discrete Calderón reproducing

Lemma 4.7. Let $S$ be as in (4.2) and $R=I-S$. Then $R$ is a Calderón-Zygmund singular integral operator, $R(1)=0=R^{*}(1)$. Moreover, for any $\epsilon_{1}^{\prime} \in\left(0, \epsilon_{1} \wedge \epsilon_{2}\right)$, there exist positive constants $C_{10}, C_{N}$, and $\delta$, depending on $\epsilon_{1}^{\prime}$, such that the kernel, $R(x, y)$, of R satisfies all the conditions of Corollary 2.22 with $\epsilon$ replaced by $\epsilon_{1}^{\prime}$ and

$$
C_{R}+\|R\|_{L^{2}(\mathcal{X}) \rightarrow L^{2}(\mathcal{X})} \leq C_{10} 2^{-\delta N}+C_{N} 2^{-j \epsilon_{1}},
$$

where $C_{R}$ is the Calderon-Zygmund constant as in Theorem 2.18, $C_{10}$ and $\delta$ are independent of $N$ and $j$, and $C_{N}$ is independent of $j$. 
Proof. From (4.11), it is easy to see that $R(1)=0=R^{*}(1)$. Moreover, Lemma 3.6 shows that $R_{N}(x, y)$, the kernel of $R_{N}$, satisfies all the conditions of Corollary 2.22 with $\epsilon$ replaced by $\epsilon_{1}^{\prime}$ and $C_{R_{N}}+\left\|R_{N}\right\|_{L^{2}(x) \rightarrow L^{2}(x)} \leq C_{10} 2^{-\delta N}$, where $C_{10}$ and $\delta$ are independent of $N$ and $j$. This combining the formula (4.11) implies that to prove Lemma 4.7, it suffices to verify that $G(x, y)$, the kernel of $G$, satisfies (2.49) and Theorem 2.18(i), (ii), and (iii) with $\epsilon$ replaced by $\epsilon_{1}^{\prime}$ and $C_{G} \leq C_{N} 2^{-j \epsilon_{1}}$, where $C_{N}$ is independent of $N$. In fact, combining Lemma 4.5(i) with Lemma 3.5 and (4.12) yields that for all $x, y \in \mathcal{X}$ with $x \neq y$,

$$
\begin{aligned}
|G(x, y)| & \leq \sum_{k=-\infty}^{\infty}\left|G_{k}(x, y)\right| \\
& \leq C_{N} 2^{-j \epsilon_{1}} \sum_{k=-\infty}^{\infty} \frac{1}{V_{2^{-k}}(x)+V(x, y)} \frac{2^{-k \epsilon_{2}}}{\left(2^{-k}+d(x, y)\right)^{\epsilon_{2}}} \\
& \leq C_{N} 2^{-j \epsilon_{1}} \frac{1}{V(x, y)}
\end{aligned}
$$

which shows $G(x, y)$ satisfies Theorem 2.18(i).

Lemma 4.5(ii) and (iii) and Lemma 3.5 together with (4.12), respectively, show that for any $\mu \in(0,1)$, all $x, y, y^{\prime} \in \mathcal{X}$ with $x \neq y$ and $d\left(y, y^{\prime}\right) \leq d(x, y) / 2$,

$$
\begin{aligned}
\left|G(x, y)-G\left(x, y^{\prime}\right)\right| \leq & \sum_{k=-\infty}^{\infty}\left|G_{k}(x, y)-G_{k}\left(x, y^{\prime}\right)\right| \\
\leq & C_{N} 2^{-j \epsilon_{1}} \frac{d\left(y, y^{\prime}\right)^{\mu \epsilon_{1}}}{d(x, y)^{\mu \epsilon_{1}}} \sum_{k=-\infty}^{\infty} \frac{1}{V_{2^{-k}}(x)+V(x, y)} \\
& \times\left\{\frac{2^{-k \epsilon_{3}}}{\left(2^{-k}+d(x, y)\right)^{\epsilon_{3}}}+\frac{2^{-k\left(\epsilon_{2}-\mu \epsilon_{1}\right)}}{\left(2^{-k}+d(x, y)\right)^{\epsilon_{2}-\mu \epsilon_{1}}}\right\} \\
\leq & C_{N} 2^{-j \epsilon_{1}} \frac{d\left(y, y^{\prime}\right)^{\mu \epsilon_{1}}}{d(x, y)^{\mu \epsilon_{1}}} \frac{1}{V(x, y)},
\end{aligned}
$$

and for any $\lambda \in(0,1)$, all $x, x^{\prime}, y \in \mathcal{X}$ with $x \neq y$ and $d\left(x, x^{\prime}\right) \leq d(x, y) / 2$,

$$
\begin{aligned}
\left|G(x, y)-G\left(x^{\prime}, y\right)\right| & \leq \sum_{k=-\infty}^{\infty}\left|G_{k}(x, y)-G_{k}\left(x^{\prime}, y\right)\right| \\
& \leq C_{N} 2^{-j \epsilon_{1}} \frac{d\left(x, x^{\prime}\right)^{\lambda \epsilon_{1}}}{d(x, y)^{\lambda \epsilon_{1}}} \sum_{k=-\infty}^{\infty} \frac{1}{V_{2^{-k}}(x)+V(x, y)} \frac{2^{-k\left(\epsilon_{2}-\lambda \epsilon_{1}\right)}}{\left(2^{-k}+d(x, y)\right)^{\epsilon_{2}-\lambda \epsilon_{1}}} \\
& \leq C_{N} 2^{-j \epsilon_{1}} \frac{d\left(x, x^{\prime}\right)^{\lambda \epsilon_{1}}}{d(x, y)^{\lambda \epsilon_{1}}} \frac{1}{V(x, y)}
\end{aligned}
$$

which proves that $G(x, y)$ satisfies Theorem 2.18(ii) and (2.49). 
By (2.49) and Theorem 2.18(ii), it is easy to see that it suffices to establish Theorem 2.18(iii) only for $d\left(x, x^{\prime}\right) \leq d(x, y) / 6$ and $d\left(y, y^{\prime}\right) \leq d(x, y) / 6$. To this end, we write

$$
\begin{aligned}
& {\left[G(x, y)-G\left(x^{\prime}, y\right)\right]-\left[G\left(x, y^{\prime}\right)-G\left(x^{\prime}, y^{\prime}\right)\right]} \\
& =\sum_{k=-\infty}^{\infty} \sum_{\tau \in I_{k}} \sum_{\nu=1}^{N(k, \tau)} \int_{Q_{\tau}^{k, v}}\left[D_{k}^{N}(x, z)-D_{k}^{N}\left(x^{\prime}, z\right)\right] \\
& \quad \times\left\{\left[D_{k}(z, y)-D_{k}\left(y_{\tau}^{k, v}, y\right)\right]-\left[D_{k}\left(z, y^{\prime}\right)-D_{k}\left(y_{\tau}^{k, v}, y^{\prime}\right)\right]\right\} d \mu(z) .
\end{aligned}
$$

If $d\left(x, x^{\prime}\right)>\left(2^{-k}+d(x, z)\right) / 2$ and $d\left(y, y^{\prime}\right)>\left(2^{-k}+d(y, z)\right) / 3$, then

$$
d\left(x, x^{\prime}\right)+d\left(y, y^{\prime}\right) \geq \frac{\left(2^{1-k}+d(x, z)+d(z, y)\right)}{3}>\frac{d(x, y)}{3}
$$

which contradicts the assumptions that $d\left(x, x^{\prime}\right) \leq d(x, y) / 6$ and $d\left(y, y^{\prime}\right) \leq d(x, y) / 6$. Thus, we still have the following three cases:

(i) $d\left(x, x^{\prime}\right) \leq\left(2^{-k}+d(x, z)\right) / 2$ and $d\left(y, y^{\prime}\right) \leq\left(2^{-k}+d(y, z)\right) / 3$;

(ii) $d\left(x, x^{\prime}\right) \leq\left(2^{-k}+d(x, z)\right) / 2$ and $d\left(y, y^{\prime}\right)>\left(2^{-k}+d(y, z)\right) / 3$;

(iii) $d\left(x, x^{\prime}\right)>\left(2^{-k}+d(x, z)\right) / 2$ and $d\left(y, y^{\prime}\right) \leq\left(2^{-k}+d(y, z)\right) / 3$.

In the case (i), by (4.19) with $j$ satisfying (4.1), the second difference regularity of $D_{k}^{N}$ and $D_{k}$, Lemma 4.4 with $\lambda, \mu \in(0,1), v_{1}=\epsilon_{2}$, and $v_{2}=\epsilon_{1}+\epsilon_{3}$, and Lemma 3.5, we obtain

$$
\begin{aligned}
& \left|\left[G(x, y)-G\left(x^{\prime}, y\right)\right]-\left[G\left(x, y^{\prime}\right)-G\left(x^{\prime}, y^{\prime}\right)\right]\right| \\
& \leq C_{N} 2^{-j \epsilon_{1}} \sum_{k=-\infty}^{\infty} \int_{x} \frac{d\left(x, x^{\prime}\right)^{\lambda \epsilon_{1}}}{\left(2^{-k}+d(x, z)\right)^{\lambda \epsilon_{1}}} \frac{1}{V_{2^{-k}}(x)+V_{2^{-k}}(z)+V(x, z)} \frac{2^{-k \epsilon_{2}}}{\left(2^{-k}+d(x, z)\right)^{\epsilon_{2}}} \frac{d\left(y, y^{\prime}\right)^{\mu \epsilon_{1}}}{\left(2^{-k}+d(z, y)\right)^{\mu \epsilon_{1}}} \\
& \times \frac{1}{V_{2^{-k}}(z)+V_{2^{-k}}(y)+V(z, y)} \frac{2^{-k\left(\epsilon_{1}+\epsilon_{3}\right)}}{\left(2^{-k}+d(z, y)\right)^{\epsilon_{1}+\epsilon_{3}}} d \mu(z) \\
& \leq C_{N} 2^{-j \epsilon_{1}} \frac{d\left(x, x^{\prime}\right)^{\lambda \epsilon_{1}}}{d(x, y)^{\lambda \epsilon_{1}}} \frac{d\left(y, y^{\prime}\right)^{\mu \epsilon_{1}}}{d(x, y)^{\mu \epsilon_{1}}} \frac{1}{V(x, y)} \text {. }
\end{aligned}
$$

In the case (ii), we also have $\left(2^{-k}+d\left(z, y^{\prime}\right)\right) / 2 \leq\left(2^{-k}+d(z, y)\right) / 2+d\left(y, y^{\prime}\right) / 2 \leq 2 d\left(y, y^{\prime}\right)$. From this, (4.19) with $j$ satisfying (4.1), the regularity of $D_{k}^{N}$ and $D_{k}$, Lemma 4.4 with $\lambda, \mu \in(0,1)$ 
and $v_{1}=v_{2}=\epsilon_{2}$, Lemmas 2.1(iii) and 3.5, it follows that

$$
\begin{aligned}
& \left|\left[G(x, y)-G\left(x^{\prime}, y\right)\right]-\left[G\left(x, y^{\prime}\right)-G\left(x^{\prime}, y^{\prime}\right)\right]\right| \\
& \leq C_{N} 2^{-j \epsilon_{1}} \sum_{k=-\infty}^{\infty} \int_{x} \frac{d\left(x, x^{\prime}\right)^{\lambda \epsilon_{1}}}{x} \frac{1}{\left(2^{-k}+d(x, z)\right)^{\lambda \epsilon_{1}}} \frac{2^{-k \epsilon_{2}}}{V_{2^{-k}}(x)+V_{2^{-k}}(z)+V(x, z)} \frac{1}{\left(2^{-k}+d(x, z)\right)^{\epsilon_{2}}} \\
& \times\left[\frac{d\left(y, y^{\prime}\right)^{\mu \epsilon_{1}}}{\left(2^{-k}+d(z, y)\right)^{\mu \epsilon_{1}}} \frac{2^{-k \epsilon_{2}}}{V_{2^{-k}}(z)+V_{2^{-k}}(y)+V(z, y)} \frac{1}{\left(2^{-k}+d(z, y)\right)^{\epsilon_{2}}}\right. \\
& \left.+\frac{d\left(y, y^{\prime}\right)^{\mu \epsilon_{1}}}{\left(2^{-k}+d\left(z, y^{\prime}\right)\right)^{\mu \epsilon_{1}}} \frac{2^{-k \epsilon_{2}}}{V_{2^{-k}}(z)+V_{2^{-k}}\left(y^{\prime}\right)+V\left(z, y^{\prime}\right)} \frac{\left.12^{-k}+d\left(z, y^{\prime}\right)\right)^{\epsilon_{2}}}{\left(2^{\prime}\right.}\right] d \mu(z) \\
& \leq C_{N} 2^{-j \epsilon_{1}} \frac{d\left(x, x^{\prime}\right)^{\lambda \epsilon_{1}}}{d(x, y)^{\lambda \epsilon_{1}}} \frac{d\left(y, y^{\prime}\right)^{\mu \epsilon_{1}}}{d(x, y)^{\mu \epsilon_{1}}} \frac{1}{V(x, y)} .
\end{aligned}
$$

For the last case (iii), we have $\left(2^{-k}+d\left(x^{\prime}, z\right)\right) / 2 \leq\left(2^{-k}+d(x, z)\right) / 2+d\left(x, x^{\prime}\right) / 2 \leq 3 d\left(x, x^{\prime}\right) / 2$. This estimate, (4.19) with $j$ satisfying (4.1), the size condition of $D_{k}^{N}$, the second difference regularity of $D_{k}$, Lemma 4.4 with $\lambda, \mu \in(0,1), v_{1}=\epsilon_{2}$, and $v_{2}=\epsilon_{1}+\epsilon_{3}$, Lemmas 2.1(iii) and 3.5 show that

$$
\begin{aligned}
& \left|\left[G(x, y)-G\left(x^{\prime}, y\right)\right]-\left[G\left(x, y^{\prime}\right)-G\left(x^{\prime}, y^{\prime}\right)\right]\right| \\
& \leq C_{N} 2^{-j \epsilon_{1}} \sum_{k=-\infty}^{\infty} \int_{x}\left[\left|D_{k}^{N}(x, z)\right|+\left|D_{k}^{N}\left(x^{\prime}, z\right)\right|\right] \\
& \quad \times \frac{d\left(y, y^{\prime}\right)^{\mu \epsilon_{1}}}{\left(2^{-k}+d(z, y)\right)^{\mu \epsilon_{1}}} \frac{1}{V_{2^{-k}}(z)+V_{2^{-k}}(y)+V(z, y)} \frac{2^{-k\left(\epsilon_{1}+\epsilon_{3}\right)}}{\left(2^{-k}+d(z, y)\right)^{\epsilon_{1}+\epsilon_{3}}} d \mu(z) \\
& \leq C_{N} 2^{-j \epsilon_{1}} \frac{d\left(x, x^{\prime}\right)^{\lambda \epsilon_{1}}}{d(x, y)^{\lambda \epsilon_{1}}} \frac{d\left(y, y^{\prime}\right)^{\mu \epsilon_{1}}}{d(x, y)^{\mu \epsilon_{1}}} \frac{1}{V(x, y)} .
\end{aligned}
$$

Thus, $G(x, y)$ satisfies Theorem 2.18(iii). This finishes the proof of Lemma 4.7.

By Lemmas 4.6 and 4.7, we obtain the boundedness of $R$ on $\mathcal{G}\left(x_{1}, r, \beta, \gamma\right)$ for any $x_{1} \in \mathcal{X}$, $r>0$ and $0<\beta, \gamma<\left(\epsilon_{1} \wedge \epsilon_{2}\right)$, and in $L^{p}(\mathcal{X})$ for $p \in(1, \infty)$. We omit the details.

Proposition 4.8. Let $R$ and the notation be as in Lemma 4.7. Then $R$ is bounded on $L^{p}(\mathcal{X})$ for $p \in$ $(1, \infty)$ and on $\mathcal{G}\left(x_{1}, r, \beta, \gamma\right)$ for any $x_{1} \in \mathcal{X}, r>0$ and $0<\beta, \gamma<\left(\epsilon_{1} \wedge \epsilon_{2}\right)$. That is, there exists $a$ constant $C_{11}>0$, only depending on $p, \beta$, and $\gamma$ such that for all $f \in L^{p}(\mathcal{X})$,

$$
\begin{gathered}
\|R(f)\|_{L^{p}(\mathcal{X})} \leq C_{11}\left(C_{10} 2^{-\delta N}+C_{N} 2^{-j \epsilon_{1}}\right)\|f\|_{L^{p}(\mathcal{X})}, \\
\|R(f)\|_{\mathcal{G}\left(x_{1}, r, \beta, \gamma\right)} \leq C_{11}\left(C_{10} 2^{-\delta N}+C_{N} 2^{-j \epsilon_{1}}\right)\|f\|_{\mathcal{G}\left(x_{1}, r, \beta, \gamma\right)} .
\end{gathered}
$$


By using Proposition 4.8 and repeating the proof of Propositions 3.7 and 3.8, we can obtain the boundedness of $S^{-1}$ on $\mathcal{G}\left(x_{1}, r, \beta, \gamma\right)$ for any $x_{1} \in \mathcal{X}, r>0$ and $0<\beta, \gamma<\epsilon$, and in $L^{p}(\mathcal{X})$ for $p \in(1, \infty)$. We also omit the details.

Corollary 4.9. Let $S$ be as in (4.2). Let $N, j \in \mathbb{N}$ such that (4.1) and

$$
C_{11}\left(C_{10} 2^{-\delta N}+C_{N} 2^{-j \epsilon_{1}}\right)<1
$$

hold. Then $S$ has a bounded inverse in $\mathcal{G}\left(x_{1}, r, \beta, \gamma\right)$ for any $x_{1} \in \mathcal{X}, r>0$, and $0<\beta, \gamma<\left(\epsilon_{1} \wedge \epsilon_{2}\right)$, and in $L^{p}(\mathcal{X})$ for $p \in(1, \infty)$. Namely, there exists a constant $C>0$ depending only on $\beta, \gamma$, and $p$ such that for all $f \in \stackrel{\mathcal{G}}{(}\left(x_{1}, r, \beta, \gamma\right)$,

$$
\left\|S^{-1}(f)\right\|_{\mathcal{G}\left(x_{1}, r, \beta, \gamma\right)} \leq C\|f\|_{\mathcal{G}\left(x_{1}, r, \beta, r\right)}
$$

and for all $f \in L^{p}(\mathcal{X})$,

$$
\left\|S^{-1}(f)\right\|_{L^{p}(\chi)} \leq C\|f\|_{L^{p}(\chi)}
$$

To establish the discrete Calderón reproducing formulae, we still need the following technical lemma.

Lemma 4.10. Let $j$ satisfy (4.1). For $k \in \mathbb{Z}$, any fixed $y_{\tau}^{k, v} \in Q_{\tau}^{k, v}$ with $\tau \in I_{k}$ and $v \in\{1, \ldots$, $N(k, \tau)\}$, and any $x \in \mathcal{X}$, let

$$
E_{k}(x, y)=\sum_{\tau \in I_{k}} \sum_{v=1}^{N(k, \tau)} \int_{Q_{\tau}^{k, \nu}} D_{k}^{N}(x, z) d \mu(z) D_{k}\left(y_{\tau}^{k, v}, y\right)
$$

Then for any $\lambda, \mu \in(0,1)$, there exists a constant $C_{N}>0$ depending only on $N, \lambda$ and $\mu$ such that

(i) for all $k \in \mathbb{Z}$ and all $x, y \in X$,

$$
\left|E_{k}(x, y)\right| \leq C_{N} \frac{1}{V_{2^{-k}}(x)+V_{2^{-k}}(y)+V(x, y)} \frac{2^{-k \epsilon_{2}}}{\left(2^{-k}+d(x, y)\right)^{\epsilon_{2}}}
$$

(ii) for all $k \in \mathbb{Z}$ and all $x, y, y^{\prime} \in X$ with $d\left(y, y^{\prime}\right) \leq\left(2^{-k}+d(x, y)\right) / 2$,

$$
\begin{aligned}
& \left|E_{k}(x, y)-E_{k}\left(x, y^{\prime}\right)\right| \\
& \quad \leq C_{N} \frac{d\left(y, y^{\prime}\right)^{\mu \epsilon_{1}}}{\left(2^{-k}+d(x, y)\right)^{\mu \epsilon_{1}}} \frac{1}{V_{2^{-k}}(x)+V_{2^{-k}}(y)+V(x, y)} \frac{2^{-k\left(\epsilon_{2}-\mu \epsilon_{1}\right)}}{\left(2^{-k}+d(x, y)\right)^{\epsilon_{2}-\mu \epsilon_{1}}}
\end{aligned}
$$

(iii) property (ii) also holds with $x$ and $y$ interchanged and $\mu$ replaced by $\lambda$; 
(iv) for all $k \in \mathbb{Z}$ and all $x, x^{\prime}, y, y^{\prime} \in X$ with $d\left(x, x^{\prime}\right) \leq\left(2^{-k}+d(x, y)\right) / 4$ and $d\left(y, y^{\prime}\right) \leq$ $\left(2^{-k}+d(x, y)\right) / 4$,

$$
\begin{aligned}
& \left|\left[E_{k}(x, y)-E_{k}\left(x^{\prime}, y\right)\right]-\left[E_{k}\left(x, y^{\prime}\right)-E_{k}\left(x^{\prime}, y^{\prime}\right)\right]\right| \\
& \leq C_{N} \frac{d\left(x, x^{\prime}\right)^{\lambda \epsilon_{1}}}{\left(2^{-k}+d(x, y)\right)^{\lambda \epsilon_{1}}} \frac{d\left(y, y^{\prime}\right)^{\mu \epsilon_{1}}}{\left(2^{-k}+d(x, y)\right)^{\mu \epsilon_{1}}} \frac{1}{V_{2^{-k}}(x)+V_{2^{-k}}(y)+V(x, y)} \\
& \quad \times\left[\frac{2^{-k\left(\epsilon_{2}-\lambda \epsilon_{1}\right)}}{\left(2^{-k}+d(x, y)\right)^{\epsilon_{2}-\lambda \epsilon_{1}}}+\frac{2^{-k\left(\epsilon_{2}-\mu \epsilon_{1}\right)}}{\left(2^{-k}+d(x, y)\right)^{\epsilon_{2}-\mu \epsilon_{1}}}\right] .
\end{aligned}
$$

Proof. The main idea for the proof of this lemma is to combine the techniques used in the proof of Lemma 3.2 with Lemma 2.1(iii). By (4.19) and Lemma 2.1(iii), for any $z \in Q_{\tau}^{k, v}$ and $y \in x$,

$$
\begin{aligned}
\frac{1}{2^{-k}+d\left(y_{\tau}^{k, v}, y\right)} & \sim \frac{1}{2^{-k}+d(z, y)} \\
\frac{1}{V_{2^{-k}}\left(y_{\tau}^{k, v}\right)+V_{2^{-k}}(y)+V\left(y_{\tau}^{k, v}, y\right)} & \sim \frac{1}{V_{2^{-k}}(z)+V_{2^{-k}}(y)+V(z, y)} .
\end{aligned}
$$

To see (i), by the size condition of $D_{k}$ together with (4.46) and (4.47), and Lemma 4.4 with $\lambda=\mu=0$ and $v_{1}=v_{2}=\epsilon_{2}$, we have

$$
\begin{aligned}
\left|E_{k}(x, y)\right| & \lesssim \int_{x}\left|D_{k}^{N}(x, z)\right| \frac{1}{V_{2^{-k}}(z)+V_{2^{-k}}(y)+V(z, y)} \frac{2^{-k \epsilon_{2}}}{\left(2^{-k}+d(z, y)\right)^{\epsilon_{2}}} d \mu(z) \\
& \lesssim \frac{1}{V_{2^{-k}}(x)+V_{2^{-k}}(y)+V(x, y)} \frac{2^{-k \epsilon_{2}}}{\left(2^{-k}+d(x, y)\right)^{\epsilon_{2}}}
\end{aligned}
$$

which verifies (i).

To verify (ii), we write

$$
\begin{aligned}
& \left|E_{k}(x, y)-E_{k}\left(x, y^{\prime}\right)\right| \\
& \leq \sum_{\tau \in I_{k}} \sum_{v=1}^{N(k, \tau)}\left|\int_{Q_{\tau}^{k, v}} D_{k}^{N}(x, z) d \mu(z)\left[D_{k}\left(y_{\tau}^{k, v}, y\right)-D_{k}\left(y_{\tau}^{k, v}, y^{\prime}\right)\right]\right| \\
& \quad \times\left[X_{\left\{d\left(y, y^{\prime}\right) \leq\left(2^{-k}+d\left(y_{\tau}^{k, v}, y\right)\right) / 2\right\}}\left(y, y^{\prime}\right)+X_{\left\{d\left(y, y^{\prime}\right)>\left(2^{-k}+d\left(y_{\tau}^{k, v}, y\right)\right) / 2\right\}}\left(y, y^{\prime}\right)\right] \\
& \equiv Y_{1}+Y_{2} .
\end{aligned}
$$


For $Y_{1}$, by the regularity of $D_{k}$ and the size condition of $D_{k}^{N}$ together with (4.46), (4.47) and Lemma 4 .4 with $\lambda=0, \mu \in(0,1)$, and $v_{1}=v_{2}=\epsilon_{2}$, we obtain

$$
\begin{aligned}
Y_{1} \lesssim \int_{x} \frac{1}{V_{2^{-k}}(x)+V_{2^{-k}}(z)+V(x, z)} \frac{2^{-k \epsilon_{2}}}{\left(2^{-k}+d(x, z)\right)^{\epsilon_{2}}} \frac{d\left(y, y^{\prime}\right)^{\mu \epsilon_{1}}}{\left(2^{-k}+d(z, y)\right)^{\mu \epsilon_{1}}} \\
\quad \times \frac{1}{V_{2^{-k}}(z)+V_{2^{-k}}(y)+V(z, y)} \frac{2^{-k \epsilon_{2}}}{\left(2^{-k}+d(z, y)\right)^{\epsilon_{2}}} d \mu(z) \\
\lesssim \frac{d\left(y, y^{\prime}\right)^{\mu \epsilon_{1}}}{\left(2^{-k}+d(x, y)\right)^{\mu \epsilon_{1}}} \frac{1}{V_{2^{-k}}(x)+V_{2^{-k}}(y)+V(x, y)} \frac{2^{-k\left(\epsilon_{2}-\mu \epsilon_{1}\right)}}{\left(2^{-k}+d(x, y)\right)^{\epsilon_{2}-\mu \epsilon_{1}}} .
\end{aligned}
$$

The property (i), Lemma 2.1(iii), and the fact that $2^{-k} \leq 2 d\left(y, y^{\prime}\right)$ yield that

$$
\begin{aligned}
Y_{2} \lesssim & \frac{1}{V_{2^{-k}}(x)+V_{2^{-k}}(y)+V(x, y)} \frac{2^{-k \epsilon_{2}}}{\left(2^{-k}+d(x, y)\right)^{\epsilon_{2}}} \\
& +\frac{1}{V_{2^{-k}}(x)+V_{2^{-k}}\left(y^{\prime}\right)+V\left(x, y^{\prime}\right)} \frac{2^{-k \epsilon_{2}}}{\left(2^{-k}+d\left(x, y^{\prime}\right)\right)^{\epsilon_{2}}} \\
& \lesssim \frac{d\left(y, y^{\prime}\right)^{\mu \epsilon_{1}}}{\left(2^{-k}+d(x, y)\right)^{\mu \epsilon_{1}}} \frac{1}{V_{2^{-k}}(x)+V_{2^{-k}}(y)+V(x, y)} \frac{2^{-k\left(\epsilon_{2}-\mu \epsilon_{1}\right)}}{\left(2^{-k}+d(x, y)\right)^{\epsilon_{2}-\mu \epsilon_{1}}}
\end{aligned}
$$

which completes the proof of (ii).

By symmetry, we can deduce (iii) from (ii).

To prove (iv), we write

$$
\begin{aligned}
& {\left[E_{k}(x, y)-E_{k}\left(x^{\prime}, y\right)\right]-\left[E_{k}\left(x, y^{\prime}\right)-E_{k}\left(x^{\prime}, y^{\prime}\right)\right]} \\
& \quad=\sum_{\tau \in I_{k}} \sum_{v=1}^{N(k, \tau)} \int_{Q_{\tau}^{k, \nu}}\left[D_{k}^{N}(x, z)-D_{k}^{N}\left(x^{\prime}, z\right)\right]\left[D_{k}\left(y_{\tau}^{k, v}, y\right)-D_{k}\left(y_{\tau}^{k, v}, y^{\prime}\right)\right] d \mu(z) .
\end{aligned}
$$

Notice that if $d\left(x, x^{\prime}\right)>\left(2^{-k}+d(x, z)\right) / 2$ with $z \in Q_{\tau}^{k, v}$ and $d\left(y, y^{\prime}\right)>\left(2^{-k}+d\left(y_{\tau}^{k, v}, y\right)\right) / 2$, then $d\left(x, x^{\prime}\right)+d\left(y, y^{\prime}\right)>\left(2^{1-k}+d(x, z)+d\left(y_{\tau}^{k, v}, y\right)\right) / 2$, which contradicts the assumptions that $d\left(x, x^{\prime}\right) \leq\left(2^{-k}+d(x, y)\right) / 4$ and $d\left(y, y^{\prime}\right) \leq\left(2^{-k}+d(x, y)\right) / 4$, since these estimates together with (4.1) and Lemma 2.19 prove that for $z \in Q_{\tau}^{k, v}, d\left(x, x^{\prime}\right)+d\left(y, y^{\prime}\right)<\left(2^{1-k}+d(x, z)+d\left(y_{\tau}^{k, v}, y\right)\right) / 2$. Thus, if we let

$$
\begin{aligned}
& W_{1}=\left\{z \in Q_{\tau}^{k, v}: d\left(x, x^{\prime}\right) \leq \frac{2^{-k}+d(x, z)}{2} \text { and } d\left(y, y^{\prime}\right) \leq \frac{2^{-k}+d\left(y_{\tau}^{k, v}, y\right)}{2}\right\}, \\
& W_{2}=\left\{z \in Q_{\tau}^{k, v}: d\left(x, x^{\prime}\right) \leq \frac{2^{-k}+d(x, z)}{2} \text { and } d\left(y, y^{\prime}\right)>\frac{2^{-k}+d\left(y_{\tau}^{k, v}, y\right)}{2}\right\}, \\
& W_{3}=\left\{z \in Q_{\tau}^{k, v}: d\left(x, x^{\prime}\right)>\frac{2^{-k}+d(x, z)}{2} \text { and } d\left(y, y^{\prime}\right) \leq \frac{2^{-k}+d\left(y_{\tau}^{k, v}, y\right)}{2}\right\},
\end{aligned}
$$


we then have

$$
\begin{aligned}
& {\left[E_{k}(x, y)-E_{k}\left(x^{\prime}, y\right)\right]-\left[E_{k}\left(x, y^{\prime}\right)-E_{k}\left(x^{\prime}, y^{\prime}\right)\right]} \\
& \quad=\sum_{i=1}^{3} \sum_{\tau \in I_{k}} \sum_{v=1}^{N(k, \tau)} \int_{Q_{\tau}^{k, v} \cap W_{i}}\left[D_{k}^{N}(x, z)-D_{k}^{N}\left(x^{\prime}, z\right)\right]\left[D_{k}\left(y_{\tau}^{k, v}, y\right)-D_{k}\left(y_{\tau}^{k, v}, y^{\prime}\right)\right] d \mu(z) \\
& \quad=\sum_{i=1}^{3} Z_{i} .
\end{aligned}
$$

For $Z_{1}$, the regularity of $D_{k}^{N}$ and $D_{k}$ together with the estimates (4.46) and (4.47) and Lemma 4.4 yield that for any $\lambda, \mu \in(0,1)$,

$$
\begin{aligned}
\left|Z_{1}\right| \lesssim & \int_{x} \frac{d\left(x, x^{\prime}\right)^{\lambda \epsilon_{1}}}{\left(2^{-k}+d(x, z)\right)^{\lambda \epsilon_{1}}} \frac{1}{V_{2^{-k}}(x)+V_{2^{-k}}(z)+V(x, z)} \frac{2^{-k \epsilon_{2}}}{\left(2^{-k}+d(x, z)\right)^{\epsilon_{2}}} \\
& \times \frac{d\left(y, y^{\prime}\right)^{\mu \epsilon_{1}}}{\left(2^{-k}+d(z, y)\right)^{\mu \epsilon_{1}}} \frac{1}{V_{2^{-k}}(z)+V_{2^{-k}}(y)+V(z, y)} \frac{2^{-k \epsilon_{2}}}{\left(2^{-k}+d(z, y)\right)^{\epsilon_{2}}} d \mu(z) \\
\lesssim & \frac{d\left(x, x^{\prime}\right)^{\lambda \epsilon_{1}}}{\left(2^{-k}+d(x, y)\right)^{\lambda \epsilon_{1}}} \frac{d\left(y, y^{\prime}\right)^{\mu \epsilon_{1}}}{\left(2^{-k}+d(x, y)\right)^{\mu \epsilon_{1}}} \frac{1}{V_{2^{-k}}(x)+V_{2^{-k}}(y)+V(x, y)} \\
& \times\left\{\frac{2^{-k\left(\epsilon_{2}-\lambda \epsilon_{1}\right)}}{\left(2^{-k}+d(x, y)\right)^{\epsilon_{2}-\lambda \epsilon_{1}}}+\frac{2^{-k\left(\epsilon_{2}-\mu \epsilon_{1}\right)}}{\left(2^{-k}+d(x, y)\right)^{\epsilon_{2}-\mu \epsilon_{1}}}\right\} .
\end{aligned}
$$

Similarly, for $Z_{2}$, from $d\left(y, y^{\prime}\right)>\left(2^{-k}+d\left(y_{\tau}^{k, v}, y\right)\right) / 2$, (4.46) and Lemma 2.1(iii) with $d\left(y, y^{\prime}\right)$ $\leq\left(2^{-k}+d(x, y)\right) / 4$, it follows that for $z \in Q_{\tau}^{k, \nu}, 1 / 2^{\mu \epsilon_{1}}<d\left(y, y^{\prime}\right)^{\mu \epsilon_{1}} /\left(2^{-k}+d\left(y_{\tau}^{k, \nu}, y\right)\right)^{\mu \epsilon_{1}} \sim$ $d\left(y, y^{\prime}\right)^{\mu \epsilon_{1}} /\left(2^{-k}+d(z, y)\right)^{\mu \epsilon_{1}}$, which together with the regularity of $D_{k}^{N}$ and the size condition of $D_{k},(4.46),(4.47)$, Lemmas 4.4 , and 2.1(iii) shows for any $\lambda, \mu \in(0,1)$,

$$
\begin{aligned}
\left|Z_{2}\right| \lesssim \int_{x} \frac{d\left(x, x^{\prime}\right)^{\lambda \epsilon_{1}}}{\left(2^{-k}+d(x, z)\right)^{\lambda \epsilon_{1}}} \frac{1}{V_{2^{-k}}(x)+V_{2^{-k}}(z)+V(x, z)} \frac{2^{-k \epsilon_{2}}}{\left(2^{-k}+d(x, z)\right)^{\epsilon_{2}}} \\
\quad \times\left[\frac{d\left(y, y^{\prime}\right)^{\mu \epsilon_{1}}}{\left(2^{-k}+d(z, y)\right)^{\mu \epsilon_{1}}} \frac{1}{V_{2^{-k}}(z)+V_{2^{-k}}(y)+V(z, y)} \frac{2^{-k \epsilon_{2}}}{\left(2^{-k}+d(z, y)\right)^{\epsilon_{2}}}\right. \\
\left.\quad+\frac{1}{V_{2^{-k}}(z)+V_{2^{-k}}\left(y^{\prime}\right)+V\left(z, y^{\prime}\right)} \frac{2^{-k \epsilon_{2}}}{\left(2^{-k}+d\left(z, y^{\prime}\right)\right)^{\epsilon_{2}}}\right] d \mu(z) \\
\lesssim \frac{d\left(x, x^{\prime}\right)^{\lambda \epsilon_{1}}}{\left(2^{-k}+d(x, y)\right)^{\lambda \epsilon_{1}} \frac{d\left(y, y^{\prime}\right)^{\mu \epsilon_{1}}}{\left(2^{-k}+d(x, y)\right)^{\mu \epsilon_{1}}} \frac{1}{V_{2^{-k}}(x)+V_{2^{-k}}(y)+V(x, y)}} \\
\quad \times\left\{\frac{2^{-k\left(\epsilon_{2}-\lambda \epsilon_{1}\right)}}{\left(2^{-k}+d(x, y)\right)^{\epsilon_{2}-\lambda \epsilon_{1}}}+\frac{2^{-k\left(\epsilon_{2}-\mu \epsilon_{1}\right)}}{\left(2^{-k}+d(x, y)\right)^{\epsilon_{2}-\mu \epsilon_{1}}}\right\} .
\end{aligned}
$$


From $Z_{2}$ and the symmetry, we can deduce the desired estimate for $Z_{3}$, which completes the proof of Lemma 4.10 .

We can now establish discrete Calderón reproducing formulae.

Theorem 4.11. Let $\epsilon_{1} \in(0,1], \epsilon_{2}>0, \epsilon_{3}>0, \epsilon \in\left(0, \epsilon_{1} \wedge \epsilon_{2}\right)$, and let $\left\{S_{k}\right\}_{k=-\infty}^{\infty}$ be an $\left(\epsilon_{1}, \epsilon_{2}, \epsilon_{3}\right)$-ATI. Set $D_{k}=S_{k}-S_{k-1}$ for any $k \in \mathbb{Z}$. Then for any fixed $j \in \mathbb{N}$ as in Corollary 4.9 , there exists a family of linear operators $\left\{\widetilde{D}_{k}\right\}_{k \in \mathbb{Z}}$ such that for any fixed $y_{\tau}^{k, v} \in Q_{\tau}^{k, v}$ with $k \in \mathbb{Z}, \tau \in I_{k}$, and $\mathcal{v}=1, \ldots, N(k, \tau)$, and all $f \in \mathcal{G}_{0}^{\epsilon}(\beta, \gamma)$ with $0<\beta, \gamma<\epsilon$,

$$
\begin{aligned}
f(x) & =\sum_{k=-\infty}^{\infty} \sum_{\tau \in I_{k}} \sum_{v=1}^{N(k, \tau)} \int_{Q_{\tau}^{k, v}} \tilde{D}_{k}(x, y) d \mu(y) D_{k}(f)\left(y_{\tau}^{k, v}\right) \\
& =\sum_{k=-\infty}^{\infty} \sum_{\tau \in I_{k}} \sum_{v=1}^{N(k, \tau)} \widetilde{D}_{k}\left(x, y_{\tau}^{k, v}\right) \int_{Q_{\tau}^{k, v}} D_{k}(f)(y) d \mu(y) \\
& =\sum_{k=-\infty}^{\infty} \sum_{\tau \in I_{k}} \sum_{v=1}^{N(k, \tau)} \mu\left(Q_{\tau}^{k, v}\right) \tilde{D}_{k}\left(x, y_{\tau}^{k, v}\right) D_{k}(f)\left(y_{\tau}^{k, v}\right),
\end{aligned}
$$

where the series converges in both the norm of $\mathcal{G}_{0}^{\epsilon}(\beta, \gamma)$ and the norm of $L^{p}(\mathcal{X})$ with $p \in(1, \infty)$. Moreover, $\tilde{D}_{k}$ satisfies the conditions as in Theorem 3.10.

Proof. We only prove the first formula in (4.57), the proof of the second formula in (4.57) being similar. Fix $N, j \in \mathbb{N}$ such that (4.1) and (4.39) hold. Thus, for such $N$ and $j$, Corollary 4.9 holds. Let $D_{k}^{N}$ for $k \in \mathbb{Z}$ be as in (3.44). For $k \in \mathbb{Z}$, let $\widetilde{D}_{k}(x, y)=S^{-1}\left[D_{k}^{N}(\cdot, y)\right](x)$. By $(4.2)$ and Corollary 4.9, similarly to the proof of Theorem 3.10, it is easy to see that we obtain all the conclusions of the theorem except for the convergence of the series in the first formula in (4.57). To prove this, we need to verify that all the series in the first summation and the second summation of the first formula in (4.57) converges in the desired ways. To simplify the presentation, by similarity, we prove this only for the series in the first summation of the first formula in (4.57).

Similarly to the proof of Theorem 3.10 , for $L \in \mathbb{N}$, we write

$$
\begin{aligned}
\sum_{|k| \leq L} & \sum_{\tau \in I_{k}} \sum_{\nu=1}^{N(k, \tau)} \int_{Q_{\tau}^{k, \nu}} \tilde{D}_{k}(x, y) d \mu(y) D_{k}(f)\left(y_{\tau}^{k, \nu}\right) \\
& =S^{-1}\left[\sum_{|k| \leq L} \sum_{\tau \in I_{k}} \sum_{\nu=1}^{N(k, \tau)} \int_{Q_{\tau}^{k, \nu}} D_{k}^{N}(\cdot, y) d \mu(y) D_{k}(f)\left(y_{\tau}^{k, \nu}\right)\right](x) \\
& =S^{-1}\left\{S(f)(\cdot)-\sum_{|k|>L} \sum_{\tau \in I_{k}} \sum_{\nu=1}^{N(k, \tau)} \int_{Q_{\tau}^{k, \nu}} D_{k}^{N}(\cdot, y) d \mu(y) D_{k}(f)\left(y_{\tau}^{k, \nu}\right)\right\}(x) \\
& =f(x)-\lim _{m \rightarrow \infty} R^{m}(f)(x)-S^{-1}\left\{\sum_{|k|>L} \sum_{\tau \in I_{k}} \sum_{\nu=1}^{N(k, \tau)} \int_{Q_{\tau}^{k, \nu}} D_{k}^{N}(\cdot, y) d \mu(y) D_{k}(f)\left(y_{\tau}^{k, v}\right)\right\}(x) .
\end{aligned}
$$


Corollary 2.24 together with Lemmas 4.6 and 4.7 shows that for all $f \in \mathcal{G}_{0}^{\epsilon}(\beta, \gamma)$ with $0<\beta$, $r<\epsilon$,

$$
\lim _{m \rightarrow \infty}\left\|R^{m}(f)\right\|_{\mathcal{C}_{0}^{e}(\beta, \gamma)} \leq \lim _{m \rightarrow \infty} C_{11}^{m}\left(C_{10} 2^{-\delta N}+C_{N} 2^{-j e}\right)^{m}\|f\|_{\mathcal{L}_{0}^{e}(\beta, \gamma)}=0,
$$

and for all $f \in L^{p}(\mathcal{X})$ with $p \in(1, \infty)$,

$$
\lim _{m \rightarrow \infty}\left\|R^{m}(f)\right\|_{L^{p}(\mathcal{X})} \leq \lim _{m \rightarrow \infty} C_{11}^{m}\left(C_{10} 2^{-\delta N}+C_{N} 2^{-j \epsilon}\right)^{m}\|f\|_{L^{p}(\mathcal{X})}=0 .
$$

To finish the proof of the theorem, we still need to verify that for all $f \in \mathcal{G}_{0}^{\epsilon}(\beta, \gamma)$ with $0<$ $\beta, \gamma<\epsilon$,

$$
\lim _{L \rightarrow \infty}\left\|S^{-1}\left\{\sum_{|k|>L} \sum_{\tau \in I_{k}} \sum_{\nu=1}^{N(k, \tau)} \int_{Q_{\tau}^{k, v}} D_{k}^{N}(\cdot, y) d \mu(y) D_{k}(f)\left(y_{\tau}^{k, v}\right)\right\}\right\|_{\mathcal{L}_{0}^{e}(\beta, \gamma)}=0,
$$

and for all $f \in L^{p}(\mathcal{X})$ with $p \in(1, \infty)$,

$$
\lim _{L \rightarrow \infty}\left\|S^{-1}\left\{\sum_{|k|>L} \sum_{\tau \in I_{k}} \sum_{\nu=1}^{N(k, \tau)} \int_{Q_{\tau}^{k, \nu}} D_{k}^{N}(\cdot, y) d \mu(y) D_{k}(f)\left(y_{\tau}^{k, \nu}\right)\right\}\right\|_{L^{p}(\mathcal{X})}=0 .
$$

We first verify (4.61). To this end, letting $\widetilde{\epsilon}=\epsilon_{1} \wedge \epsilon_{2}$, similarly to the proof of Theorem 3.10, by Corollary 4.9, it suffices to prove that there exists some $\sigma>0$ such that for all $0<\beta<\beta^{\prime}<$ $\widetilde{\epsilon}, 0<\gamma<\gamma^{\prime}<\widetilde{\epsilon}$, all $L \in \mathbb{N}$, and all $f \in \mathcal{G}_{(}\left(\beta^{\prime}, \gamma^{\prime}\right)$,

$$
\left\|\sum_{|k|>L} \sum_{\tau \in I_{k}} \sum_{\nu=1}^{N(k, \tau)} \int_{Q_{\tau}^{k, \nu}} D_{k}^{N}(\cdot, y) d \mu(y) D_{k}(f)\left(y_{\tau}^{k, \nu}\right)\right\|_{\mathcal{G}(\beta, \gamma)} \leq C 2^{-\sigma L}\|f\|_{\mathcal{G}\left(\beta^{\prime}, \gamma^{\prime}\right)},
$$

where $C>0$ is independent of $L$ and $f$. An argument similar to the proof of (3.3), via Lemma 2.1(iii) and geometric mean, reduces the proof of this estimate to verifying the following two estimates that there exists some $\sigma>0$ such that for all $f \in \mathcal{G}\left(\beta^{\prime}, \gamma^{\prime}\right)$ and 
all $x \in \mathcal{X}$

$$
\begin{aligned}
\mid \sum_{|k|>L} & \sum_{\tau \in I_{k}} \sum_{\nu=1}^{N(k, \tau)} \int_{Q_{\tau}^{k, \nu}} D_{k}^{N}(x, y) d \mu(y) D_{k}(f)\left(y_{\tau}^{k, v}\right) \mid \\
& \lesssim 2^{-\sigma L}\|f\|_{\mathcal{G}\left(\beta^{\prime}, \gamma^{\prime}\right)} \frac{1}{V_{1}\left(x_{1}\right)+V\left(x_{1}, x\right)} \frac{1}{\left(1+d\left(x, x_{1}\right)\right)^{\gamma}}
\end{aligned}
$$

and for all $x, x^{\prime} \in \mathcal{X}$ with $d\left(x, x^{\prime}\right) \leq(1 / 2)\left(1+d\left(x, x_{1}\right)\right)$,

$$
\begin{aligned}
& \mid \sum_{|k|>L} \sum_{\tau \in I_{k}} \sum_{\nu=1}^{N(k, \tau)} \int_{Q_{\tau}^{k, v}} D_{k}^{N}(x, y) d \mu(y) D_{k}(f)\left(y_{\tau}^{k, v}\right) \\
& \quad-\sum_{|k|>L} \sum_{\tau \in I_{k}} \sum_{\nu=1}^{N(k, \tau)} \int_{Q_{\tau}^{k, v}} D_{k}^{N}\left(x^{\prime}, y\right) d \mu(y) D_{k}(f)\left(y_{\tau}^{k, v}\right) \mid \\
& \lesssim\|f\|_{\mathcal{G}\left(\beta^{\prime}, \gamma^{\prime}\right)} \frac{d\left(x, x^{\prime}\right)^{\beta^{\prime}}}{\left(1+d\left(x, x_{1}\right)\right)^{\beta^{\prime}}} \frac{1}{V_{1}\left(x_{1}\right)+V\left(x_{1}, x\right)} \frac{1}{\left(1+d\left(x, x_{1}\right)\right)^{\gamma^{\prime}}} .
\end{aligned}
$$

For $L \in \mathbb{N}$, let $T_{L}$ be the operator associated with the kernel

$$
K_{L}(x, y)=\sum_{|k|>L} \sum_{\tau \in I_{k}} \sum_{v=1}^{N(k, \tau)} \int_{Q_{\tau}^{k, v}} D_{k}^{N}(x, z) d \mu(z) D_{k}\left(y_{\tau}^{k, v}, y\right)
$$

with $x, y \in \mathcal{X}$. By an argument similar to the proof of Lemma 4.7 together with Lemmas 4.10 and 3.5, we know $T_{L}$ satisfies all the conditions of Corollary 2.22 with $\epsilon$ replaced by $\tilde{\epsilon}$ and $C_{T_{L}}+\left\|T_{L}\right\|_{L^{2}(x) \rightarrow L^{2}(x)} \lesssim 1$. Thus, Corollary 2.22 then shows that $T_{L}$ is bounded on $\mathcal{G}\left(\beta^{\prime}, \gamma^{\prime}\right)$ for any $0<\beta^{\prime}, \gamma^{\prime}<\tilde{\epsilon}$. In particular, (4.65) holds. Using Lemma 4.10 and an argument similar to the proof of (3.75) also gives (4.64). Thus, (4.61) holds.

We now prove (4.62). To this end, Corollary 4.9 shows that it suffices to verify that for all $f \in L^{p}(\mathcal{X})$ with $p \in(1, \infty), \lim _{L \rightarrow \infty}\left\|T_{L}(f)\right\|_{L^{p}(\mathcal{X})}=0$. By Theorem 3.10, for $f \in L^{p}(\mathcal{X})$ and $h \in L^{p^{\prime}}(\mathcal{X}), f=\sum_{l=-\infty}^{\infty} \widetilde{D}_{l} D_{l}(f)$ and $h=\sum_{l=-\infty}^{\infty} \widetilde{D}_{l} D_{l}(h)$, respectively, in $L^{p}(\mathcal{X})$ and $L^{p^{\prime}}(\mathcal{X})$, where $\widetilde{D}_{k}$ for $k \in \mathbb{Z}$ are as in Theorem 3.10. From Remark 3.3, it is easy to deduce that there exists $\epsilon_{1}^{\prime} \in\left(0, \epsilon_{1} \wedge \epsilon_{2}\right)$ such that for all $y \in Q_{\tau}^{k, v}$ and $z \in \mathcal{X}$,

$$
\begin{gathered}
\left|D_{k} \widetilde{D}_{l}\left(y_{\tau}^{k, v}, z\right)\right| \lesssim 2^{-|k-l| \epsilon_{1}^{\prime}} \frac{1}{V_{2^{-k}}(y)+V_{2^{-k}}(z)+V(y, z)} \frac{2^{-(k \wedge l) \epsilon_{1}^{\prime}}}{\left(2^{-(k \wedge l)}+d(y, z)\right)^{\epsilon_{1}^{\prime}}}, \\
\left|\left(D_{k}^{N}\right)^{t} \widetilde{D}_{l}(y, z)\right| \lesssim 2^{-|k-l| \epsilon_{1}^{\prime}} \frac{1}{V_{2^{-k}}(y)+V_{2^{-k}}(z)+V(y, z)} \frac{2^{-(k \wedge l) \epsilon_{1}^{\prime}}}{\left(2^{-(k \wedge l)}+d(y, z)\right)^{\epsilon_{1}^{\prime}}} .
\end{gathered}
$$


These estimates and Lemma 2.1(iv) together with Hölder's inequality, Lemma 3.14, and Proposition 3.15 yield that

$$
\begin{aligned}
\left\|T_{L}(f)\right\|_{L^{p}(x)} & =\sup _{\|h\|_{L^{p^{\prime}}(x)} \leq 1}\left|\left\langle T_{L}(f), h\right\rangle\right| \\
= & \sup _{\|h\|_{L^{p^{\prime}}(x)} \leq 1}\left|\sum_{|k|>L} \sum_{\tau \in I_{k}} \sum_{v=1}^{N(k, \tau)} \int_{Q_{\tau}^{k, \nu}} D_{k}(f)\left(y_{\tau}^{k, \nu}\right)\left(D_{k}^{N}\right)^{t}(h)(y) d \mu(y)\right| \\
& \lesssim \sup _{\|h\|_{L^{p^{\prime}}(x)} \leq 1}\left\|\left\{\sum_{|k|>L}\left[\sum_{l=-\infty}^{\infty} 2^{-|k-l| \epsilon_{1}^{\prime}} M\left(\left|D_{l}(f)\right|\right)\right]^{2}\right\}^{1 / 2}\right\|_{L^{p}(x)} \\
& \times\left\|\left\{\sum_{|k|>L}\left[\sum_{l=-\infty}^{\infty} 2^{-|k-l| \epsilon_{1}^{\prime}} M\left(\left|\left(D_{k}^{N}\right)^{t}(h)\right|\right)\right]^{2}\right\}^{1 / 2}\right\|_{L^{p^{\prime}}(\mathcal{x})} \\
& \lesssim 2^{-\epsilon_{1}^{\prime} L / 2}\left\|\left\{\sum_{|l|<L / 2}\left[M\left(\left|D_{l}(f)\right|\right)\right]^{2}\right\}^{1 / 2}\right\| \|_{L^{p}(\mathcal{x})} \\
& +\left\|\left\{\sum_{|l| \geq L / 2}\left[M\left(\left|D_{l}(f)\right|\right)\right]^{2}\right\}^{1 / 2}\right\| \\
& \longrightarrow 0,
\end{aligned}
$$

as $L \rightarrow \infty$. That is, (4.62) also holds, which completes the proof of Theorem 4.11.

By an argument similar to the proof of Theorem 4.11, we can establish the following variants of the discrete Calderon reproducing formulae (we omit the details).

Theorem 4.12. Let $\epsilon_{1} \in(0,1], \epsilon_{2}>0, \epsilon_{3}>0, \epsilon \in\left(0, \epsilon_{1} \wedge \epsilon_{2}\right)$, and let $\left\{S_{k}\right\}_{k=-\infty}^{\infty}$ be an $\left(\epsilon_{1}, \epsilon_{2}, \epsilon_{3}\right)$-ATI. Set $D_{k}=S_{k}-S_{k-1}$ for any $k \in \mathbb{Z}$. Then for any fixed $j \in \mathbb{N}$ as in Corollary 4.9, there exists a family of linear operators $\left\{\bar{D}_{k}\right\}_{k \in \mathbb{Z}}$ such that for any fixed $y_{\tau}^{k, v} \in Q_{\tau}^{k, v}$ with $k \in \mathbb{Z}, \tau \in I_{k}$, and $v=1, \ldots, N(k, \tau)$, and all $f \in \mathcal{G}_{0}^{\epsilon}(\beta, \gamma)$ with $0<\beta, \gamma<\epsilon$,

$$
\begin{aligned}
f(x) & =\sum_{k=-\infty}^{\infty} \sum_{\tau \in I_{k}} \sum_{v=1}^{N(k, \tau)} \int_{Q_{\tau}^{k, v}} D_{k}(x, y) d \mu(y) \bar{D}_{k}(f)\left(y_{\tau}^{k, \nu}\right) \\
& =\sum_{k=-\infty}^{\infty} \sum_{\tau \in I_{k}} \sum_{v=1}^{N(k, \tau)} D_{k}\left(x, y_{\tau}^{k, v}\right) \int_{Q_{\tau}^{k, v}} \bar{D}_{k}(f)(y) d \mu(y) \\
& =\sum_{k=-\infty}^{\infty} \sum_{\tau \in I_{k}} \sum_{v=1}^{N(k, \tau)} \mu\left(Q_{\tau}^{k, v}\right) D_{k}\left(x, y_{\tau}^{k, v}\right) \bar{D}_{k}(f)\left(y_{\tau}^{k, v}\right),
\end{aligned}
$$

where the series converges in both the norm of $\mathcal{G}_{0}^{\epsilon}(\beta, \gamma)$ and the norm of $L^{p}(\mathcal{X})$ with $p \in(1, \infty)$. Moreover, $\bar{D}_{k}$ satisfies the conditions as in Theorem 3.11. 
Theorems 4.11 and 4.12 in combination with a duality argument show that discrete Calderón reproducing formulae on spaces of distributions.

Theorem 4.13. Let all the notation be as in Theorems 4.11 and 4.12. Then for all $f \in\left(\mathcal{G}_{0}^{\epsilon}(\beta, \gamma)\right)^{\prime}$ with $0<\beta, \gamma<\epsilon,(4.57)$ and (4.69) hold in $\left(\mathcal{G}_{0}^{\epsilon}(\beta, \gamma)\right)^{\prime}$.

\subsection{Inhomogeneous discrete Calderón reproducing formulae}

Similarly to Subsection 3.2, we can establish the following inhomogeneous discrete Calderón reproducing formulae (we omit the details). Here, again, we have no restriction on $\operatorname{diam}(\mathcal{X})$.

Theorem 4.14. Let $\epsilon_{1} \in(0,1], \epsilon_{2}>0, \epsilon_{3}>0, \epsilon \in\left(0, \epsilon_{1} \wedge \epsilon_{2}\right)$, and let $\left\{S_{k}\right\}_{k \in \mathbb{Z}_{+}}$be an $\left(\epsilon_{1}, \epsilon_{2}, \epsilon_{3}\right)$-IATI. Set $D_{0}=S_{0}$ and $D_{k}=S_{k}-S_{k-1}$ for $k \in \mathbb{N}$. Then for any fixed $j, N \in \mathbb{N}$ such that (4.1) and (4.39) hold, there exists a family of functions $\left\{\widetilde{D}_{k}(x, y)\right\}_{k \in \mathbb{Z}_{+}}$such that for any fixed $y_{\tau}^{k, v} \in Q_{\tau}^{k, v}$ with $k \in \mathbb{N}, \tau \in I_{k}$ and $v=1, \ldots, N(k, \tau)$ and all $f \in \mathcal{G}_{0}^{\epsilon}(\beta, \gamma)$ with $0<\beta, \gamma<\epsilon$,

$$
\begin{aligned}
f(x)= & \sum_{k=0}^{N} \sum_{\tau \in I_{k}} \sum_{\nu=1}^{N(k, \tau)} \int_{Q_{\tau}^{k, v}} \widetilde{D}_{k}(x, y) d \mu(y) D_{\tau, 1}^{k, v}(f)+\sum_{k=N+1}^{\infty} \sum_{\tau \in I_{k}} \sum_{v=1}^{N(k, \tau)} \int_{Q_{\tau}^{k, \nu}} \widetilde{D}_{k}(x, y) d \mu(y) D_{k}(f)\left(y_{\tau}^{k, v}\right) \\
= & \sum_{\tau \in I_{0}} \sum_{v=1}^{N(0, \tau)} \int_{Q_{\tau}^{0, v}} \widetilde{D}_{0}(x, y) d \mu(y) D_{\tau, 1}^{0, v}(f)+\sum_{k=1}^{\infty} \sum_{\tau \in I_{k}} \sum_{v=1}^{N(k, \tau)} \mu\left(Q_{\tau}^{k, v}\right) \widetilde{D}_{k}\left(x, y_{\tau}^{k, v}\right) D_{\tau, 1}^{k, v}(f) \\
= & \sum_{\tau \in I_{0}} \sum_{v=1}^{N(0, \tau)} \int_{Q_{\tau}^{0, v}} \tilde{D}_{0}(x, y) d \mu(y) D_{\tau, 1}^{0, v}(f)+\sum_{k=1}^{N} \sum_{\tau \in I_{k}} \sum_{v=1}^{N(k, \tau)} \mu\left(Q_{\tau}^{k, v}\right) \widetilde{D}_{k}\left(x, y_{\tau}^{k, v}\right) D_{\tau, 1}^{k, v}(f) \\
& +\sum_{k=N+1}^{\infty} \sum_{\tau \in I_{k}} \sum_{v=1}^{N(k, \tau)} \mu\left(Q_{\tau}^{k, v}\right) \widetilde{D}_{k}\left(x, y_{\tau}^{k, v}\right) D_{k}(f)\left(y_{\tau}^{k, v}\right),
\end{aligned}
$$

where the series converges in both the norm of $\mathcal{G}_{0}^{\epsilon}(\beta, \gamma)$ and the norm of $L^{p}(\mathcal{X})$ with $p \in(1, \infty)$, and $D_{\tau, 1}^{k, v}$ for $k \in \mathbb{Z}_{+}, \tau \in I_{k}$, and $v=1, \ldots, N(k, \tau)$ is the corresponding integral operator with the kernel $D_{\tau, 1}^{k, v}(z)=\left(1 / \mu\left(Q_{\tau}^{k, v}\right)\right) \int_{Q_{\tau}^{k, v}} D_{k}(u, z) d \mu(u)$. Moreover, $\widetilde{D}_{k}$ for $k \geq N+1$ satisfies the conditions ( $\left.i\right)$ and (ii) of Definition 2.2 with $\epsilon_{1}$ and $\epsilon_{2}$ replaced by $\epsilon^{\prime} \in\left(\epsilon, \epsilon_{1} \wedge \epsilon_{2}\right)$; and there exists a constant $C>0$ depending on $\epsilon^{\prime}$ such that the function $\widetilde{D}_{k}(x, y)$ for $k=0,1, \ldots, N$ satisfies that

(i) for all $x, y \in X,\left|\widetilde{D}_{k}(x, y)\right| \leq C\left(\left(1 /\left(V_{1}(x)+V_{1}(y)+V(x, y)\right)\right)\left(1 /(1+d(x, y))^{\epsilon^{\prime}}\right)\right)$,

(ii) for all $x, x^{\prime}, y \in \mathcal{x}$ with $d\left(x, x^{\prime}\right) \leq(1+d(x, y)) / 2$,

$$
\begin{aligned}
& \left|\widetilde{D}_{k}(x, y)-\tilde{D}_{k}\left(x^{\prime}, y\right)\right| \\
& \quad \leq C\left(\frac{d\left(x, x^{\prime}\right)}{1+d(x, y)}\right)^{\epsilon^{\prime}} \frac{1}{V_{1}(x)+V_{1}(y)+V(x, y)} \frac{1}{(1+d(x, y))^{\prime^{\prime}}}
\end{aligned}
$$

$$
\text { and } \int_{x} \widetilde{D}_{k}(x, y) d \mu(x)=\int_{x} \widetilde{D}_{k}(x, y) d \mu(y)=1 \text { when } 0 \leq k \leq N ;=0 \text { when } k>N \text {. }
$$


Theorem 4.15. Let $\epsilon_{1} \in(0,1], \epsilon_{2}>0, \epsilon_{3}>0, \epsilon \in\left(0, \epsilon_{1} \wedge \epsilon_{2}\right)$, and let $\left\{S_{k}\right\}_{k \in \mathbb{Z}_{+}}$be an $\left(\epsilon_{1}, \epsilon_{2}, \epsilon_{3}\right)$-IATI. Set $D_{0}=S_{0}$ and $D_{k}=S_{k}-S_{k-1}$ for $k \in \mathbb{N}$. Then for any fixed $j, N \in \mathbb{N}$ such that (4.1) and (4.39) hold, there exists a family of functions $\left\{\bar{D}_{k}(x, y)\right\}_{k \in \mathbb{Z}_{+}}$such that for any fixed $y_{\tau}^{k, v} \in Q_{\tau}^{k, v}$ with $k \in \mathbb{N}, \tau \in I_{k}$, and $v=1, \ldots, N(k, \tau)$ and all $f \in \mathcal{G}_{0}^{\epsilon}(\beta, \gamma)$ with $0<\beta, \gamma<\epsilon$,

$$
\begin{aligned}
f(x)= & \sum_{k=0}^{N} \sum_{\tau \in I_{k}} \sum_{\nu=1}^{N(k, \tau)} \int_{Q_{\tau}^{k, v}} D_{k}(x, y) d \mu(y) \overline{D_{\tau, 1}^{k, v}}(f)+\sum_{k=N+1}^{\infty} \sum_{\tau \in I_{k}} \sum_{v=1}^{N(k, \tau)} \int_{Q_{\tau}^{k, v}} D_{k}(x, y) d \mu(y) \bar{D}_{k}(f)\left(y_{\tau}^{k, v}\right) \\
= & \sum_{\tau \in I_{0}} \sum_{v=1}^{N(0, \tau)} \int_{Q_{\tau}^{o, v}} D_{0}(x, y) d \mu(y) \overline{D_{\tau, 1}^{0, v}}(f)+\sum_{k=1}^{\infty} \sum_{\tau \in I_{k}} \sum_{v=1}^{N(k, \tau)} \mu\left(Q_{\tau}^{k, v}\right) D_{k}\left(x, y_{\tau}^{k, v}\right) \overline{D_{\tau, 1}^{k, v}}(f) \\
= & \sum_{\tau \in I_{0}} \sum_{\nu=1}^{N(0, \tau)} \int_{Q_{\tau}^{o, v}} D_{0}(x, y) d \mu(y) \overline{D_{\tau, 1}^{0, v}}(f)+\sum_{k=1}^{N} \sum_{\tau \in I_{k}} \sum_{v=1}^{N(k, \tau)} \mu\left(Q_{\tau}^{k, v}\right) D_{k}\left(x, y_{\tau}^{k, v}\right) \overline{D_{\tau, 1}^{k, v}}(f) \\
& +\sum_{k=N+1}^{\infty} \sum_{\tau \in I_{k}} \sum_{v=1}^{N(k, \tau)} \mu\left(Q_{\tau}^{k, v}\right) D_{k}\left(x, y_{\tau}^{k, v}\right) \bar{D}_{k}(f)\left(y_{\tau}^{k, v}\right),
\end{aligned}
$$

where the series converges in both the norm of $\mathcal{G}_{0}^{\epsilon}(\beta, \gamma)$ and the norm of $L^{p}(\mathcal{X})$ with $p \in(1, \infty)$, and $\overline{D_{\tau, 1}^{k, v}}$ for $k \in \mathbb{Z}_{+}, \tau \in I_{k}$, and $v=1, \ldots, N(k, \tau)$ is the corresponding integral operator with the kernel $\overline{D_{\tau, 1}^{k, v}}(z)$ as in (4.70) with $D_{k}$ replaced by $\bar{D}_{k}$. Moreover, $\bar{D}_{k}$ for $k \geq N+1$ satisfies the conditions (i) and (iii) of Definition 2.2 with $\epsilon_{1}$ and $\epsilon_{2}$ replaced by $\epsilon^{\prime} \in\left(\epsilon, \epsilon_{1} \wedge \epsilon_{2}\right)$; and there exists a constant $C>0$ depending on $\epsilon^{\prime}$ such that the function $\bar{D}_{k}(x, y)$ for $k=0,1, \ldots, N$ satisfies that

(i) for all $x, y \in X,\left|\bar{D}_{k}(x, y)\right| \leq C\left(\left(1 /\left(V_{1}(x)+V_{1}(y)+V(x, y)\right)\right)\left(1 /(1+d(x, y))^{\epsilon^{\prime}}\right)\right)$,

(ii) for all $x, x^{\prime}, y \in X$ with $d\left(x, x^{\prime}\right) \leq(1+d(x, y)) / 2$,

$$
\begin{aligned}
& \left|\bar{D}_{k}(x, y)-\bar{D}_{k}\left(x^{\prime}, y\right)\right| \\
& \leq C\left(\frac{d\left(x, x^{\prime}\right)}{1+d(x, y)}\right)^{\epsilon^{\prime}} \frac{1}{V_{1}(x)+V_{1}(y)+V(x, y)} \frac{1}{(1+d(x, y))^{\epsilon^{\prime}}} \\
& \text { and } \int_{x} \bar{D}_{k}(x, y) d \mu(x)=\int_{x} \bar{D}_{k}(x, y) d \mu(y)=1 \text { when } 0 \leq k \leq N ;=0 \text { when } k>N .
\end{aligned}
$$

Theorem 4.16. Use the same notation as in Theorems 4.14 and 4.15. Then for all $f \in\left(\mathcal{G}_{0}^{\epsilon}(\beta, \gamma)\right)^{\prime}$ with $0<\beta, \gamma<\epsilon,(4.70)$ and (4.72) hold in $\left(\mathcal{G}_{0}^{\epsilon}(\beta, \gamma)\right)^{\prime}$.

Remark 4.17. Similarly to Remark 3.27, in the sequel, to simplify the representation of the results, we will always assume that $N=0$ in Theorems 4.14, 4.15, and 4.16. 


\section{Besov spaces and Triebel-Lizorkin spaces}

In this section, we consider Besov and Triebel-Lizorkin spaces on RD-spaces and study their relations. As applications, we obtain boundedness results on these spaces for singular integrals considered by Nagel and Stein [44]. Finally, we establish a variant of the T(1)theorem of David and Journé in these settings, and a variant of the T(1)-theorem of Stein in [75] is also presented.

To develop a theory of these function spaces, we need two basic tools: the Calderón reproducing formulae from Sections 3 and 4 and Plancherel-Pôlya inequalities, which will be established in this section.

\subsection{Plancherel-Pôlya inequalities and definition of $\dot{B}_{p, q}^{s}(\mathcal{X})$ and $\dot{F}_{p, q}^{s}(X)$}

Throughout this and the next subsection, we will always assume that $\mu(\mathcal{X})=\infty$. We first introduce the norms in $\dot{B}_{p, q}^{s}(\mathcal{X})$ and $\dot{F}_{p, q}^{s}(\mathcal{X})$ via certain ATIs. We then prove that they are independent of the choices of ATIs and spaces of distributions. To this end, we need to establish homogeneous Plancherel-Pôlya inequalities; see also [79].

Adapting Triebel's approach to homogeneous Besov and Triebel-Lizorkin spaces on $\mathbb{R}^{n}$ in [3], we make the following.

Definition 5.1. Let $\epsilon_{1} \in(0,1], \epsilon_{2}>0, \epsilon_{3}>0, \epsilon \in\left(0, \epsilon_{1} \wedge \epsilon_{2}\right)$, and let $\left\{S_{k}\right\}_{k \in \mathbb{Z}}$ be an $\left(\epsilon_{1}, \epsilon_{2}, \epsilon_{3}\right)$ ATI. For $k \in \mathbb{Z}$, set $D_{k}=S_{k}-S_{k-1}$.

(i) For all $f \in\left(\mathcal{G}_{0}^{\epsilon}(\beta, \gamma)\right)^{\prime}$ with $0<\beta, \gamma<\epsilon,|s|<\epsilon, p(s, \epsilon)<p \leq \infty$, and $0<q \leq \infty$, one sets $\|f\|_{\dot{B}_{p, q}^{s}(\mathcal{X})} \equiv\left\{\sum_{k \in \mathbb{Z}} 2^{k s q}\left\|D_{k}(f)\right\|_{L^{p}(\mathcal{X})}^{q}\right\}^{1 / q}$ with the usual modification made when $p=\infty$ or $q=\infty$.

(ii) For all $f \in\left(\mathcal{G}_{0}^{\epsilon}(\beta, \gamma)\right)^{\prime}$ with $0<\beta, \gamma<\epsilon,|s|<\epsilon, p(s, \epsilon)<p<\infty$, and $p(s, \epsilon)<q \leq \infty$, one defines $\|f\|_{\dot{F}_{p, q}^{s}(x)} \equiv\left\|\left\{\sum_{k \in \mathbb{Z}} 2^{k s q}\left|D_{k}(f)\right|^{q}\right\}^{1 / q}\right\|_{L^{p}(x)}$ with the usual modification made when $q=\infty$.

To verify that the definitions of $\|\cdot\|_{\dot{B}_{p, q}^{s}(x)}$ and $\|\cdot\|_{\dot{F}_{p, q}^{s}}(x)$ are independent of the choice of ATIs, we need two technical lemmas, which have independent interest.

Lemma 5.2. Let $\epsilon>0, k^{\prime}, k \in \mathbb{Z}$, and $y_{\tau}^{k, v}$ be any point in $Q_{\tau}^{k, v}$ for $\tau \in I_{k}$ and $v=1, \ldots, N(k, \tau)$. If $n /(n+e)<p \leq \infty$, then for any $x \in \mathcal{X}$,

$$
\sum_{\tau \in I_{k}} \sum_{\nu=1}^{N(k, \tau)} \mu\left(Q_{\tau}^{k, v}\right)\left[\frac{1}{V_{2^{-\left(k^{\prime} \wedge k\right)}}(x)+V\left(x, y_{\tau}^{k, \nu}\right)}\right]^{(p \wedge 1)}\left[\frac{2^{-\left(k \wedge k^{\prime}\right)}}{2^{-\left(k \wedge k^{\prime}\right)}+d\left(x, y_{\tau}^{k, v}\right)}\right]^{\epsilon(p \wedge 1)} \leq C\left[V_{2^{-\left(k^{\prime} \wedge k\right)}}(x)\right]^{1-(p \wedge 1)},
$$

where $C>0$ is independent of $x \in \mathcal{X}, k, k^{\prime}, \tau$, and $v$. 
Proof. Notice that for any $z \in Q_{\tau}^{k, v}$, by Lemma 2.1(iii), we have

$$
\begin{gathered}
V_{2^{-\left(k^{\prime} \wedge k\right)}}\left(y_{\tau}^{k, v}\right)+V\left(x, y_{\tau}^{k, v}\right) \sim V_{2^{-\left(k^{\prime} \wedge k\right)}}(z)+V(x, z), \\
2^{-\left(k \wedge k^{\prime}\right)}+d\left(x, y_{\tau}^{k, v}\right) \sim 2^{-\left(k \wedge k^{\prime}\right)}+d(x, z) .
\end{gathered}
$$

These estimates together with Lemma 2.19 and the second inequality in (1.3) yield that the left-hand side of (5.1) is, up to a bounded multiplicative constant, controlled by

$$
\begin{aligned}
\int_{x}[ & \left.\frac{1}{V_{2^{-\left(k^{\prime} \wedge k\right)}}(x)+V(x, z)}\right]^{(p \wedge 1)}\left[\frac{2^{-\left(k \wedge k^{\prime}\right)}}{2^{-\left(k \wedge k^{\prime}\right)}+d(x, z)}\right]^{\epsilon(p \wedge 1)} d \mu(z) \\
& \lesssim\left[V_{2^{-\left(k^{\prime} \wedge k\right)}}(x)\right]^{1-(p \wedge 1)} \sum_{l=0}^{\infty} 2^{l[n-n(p \wedge 1)-\epsilon(p \wedge 1)]} \\
& \lesssim\left[V_{2^{-\left(k^{\prime} \wedge k\right)}}(x)\right]^{1-(p \wedge 1)},
\end{aligned}
$$

which completes the proof of Lemma 5.2.

Lemma 5.3. Let $\epsilon>0, k^{\prime}, k \in \mathbb{Z}$, and $y_{\tau}^{k, v}$ be any point in $Q_{\tau}^{k, v}$ for $\tau \in I_{k}$ and $v=1, \ldots, N(k, \tau)$. If $n /(n+\epsilon)<r \leq 1$, then there exists a constant $C>0$ depending on $r$ such that for all $a_{\tau}^{k, v} \in \mathbb{C}$ and all $x \in \mathcal{X}$,

$$
\begin{gathered}
\sum_{\tau \in I_{k}} \sum_{\nu=1}^{N(k, \tau)} \mu\left(Q_{\tau}^{k, v}\right) \frac{1}{V_{2^{-\left(k^{\prime} \wedge k\right)}}(x)+V\left(x, y_{\tau}^{k, \nu}\right)} \frac{2^{-\left(k \wedge k^{\prime}\right) \epsilon}}{\left(2^{-\left(k \wedge k^{\prime}\right)}+d\left(x, y_{\tau}^{k, \nu}\right)\right)^{\epsilon}}\left|a_{\tau}^{k, v}\right| \\
\leq C 2^{\left[\left(k \wedge k^{\prime}\right)-k\right] n(1-1 / r)}\left\{M\left(\sum_{\tau \in I_{k}} \sum_{\nu=1}^{N(k, \tau)}\left|a_{\tau}^{k, v}\right|^{r} X_{Q_{\tau}^{k, v}}\right)(x)\right\}^{1 / r},
\end{gathered}
$$

where $C>0$ is also independent of $k, k^{\prime}, \tau$, and $v$.

Proof. We first recall the following well-known inequality that for all $r \in(0,1]$ and all $a_{j} \in \mathbb{C}$ with $j$ in some countable set of indices,

$$
\left(\sum_{j}\left|a_{j}\right|\right)^{r} \leq \sum_{j}\left|a_{j}\right|^{r} .
$$

From this inequality, the fact that for all $\tau \in I_{k}$ and $v=1, \ldots, N(k, \tau)$, and all $z \in \mathcal{X}$, $\mu\left(Q_{\tau}^{k, v}\right) \chi_{Q_{\tau}^{k, v}}(z) \sim V_{2^{-k}}(z) \chi_{Q_{\tau}^{k, v}}(z)$, and the fact that for all $z \in \mathcal{X}$ and all $k, k^{\prime} \in \mathbb{Z}$, by (1.3), $V_{2^{-\left(k \wedge k^{\prime}\right)}}(z) \lesssim 2^{\left[k-\left(k \wedge k^{\prime}\right)\right] n} V_{2^{-k}}(z)$, together with (5.2), Lemma 2.1(vi), and the second inequality of (1.3), it follows that the left-hand side of (5.4) is, up to a bounded multiplicative constant, 
controlled by

$$
\begin{aligned}
& \left\{\sum_{\tau \in I_{k}} \sum_{\nu=1}^{N(k, \tau)} \mu\left(Q_{\tau}^{k, \nu}\right)^{r}\left(\frac{1}{V_{2^{-\left(k^{\prime} \wedge k\right)}}(x)+V\left(x, y_{\tau}^{k, v}\right)}\right)^{r} \frac{2^{-\left(k \wedge k^{\prime}\right) r \epsilon}}{\left(2^{-\left(k \wedge k^{\prime}\right)}+d\left(x, y_{\tau}^{k, v}\right)\right)^{r \epsilon}}\left|a_{\tau}^{k, v}\right|^{r}\right\}^{1 / r} \\
& \lesssim 2^{\left[\left(k \wedge k^{\prime}\right)-k\right] n(1-1 / r)}\left\{\frac{1}{V_{2^{-\left(k^{\prime} \wedge k\right)}}(x)} \int_{d(x, z)<2^{-\left(k^{\prime} \wedge k\right)}}\left(\sum_{\tau \in I_{k}} \sum_{\nu=1}^{N(k, \tau)}\left|a_{\tau}^{k, v}\right|^{r} \chi_{\mathrm{Q}_{\tau}^{k_{\tau}, v}}(z)\right) d \mu(z)\right. \\
& +\sum_{l=0}^{\infty} \frac{1}{2^{l[r \epsilon+n(r-1)]}} \frac{1}{V_{2^{l+1} 2^{-\left(k^{\prime} \wedge k\right)}(x)}} \\
& \left.\times \int_{d(x, z)<2^{l+1} 2^{-\left(k^{\prime} \wedge k\right)}}\left(\sum_{\tau \in I_{k}} \sum_{\nu=1}^{N(k, \tau)}\left|a_{\tau}^{k, v}\right|^{r} X_{Q_{\tau}^{k, v}}(z)\right) d \mu(z)\right\}^{1 / r},
\end{aligned}
$$

which implies the desired conclusion.

Using these technical lemmas and the discrete Calderón reproducing formulae, we can now establish the Plancherel-Pôlya inequality.

Proposition 5.4. Let $\epsilon_{1} \in(0,1], \epsilon_{2}>0, \epsilon_{3}>0, \epsilon \in\left(0, \epsilon_{1} \wedge \epsilon_{2}\right)$, and let $\left\{S_{k}\right\}_{k \in \mathbb{Z}}$ and $\left\{P_{k}\right\}_{k \in \mathbb{Z}}$ be two $\left(\epsilon_{1}, \epsilon_{2}, \epsilon_{3}\right)$-ATIs. For $k \in \mathbb{Z}$, set $D_{k}=S_{k}-S_{k-1}$ and $Q_{k}=P_{k}-P_{k-1}$.

(i) For all $f \in\left(\mathcal{G}_{0}^{\epsilon}(\beta, \gamma)\right)^{\prime}$ with $0<\beta, \gamma<\epsilon,|s|<\epsilon, p(s, \epsilon)<p \leq \infty$, and $0<q \leq \infty$,

$$
\begin{gathered}
\left\{\sum_{k \in \mathbb{Z}} 2^{k s q}\left(\sum_{\tau \in I_{k}} \sum_{v=1}^{N(k, \tau)} \mu\left(Q_{\tau}^{k, v}\right)\left[\sup _{z \in Q_{\tau}^{k, v}}\left|D_{k}(f)(z)\right|\right]^{p}\right)^{q / p}\right\}^{1 / q} \\
\sim\left\{\sum_{k \in \mathbb{Z}} 2^{k s q}\left(\sum_{\tau \in I_{k}} \sum_{v=1}^{N(k, \tau)} \mu\left(Q_{\tau}^{k, v}\right)\left[\inf _{z \in Q_{\tau}^{k, \nu}}\left|Q_{k}(f)(z)\right|\right]^{p}\right)^{q / p}\right\}^{1 / q} .
\end{gathered}
$$

(ii) For all $f \in\left(\mathcal{G}_{0}^{\epsilon}(\beta, \gamma)\right)^{\prime}$ with $0<\beta, \gamma<\epsilon,|s|<\epsilon, p(s, \epsilon)<p<\infty$, and $p(s, \epsilon)<q \leq \infty$,

$$
\begin{gathered}
\left\|\left\{\sum_{k \in \mathbb{Z}} \sum_{\tau \in I_{k}} \sum_{\nu=1}^{N(k, \tau)} 2^{k s q}\left[\sup _{z \in Q_{\tau}^{k, \nu}}\left|D_{k}(f)(z)\right|\right]^{q} x_{Q_{\tau}^{k, \nu}}\right\}^{1 / q}\right\|_{L^{p}(\mathcal{X})} \\
\sim\left\|\left\{\sum_{k \in \mathbb{Z}} \sum_{\tau \in I_{k}} \sum_{\nu=1}^{N(k, \tau)} 2^{k s q}\left[\inf _{z \in Q_{\tau}^{k, \nu}}\left|Q_{k}(f)(z)\right|\right]^{q} X_{Q_{\tau}^{k_{\nu}, \nu}}\right\}^{1 / q}\right\|_{L^{p}(\mathcal{x})} .
\end{gathered}
$$

Proof. We first verify (5.7). By Theorem 4.13, there exist functions $\left\{\widetilde{D}_{k}\right\}_{k \in \mathbb{Z}}$ such that for all $f \in\left(\mathcal{G}_{0}^{\epsilon}(\beta, \gamma)\right)^{\prime}$ with $0<\beta, \gamma<\epsilon$ and all $z \in \mathcal{X}$,

$$
f(z)=\sum_{k^{\prime} \in \mathbb{Z}} \sum_{\tau^{\prime} \in I_{k^{\prime}}} \sum_{v^{\prime}=1}^{N\left(k^{\prime}, \tau^{\prime}\right)} \mu\left(Q_{\tau^{\prime}}^{k^{\prime}, v^{\prime}}\right) \widetilde{D}_{k^{\prime}}\left(z, y_{\tau^{\prime}}^{k^{\prime}, v^{\prime}}\right) Q_{k^{\prime}}(f)\left(y_{\tau^{\prime}}^{k^{\prime}, v^{\prime}}\right)
$$


holds in $\left(\mathcal{G}_{0}^{\epsilon}(\beta, \gamma)\right)^{\prime}$, where $\widetilde{D}_{k^{\prime}}$ satisfies the same conditions as $\widetilde{D}_{k}$ in Theorem 4.13 and $y_{\tau^{\prime}}^{k^{\prime}, \nu^{\prime}}$ is any point in $Q_{\tau^{\prime}}^{k^{\prime}, v^{\prime}}$. For any $\epsilon^{\prime} \in(0, \epsilon)$, by Lemma 3.2 (see also Remark 3.3), for all $z \in \mathcal{X}$,

$$
\begin{aligned}
& \left|\left(D_{k} \tilde{D}_{k^{\prime}}\right)\left(z, y_{\tau^{\prime}}^{k^{\prime}, v^{\prime}}\right)\right| \\
& \quad \lesssim 2^{-\left|k-k^{\prime}\right| \epsilon^{\prime}} \frac{1}{V_{2^{-\left(k \wedge k^{\prime}\right)}}(z)+V_{2^{-\left(k \wedge k^{\prime}\right)}}\left(y_{\tau^{\prime}}^{k^{\prime}, v^{\prime}}\right)+V\left(z, y_{\tau^{\prime}}^{k^{\prime}, v^{\prime}}\right)} \frac{2^{-\left(k \wedge k^{\prime}\right) \epsilon}}{\left(2^{-\left(k \wedge k^{\prime}\right)}+d\left(z, y_{\tau^{\prime}}^{k^{\prime}, v^{\prime}}\right)\right)} .
\end{aligned}
$$

If $p(s, \epsilon)<p \leq 1$, by applying $D_{k}$ to (5.9), and making use of (5.10), (5.5), and (5.2) together with Lemma 5.2, we obtain

$$
\begin{aligned}
\sum_{\tau \in I_{k}} & \sum_{v=1}^{N(k, \tau)} \mu\left(Q_{\tau}^{k, v}\right)\left[\sup _{z \in Q_{\tau}^{k, v}}\left|D_{k}(f)(z)\right|\right]^{p} \\
& \lesssim \sum_{k^{\prime} \in \mathbb{Z}} \sum_{\tau^{\prime} \in I_{k^{\prime}}} \sum_{v^{\prime}=1}^{N\left(k^{\prime}, \tau^{\prime}\right)} 2^{-\left|k-k^{\prime}\right| \epsilon^{\prime} p} \mu\left(Q_{\tau^{\prime}}^{k^{\prime}, v^{\prime}}\right)^{p}\left[V_{2^{-\left(k \wedge k^{\prime}\right)}}\left(y_{\tau^{\prime}}^{k^{\prime}, v^{\prime}}\right)\right]^{1-p}\left|Q_{k^{\prime}}(f)\left(y_{\tau^{\prime}}^{k^{\prime}, v^{\prime}}\right)\right|^{p}
\end{aligned}
$$

From this and the fact that

$$
V_{2^{-\left(k \wedge k^{\prime}\right)}}\left(y_{\tau^{\prime}}^{k^{\prime}, v^{\prime}}\right) \lesssim 2^{\left[k^{\prime}-\left(k \wedge k^{\prime}\right)\right] n} V_{2^{-k^{\prime}}}\left(y_{\tau^{\prime}}^{k^{\prime}, v^{\prime}}\right) \sim 2^{\left[k^{\prime}-\left(k \wedge k^{\prime}\right)\right] n} \mu\left(Q_{\tau^{\prime}}^{k^{\prime}, v^{\prime}}\right)
$$

together with Hölder's inequality when $q / p \geq 1$ and (5.5) when $q / p<1$, it follows that if we choose $\epsilon^{\prime} \in(0, \epsilon)$ such that $\max \left\{n /\left(n+\epsilon^{\prime}\right), n /\left(n+\epsilon^{\prime}+s\right)\right\}<p \leq 1$ and $|s|<\epsilon^{\prime}$, the left-hand side of (5.7) is, up to a bounded multiplicative constant, dominated by

$$
\begin{aligned}
\left\{\sum_{k \in \mathbb{Z}}\right. & {\left.\left[\sum_{k^{\prime} \in \mathbb{Z}} \sum_{\tau^{\prime} \in I_{k^{\prime}}} \sum_{v^{\prime}=1}^{N\left(k^{\prime}, \tau^{\prime}\right)} 2^{-\left|k-k^{\prime}\right| \epsilon^{\prime} p} 2^{\left(k-k^{\prime}\right) s p} \mu\left(Q_{\tau^{\prime}}^{k^{\prime}, v^{\prime}}\right)^{p}\left[V_{2^{-\left(k N k^{\prime}\right)}}\left(y_{\tau^{\prime}}^{k^{\prime}, v^{\prime}}\right)\right]^{1-p} 2^{k^{\prime} s p}\left|Q_{k^{\prime}}(f)\left(y_{\tau^{\prime}}^{k^{\prime}, v^{\prime}}\right)\right|^{p}\right]^{q / p}\right\}^{1 / q} } \\
& \lesssim\left\{\sum_{k^{\prime} \in \mathbb{Z}} 2^{k^{\prime} s q}\left(\sum_{\tau^{\prime} \in I_{k^{\prime}}} \sum_{v^{\prime}=1}^{N\left(k^{\prime}, \tau^{\prime}\right)} \mu\left(Q_{\tau^{\prime}}^{k^{\prime}, v^{\prime}}\right)\left|Q_{k^{\prime}}(f)\left(y_{\tau^{\prime}}^{k^{\prime}, v^{\prime}}\right)\right|^{p}\right)^{q / p}\right\}^{1 / q},
\end{aligned}
$$

which together with the arbitrary choice of $y_{\tau^{\prime}}^{k^{\prime}, v^{\prime}}$ yields

$$
\begin{gathered}
\left\{\sum_{k \in \mathbb{Z}} 2^{k s q}\left(\sum_{\tau \in I_{k}} \sum_{v=1}^{N(k, \tau)} \mu\left(Q_{\tau}^{k, v}\right)\left[\sup _{z \in Q_{\tau}^{k, v}}\left|D_{k}(f)(z)\right|\right]^{p}\right)^{q / p}\right\}^{1 / q} \\
\lesssim\left\{\sum_{k \in \mathbb{Z}} 2^{k s q}\left(\sum_{\tau \in I_{k}} \sum_{\nu=1}^{N(k, \tau)} \mu\left(Q_{\tau}^{k, v}\right)\left[\inf _{z \in Q_{\tau}^{k, v}}\left|Q_{k}(f)(z)\right|\right]^{p}\right)^{q / p}\right\}^{1 / q} .
\end{gathered}
$$

By symmetry, we then obtain (5.7) when $p(s, \epsilon)<p \leq 1$. 
If $p \in(1, \infty]$ and if we choose $\epsilon^{\prime} \in(0, \epsilon)$ such that $|s|<\epsilon^{\prime}$, by Hölder's inequality and Lemma 5.2, we obtain

$$
\begin{aligned}
\sum_{\tau \in I_{k}} & \sum_{v=1}^{N(k, \tau)} \mu\left(Q_{\tau}^{k, v}\right)\left[\sup _{z \in Q_{\tau}^{k, v}}\left|D_{k}(f)(z)\right|\right]^{p} \\
& \lesssim \sum_{k^{\prime} \in \mathbb{Z}} \sum_{\tau^{\prime} \in I_{k^{\prime}}} \sum_{v^{\prime}=1}^{N\left(k^{\prime}, \tau^{\prime}\right)} 2^{-\left|k-k^{\prime}\right| \epsilon^{\prime}} 2^{\left(k^{\prime}-k\right) s(p-1)} \mu\left(Q_{\tau^{\prime}}^{k^{\prime}, v^{\prime}}\right)\left|Q_{k^{\prime}}(f)\left(y_{\tau^{\prime}}^{k^{\prime}, v^{\prime}}\right)\right|^{p}
\end{aligned}
$$

which together with Hölder's inequality when $q / p \geq 1$ and (5.5) when $q / p<1$ yields that the left-hand side of (5.7) is, up to a bounded multiplicative constant, controlled by

$$
\begin{gathered}
\left\{\sum_{k \in \mathbb{Z}}\left[\sum_{k^{\prime} \in \mathbb{Z}} \sum_{\tau^{\prime} \in I_{k^{\prime}}} \sum_{v^{\prime}=1}^{N\left(k^{\prime}, \tau^{\prime}\right)} 2^{-\left|k-k^{\prime}\right| \epsilon^{\prime}} 2^{\left(k-k^{\prime}\right) s} \mu\left(Q_{\tau^{\prime}}^{k^{\prime}, v^{\prime}}\right) 2^{k^{\prime} s p}\left|Q_{k^{\prime}}(f)\left(y_{\tau^{\prime}}^{k^{\prime}, v^{\prime}}\right)\right|^{p}\right]^{q / p}\right\}^{1 / q} \\
\lesssim\left\{\sum_{k^{\prime} \in \mathbb{Z}} 2^{k^{\prime} s q}\left(\sum_{\tau^{\prime} \in I_{k^{\prime}}} \sum_{v^{\prime}=1}^{N\left(k^{\prime}, \tau^{\prime}\right)} \mu\left(Q_{\tau^{\prime}}^{k^{\prime}, v^{\prime}}\right)\left|Q_{k^{\prime}}(f)\left(y_{\tau^{\prime}}^{k^{\prime}, v^{\prime}}\right)\right|^{p}\right)^{q / p}\right\}^{1 / q} .
\end{gathered}
$$

Then, since $y_{\tau^{\prime}}^{k^{\prime}, v^{\prime}}$ was an arbitrary point in $Q_{\tau^{\prime}}^{k^{\prime}, v^{\prime}}$, we see that the estimate (5.14) also holds when $p \in(1, \infty]$, which by symmetry then completes the proof of (5.7).

We now verify (5.8). By applying $D_{k}$ to (5.9), and making use of (5.10) together with (5.2), and Lemma 5.3, we obtain that for $\epsilon^{\prime}>|s|$ and $r>\max \left\{p, p\left(s, \epsilon^{\prime}\right), q\right\}$,

$$
\begin{aligned}
\left\{\sum_{k \in \mathbb{Z}} \sum_{\tau \in I_{k}} \sum_{v=1}^{N(k, \tau)} 2^{k s q}\left[\sup _{z \in Q_{\tau}^{k, v}}\left|D_{k}(f)(z)\right|\right]^{q} X_{Q_{\tau}^{k, v}}(x)\right\}^{1 / q} \\
\lesssim\left\{\sum _ { k \in \mathbb { Z } } \left[\sum_{k^{\prime} \in \mathbb{Z}} 2^{\left(k-k^{\prime}\right) s-\left|k-k^{\prime}\right| \epsilon^{\prime}} 2^{\left[\left(k \wedge k^{\prime}\right)-k^{\prime}\right] n(1-1 / r)}\right.\right. \\
\left.\left.\times\left\{M\left(\sum_{\tau^{\prime} \in I_{k^{\prime}}} \sum_{\nu^{\prime}=1}^{N\left(k^{\prime}, \tau^{\prime}\right)} 2^{k^{\prime} s r}\left|Q_{k^{\prime}}(f)\left(y_{\tau^{\prime}}^{k^{\prime}, v^{\prime}}\right)\right|^{r} X_{Q_{\tau^{\prime}}^{k^{\prime}, \nu^{\prime}}}\right)(x)\right\}^{1 / r}\right]^{q}\right\}^{1 / q},
\end{aligned}
$$

which together with Hölder's inequality when $q \in(1, \infty]$ or (5.5) when $q \leq 1$, and Lemma 3.14 further implies that the left-hand side of (5.8) is, up to a bounded multiplicative constant, dominated by

$$
\begin{gathered}
\left\|\left\{\sum_{k^{\prime} \in \mathbb{Z}}\left[M\left(\sum_{\tau^{\prime} \in I_{k^{\prime}}} \sum_{v^{\prime}=1}^{N\left(k^{\prime}, \tau^{\prime}\right)} 2^{k^{\prime} s r}\left|Q_{k^{\prime}}(f)\left(y_{\tau^{\prime}}^{k^{\prime}, v^{\prime}}\right)\right|^{r} X_{Q_{\tau^{\prime}}^{k^{\prime}, v^{\prime}}}\right)\right]^{q / r}\right\}^{r / q}\right\|_{L^{p / r}(x)}^{1 / r} \\
\lesssim\left\|\left\{\sum_{k^{\prime} \in \mathbb{Z}} \sum_{\tau^{\prime} \in I_{k^{\prime}}} \sum_{v^{\prime}=1}^{N\left(k^{\prime}, \tau^{\prime}\right)} 2^{k^{\prime} s q}\left|Q_{k^{\prime}}(f)\left(y_{\tau^{\prime}}^{k^{\prime}, v^{\prime}}\right)\right|^{q} X_{Q_{\tau^{\prime}}^{k^{\prime}, v^{\prime}}}\right\}^{1 / q}\right\|_{L^{p}(x)} .
\end{gathered}
$$


Then, by symmetry and the fact that $y_{\tau^{\prime}}^{k^{\prime}, v^{\prime}}$ was an arbitrary point in $Q_{\tau^{\prime}}^{k^{\prime}, v^{\prime}}$, we obtain (5.8), which completes the proof of Proposition 5.4.

The following remark is useful in applications.

Remark 5.5. Let all the notation be as in Proposition 5.4, except that $S_{k}$ (and therefore $D_{k}$ ) for $k \in \mathbb{Z}$ has regularity only in the second variable. Then, there exists a constant $C>0$ such that

(i) for all $f \in\left(\mathcal{G}_{0}^{\epsilon}(\beta, \gamma)\right)^{\prime}$ with $0<\beta, \gamma<\epsilon,|s|<\epsilon, p(s, \epsilon)<p \leq \infty$, and $0<q \leq \infty$,

$$
\begin{aligned}
& \left\{\sum_{k \in \mathbb{Z}} 2^{k s q}\left(\sum_{\tau \in I_{k}} \sum_{v=1}^{N(k, \tau)} \mu\left(Q_{\tau}^{k, v}\right)\left[\sup _{z \in Q_{\tau}^{k, v}}\left|D_{k}(f)(z)\right|\right]^{p}\right)^{q / p}\right\}^{1 / q} \\
& \leq C\left\{\sum_{k \in \mathbb{Z}} 2^{k s q}\left(\sum_{\tau \in I_{k}} \sum_{v=1}^{N(k, \tau)} \mu\left(Q_{\tau}^{k, \nu}\right)\left[\inf _{z \in Q_{\tau}^{k, v}}\left|Q_{k}(f)(z)\right|\right]^{p}\right)^{q / p}\right\}^{1 / q} ;
\end{aligned}
$$

(ii) for all $f \in\left(\mathcal{G}_{0}^{\epsilon}(\beta, \gamma)\right)^{\prime}$ with $0<\beta, \gamma<\epsilon,|s|<\epsilon, p(s, \epsilon)<p<\infty$, and $p(s, \epsilon)<q \leq \infty$,

$$
\begin{gathered}
\left\|\left\{\sum_{k \in \mathbb{Z}} \sum_{\tau \in I_{k}} \sum_{\nu=1}^{N(k, \tau)} 2^{k s q}\left[\sup _{z \in Q_{\tau}^{k, \nu}}\left|D_{k}(f)(z)\right|\right]^{q} X_{Q_{\tau}^{k, v}}\right\}^{1 / q}\right\|_{L^{p}(\mathcal{X})} \\
\leq C\left\|\left\{\sum_{k \in \mathbb{Z}} \sum_{\tau \in I_{k}} \sum_{\nu=1}^{N(k, \tau)} 2^{k s q}\left[\inf _{z \in Q_{\tau}^{k, \nu}}\left|Q_{k}(f)(z)\right|\right]^{q} X_{Q_{\tau}^{k, \nu}}\right\}^{1 / q}\right\|_{L^{p}(\mathcal{X})} .
\end{gathered}
$$

Using Proposition 5.4, we can easily verify that the definitions of $\|\cdot\|_{\dot{B}_{p, q}^{s}(x)}$ and $\|\cdot\|_{\dot{F}_{p, q}^{s}(x)}$ are independent of the choices of ATIs. We omit the details.

Proposition 5.6. Let all the notation be as in Proposition 5.4.

(i) For all $f \in\left(\mathcal{G}_{0}^{\epsilon}(\beta, \gamma)\right)^{\prime}$ with $0<\beta, \gamma<\epsilon,|s|<\epsilon, p(s, \epsilon)<p \leq \infty$, and $0<q \leq \infty$,

$$
\left\{\sum_{k \in \mathbb{Z}} 2^{k s q}\left\|D_{k}(f)\right\|_{L^{p}(\mathcal{X})}^{q}\right\}^{1 / q} \sim\left\{\sum_{k \in \mathbb{Z}} 2^{k s q}\left\|Q_{k}(f)\right\|_{L^{p}(\mathcal{X})}^{q}\right\}^{1 / q} .
$$

(ii) For all $f \in\left(\mathcal{G}_{0}^{\epsilon}(\beta, \gamma)\right)^{\prime}$ with $0<\beta, \gamma<\epsilon,|s|<\epsilon, p(s, \epsilon)<p<\infty$, and $p(s, \epsilon)<q \leq \infty$,

$$
\left\|\left\{\sum_{k \in \mathbb{Z}} 2^{k s q}\left|D_{k}(f)\right|^{q}\right\}^{1 / q}\right\|_{L^{p}(\mathcal{X})} \sim\left\|\left\{\sum_{k \in \mathbb{Z}} 2^{k s q}\left|Q_{k}(f)\right|^{q}\right\}^{1 / q}\right\|_{L^{p}(\mathcal{X})} .
$$

We now verify that the definition of the norm $\|\cdot\|_{\dot{B}_{p, q}^{s}(\mathcal{X})}$ and the norm $\|\cdot\|_{\dot{F}_{p, q}^{s}(\mathcal{X})}$ is independent of the choice of the underlying space of distributions. We recall that $a_{+}=$ $\max \{a, 0\}$ for any $a \in \mathbb{R}$. 
Proposition 5.7. Let all the notation be as in Definition 5.1.

(i) Let $|s|<\epsilon, p(s, \epsilon)<p \leq \infty$, and $0<q \leq \infty$. If $f \in\left(\mathcal{C}_{0}^{e}\left(\beta_{1}, \gamma_{1}\right)\right)^{\prime}$ with

$$
\max \left\{0,-s+n\left(\frac{1}{p}-1\right)_{+}\right\}<\beta_{1}<\epsilon, \quad \max \left\{n\left(\frac{1}{p}-1\right)_{+}, s-\frac{\kappa}{p}\right\}<\gamma_{1}<\epsilon,
$$

and if $\|f\|_{B_{p, q}^{s}(x)}<\infty$, then $f \in\left(\mathcal{G}_{0}^{\epsilon}\left(\beta_{2}, \gamma_{2}\right)\right)^{\prime}$ for every $\beta_{2}$, $\gamma_{2}$ satisfying (5.23).

(ii) Let $|s|<\epsilon, p(s, \epsilon)<p<\infty$, and $p(s, \epsilon)<q \leq \infty$. If $f \in\left(\mathcal{G}_{0}^{\epsilon}\left(\beta_{1}, \gamma_{1}\right)\right)^{\prime}$ with $\beta_{1}, \gamma_{1}$ as in (5.23), and if $\|f\|_{\dot{F}_{p, q}^{s}(x)}<\infty$, then $f \in\left(\mathcal{G}_{0}^{\epsilon}\left(\beta_{2}, \gamma_{2}\right)\right)^{\prime}$ for every $\beta_{2}, \gamma_{2}$ satisfying (5.23).

Proof. Let $\psi \in \mathcal{G}(\epsilon, \epsilon)$. Adopting the notation from Theorem 4.11, we first claim that for $k \in \mathbb{Z}_{+}$,

$$
\left|\left\langle\widetilde{D}_{k}(\cdot, y), \psi\right\rangle\right| \lesssim 2^{-k \beta_{2}}\|\psi\|_{\mathcal{G}\left(\beta_{2}, \gamma_{2}\right)} \frac{1}{V_{1}\left(x_{1}\right)+V\left(x_{1}, y\right)} \frac{1}{\left(1+d\left(x_{1}, y\right)\right)^{\gamma_{2}}}
$$

and that for $k=-1,-2, \ldots$,

$$
\left|\left\langle\tilde{D}_{k}(\cdot, y), \psi\right\rangle\right| \lesssim 2^{k \gamma_{2}^{\prime}}\|\psi\|_{\mathcal{G}\left(\beta_{2}, \gamma_{2}\right)} \frac{1}{V_{2^{-k}}\left(x_{1}\right)+V\left(x_{1}, y\right)} \frac{2^{-k \gamma_{2}}}{\left(2^{-k}+d\left(x_{1}, y\right)\right)^{\gamma_{2}}},
$$

where $\gamma_{2}^{\prime}$ can be any positive number in $\left(0, \gamma_{2}\right)$.

In fact, to verify (5.24), by the vanishing moment of $\widetilde{D}_{k}$, Lemma 2.1(ii), we have

$$
\begin{aligned}
& \left|\left\langle\widetilde{D}_{k}(\cdot, y), \psi\right\rangle\right| \\
& =\left|\int_{x} \widetilde{D}_{k}(z, y)[\psi(z)-\psi(y)] d \mu(z)\right| \\
& \quad \lesssim\|\psi\|_{\mathcal{G}\left(\beta_{2}, \gamma_{2}\right)}\left\{\int_{d(z, y) \leq\left(1+d\left(y, x_{1}\right)\right) / 2}\left|\widetilde{D}_{k}(z, y)\right| \frac{1}{V_{1}\left(x_{1}\right)+V\left(x_{1}, y\right)} \frac{d(z, y)^{\beta_{2}}}{\left(1+d\left(x_{1}, y\right)\right)^{\beta_{2}+\gamma_{2}}} d \mu(z)\right. \\
& \quad+\int_{d(z, y)>\left(1+d\left(y, x_{1}\right)\right) / 2}\left|\widetilde{D}_{k}(z, y)\right|\left[\frac{1}{V_{1}\left(x_{1}\right)+V\left(x_{1}, z\right)} \frac{1}{\left(1+d\left(x_{1}, z\right)\right)^{\gamma_{2}}}\right. \\
& \left.\left.\quad \lesssim 2^{-k \beta_{2}}\|\psi\|_{\mathcal{G}\left(\beta_{2}, \gamma_{2}\right)} \frac{1}{V_{1}\left(x_{1}\right)+V\left(x_{1}, y\right)} \frac{1}{\left(1+d\left(x_{1}, y\right)\right)^{\gamma_{2}}}\right] d \mu(z)\right\} \\
& \quad \times\left\{1+\int_{x} \frac{1}{V_{1}\left(x_{1}\right)+V\left(x_{1}, y\right)+V\left(x_{1}, z\right)} \frac{1}{\left(1+d\left(x_{1}, z\right)\right)^{\gamma_{2}}} d \mu(z)\right. \\
& \left.\quad+\int_{x} \frac{1}{V_{2^{-k}(y)+V(z, y)}} \frac{2^{-k\left(e-\beta_{2}\right)}}{\left(2^{-k}+d(z, y)\right)^{\epsilon-\beta_{2}}} d \mu(z)\right\}
\end{aligned}
$$


where the last quantity is, up to a bounded multiplicative constant, controlled by the righthand side of (5.24), and in the last inequality, we used the fact that for $d(z, y)>(1+$ $\left.d\left(y, x_{1}\right)\right) / 2, V(z, y) \gtrsim V_{1}(y)+V\left(x_{1}, y\right) \sim V_{1}\left(x_{1}\right)+V\left(x_{1}, y\right)$; see also Lemma 2.1(vii). Thus, (5.24) holds.

To see (5.25), by $\int_{x} \psi(z) d \mu(z)=0$, (i) and (ii) of Lemma 2.1, and the fact that for $d\left(z, x_{1}\right)>\left(2^{-k}+d\left(x_{1}, y\right)\right) / 2, V\left(x_{1}, z\right) \gtrsim V_{2^{-k}}\left(x_{1}\right)+V\left(x_{1}, y\right)$, we obtain that for $k=-1$, $-2, \ldots$,

$$
\begin{aligned}
& \left|\left\langle\widetilde{D}_{k}(\cdot, y), \psi\right\rangle\right| \\
& =\left|\int_{x}\left[\tilde{D}_{k}(z, y)-\tilde{D}_{k}\left(x_{1}, y\right)\right] \psi(z) d \mu(z)\right| \\
& \lesssim\|\psi\|_{\mathcal{G}\left(\beta_{2}, \gamma_{2}\right)}\left\{\int_{d\left(z, x_{1}\right) \leq\left(2^{-k}+d\left(y, x_{1}\right)\right) / 2} \frac{d\left(z, x_{1}\right)^{\epsilon}}{\left(2^{-k}+d\left(x_{1}, y\right)\right)^{\epsilon}} \frac{2^{-k \epsilon}}{\left(2^{-k}+d\left(x_{1}, y\right)\right)^{\epsilon}}\right. \\
& \times \frac{1}{V_{2^{-k}}\left(x_{1}\right)+V_{2^{-k}}(y)+V\left(x_{1}, y\right)} \\
& \times \frac{1}{V_{1}\left(x_{1}\right)+V\left(x_{1}, z\right)} \frac{1}{\left(1+d\left(x_{1}, z\right)\right)^{\gamma_{2}}} d \mu(z) \\
& +\int_{d\left(z, x_{1}\right)>\left(2^{-k}+d\left(y, x_{1}\right)\right) / 2}\left[\frac{1}{V_{2^{-k}}(z)+V_{2^{-k}}(y)+V(z, y)} \frac{2^{-k \epsilon}}{\left(2^{-k}+d(z, y)\right)^{\epsilon}}\right. \\
& \left.+\frac{1}{V_{2^{-k}}\left(x_{1}\right)+V_{2^{-k}}(y)+V\left(x_{1}, y\right)} \frac{2^{-k \epsilon}}{\left(2^{-k}+d\left(x_{1}, y\right)\right)^{\epsilon}}\right] \\
& \left.\times \frac{1}{V_{1}\left(x_{1}\right)+V\left(x_{1}, z\right)} \frac{1}{\left(1+d\left(x_{1}, z\right)\right)^{\gamma_{2}}} d \mu(z)\right\} \\
& \lesssim \frac{1}{V_{2^{-k}}\left(x_{1}\right)+V\left(x_{1}, y\right)} \\
& \times\left\{\frac{1}{\left(2^{-k}+d\left(x_{1}, y\right)\right)^{\gamma_{2}}}+\frac{2^{-k \epsilon}}{\left(2^{-k}+d\left(x_{1}, y\right)\right)^{\epsilon}} \frac{1}{\left(2^{-k}+d\left(x_{1}, y\right)\right)^{r_{2}^{\prime}}}\right\},
\end{aligned}
$$

where the last quantity is, up to a bounded multiplicative constant, dominated by the righthand side of (5.25); namely, (5.25) holds. 
Thus, Theorem 4.13 together with (5.24), (5.25), and Lemma 2.1(iii) yield that

$$
\begin{aligned}
|\langle f, \psi\rangle|=\left|\sum_{k=-\infty}^{\infty} \sum_{\tau \in I_{k}} \sum_{\nu=1}^{N(k, \tau)} \mu\left(Q_{\tau}^{k, v}\right) D_{k}(f)\left(y_{\tau}^{k, v}\right)\left\langle\tilde{D}_{k}\left(\cdot, y_{\tau}^{k, v}\right), \psi\right\rangle\right| \\
\lesssim\|\psi\|_{\mathcal{G}\left(\beta_{2}, \gamma_{2}\right)}\left\{\sum_{k=0}^{\infty} \sum_{\tau \in I_{k}} \sum_{\nu=1}^{N(k, \tau)} 2^{-k \beta_{2}} \mu\left(Q_{\tau}^{k, v}\right)\left|D_{k}(f)\left(y_{\tau}^{k, v}\right)\right|\right. \\
\quad \times \frac{1}{V_{1}\left(x_{1}\right)+V\left(x_{1}, y_{\tau}^{k, v}\right)} \frac{1}{\left(1+d\left(x_{1}, y_{\tau}^{k, v}\right)\right)^{\gamma_{2}}} \\
+\sum_{k=-\infty} \sum_{\tau \in I_{k}} \sum_{v=1}^{N(k, \tau)} 2^{k \gamma_{2}^{\prime}} \mu\left(Q_{\tau}^{k, v}\right)\left|D_{k}(f)\left(y_{\tau}^{k, v}\right)\right| \\
\left.\times \frac{1}{V_{2^{-k}}\left(x_{1}\right)+V\left(x_{1}, y_{\tau}^{k, v}\right)} \frac{2^{-k \gamma_{2}}}{\left(2^{-k}+d\left(x_{1}, y_{\tau}^{k, v}\right)\right)^{\gamma_{2}}}\right\} .
\end{aligned}
$$

If $p \leq 1$, by (5.5),

$$
\begin{aligned}
|\langle f, \psi\rangle| \lesssim\|\psi\|_{\mathcal{G}\left(\beta_{2}, \gamma_{2}\right)}\left\{\sum _ { k = 0 } ^ { \infty } 2 ^ { - k ( \beta _ { 2 } + s ) } \left[\sum_{\tau \in I_{k}} \sum_{\nu=1}^{N(k, \tau)} 2^{k s p} \mu\left(Q_{\tau}^{k, v}\right)^{p}\left|D_{k}(f)\left(y_{\tau}^{k, v}\right)\right|^{p}\right.\right. & \left.\times \frac{1}{\left(V_{1}\left(x_{1}\right)+V\left(x_{1}, y_{\tau}^{k, v}\right)\right)^{p}} \frac{1}{\left(1+d\left(x_{1}, y_{\tau}^{k, v}\right)\right)^{\gamma_{2} p}}\right]^{1 / p} \\
+\sum_{k=-\infty}^{-1} 2^{k\left(\gamma_{2}^{\prime}-s\right)}\left[\sum_{\tau \in I_{k}}^{N(k, \tau)} \mu\left(Q_{v=1}^{k, v}\right)^{p} 2^{k s p}\left|D_{k}(f)\left(y_{\tau}^{k, v}\right)\right|^{p}\right. & \left.\left.\times \frac{1}{\left(V_{2^{-k}}\left(x_{1}\right)+V\left(x_{1}, y_{\tau}^{k, v}\right)\right)^{p}} \frac{2^{-k \gamma_{2} p}}{\left(2^{-k}+d\left(x_{1}, y_{\tau}^{k, v}\right)\right)^{\gamma_{2} p}}\right]^{1 / p}\right\} .
\end{aligned}
$$

Notice that when $p \leq 1$, by Lemma 2.1(vii) and $\gamma_{2}>n(1 / p-1)$, for $k \in \mathbb{Z}_{+}, \tau \in I_{k}$ and $v=1, \ldots, N(k, \tau)$,

$$
\begin{aligned}
& \frac{\mu\left(Q_{\tau}^{k, v}\right)^{p-1}}{\left(V_{1}\left(x_{1}\right)+V\left(x_{1}, y_{\tau}^{k, v}\right)\right)^{p}} \frac{1}{\left(1+d\left(x_{1}, y_{\tau}^{k, v}\right)\right)^{\gamma_{2} p}} \\
& \lesssim \frac{1}{V_{1}\left(x_{1}\right)}\left[\frac{V_{1}\left(y_{\tau}^{k, v}\right)+V\left(x_{1}, y_{\tau}^{k, v}\right)}{\mu\left(Q_{\tau}^{k, v}\right)}\right]^{1-p} \frac{1}{\left(1+d\left(x_{1}, y_{\tau}^{k, v}\right)\right)^{\gamma_{2} p}} \lesssim 2^{k n(1-p)} \frac{1}{V_{1}\left(x_{1}\right)}
\end{aligned}
$$

and similarly, by the first inequality of (1.3), for $k=-1,-2, \ldots, \tau \in I_{k}$ and $v=1, \ldots, N(k, \tau)$,

$$
\begin{aligned}
& \frac{\mu\left(Q_{\tau}^{k, v}\right)^{p-1}}{\left(V_{2^{-k}}\left(x_{1}\right)+V\left(x_{1}, y_{\tau}^{k, v}\right)\right)^{p}} \frac{2^{-k \gamma_{2} p}}{\left(2^{-k}+d\left(x_{1}, y_{\tau}^{k, v}\right)\right)^{\gamma_{2} p}} \\
& \lesssim 2^{k \kappa} \frac{1}{V_{1}\left(x_{1}\right)}\left[\frac{V_{2^{-k}}\left(y_{\tau}^{k, v}\right)+V\left(x_{1}, y_{\tau}^{k, v}\right)}{\mu\left(Q_{\tau}^{k, v}\right)}\right]^{1-p} \frac{2^{-k \gamma_{2} p}}{\left(2^{-k}+d\left(x_{1}, y_{\tau}^{k, v}\right)\right)^{\gamma_{2} p}} \lesssim 2^{k \kappa} \frac{1}{V_{1}\left(x_{1}\right)} .
\end{aligned}
$$


Thus, if $p \leq 1$, then by Hölder's inequality when $q \geq 1$ or (5.5) when $q<1$ together with Proposition 5.4,

$$
\begin{aligned}
|\langle f, \psi\rangle| \lesssim & \frac{1}{V_{1}\left(x_{1}\right)^{1 / p}}\|\psi\|_{\mathcal{G}\left(\beta_{2}, \gamma_{2}\right)}\left\{\sum_{k=0}^{\infty} 2^{k\left[n(1 / p-1)-\left(\beta_{2}+s\right)\right]}+\sum_{k=-\infty}^{-1} 2^{k\left[\kappa / p+\gamma_{2}^{\prime}-s\right]}\right\} \\
& \times\left[\sum_{\tau \in I_{k}} \sum_{v=1}^{N(k, \tau)} \mu\left(Q_{\tau}^{k, v}\right) 2^{k s p}\left|D_{k}(f)\left(y_{\tau}^{k, v}\right)\right|^{p}\right]^{1 / p} \\
\lesssim & \frac{1}{V_{1}\left(x_{1}\right)^{1 / p}}\|\psi\|_{\mathcal{G}\left(\beta_{2}, \gamma_{2}\right)}\|f\|_{\dot{B}_{p, q}^{s}(x),}
\end{aligned}
$$

where we used the assumption that $\beta_{2}>-s+n(1 / p-1)$ and we chose $\gamma_{2}^{\prime} \in\left((s-\kappa / p)_{+}, \gamma_{2}\right)$.

Similarly, if $p>1$, by Lemma 2.1(ii), we have

$$
\begin{aligned}
|\langle f, \psi\rangle| \lesssim & \frac{1}{V_{1}\left(x_{1}\right)^{1 / p}}\|\psi\|_{\mathcal{G}\left(\beta_{2}, \gamma_{2}\right)} \\
& \times\left\{\sum_{k=0}^{\infty} 2^{-k\left(\beta_{2}+s\right)}\left[\int_{x} \frac{1}{V_{1}\left(x_{1}\right)+V\left(x_{1}, y\right)} \frac{1}{\left(1+d\left(x_{1}, y\right)\right)^{\gamma_{2} p^{\prime}}} d \mu(y)\right]^{1 / p^{\prime}}\right. \\
& \left.+\sum_{k=0}^{\infty} 2^{k\left(\kappa / p-\gamma_{2}^{\prime}-s\right)}\left[\int_{x} \frac{1}{V_{2^{-k}}\left(x_{1}\right)+V\left(x_{1}, y\right)} \frac{2^{-k \gamma_{2} p_{2}^{\prime}}}{\left(2^{-k}+d\left(x_{1}, y\right)\right)^{\gamma_{2} p^{\prime}}} d \mu(y)\right]^{1 / p^{\prime}}\right\} \\
& \times\left[\sum_{\tau \in I_{k}} \sum_{\nu=1}^{N(k, \tau)} \mu\left(Q_{\tau}^{k, v}\right) 2^{k s p}\left|D_{k}(f)\left(y_{\tau}^{k, v}\right)\right|^{p}\right]^{1 / p} \\
\lesssim & \frac{1}{V_{1}\left(x_{1}\right)^{1 / p}}\|\psi\|_{\mathcal{G}\left(\beta_{2}, \gamma_{2}\right)}\|f\|_{\dot{B}_{p, q}^{s}(x),}
\end{aligned}
$$

where we used $\beta_{2}>-s$ and chose $\gamma_{2}^{\prime}>\max \{0, s-\kappa / p\}$.

Let now $h \in \mathcal{G}_{0}^{\epsilon}\left(\beta_{2}, \gamma_{2}\right)$. Then there exists $\left\{h_{n}\right\}_{n=1}^{\infty} \subset \mathcal{G}(\epsilon, \epsilon)$ such that as $n \rightarrow \infty, \| h-$ $h_{n} \|_{\mathcal{G}\left(\beta_{2}, \gamma_{2}\right)} \rightarrow 0$. By (5.32) and (5.33), we obtain

$$
\left|\left\langle f, h_{n}-h_{m}\right\rangle\right| \lesssim\|f\|_{\dot{B}_{p, q}^{s}(\mathcal{X})}\left\|h_{n}-h_{m}\right\|_{\mathcal{G}\left(\beta_{2}, \gamma_{2}\right)}
$$

which shows that $\lim _{n \rightarrow \infty}\left\langle f, h_{n}\right\rangle$ exists and the limit is independent of the choice of $\left\{h_{n}\right\}$. Thus, if we define $\langle f, h\rangle=\lim _{n \rightarrow \infty}\left\langle f, h_{n}\right\rangle$, by (5.32) and (5.33), we have $|\langle f, h\rangle| \lesssim$ $\|f\|_{\dot{B}_{p, q}^{s}(x)}\|h\|_{\mathcal{G}_{0}^{\epsilon}\left(\beta_{2}, \gamma_{2}\right)}$. That is, $f \in\left(\dot{\mathcal{G}}_{0}^{\epsilon}\left(\beta_{2}, \gamma_{2}\right)\right)^{\prime}$, which completes the proof of (i).

The conclusion (ii) can be deduced from (i) and the fact that $\|f\|_{\dot{B}_{p, \max (p, q)}^{s}}(\mathcal{}) \lesssim\|f\|_{\dot{F}_{p, q}^{s}(\mathcal{x})}$; see [3, Proposition 2.3.2/2] or Proposition 5.10(ii) below.

Now we can introduce the Besov spaces, $\dot{B}_{p, q}^{s}(\mathcal{x})$, and the Triebel-Lizorkin spaces, $\dot{F}_{p, q}^{s}(X)$. 
Definition 5.8. Let $\epsilon_{1} \in(0,1], \epsilon_{2}>0, \epsilon_{3}>0, \epsilon \in\left(0, \epsilon_{1} \wedge \epsilon_{2}\right)$, and let $\left\{S_{k}\right\}_{k \in \mathbb{Z}}$ be an $\left(\epsilon_{1}, \epsilon_{2}, \epsilon_{3}\right)$ ATI. For $k \in \mathbb{Z}$, set $D_{k}=S_{k}-S_{k-1}$.

(i) Let $|s|<\epsilon, p(s, \epsilon)<p \leq \infty$, and $0<q \leq \infty$. The space $\dot{B}_{p, q}^{s}(\mathcal{X})$ is defined to be the set of all $f \in\left(\mathcal{G}_{0}^{\epsilon}(\beta, \gamma)\right)^{\prime}$, for some $\beta, \gamma$ satisfying

$$
\begin{gathered}
\max \left\{s, 0,-s+n\left(\frac{1}{p}-1\right)_{+}\right\}<\beta<\epsilon \\
\max \left\{s-\frac{\kappa}{p}, n\left(\frac{1}{p}-1\right)_{+},-s+n\left(\frac{1}{p}-1\right)_{+}-\kappa\left(1-\frac{1}{p}\right)_{+}\right\}<\gamma<\epsilon
\end{gathered}
$$

such that $\|f\|_{\dot{B}_{p, q}^{s}(x)}=\left\{\sum_{k=-\infty}^{\infty} 2^{k s q}\left\|D_{k}(f)\right\|_{L^{p}(\mathcal{X})}^{q}\right\}^{1 / q}<\infty$ with the usual modifications made when $p=\infty$ or $q=\infty$.

(ii) Let $|s|<\epsilon, p(s, \epsilon)<p<\infty$, and $p(s, \epsilon)<q \leq \infty$. The space $\dot{F}_{p, q}^{s}(\mathcal{X})$ is defined to be the set of all $f \in\left(\dot{\mathcal{C}}_{0}^{e}(\beta, \gamma)\right)^{\prime}$ for some $\beta, \gamma$ satisfying (5.35) such that $\|f\|_{\dot{F}_{p, q}^{s}(x)}=$ $\left\|\left\{\sum_{k=-\infty}^{\infty} 2^{k s q}\left|D_{k}(f)\right|^{q}\right\}^{1 / q}\right\|_{L^{p}(x)}<\infty$ with the usual modification made when $q=\infty$.

Propositions 5.6 and 5.7 show that the definitions of the spaces $\dot{B}_{p, q}^{s}(\mathcal{X})$ and $\dot{F}_{p, q}^{s}(\mathcal{X})$ are independent of the choice of the approximations of the identity and the distribution space, $\left(\mathcal{G}_{0}^{\epsilon}(\beta, \gamma)\right)^{\prime}$, with $\beta, \gamma$ as in (5.35).

Remark 5.9. To guarantee that the definitions of the spaces $\dot{B}_{p, q}^{s}(\mathcal{X})$ and $\dot{F}_{p, q}^{s}(\mathcal{X})$ are independent of the choice of the distribution space $\left(\mathcal{C}_{0}^{\epsilon}(\beta, \gamma)\right)^{\prime}$, we only need the restriction that $\beta, \gamma$ satisfy (5.23); see Proposition 5.7. Moreover, if we assume that $\max \{s, 0\}<\beta<\epsilon$ and $\max \left\{n(1 / p-1)_{+}-s+n(1 / p-1)_{+}-\kappa(1-1 / p)_{+}\right\}<\gamma<\epsilon$, we can verify that the space of test functions $\mathcal{G}(\beta, \gamma)$ is contained in $\dot{B}_{p, q}^{s}(\mathcal{X})$ and $\dot{F}_{p, q}^{s}(\mathcal{X})$; see Proposition 5.10 below. Thus, the spaces $\dot{B}_{p, q}^{s}(\mathcal{X})$ and $\dot{F}_{p, q}^{s}(\mathcal{X})$ are nonempty if we restrict $\beta, \gamma$ as in (5.35).

\subsection{Properties of $\dot{B}_{p, q}^{s}(\mathcal{X})$ and $\dot{F}_{p, q}^{s}(X)$ and boundedness of singular integrals}

In this subsection, we first present some basic properties of $\dot{B}_{p, q}^{s}(\mathcal{X})$ and $\dot{F}_{p, q}^{s}(\mathcal{X})$. Then we establish a Lusin-area characterization of $\dot{F}_{p, q}^{s}(\mathcal{X})$, and as an application, we discuss the relation between the spaces $\dot{F}_{p, q}^{s}(\mathcal{X})$ and the atomic Hardy spaces $H_{\text {at }}^{p}(\mathcal{X})$ of Coifman and Weiss in [28] and the Hardy spaces $H^{p}(\mathcal{X})$ in [48]. Finally, we show that the singular integrals considered by Nagel and Stein in [44] act boundedly on $\dot{B}_{p, q}^{s}(\mathcal{X})$ and $\dot{F}_{p, q}^{s}(\mathcal{X})$.

Proposition 5.10. Let $\epsilon_{1} \in(0,1], \epsilon_{2}>0, \epsilon \in\left(0, \epsilon_{1} \wedge \epsilon_{2}\right)$, and $|s|<\epsilon$.

(i) For $p(s, \epsilon)<p \leq \infty$ and $0<q_{0} \leq q_{1} \leq \infty, \dot{B}_{p, q_{0}}^{s}(\mathcal{X}) \subset \dot{B}_{p, q_{1}}^{s}(\mathcal{X})$; and for $p(s, \epsilon)<p<\infty$ and $p(s, \epsilon)<q_{0} \leq q_{1} \leq \infty, \dot{F}_{p, q_{0}}^{s}(\mathcal{X}) \subset \dot{F}_{p, q_{1}}^{s}(\mathcal{X})$.

(ii) If $p(s, \epsilon)<p<\infty$ and $p(s, \epsilon)<q \leq \infty$, then $\dot{B}_{p, \min (p, q)}^{s}(\mathcal{X}) \subset \dot{F}_{p, q}^{s}(\mathcal{X}) \subset \dot{B}_{p, \max (p, q)}^{s}(\mathcal{X})$.

(iii) If $\beta, \gamma$ as in (5.23), then $\dot{B}_{p, q}^{s}(\mathcal{X}) \subset\left(\mathcal{G}_{0}^{\epsilon}(\beta, \gamma)\right)^{\prime}$ when $p(s, \epsilon)<p \leq \infty$ and $0<q \leq \infty$, and $\dot{F}_{p, q}^{s}(\mathcal{X}) \subset\left(\dot{\mathcal{C}}_{0}^{\epsilon}(\beta, \gamma)\right)^{\prime}$ when $p(s, \epsilon)<p<\infty$ and $p(s, \epsilon)<q \leq \infty$. 
(iv) If $\max \{s, 0\}<\beta<\epsilon$ and $\max \left\{n(1 / p-1)_{+^{\prime}}-s+n(1 / p-1)_{+}-\kappa(1-1 / p)_{+}\right\}<\gamma<\epsilon$, then $\mathcal{G}(\beta, \gamma) \subset \dot{B}_{p, q}^{s}(X)$ when $p(s, \epsilon)<p \leq \infty$ and $0<q \leq \infty$, and $\mathcal{G}(\beta, \gamma) \subset \dot{F}_{p, q}^{s}(X)$ when $p(s, \epsilon)<p<\infty$ and $p(s, \epsilon)<q \leq \infty$.

(v) If $1<p<\infty$, then $\dot{F}_{p, 2}^{0}(\mathcal{X})=L^{p}(\mathcal{X})$ with equivalent norms.

(vi) The spaces $\dot{B}_{p, q}^{s}(\mathcal{X}) / \mathcal{N}$ with $p(s, \epsilon)<p \leq \infty$ and $0<q \leq \infty$ and the spaces $\dot{F}_{p, q}^{s}(\mathcal{X}) / \mathcal{N}$ with $p(s, \epsilon)<p<\infty$ and $p(s, \epsilon)<q \leq \infty$ are complete.

Proof. Property (i) is a simple corollary of (5.5). Property (ii) can be deduced from Minkowski's inequality, (5.5), and the following generalized Minkowski inequality that for all $1 \leq q \leq \infty$,

$$
\left\{\sum_{k=-\infty}^{\infty}\left[\int_{x}\left|a_{k}(x)\right| d \mu(x)\right]^{q}\right\}^{1 / q} \lesssim \int_{\chi}\left\{\sum_{k=-\infty}^{\infty}\left|a_{k}(x)\right|^{q}\right\}^{1 / q} d \mu(x)
$$

see also [3, Proposition 2.3.2/2] and [6, Proposition 2.3].

Property (iii) is implied by the proof of Proposition 5.7 and Property (vi) can be easily deduced from Property (iii). for $k \in \mathbb{Z}_{+}$

To verify (iv), similarly to the proofs of (5.24) and (5.25), for $f \in \mathcal{G}(\beta, \gamma)$, we have that

$$
\left|D_{k}(f)(x)\right| \lesssim 2^{-k \beta}\|f\|_{\mathcal{G}(\beta, \gamma)} \frac{1}{V_{1}\left(x_{1}\right)+V\left(x_{1}, x\right)} \frac{1}{\left(1+d\left(x_{1}, x\right)\right)^{\gamma}},
$$

and that for $k=-1,-2, \ldots$,

$$
\left|D_{k}(f)(x)\right| \lesssim 2^{k \gamma^{\prime}}\|f\|_{\mathcal{G}(\beta, \gamma)} \frac{1}{V_{2^{-k}}\left(x_{1}\right)+V\left(x_{1}, x\right)} \frac{2^{-k \gamma}}{\left(2^{-k}+d\left(x_{1}, x\right)\right)^{\gamma}},
$$

where $\gamma^{\prime}$ can be any positive number in $(0, \gamma)$. Moreover, since $\gamma>n(1 / p-1)_{+}$

$$
\begin{aligned}
\left\{\int_{x}\right. & \left.\frac{1}{\left(V_{1}\left(x_{1}\right)+V\left(x_{1}, x\right)\right)^{p}} \frac{1}{\left(1+d\left(x_{1}, x\right)\right)^{\gamma p}} d \mu(x)\right\}^{1 / p} \\
& \lesssim\left\{\frac{1}{V_{1}\left(x_{1}\right)^{p-1}}+\sum_{l=0}^{\infty} \frac{1}{V_{2^{l} r}\left(x_{1}\right)^{p-1}} \frac{1}{2^{l \gamma p}}\right\}^{1 / p} \lesssim \frac{1}{V_{1}\left(x_{1}\right)^{1-1 / p}}
\end{aligned}
$$

and for $k=-1,-2, \ldots$,

$$
\begin{aligned}
& \left\{\int_{x} \frac{1}{\left(V_{2^{-k}}\left(x_{1}\right)+V\left(x_{1}, x\right)\right)^{p}} \frac{2^{-k \gamma p}}{\left(2^{-k}+d\left(x_{1}, x\right)\right)^{\gamma p}} d \mu(x)\right\}^{1 / p} \\
& \quad \lesssim\left\{\frac{1}{V_{2^{-k}}\left(x_{1}\right)^{p-1}}+\sum_{l=0}^{\infty} \frac{1}{V_{2^{l} 2^{-k}}\left(x_{1}\right)^{p-1}} \frac{1}{2^{l \gamma p}}\right\}^{1 / p} \lesssim 2^{k \kappa(1-1 / p)_{+}-k n(1 / p-1)_{+}} V_{1}\left(x_{1}\right)^{1 / p-1}
\end{aligned}
$$


Choose $\gamma^{\prime} \in(0, \gamma)$ such that $\gamma^{\prime}>n(1 / p-1)_{+}-s-\kappa(1-1 / p)_{+}$. From the above estimates, it follows that

$$
\begin{aligned}
\|f\|_{\dot{B}_{p, q}^{s}(x)} & \lesssim\|f\|_{\mathcal{G}(\beta, \gamma)} \frac{1}{V_{1}\left(x_{1}\right)^{1-1 / p}}\left\{\sum_{k=0}^{\infty} 2^{k(s-\beta) q}+\sum_{k=-\infty}^{-1} 2^{k\left[s+\gamma^{\prime}-n(1 / p-1)_{+}+\kappa(1-1 / p)_{+}\right] q}\right\}^{1 / q} \\
& \lesssim\|f\|_{\mathcal{G}(\beta, \gamma)} .
\end{aligned}
$$

Thus, $\mathcal{G}(\beta, \gamma) \subset \dot{B}_{p, q}^{s}(\mathcal{X})$, which together with (ii) implies that $\mathcal{G}(\beta, \gamma) \subset \dot{F}_{p, q}^{s}(\mathcal{X})$.

Property (v) is a simple conclusion of Proposition 3.15, which completes the proof of Proposition 5.10.

We next give a Lusin-area characterization for Triebel-Lizorkin spaces $\dot{F}_{p, q}^{s}(\mathcal{X})$, which will allow to establish some relations between the Triebel-Lizorkin spaces $\dot{F}_{p, q}^{s}(\mathcal{X})$, the atomic Hardy spaces of Coifman and Weiss in [28], and the Hardy spaces $H^{p}(\mathcal{X})$ in [48].

Definition 5.11. Let $s \in \mathbb{R}, a>0, q \in(0, \infty]$, and let $\epsilon_{1} \in(0,1], \epsilon_{2}>0, \epsilon_{3}>0, \epsilon \in\left(0, \epsilon_{1} \wedge \epsilon_{2}\right]$. Let $\left\{S_{k}\right\}_{k \in \mathbb{Z}}$ be an $\left(\epsilon_{1}, \epsilon_{2}, \epsilon_{3}\right)$-ATI. For $k \in \mathbb{Z}$, set $D_{k}=S_{k}-S_{k-1}$. The Lusin-area function (also called the Littlewood-Paley S-function) $\dot{S}_{q, a}^{s}(f)$ for any $f \in\left(\mathcal{G}_{0}^{\epsilon}(\beta, \gamma)\right)^{\prime}$ with $0<\beta, \gamma \leq \epsilon$ and $x \in \mathcal{X}$ is given by

$$
\dot{S}_{q, a}^{s}(f)(x)=\left\{\sum_{k \in \mathbb{Z}} \int_{d(x, y)<a 2^{-k}} 2^{k s q}\left|D_{k}(f)(y)\right|^{q} \frac{d \mu(y)}{V_{a 2^{-k}}(x)}\right\}^{1 / q},
$$

where the usual modification is made when $q=\infty$.

Remark 5.12. (i) By Lemma 2.1(vi), if we replace $V_{a 2^{-k}}(x)$, respectively, by $V_{a 2^{-k}}(y), V_{a 2^{-k}}(y)+$ $V_{a 2^{-k}}(x), V_{2^{-k}}(x), V_{2^{-k}}(y)$, or $V_{2^{-k}}(x)+V_{2^{-k}}(y)$ in the definition of $\dot{S}_{q, a}^{s}(f)$, then the corresponding Littlewood-Paley $S$-functions $\dot{S}_{q, a}^{s}(f)$ are pointwise equivalent. This is often useful in applications.

(ii) It is easy to see that $\dot{S}_{2, a}^{0}(f)$ is a discrete version of the corresponding LittlewoodPaley $S$-function in [48].

Theorem 5.13. Let $\epsilon_{1} \in(0,1], \epsilon_{2}>0, \epsilon_{3}>0, \epsilon \in\left(0, \epsilon_{1} \wedge \epsilon_{2}\right)$, and let $\left\{S_{k}\right\}_{k \in \mathbb{Z}}$ be an $\left(\epsilon_{1}, \epsilon_{2}, \epsilon_{3}\right)$ ATI. For $k \in \mathbb{Z}$, set $D_{k}=S_{k}-S_{k-1}$. Let $a>0,|s|<\epsilon, p(s, \epsilon)<p<\infty, p(s, \epsilon)<q \leq \infty$, and let $\dot{S}_{q, a}^{s}(f)$ be as in Definition 5.11 for any $f \in\left(\mathcal{G}_{0}^{e}(\beta, \gamma)\right)^{\prime}$ with $\beta, \gamma$ as in (5.35). Then $f \in \dot{F}_{p, q}^{s}(\mathcal{X})$ if and only if $f \in\left(\mathcal{G}_{0}^{\epsilon}(\beta, \gamma)\right)^{\prime}$ for some $\beta, \gamma$ as in (5.35), and $\dot{S}_{q, a}^{s}(f) \in L^{p}(\mathcal{X})$. Moreover, in this case, $\|f\|_{\dot{F}_{p, q}^{s}(x)} \sim\left\|\dot{S}_{q, a}^{s}(f)\right\|_{L^{p}(x)}$.

Proof. We use the notation as in the proof of Proposition 5.4. It is easy to check that for any fixed constant $C>0$,

$$
\begin{aligned}
& \sup _{z \in B\left(z_{\tau}^{k, v}, \mathrm{C} 2^{-k}\right)}\left|\left(D_{k} \widetilde{D}_{k^{\prime}}\right)\left(z, y_{\tau^{\prime}}^{k^{\prime}, v^{\prime}}\right)\right| \\
& \quad \lesssim 2^{-\left|k-k^{\prime}\right| \epsilon^{\prime}} \frac{1}{V_{2^{-\left(k \wedge k^{\prime}\right)}}\left(z_{\tau}^{k, v}\right)+V_{2^{-\left(k \wedge k^{\prime}\right)}}\left(y_{\tau^{\prime}}^{k^{\prime}, \nu^{\prime}}\right)+V\left(z_{\tau}^{k, v}, y_{\tau^{\prime}}^{k^{\prime}, v^{\prime}}\right)} \frac{2^{-\left(k \wedge k^{\prime}\right) \epsilon}}{\left(2^{-\left(k \wedge k^{\prime}\right)}+d\left(z_{\tau}^{k, v}, y_{\tau^{\prime}}^{k^{\prime}, v^{\prime}}\right)\right)} .
\end{aligned}
$$


Using this to replace (5.10) and then repeating the proof of (5.8) in Proposition 5.4 yield

$$
\begin{aligned}
& \|\left\{\sum_{k \in \mathbb{Z}}\right.\left.\sum_{\tau \in I_{k}} \sum_{\nu=1}^{N(k, \tau)} 2^{k s q}\left[\sup _{z \in B\left(z_{\tau}^{k, v}, C 2^{-k}\right)}\left|D_{k}(f)(z)\right|\right]^{q} x_{Q_{\tau}^{k, \nu}}\right\}^{1 / q} \|_{L^{p}(\mathcal{X})} \\
& \sim\left\|\left\{\sum_{k \in \mathbb{Z}} \sum_{\tau \in I_{k}} \sum_{\nu=1}^{N(k, \tau)} 2^{k s q}\left[\inf _{z \in Q_{\tau}^{k, v}}\left|D_{k}(f)(z)\right|\right]^{q} x_{Q_{\tau}^{k, \nu}}\right\}^{1 / q}\right\|_{L^{p}(\mathcal{X})} \\
& \sim\|f\|_{\dot{F}_{p, q}^{s}(\mathcal{X}),}
\end{aligned}
$$

which shows that

$$
\begin{aligned}
& \left\|\dot{S}_{q, a}^{s}(f)\right\|_{L^{p}(\mathcal{X})} \\
& \quad \leq\left\|\left\{\sum_{k \in \mathbb{Z}} \sum_{\tau \in I_{k}} \sum_{\nu=1}^{N(k, \tau)} 2^{k s q}\left[\sup _{z \in B\left(z_{\tau}^{k, \nu}, C 2^{-k}\right)}\left|D_{k}(f)(z)\right|\right]^{q} X_{Q_{\tau}^{k, \nu}}\right\}^{1 / q}\right\|_{L^{p}(\mathcal{X})} \sim\|f\|_{F_{p, q}^{s}(\mathcal{X})} .
\end{aligned}
$$

On the other hand, noticing that if $x \in B\left(z_{\tau}^{k, v}, \min \{a, 1\} C_{7} 2^{-(k+j)}\right)$, then

$$
B\left(z_{\tau}^{k, v}, \min \{a, 1\} C_{7} 2^{-(k+j)}\right) \subset\left\{y \in X: d(x, y)<a 2^{-k}\right\}
$$

therefore, by Lemma 2.19,

$$
\begin{aligned}
S_{q, a}^{s}(f)(x) & =\left\{\sum_{k \in \mathbb{Z}} \sum_{\tau \in I_{k}} \sum_{\nu=1}^{N(k, \tau)} \int_{d(x, y)<a 2^{-k}} 2^{k s q}\left|D_{k}(f)(y)\right|^{q} X_{Q_{\tau}^{k, v}}(x) \frac{d \mu(y)}{V_{a 2^{-k}}(x)}\right\}^{1 / q} \\
& \geq\left\{\sum_{k \in \mathbb{Z}} \sum_{\tau \in I_{k}} \sum_{\nu=1}^{N(k, \tau)} 2^{k s q}\left[\inf _{y \in B\left(z_{\tau}^{k, v}, \min \{a, 1\} C_{7} 2^{-(k+j))}\right.}\left|D_{k}(f)(y)\right|\right]^{q} X_{B\left(z_{\tau}^{k, v}, \min \{a, 1\} C_{7} 2^{-(k+j)}\right)}(x)\right\}^{1 / q},
\end{aligned}
$$

which together with the following estimate

$$
\begin{aligned}
& \left\|\left\{\sum_{k \in \mathbb{Z}} 2^{k s q}\left|D_{k}(f)\right|^{q}\right\}^{1 / q}\right\| \|_{L^{p}(\mathcal{X})} \\
& \quad \lesssim\left\|\left\{\sum_{k \in \mathbb{Z}} \sum_{\tau \in I_{k}} \sum_{v=1}^{N(k, \tau)} 2^{k s q}\left[\inf _{y \in B\left(z_{\tau}^{k, v}, \min \{a, 1\} C_{7} 2^{-(k+j))}\right.}\left|D_{k}(f)(y)\right|\right]^{q} x_{B\left(z_{\tau}^{k, v}, \min \{a, 1\} C_{7} 2^{-(k+j)}\right)}\right\}^{1 / q}\right\| \|_{L^{p}(\mathcal{X})}
\end{aligned}
$$

yields that $\left\|\dot{S}_{q, a}^{s}(f)\right\|_{L^{p}(\mathcal{X})} \gtrsim\|f\|_{\dot{F}_{p, q}^{s}(\mathcal{X})}$. Using the notation as in Lemma 5.3, we can verify the estimate (5.48) by repeating the proof of (5.8) in Proposition 5.4 and replacing Lemma 5.3 by 
the following estimate that for any fixed constant $C>0$,

$$
\begin{aligned}
\sum_{\tau \in I_{k}} & \sum_{\nu=1}^{N(k, \tau)} \mu\left(Q_{\tau}^{k, v}\right) \frac{1}{V_{2^{-\left(k^{\prime} \wedge k\right)}}(x)+V_{2^{-\left(k^{\prime} \wedge k\right)}}\left(y_{\tau}^{k, v}\right)+V\left(x, y_{\tau}^{k, v}\right)} \frac{2^{-\left(k \wedge k^{\prime}\right) \epsilon}}{\left(2^{-\left(k \wedge k^{\prime}\right)}+d\left(x, y_{\tau}^{k, v}\right)\right)}\left|a_{\tau}^{k, v}\right| \\
& \lesssim 2^{\left[\left(k \wedge k^{\prime}\right)-k\right] n(1-1 / r)}\left\{M\left(\sum_{\tau \in I_{k}} \sum_{\nu=1}^{N(k, \tau)}\left|a_{\tau}^{k, v}\right|^{r} X_{B\left(z_{\tau}^{k, v}, C 2^{-k}\right)}\right)(x)\right\}^{1 / r},
\end{aligned}
$$

which can be proved by the same way as the proof of Lemma 5.3 via the following facts $\mu\left(B\left(z_{\tau}^{k, v}, C 2^{-k}\right)\right) \sim \mu\left(Q_{\tau}^{k, v}\right)$ and

$$
\mu\left(B\left(z_{\tau}^{k, v}, C 2^{-k}\right)\right) X_{B\left(z_{\tau}^{k, v}, C 2^{-k}\right)}(z) \sim V_{2^{-k}}(z) X_{B\left(z_{\tau}^{k, v}, C 2^{-k}\right)}(z)
$$

This finishes the proof of Theorem 5.13.

Definition 5.14. Let all the notation be as in Definition 5.8. The Hardy space $H^{p}(x)$ when $n /(n+$ $\epsilon)<p \leq 1$ is defined to be the Triebel-Lizorkin spaces $\dot{F}_{p, 2}^{0}(\mathcal{X})$, with norm $\|f\|_{H^{p}(\mathcal{X})} \equiv\|f\|_{\dot{F}_{p, 2}^{0}(\mathcal{X})}$.

We now recall the definition of atoms on spaces of homogeneous type in [28].

Definition 5.15. A function $a$ on $\mathcal{X}$ is called an $H^{p}(\mathcal{X})$-atom if

(i) supp $a \subset B\left(x_{0}, r\right)$ for some $x_{0} \in \mathcal{X}$ and some $r>0$;

(ii) $\|a\|_{L^{2}(x)} \leq\left[\mu\left(B\left(x_{0}, r\right)\right)\right]^{1 / 2-1 / p}$;

(iii) $\int_{x} a(x) d \mu(x)=0$.

If (i) to (iii) apply, we also say that $a$ is an $H^{p}(\mathcal{X})$-atom supported on $B\left(x_{0}, r\right)$.

Theorem 5.16. Assume that $\epsilon$ and $\beta, \gamma$ are as in Definition 5.8. If $n /(n+\epsilon)<p \leq 1$, then $f \in H^{p}(\mathcal{X})$ if and only if there exist a sequence of numbers $\left\{\lambda_{k}\right\}_{k=0}^{\infty} \subset \mathbb{C}$ with $\sum_{k=0}^{\infty}\left|\lambda_{k}\right|^{p}<\infty$ and a sequence of $H^{p}(\mathcal{X})$-atoms $\left\{a_{k}\right\}_{k=0}^{\infty}$ such that $f=\sum_{k=0}^{\infty} \lambda_{k} a_{k}$ in $\left(\mathcal{G}_{0}^{\epsilon}(\beta, \gamma)\right)^{\prime}$. Moreover, in this case, $\|f\|_{H^{p}(\mathcal{X})} \sim \inf \left\{\left(\sum_{k=0}^{\infty}\left|\mathcal{\lambda}_{k}\right|^{p}\right)^{1 / p}\right\}$, where the infimum is taken over all the above decompositions of $f$.

This theorem can be proved by a literal repetition of Theorem 2.21 in [48], with Lemma 2.22 therein replaced by Theorem 2.6 in this paper. We omit the details.

Remark 5.17. Theorem 5.16 shows that the Hardy spaces defined here are the same as those in [48]. Moreover, by [48, Remark 2.27], we know that the Hardy space $H^{1}(\mathcal{X})$ also coincides with the atomic Hardy space $H_{\mathrm{at}}^{1}(\mathcal{X})$ of Coifman and Weiss in [28]. Moreover, when $n /(n+$ $\epsilon)<p<1$ and $\mathcal{X}$ is an Ahlfors $n$-regular metric measure space, $H^{p}(\mathcal{X})$ also coincides with the atomic Hardy space $H_{\mathrm{at}}^{p}(\mathcal{X})$ of Coifman and Weiss in [28]. However, if $\mathcal{X}$ is a general space of homogeneous type, it is still unclear so far to us if $H^{p}(\mathcal{X})=H_{\mathrm{at}}^{p}(\mathcal{X})$ when $p<1$; see [48, Remark 2.30].

We now recall the definition of the Lipschitz $\operatorname{space~} \operatorname{Lip}_{s}(\mathcal{X})$ with $s>0$; see [28]. 
Definition 5.18. Let $s>0$. The Lipschitz (or Hölder) space $\operatorname{Lip}_{s}(\mathcal{X})$ is defined to be the set of all functions $f$ on $X$ such that

$$
\|f\|_{\operatorname{Lip}_{s}(x)}=\sup _{x \neq y} \frac{|f(x)-f(y)|}{V(x, y)^{s}}<\infty
$$

Observe that these classes are rather Lipschitz (or Hölder) classes with respect to the measure distance $\rho(x, y) \equiv \inf \{\mu(B): x, y \in B, B$ a ball $\}$, not the distance $d$.

Following Coifman and Weiss [28], we immediately obtain from Theorem 5.16 in this paper and [48, Remark 2.27] the duality between $H^{1}(\mathcal{X})$ and $\mathrm{BMO}(\mathcal{X})$ and between $H_{\mathrm{at}}^{p}(\mathcal{x})$ and $\operatorname{Lip}_{1 / p-1}(\mathcal{X})$ when $p<1$, which is [48, Corollary 2.29].

Theorem 5.19. (i) The space $\operatorname{BMO}(x) / \mathcal{N}$ is the dual space of $H^{1}(x)$, in the following sense: if $f=\sum_{k=0}^{\infty} \lambda_{k} a_{k} \in H^{1}(\mathcal{X})$ is as in Theorem 5.16, then for each $g \in \operatorname{BMO}(\mathcal{X})$, $\lim _{N \rightarrow \infty} \sum_{k=0}^{N} \lambda_{k} \int_{x} a_{k}(x) g(x) d \mu(x)$ is a well-defined continuous linear functional $\mathcal{L}_{g}: f \mapsto\langle f, g\rangle$ with norm $\lesssim\|g\|_{\mathrm{BMO}(x)}$.

Conversely, each continuous linear functional $\mathcal{L}$ on $H^{1}(\mathcal{X})$ has the form $\mathcal{L}=\mathcal{L}_{\mathrm{g}}$ for some $g \in \mathrm{BMO}(\mathcal{X})$ with $\|g\|_{\mathrm{BMO}(\mathcal{x})} \lesssim\|\mathcal{L}\|$.

Moreover, $\mathcal{L}_{g}=0$ if and only if $g \in \mathcal{N}$.

(ii) Assume that $\epsilon$ is as in Definition 5.8, $n /(n+\epsilon)<p<1$, and $s=1 / p-1$. Then $\operatorname{Lip}_{s}(x)$ (more precisely, $\left.\operatorname{Lip}_{s}(\mathcal{X}) / \mathcal{N}\right)$ is the dual space of $H^{p}(\mathcal{X})$ in the sense of $(i)$.

Remark 5.20. It is easy to see that if $\chi=\mathbb{R}^{n}$ and $\mu$ is the $n$-dimensional Lebesgue measure, then $\dot{C}^{n s}(\mathcal{X})=\operatorname{Lip}_{s}(\mathcal{X})$ with equivalent norms. Thus,

$$
\dot{B}_{\infty, \infty}^{n s}(\mathcal{X})=\dot{F}_{\infty, \infty}^{n s}(\mathcal{X})=\dot{\operatorname{Lip}}_{s}(\mathcal{X})
$$

with equivalent norms. Therefore, the dual space of $H^{p}(\mathcal{X})$ with $n /(n+\epsilon)<p<1$ is the space $\dot{B}_{\infty, \infty}^{n(1 / p-1)}(\mathcal{X})$; see also [3, Theorem 2.11.3(ii)]. However, for a general space $\mathcal{X}$ of homogeneous type, it seems that $\dot{C}^{n s}(\mathcal{X}) \neq \operatorname{Lip}_{s}(\mathcal{X})$, unless $\mu(B(x, r)) \sim r^{n}$ for all $x \in \mathcal{X}$ and $r>0$, namely, $\mathcal{X}$ is an Ahlfors $n$-regular metric measure space. Thus, one cannot expect that $[3$, Theorem 2.11.3] still holds when $X$ is a general space of homogeneous type considered in this paper, which demonstrates an essential difference between function spaces on general spaces of homogeneous type considered in this paper and those on Ahlfors regular metric measure space.

Using Proposition 5.10 and Theorem 2.6, we obtain the following density property of $\dot{B}_{p, q}^{s}(\mathcal{X})$ and $\dot{F}_{p, q}^{s}(\mathcal{X})$.

Proposition 5.21. Let $\epsilon_{1}, \epsilon_{2}, \epsilon$, and let $|s|<\epsilon$ be as in Definition 5.8. Then $\dot{\mathcal{G}}_{b}\left(\epsilon_{1}, \epsilon_{2}\right)$ is dense in $\dot{B}_{p, q}^{s}(\mathcal{X})$ when $p(s, \epsilon)<p<\infty$ and $0<q<\infty$, and in $\dot{F}_{p, q}^{s}(\mathcal{X})$ when $p(s, \epsilon)<p, q<\infty$.

Proof. By similarity, we only verify the conclusion on $\dot{B}_{p, q}^{s}(\mathcal{X})$. Let $f \in \dot{B}_{p, q}^{s}(\mathcal{X})$ with $s, p, q$ as in the proposition. By Proposition 5.10(iii), we know $f \in\left(\mathcal{G}_{0}^{\epsilon}(\beta, \gamma)\right)^{\prime}$ with $\beta, \gamma$ as in (5.23). Let 
$\left\{D_{k}\right\}_{k \in \mathbb{Z}}$ be an ATI with bounded support as constructed in Theorem 2.6. By Theorem 4.13 and using the notation as in Theorem 4.12, we have

$$
f(x)=\sum_{k^{\prime}=-\infty}^{\infty} \sum_{\tau^{\prime} \in I_{k^{\prime}}} \sum_{v^{\prime}=1}^{N\left(k^{\prime}, \tau^{\prime}\right)} \mu\left(Q_{\tau^{\prime}}^{k^{\prime}, v^{\prime}}\right) D_{k^{\prime}}\left(x, y_{\tau^{\prime}}^{k^{\prime}, v^{\prime}}\right) \bar{D}_{k^{\prime}}(f)\left(y_{\tau^{\prime}}^{k^{\prime}, v^{\prime}}\right)
$$

in $\left(\mathcal{G}_{0}^{\epsilon}(\beta, \gamma)\right)^{\prime}$. It is easy to check that for any fixed $k^{\prime} \in \mathbb{Z}$ and $\tau^{\prime} \in I_{k^{\prime}}, N\left(k^{\prime}, \tau^{\prime}\right)$ is a finite set of indices. For any fixed $k^{\prime} \in \mathbb{Z}$, choose $\left\{I_{k^{\prime}}^{N}\right\}_{N \in \mathbb{N}}$ such that $I_{k^{\prime}}^{N} \subset I_{k^{\prime}}^{N+1}, I_{k^{\prime}}^{N}$ is a finite set of indices, and $I_{k^{\prime}}^{N} \rightarrow I_{k^{\prime}}$ as $N \rightarrow \infty$. If for any $N \in \mathbb{N}$, setting

$$
f_{N}(x)=\sum_{\left|k^{\prime}\right| \leq N} \sum_{\tau^{\prime} \in I_{k^{\prime}}^{N}} \sum_{v^{\prime}=1}^{N\left(k^{\prime}, \tau^{\prime}\right)} \mu\left(Q_{\tau^{\prime}}^{k^{\prime}, v^{\prime}}\right) D_{k^{\prime}}\left(x, y_{\tau^{\prime}}^{k^{\prime}, v^{\prime}}\right) \bar{D}_{k^{\prime}}(f)\left(y_{\tau^{\prime}}^{k^{\prime}, v^{\prime}}\right)
$$

then it is easy to check $f_{N} \in \mathcal{G}_{b}\left(\epsilon_{1}, \epsilon_{2}\right)$. From the proof of Proposition 5.4 together with Remark 5.5, it is easy to see that $\left\|f-f_{N}\right\|_{\dot{B}_{p, q}^{s}(x)} \rightarrow 0$, as $N \rightarrow \infty$ (here we need $p, q<\infty$ ). Thus, $\mathcal{G}_{b}\left(\epsilon_{1}, \epsilon_{2}\right)$ is dense in $\dot{B}_{p, q}^{s}(\mathcal{X})$ with $s, p, q$ as in the proposition, which completes the proof of Proposition 5.21.

We now discuss the boundedness on $\dot{B}_{p, q}^{s}(\mathcal{X})$ and $\dot{F}_{p, q}^{s}(\mathcal{X})$ of the singular integral operators introduced by Nagel and Stein in [44]. To state their definition, we need first to recall the notion of a normalized bump function on $\mathcal{X}$. A function $\varphi$ on $\mathcal{X}$ is said to be an $\epsilon$-bump function associated to a ball $B\left(x_{0}, \delta\right)$ for some $x_{0} \in \mathcal{X}$ and $\delta>0$, if it is supported in that ball, and if there exists a constant $C>0$ independent of $\varphi$ such that $\|\varphi\|_{C^{\eta}(x)} \leq C \delta^{-\eta}$ for all $\eta \in(0, \epsilon]$. Notice that then $\|\varphi\|_{L^{\infty}(\mathcal{X})} \leq C$ (see Remark 2.14(ii)). If $C=1$ in this definition, then such a bump function is called a normalized $\epsilon$-bump function for the ball $B\left(x_{0}, \delta\right)$.

Let $\epsilon \in(0,1]$. A linear operator $T$, which is initially assumed to be continuous from $C_{b}^{\eta}(\mathcal{X})$ to $\left(C_{b}^{\eta}(\mathcal{X})\right)^{\prime}$ for all $\eta \in(0, \epsilon)$, is called a singular integral of order $\epsilon$ if $T$ has a distributional kernel $K(x, y)$ which is locally integrable away from the diagonal of $\mathcal{X} \times \mathcal{X}$, and satisfies the following conditions.

(I-1) If $\varphi, \psi \in C_{b}^{\eta}(\mathcal{X})$ have disjoint supports, then

$$
\langle T \varphi, \psi\rangle=\iint_{\chi \times \mathcal{X}} K(x, y) \varphi(y) \psi(x) d \mu(x) d \mu(y) .
$$

(I-2) If $\varphi$ is a normalized $\epsilon$-bump function associated to a ball of radius $r$, then $\|T \varphi\|_{C^{\eta} \eta(\mathcal{X})} \leq C r^{-\eta}$ for all $\eta \in(0, \epsilon)$, where $C>0$ is independent of $\varphi$. More precisely, for each $\eta \in(0, \epsilon)$, there is another $\tilde{\eta} \in(0, \epsilon)$ and a constant $C_{\eta, \tilde{\eta}}>0$ so that whenever $\varphi$ is a $C_{b}^{\tilde{\eta}}(\mathcal{X})$ function supported in a ball $B\left(x_{0}, r\right)$, then $r^{\eta}\|T \varphi\|_{\dot{C}^{\eta}(\mathcal{X})} \leq$ $C_{\eta, \tilde{\eta}} \sup _{\eta_{0} \leq \tilde{\eta}} r^{\eta_{0}}\|\varphi\|_{\dot{C}^{\eta_{0}}(x)}$.

(I-3) There exists a constant $C>0$ such that

(I-3) ${ }_{1}$ for all $x, y \in X$ with $x \neq y,|K(x, y)| \leq C(1 / V(x, y))$; 
(I-3) $)_{2}$ for all $x, x^{\prime}, y \in \mathcal{X}$ with $d\left(x, x^{\prime}\right) \leq d(x, y) / 2$ and $x \neq y$,

$$
\left|K(x, y)-K\left(x^{\prime}, y\right)\right|+\left|K(y, x)-K\left(y, x^{\prime}\right)\right| \leq C \frac{d\left(x, x^{\prime}\right)^{\epsilon}}{V(x, y) d(x, y)^{\epsilon}} .
$$

(I-4) Properties (I-1) through (I-3) also hold with $x$ and $y$ interchanged. That is, these properties also hold for the adjoint operator $T^{t}$ defined by $\left\langle T^{t} \varphi, \psi\right\rangle=\langle\varphi, T \psi\rangle$.

Remark 5.22. We remark that if $T$ is a singular integral operator of order $\epsilon$, then $T$ extends to a continuous linear operator from $C^{\eta}(\mathcal{X})$ to $\left(\dot{\mathcal{G}}_{b}(\eta, \gamma)\right)^{\prime}$ for all $\eta \in(0, \epsilon]$ and all $\gamma>0$ by Proposition 2.12.

We also claim that for $f \in \mathcal{G}_{b}(\eta, \delta)$ with $\eta \in(0, \epsilon]$ and $\delta>0, T f$ can be defined as a distribution in $\left(\mathcal{G}_{0}^{\epsilon}(\beta, \gamma)\right)^{\prime}$ with $0<\beta, \gamma \leq \epsilon$. We first define $T f$ as a distribution in $\left(\mathcal{G}_{\mathcal{G}}(\beta, \gamma)\right)^{\prime}$ with $0<\beta \leq \epsilon$ and $\gamma>0$. In fact, for any given $\beta \in(0, \epsilon]$, noticing that $\dot{\mathcal{G}}_{b}\left(\eta_{1}, \delta\right) \subset \dot{\mathcal{G}}_{b}\left(\eta_{2}, \delta\right)$ when $\eta_{1} \geq \eta_{2}$, without loss of generality, we may assume that $\eta \leq \beta$. Assume that supp $f \subset$ $B\left(x_{0}, r\right)$ for some $x_{0} \in \mathcal{X}$ and $r>0$. Let $\psi \in C_{b}^{\eta}(\mathcal{X})$ such that $\psi(x)=1$ when $x \in B\left(x_{0}, 2 r\right)$ and $\psi(x)=0$ when $x \notin B\left(x_{0}, 4 r\right)$. Choose any $g \in \mathcal{G}(\beta, \gamma)$ with $0<\beta \leq \epsilon$ and $\gamma>0$. It is easy to check that $\psi g \in C_{b}^{\eta}(\mathcal{X})$. Notice that $\dot{\mathcal{G}}_{b}(\eta, \delta) \subset C_{b}^{\eta}(\mathcal{X})$. Both facts show that $\langle T f, \psi g\rangle$ is well defined. On the other hand, we define $\langle T f,(1-\psi) g\rangle$ by

$$
\langle T f,(1-\psi) g\rangle=\iint_{x_{\times} \times \mathcal{X}}\left[K(x, y)-K\left(x, x_{0}\right)\right] f(y)(1-\psi(x)) g(x) d \mu(x) d \mu(y) .
$$

By (I-3) $)_{2}$ and Lemma 2.1(i), it is easy to check that

$$
|\langle T f,(1-\psi) g\rangle| \lesssim\|f\|_{L^{1}(\mathcal{X})}\|g\|_{L^{\infty}(\mathcal{X})} \lesssim\|f\|_{\mathcal{G}(\eta, \delta)}\|g\|_{\mathcal{G}(\beta, \gamma)} .
$$

Moreover, if $g \in \mathcal{G}_{b}(\beta, \gamma)$, since $\int_{x} f(y) d \mu(y)=0$, we then obtain

$$
\langle T f,(1-\psi) g\rangle=\iint_{x \times x} K(x, y) f(y)(1-\psi(x)) g(x) d \mu(x) d \mu(y),
$$

which coincides with (I-1). Furthermore, it is easy to verify that $\langle T f, \psi g\rangle+\langle T f,(1-\psi) g\rangle$ is independent of the choice of $\psi$. Thus, we can define $T f$ by

$$
\langle T f, g\rangle=\langle T f, \psi g\rangle+\langle T f,(1-\psi) g\rangle \text {. }
$$

In this sense, we have $T f \in(\mathcal{G}(\beta, \gamma))^{\prime}$ with $0<\beta \leq \epsilon$ and $\gamma>0$. Now for any $g \in \mathcal{G}_{0}^{\epsilon}(\beta, \gamma)$ with $0<\beta, \gamma \leq \epsilon$, let $\left\{g_{n}\right\}_{n \in \mathbb{N}} \subset \mathcal{G}(\epsilon, \epsilon)$ such that $\left\|g_{n}-g\right\|_{\mathcal{G}(\beta, \gamma)} \rightarrow 0$ as $n \rightarrow \infty$. We then define $T f \in\left(\mathcal{G}_{0}^{\epsilon}(\beta, \gamma)\right)^{\prime}$ with $0<\beta, \gamma \leq \epsilon$ by $\langle T f, g\rangle=\lim _{n \rightarrow \infty}\left\langle T f, g_{n}\right\rangle$. It is easy to check that $\langle T f, g\rangle$ is independent of the choice of $\left\{g_{n}\right\}_{n \in \mathbb{N}} \subset \mathcal{G}(\epsilon, \epsilon)$. In this sense, we have $T f \in\left(\mathcal{G}_{0}^{\epsilon}(\beta, \gamma)\right)^{\prime}$ with $0<\beta, \gamma \leq \epsilon$.

In what follows, for any $\epsilon \in(0,1]$ and $0<\beta, \gamma \leq \epsilon$, we set

$$
\mathcal{\mathcal { G }}_{0, b}^{e}(\beta, \gamma)=\left\{f \in \dot{\mathcal{C}}_{0}^{e}(\beta, \gamma): f \text { has bounded support }\right\} .
$$


Theorem 5.23. Let $\epsilon_{1}, \epsilon_{2}, \epsilon$, and $|s|<\epsilon$ be as in Definition 5.8. Let $T$ be a singular integral of order $\epsilon$ satisfying (I-1) through (I-4). Then $T$ is bounded on $\dot{B}_{p, q}^{s}(\mathcal{X})$ when $p(s, \epsilon)<p<\infty$ and $0<q<\infty$ and bounded from $\dot{B}_{p, q}^{s}(\mathcal{X}) \cap \dot{\mathcal{G}}_{b}\left(\epsilon_{1}, \epsilon_{2}\right)$ to $\dot{B}_{p, q}^{s}(\mathcal{X})$ when $\max \{p, q\}=\infty$, and $T$ is also bounded on $\dot{F}_{p, q}^{s}(\mathcal{X})$ when $p(s, \epsilon)<p, q<\infty$, and bounded from $\dot{F}_{p, q}^{s}(\mathcal{X}) \cap \mathcal{G}_{b}\left(\epsilon_{1}, \epsilon_{2}\right)$ to $\dot{F}_{p, q}^{s}(\mathcal{X})$ when $p(s, \epsilon)<p<\infty$ and $q=\infty$.

Proof. By Propositions 5.21 and 5.10(vi) together with a density argument, to prove the theorem, it suffices to verify the conclusions of the theorem only for all $f \in \mathcal{G}_{0, b}^{e}(\beta, \gamma)$ with $0<\beta, \gamma<\epsilon$ as in (5.35). Let $\left\{S_{k}\right\}_{k \in \mathbb{Z}}$ be an ATI with bounded support as constructed in Theorem 2.6. Put $D_{k}=S_{k}-S_{k-1}$ for $k \in \mathbb{Z}$. We then interpret $D_{k} T=T^{t}\left(D_{k}^{t}\right)$, where $D_{k}^{t}(x, y)=$ $D_{k}(y, x)$ for all $x, y \in \mathcal{X}$ (cf. Lemma 3.12). By Remark 5.22, we have $D_{k} T \in\left(\mathcal{G}_{0}^{\epsilon}(\beta, \gamma)\right)^{\prime}$. On the other hand, let $f \in \mathcal{G}_{0, b}^{e}(\beta, \gamma)$. By Theorem 4.12, we have

$$
f(z)=\sum_{k^{\prime} \in \mathbb{Z}} \sum_{\tau^{\prime} \in I_{k^{\prime}}} \sum_{v^{\prime}=1}^{N\left(k^{\prime}, \tau^{\prime}\right)} \mu\left(Q_{\tau^{\prime}}^{k^{\prime}, v^{\prime}}\right) D_{k^{\prime}}\left(z, y_{\tau^{\prime}}^{k^{\prime}, v^{\prime}}\right) \bar{D}_{k^{\prime}}(f)\left(y_{\tau^{\prime}}^{k^{\prime}, v^{\prime}}\right)
$$

where the series converges in $\mathcal{G}_{0}^{\epsilon}(\beta, \gamma)$ and all the notation is as in Theorem 4.12. Thus, for all $x \in \mathcal{x}$, we have

$$
D_{k} T f(x)=\sum_{k^{\prime} \in \mathbb{Z}} \sum_{\tau^{\prime} \in I_{k^{\prime}}} \sum_{v^{\prime}=1}^{N\left(k^{\prime}, \tau^{\prime}\right)} \mu\left(Q_{\tau^{\prime}}^{k^{\prime}, v^{\prime}}\right)\left(D_{k} T D_{k^{\prime}}\left(\cdot, y_{\tau^{\prime}}^{k^{\prime}, v^{\prime}}\right)\right)(x) \bar{D}_{k^{\prime}}(f)\left(y_{\tau^{\prime}}^{k^{\prime}, v^{\prime}}\right)
$$

For $k, k^{\prime} \in \mathbb{Z}, \tau^{\prime} \in I_{k^{\prime}}, v^{\prime}=1, \ldots, N\left(k^{\prime}, \tau^{\prime}\right), y_{\tau^{\prime}}^{k^{\prime}, v^{\prime}} \in Q_{\tau^{\prime}}^{k^{\prime}, v^{\prime}}$, and $x \in \mathcal{X}$, let

$$
J\left(x, y_{\tau^{\prime}}^{k^{\prime}, v^{\prime}}\right) \equiv\left(D_{k} T D_{k^{\prime}}\left(\cdot, y_{\tau^{\prime}}^{k^{\prime}, v^{\prime}}\right)\right)(x)
$$

We now claim that for any fixed $\eta \in(0, \epsilon)$,

$$
\begin{aligned}
& \left|J\left(x, y_{\tau^{\prime}}^{k^{\prime}, v^{\prime}}\right)\right| \\
& \quad \lesssim 2^{-\left|k-k^{\prime}\right| \eta}\left(\frac{2^{-\left(k \wedge k^{\prime}\right)}}{2^{-\left(k \wedge k^{\prime}\right)}+d\left(x, y_{\tau^{\prime}}^{k^{\prime}, v^{\prime}}\right)}\right)^{\eta} \frac{1}{V_{2^{-\left(k \wedge k^{\prime}\right)}}(x)+V_{2^{-\left(k \wedge k^{\prime}\right)}}\left(y_{\tau^{\prime}}^{k^{\prime}, \nu^{\prime}}\right)+V\left(x, y_{\tau^{\prime}}^{k^{\prime}, v^{\prime}}\right)} .
\end{aligned}
$$

To verify (5.65), by symmetry, we only need to verify that if $k \leq k^{\prime}$, then

$$
\left|J\left(x, y_{\tau^{\prime}}^{k^{\prime}, v^{\prime}}\right)\right| \lesssim 2^{\left(k-k^{\prime}\right) \eta} \frac{1}{V_{2^{-k}}(x)}
$$

and moreover, if $d\left(x, y_{\tau^{\prime}}^{k^{\prime}, v^{\prime}}\right) \geq 2^{5-k}$, then

$$
\left|J\left(x, y_{\tau^{\prime}}^{k^{\prime}, v^{\prime}}\right)\right| \lesssim\left(\frac{2^{-k^{\prime}}}{d\left(x, y_{\tau^{\prime}}^{k^{\prime}, v^{\prime}}\right)}\right)^{\eta} \frac{1}{V\left(x, y_{\tau^{\prime}}^{k^{\prime}, v^{\prime}}\right)}
$$


To see this, for $y \in \mathcal{X}$, set

$$
\Psi(y)=\int_{x} K(z, y) D_{k}(x, z) d \mu(z) .
$$

Notice that for any fixed $x \in \mathcal{X}, D_{k}(x, z)$ is an adjusted bump function in $z$ associated with the ball $B\left(x, 2^{2-k}\right)$. Conditions (I-2) and (I-4) show that for any fixed $\eta \in(0, \epsilon)$,

$$
2^{-k \eta}\|\Psi\|_{\dot{C}^{\eta}(x)} \lesssim \frac{1}{V_{2^{-k}}(x)},
$$

which further implies that for all $y \in \mathcal{X}$,

$$
\left|\Psi(y)-\Psi\left(y_{\tau^{\prime}}^{k^{\prime}, v^{\prime}}\right)\right| \lesssim \frac{d\left(y, y_{\tau^{\prime}}^{k^{\prime}, v^{\prime}}\right)^{\eta}}{2^{-k \eta}} \frac{1}{V_{2^{-k}}(x)}
$$

Thus,

$$
\begin{aligned}
\left|J\left(x, y_{\tau^{\prime}}^{k^{\prime}, v^{\prime}}\right)\right| & =\left|\int_{x} \Psi(y) D_{k^{\prime}}\left(y, y_{\tau^{\prime}}^{k^{\prime}, v^{\prime}}\right) d \mu(y)\right| \\
& =\left|\int_{x}\left[\Psi(y)-\Psi\left(y_{\tau^{\prime}}^{k^{\prime}, v^{\prime}}\right)\right] D_{k^{\prime}}\left(y, y_{\tau^{\prime}}^{k^{\prime}, v^{\prime}}\right) d \mu(y)\right| \\
& \lesssim 2^{\left(k-k^{\prime}\right) \eta} \frac{1}{V_{2^{-k}}(x)} .
\end{aligned}
$$

That is, (5.66) holds.

On the other hand, when $k \leq k^{\prime}, z \in B\left(x, 2^{2-k}\right), y \in B\left(y_{\tau^{\prime}}^{k^{\prime}, v^{\prime}}, 2^{2-k^{\prime}}\right)$, and $d\left(x, y_{\tau^{\prime}}^{k^{\prime}, v^{\prime}}\right) \geq$ $2^{5-k}$, then by Condition (I-3), we have

$$
\left|K(z, y)-K\left(z, y_{\tau^{\prime}}^{k^{\prime}, v^{\prime}}\right)\right| \lesssim \frac{2^{-k^{\prime} \epsilon}}{d\left(x, y_{\tau^{\prime}}^{k^{\prime}, v^{\prime}}\right)^{\epsilon} V\left(x, y_{\tau^{\prime}}^{k^{\prime}, v^{\prime}}\right)}
$$

which yields that

$$
\begin{aligned}
\left|J\left(x, y_{\tau^{\prime}}^{k^{\prime}, v^{\prime}}\right)\right| & =\left|\iint_{x \times x}\left[K(z, y)-K\left(z, y_{\tau^{\prime}}^{k^{\prime}, v^{\prime}}\right)\right] D_{k}(z, x) D_{k^{\prime}}\left(y, y_{\tau^{\prime}}^{k^{\prime}, v^{\prime}}\right) d \mu(y) d \mu(z)\right| \\
& \lesssim\left(\frac{2^{-k^{\prime}}}{d\left(x, y_{\tau^{\prime}}^{k^{\prime}, v^{\prime}}\right)}\right)^{\eta} \frac{1}{V\left(x, y_{\tau^{\prime}}^{k^{\prime}, v^{\prime}}\right)}
\end{aligned}
$$

namely, (5.67) holds. Thus, (5.65) holds.

Notice that the estimate (5.65) is analogous to the estimate (5.10) in the proof of Proposition 5.4. We can further proceed from here on exactly as in the proof of Proposition 5.4 in order to obtain the boundedness of $T$ on $\dot{B}_{p, q}^{s}(\mathcal{X})$ and $\dot{F}_{p, q}^{s}(\mathcal{X})$. This finishes the proof of Theorem 5.16. 


\subsection{Inhomogeneous Plancherel-Pôlya inequalities and definition of $B_{p, q}^{s}(X)$ and $F_{p, q}^{s}(X)$}

Throughout this and the next subsection, $\mu(\mathcal{X})$ can be finite or infinite. We first introduce the norms in $B_{p, q}^{s}(\mathcal{X})$ and $F_{p, q}^{s}(\mathcal{X})$ via certain IATI and then verify that these norms are independent of the choices of IATIs and spaces of distributions; see [37]. To this end, we need to establish inhomogeneous Plancherel-Pôlya inequalities; see [80]. In what follows, for any dyadic cube $Q$, we set $m_{Q}(f)=(1 / \mu(Q)) \int_{Q} f(x) d \mu(x)$.

Definition 5.24. Let $\epsilon_{1} \in(0,1], \epsilon_{2}>0, \epsilon_{3}>0, \epsilon \in\left(0, \epsilon_{1} \wedge \epsilon_{2}\right)$, and let $\left\{S_{k}\right\}_{k \in \mathbb{Z}_{+}}$be an $\left(\epsilon_{1}, \epsilon_{2}, \epsilon_{3}\right)$ IATI. Set $D_{0}=S_{0}$ and $D_{k}=S_{k}-S_{k-1}$ for $k \in \mathbb{N}$. Let $\left\{Q_{\tau}^{0, v}: \tau \in I_{0}, v=1, \ldots, N(0, \tau)\right\}$ with a fixed large $j \in \mathbb{N}$ be dyadic cubes as in Section 4 .

(i) For all $f \in\left(\mathcal{G}_{0}^{\epsilon}(\beta, \gamma)\right)^{\prime}$ with $0<\beta, \gamma<\epsilon,|s|<\epsilon, p(s, \epsilon)<p \leq \infty$, and $0<q \leq \infty$, we define

$$
\|f\|_{B_{p, q}^{s}(\mathcal{X})}=\left\{\sum_{\tau \in I_{0}} \sum_{\nu=1}^{N(0, \tau)} \mu\left(Q_{\tau}^{0, v}\right)\left[m_{Q_{\tau}^{0, v}}\left(\left|D_{0}(f)\right|\right)\right]^{p}\right\}^{1 / p}+\left\{\sum_{k=1}^{\infty} 2^{k s q}\left\|D_{k}(f)\right\|_{L^{p}(\mathcal{X})}^{q}\right\}^{1 / q}
$$

with the usual modification made when $p=\infty$ or $q=\infty$.

(ii) For all $f \in\left(\mathcal{G}_{0}^{\epsilon}(\beta, \gamma)\right)^{\prime}$ with $0<\beta, \gamma<\epsilon,|s|<\epsilon, p(s, \epsilon)<p<\infty$, and $p(s, \epsilon)<q \leq$ $\infty$, we define

$$
\|f\|_{F_{p, q}^{s}(x)}=\left\{\sum_{\tau \in I_{0}} \sum_{\nu=1}^{N(0, \tau)} \mu\left(Q_{\tau}^{0, v}\right)\left[m_{Q_{\tau}^{0, v}}\left(\left|D_{0}(f)\right|\right)\right]^{p}\right\}^{1 / p}+\left\|\left\{\sum_{k=1}^{\infty} 2^{k s q}\left|D_{k}(f)\right|^{q}\right\}^{1 / q}\right\|_{L^{p}(\mathcal{X})}
$$

with the usual modification made when $q=\infty$.

The following theorem is the inhomogeneous Plancherel-Pôlya inequalities.

Proposition 5.25. Let $\epsilon_{1} \in(0,1], \epsilon_{2}>0, \epsilon_{3}>0, \epsilon \in\left(0, \epsilon_{1} \wedge \epsilon_{2}\right)$, and let $\left\{S_{k}\right\}_{k \in \mathbb{Z}_{+}}$and $\left\{P_{k}\right\}_{k \in \mathbb{Z}_{+}}$ be two $\left(\epsilon_{1}, \epsilon_{2}, \epsilon_{3}\right)$-IATIs. Set $D_{0}=S_{0}$ and $Q_{0}=P_{0}$, and $D_{k}=S_{k}-S_{k-1}$ and $Q_{k}=P_{k}-P_{k-1}$ for $k \in \mathbb{N}$. Let $\left\{Q_{\tau}^{0, v}: \tau \in I_{0}, v=1, \ldots, N(0, \tau)\right\}$ with a fixed large $j \in \mathbb{N}$ be dyadic cubes as in Section 4.

(i) For all $f \in\left(\mathcal{G}_{0}^{\epsilon}(\beta, \gamma)\right)^{\prime}$ with $0<\beta, \gamma<\epsilon,|s|<\epsilon, p(s, \epsilon)<p \leq \infty$, and $0<q \leq \infty$,

$$
\begin{aligned}
\left\{\sum_{\tau \in I_{0}}\right. & \left.\sum_{\nu=1}^{N(0, \tau)} \mu\left(Q_{\tau}^{0, v}\right)\left[m_{Q_{\tau}^{0, v}}\left(\left|D_{0}(f)\right|\right)\right]^{p}\right\}^{1 / p} \\
& +\left\{\sum_{k=1}^{\infty} 2^{k s q}\left(\sum_{\tau \in I_{k}} \sum_{\nu=1}^{N(k, \tau)} \mu\left(Q_{\tau}^{k, v}\right)\left[\sup _{z \in Q_{\tau}^{k, \nu}}\left|D_{k}(f)(z)\right|\right]^{p}\right)^{q / p}\right\}^{1 / q}
\end{aligned}
$$


Yongsheng Han et al.

$$
\begin{aligned}
& \sim\left\{\sum_{\tau \in I_{0}} \sum_{\nu=1}^{N(0, \tau)} \mu\left(Q_{\tau}^{0, v}\right)\left[m_{Q_{\tau}^{0, v}}\left(\left|Q_{0}(f)\right|\right)\right]^{p}\right\}^{1 / p} \\
& +\left\{\sum_{k=1}^{\infty} 2^{k s q}\left(\sum_{\tau \in I_{k}} \sum_{\nu=1}^{N(k, \tau)} \mu\left(Q_{\tau}^{k, v}\right)\left[\inf _{z \in Q_{\tau}^{k, v}}\left|Q_{k}(f)(z)\right|\right]^{p}\right)^{q / p}\right\}^{1 / q}
\end{aligned}
$$

with the usual modification made when $p=\infty$ or $q=\infty$.

(ii) For all $f \in\left(\mathcal{G}_{0}^{\epsilon}(\beta, \gamma)\right)^{\prime}$ with $0<\beta, \gamma<\epsilon,|s|<\epsilon, p(s, \epsilon)<p<\infty$, and $p(s, \epsilon)<q \leq \infty$,

$$
\begin{aligned}
\left\{\sum_{\tau \in I_{0}}\right. & \left.\left.\sum_{\nu=1}^{N(0, \tau)} \mu\left(Q_{\tau}^{0, v}\right)\left[m_{Q_{\tau}^{0, v}}|| D_{0}(f) \mid\right)\right]^{p}\right\}^{1 / p} \\
& +\left\|\left\{\sum_{k=1}^{\infty} \sum_{\tau \in I_{k}} \sum_{v=1}^{N(k, \tau)} 2^{k s q}\left[\sup _{z \in Q_{\tau}^{k, v}}\left|D_{k}(f)(z)\right|\right]^{q} x_{Q_{\tau}^{k, v}}\right\}^{1 / q}\right\| \|_{L^{p}(x)} \\
\sim & \left.\left\{\sum_{\tau \in I_{0}} \sum_{v=1}^{N(0, \tau)} \mu\left(Q_{\tau}^{0, v}\right)\left[m_{Q_{\tau}^{0, v}}|| Q_{0}(f) \mid\right)\right]^{p}\right\}^{1 / p} \\
& +\left\|\left\{\sum_{k=1}^{\infty} \sum_{\tau \in I_{k}} \sum_{v=1}^{N(k, \tau)} 2^{k s q}\left[\inf _{z \in Q_{\tau}^{k, v}}\left|Q_{k}(f)(z)\right|\right]^{q} X_{Q_{\tau}^{k, \nu}}\right\}^{1 / q}\right\| \|_{L^{p}(x)}
\end{aligned}
$$

with the usual modification made when $q=\infty$.

Proof. We first verify (5.76). By Theorem 4.16 together with Remark 4.17, there exists a family of functions $\left\{\widetilde{D}_{k^{\prime}}(x, y)\right\}_{k^{\prime} \in \mathbb{Z}_{+}}$such that for all $f \in\left(\mathcal{G}_{0}^{\epsilon}(\beta, \gamma)\right)^{\prime}$ with $0<\beta, \gamma<\epsilon$ and all $z \in \mathcal{X}$,

$$
\begin{aligned}
f(z)= & \sum_{\tau^{\prime} \in I_{0}} \sum_{v^{\prime}=1}^{N\left(0, \tau^{\prime}\right)} \int_{Q_{\tau^{\prime}}^{0, v^{\prime}}} \tilde{D}_{0}(x, y) d \mu(y) Q_{\tau^{\prime}, 1}^{0, v^{\prime}}(f) \\
& +\sum_{k^{\prime}=1}^{\infty} \sum_{\tau^{\prime} \in I_{k^{\prime}}} \sum_{v^{\prime}=1}^{N\left(k^{\prime}, \tau^{\prime}\right)} \mu\left(Q_{\tau^{\prime}}^{k^{\prime}, v^{\prime}}\right) \tilde{D}_{k^{\prime}}\left(z, y_{\tau^{\prime}}^{k^{\prime}, v^{\prime}}\right) Q_{k^{\prime}}(f)\left(y_{\tau^{\prime}}^{k^{\prime}, v^{\prime}}\right),
\end{aligned}
$$

where $Q_{\tau^{\prime}, 1}^{0, v^{\prime}}$ denotes the integral operator with kernel

$$
Q_{\tau^{\prime}, 1}^{0, v^{\prime}}(z)=\frac{1}{\mu\left(Q_{\tau^{\prime}}^{0, v^{\prime}}\right)} \int_{Q_{\tau^{\prime}}^{0, y^{\prime}}} Q_{0}(u, z) d \mu(u)
$$


and $\left\{\widetilde{D}_{k^{\prime}}\right\}_{k^{\prime} \in \mathbb{Z}_{+}}$satisfy the same conditions as $\left\{\widetilde{D}_{k}\right\}_{k \in \mathbb{Z}_{+}}$in Theorem 4.14. From (5.78) together with

$$
\begin{aligned}
\left|Q_{\tau^{\prime}, 1}^{0, v^{\prime}}(f)\right| & =\left|\int_{\chi} Q_{\tau^{\prime}, 1}^{0, v^{\prime}}(z) f(z) d \mu(z)\right| \\
& =\left|\frac{1}{\mu\left(Q_{\tau^{\prime}}^{0, v^{\prime}}\right)} \int_{Q_{\tau^{\prime}}^{0, v^{\prime}}} Q_{0}(f)(u) d \mu(u)\right| \\
& \leq m_{Q_{\tau^{\prime}}^{0, v^{\prime}}}\left(\left|Q_{0}(f)\right|\right),
\end{aligned}
$$

it follows that

$$
\begin{aligned}
m_{Q_{\tau}^{0, v}}\left(\left|D_{0}(f)\right|\right)= & \frac{1}{\mu\left(Q_{\tau}^{0, v}\right)} \int_{Q_{\tau}^{0, v}}\left|D_{0}(f)(z)\right| d \mu(z) \\
\leq & \left.\sum_{\tau^{\prime} \in I_{0}} \sum_{v^{\prime}=1}^{N\left(0, \tau^{\prime}\right)} m_{Q_{\tau^{\prime}}^{0, v^{\prime}}}\left|Q_{0}(f)\right|\right) \frac{1}{\mu\left(Q_{\tau}^{0, v}\right)} \int_{Q_{\tau^{\prime}, v}^{0, v}} \int_{Q_{\tau^{\prime}}^{0, v^{\prime}}}\left|\left(D_{0} \tilde{D}_{0}\right)(z, y)\right| d \mu(y) d \mu(z) \\
& +\sum_{k^{\prime}=1}^{\infty} \sum_{\tau^{\prime} \in I_{k^{\prime}}} \sum_{v^{\prime}=1}^{N\left(k^{\prime}, \tau^{\prime}\right)}\left|Q_{k^{\prime}}(f)\left(y_{\tau^{\prime}}^{k^{\prime}, v^{\prime}}\right)\right| \mu\left(Q_{\tau^{\prime}}^{k^{\prime}, v^{\prime}}\right) \frac{1}{\mu\left(Q_{\tau}^{0, v}\right)} \int_{Q_{\tau}^{0, v}}\left|\left(D_{0} \tilde{D}_{k^{\prime}}\right)\left(z, y_{\tau^{\prime}}^{k^{\prime}, v^{\prime}}\right)\right| d \mu(z) \\
& \equiv Z_{1}+Z_{2} .
\end{aligned}
$$

By Lemma 3.19 together with Remark 3.3, we have that for any $\epsilon \in\left(0, \epsilon_{1} \wedge \epsilon_{2}\right)$, all $k^{\prime} \in \mathbb{Z}_{+}$and all $z, y \in \mathcal{X}$,

$$
\left|\left(D_{0} \widetilde{D}_{k^{\prime}}\right)(z, y)\right| \lesssim 2^{-k^{\prime} \epsilon} \frac{1}{V_{1}(z)+V_{1}(y)+V(z, y)} \frac{1}{(1+d(z, y))^{\epsilon}}
$$

From this together with Lemma 2.1(iii), it follows that

$$
\sup _{z \in Q_{\tau^{\prime}}^{0, v}} \sup _{y \in Q_{\tau^{\prime}}^{0, y^{\prime}}}\left|\left(D_{0} \widetilde{D}_{0}\right)(z, y)\right| \lesssim \inf _{z \in Q_{\tau}^{0, v}} \inf _{y \in Q_{\tau^{\prime}}^{0, y^{\prime}}} \frac{1}{V_{1}(z)+V_{1}(y)+V(z, y)} \frac{1}{(1+d(z, y))^{\epsilon}}
$$

Thus,

$$
Z_{1} \lesssim \sum_{\tau^{\prime} \in I_{0}} \sum_{v^{\prime}=1}^{N\left(0, \tau^{\prime}\right)} m_{Q_{\tau^{\prime}}^{0, v^{\prime}}}\left(\left|Q_{0}(f)\right|\right) \mu\left(Q_{\tau^{\prime}}^{0, v^{\prime}}\right) \inf _{z \in Q_{\tau}^{0, v}} \inf _{y \in Q_{\tau^{\prime}}^{0, v^{\prime}}} \frac{1}{V_{1}(z)+V_{1}(y)+V(z, y)} \frac{1}{(1+d(z, y))^{\epsilon}}
$$


Therefore, if $n /(n+\epsilon)<p \leq 1$, by (5.5) and Lemma 5.2, we then have

$$
\begin{aligned}
\left\{\sum_{\tau \in I_{0}} \sum_{\nu=1}^{N(0, \tau)} \mu\left(Q_{\tau}^{0, v}\right)\left[Z_{1}\right]^{p}\right\}^{1 / p} & \lesssim\left\{\sum_{\tau^{\prime} \in I_{0}} \sum_{v^{\prime}=1}^{N\left(0, \tau^{\prime}\right)}\left[m_{Q_{\tau^{\prime}}^{0, v^{\prime}}}\left(\left|Q_{0}(f)\right|\right)\right]^{p}\left[\mu\left(Q_{\tau^{\prime}}^{0, v^{\prime}}\right)\right]^{p}\left[V_{1}\left(z_{\tau^{\prime}}^{0, v^{\prime}}\right)\right]^{1-p}\right\}^{1 / p} \\
& \lesssim\left\{\sum_{\tau^{\prime} \in I_{0}} \sum_{v^{\prime}=1}^{N\left(0, \tau^{\prime}\right)} \mu\left(Q_{\tau^{\prime}}^{0, v^{\prime}}\right)\left[m_{Q_{\tau^{\prime}}^{0, v^{\prime}}}\left(\left|Q_{0}(f)\right|\right)\right]^{p}\right\}^{1 / p},
\end{aligned}
$$

where in the last step, we used the fact that for any $y_{\tau^{\prime}}^{0, v^{\prime}} \in Q_{\tau^{\prime}}^{0, v^{\prime}}$,

$$
V_{1}\left(y_{\tau^{\prime}}^{0, v^{\prime}}\right) \sim \mu\left(Q_{\tau^{\prime}}^{0, v^{\prime}}\right)
$$

If $1<p \leq \infty$, by Hölder's inequality and Lemma 5.2, we have

$$
Z_{1} \lesssim\left\{\sum_{\tau^{\prime} \in I_{0}} \sum_{\nu^{\prime}=1}^{N\left(0, \tau^{\prime}\right)}\left[m_{Q_{\tau^{\prime}}^{0, v^{\prime}}}\left(\left|Q_{0}(f)\right|\right)\right]^{p} \mu\left(Q_{\tau^{\prime}}^{0, v^{\prime}}\right) \inf _{z \in Q_{\tau^{\prime}}^{0, p}} \inf _{y \in Q_{\tau^{\prime}}^{0, y^{\prime}}} \frac{1}{V_{1}(z)+V_{1}(y)+V(z, y)} \frac{1}{(1+d(z, y))^{\epsilon}}\right\}^{1 / p},
$$

which together with Lemma 5.2 again yields that

$$
\left\{\sum_{\tau \in I_{0}} \sum_{\nu=1}^{N(0, \tau)} \mu\left(Q_{\tau}^{0, v}\right)\left[Z_{1}\right]^{p}\right\}^{1 / p} \lesssim\left\{\sum_{\tau^{\prime} \in I_{0}} \sum_{\nu^{\prime}=1}^{N\left(0, \tau^{\prime}\right)} \mu\left(Q_{\tau^{\prime}}^{0, v^{\prime}}\right)\left[m_{Q_{\tau^{\prime}}^{0, \nu^{\prime}}}\left(\left|Q_{0}(f)\right|\right)\right]^{p}\right\}^{1 / p}
$$

This is the desired estimate.

From (5.82), it also follows that

$$
\sup _{z \in Q_{\tau}^{0, v}}\left|\left(D_{0} \widetilde{D}_{k^{\prime}}\right)\left(z, y_{\tau^{\prime}}^{k^{\prime}, v^{\prime}}\right)\right| \lesssim 2^{-k^{\prime} \epsilon} \inf _{z \in Q_{\tau}^{0, \nu}} \frac{1}{V_{1}(z)+V_{1}\left(y_{\tau^{\prime}}^{k^{\prime}, v^{\prime}}\right)+V\left(z, y_{\tau^{\prime}}^{k^{\prime}, v^{\prime}}\right)} \frac{1}{\left(1+d\left(z, y_{\tau^{\prime}}^{k^{\prime}, v^{\prime}}\right)\right)^{\epsilon}}
$$

which proves that

$$
\begin{aligned}
Z_{2} \lesssim \sum_{k^{\prime}=1}^{\infty} 2^{-k^{\prime} \epsilon} \sum_{\tau^{\prime} \in I_{k^{\prime}}} \sum_{v^{\prime}=1}^{N\left(k^{\prime}, \tau^{\prime}\right)} & \mu\left(Q_{\tau^{\prime}}^{k^{\prime}, v^{\prime}}\right)\left|Q_{k^{\prime}}(f)\left(y_{\tau^{\prime}}^{k^{\prime}, v^{\prime}}\right)\right| \\
& \times \inf _{z \in Q_{\tau}^{0, v}} \frac{1}{V_{1}(z)+V_{1}\left(y_{\tau^{\prime}}^{k^{\prime}, v^{\prime}}\right)+V\left(z, y_{\tau^{\prime}}^{k^{\prime}, v^{\prime}}\right)} \frac{1}{\left(1+d\left(z, y_{\tau^{\prime}}^{k^{\prime}, v^{\prime}}\right)\right)^{\epsilon}}
\end{aligned}
$$


Notice also that by Lemma 2.19 and (1.3), for any $y_{\tau^{\prime}}^{k^{\prime}, v^{\prime}} \in Q_{\tau^{\prime}}^{k^{\prime}, v^{\prime}}, V_{1}\left(y_{\tau^{\prime}}^{k^{\prime}, v^{\prime}}\right) \lesssim 2^{-k^{\prime} n} \mu\left(Q_{\tau^{\prime}}^{k^{\prime}, v^{\prime}}\right)$. If $p(s, \epsilon)<p \leq 1$, then this fact together with (5.5) and Lemma 5.2 proves that

$$
\left\{\sum_{\tau \in I_{0}} \sum_{\nu=1}^{N(0, \tau)} \mu\left(Q_{\tau}^{0, v}\right)\left[Z_{2}\right]^{p}\right\}^{1 / p} \lesssim\left\{\sum_{k^{\prime}=1}^{\infty} 2^{k^{\prime} s q}\left(\sum_{\tau^{\prime} \in I_{k^{\prime}}} \sum_{\nu^{\prime}=1}^{N\left(k^{\prime}, \tau^{\prime}\right)} \mu\left(Q_{\tau^{\prime}}^{k^{\prime}, \nu^{\prime}}\right)\left|Q_{k^{\prime}}(f)\left(y_{\tau^{\prime}}^{k^{\prime}, v^{\prime}}\right)\right|^{p}\right)^{q / p}\right\}^{1 / q}
$$

where in the last step, we used (5.5) when $q / p \leq 1$ or Hölder's inequality when $q / p>1$.

If $1<p \leq \infty$, then by Hölder's inequality and Lemma 5.2, we first have

$$
\begin{aligned}
Z_{2} \lesssim\left\{\sum_{k^{\prime}=1}^{\infty} 2^{-k^{\prime} \epsilon^{\prime} p} \sum_{\tau^{\prime} \in I_{k^{\prime}}} \sum_{v^{\prime}=1}^{N\left(k^{\prime}, \tau^{\prime}\right)}\right. & \mu\left(Q_{\tau^{\prime}}^{k^{\prime}, v^{\prime}}\right)\left|Q_{k^{\prime}}(f)\left(y_{\tau^{\prime}}^{k^{\prime}, v^{\prime}}\right)\right|^{p} \\
& \left.\quad \times \inf _{z \in Q_{\tau}^{0, v}} \frac{1}{V_{1}(z)+V_{1}\left(y_{\tau^{\prime}}^{k^{\prime}, v^{\prime}}\right)+V\left(z, y_{\tau^{\prime}}^{k^{\prime}, v^{\prime}}\right)} \frac{1}{\left(1+d\left(z, y_{\tau^{\prime}}^{k^{\prime}, v^{\prime}}\right)\right)^{\epsilon}}\right\}^{1 / p},
\end{aligned}
$$

where $\max \{-s, 0\}<\epsilon^{\prime}<\epsilon$. This together with Lemma 5.2 again shows that

$$
\left\{\sum_{\tau \in I_{0}} \sum_{\nu=1}^{N(0, \tau)} \mu\left(Q_{\tau}^{0, v}\right)\left[Z_{2}\right]^{p}\right\}^{1 / p} \lesssim\left\{\sum_{k^{\prime}=1}^{\infty} 2^{k^{\prime} s q}\left(\sum_{\tau^{\prime} \in I_{k^{\prime}}} \sum_{\nu^{\prime}=1}^{N\left(k^{\prime}, \tau^{\prime}\right)} \mu\left(Q_{\tau^{\prime}}^{k^{\prime}, v^{\prime}}\right)\left|Q_{k^{\prime}}(f)\left(y_{\tau^{\prime}}^{k^{\prime}, v^{\prime}}\right)\right|^{p}\right)^{q / p}\right\}^{1 / q}
$$

where we used (5.5) when $q / p \leq 1$ or Hölder's inequality when $q / p>1$.

All the above estimates together with the arbitrary choice of $y_{\tau^{\prime}}^{k^{\prime}, v^{\prime}}$ yield that the first term in the left-hand side of (5.76) is controlled by its right-hand side.

We now verify that the second term of the left-hand side of (5.76) is also controlled by its right-hand side. To this end, by (5.78),

$$
\begin{aligned}
\left|D_{k}(f)(z)\right| \lesssim & \sum_{\tau^{\prime} \in I_{0}} \sum_{v^{\prime}=1}^{N\left(0, \tau^{\prime}\right)} m_{Q_{\tau^{\prime}}^{0, v^{\prime}}}\left(\left|Q_{0}(f)\right|\right) \int_{Q_{\tau^{\prime}}^{0, v^{\prime}}}\left|\left(D_{k} \widetilde{D}_{0}\right)(z, y)\right| d \mu(y) \\
& +\sum_{k^{\prime}=1}^{\infty} \sum_{\tau^{\prime} \in I_{k^{\prime}}} \sum_{v^{\prime}=1}^{N\left(k^{\prime}, \tau^{\prime}\right)} \mu\left(Q_{\tau^{\prime}}^{k^{\prime}, v^{\prime}}\right)\left|\left(D_{k} \widetilde{D}_{k^{\prime}}\right)\left(z, y_{\tau^{\prime}}^{k^{\prime}, v^{\prime}}\right)\right|\left|Q_{k^{\prime}}(f)\left(y_{\tau^{\prime}}^{k^{\prime}, v^{\prime}}\right)\right| \\
\equiv & Y_{1}+Y_{2} .
\end{aligned}
$$


The estimate for $Y_{2}$ is as in (5.7), and we only need to estimate $Y_{1}$. In this case, by Lemma 3.19 together with Remark 3.3, for any $\epsilon \in\left(0, \epsilon_{1} \wedge \epsilon_{2}\right)$, we have

$$
\begin{aligned}
& \sup _{z \in Q_{\tau}^{k, v}} Y_{1} \\
& \quad \lesssim 2^{-k \epsilon} \sum_{\tau^{\prime} \in I_{0}} \sum_{v^{\prime}=1}^{N\left(0, \tau^{\prime}\right)} m_{Q_{\tau^{\prime}}^{0, v^{\prime}}}\left(\left|Q_{0}(f)\right|\right) \mu\left(Q_{\tau^{\prime}}^{0, v^{\prime}}\right) \inf _{z \in Q_{\tau}^{k, v},} \inf _{y \in Q_{\tau^{\prime}}^{0, y^{\prime}}} \frac{1}{V_{1}(z)+V_{1}(y)+V(z, y)} \frac{1}{(1+d(z, y))^{\epsilon}},
\end{aligned}
$$

and therefore, if $n /(n+\epsilon)<p \leq 1$ and $s<\epsilon$, by (5.5) and Lemma 5.2 together with (5.86), we obtain

$$
\begin{gathered}
\left\{\sum_{k=1}^{\infty} 2^{k s q}\left(\sum_{\tau \in I_{k}} \sum_{v=1}^{N(k, \tau)} \mu\left(Q_{\tau}^{k, v}\right)\left[\sup _{z \in Q_{\tau^{k}}^{k, v}} Y_{1}\right]^{p}\right)^{q / p}\right\}^{1 / q} \\
\lesssim\left\{\sum_{\tau^{\prime} \in I_{0}} \sum_{v^{\prime}=1}^{N\left(0, \tau^{\prime}\right)} \mu\left(Q_{\tau^{\prime}}^{0, v^{\prime}}\right)\left[m_{Q_{\tau^{\prime}}^{0, v^{\prime}}}\left(\left|Q_{0}(f)\right|\right)\right]^{p}\right\}^{1 / p},
\end{gathered}
$$

while when $1<p \leq \infty$, by Hölder's inequality and Lemma 5.2,

$$
\begin{gathered}
\left\{\sum_{k=1}^{\infty} 2^{k s q}\left(\sum_{\tau \in I_{k}} \sum_{\nu=1}^{N(k, \tau)} \mu\left(Q_{\tau}^{k, \nu}\right)\left[\sup _{z \in Q_{\tau^{k}, \nu}^{k}} Y_{1}\right]^{p}\right)^{q / p}\right\}^{1 / q} \\
\lesssim\left\{\sum_{\tau^{\prime} \in I_{0}} \sum_{\nu^{\prime}=1}^{N\left(0, \tau^{\prime}\right)} \mu\left(Q_{\tau^{\prime}}^{0, v^{\prime}}\right)\left[m_{Q_{\tau^{\prime}}^{0, v^{\prime}}}\left(\left|Q_{0}(f)\right|\right)\right]^{p}\right\}^{1 / p} .
\end{gathered}
$$

All these estimates imply that the left-hand side of (5.76) is controlled by its right-hand side, which together with the symmetry verifies (5.76).

Similarly, to establish (5.77), we only need to verify that its left-hand side is controlled by its right-hand side. To this end, the estimates for $Z_{1}$ are still valid for the current case. To estimate $Z_{2}$, by Lemma 5.3 and (5.5) when $q \leq 1$ or Hölder's inequality when $1<q \leq \infty$, we have that for $p(s, \epsilon)<r<\min \{1, p, q\}$,

$$
\begin{aligned}
\left\{\sum_{\tau \in I_{0}}\right. & \left.\sum_{\nu=1}^{N(0, \tau)} \mu\left(Q_{\tau}^{0, v}\right)\left[Z_{2}\right]^{p}\right\}^{1 / p} \\
& \lesssim\left\|\left\{\sum_{k^{\prime}=1}^{\infty}\left[M\left(\sum_{\tau^{\prime} \in I_{k^{\prime}}} \sum_{v^{\prime}=1}^{N\left(k^{\prime}, \tau^{\prime}\right)} 2^{k^{\prime} s r}\left|Q_{k^{\prime}}(f)\left(y_{\tau^{\prime}}^{k^{\prime}, v^{\prime}}\right)\right|^{r} X_{Q_{\tau^{\prime}}^{k^{\prime}, v^{\prime}}}\right)\right]^{q / r}\right\}^{1 / q}\right\|_{L^{p}(x)} \\
& \lesssim\left\|\left\{\sum_{k^{\prime}=1}^{\infty} \sum_{\tau^{\prime} \in I_{k^{\prime}}} \sum_{v^{\prime}=1}^{N\left(k^{\prime}, \tau^{\prime}\right)} 2^{k^{\prime} s q}\left|Q_{k^{\prime}}(f)\left(y_{\tau^{\prime}}^{k^{\prime}, v^{\prime}}\right)\right|^{q} X_{Q_{\tau^{\prime}}^{k^{\prime}, v^{\prime}}}\right\}^{1 / q}\right\|_{L^{p}(x)^{\prime}},
\end{aligned}
$$

where in the last step, we used Lemma 3.14. This is the desired estimate. 
The estimation for $Y_{2}$ is as in the proof of (5.8). To finish the proof of (5.77), we still need to estimate $Y_{1}$. In what follows, we set $a=1$ when $p / q \leq 1$ and $a=q / p$ when $p / q>1$. The estimates (5.95) and (5.5) when $p / q \leq 1$ or Hölder's inequality when $p / q>1$ show that

$$
\begin{aligned}
\|\left\{\sum_{k=1}^{\infty}\right. & \left.\sum_{\tau \in I_{k}} \sum_{v=1}^{N(k, \tau)} 2^{k s q}\left[\sup _{z \in Q_{\tau}^{k, v}} Y_{1}\right]^{q} X_{Q_{\tau}^{k, v}}\right\}^{1 / q} \|_{L^{p}(x)}^{p} \\
\lesssim & \sum_{k=1}^{\infty} 2^{k(s-e) p a} \sum_{\tau \in I_{k}} \sum_{\nu=1}^{N(k, \tau)} \mu\left(Q_{\tau}^{k, v}\right) \\
& \times\left[\sum_{\tau^{\prime} \in I_{0}} \sum_{v^{\prime}=1}^{N\left(0, \tau^{\prime}\right)} \mu\left(Q_{\tau^{\prime}}^{0, v^{\prime}}\right) m_{Q_{\tau^{\prime}}^{0, \gamma^{\prime}}}\left(\left|Q_{0}(f)\right|\right) \inf _{z \in Q_{\tau}^{k, v}} \inf _{y \in Q_{\tau^{\prime}}^{0, v^{\prime}}} \frac{1}{V_{1}(z)+V_{1}(y)+V(z, y)} \frac{1}{(1+d(z, y))^{\epsilon}}\right]^{p} .
\end{aligned}
$$

If $n /(n+\epsilon)<p \leq 1$, by (5.5), $s<\epsilon$, and Lemma 5.2 together with (5.82),

$$
\left\|\left\{\sum_{k=1}^{\infty} \sum_{\tau \in I_{k}} \sum_{\nu=1}^{N(k, \tau)} 2^{k s q}\left[\sup _{z \in Q_{\tau}^{k, \nu}} Y_{1}\right]^{q} X_{Q_{\tau}^{k, \nu}}\right\}^{1 / q}\right\|_{L^{p}(\mathcal{X})}^{p} \lesssim \sum_{\tau^{\prime} \in I_{0}} \sum_{\nu^{\prime}=1}^{N\left(0, \tau^{\prime}\right)} \mu\left(Q_{\tau^{\prime}}^{0, v^{\prime}}\right)\left[m_{Q_{\tau^{\prime}}^{0, v^{\prime}}}\left(\left|Q_{0}(f)\right|\right)\right]^{p}
$$

and if $1<p \leq \infty$, by Hölder's inequality and Lemma 5.2,

$$
\left\|\left\{\sum_{k=1}^{\infty} \sum_{\tau \in I_{k}} \sum_{\nu=1}^{N(k, \tau)} 2^{k s q}\left[\sup _{z \in Q_{\tau^{k}}^{k, \nu}} Y_{1}\right]^{q} x_{Q_{\tau}^{k, \nu}}\right\}^{1 / q}\right\|_{L^{p}(\boldsymbol{X})}^{p} \lesssim \sum_{\tau^{\prime} \in I_{0}} \sum_{\nu^{\prime}=1}^{N\left(0, \tau^{\prime}\right)} \mu\left(Q_{\tau^{\prime}}^{0, \nu^{\prime}}\right)\left[m_{Q_{\tau^{\prime}}^{0, \nu^{\prime}}}\left(\left|Q_{0}(f)\right|\right)\right]^{p},
$$

which completes the proof of (5.77), and hence, that of Proposition 5.25.

Remark 5.26. We point out that Remark 5.5 applies in a similar way to Proposition 5.25.

Similarly to Proposition 5.6, using Proposition 5.4, we can verify that the definitions of $\|\cdot\|_{B_{p, q}^{s}(\mathcal{x})}$ and $\|\cdot\|_{F_{p, q}^{s}(\mathcal{X})}$ are independent of the choices of IATIs. We omit the details.

Proposition 5.27. Let all the notation be as in Proposition 5.25.

(i) For all $f \in\left(\mathcal{G}_{0}^{\epsilon}(\beta, \gamma)\right)^{\prime}$ with $0<\beta, \gamma<\epsilon,|s|<\epsilon, p(s, \epsilon)<p \leq \infty$, and $0<q \leq \infty$,

$$
\begin{aligned}
& \left\{\sum_{\tau \in I_{0}} \sum_{\nu=1}^{N(0, \tau)} \mu\left(Q_{\tau}^{0, v}\right)\left[m_{Q_{\tau}^{0, v}}\left(\left|D_{0}(f)\right|\right)\right]^{p}\right\}^{1 / p}+\left\{\sum_{k=1}^{\infty} 2^{k s q}\left\|D_{k}(f)\right\|_{L^{p}(\mathcal{q})}^{q}\right\}^{1 / q} \\
& \sim\left\{\sum_{\tau \in I_{0}} \sum_{\nu=1}^{N(0, \tau)} \mu\left(Q_{\tau}^{0, v}\right)\left[m_{Q_{\tau}^{0, v}}\left(\left|Q_{0}(f)\right|\right)\right]^{p}\right\}^{1 / p}+\left\{\sum_{k=1}^{\infty} 2^{k s q}\left\|Q_{k}(f)\right\|_{L^{p}(\mathcal{x})}^{q}\right\}^{1 / q}
\end{aligned}
$$

with the usual modification made when $p=\infty$ or $q=\infty$. 
(ii) For all $f \in\left(\mathcal{G}_{0}^{\epsilon}(\beta, \gamma)\right)^{\prime}$ with $0<\beta, \gamma<\epsilon,|s|<\epsilon, p(s, \epsilon)<p<\infty$, and $p(s, \epsilon)<q \leq \infty$,

$$
\begin{aligned}
\left\{\sum_{\tau \in I_{0}}\right. & \left.\sum_{v=1}^{N(0, \tau)} \mu\left(Q_{\tau}^{0, v}\right)\left[m_{Q_{\tau}^{0, v}}\left(\left|D_{0}(f)\right|\right)\right]^{p}\right\}^{1 / p}+\left\|\left\{\sum_{k=1}^{\infty} 2^{k s q}\left|D_{k}(f)\right|^{q}\right\}^{1 / q}\right\|_{L^{p}(\mathcal{X})} \\
& \left.\sim\left\{\sum_{\tau \in I_{0}} \sum_{\nu=1}^{N(0, \tau)} \mu\left(Q_{\tau}^{0, v}\right)\left[m_{Q_{\tau}^{0, v}}\left|Q_{0}(f)\right|\right)\right]^{p}\right\}^{1 / p}+\left\|\left\{\sum_{k=1}^{\infty} 2^{k s q}\left|Q_{k}(f)\right|^{q}\right\}^{1 / q}\right\|_{L^{p}(\mathcal{X})}
\end{aligned}
$$

with the usual modification made when $q=\infty$.

We now verify that the definitions of the norm $\|\cdot\|_{B_{p, q}^{s}(x)}$ and the norm $\|\cdot\|_{F_{p, q}^{s}(x)}$ are independent of the choice of the underlying space of distributions as follows.

Proposition 5.28. Let all the notation be as in Definition 5.24.

(i) Let $|s|<\epsilon, p(s, \epsilon)<p \leq \infty$, and $0<q \leq \infty$. If $f \in\left(\mathcal{G}_{0}^{\epsilon}\left(\beta_{1}, \gamma_{1}\right)\right)^{\prime}$ with

$$
\max \left\{0,-s+n\left(\frac{1}{p}-1\right)_{+}\right\}<\beta_{1}<\epsilon, \quad n\left(\frac{1}{p}-1\right)_{+}<\gamma_{1}<\epsilon,
$$

and if $\|f\|_{B_{p, q}^{s}(x)}<\infty$, then $f \in\left(\mathcal{G}_{0}^{\epsilon}\left(\beta_{2}, \gamma_{2}\right)\right)^{\prime}$ for every $\beta_{2}, \gamma_{2}$ satisfying (5.104).

(ii) Let $|s|<\epsilon, p(s, \epsilon)<p<\infty$, and $p(s, \epsilon)<q \leq \infty$. If $f \in\left(\mathcal{G}_{0}^{\epsilon}\left(\beta_{1}, \gamma_{1}\right)\right)^{\prime}$ with $\beta_{1}, \gamma_{1}$ as in (5.104), and if $\|f\|_{F_{p, q}^{s}(x)}<\infty$, then $f \in\left(\mathcal{G}_{0}^{\epsilon}\left(\beta_{2}, \gamma_{2}\right)\right)^{\prime}$ for every $\beta_{2}, \gamma_{2}$ satisfying (5.104).

Proof. Similarly to the proof of Proposition 5.7, we only need to verify (i). Let $\psi \in \mathcal{G}(\epsilon, \epsilon)$ and all the notation as in Theorem 4.14 with $N=0$. We first claim that for $k \in \mathbb{Z}_{+}$,

$$
\left|\left\langle\widetilde{D}_{k}(\cdot, y), \psi\right\rangle\right| \lesssim 2^{-k \beta_{2}}\|\psi\|_{\mathcal{G}\left(\beta_{2}, \gamma_{2}\right)} \frac{1}{V_{1}\left(x_{1}\right)+V\left(x_{1}, y\right)} \frac{1}{\left(1+d\left(x_{1}, y\right)\right)^{\gamma_{2}}}
$$

In fact, if $k \in \mathbb{N}$, (5.105) is just (5.24). If $k=0$, then

$$
\begin{aligned}
& \left|\left\langle\widetilde{D}_{0}(\cdot, y), \psi\right\rangle\right| \\
& \quad=\left|\int_{x} \tilde{D}_{0}(z, y) \psi(z) d \mu(z)\right| \\
& \quad \lesssim\|\psi\|_{\mathcal{G}\left(\beta_{2}, \gamma_{2}\right)} \int_{x} \frac{1}{V_{1}(z)+V_{1}(y)+V(z, y)} \frac{1}{(1+d(z, y))^{\epsilon}} \frac{1}{V_{1}\left(x_{1}\right)+V\left(x_{1}, z\right)} \frac{1}{\left(1+d\left(x_{1}, z\right)\right)^{\gamma_{2}}} d \mu(z) .
\end{aligned}
$$


Notice that $V_{1}\left(x_{1}\right)+V\left(x_{1}, z\right) \sim V_{1}\left(x_{1}\right)+V_{1}(z)+V\left(x_{1}, z\right)$. By Lemma 4.4, we have

$$
\begin{aligned}
\left|\left\langle\widetilde{D}_{0}(\cdot, y), \psi\right\rangle\right| & \lesssim\|\psi\|_{\mathcal{G}\left(\beta_{2}, \gamma_{2}\right)} \frac{1}{V_{1}\left(x_{1}\right)+V_{1}(y)+V\left(x_{1}, y\right)}\left\{\frac{1}{\left(1+d\left(x_{1}, y\right)\right)^{\epsilon}}+\frac{1}{\left(1+d\left(x_{1}, y\right)\right)^{\gamma_{2}}}\right\} \\
& \lesssim\|\psi\|_{\mathcal{G}\left(\beta_{2}, \gamma_{2}\right)} \frac{1}{V_{1}\left(x_{1}\right)+V\left(x_{1}, y\right)} \frac{1}{\left(1+d\left(x_{1}, y\right)\right)^{\gamma_{2}}},
\end{aligned}
$$

which shows that (5.105) also holds when $k=0$.

From Theorem 4.16 together with (5.80), (5.105), and Lemma 2.1(iii), it follows that

$$
\begin{aligned}
& |\langle f, \psi\rangle| \\
& =\left|\sum_{\tau \in I_{0}} \sum_{\nu=1}^{N(0, \tau)} \int_{Q_{\tau}^{0, v}}\left\langle\widetilde{D}_{0}(\cdot, y), \psi\right\rangle d \mu(y) D_{\tau, 1}^{0, v}(f)+\sum_{k=1}^{\infty} \sum_{\tau \in I_{k}} \sum_{\nu=1}^{N(k, \tau)} \mu\left(Q_{\tau}^{k, v}\right)\left\langle\widetilde{D}_{k}\left(\cdot, y_{\tau}^{k, v}\right), \psi\right\rangle D_{k}(f)\left(y_{\tau}^{k, v}\right)\right| \\
& \lesssim\|\psi\|_{\mathcal{G}\left(\beta_{2}, \gamma_{2}\right)}\left\{\sum_{\tau \in I_{0}} \sum_{\nu=1}^{N(0, \tau)} \mu\left(Q_{\tau}^{0, v}\right) m_{Q_{\tau}^{0, v}}\left(\left|D_{0}(f)\right|\right) \frac{1}{V_{1}\left(x_{1}\right)+V\left(x_{1}, y_{\tau}^{0, \nu}\right)} \frac{1}{\left(1+d\left(x_{1}, y_{\tau}^{0, \nu}\right)\right)^{\gamma_{2}}}\right. \\
& \left.+\sum_{k=1}^{\infty} 2^{-k \beta_{2}} \sum_{\tau \in I_{k}} \sum_{\nu=1}^{N(k, \tau)} \mu\left(Q_{\tau}^{k, \nu}\right)\left|D_{k}(f)\left(y_{\tau}^{k, v}\right)\right| \frac{1}{V_{1}\left(x_{1}\right)+V\left(x_{1}, y_{\tau}^{k, v}\right)} \frac{1}{\left(1+d\left(x_{1}, y_{\tau}^{k, v}\right)\right)^{\gamma_{2}}}\right\} .
\end{aligned}
$$

Notice that if $p \leq 1$ and $\gamma_{2}>n(1 / p-1)$, then (5.30) with $\beta_{2}=0$ still holds. Thus, when $p \leq 1$, by this fact and (5.5), we have

$$
\begin{aligned}
|\langle f, \psi\rangle| \lesssim\|\psi\|_{\mathcal{G}\left(\beta_{2}, \gamma_{2}\right)} & \left\{\sum_{\tau \in I_{0}} \sum_{\nu=1}^{N(0, \tau)} \mu\left(Q_{\tau}^{0, v}\right)\left[m_{Q_{\tau}^{0, v}}\left(\left|D_{0}(f)\right|\right)\right]^{p}\right. \\
& \left.+\sum_{k=1}^{\infty} 2^{-k\left[\beta_{2} p-n(1-p)+s p\right]} 2^{k s p}\left(\sum_{\tau \in I_{k}} \sum_{v=1}^{N(k, \tau)} \mu\left(Q_{\tau}^{k, v}\right)\left|D_{k}(f)\left(y_{\tau}^{k, v}\right)\right|^{p}\right)\right\}^{1 / p}
\end{aligned}
$$

$\lesssim\|\psi\|_{\mathcal{G}\left(\beta_{2}, \gamma_{2}\right)}\|f\|_{B_{p, q}^{s}(x)}$,

where in the last inequality, we used the assumption that $\beta_{2}>-s+n(1 / p-1)$ when $p \leq 1$, and (5.5) when $q / p \leq 1$ or Hölder's inequality when $q / p>1$. 
If $1<p \leq \infty$, by Hölder's inequality, we have

$$
\begin{aligned}
&|\langle f, \psi\rangle| \lesssim\|\psi\|_{\mathcal{G}\left(\beta_{2}, \gamma_{2}\right)}\{\left(\sum_{\tau \in I_{0}} \sum_{\nu=1}^{N(0, \tau)} \mu\left(Q_{\tau}^{0, \nu}\right)\left[m_{Q_{\tau}^{0, v}}\left(\left|D_{0}(f)\right|\right)\right]^{p}\right)^{1 / p} \\
& \times\left[\int_{\chi} \frac{1}{V_{1}\left(x_{1}\right)+V\left(x_{1}, y\right)} \frac{1}{\left(1+d\left(x_{1}, y\right)\right)^{\gamma_{2}}} d \mu(y)\right]^{1 / p^{\prime}} \\
&+\sum_{k=1}^{\infty} 2^{-k \beta_{2}}\left(\sum_{\tau \in I_{k}} \sum_{v=1}^{N(k, \tau)} \mu\left(Q_{\tau}^{k, v}\right)\left|D_{k}(f)\left(y_{\tau}^{k, v}\right)\right|^{p}\right)^{1 / p} \\
&\left.\times\left[\int_{\chi} \frac{1}{V_{1}\left(x_{1}\right)+V\left(x_{1}, y\right)} \frac{1}{\left(1+d\left(x_{1}, y\right)\right)^{\gamma_{2}}} d \mu(y)\right]^{1 / p^{\prime}}\right\} \\
& \lesssim\|\psi\|_{\mathcal{G}\left(\beta_{2}, \gamma_{2}\right)}\|f\|_{B_{,, q}^{s}(\mathcal{x}),}
\end{aligned}
$$

here, again, we used the assumption $\beta_{2}>-s$ in this case, and (5.5) when $q \leq 1$ or Hölder's inequality when $q>1$.

Using (5.109) and (5.110) together with an argument similar to that used in the proof of Proposition 5.7 then completes the proof of Proposition 5.28.

We can now introduce the Besov spaces $B_{p, q}^{s}(\mathcal{X})$ and Triebel-Lizorkin spaces $F_{p, q}^{s}(\mathcal{X})$.

Definition 5.29. Let $\epsilon_{1} \in(0,1], \epsilon_{2}>0, \epsilon_{3}>0, \epsilon \in\left(0, \epsilon_{1} \wedge \epsilon_{2}\right)$, and let $\left\{S_{k}\right\}_{k \in \mathbb{Z}_{+}}$be an $\left(\epsilon_{1}, \epsilon_{2}, \epsilon_{3}\right)$ IATI. Set $D_{0}=S_{0}$ and $D_{k}=S_{k}-S_{k-1}$ for $k \in \mathbb{N}$. Let $\left\{Q_{\tau}^{0, v}: \tau \in I_{0}, v=1, \ldots, N(0, \tau)\right\}$ with a fixed large $j \in \mathbb{N}$ be dyadic cubes as in Section 4 .

(i) Let $|s|<\epsilon, p(s, \epsilon)<p \leq \infty$, and $0<q \leq \infty$. The space $B_{p, q}^{s}(\mathcal{X})$ is defined to be the set of all $f \in\left(\mathcal{G}_{0}^{\epsilon}(\beta, \gamma)\right)^{\prime}$, for some $\beta, \gamma$ satisfying

$$
\max \left\{s, 0,-s+n\left(\frac{1}{p}-1\right)_{+}\right\}<\beta<\epsilon, \quad n\left(\frac{1}{p}-1\right)_{+}<\gamma<\epsilon
$$

such that

$$
\|f\|_{B_{p, q}^{s}(\mathcal{X})}=\left\{\sum_{\tau \in I_{0}} \sum_{\nu=1}^{N(0, \tau)} \mu\left(Q_{\tau}^{0, v}\right)\left[m_{Q_{\tau}^{0, v}}\left(\left|D_{0}(f)\right|\right)\right]^{p}\right\}^{1 / p}+\left\{\sum_{k=1}^{\infty} 2^{k s q}\left\|D_{k}(f)\right\|_{L^{p}(\mathcal{X})}^{q}\right\}^{1 / q}<\infty
$$

with the usual modifications made when $p=\infty$ or $q=\infty$. 
(ii) Let $|s|<\epsilon, p(s, \epsilon)<p<\infty$, and $p(s, \epsilon)<q \leq \infty$. The space $F_{p, q}^{s}(\mathcal{X})$ is defined to be the set of all $f \in\left(\mathcal{G}_{0}^{\epsilon}(\beta, \gamma)\right)^{\prime}$ for some $\beta, \gamma$ satisfying (5.111) such that

$$
\|f\|_{F_{p, q}^{s}(\mathcal{X})}=\left\{\sum_{\tau \in I_{0}} \sum_{v=1}^{N(0, \tau)} \mu\left(Q_{\tau}^{0, v}\right)\left[m_{Q_{\tau}^{0, v}}\left(\left|D_{0}(f)\right|\right)\right]^{p}\right\}^{1 / p}+\left\|\left\{\sum_{k=1}^{\infty} 2^{k s q}\left|D_{k}(f)\right|^{q}\right\}^{1 / q}\right\|_{L^{p}(\mathcal{X})}<\infty
$$

with the usual modification made when $q=\infty$.

Remark 5.30. Propositions 5.27 and 5.28 show that the definitions of the spaces $B_{p, q}^{s}(\mathcal{X})$ and $F_{p, q}^{s}(\mathcal{X})$ are independent of the choice of inhomogeneous approximations of the identity as in Definition 2.2 and the distribution space $\left(\mathcal{G}_{0}^{\epsilon}(\beta, \gamma)\right)^{\prime}$ with $\beta$ and $\gamma$ satisfying (5.111). We also remark that to guarantee that the definitions of the spaces $B_{p, q}^{s}(\mathcal{X})$ and $F_{p, q}^{s}(\mathcal{X})$ are independent of the choice of the distribution space $\left(\mathcal{G}_{0}^{\epsilon}(\beta, \gamma)\right)^{\prime}$, we only need the restriction that $\beta$ and $\gamma$ satisfy (5.104). Moreover, if we assume that $\max \{0, s\}<\beta<\epsilon$ and $n(1 / p-1)_{+}<$ $\gamma<\epsilon$, then we can verify that $\mathcal{G}(\beta, \gamma)$ is contained in $B_{p, q}^{s}(\mathcal{X})$ and $F_{p, q}^{s}(\mathcal{X})$; see Proposition 5.31 below.

\subsection{Properties of $B_{p, q}^{s}(X)$ and $F_{p, q}^{s}(X)$ and boundedness of singular integrals}

In this subsection, we first present some basic properties of $B_{p, q}^{s}(\mathcal{X})$ and $F_{p, q}^{s}(\mathcal{X})$. Then we establish a Lusin-area characterization of the spaces $F_{p, q}^{s}(\mathcal{X})$ and the relations between the spaces $\dot{B}_{p, q}^{s}(\mathcal{X})$ and $B_{p, q}^{s}(\mathcal{X})$ and between the spaces $\dot{F}_{p, q}^{s}(\mathcal{X})$ and $F_{p, q}^{s}(\mathcal{X})$. Using the Lusin-area characterization of $F_{p, q}^{s}(\mathcal{X})$, we also obtain the relation between the spaces $F_{p, q}^{s}(\mathcal{X})$ and the local Hardy spaces $h^{p}(\mathcal{X})$ in the sense of Goldberg [73]. Finally, we obtain the boundedness on $B_{p, q}^{s}(\mathcal{X})$ and $F_{p, q}^{s}(\mathcal{X})$ of singular integrals with some natural extra size and regularity conditions of Nagel-Stein type in [44].

The following proposition is an inhomogeneous version of Proposition 5.10.

Proposition 5.31. Let $\epsilon_{1} \in(0,1], \epsilon_{2}>0, \epsilon \in\left(0, \epsilon_{1} \wedge \epsilon_{2}\right)$, and $|s|<\epsilon$.

(i) For $p(s, \epsilon)<p \leq \infty$ and $0<q_{0} \leq q_{1} \leq \infty, B_{p, q_{0}}^{s}(\mathcal{X}) \subset B_{p, q_{1}}^{s}(\mathcal{X})$; and for $p(s, \epsilon)<p<\infty$ and $p(s, \epsilon)<q_{0} \leq q_{1} \leq \infty, F_{p, q_{0}}^{s}(\mathcal{X}) \subset F_{p, q_{1}}^{s}(\mathcal{X})$.

(ii) Let $-\epsilon<s+\theta<\epsilon$ and $\theta>0$. Then for $p(s, \epsilon)<p \leq \infty$ and $0<q_{0}, q_{1} \leq \infty, B_{p, q_{0}}^{s+\theta}(\mathcal{X}) \subset$ $B_{p, q_{1}}^{s}(\mathcal{X})$; and for $p(s, \epsilon)<p<\infty$ and $p(s, \epsilon)<q_{0}, q_{1} \leq \infty, F_{p, q_{0}}^{s+\theta}(\mathcal{X}) \subset F_{p, q_{1}}^{s}(\mathcal{X})$.

(iii) If $p(s, \epsilon)<p<\infty$ and $p(s, \epsilon)<q \leq \infty$, then $B_{p, \min (p, q)}^{s}(\mathcal{X}) \subset F_{p, q}^{s}(\mathcal{X}) \subset B_{p, \max (p, q)}^{s}(\mathcal{X})$.

(iv) If $\beta, \gamma$ as in (5.104), then $B_{p, q}^{s}(\mathcal{X}) \subset\left(\mathcal{G}_{0}^{\epsilon}(\beta, \gamma)\right)^{\prime}$ when $p(s, \epsilon)<p \leq \infty$ and $0<q \leq \infty$, and $F_{p, q}^{s}(\mathcal{X}) \subset\left(\mathcal{G}_{0}^{\epsilon}(\beta, \gamma)\right)^{\prime}$ when $p(s, \epsilon)<p<\infty$ and $p(s, \epsilon)<q \leq \infty$.

(v) If $\max \{s, 0\}<\beta<\epsilon$ and $n(1 / p-1)_{+}<\gamma<\epsilon$, then $\mathcal{G}(\beta, \gamma) \subset B_{p, q}^{s}(\mathcal{X})$ when $p(s, \epsilon)<$ $p \leq \infty$ and $0<q \leq \infty$, and $\mathcal{G}(\beta, \gamma) \subset F_{p, q}^{s}(\mathcal{X})$ when $p(s, \epsilon)<p<\infty$ and $p(s, \epsilon)<q \leq \infty$.

(vi) If $1<p<\infty$, then $F_{p, 2}^{0}(X)=L^{p}(X)$ with equivalent norms.

(vii) The spaces $B_{p, q}^{s}(\mathcal{X})$ with $p(s, \epsilon)<p \leq \infty$ and $0<q \leq \infty$ and the spaces $F_{p, q}^{s}(\mathcal{X})$ with $p(s, \epsilon)<p<\infty$ and $p(s, \epsilon)<q \leq \infty$ are complete. 
Proof. Property (i) is a simple corollary of (5.5). To see (ii), we notice that

$$
\left\{\sum_{k=0}^{\infty} 2^{s k q_{1}}\left|b_{k}\right|^{q_{1}}\right\}^{1 / q_{1}} \leq \sup _{k \in \mathbb{Z}_{+}} 2^{(s+\theta) k}\left|b_{k}\right|\left\{\sum_{l=0}^{\infty} 2^{-\theta l q_{1}}\right\}^{1 / q_{1}} \lesssim \sup _{k \in \mathbb{Z}_{+}} 2^{(s+\theta) k}\left|b_{k}\right|,
$$

which combined with (i) verifies (ii); see also [3, the proof of Proposition 2.3.2/2].

The proof of (iii) is similar to that of Property (ii) in Proposition 5.10; see also [3, 6].

Property (iv) is implied by the proof of Proposition 5.28, and Property (vii) can be easily deduced from Property (iv) and Property (vi) is just Proposition 3.30.

To see Property (v), similarly to the proof of (5.105), for $f \in \mathcal{G}(\beta, \gamma)$, we have that for all $k \in \mathbb{Z}_{+}$and $x \in \mathcal{X}$,

$$
\left|D_{k}(f)(x)\right| \lesssim 2^{-k \beta}\|f\|_{\mathcal{G}(\beta, \gamma)} \frac{1}{V_{1}\left(x_{1}\right)+V\left(x_{1}, x\right)} \frac{1}{\left(1+d\left(x_{1}, x\right)\right)^{\gamma}} .
$$

Notice that (5.39) with $\beta=0$ is still true when $\gamma>n(1 / p-1)_{+}$. From this fact and $\beta>s$, it follows that

$$
\begin{aligned}
&\|f\|_{B_{p, q}^{s}(x)} \sim\left\{\sum_{\tau \in I_{0}} \sum_{v=1}^{N(0, \tau)} \mu\left(Q_{\tau}^{0, v}\right)\left[m_{Q_{\tau}^{0, v}}\left(\left|D_{0}(f)\right|\right)\right]^{p}\right\}^{1 / p}+\left\{\sum_{k=1}^{\infty} 2^{k s q}\left\|D_{k}(f)\right\|_{L^{p}(\mathcal{q})}^{q}\right\}^{1 / q} \\
& \lesssim\|f\|_{\mathcal{G}(\beta, \gamma)}\left\{\left[\sum_{\tau \in I_{0}} \sum_{\nu=1}^{N(0, \tau)} \mu\left(Q_{\tau}^{0, v}\right) \frac{1}{\left(V_{1}\left(x_{1}\right)+V\left(x_{1}, y_{\tau}^{0, v}\right)\right)^{p}} \frac{1}{\left(1+d\left(x_{1}, y_{\tau}^{0, v}\right)\right)^{\gamma p}}\right]^{1 / p}\right. \\
&\left.+\left[\sum_{k=1}^{\infty} 2^{k s q} 2^{-k \beta q}\right]^{1 / q}\right\} \\
& \lesssim\|f\|_{\mathcal{G}(\beta, \gamma)} .
\end{aligned}
$$

Thus, $\mathcal{G}(\beta, \gamma) \subset B_{p, q}^{s}(\mathcal{X})$, which together with (i) also proves that $\mathcal{G}(\beta, \gamma) \subset F_{p, q}^{s}(\mathcal{X})$. This verifies (v) and hence, finishes the proof of Proposition 5.31.

When $p, q \geq 1$, the following theorem implies that the norms of $\|\cdot\|_{B_{p, q}^{s}(\mathcal{x})}$ and $\|\cdot\|_{F_{p, q}^{s}(\mathcal{x})}$ have the following equivalent and simple version.

Proposition 5.32. Let all the notation be as in Proposition 5.25.

(i) For all $f \in\left(\mathcal{G}_{0}^{\epsilon}(\beta, \gamma)\right)^{\prime}$ with $0<\beta, \gamma<\epsilon,|s|<\epsilon, 1 \leq p \leq \infty$, and $0<q \leq \infty$,

$$
\begin{gathered}
\left\{\sum_{\tau \in I_{0}} \sum_{v=1}^{N(0, \tau)} \mu\left(Q_{\tau}^{0, v}\right)\left[m_{Q_{\tau}^{0, v}}\left(\left|D_{0}(f)\right|\right)\right]^{p}\right\}^{1 / p}+\left\{\sum_{k=1}^{\infty} 2^{k s q}\left\|D_{k}(f)\right\|_{L^{p}(x)}^{q}\right\}^{1 / q} \\
\sim\left\{\sum_{k=0}^{\infty} 2^{k s q}\left\|D_{k}(f)\right\|_{L^{p}(x)}^{q}\right\}^{1 / q}
\end{gathered}
$$

with the usual modification made when $p=\infty$ or $q=\infty$. 
(ii) For all $f \in\left(\mathcal{G}_{0}^{\epsilon}(\beta, \gamma)\right)^{\prime}$ with $0<\beta, \gamma<\epsilon,|s|<\epsilon, 1 \leq p<\infty$, and $p(s, \epsilon)<q \leq \infty$,

$$
\begin{gathered}
\left.\left\{\sum_{\tau \in I_{0}} \sum_{v=1}^{N(0, \tau)} \mu\left(Q_{\tau}^{0, v}\right)\left[m_{Q_{\tau}^{0, v}}|| D_{0}(f) \mid\right)\right]^{p}\right\}^{1 / p}+\left\|\left\{\sum_{k=1}^{\infty} 2^{k s q}\left|D_{k}(f)\right|^{q}\right\}^{1 / q}\right\|_{L^{p}(\mathcal{X})} \\
\sim\left\|\left\{\sum_{k=0}^{\infty} 2^{k s q}\left|D_{k}(f)\right|^{q}\right\}^{1 / q}\right\| \|_{L^{p}(\mathcal{X})}
\end{gathered}
$$

with the usual modification made when $q=\infty$.

Proof. We first verify (5.117). To see this, by $p \geq 1$, Hölder's inequality, and Lemma 2.19, we have

$$
\begin{aligned}
\left\{\sum_{\tau \in I_{0}} \sum_{\nu=1}^{N(0, \tau)} \mu\left(Q_{\tau}^{0, \nu}\right)\left[m_{Q_{\tau}^{0, v}}\left(\left|D_{0}(f)\right|\right)\right]^{p}\right\}^{1 / p} & \leq\left\{\sum_{\tau \in I_{0}} \sum_{\nu=1}^{N(0, \tau)} \mu\left(Q_{\tau}^{0, v}\right) m_{Q_{\tau}^{0, v}}\left(\left|D_{0}(f)\right|^{p}\right)\right\}^{1 / p} \\
& =\left\|D_{0}(f)\right\|_{L^{p}(\mathcal{X})^{\prime}}
\end{aligned}
$$

which shows that the left-hand side of (5.117) is controlled by its right-hand side.

To see the converse, by Lemma 2.19 and Theorem 4.16 together with a proof similar to that of (5.76) in Proposition 5.25, we have that for all $f \in\left(\mathcal{G}_{0}^{\epsilon}(\beta, \gamma)\right)^{\prime}$ with $0<\beta, \gamma<\epsilon$, and $|s|<\epsilon$,

$$
\begin{aligned}
\left\|D_{0}(f)\right\|_{L^{p}(x)} & \left\{\sum_{\tau \in I_{0}} \sum_{v=1}^{N(0, \tau)} \int_{Q_{\tau}^{0, v}}\left|D_{0}(f)(z)\right|^{p} d \mu(z)\right\}^{1 / p} \\
\leq & \left\{\sum_{\tau \in I_{0}} \sum_{v=1}^{N(0, \tau)} \mu\left(Q_{\tau}^{0, v}\right)\left[\sup _{z \in Q_{\tau}^{0, v}}\left|D_{0}(f)(z)\right|\right]^{p}\right\}^{1 / p} \\
\lesssim & \left\{\sum_{\tau \in I_{0}} \sum_{v=1}^{N(0, \tau)} \mu\left(Q_{\tau}^{0, v}\right)\left[m_{Q_{\tau}^{0, v}}\left(\left|D_{0}(f)\right|\right)\right]^{p}\right\}^{1 / p} \\
& +\left\{\sum_{k=1}^{\infty} 2^{k s q}\left(\sum_{\tau \in I_{k}} \sum_{v=1}^{N(k, \tau)} \mu\left(Q_{\tau}^{k, v}\right)\left[\inf _{z \in Q_{\tau}^{k, v}}\left|D_{k}(f)(z)\right|\right]^{p}\right)^{q / p}\right\}^{1 / q},
\end{aligned}
$$

which is, up to a bounded multiplicative constant, controlled by the left-hand side of (5.117). This completes the proof of (5.117).

The estimate (5.119) also proves that the left-hand side of (5.118) is controlled by its right-hand side. The converse inequality can be proved by a way similar to that of (5.77) in Proposition 5.25, which completes the proof of Proposition 5.32.

We next give an inhomogeneous Lusin-area characterization for the Triebel-Lizorkin spaces $F_{p, q}^{s}(\mathcal{X})$. 
Definition 5.33. Let $s \in \mathbb{R}, a>0, q \in(0, \infty]$, and let $\epsilon_{1} \in(0,1], \epsilon_{2}>0, \epsilon_{3}>0, \epsilon \in\left(0, \epsilon_{1} \wedge \epsilon_{2}\right]$. Let $\left\{S_{k}\right\}_{k \in \mathbb{Z}_{+}}$be an $\left(\epsilon_{1}, \epsilon_{2}, \epsilon_{3}\right)$-IATI. Set $D_{0}=S_{0}$ and $D_{k}=S_{k}-S_{k-1}$ for $k \in \mathbb{N}$. The inhomogeneous Lusin-area function (also called the inhomogeneous Littlewood-Paley $S$-function) $S_{q, a}^{s}(f)(x)$ for any $f \in\left(\mathcal{G}_{0}^{\epsilon}(\beta, \gamma)\right)^{\prime}$ with $0<\beta, \gamma \leq \epsilon$ and $x \in \mathcal{X}$ is given by

$$
S_{q, a}^{s}(f)(x)=\left\{\sum_{k=0}^{\infty} \int_{d(x, y)<a 2^{-k}} 2^{k s q}\left|D_{k}(f)(y)\right|^{q} \frac{d \mu(y)}{V_{a 2^{-k}}(x)}\right\}^{1 / q},
$$

where the usual modification is made when $q=\infty$.

Theorem 5.34. Let $a>0, \epsilon_{1} \in(0,1], \epsilon_{2}>0, \epsilon_{3}>0, \epsilon \in\left(0, \epsilon_{1} \wedge \epsilon_{2}\right)$, and let $\left\{S_{k}\right\}_{k \in \mathbb{Z}_{+}}$be an $\left(\epsilon_{1}, \epsilon_{2}, \epsilon_{3}\right)$-IATI with $C_{6} 2^{1-j} \leq a$. Set $D_{0}=S_{0}$ and $D_{k}=S_{k}-S_{k-1}$ for $k \in \mathbb{Z}$. Let $|s|<\epsilon$, $p(s, \epsilon)<p<\infty, 1 \leq q \leq \infty$, and let $S_{q, a}^{s}(f)$ be as in Definition 5.33 for any $f \in\left(\mathcal{G}_{0}^{\epsilon}(\beta, \gamma)\right)^{\prime}$ with $\beta, \gamma$ as in (5.111). Then $f \in F_{p, q}^{s}(\mathcal{X})$ if and only if $f \in\left(\mathcal{G}_{0}^{\epsilon}(\beta, \gamma)\right)^{\prime}$ for some $\beta, \gamma$ as in (5.111) and $S_{q, a}^{s}(f) \in L^{p}(\mathcal{X})$. Moreover, in this case,

$$
\|f\|_{F_{p, q}^{s}(\chi)} \sim\left\|S_{q, a}^{s}(f)\right\|_{L^{p}(\mathcal{X})} .
$$

Proof. Similarly to the proof of Theorem 5.13, there exists a constant $C>0$ such that

$$
\begin{aligned}
& \left\|S_{q, a}^{s}(f)\right\|_{L^{p}(x)}=\left\|\left\{\sum_{k=0}^{\infty} \sum_{\tau \in I_{k}} \sum_{\nu=0}^{N(k, \tau)} 2^{k s q} \int_{d(\cdot, y)<a 2^{-k}}\left|D_{k}(f)(y)\right|^{q} \frac{d \mu(y)}{V_{a 2^{-k}}(\cdot)} x_{Q_{\tau}^{k, \nu}}(\cdot)\right\}^{1 / q}\right\|_{L^{p}(x)} \\
& \lesssim\left\|\left\{\sum_{k=0}^{\infty} \sum_{\tau \in I_{k}} \sum_{\nu=0}^{N(k, \tau)} 2^{k s q}\left[\sup _{y \in B\left(z_{\tau}^{k, \nu}, \mathrm{C} 2^{-k}\right)}\left|D_{k}(f)(y)\right|\right]^{q} x_{Q_{\tau}^{k, \nu}}\right\}^{1 / q}\right\|_{L^{p}(x)} \\
& \lesssim\left\{\sum_{\tau \in I_{0}} \sum_{\nu=1}^{N(0, \tau)} \mu\left(Q_{\tau}^{0, v}\right)\left[\sup _{z \in B\left(z_{\tau}^{0, v}, C\right)}\left|D_{0}(f)(z)\right|\right]^{p}\right\}^{1 / p} \\
& +\left\|\left\{\sum_{k=1}^{\infty} \sum_{\tau \in I_{k}} \sum_{\nu=0}^{N(k, \tau)} 2^{k s q}\left[\sup _{z \in B\left(z_{\tau}^{k, \nu}, C 2^{-k}\right)}\left|D_{k}(f)(z)\right|\right]^{q} x_{Q_{\tau}^{k, v}}\right\}^{1 / q}\right\|_{L^{p}(\mathcal{X})} \\
& \lesssim\left\{\sum_{\tau \in I_{0}} \sum_{\nu=1}^{N(0, \tau)} \mu\left(Q_{\tau}^{0, v}\right)\left[m_{Q_{\tau}^{0, v}}\left(\left|D_{0}(f)\right|\right)\right]^{p}\right\}^{1 / p} \\
& +\left\|\left\{\sum_{k=1}^{\infty} \sum_{\tau \in I_{k}} \sum_{\nu=0}^{N(k, \tau)} 2^{k s q}\left[\inf _{z \in Q_{\tau}^{k, \nu}}\left|D_{k}(f)(z)\right|\right]^{q} x_{Q_{\tau}^{k, \nu}}\right\}^{1 / q}\right\|_{L^{p}(\mathcal{X})} \\
& \sim\|f\|_{F_{p, q}^{s}(\mathcal{X})} \text {, }
\end{aligned}
$$

where the last inequality can be proved by a way similar to the proof of (5.76) in Proposition 5.25; see also the proof of Theorem 5.13. 
On the other hand, since $1 \leq q \leq \infty$, by Hölder's inequality and Lemma 2.19 together with $C_{6} 2^{1-j} \leq a$, we have that for $x \in Q_{\tau}^{k, v}$ with $k \in \mathbb{Z}_{+}, Q_{\tau}^{k, v} \subset\left\{y \in \mathcal{X}: d(y, x) \leq a 2^{-k}\right\}$ and $V_{a}(x) \sim \mu\left(Q_{\tau}^{0, v}\right)$, and

$$
\begin{aligned}
\left\{\int_{d(x, y)<a}\left|D_{0}(f)(y)\right|^{q} \frac{d \mu(y)}{V_{a}(x)}\right\}^{1 / q} & \geq \int_{d(x, y)<a}\left|D_{0}(f)(y)\right| \frac{d \mu(y)}{V_{a}(x)} \\
& =\sum_{\tau \in I_{0}} \sum_{v=1}^{N(0, \tau)} \int_{d(x, y)<a}\left|D_{0}(f)(y)\right| \frac{d \mu(y)}{V_{a}(x)} \chi_{Q_{\tau}^{0, v}}(x) \\
& \gtrsim \sum_{\tau \in I_{0}} \sum_{v=1}^{N(0, \tau)} m_{Q_{\tau}^{0, v}}\left(\left|D_{0}(f)\right|\right) \chi_{Q_{\tau}^{0, v}}(x) .
\end{aligned}
$$

Therefore, similarly to the proof of Theorem 5.13, by the estimate as above and Lemma 2.19 again,

$$
\begin{aligned}
S_{q, a}^{s}(f)(x) & =\left\{\sum_{k=0}^{\infty} \int_{d(x, y)<a 2^{-k}} 2^{k s q}\left|D_{k}(f)(y)\right|^{q} \frac{d \mu(y)}{V_{a 2^{-k}}(x)}\right\}^{1 / q} \\
& \gtrsim \sum_{\tau \in I_{0}} \sum_{v=1}^{N(0, \tau)} m_{Q_{\tau}^{0, v}}\left(\left|D_{0}(f)\right|\right) X_{Q_{\tau}^{0, v}}(x)+\left\{\sum_{k=1}^{\infty} \sum_{\tau \in I_{k}} \sum_{v=1}^{N(k, \tau)} 2^{k s q}\left[\inf _{y \in Q_{\tau}^{k, v}}\left|D_{k}(f)(y)\right|\right]^{q} X_{Q_{\tau}^{k_{v}, v}}(x)\right\}^{1 / q},
\end{aligned}
$$

which together with Proposition 5.25 and Lemma 2.19 proves that

$$
\begin{aligned}
& \left\|S_{q, a}^{s}(f)\right\|_{L^{p}(\mathcal{X})} \\
& \left.\quad \gtrsim\left\{\sum_{\tau \in I_{0}} \sum_{\nu=1}^{N(0, \tau)} \mu\left(Q_{\tau}^{0, v}\right)\left[m_{Q_{\tau}^{0, v}}|| D_{0}(f) \mid\right)\right]^{p}\right\}^{1 / p}+\left\|\left\{\sum_{k=1}^{\infty} 2^{k s q}\left|D_{k}(f)\right|^{q}\right\}^{1 / q}\right\|_{L^{p}(\mathcal{X})} \sim\|f\|_{F_{p, q}^{s}(\mathcal{X}) .}
\end{aligned}
$$

This finishes the proof of Theorem 5.34.

Remark 5.35. Comparing Theorem 5.34 with Theorem 5.13, we here need to require that $1 \leq$ $q \leq \infty$ and $C_{6} 2^{1-j} \leq a$ due to the inhomogeneity of Triebel-Lizorkin spaces $F_{p, q}^{s}(\mathcal{X})$.

From Theorem 2.29, we immediately obtain the following technical lemma, which will be useful in applications.

Lemma 5.36. Let $S_{0}$ be as in Definition 2.2 and $\epsilon \in\left(0, \epsilon_{1} \wedge \epsilon_{2}\right]$. Let $0<\beta, \gamma \leq \epsilon$, and let $S_{0}^{t}$ be the integral operator with the kernel $S_{0}^{t}(x, y)=S_{0}(y, x)$ for all $x, y \in X$. Then $S_{0}^{t}$ is bounded on $\mathcal{G}(\beta, \gamma)$, namely, there exists a constant $C>0$ such that for all $g \in \mathcal{G}(\beta, \gamma), S_{0}^{t}(g) \in \mathcal{G}(\beta, \gamma)$, and

$$
\left\|S_{0}^{t}(g)\right\|_{\mathcal{G}(\beta, \gamma)} \leq C\|g\|_{\mathcal{G}(\beta, \gamma)}
$$


We now establish some relations between homogeneous Besov and Triebel-Lizorkin spaces with the corresponding inhomogeneous ones.

Proposition 5.37. Let all the notation be as in Definition 5.29 and $\mu(\mathcal{X})=\infty$. Then there exists a constant $C>0$ such that if $f \in B_{p, q}^{s}(\mathcal{X})$ or $F_{p, q}^{s}(\mathcal{X})$, then $f-S_{0}(f) \in \dot{B}_{p, q}^{s}(\mathcal{X})$ or $\dot{F}_{p, q}^{s}(\mathcal{X})$, and

$$
\left\|f-S_{0}(f)\right\|_{\dot{B}_{p, q}^{s}(x)} \leq C\|f\|_{B_{p, q}^{s}(x)}
$$

or

$$
\left\|f-S_{0}(f)\right\|_{\dot{F}_{p, q}^{s}(x)} \leq C\|f\|_{F_{p, q}^{s}(x)}
$$

respectively.

Proof. Let $f \in B_{p, q}^{s}(x)$ or $f \in F_{p, q}^{s}(x)$. By Proposition 5.28, without loss of generality, we may assume that $f \in\left(\mathcal{G}_{0}^{\epsilon}(\beta, \gamma)\right)^{\prime}$ with $\beta, \gamma$ as in (5.35). From $\mathcal{G}(\beta, \gamma) \subset \mathcal{G}(\beta, \gamma)$, it follows that $f \in$ $\left(\mathcal{G}_{0}^{\epsilon}(\beta, \gamma)\right)^{\prime}$. On the other hand, for any $g \in \mathcal{G}_{0}^{\epsilon}(\beta, \gamma)$, we have $\left\langle S_{0}(f), g\right\rangle=\left\langle f, S_{0}^{t}(g)\right\rangle$, which together with Lemma 5.36 also shows that $S_{0}(f) \in\left(\mathcal{G}_{0}^{\epsilon}(\beta, \gamma)\right)^{\prime}$. Thus, $f-S_{0}(f) \in\left(\mathcal{G}_{0}^{\epsilon}(\beta, \gamma)\right)^{\prime}$ with $\beta, \gamma$ as in (5.35).

To verify the norm inequalities in the proposition, in what follows, we let $I$ be the identity operator on $B_{p, q}^{s}(\mathcal{X})$ or $F_{p, q}^{s}(\mathcal{X})$. Let $\beta$ and $\gamma$ be as in (5.35), let $\left\{D_{k}\right\}_{k \in \mathbb{Z}}$ be as in Definition 5.8, and let $\left\{\widetilde{D}_{k^{\prime}}\right\}_{k^{\prime} \in \mathbb{Z}_{+}}$be as in Theorem 4.14. We first claim that for all $k \in \mathbb{Z}$ and $k^{\prime} \in \mathbb{Z}_{+}$,

$$
\begin{aligned}
& \left|\left(D_{k}\left(I-S_{0}\right) \tilde{D}_{k^{\prime}}\right)(x, y)\right| \\
& \quad \lesssim 2^{-\left|k-k^{\prime}\right| \epsilon} \frac{1}{V_{2^{-\left(k \wedge k^{\prime}\right)}}(x)+V_{2^{-\left(k \wedge k^{\prime}\right)}}(y)+V(x, y)} \frac{2^{-\left(k \wedge k^{\prime}\right) \epsilon}}{\left(2^{-\left(k \wedge k^{\prime}\right)}+d(x, y)\right)} .
\end{aligned}
$$

We verify (5.130) by considering the following three cases.

Case $1\left(k^{\prime} \geq 0 \geq k\right)$. In this case, we have

$$
\begin{aligned}
& \left|\left(D_{k}\left(I-S_{0}\right) \tilde{D}_{k^{\prime}}\right)(x, y)\right| \\
& \quad=\left|\int_{x}\left[D_{k}(x, z)-D_{k}(x, y)\right]\left(\left(I-S_{0}\right) \widetilde{D}_{k^{\prime}}\right)(z, y) d \mu(z)\right| \\
& \quad \leq\left|\int_{x}\left[D_{k}(x, z)-D_{k}(x, y)\right] \widetilde{D}_{k^{\prime}}(z, y) d \mu(z)\right|+\left|\int_{\chi}\left[D_{k}(x, z)-D_{k}(x, y)\right]\left(S_{0} \widetilde{D}_{k^{\prime}}\right)(z, y) d \mu(z)\right| .
\end{aligned}
$$

On the first term, an argument similar to the proof of (3.2) in Lemma 3.2 gives the desired estimate. For the second term, by Lemma 3.19, we first have

$$
\left|\left(S_{0} \tilde{D}_{k^{\prime}}\right)(z, y)\right| \lesssim 2^{-k^{\prime} \epsilon} \frac{1}{V_{1}(z)+V_{1}(y)+V(z, y)} \frac{1}{(1+d(z, y))^{\epsilon}}
$$


which together with an argument similar to the proof of (3.2) in Lemma 3.2 also gives the desired estimate for the second term.

Case $2\left(k^{\prime} \geq k>0\right)$. In this case, we write

$$
\left|\left(D_{k}\left(I-S_{0}\right) \tilde{D}_{k^{\prime}}\right)(x, y)\right| \leq\left|\left(D_{k} \tilde{D}_{k^{\prime}}\right)(x, y)\right|+\left|\left(D_{k} S_{0} \widetilde{D}_{k^{\prime}}\right)(x, y)\right|
$$

The estimate (3.2) in Lemma 3.2 directly gives the desired estimate for the first term. Denote the second term by $J$ and write

$$
\begin{aligned}
J \equiv & \left|\iint_{\chi \times \chi} D_{k}(x, z)\left[S_{0}(z, u)-S_{0}(z, y)\right] \widetilde{D}_{k^{\prime}}(u, y) d \mu(u) d \mu(z)\right| \\
\leq & \int_{x} \int_{d(u, y) \leq(1+d(z, y)) / 2}\left|D_{k}(x, z)\right|\left|\left[S_{0}(z, u)-S_{0}(z, y)\right] \widetilde{D}_{k^{\prime}}(u, y)\right| d \mu(u) d \mu(z) \\
& +\int_{x} \int_{d(u, y)>(1+d(z, y)) / 2}\left|D_{k}(x, z)\right|\left|S_{0}(z, u) \widetilde{D}_{k^{\prime}}(u, y)\right| d \mu(u) d \mu(z) \\
& +\int_{x} \int_{d(u, y)>(1+d(z, y)) / 2}\left|D_{k}(x, z)\right|\left|S_{0}(z, y) \widetilde{D}_{k^{\prime}}(u, y)\right| d \mu(u) d \mu(z) \\
\equiv & J_{1}+J_{2}+J_{3} .
\end{aligned}
$$

The regularity of $S_{0}$ together with Lemma 2.1(ii) yields that

$$
\begin{gathered}
J_{1} \lesssim \int_{x} \int_{d(u, y) \leq(1+d(z, y)) / 2}\left|D_{k}(x, z)\right|\left(\frac{d(u, y)}{1+d(z, y)}\right)^{\epsilon} \frac{1}{V_{1}(z)+V_{1}(y)+V(z, y)} \\
\times \frac{1}{(1+d(z, y))^{e}}\left|\tilde{D}_{k^{\prime}}(u, y)\right| d \mu(u) d \mu(z) \\
\lesssim 2^{-k^{\prime} \epsilon} \int_{x}\left|D_{k}(x, z)\right| \frac{1}{V_{1}(z)+V_{1}(y)+V(z, y)} \frac{1}{(1+d(z, y))^{\epsilon}} d \mu(z) .
\end{gathered}
$$

If $d(x, y) \leq 2^{-k}$, then by Lemma 2.1(ii) again, $J_{1} \lesssim 2^{-k^{\prime} \epsilon}\left(1 / V_{2^{-k}}(x)\right)$, which is the desired estimate. When $d(x, y)>2^{-k}$, we further control $J_{1}$ by

$$
\begin{aligned}
J_{1} \lesssim 2^{-k^{\prime} \epsilon}\left\{\int_{d(x, z) \geq d(x, y) / 2}\right. & \frac{1}{V_{2^{-k}}(x)+V_{2^{-k}}(z)+V(x, z)} \frac{2^{-k \epsilon}}{\left(2^{-k}+d(x, z)\right)^{\epsilon}} \\
& \left.\times \frac{1}{V_{1}(z)+V_{1}(y)+V(z, y)} \frac{1}{(1+d(z, y))^{\epsilon}} d \mu(z)+\int_{d(x, z)<d(x, y) / 2} \ldots\right\} .
\end{aligned}
$$


Notice that $d(x, z)<d(x, y) / 2$ also implies that $d(z, y) \geq d(x, y) / 2$. From this together with Lemma 2.1(ii), it follows that

$$
J_{1} \lesssim 2^{-k^{\prime} \epsilon}\left\{\frac{1}{V(x, y)} \frac{2^{-k \epsilon}}{d(x, y)^{\epsilon}}+\frac{1}{V(x, y)} \frac{1}{d(x, y)^{\epsilon}}\right\} \lesssim 2^{-\left(k^{\prime}-k\right) \epsilon} \frac{1}{V(x, y)} \frac{2^{-k \epsilon}}{d(x, y)^{\epsilon}}
$$

which is also the desired estimate.

The estimate for $J_{3}$ is similar to that for $J_{1}$.

To estimate $J_{2}$, choosing $\epsilon^{\prime}>\epsilon$ and using Lemma 2.1(ii), we first have

$$
\int_{d(u, y)>(1+d(z, y)) / 2}\left|S_{0}(z, u) \tilde{D}_{k^{\prime}}(u, y)\right| d \mu(u) \lesssim \frac{1}{V_{1}(z)} \frac{2^{-k^{\prime} \epsilon}}{(1+d(z, y))^{\epsilon}}
$$

and by the fact that for $d(u, y)>(1+d(z, y)) / 2, V(u, y) \sim V(y, u) \gtrsim V_{1}(y)+V(y, z)$, we also obtain

$$
\int_{d(u, y)>(1+d(z, y)) / 2}\left|S_{0}(z, u) \widetilde{D}_{k^{\prime}}(u, y)\right| d \mu(u) \lesssim \frac{1}{V_{1}(y)+V(y, z)} \frac{2^{-k^{\prime} \epsilon}}{(1+d(z, y))^{\epsilon}}
$$

Therefore, combining these estimates gives

$$
J_{2} \lesssim 2^{-k^{\prime} \epsilon} \int_{x}\left|D_{k}(x, z)\right| \frac{1}{V_{1}(z)+V_{1}(y)+V(z, y)} \frac{1}{(1+d(z, y))^{\epsilon}} d \mu(z)
$$

which together with some computations the same as for $J_{1}$ gives the desired estimate for $J_{2}$.

Case $3\left(k>k^{\prime} \geq 0\right)$. The proof for this case is similar to Case 2 by symmetry. We omit the details for simplicity, which completes the proof of (5.130).

Theorem 4.16 together with (5.80), (5.130), and Lemma 5.3 yields that for $n /(n+\epsilon)<$ $r \leq 1, k \in \mathbb{Z}$, and $x \in \mathcal{X}$,

$$
\begin{aligned}
\mid D_{k}(f & \left.-S_{0}(f)\right)(x) \mid \\
\lesssim & 2^{-|k| \epsilon} 2^{(k \wedge 0) n(1-1 / r)}\left\{M\left(\sum_{\tau^{\prime} \in I_{0}} \sum_{v^{\prime}=1}^{N\left(0, \tau^{\prime}\right)}\left[m_{Q_{\tau^{\prime}}^{0, v^{\prime}}}\left(\left|D_{0}(f)\right|\right)\right]^{r} X_{Q_{\tau^{\prime}}^{0, v^{\prime}}}\right)(x)\right\}^{1 / r} \\
& +\sum_{k^{\prime}=1}^{\infty} 2^{-\left|k-k^{\prime}\right| \epsilon} 2^{\left[\left(k \wedge k^{\prime}\right)-k^{\prime}\right] n(1-1 / r)}\left\{M\left(\sum_{\tau^{\prime} \in I_{k^{\prime}}} \sum_{v^{\prime}=1}^{N\left(k^{\prime}, \tau^{\prime}\right)}\left|D_{k^{\prime}}(f)\left(y_{\tau^{\prime}}^{k^{\prime}, v^{\prime}}\right)\right|^{r} X_{Q_{\tau^{\prime}}^{k^{\prime}, v^{\prime}}}\right)(x)\right\}^{1 / r} \\
\equiv & Z_{1}+Z_{2} .
\end{aligned}
$$


We now first consider the case of Besov spaces. Choosing $p(s, \epsilon)<r<\min \{1, p\}$ and using $s<\epsilon$ and the boundedness of $M$ yield

$$
\begin{aligned}
\left\{\sum_{k \in \mathbb{Z}} 2^{k s q}\left\|Z_{1}\right\|_{L^{p}(\mathcal{X})}^{q}\right\}^{1 / q} & \lesssim\left\{\sum_{\tau^{\prime} \in I_{0}} \sum_{v^{\prime}=1}^{N\left(0, \tau^{\prime}\right)} \mu\left(Q_{\tau^{\prime}}^{0, v^{\prime}}\right)\left[m_{Q_{\tau^{\prime}}^{0, v^{\prime}}}\left(\left|D_{0}(f)\right|\right)\right]^{p}\right\}^{1 / p} \\
& \lesssim\|f\|_{B_{p, q}^{s}(\mathcal{X}),}
\end{aligned}
$$

which is the desired estimate.

Similarly, choosing $r$ as above, when $1 \leq p \leq \infty$, by Minkowski's inequality and the boundedness of $M$ together with the assumption $s<\epsilon$, we have

$$
\begin{aligned}
\left\{\sum_{k \in \mathbb{Z}} 2^{k s q}\left\|Z_{2}\right\|_{L^{p}(\mathcal{x})}^{q}\right\}^{1 / q} & \lesssim\left\{\sum_{k \in \mathbb{Z}} \| \sum_{k^{\prime}=1}^{\infty} 2^{\left(k-k^{\prime}\right) s-\left|k-k^{\prime}\right| \epsilon} 2^{\left[\left(k \wedge k^{\prime}\right)-k^{\prime}\right] n(1-1 / r)} 2^{k^{\prime} s}\right. \\
& \left.\times\left\{M\left(\sum_{\tau^{\prime} \in I_{k^{\prime}}} \sum_{v^{\prime}=1}^{N\left(k^{\prime}, \tau^{\prime}\right)}\left|D_{k^{\prime}}(f)\left(y_{\tau^{\prime}}^{k^{\prime}, v^{\prime}}\right)\right|^{r} X_{Q_{\tau^{\prime}}^{k^{\prime}, \prime^{\prime}}}\right)\right\}^{1 / r} \|_{L^{p}(\mathcal{x})}^{q}\right\}^{1 / q} \\
& \lesssim\left\{\sum_{k^{\prime}=1}^{\infty} 2^{k^{\prime} s q}\left\|D_{k^{\prime}}(f)\right\|_{L^{p}(\mathcal{x})}^{q}\right\}^{1 / q} \\
& \lesssim\|f\|_{B_{p, q}^{s}(x),}
\end{aligned}
$$

where in the second-to-last inequality, we used (5.5) when $q \leq 1$ and Hölder's inequality when $q>1$, while when $r<p<1$, instead of Minkowski's inequality by (5.5), we have

$$
\begin{aligned}
\left\{\sum_{k \in \mathbb{Z}} 2^{k s q}\left\|Z_{2}\right\|_{L^{p}(\mathcal{X})}^{q}\right\}^{1 / q} & \lesssim\left\{\sum_{k^{\prime}=1}^{\infty} 2^{k^{\prime} s q}\left\|D_{k^{\prime}}(f)\right\|_{L^{p}(\mathcal{X})}^{q}\right\}^{1 / q} \\
& \lesssim\|f\|_{B_{p, q}^{s}(\mathcal{X})}
\end{aligned}
$$

where in the second-to-last inequality, we used (5.5) when $q / p \leq 1$ and Hölder's inequality when $q / p>1$. Combining the above estimates completes the proof of Besov spaces.

We now turn to the case of Triebel-Lizorkin spaces. In this case, we also choose $p(s, \epsilon)<$ $r<\min \{1, p\}$ and use $s<\epsilon$ and the boundedness of $M$ to obtain

$$
\begin{aligned}
\left\|\left\{\sum_{k \in \mathbb{Z}} 2^{k s q}\left|Z_{1}\right|^{q}\right\}^{1 / q}\right\|_{L^{p}(x)} & \lesssim\left\{\sum_{\tau^{\prime} \in I_{0}} \sum_{v^{\prime}=1}^{N\left(0, \tau^{\prime}\right)} \mu\left(Q_{\tau^{\prime}}^{0, v^{\prime}}\right)\left[m_{Q_{\tau^{\prime}}^{0, v^{\prime}}}\left(\left|D_{0}(f)\right|\right)\right]^{p}\right\}^{1 / p} \\
& \lesssim\|f\|_{F_{p, q}^{s}(x),}
\end{aligned}
$$


while some computations similar to the proof of (5.8) in Proposition 5.4 also show that

$$
\left\|\left\{\sum_{k \in \mathbb{Z}} 2^{k s q}\left|Z_{2}\right|^{q}\right\}^{1 / q}\right\|_{L^{p}(\mathcal{X})} \lesssim\left\|\left\{\sum_{k^{\prime}=1}^{\infty} 2^{k^{\prime} s q}\left|D_{k^{\prime}}(f)\right|^{q}\right\}^{1 / q}\right\|_{L^{p}(\mathcal{X})} \lesssim\|f\|_{F_{p, q}^{s}(\mathcal{X})}
$$

which completes the proof of Proposition 5.37.

Remark 5.38. Obviously, $S_{0}$ in Proposition 5.37 can be replaced by $S_{k_{0}}$ with $k_{0} \in \mathbb{Z}$ or any integral operator with a kernel having similar properties.

Proposition 5.39. Let $\epsilon$ be as in Definition 5.29, $0<s<\epsilon$, and $\mu(\mathcal{X})=\infty$. Then,

(i) if $1 \leq p \leq \infty$ and $0<q \leq \infty$, then $B_{p, q}^{s}(\mathcal{X})=\dot{B}_{p, q}^{s}(\mathcal{X}) \cap L^{p}(\mathcal{X})$ and moreover, for any $f \in B_{p, q}^{s}(x)$,

$$
\|f\|_{B_{p, q}^{s}(\mathcal{X})} \sim\|f\|_{\dot{B}_{p, q}^{s}(\mathcal{X})}+\|f\|_{L^{p}(\mathcal{X})}
$$

(ii) if $1 \leq p<\infty$ and $p(s, \epsilon)<q \leq \infty$, then $F_{p, q}^{s}(\mathcal{X})=\dot{F}_{p, q}^{s}(\mathcal{X}) \cap L^{p}(\mathcal{X})$ and moreover, for any $f \in F_{p, q}^{s}(x)$,

$$
\|f\|_{F_{p, q}^{s}(\mathcal{X})} \sim\|f\|_{F_{p, q}^{s}(\mathcal{X})}+\|f\|_{L^{p}(\mathcal{X})} .
$$

Proof. We use the same notation as in Definition 5.8. Let $f \in B_{p, q}^{s}(\mathcal{X})$ or $f \in F_{p, q}^{s}(\mathcal{X})$. Then, by Proposition 5.28, it is easy to see that $f \in\left(\dot{\mathcal{C}}_{0}^{\epsilon}(\beta, \gamma)\right)^{\prime}$ with $\beta, \gamma$ as in (5.35).

To verify (i), let $f \in B_{p, q}^{s}(x)$. If $1 \leq p<\infty$, Proposition 3.18, Minkowski's inequality, and Hölder's inequality when $1 \leq q \leq \infty$ or (5.5) when $0<q<1$ show that

$$
\begin{aligned}
\|f\|_{L^{p}(\mathcal{X})} & \leq\left\|S_{0}(f)\right\|_{L^{p}(\mathcal{X})}+\sum_{k=1}^{\infty}\left\|D_{k}(f)\right\|_{L^{p}(\mathcal{X})} \\
& \lesssim\left\{\left\|S_{0}(f)\right\|_{L^{p}(\mathcal{X})}^{q}+\sum_{k=1}^{\infty} 2^{k s q}\left\|D_{k}(f)\right\|_{L^{p}(\mathcal{X})}^{q}\right\}^{1 / q} \\
& \lesssim\|f\|_{B_{p, q}^{s}(\mathcal{x}) .}
\end{aligned}
$$

If $p=\infty$, by Theorem 3.29, we have that $f=\widetilde{D}_{0} S_{0}(f)+\sum_{k=1}^{\infty} \widetilde{D}_{k} D_{k}(f)$ holds in $(\mathcal{G}(\beta, \gamma))^{\prime}$ with $s<\beta<\epsilon$ and $0<\gamma<\epsilon$, where $\widetilde{D}_{k}$ with $k \in \mathbb{Z}_{+}$is as in Theorem 3.26. From this and $f \in B_{\infty, q}^{s}(\mathcal{X})$, it follows that for almost all $x \in \mathcal{X}$,

$$
\begin{aligned}
|f(x)| & \lesssim\|f\|_{B_{\infty, q}^{s}(x)}\left\{\int_{x}\left|\tilde{D}_{0}(x, y)\right| d \mu(y)+\sum_{k=1}^{\infty} 2^{-k s} \int_{x}\left|\tilde{D}_{k}(x, y)\right| d \mu(y)\right\} \\
& \lesssim\|f\|_{B_{\infty, q}^{s}(x) .}
\end{aligned}
$$


Moreover, when $1 \leq p \leq \infty$, Proposition 2.7(iii) further yields

$$
\begin{aligned}
\|f\|_{\dot{B}_{p, q}^{s}(x)} & \sim\left\{\sum_{k=-\infty}^{\infty} 2^{k s q}\left\|D_{k}(f)\right\|_{L^{p}(x)}^{q}\right\}^{1 / q} \\
& \lesssim\left\{\sum_{k=-\infty}^{0} 2^{k s q}\left\|D_{k}(f)\right\|_{L^{p}(x)}^{q}\right\}^{1 / q}+\left\{\sum_{k=1}^{\infty} 2^{k s q}\left\|D_{k}(f)\right\|_{L^{p}(x)}^{q}\right\}^{1 / q} \\
& \lesssim\|f\|_{B_{p, q}^{s}(x) .}
\end{aligned}
$$

Thus, $f \in \dot{B}_{p, q}^{s}(\mathcal{X}) \cap L^{p}(\mathcal{X})$ and $\|f\|_{L^{p}(\mathcal{X})}+\|f\|_{\dot{B}_{p, q}^{s}(\mathcal{X})} \lesssim\|f\|_{B_{p, q}^{s}(\mathcal{X})}$.

Conversely, if $f \in \dot{B}_{p, q}^{s}(\mathcal{X}) \cap L^{p}(\mathcal{X})$, it is obvious that $f \in(\mathcal{G}(\beta, \gamma))^{\prime}$ with $\beta, \gamma$ as in (5.111); and moreover, Proposition 2.7(iii) also proves

$$
\begin{aligned}
\|f\|_{B_{p, q}^{s}(\mathcal{X})} & \sim\left\{\left\|S_{0}(f)\right\|_{L^{p}(\mathcal{X})}^{q}+\sum_{k=1}^{\infty} 2^{k s q}\left\|D_{k}(f)\right\|_{L^{p}(\mathcal{X})}^{q}\right\}^{1 / q} \\
& \lesssim\|f\|_{L^{p}(\mathcal{X})}+\|f\|_{\dot{B}_{p, q}^{s}(\mathcal{X})}
\end{aligned}
$$

which completes the proof of (i).

To prove (ii), let $f \in F_{p, q}^{s}(x)$. From Proposition 3.18 and Hölder's inequality when $1 \leq q \leq \infty$ or (5.5) when $p(s, \epsilon)<q<1$, it follows that

$$
\begin{aligned}
& \|f\|_{L^{p}(\mathcal{X})} \leq\left\|\left|S_{0}(f)\right|+\sum_{k=1}^{\infty}\left|D_{k}(f)\right|\right\|_{L^{p}(\mathcal{X})} \\
& \lesssim\left\|\left\{\left|S_{0}(f)\right|+\sum_{k=1}^{\infty} 2^{k s q}\left|D_{k}(f)\right|^{q}\right\}^{1 / q}\right\|_{L^{p}(x)} \\
& \lesssim\|f\|_{F_{p, q}^{s}(\mathcal{X})}, \\
& \|f\|_{\dot{F}_{p, q}^{s}(x)} \sim\left\|\left\{\sum_{k \in \mathbb{Z}} 2^{k s q}\left|D_{k}(f)\right|^{q}\right\}^{1 / q}\right\|_{L^{p}(x)} \\
& \lesssim\left\|\left\{\sum_{k=-\infty}^{0} 2^{k s q}\left|D_{k}(f)\right|^{q}\right\}^{1 / q}\right\|_{L^{p}(\mathcal{X})}+\left\|\left\{\sum_{k=1}^{\infty} 2^{k s q}\left|D_{k}(f)\right|^{q}\right\}^{1 / q}\right\|_{L^{p}(\mathcal{X})} \\
& \lesssim Z+\|f\|_{F_{p, q}^{s}(x)} .
\end{aligned}
$$

If $p / q \leq 1$, by (5.5), Proposition 2.7(iii), and $s>0$,

$$
(Z)^{p} \lesssim \sum_{k=-\infty}^{0} 2^{k s p}\left\|D_{k}(f)\right\|_{L^{p}(\mathcal{X})}^{p} \lesssim\|f\|_{L^{p}(\mathcal{X})}^{p} \sum_{k=-\infty}^{0} 2^{k s p} \lesssim\|f\|_{L^{p}(\mathcal{X})^{\prime}}^{p}
$$


while when $p / q>1$, by Minkowski's inequality, Proposition 2.7(iii), and $s>0$,

$$
Z \lesssim\left\{\sum_{k=-\infty}^{0} 2^{k s q}\left\|D_{k}(f)\right\|_{L^{p}(\mathcal{X})}^{q}\right\}^{1 / q} \lesssim\|f\|_{L^{p}(\mathcal{X})}\left\{\sum_{k=-\infty}^{0} 2^{k s q}\right\}^{1 / q} \lesssim\|f\|_{L^{p}(\mathcal{X})} .
$$

Thus, $f \in \dot{F}_{p, q}^{s}(\mathcal{X}) \cap L^{p}(\mathcal{X})$ and $\|f\|_{L^{p}(\mathcal{X})}+\|f\|_{\dot{F}_{p, q}^{s}(\mathcal{X})} \lesssim\|f\|_{F_{p, q}^{s}(\mathcal{X})}$.

Conversely, if $f \in \dot{F}_{p, q}^{s}(\mathcal{X}) \cap L^{p}(\mathcal{X})$, it is again obvious that $f \in(\mathcal{G}(\beta, \gamma))^{\prime}$ with $\beta, \gamma$ as in (5.111); and moreover, Proposition 2.7(iii) yields that

$$
\begin{aligned}
\|f\|_{F_{p, q}^{s}(x)} & \sim\left\|\left\{\left|S_{0}(f)\right|^{q}+\sum_{k=1}^{\infty} 2^{k s q}\left|D_{k}(f)\right|^{q}\right\}^{1 / q}\right\|_{L^{p}(\mathcal{X})} \\
& \lesssim\|f\|_{L^{p}(\mathcal{X})}+\|f\|_{\dot{F}_{p, q}^{s}(x)}
\end{aligned}
$$

which completes the proof of (ii) and hence, the proof of Proposition 5.39.

We now introduce the local Hardy spaces in the sense of Goldberg [73].

Definition 5.40. Let all the notation be as in Definition 5.29. The local Hardy space $h^{p}(\mathcal{X})$, for $n /(n+\epsilon)<p \leq 1$, is defined to be the inhomogeneous Triebel-Lizorkin space $F_{p, 2}^{0}(\mathcal{X})$, with norm $\|f\|_{h^{p}(\mathcal{X})} \equiv\|f\|_{F_{p, 2}^{0}(\mathcal{x})}$.

We now introduce the definitions of $h^{p}(\mathcal{X})$-atoms and $h^{p}(\mathcal{X})$-blocks.

Definition 5.41. Let $j \in \mathbb{N}$ be as in Theorem 5.34 and $0<p \leq 1$. A function $a$ on $\mathcal{X}$ is called an $h^{p}(\mathcal{X})$-atom if $a$ satisfies (i) through (iii) of Definition 5.15 with $r<C_{6} 2^{-j}$, and $a$ is called an $h^{p}(\mathcal{X})$-block if $a$ satisfies (i) and (ii) of Definition 5.15 with $r \geq C_{6} 2^{-j}$.

Applying Theorem 5.34 and Proposition 5.37, we obtain the following atomic and block decomposition characterization for the local Hardy spaces $h^{p}(\mathcal{X})$, which is similar to Theorem 5.16.

Theorem 5.42. Let $\epsilon$ and $\beta, \gamma$ be as in Definition 5.29. If $n /(n+\epsilon)<p \leq 1$, then $f \in h^{p}(\mathcal{X})$ if and only if there exist two sequences of numbers $\left\{\lambda_{k}\right\}_{k \in \mathbb{Z}_{+}}$and $\left\{\mu_{k}\right\}_{k \in \mathbb{Z}_{+}}$with $\sum_{k=0}^{\infty}\left|\lambda_{k}\right|^{p}<\infty$ and $\sum_{k=0}^{\infty}\left|\mu_{k}\right|^{p}<\infty$, a sequence of $h^{p}(X)$-atoms $\left\{a_{k}\right\}_{k \in \mathbb{Z}_{+}}$and a sequence of $h^{p}(X)$-blocks $\left\{b_{k}\right\}_{k \in \mathbb{Z}_{+}}$such that $f=\sum_{k=0}^{\infty} \lambda_{k} a_{k}+\sum_{k=0}^{\infty} \mu_{k} b_{k}$ in $\left(\mathcal{G}_{0}^{\epsilon}(\beta, \gamma)\right)^{\prime}$. Moreover, in this case,

$$
\|f\|_{h^{p}(\mathcal{X})} \sim \inf \left\{\left(\sum_{k=0}^{\infty}\left|\lambda_{k}\right|^{p}\right)^{1 / p}+\left(\sum_{k=0}^{\infty}\left|\mu_{k}\right|^{p}\right)^{1 / p}\right\}
$$

where the infimum is taken over all the above decompositions of $f$.

Proof. Let $f \in h^{p}(\mathcal{X})$. In order to derive the decomposition of $f$ into atoms and blocks, we will assume for simplicity that $\mu(x)=\infty$. The case where $\mu(X)<\infty$ can be proved by using Theorems 5.34, 2.6, and 3.29 together with an argument similar to that used for the proof of the necessity of Theorem 2.21 in [48]. 
By Proposition 5.37, we have $f-S_{0}(f) \in H^{p}(\mathcal{X})$ and moreover,

$$
\left\|f-S_{0}(f)\right\|_{H^{p}(\mathcal{X})} \lesssim\|f\|_{h^{p}(\mathcal{X})},
$$

where $S_{0}$ is as in Definition 5.29. By Theorem 5.16, there exist a sequence of numbers $\left\{\lambda_{k}\right\}_{k=0}^{\infty} \subset$ $\mathbb{C}$ with $\sum_{k=0}^{\infty}\left|\lambda_{k}\right|^{p}<\infty$ and a sequence of $H^{p}(\mathcal{X})$-atoms $\left\{a_{k}\right\}_{k=0}^{\infty}$ such that in $\left(\dot{\mathcal{C}}_{0}^{e}(\beta, \gamma)\right)^{\prime}$,

$$
\begin{gathered}
f-S_{0}(f)=\sum_{k=0}^{\infty} \lambda_{k} a_{k} \\
\left(\sum_{k=0}^{\infty}\left|\lambda_{k}\right|^{p}\right)^{1 / p} \lesssim\left\|f-S_{0}(f)\right\|_{H^{p}(\mathcal{x})}
\end{gathered}
$$

Let $S_{0}^{t}$ denote the adjoint operator to $S_{0}$ with integral kernel $S_{0}^{t}(x, y)=S_{0}(y, x)$. Notice that if $g \in \mathcal{G}_{0}^{\epsilon}(\beta, \gamma)$, then $g-S_{0}^{t}(g) \in \dot{\mathcal{G}}_{0}^{\epsilon}(\beta, \gamma)$ by Lemma 5.36, which together with (5.159) shows that

$$
\langle f, g\rangle=\left\langle 2 S_{0}(f)-S_{0} S_{0}(f), g\right\rangle+\sum_{k=0}^{\infty} \lambda_{k}\left\langle a_{k}-S_{0}\left(a_{k}\right), g\right\rangle
$$

Using Lemma 2.19, we have

$$
\begin{aligned}
2 S_{0}(f)(x)-S_{0} S_{0}(f)(x) & =\sum_{\tau \in I_{0}} \sum_{\nu=1}^{N(0, \tau)}\left[2 S_{0}(f)(x)-S_{0} S_{0}(f)(x)\right] \chi_{Q_{\tau}^{0, v}}(x) \\
& \equiv \sum_{\tau \in I_{0}} \sum_{\nu=1}^{N(0, \tau)} \lambda_{\tau}^{0, v} b_{\tau}^{0, v}(x),
\end{aligned}
$$

where

$$
\lambda_{\tau}^{0, v} \equiv\left[\mu\left(Q_{\tau}^{0, v}\right)\right]^{1 / p} \sup _{z \in Q_{\tau}^{0, v}}\left|2 S_{0}(f)(z)-S_{0} S_{0}(f)(z)\right|
$$

and $b_{\tau}^{0, v}(x) \equiv 0$ when $\lambda_{\tau}^{0, v}=0$, otherwise,

$$
b_{\tau}^{0, v}(x) \equiv \frac{1}{\lambda_{\tau}^{0, v}}\left[2 S_{0}(f)(x)-S_{0} S_{0}(f)(x)\right] \chi_{Q_{\tau}^{0, v}}(x) .
$$

It is easy to see that supp $b_{\tau}^{0, v} \subset Q_{\tau}^{0, v} \subset B\left(z_{\tau}^{0, v}, C_{6} 2^{-j}\right)$ and

$$
\begin{aligned}
\left\|b_{\tau}^{0, v}\right\|_{L^{2}(\chi)} & \leq\left[\mu\left(Q_{\tau}^{0, v}\right)\right]^{1 / 2} \frac{1}{\lambda_{\tau}^{0, v}} \sup _{z \in Q_{\tau}^{0, v}}\left|2 S_{0}(f)(z)-S_{0} S_{0}(f)(z)\right| \\
& \lesssim\left[\mu\left(B\left(z_{\tau}^{0, v}, C_{6} 2^{-j}\right)\right)\right]^{1 / 2-1 / p}
\end{aligned}
$$


Thus, $b_{\tau}^{0, v}$ is an $h^{p}(\mathcal{X})$-block multiplied with a normalizing constant. Moreover, noticing that the kernel of $S_{0} S_{0}$ has the properties similar to $S_{0}$ by Lemma 3.19, applying Theorem 4.16, and using an argument similar to the proof of (5.77) in Proposition 5.25, we obtain

$$
\begin{aligned}
\left\{\sum_{\tau \in I_{0}} \sum_{\nu=1}^{N(0, \tau)}\left|\lambda_{\tau}^{0, v}\right|^{p}\right\}^{1 / p} & \lesssim\left\{\sum_{\tau \in I_{0}} \sum_{\nu=1}^{N(0, \tau)} \mu\left(Q_{\tau}^{0, v}\right)\left[\sup _{z \in Q_{\tau}^{0, v}}\left|2 S_{0}(f)(z)-S_{0} S_{0}(f)(z)\right|\right]^{p}\right\}^{1 / p} \\
& \lesssim\left\{\sum_{\tau \in I_{0}} \sum_{\nu=1}^{N(0, \tau)} \mu\left(Q_{\tau}^{0, v}\right)\left[m_{Q_{\tau}^{0, v}}\left(\left|D_{0}(f)\right|\right)\right]^{p}\right\}^{1 / p} \\
& +\left\|\left\{\sum_{k=1}^{\infty} \sum_{\tau \in I_{k}} \sum_{\nu=1}^{N(k, \tau)}\left[\inf _{z \in Q_{\tau}^{k, \nu}}\left|D_{k}(f)(z)\right|\right]^{2} X_{Q_{\tau}^{\alpha_{\nu}, v}}\right\}^{1 / 2}\right\|_{L^{p}(\boldsymbol{x})} \\
& \sim\|f\|_{h^{p}(\boldsymbol{X}) .}
\end{aligned}
$$

We next show that

$$
\left\langle 2 S_{0}(f)-S_{0} S_{0}(f), g\right\rangle=\sum_{\tau \in I_{0}} \sum_{\nu=0}^{N(0, \tau)} \lambda_{\tau}^{0, v}\left\langle b_{\tau}^{0, v}, g\right\rangle,
$$

which can be deduced by $\left|\left\langle b_{\tau}^{0, v}, g\right\rangle\right| \lesssim 1$. To see this, by Hölder's inequality, Lemma 2.1(iii), and (5.30) in the proof of Proposition 5.7 together with the assumption $\gamma>n(1 / p-1)$, we have

$$
\begin{aligned}
\left|\left\langle b_{\tau}^{0, v}, g\right\rangle\right| & \leq\left\|b_{\tau}^{0, v}\right\|_{L^{2}(x)}\left\|g X_{Q_{\tau}^{0, v}}\right\|_{L^{2}(x)} \\
& \leq\left[\mu\left(Q_{\tau}^{0, v}\right)\right]^{1 / 2-1 / p}\left\{\int_{Q_{\tau}^{0, v}}\left[\frac{1}{V_{1}\left(x_{1}\right)+V\left(x_{1}, x\right)} \frac{1}{\left(1+d\left(x_{1}, x\right)\right)^{\gamma}}\right]^{2} d \mu(x)\right\}^{1 / 2} \\
& \lesssim \frac{1}{\left[V_{1}\left(x_{1}\right)\right]^{1 / p}} \\
& \lesssim 1
\end{aligned}
$$

Notice that by (5.168) below, we have $\left\|a_{k}\right\|_{h^{p}(\mathcal{X})} \lesssim 1$. We can obtain a desired $h^{p}(\mathcal{X})$ block decomposition of $S_{0}\left(a_{k}\right)$ in the same way as $2 S_{0}(f)-S_{0} S_{0}(f)$, which completes the proof of the necessity.

We now use Theorem 5.34 to verify the sufficiency of the condition in the theorem. To this end, by Fatou's lemma, it suffices to prove that for any $h^{p}(\mathcal{X})$-atom or any $h^{p}(\mathcal{X})$-block $b$,

$$
\left\|S_{2, a}^{0}(b)\right\|_{L^{p}(\chi)} \lesssim 1
$$

where $a \geq C_{6} 2^{1-j}$ is as in Theorem 5.34. If $b$ is an $h^{p}(\mathcal{X})$-atom, the estimate (5.168) can be established by an argument similar to the proof of the sufficiency of Theorem 2.21 in [48]. 
We omit the details. Now, we suppose $b$ is an $h^{p}(\mathcal{X})$-block supported on $B\left(x_{0}, r\right)$ with $r \geq$ $C_{6} 2^{-j}$. Choose $N \in \mathbb{N}$ such that $N \geq \max \{2,2 / a\}$ and write

$$
\begin{aligned}
\left\|S_{2, a}^{0}(b)\right\|_{L^{p}(\mathcal{X})}^{p} & =\int_{B\left(x_{0}, N a r\right)}\left[S_{2, a}^{0}(b)(x)\right]^{p} d \mu(x)+\int_{\chi \backslash B\left(x_{0}, N a r\right)} \ldots \\
& \equiv J_{1}+J_{2} .
\end{aligned}
$$

Hölder's inequality together with the size condition of $b$ shows that

$$
J_{1} \leq\left[\mu\left(B\left(x_{0}, N a r\right)\right)\right]^{1-p / 2}\left\|S_{2, a}^{0}(b)\right\|_{L^{2}(\mathcal{X})}^{p} \lesssim\left[\mu\left(B\left(x_{0}, r\right)\right)\right]^{1-p / 2}\|b\|_{L^{2}(\mathcal{X})}^{p} \lesssim 1 .
$$

Now if $x \in X \backslash B\left(x_{0}, N a r\right), d(y, x) \leq a 2^{-k}$ with $k \in \mathbb{Z}_{+}$and $u \in B\left(x_{0}, r\right)$, we then have $d\left(u, x_{0}\right) \leq(1 / 2)\left(2^{-k}+d\left(y, x_{0}\right)\right)$, which together with the size conditions of $D_{k}$ and $b$, Lemma 2.1(iii), and Hölder's inequality yields that

$$
\left|D_{k}(b)(y)\right| \lesssim\left[\mu\left(B\left(x_{0}, r\right)\right)\right]^{1-1 / p} \frac{1}{V_{2^{-k}}(y)+V_{2^{-k}}\left(x_{0}\right)+V\left(y, x_{0}\right)} \frac{2^{-k \epsilon}}{\left(2^{-k}+d\left(y, x_{0}\right)\right)^{\epsilon}} .
$$

From this, it follows that

$$
S_{2, a}^{0}(b)(x) \lesssim\left[\mu\left(B\left(x_{0}, r\right)\right)\right]^{1-1 / p}\left\{\sum_{k=0}^{\infty}\left[\frac{1}{V_{2^{-k}}(x)+V_{2^{-k}}\left(x_{0}\right)+V\left(x, x_{0}\right)} \frac{2^{-k \epsilon}}{\left(2^{-k}+d\left(x, x_{0}\right)\right)^{\epsilon}}\right]^{2}\right\}^{1 / 2}
$$

Therefore, if we choose $\epsilon^{\prime} \in(0, \epsilon)$ such that $p>n /\left(n+\epsilon^{\prime}\right)$, by (5.5), we then have

$$
\begin{aligned}
J_{2} \lesssim & \frac{1}{r^{\left(\epsilon-\epsilon^{\prime}\right) p}}\left[\mu\left(B\left(x_{0}, r\right)\right)\right]^{p-1} \sum_{k=0}^{\infty} 2^{-k\left(\epsilon-\epsilon^{\prime}\right) p} \\
& \times \int_{x \backslash B\left(x_{0}, N a r\right)}\left(\frac{1}{V_{2^{-k}}(x)+V_{2^{-k}}\left(x_{0}\right)+V\left(x, x_{0}\right)}\right)^{p} \frac{2^{-k \epsilon^{\prime} p}}{\left(2^{-k}+d\left(x, x_{0}\right)\right)^{\epsilon^{\prime} p}} d \mu(x) \\
\lesssim & \frac{1}{r^{\left(\epsilon-\epsilon^{\prime}\right) p}}\left[\mu\left(B\left(x_{0}, r\right)\right)\right]^{p-1} \sum_{k=0}^{\infty} 2^{-k\left(\epsilon-\epsilon^{\prime}\right) p} \\
& \times \sum_{l=k}^{\infty} \int_{2^{l} 2^{-k} N a r \leq d\left(x, x_{0}\right)<2^{l+1} 2^{-k} N a r}\left(\frac{1}{V_{2^{-k}}(x)+V_{2^{-k}}\left(x_{0}\right)+V\left(x, x_{0}\right)}\right)^{p} \frac{2^{-k \epsilon^{\prime} p}}{\left(2^{-k}+d\left(x, x_{0}\right)\right)^{\epsilon^{\prime} p}} d \mu(x) \\
\lesssim & 1,
\end{aligned}
$$

which completes the proof of Theorem 5.42.

We now recall the definition of the Lipschitz $\operatorname{space}_{\operatorname{Lip}}(\mathcal{X})$ with $s>0$; see [74]. 
Definition 5.43. Let $s>0$. The Lipschitz (or Hölder) space $\operatorname{Lip}_{s}(\boldsymbol{X})$ is defined to be the set of all functions $f$ on $X$ such that

$$
\|f\|_{\operatorname{Lip}_{s}(x)}=\sup _{x \in B, r_{B} \geq 1} \frac{|f(x)|}{\mu(B)^{s}}+\sup _{x \neq y} \frac{|f(x)-f(y)|}{V(x, y)^{s}}<\infty,
$$

where the first supremum is taken over all balls $B$ of $\mathcal{X}$ with radius $r_{B} \geq 1$.

Observe that these classes are rather Lipschitz (or Hölder) classes with respect to the measure distance $\rho(x, y) \equiv \inf \{\mu(B): x, y \in B, B$ a ball $\}$, not the distance $d$.

The dual spaces of $h^{1}(\mathcal{X})$ and $h^{p}(\mathcal{X})$ when $p<1$ are proved, respectively, to be bmo $(\mathcal{X})$ and $\operatorname{Lip}_{1 / p-1}(\mathcal{X})$ in [74] as follows.

Theorem 5.44. (i) The space $b m o(x)$ is the dual space of $h^{1}(\mathcal{X})$, in the following sense: if $f=$ $\sum_{k=0}^{\infty} \lambda_{k} a_{k}+\sum_{k=0}^{\infty} \mu_{k} b_{k} \in h^{1}(X)$, with atom's $a_{k}$ and block's $b_{k}$, is as in Theorem 5.42, then for each $g \in \operatorname{bmo}(x)$,

$$
\lim _{N \rightarrow \infty}\left\{\sum_{k=0}^{N} \lambda_{k} \int_{\chi} a_{k}(x) g(x) d \mu(x)+\sum_{k=0}^{N} \mu_{k} \int_{x} b_{k}(x) g(x) d \mu(x)\right\}
$$

is a well-defined continuous linear functional $\mathcal{L}_{g}: f \mapsto\langle f, g\rangle$ with norm $\lesssim\|g\|_{\mathrm{bmo}(\boldsymbol{x})}$.

Conversely, each continuous linear functional $\mathcal{L}$ on $h^{1}(\mathcal{X})$ has the form $\mathcal{L}=\mathcal{L}_{g}$ for some $g \in \operatorname{bmo}(x)$ with $\|g\|_{\mathrm{bmo}(x)} \lesssim\|\mathcal{L}\|$.

(ii) Assume that $\epsilon$ is as in Definition 5.29, $n /(n+\epsilon)<p<1$, and $s=1 / p-1$. Then $\operatorname{Lip}_{s}(x)$ is the dual space of $h^{p}(x)$ in the sense of $(i)$.

Remark 5.45. We point out that Remark 5.20 applies in a similar way to Theorem 5.44.

In what follows, for any $\beta, \gamma>0$, we let

$$
\mathcal{G}_{b}(\beta, \gamma)=\{f \in \mathcal{G}(\beta, \gamma): f \text { has bounded support }\}
$$

Using Proposition 5.31, by an argument similar to the proof of Proposition 5.21, we establish the following density result for $B_{p, q}^{s}(\mathcal{X})$ and $F_{p, q}^{s}(\mathcal{X})$. We omit the details.

Proposition 5.46. Let $\epsilon_{1}, \epsilon_{2}, \epsilon$, and let $|s|<\epsilon$ be as in Definition 5.29. Then $\mathcal{G}_{b}\left(\epsilon_{1}, \epsilon_{2}\right)$ is dense in $B_{p, q}^{s}(X)$ when $p(s, \epsilon)<p<\infty$ and $0<q<\infty$, and in $F_{p, q}^{s}(X)$ when $p(s, \epsilon)<p, q<\infty$.

We now turn to boundedness results for singular integral operators on $B_{p, q}^{s}(\mathcal{X})$ and $F_{p, q}^{s}(\mathcal{X})$ spaces. In what follows, it will be convenient to put $\|f\|_{\mathcal{C}^{\eta}(\mathcal{X})} \equiv\|f\|_{L^{\infty}(\mathcal{X})}$ when $\eta=0$.

Let $\epsilon>0$ and $\sigma>0$. A linear operator $T$, which is initially assumed to be continuous from $C_{b}^{\eta}(\mathcal{X})$ to $\left(C_{b}^{\eta}(\mathcal{X})\right)^{\prime}$ for all $\eta \in(0, \epsilon)$, is called an inhomogeneous singular integral of order $(\epsilon, \sigma)$ if $T$ has a distributional kernel $K$ which satisfies the conditions (I-1) through (I-4) of 
the kernel of a singular integral of order $\epsilon$ in Subsection 5.2, and the following additional "vanishing" condition that

(I-2) $)_{1}$ the property (I-2) also holds in the limiting case $\eta=\widetilde{\eta}=0$, for $T$ and its adjoint operator $T^{t}$, that is, there exists a constant $C>0$ such that for every normalized $\epsilon$-bump function $\varphi,\|T \varphi\|_{L^{\infty}(\mathcal{X})}+\left\|T^{t} \varphi\right\|_{L^{\infty}(\mathcal{X})} \lesssim C$, as well as the following additional size condition that

$(\mathrm{I}-3)_{3}$ for all $x, y \in \mathcal{X}$ with $d(x, y) \geq 1,|K(x, y)| \leq C(1 / V(x, y))\left(1 / d(x, y)^{\sigma}\right)$.

Remark 5.47. We point out that if $T$ is a singular integral operator of order $(\epsilon, \sigma)$, then $T$ extends to a continuous linear operator from $C^{\eta}(\mathcal{X})$ to $\left(\mathcal{G}_{b}(\eta, \gamma)\right)^{\prime}$ for all $\eta \in(0, \epsilon]$ and all $\gamma>0$; see Proposition 2.25.

We also claim that for $f \in \mathcal{G}_{b}(\eta, \delta)$ with $\eta \in(0, \epsilon]$ and $\delta>0, T f$ can be defined as a distribution in $\left(\mathcal{G}_{0}^{\epsilon}(\beta, \gamma)\right)^{\prime}$ with $0<\beta, \gamma \leq \epsilon$. We first define $T f$ as a distribution in $(\mathcal{G}(\beta, \gamma))^{\prime}$ with $0<\beta \leq \epsilon$ and $\gamma>0$. In fact, for any given $\beta \in(0, \epsilon]$, noticing that $\mathcal{G}_{b}\left(\eta_{1}, \delta\right) \subset \mathcal{G}_{b}\left(\eta_{2}, \delta\right)$ when $\eta_{1} \geq \eta_{2}$, without loss of generality, we may assume that $\eta \leq \beta$. Assume that supp $f \subset$ $B\left(x_{0}, r\right)$ for some $x_{0} \in \mathcal{X}$ and $r>0$. Let $\psi \in C_{b}^{\eta}(\mathcal{X})$ such that $\psi(x)=1$ when $x \in B\left(x_{0}, 2 r\right)$ and $\psi(x)=0$ when $x \notin B\left(x_{0}, 4 r\right)$. For any $g \in \mathcal{G}(\beta, \gamma)$ with $\eta \leq \beta \leq \epsilon$ and $\gamma>0$, it is easy to see that $\psi g \in C_{b}^{\eta}(\mathcal{X})$. From this and $\mathcal{G}_{b}(\eta, \delta) \subset C_{b}^{\eta}(x)$, it follows that $\langle T f, \psi g\rangle$ is well defined. On the other hand, we define $\langle T f,(1-\psi) g\rangle$ by

$$
\langle T f,(1-\psi) g\rangle=\iint_{\chi_{\times} \times} K(x, y) f(y)(1-\psi(x)) g(x) d \mu(x) d \mu(y) .
$$

Clearly, if supp $f \cap \operatorname{supp}\{(1-\psi) g\}=\varnothing$, this definition coincides with (I-1). Moreover, by $(\mathrm{I}-3)_{3}$ and Lemma 2.1(i), we have

$$
|\langle T f,(1-\psi) g\rangle| \lesssim\|f\|_{L^{\infty}(\chi)}\|g\|_{L^{1}(\chi)} \lesssim\|f\|_{\mathcal{G}_{(\eta, \delta)}}\|g\|_{\mathcal{G}_{(\beta, \gamma)}}
$$

It is also easy to verify that $\langle T f, \psi g\rangle+\langle T f,(1-\psi) g\rangle$ is independent of the choice of $\psi$. Thus, we can define $T f$ by $\langle T f, g\rangle=\langle T f, \psi g\rangle+\langle T f,(1-\psi) g\rangle$, so that $T f \in(\mathcal{G}(\beta, \gamma))^{\prime}$ with $0<$ $\beta \leq \epsilon$ and $\gamma>0$. Now for any $g \in \mathcal{G}_{0}^{\epsilon}(\beta, \gamma)$ with $0<\beta, \gamma \leq \epsilon$, let $\left\{g_{n}\right\}_{n \in \mathbb{N}} \subset \mathcal{G}(\epsilon, \epsilon)$ such that $\left\|g_{n}-g\right\|_{\mathcal{G}(\beta, \gamma)} \rightarrow 0$ as $n \rightarrow \infty$. We then define $T f \in\left(\mathcal{G}_{0}^{\epsilon}(\beta, \gamma)\right)^{\prime}$ with $0<\beta, \gamma \leq \epsilon$ by $\langle T f, g\rangle=\lim _{n \rightarrow \infty}\left\langle T f, g_{n}\right\rangle$. It is easy to check that $\langle T f, g\rangle$ is independent of the choice of $\left\{g_{n}\right\}_{n \in \mathbb{N}} \subset \mathcal{G}(\epsilon, \epsilon)$. In this sense, we have $T f \in\left(\mathcal{G}_{0}^{\epsilon}(\beta, \gamma)\right)^{\prime}$ with $0<\beta, \gamma \leq \epsilon$.

In what follows, for $\epsilon \in(0,1]$ and $0<\beta, \gamma \leq \epsilon$, put

$$
\mathcal{G}_{0, b}^{\epsilon}(\beta, \gamma)=\left\{f \in \mathcal{G}_{0}^{\epsilon}(\beta, \gamma): f \text { has bounded support }\right\}
$$

Theorem 5.48. Let $\epsilon_{1}, \epsilon_{2}, \epsilon$, and $|s|<\epsilon$ be as in Definition 5.29. Let $\sigma>0$ and let $T$ be a singular integral of order $(\epsilon, \sigma)$ with $\sigma>n(1 / p-1)_{+}$. Then $T$ is bounded on $B_{p, q}^{s}(x)$ when $p(s, \epsilon)<p<\infty$ and $0<q<\infty$ and bounded from $B_{p, q}^{s}(\mathcal{X}) \cap \mathcal{G}_{b}\left(\epsilon_{1}, \epsilon_{2}\right)$ to $B_{p, q}^{s}(\mathcal{X})$ when $\max \{p, q\}=\infty$, and $T$ is also bounded on $F_{p, q}^{s}(\mathcal{X})$ when $p(s, \epsilon)<p, q<\infty$, and bounded from $F_{p, q}^{s}(\mathcal{X}) \cap \mathcal{G}_{b}\left(\epsilon_{1}, \epsilon_{2}\right)$ to $F_{p, q}^{s}(\mathcal{X})$ when $p(s, \epsilon)<p<\infty$ and $q=\infty$. 
Proof. By Propositions 5.46 and 5.31(vii) together with a density argument, it suffices to verify the conclusions of the theorem for all $f \in \mathcal{G}_{0, b}^{\epsilon}(\beta, \gamma)$ with $\beta, \gamma$ as in (5.111).

Let $\left\{S_{k}\right\}_{k \in \mathbb{Z}_{+}}$be an IATI with bounded support as constructed in Theorem 2.6. Put $D_{0}=$ $S_{0}$ and $D_{k}=S_{k}-S_{k-1}$ for $k \in \mathbb{N}$. Using Remark 5.47 and Theorem 4.15 together with an argument similar to the proof of Theorem 5.23, we see that for all $k \in \mathbb{Z}_{+}$and $x \in \mathcal{X}$,

$$
\begin{aligned}
D_{k} T f(x)= & \sum_{\tau^{\prime} \in I_{0}} \sum_{v^{\prime}=1}^{N\left(0, \tau^{\prime}\right)} \bar{D}_{\tau^{\prime}, 1}^{0, v^{\prime}}(f) \int_{Q_{\tau^{\prime}}^{0, v^{\prime}}}\left(D_{k} T D_{0}(\cdot, y)\right)(x) d \mu(y) \\
& +\sum_{k^{\prime}=1}^{\infty} \sum_{\tau^{\prime} \in I_{k^{\prime}}}^{N\left(k^{\prime}, \tau^{\prime}\right)} \mu\left(Q_{v^{\prime}=1}^{k^{\prime}, v^{\prime}}\right)\left(D_{k} T D_{k^{\prime}}\left(\cdot, y_{\tau^{\prime}}^{k^{\prime}, \nu^{\prime}}\right)\right)(x) \bar{D}_{k^{\prime}}(f)\left(y_{\tau^{\prime}}^{k^{\prime}, v^{\prime}}\right),
\end{aligned}
$$

where all the notation is as in Theorem 4.15.

For $k, k^{\prime} \in \mathbb{Z}_{+}, \tau^{\prime} \in I_{k^{\prime}}, v^{\prime}=1, \ldots, N\left(k^{\prime}, \tau^{\prime}\right), y \in Q_{\tau^{\prime}}^{k^{\prime}, v^{\prime}}$, and $x \in \mathcal{x}$, let $Z(x, y) \equiv$ $\left(D_{k} T D_{k^{\prime}}(\cdot, y)\right)(x)$. We now claim that when $k=k^{\prime}=0$,

$$
|Z(x, y)| \lesssim\left(\frac{1}{1+d(x, y)}\right)^{\sigma} \frac{1}{V_{1}(x)+V_{1}(y)+V(x, y)}
$$

when $k=0$ and $k^{\prime} \in \mathbb{N}$, for any fixed $\eta \in(0, \epsilon)$,

$$
|Z(x, y)| \lesssim 2^{-k^{\prime} \eta}\left(\frac{1}{1+d(x, y)}\right)^{\eta} \frac{1}{V_{1}(x)+V_{1}(y)+V(x, y)}
$$

when $k \in \mathbb{N}$ and $k^{\prime}=0$, for any fixed $\eta \in(0, \epsilon)$,

$$
|Z(x, y)| \lesssim 2^{-k \eta}\left(\frac{1}{1+d(x, y)}\right)^{\eta} \frac{1}{V_{1}(x)+V_{1}(y)+V(x, y)}
$$

and when $k, k^{\prime} \in \mathbb{N}$, for any fixed $\eta \in(0, \epsilon)$,

$$
|Z(x, y)| \lesssim 2^{-\left|k-k^{\prime}\right| \eta}\left(\frac{2^{-\left(k \wedge k^{\prime}\right)}}{2^{-\left(k \wedge k^{\prime}\right)}+d(x, y)}\right)^{\eta} \frac{1}{V_{2^{-\left(k \wedge k^{\prime}\right)}}(x)+V_{2^{-\left(k \wedge k^{\prime}\right)}}(y)+V(x, y)} .
$$

Obviously, (5.184) is just (5.65). The estimate (5.183) is easily deduced from (5.182) by symmetry. Thus, we only need to verify (5.181) and (5.182). Assume that $k^{\prime} \in \mathbb{Z}_{+}$. To prove (5.181) and (5.182), it suffices to prove that

$$
|Z(x, y)| \lesssim 2^{-k^{\prime} \eta_{1}} \frac{1}{V_{1}(x)},
$$

and that when $d(x, y) \geq 2^{5}$, then

$$
|Z(x, y)| \lesssim\left(\frac{2^{-k^{\prime}}}{d(x, y)}\right)^{\eta_{2}} \frac{1}{V(x, y)}
$$


where when $k^{\prime}=0, \eta_{1}=0$, and $\eta_{2}=\sigma$, and when $k^{\prime} \in \mathbb{N}, \eta_{1}=\eta_{2}=\eta$. To see (5.185), for $z \in \mathcal{X}$, set $\Psi(z) \equiv \int_{\mathcal{X}} K(w, z) D_{0}(w, x) d \mu(w)$. From the properties of $S_{0}$ in Theorem 2.6, Condition (I-2), Condition (I-4), and Condition (I-2) $)_{1}$, it follows that for any fixed $\eta \in[0, \epsilon)$, $\|\Psi\|_{C^{\eta}(x)} \lesssim 1 / V_{1}(x)$, which shows that for all $z \in \mathcal{X},|\Psi(z)| \lesssim 1 / V_{1}(x)$, and for $\eta \in(0, \epsilon)$ and all $z, y \in \mathcal{X}$,

$$
|\Psi(z)-\Psi(y)| \lesssim d(z, y)^{\eta} \frac{1}{V_{1}(x)}
$$

Thus, when $k^{\prime}=0$, by size conditions of both $\Psi$ and $S_{0}$, then

$$
|Z(x, y)|=\left|\int_{\chi} \Psi(z) S_{0}(z, y) d \mu(z)\right| \lesssim \frac{1}{V_{1}(x)}
$$

while when $k^{\prime} \in \mathbb{N}$, by the vanishing moment and the size condition of $D_{k^{\prime}}$ together with the regularity of $\Psi$, for any fixed $\eta \in(0, \epsilon)$,

$$
|Z(x, y)|=\left|\int_{x}[\Psi(z)-\Psi(y)] D_{k^{\prime}}(z, y) d \mu(z)\right| \lesssim 2^{-k^{\prime} \eta} \frac{1}{V_{1}(x)}
$$

which verifies (5.185).

To see (5.186), we first notice that when $d(z, y)<2^{2-k^{\prime}}, d(w, x)<2^{2}$, and $d(x, y) \geq 2^{5}$, then $d(w, z) \geq d(x, y)-d(w, x)-d(z, y)>\max \{d(x, y) / 2,8,8 d(z, y)\}, d(w, y)>d(x, y) / 2$, and $d(w, z) \geq d(w, y)-d(z, y)>d(w, y) / 2$. Therefore,

$$
V(w, z) \gtrsim V(w, y) \gtrsim V(y, x) \sim V(x, y)
$$

Thus, if $k^{\prime}=0$, by $(\mathrm{I}-3)_{3}$, we have

$$
\begin{aligned}
|Z(x, y)| & \lesssim \iint_{x \times x}\left|D_{0}(w, x)\right| \frac{1}{V(w, z)} \frac{1}{d(z, y)^{\sigma}}\left|D_{0}(z, y)\right| d \mu(w) d \mu(z) \\
& \lesssim \frac{1}{V(x, y)} \frac{1}{d(x, y)^{\sigma}}
\end{aligned}
$$

while when $k^{\prime} \in \mathbb{N}$, by the regularity on $K$ and the vanishing moment of $D_{k^{\prime}}$, for any fixed $\eta \in(0, \epsilon)$, we have

$$
\begin{aligned}
|Z(x, y)| & \lesssim \iint_{\chi \times x}\left|D_{0}(w, x)\right| \frac{d(z, y)^{\epsilon}}{V(w, z) d(w, z)^{\epsilon}}\left|D_{k^{\prime}}(z, y)\right| d \mu(w) d \mu(z) \\
& \lesssim\left(\frac{2^{-k^{\prime}}}{d(x, y)}\right)^{\epsilon} \frac{1}{V(x, y)}
\end{aligned}
$$

which implies (5.186). Thus, the estimates (5.181) and (5.182) hold. 
Using these estimates and Remark 5.26, by a procedure essentially similar to the proof of Proposition 5.25, we then obtain the boundedness on $B_{p, q}^{s}(\mathcal{X})$ and $F_{p, q}^{s}(\mathcal{X})$ of $T$. The details are left to the reader.

\section{5. $T(1)$-theorems}

In this subsection, we will establish analogues of David and Journé's T(1)-theorem [68] and of Stein's variant of this theorem in [75] for RD-spaces.

We begin with generalizing [71, Theorem 1, page 114] on $\mathbb{R}^{n}$ to the setting of spaces of homogeneous type which is of independent of interest; see also [69, Proposition 2].

Theorem 5.49. Let $\epsilon \in(0,1], \beta \in(0, \epsilon)$, and let $T$ be as in Proposition 2.12 with a distributional kernel $K$ satisfying the size condition (2.59). Then $T$ can be extended as a continuous linear operator on $\dot{C}^{\beta}(\mathcal{X})$ if and only if $T \in \operatorname{WBP}(\beta)$ and $T(1)=0$ in $\left(\dot{C}_{b}^{\beta}(\mathcal{X})\right)^{\prime}$.

Proof. We first prove the sufficiency. To this end, for any $k_{1} \in \mathbb{Z}$, let

$$
B_{k_{1}}=\left\{x \in X: 2^{k_{1}-1} \leq d\left(x, x_{1}\right)<2^{k_{1}}\right\}
$$

Fix any $x_{0} \in B_{k_{1}}$. Let $\theta$ be as in Lemma 2.15. For any $k_{2} \in \mathbb{Z}$, put $\theta_{k_{2}}(y)=\theta\left(d\left(x_{0}, y\right) / 3 \cdot 2^{k_{2}}\right)$, and define $\omega_{k_{2}}=1-\theta_{k_{2}}$. For any $f \in C^{\beta}(\mathcal{X})$, following an argument as in the proof of Lemma 2.20 (see also [69, the proof of Proposition 2]), for a.e. $x \in B\left(x_{0},(3 / 2) 2^{k_{2}}\right)$, we have

$$
\begin{aligned}
T f(x)= & \int_{x} K(x, y)[f(y)-f(x)] \theta_{k_{2}}(y) d \mu(y) \\
& +\left[\int_{x} K(x, y) f(y) \omega_{k_{2}}(y) d \mu(y)+f(x) T\left(\theta_{k_{2}}\right)(x)\right] \\
= & \Gamma_{1}(x)+\Gamma_{2}(x) .
\end{aligned}
$$

By Lemma 2.15, for a.e. $x \in B\left(x_{0},(3 / 2) 2^{k_{2}}\right)$, we also have

$$
\begin{gathered}
\left|T\left(\theta_{k_{2}}\right)(x)\right| \lesssim C_{T}+\|T\|_{\mathrm{WBP}(\beta),} \\
T\left(\theta_{k_{2}}\right)(x)=C_{k_{2}}-\int_{x}\left[K(x, y)-K\left(x_{0}, y\right)\right] \omega_{k_{2}}(y) d \mu(y),
\end{gathered}
$$

where $C_{k_{2}}$ is a constant independent of $x$.

For any $x \in B\left(x_{0}, 2^{k_{2}} / 2\right)$, we then consider $x^{\prime} \in \mathcal{X}$ satisfying $2^{k_{2}-1} \leq d\left(x, x^{\prime}\right)<2^{k_{2}}$. Notice that if $x \in B\left(x_{0}, 2^{k_{2}} / 2\right)$ and $d\left(x, x^{\prime}\right)<2^{k_{2}}$, then $x^{\prime} \in B\left(x_{0},(3 / 2) 2^{k_{2}}\right)$, and that $\theta_{k_{2}}(y) \neq 0$ implies $d(x, y)<14 d\left(x, x^{\prime}\right)$. Thus, if $x, x^{\prime}$ satisfy (5.194), (5.195), and (5.196), by the size condition (2.59) of the kernel $K$, the definition of $\dot{C}^{\beta}(\mathcal{X})$ and Lemma 2.1(i), we then have

$$
\left|\Gamma_{1}(x)\right| \leq \int_{d(x, y)<14 d\left(x, x^{\prime}\right)}|K(x, y)||f(y)-f(x)| d \mu(y) \lesssim d\left(x, x^{\prime}\right)^{\beta} C_{T}\|f\|_{\dot{C}^{\beta}(x)}
$$


and a similar estimate also holds for $\Gamma_{1}\left(x^{\prime}\right)$, which clearly implies

$$
\left|\Gamma_{1}(x)-\Gamma_{1}\left(x^{\prime}\right)\right| \leq\|f\|_{\mathcal{C}^{\beta}(x)} C_{T} d\left(x, x^{\prime}\right)^{\beta}
$$

Moreover, by (5.196), we also have

$$
\begin{aligned}
\Gamma_{2}(x)-\Gamma_{2}\left(x^{\prime}\right)= & \int_{x}\left[K(x, y)-K\left(x^{\prime}, y\right)\right][f(y)-f(x)] \omega_{k_{2}}(y) d \mu(y) \\
& +\left[f(x)-f\left(x^{\prime}\right)\right] \int_{x} K\left(x^{\prime}, y\right) \theta_{k_{2}}(y) d \mu(y) \\
= & \Gamma_{2,1}+\Gamma_{2,2} .
\end{aligned}
$$

The estimate (5.195) yields

$$
\left|\Gamma_{2,2}\right| \lesssim d\left(x, x^{\prime}\right)^{\beta}\left(C_{T}+\|T\|_{\mathrm{BMO}(\beta)}\right)\|f\|_{\dot{C}^{\beta}(x)}
$$

Notice that $\omega_{k_{2}}(y) \neq 0$ implies that $d(x, y)>2 d\left(x, x^{\prime}\right)$. The regularity (2.49) on $K$ and the definition of $\dot{C}^{\beta}(\mathcal{X})$ together with Lemma 2.1(i) and $\beta<\epsilon$ then yield

$$
\begin{aligned}
\left|\Gamma_{2,1}\right| & \lesssim C_{T}\|f\|_{\dot{C}^{\beta}(x)} \int_{d(x, y)>2 d\left(x, x^{\prime}\right)} \frac{d\left(x, x^{\prime}\right)^{\epsilon}}{V(x, y) d(x, y)^{\epsilon}} d(x, y)^{\beta} d \mu(y) \\
& \lesssim d\left(x, x^{\prime}\right)^{\beta} C_{T}\|f\|_{\dot{C}^{\beta}(x)} .
\end{aligned}
$$

Combining all the above estimates shows that for a.e. $x \in B\left(x_{0}, 2^{k_{2}} / 4\right)$ and a.e. $x^{\prime} \in x$ satisfying $2^{k_{2}-1} \leq d\left(x, x^{\prime}\right)<2^{k_{2}}$, we have

$$
\left|T f(x)-T f\left(x^{\prime}\right)\right| \lesssim d\left(x, x^{\prime}\right)^{\beta}\left(C_{T}+\|T\|_{\mathrm{WBP}(\beta)}\right)\|f\|_{\dot{C}^{\beta}(x)} .
$$

Then an argument via the Besicovitch covering lemma further shows that there exists an extension of $T f$ such that

$$
\|T f\|_{\mathcal{C}^{\beta}(\mathcal{X})} \lesssim\left(C_{T}+\|T\|_{\mathrm{WBP}(\beta)}\right)\|f\|_{\mathcal{C}^{\beta}(\mathcal{X})}
$$

which completes the proof of the sufficiency.

We now prove the necessity. Since $T$ is extended as a continuous linear operator on $\dot{C}^{\beta}(\mathcal{X})$ and $1=0$ in $\dot{C}^{\beta}(\mathcal{X})$. Thus, $T(1)=0$ in $\dot{C}^{\beta}(\mathcal{X})$. Since $\dot{C}^{\beta}(\mathcal{X})=\dot{B}_{\infty, \infty}^{\beta}(\mathcal{X})=\left(\dot{B}_{1,1}^{-\beta}(\mathcal{X})\right)^{\prime}$ by Theorems 6.11 and $8.11(\mathrm{i})$, and $\dot{C}_{b}^{\beta}(\mathcal{X}) \subset \dot{B}_{1,1}^{-\beta}(\mathcal{X})$ by Proposition 5.10(iv), we then have that for all $f \in \dot{C}_{b}^{\beta}(\mathcal{X}),\langle T(1), f\rangle=0$, which just means that $T(1)=0$ in $\left(\stackrel{C}{C}_{b}^{\beta}(\mathcal{X})\right)^{\prime}$. 
Let now $\phi, \psi \in C_{b}^{\beta}(\mathcal{X})$ as in Definition 2.13. Fix $x_{0}^{\prime} \in \mathcal{X}$ such that $2 r \leq d\left(x_{0}^{\prime}, x_{0}\right)<3 r$. Since $x_{0}^{\prime} \notin \operatorname{supp} \phi$, by the size condition (2.59) of the kernel $K$ and (2.55), we then have

$$
\begin{aligned}
\left|T(\phi)\left(x_{0}^{\prime}\right)\right| & =\left|\int_{B\left(x_{0}, r\right)} K\left(x_{0}^{\prime}, y\right) \phi(y) d \mu(y)\right| \\
& \lesssim \frac{1}{V_{r}\left(x_{0}^{\prime}\right)}\|\phi\|_{L^{\infty}(x)} \mu\left(B\left(x_{0}, r\right)\right) \\
& \lesssim 1
\end{aligned}
$$

Since $T$ is bounded on $\dot{C}^{\beta}(\mathcal{X})$, when $d\left(x, x_{0}^{\prime}\right) \leq 5 r$, we then have

$$
|T(\phi)(x)| \lesssim\left|T(\phi)\left(x_{0}^{\prime}\right)\right|+d\left(x, x_{0}^{\prime}\right)^{\beta}\|\phi\|_{\dot{C}^{\beta}(x)} \lesssim 1
$$

When $d\left(x, x_{0}^{\prime}\right)>5 r$, by the size condition (2.59) of the kernel $K$ and (2.55) again, we also have

$$
|T(\phi)(x)|=\left|\int_{B\left(x_{0}, r\right)} K(x, y) \phi(y) d \mu(y)\right| \lesssim \frac{1}{V\left(x, x_{0}^{\prime}\right)}\|\phi\|_{L^{\infty}(x)} \mu\left(B\left(x_{0}, r\right)\right) \lesssim 1 .
$$

Thus, $\|T(\phi)\|_{L^{\infty}(\mathcal{X})} \lesssim 1$, which gives that $|\langle T(\phi), \psi\rangle| \lesssim\|\psi\|_{L^{1}(\mathcal{X})} \lesssim \mu\left(B\left(x_{0}, r\right)\right)$. That is, $T \in$ $\mathrm{WBP}(\beta)$, which completes the proof of Theorem 5.49.

Remark 5.50. (i) The proof of Theorem 5.49 in combination with Corollary 2.23 shows that if $T$ is bounded on $\dot{C}^{\beta}(\mathcal{X})$, then there exists a constant $C>0$ such that for all $\phi \in \dot{C}_{b}^{\beta}(\mathcal{X})$ and all $x \in X$,

$$
|T(\phi)(x)| \leq C\left(C_{T}+\|T\|_{\dot{C}^{\beta}(\mathcal{X}) \rightarrow \dot{C}^{\beta}(\mathcal{X})}\right)[\operatorname{diam}(\operatorname{supp} \phi)]^{\beta}\|\phi\|_{\dot{C}^{\beta}(\mathcal{X})} .
$$

(ii) Let $T$ be as in Theorem 5.49 and $T(1)=0$ in $\left(\mathcal{C}_{b}^{\beta}(\mathcal{X})\right)^{\prime}$. Then from (i) and Theorem 5.49, it is easy to see that $T \in \mathrm{WBP}(\beta)$ if and only if (5.207) holds.

Now we recall the notion of Carleson measures and establish their connection with $\operatorname{BMO}(x)$ functions.

Definition 5.51. A positive measure $v$ on $\mathcal{X} \times(0, \infty)$ is said to be a Carleson measure if there exists a constant $C>0$ such that for every ball $B(x, r)$ for some $x \in \mathcal{X}$ and $r>0$,

$$
\mathcal{v}(B(x, r) \times(0, r)) \leq C \mu(B(x, r)) .
$$

The smallest bound $C$ as above is defined to be the Carleson norm of $v$ and is denoted by $\|v\|_{\mathfrak{c}}$.

For any given open set $E$ of $\mathcal{X}$, let

$$
\widehat{E}=\{(x, t) \in X \times(0, \infty): B(x, t) \subset E\} .
$$


We first establish a basic property of Carleson measures; see [75, pages 59-60] or [81, page 198] for the case of $\mathbb{R}^{n}$.

Lemma 5.52. If $v$ is a Carleson measure in $\mathcal{X} \times(0, \infty)$ and $E \subset \mathcal{X}$ is open, then $v(\widehat{E}) \leq C\|v\|_{\mathfrak{c}} \mu(E)$.

Proof. Without loss of generality, we may assume that $E$ is a bounded open set of $\mathcal{X}$. By the Whitney-type covering lemma (see [28, Theorem (3.2)]), there exist a constant $C>0$ and a sequence of balls $\left\{B\left(y_{j}, r_{j}\right)\right\}_{j}$ satisfying $E=\cup_{j} B\left(y_{j}, r_{j}\right), \sum_{j} X B\left(y_{j}, r_{j}\right) \leq C$ and $B\left(y_{j}, 3 r_{j}\right) \cap(X \backslash$ $E) \neq \varnothing$ for each $j$. If $(x, t) \in \widehat{E}$, then there exists $j_{0}$ such that $x \in B\left(y_{j_{0}}, r_{j_{0}}\right), B(x, t) \subset E$, and $B\left(y_{j_{0}}, 3 r_{j_{0}}\right) \cap(x \backslash E) \neq \varnothing$. From this, it follows that $t<6 r_{j_{0}}$, and therefore, $(x, t) \in B\left(y_{j_{0}}, r_{j_{0}}\right) \times$ $\left(0,6 r_{j_{0}}\right)$. Thus, $\widehat{E} \subset \cup_{j}\left\{B\left(y_{j}, r_{j}\right) \times\left(0,6 r_{j}\right)\right\}$, which together with the definition of Carleson measures implies that

$$
v(\widehat{E}) \leq \sum_{j} v\left(B\left(y_{j}, r_{j}\right) \times\left(0,6 r_{j}\right)\right) \lesssim\|v\|_{\mathfrak{C}} \sum_{j} \mu\left(B\left(y_{j}, r_{j}\right)\right)
$$

On the other hand, from $E=\cup_{j} B\left(y_{j}, r_{j}\right)$ and $\sum_{j} X_{B\left(y_{j}, r_{j}\right)} \leq C$, it follows that

$$
\mu(E)=\mu\left(\bigcup_{j} B\left(y_{j}, r_{j}\right)\right) \gtrsim \int_{x} \sum_{j} X_{B\left(y_{j}, r_{j}\right)}(y) d \mu(y) \sim \sum_{j} \mu\left(B\left(y_{j}, r_{j}\right)\right) .
$$

Combining both estimates yields $v(\widehat{E}) \lesssim\|v\|_{\mathfrak{C}} \mu(E)$, which completes the proof of Lemma 5.52.

Let $\left\{S_{k}\right\}_{k \in \mathbb{Z}}$ be an $\left(\epsilon_{1}, \epsilon_{2}, \epsilon_{3}\right)$-ATI as in Definition 2.2. For $(x, t) \in \mathcal{X} \times(0, \infty)$, we define

$$
\mathfrak{S}(f)(x, t)=\sum_{j=-\infty}^{\infty} S_{j}(f)(x) \chi_{\left(2^{-j-1}, 2^{-j}\right]}(t)
$$

Proposition 5.53. Let $\mathfrak{S}$ be as in (5.212). For any $p \in(1, \infty)$, there exists a constant $C_{p}>0$ such that for all $f \in L^{p}(\mathcal{X})$ and all Carleson measures $\mathcal{v}$,

$$
\int_{x \times(0, \infty)}|\mathfrak{S}(f)(x, t)|^{p} d v(x, t) \leq C_{p}\|v\|_{\mathfrak{C}} \int_{x}|f(x)|^{p} d \mu(x) .
$$

Proof. Let $\mathfrak{S}$ be as in above. For any $x \in \mathcal{X}$, we define

$$
M_{\mathfrak{S}} f(x)=\sup \{|\mathfrak{S}(f)(y, t)|: d(x, y)<t\}
$$


We first claim that for all $x \in \mathcal{X}, M_{\mathfrak{S}} f(x) \lesssim M f(x)$, where $M$ is the Hardy-Littlewood maximal function. To see this, for any $(y, t) \in \mathcal{X} \times(0, \infty)$ satisfying $d(x, y)<t$, assuming $2^{-j_{0}-1}<t \leq 2^{-j_{0}}$ for some $j_{0} \in \mathbb{Z}$, by Lemma 2.1(vi), we then have

$|\mathfrak{S}(f)(y, t)|$

$$
\begin{aligned}
& \lesssim \int_{d(y, z)<2^{-j_{0}}} \frac{1}{V_{2^{-j_{0}}}(y)+V_{2^{-j_{0}}}(z)+V(y, z)}\left(\frac{2^{-j_{0}}}{2^{-j_{0}}+d(y, z)}\right)^{\epsilon_{2}}|f(z)| d \mu(z)+\sum_{l=1}^{\infty} \int_{2^{l-12^{-j} j_{0} \leq d(y, z)<2^{2} 2^{-j_{0}}}} \ldots \\
& \lesssim M f(x),
\end{aligned}
$$

which implies the claim.

Notice that

$$
\int_{\chi \times(0, \infty)}|\mathfrak{S}(f)(x, t)|^{p} d v(x, t)=p \int_{0}^{\infty} \lambda^{p-1} \mathcal{v}(\{(x, t) \in \mathcal{X} \times(0, \infty):|\mathfrak{S}(f)(x, t)|>\lambda\}) d \lambda .
$$

Let $E_{\lambda}=\left\{x \in \mathcal{X}: M_{\mathfrak{S}} f(x)>\lambda\right\}$. We then claim

$$
\{(x, t) \in \mathcal{X} \times(0, \infty):|\mathfrak{S}(f)(x, t)|>\lambda\} \subset \widehat{E}_{\lambda}
$$

In fact, for any $(x, t) \in \mathcal{X} \times(0, \infty)$ such that $|\mathfrak{S}(f)(x, t)|>\lambda$, assume that $2^{-j_{0}-1}<t \leq 2^{-j_{0}}$ for some $j_{0} \in \mathbb{Z}$. Then $|\mathfrak{S}(f)(x, t)|>\lambda$ if and only if $\left|S_{j_{0}}(f)(x)\right|>\lambda$. If $d(y, x)<t$, then $M_{\mathfrak{S}} f(y)>\lambda$ and hence $B(x, t) \subset E_{\lambda}$, which implies the claim.

From this claim and Lemma 5.52, it follows that

$$
v(\{(x, t) \in \mathcal{X} \times(0, \infty):|\mathfrak{S}(f)(x, t)|>\lambda\}) \leq v\left(\widehat{E}_{\curlywedge}\right) \lesssim\|v\|_{\mathfrak{C}} \mu\left(E_{\curlywedge}\right),
$$

which together with the $L^{p}(\mathcal{X})$-boundedness for $p \in(1, \infty)$ of $M$ yields

$$
\begin{aligned}
\int_{x \times(0, \infty)}|\mathfrak{S}(f)(x, t)|^{p} d v(x, t) & \lesssim\|v\|_{\mathfrak{C}} \int_{0}^{\infty} \lambda^{p-1} \mu\left(E_{\curlywedge}\right) d \lambda \\
& \sim\|v\|_{\mathfrak{C}} \int_{x}\left|M_{\mathfrak{S}}(f)(x)\right|^{p} d \mu(x) \\
& \lesssim\|v\|_{\mathfrak{C}} \int_{x}|M(f)(x)|^{p} d \mu(x) \\
& \lesssim\|v\|_{\mathfrak{C}} \int_{x}|f(x)|^{p} d \mu(x),
\end{aligned}
$$

which completes the proof of Proposition 5.53. below.

The relation between Carleson measures and $\operatorname{BMO}(\mathcal{X})$ functions can be stated as 
Proposition 5.54. Let $b \in \mathrm{BMO}(x)$ and let $\left\{S_{k}\right\}_{k \in \mathbb{Z}}$ be an $\left(\epsilon_{1}, \epsilon_{2}, \epsilon_{3}\right)$-ATI as in Definition 2.2. For $k \in \mathbb{Z}$, let $D_{k}=S_{k}-S_{k-1}$. Then the measure $v$ defined by

$$
d v(x, t)=\sum_{j=-\infty}^{\infty}\left|D_{j}(b)(x)\right|^{2} x_{\left(2^{-j-1}, 2^{-j}\right]}(t) d \mu(x) \frac{d t}{t}
$$

is a Carleson measure such that $\|v\|_{\mathfrak{C}}$ is dominated by $\|b\|_{\mathrm{BMO}(x)}^{2}$.

Proof. For any ball $B=B\left(x_{0}, r\right)$ with some $x_{0} \in \mathcal{X}$ and $r>0$, assume that $2^{-j_{0}-1}<r \leq 2^{-j_{0}}$ for some $j_{0} \in \mathbb{Z}$. We then have

$$
\begin{aligned}
v\left(B\left(x_{0}, r\right) \times(0, r)\right) & =\int_{B\left(x_{0}, r\right)} \int_{0}^{r} d v(x, t) \\
& =\sum_{j=-\infty}^{\infty} \int_{B\left(x_{0}, r\right)} \int_{0}^{r}\left|D_{j}(b)(x)\right|^{2} \chi_{\left(2^{-j-1}, 2^{-j}\right]}(t) d \mu(x) \frac{d t}{t} \\
& \lesssim \sum_{j=j_{0}-1}^{\infty} \int_{B\left(x_{0}, r\right)}\left|D_{j}(b)(x)\right|^{2} d \mu(x) .
\end{aligned}
$$

Let $\widetilde{B}=B\left(x_{0}, 3 r\right), \widetilde{B}_{0}=\widetilde{B}$, and $\widetilde{B}_{k}=B\left(x_{0}, 2^{k} 3 r\right)$ for $k \in \mathbb{N}$. Set also

$$
b_{\widetilde{B}}=\frac{1}{\mu(\widetilde{B})} \int_{\widetilde{B}} b(y) d \mu(y) .
$$

Since $\int_{x} D_{j}(x, y) d \mu(y)=0$, we then have

$$
\begin{aligned}
v\left(B\left(x_{0}, r\right) \times(0, r)\right) \lesssim & \sum_{j=j_{0}-1}^{\infty} \int_{B\left(x_{0}, r\right)}\left|D_{j}\left(\left(b-b_{\tilde{B}}\right) X_{\widetilde{B}}\right)(x)\right|^{2} d \mu(x) \\
& +\sum_{j=j_{0}-1}^{\infty} \int_{B\left(x_{0}, r\right)}\left|D_{j}\left(\left(b-b_{\tilde{B}}\right) x_{x \backslash \tilde{B}}\right)(x)\right|^{2} d \mu(x) \\
& \equiv Y_{1}+Y_{2} .
\end{aligned}
$$

By Lemma 3.9, we have

$$
\begin{aligned}
Y_{1} & \lesssim \sum_{j=j_{0}-1}^{\infty} \int_{x}\left|D_{j}\left(\left(b-b_{\tilde{B}}\right) X_{\tilde{B}}\right)(x)\right|^{2} d \mu(x) \\
& \lesssim \int_{x}\left|\left(\left(b(x)-b_{\widetilde{B}}\right) X_{\widetilde{B}}(x)\right)\right|^{2} d \mu(x) \\
& \lesssim\|b\|_{\mathrm{BMO}(\mathcal{X})}^{2} \mu\left(B\left(x_{0}, r\right)\right) .
\end{aligned}
$$


To estimate $Y_{2}$, we first notice that if $d\left(x, x_{0}\right)<r$ and $y \notin \widetilde{B}_{k}$ for $k \in \mathbb{Z}_{+}$, then $d(y, x) \geq$ $2^{k} 3 r-r>2^{k-1} 3 r$ and hence

$$
\begin{aligned}
\left|D_{j}(x, y)\right| & \lesssim \frac{1}{V_{2^{-j}}(x)+V_{2^{-j}}(y)+V(x, y)}\left(\frac{2^{-j}}{2^{-j}+d(x, y)}\right)^{\epsilon_{2}} \\
& \lesssim \frac{1}{V_{2^{k+2} 3 r}(x)}\left(\frac{2^{-j}}{2^{k} r}\right)^{\epsilon_{2}} .
\end{aligned}
$$

From this, it follows that

$$
\begin{aligned}
Y_{2} & \lesssim \sum_{j=j_{0}-1}^{\infty} \int_{B\left(x_{0}, r\right)}\left[\sum_{k=0}^{\infty} \int_{\tilde{B}_{k+1} \backslash \tilde{B}_{k}}\left|b(y)-b_{\tilde{B}}\right|\left|D_{j}(x, y)\right| d \mu(y)\right]^{2} d \mu(x) \\
& \lesssim \frac{1}{r^{2 \epsilon_{2}}} \sum_{j=j_{0}-1}^{\infty} 2^{-2 j \epsilon_{2}} \int_{B\left(x_{0}, r\right)}\left[\sum_{k=0}^{\infty} \frac{1}{2^{k \epsilon_{2}}} \frac{1}{V_{2^{k+2} 3 r}(x)} \int_{B\left(x, 2^{k+2} 3 r\right)}\left|b(y)-b_{\tilde{B}}\right| d \mu(y)\right]^{2} d \mu(x) \\
& \lesssim\|b\|_{\mathrm{BMO}(x)}^{2} \mu\left(B\left(x_{0}, r\right)\right)
\end{aligned}
$$

where we used the well-known fact that

$$
\|b\|_{\mathrm{BMO}(\mathcal{X})} \sim \inf _{C \in \mathbb{C}} \sup _{x \in \mathcal{X}, r>0} \frac{1}{\mu(B(x, r))} \int_{B(x, r)}|f(y)-C| d \mu(y) .
$$

This finishes the proof of Proposition 5.54.

Combining Proposition 5.53 with Proposition 5.54 yields the following conclusion, which will be used in the proof of $T(1)$-theorem.

Corollary 5.55. Let all the notation be as in Propositions 5.53 and 5.54. For any $p \in(1, \infty)$, there exists a constant $C_{p}>0$ such that for all $f \in L^{p}(\mathcal{X})$ and $b \in \mathrm{BMO}(\mathcal{X})$,

$$
\sum_{j=-\infty}^{\infty} \int_{x}\left|S_{j}(f)(x)\right|^{p}\left|D_{j}(b)(x)\right|^{2} d \mu(x) \leq C_{p}\|b\|_{\mathrm{BMO}(x)}^{2} \int_{x}|f(x)|^{p} d \mu(x) .
$$

Proof. From Propositions 5.53 and 5.54, it follows that

$$
\int_{x \times(0, \infty)}|\mathfrak{S}(f)(x, t)|^{p} d v(x, t) \lesssim\|b\|_{\mathrm{BMO}(x)}^{2} \int_{x}|f(x)|^{p} d \mu(x),
$$

where $\mathfrak{S}(f)$ is as in Proposition 5.53 and $d v(x, t)$ is as in Proposition 5.54. Moreover, 
we have

$$
\begin{aligned}
\int_{x \times(0, \infty)} & |\mathfrak{S}(f)(x, t)|^{p} d v(x, t) \\
= & \sum_{k=-\infty}^{\infty} \int_{x} \int_{2^{-k-1}}^{2^{-k}}|\mathfrak{S}(f)(x, t)|^{p} d v(x, t) \\
= & \sum_{k=-\infty}^{\infty} \int_{x}\left|S_{k}(f)(x)\right|^{p} \int_{0}^{\infty} x_{\left(2^{-k-1}, 2^{-k}\right]}(t)\left(\sum_{j=-\infty}^{\infty}\left|D_{j}(b)(x)\right|^{2} x_{\left(2^{-j-1}, 2^{-j}\right]}(t)\right) d \mu(x) \frac{d t}{t} \\
= & \log 2\left\{\sum_{k=-\infty}^{\infty} \int_{x}\left|S_{k}(f)(x)\right|^{p}\left|D_{k}(b)(x)\right|^{2} d \mu(x)\right\} .
\end{aligned}
$$

Combining both estimates completes the proof of Corollary 5.55 .

We now can state a variant of David-Journé T(1)-theorem on spaces of homogeneous type. In what follows, for any $\epsilon \in(0,1]$, a continuous function on $\mathcal{X} \times \mathcal{X} \backslash\{(x, x): x \in \mathcal{X}\}$ is said to be a standard kernel of order $\epsilon$ if it satisfies (I-3) in Subsection 5.2. Let $\beta \in(0, \epsilon)$. A continuous linear operator $T$ from $C_{b}^{\beta}(x)$ to $\left(C_{b}^{\beta}(x)\right)^{\prime}$ is said to have a standard distributional kernel $K$ of order $\epsilon$ if $T$ and $K$ satisfy (2.48). Also, the adjoint $T^{*}$ of $T$ is given by that for all $f, g \in C_{b}^{\beta}(\mathcal{X}),(T f, g)=\left(f, T^{*} g\right)$. Then $T^{*}: C_{b}^{\beta}(\mathcal{X}) \rightarrow\left(C_{b}^{\beta}(\mathcal{X})\right)^{\prime}$ is a continuous mapping; moreover, $T^{*}$ is associated to the kernel $K^{*}(x, y)=\bar{K}(y, x)$ for all $x, y \in \mathcal{X}$.

Theorem 5.56. Let $\epsilon \in(0,1], \beta \in(0, \epsilon)$, and let $T$ be a continuous linear operator from $C_{b}^{\beta}(x)$ to $\left(C_{b}^{\beta}(x)\right)^{\prime}$. Assume that $T$ has a standard distributional kernel $K$ of order $\epsilon$ as in (2.48). Then $T$ extends to a bounded operator on $L^{2}(X)$ if and only if the following conditions are true:

(i) $T(1) \in \operatorname{BMO}(x)$,

(ii) $T^{*}(1) \in \operatorname{BMO}(\mathcal{X})$,

(iii) $T \in \operatorname{WBP}(\beta)$.

Proof. We first verify the sufficiency. By Proposition 2.12, both $T$ and $T^{*}$ can be extended to a continuous linear operator from $C^{\beta}(\mathcal{X})$ to $\left(\check{C}_{b}^{\beta}(\mathcal{X})\right)^{\prime}$. If $T(1)=T^{*}(1)=0$ in $\left(\check{C}_{b}^{\beta}(\mathcal{X})\right)^{\prime}$, then by Theorem 5.49, $T$ and $T^{*}$ can be extended as continuous linear operators on $\dot{C}^{\beta}(\mathcal{X})$. By this fact, Theorem 5.23 and Proposition 5.10(v), we know that $T$ extends to a bounded operator on $L^{2}(\mathcal{X})$.

We now consider the general case. Let $\left\{S_{j}\right\}_{j \in \mathbb{Z}}$ be as in Definition 2.2 and $D_{j}=S_{j}-S_{j-1}$ for $j \in \mathbb{Z}$. Let $\left\{\widetilde{D}_{j}\right\}_{j \in \mathbb{Z}}$ be as in Theorem 3.10. Let $b \in \operatorname{BMO}(\mathcal{X})$. For any $f \in C_{b}^{\beta}(\mathcal{X})$, we define the paraproduct

$$
P_{b}(f)(x)=\sum_{j=-\infty}^{\infty} \tilde{D}_{j}\left(D_{j}(b) S_{j}(f)\right)(x)
$$

We will show that the kernel of $P_{b}$ is a standard kernel of order $\epsilon$, that $P_{b}$ is bounded on $L^{2}(\mathcal{X})$, and that $P_{b}(1)=b$ and $P_{b}^{*}(1)=0$. In order to be rigorous in the following calculations, we 
should consider $\sum_{j=-N}^{N}$ instead of $\sum_{j=-\infty}^{\infty}$ and then let $N \rightarrow \infty$. However, we will omit these details; see [75, pages 302-305].

(1) The size of the kernel. The kernel of $P_{b}$ is

$$
K(x, y)=\sum_{j=-\infty}^{\infty} \int_{x} \tilde{D}_{j}(x, z) D_{j}(b)(z) S_{j}(z, y) d \mu(z)
$$

Let $B_{k}=B\left(z, 2^{k+1} 2^{-j}\right)$, for $k \in \mathbb{Z}_{+}$, and $b_{B_{0}}=\left(1 / \mu\left(B_{0}\right)\right) \int_{B_{0}} b(z) d \mu(z)$. Since

$$
\int_{x} D_{j}(z, y) d \mu(y)=0,
$$

by the size condition of $D_{j}$ and (5.227), for any $z \in \mathcal{X}$, we have

$$
\begin{aligned}
\left|D_{j}(b)(z)\right| & \leq \int_{B_{0}}\left|D_{j}(z, y)\right|\left|b(y)-b_{B_{0}}\right| d \mu(y)+\sum_{k=1}^{\infty} \int_{B_{k} \backslash B_{k-1}}\left|D_{j}(z, y)\right|\left|b(y)-b_{B_{0}}\right| d \mu(y) \\
& \lesssim \sum_{k=0}^{\infty} \frac{1}{2^{k \epsilon_{2}}} \frac{1}{\mu\left(B_{k}\right)} \int_{B_{k}}\left|b(y)-b_{B_{0}}\right| d \mu(y) \\
& \lesssim\|b\|_{\mathrm{BMO}(x) .}
\end{aligned}
$$

Let $\epsilon^{\prime} \in\left(\epsilon, \epsilon_{1} \wedge \epsilon_{2}\right)$. By the size conditions of $\widetilde{D}_{j}$ and $S_{j}$, (5.234), Lemmas 4.4, and 3.5, we have

$$
\begin{aligned}
&|K(x, y)| \lesssim\|b\|_{\mathrm{BMO}(x)} \sum_{j=-\infty}^{\infty} \int_{x} \frac{1}{V_{2^{-j}}(x)+V_{2^{-j}}(z)+V(x, z)}\left(\frac{2^{-j}}{2^{-j}+d(x, z)}\right)^{\epsilon^{\prime}} \\
& \quad \times \frac{1}{V_{2^{-j}}(z)+V_{2^{-j}}(y)+V(z, y)}\left(\frac{2^{-j}}{2^{-j}+d(z, y)}\right)^{\epsilon_{2}} d \mu(z) \\
& \lesssim\|b\|_{\mathrm{BMO}(x)} \sum_{j=-\infty}^{\infty} \frac{1}{V_{2^{-j}}(x)+V_{2^{-j}}(y)+V(x, y)}\left(\frac{2^{-j}}{2^{-j}+d(x, y)}\right)^{\epsilon^{\prime}} \\
& \lesssim\|b\|_{\mathrm{BMO}(x)} \frac{1}{V(x, y)^{\prime}},
\end{aligned}
$$

which verifies the size condition of the kernel $K$. 
If $d\left(x, x^{\prime}\right) \leq d(x, y) / 2$ with $x \neq y$, by (5.234),

$$
\begin{aligned}
& \left|K(x, y)-K\left(x^{\prime}, y\right)\right| \\
& =\left|\sum_{j=-\infty}^{\infty} \int_{x}\left[\widetilde{D}_{j}(x, z)-\tilde{D}_{j}\left(x^{\prime}, z\right)\right] D_{j}(b)(z) S_{j}(z, y) d \mu(z)\right| \\
& \lesssim\|b\|_{\mathrm{BMO}(x)} \sum_{j=-\infty}^{\infty}\left\{\int_{d\left(x, x^{\prime}\right) \leq\left(2^{-j}+d(x, z)\right) / 2}\left|\tilde{D}_{j}(x, z)-\tilde{D}_{j}\left(x^{\prime}, z\right)\right|\left|S_{j}(z, y)\right| d \mu(z)\right. \\
& +\int_{d\left(x, x^{\prime}\right)>\left(2^{-j}+d(x, z)\right) / 2}\left|\tilde{D}_{j}(x, z)\right|\left|S_{j}(z, y)\right| d \mu(z) \\
& \left.+\int_{d\left(x, x^{\prime}\right)>\left(2^{-j}+d(x, z)\right) / 2}\left|\widetilde{D}_{j}\left(x^{\prime}, z\right)\right|\left|S_{j}(z, y)\right| d \mu(z)\right\} \\
& \equiv\|b\|_{\mathrm{BMO}(x)}\left\{Y_{1}+Y_{2}+Y_{3}\right\} \text {. }
\end{aligned}
$$

The regularity of $\widetilde{D}_{j}$ and the size condition of $S_{j}$ together with Lemmas 4.4 and 3.5 give

$$
\begin{aligned}
& Y_{1} \lesssim \sum_{j=-\infty}^{\infty} \int_{x}\left(\frac{d\left(x, x^{\prime}\right)}{2^{-j}+d(x, z)}\right)^{\epsilon^{\prime}} \frac{1}{V_{2^{-j}}(x)+V_{2^{-j}}(z)+V(x, z)}\left(\frac{2^{-j}}{2^{-j}+d(x, z)}\right)^{\epsilon^{\prime}} \\
& \quad \times \frac{1}{V_{2^{-j}}(z)+V_{2^{-j}}(y)+V(z, y)}\left(\frac{2^{-j}}{2^{-j}+d(z, y)}\right)^{\epsilon_{2}} d \mu(z) \\
& \lesssim \sum_{j=-\infty}^{\infty}\left(\frac{d\left(x, x^{\prime}\right)}{2^{-j}+d(x, y)}\right)^{\epsilon} \frac{1}{V_{2^{-j}}(x)+V_{2^{-j}}(y)+V(x, y)}\left(\frac{2^{-j}}{2^{-j}+d(x, y)}\right)^{\epsilon^{\prime}-\epsilon} \\
& \lesssim \frac{d\left(x, x^{\prime}\right)^{\epsilon}}{V(x, y) d(x, y)^{\epsilon}} .
\end{aligned}
$$

The size conditions of $\widetilde{D}_{j}$ and $S_{j}$ together with Lemmas 4.4 and 3.5 also yield

$$
\begin{aligned}
Y_{2} & \lesssim \sum_{j=-\infty}^{\infty} \int_{d\left(x, x^{\prime}\right)>\left(2^{-j}+d(x, z)\right) / 2} \frac{1}{V_{2^{-j}}(x)+V_{2^{-j}}(z)+V(x, z)}\left(\frac{2^{-j}}{2^{-j}+d(x, z)}\right)^{\epsilon^{\prime}} \\
& \times \frac{1}{V_{2^{-j}}(z)+V_{2^{-j}}(y)+V(z, y)}\left(\frac{2^{-j}}{2^{-j}+d(z, y)}\right)^{\epsilon_{2}} d \mu(z) \\
& \lesssim \sum_{j=-\infty}^{\infty} \frac{1}{V_{2^{-j}(x)+V_{2^{-j}}(y)+V(x, y)} \frac{d\left(x, x^{\prime}\right)^{\epsilon} 2^{-j\left(\epsilon^{\prime}-\epsilon\right)}}{\left(2^{-j}+d(x, y)\right)^{\epsilon^{\prime}}}} \\
& \lesssim \frac{d\left(x, x^{\prime}\right)^{\epsilon}}{V(x, y) d(x, y)^{\epsilon}} .
\end{aligned}
$$


Similarly, we have

$$
Y_{3} \lesssim \frac{d\left(x, x^{\prime}\right)^{\epsilon}}{V(x, y) d(x, y)^{\epsilon}}
$$

which shows that $K$ has the desired regularity on the first variable.

An argument similar to above also proves that if $d\left(y, y^{\prime}\right) \leq d(x, y) / 2$ with $x \neq y$,

$$
\left|K(x, y)-K\left(x, y^{\prime}\right)\right| \lesssim\|b\|_{\mathrm{BMO}(x)} \frac{d\left(y, y^{\prime}\right)^{\epsilon}}{V(x, y) d(x, y)^{\epsilon}} .
$$

Thus, $K$ is a standard kernel of order $\epsilon$.

(2) Boundedness of $P_{b}$ on $L^{2}(\mathcal{X})$. For any $f, g \in L^{2}(\mathcal{X})$, by Hölder's inequality, Corollary 5.55, and Lemma 3.9 together with Remark 3.16, we have

$$
\begin{aligned}
\left|\left\langle P_{b}(f), g\right\rangle\right| & =\left|\sum_{j=-\infty}^{\infty} \int_{x} D_{j}(b)(x) S_{j}(f)(x) \tilde{D}_{j}^{*}(g)(x) d \mu(x)\right| \\
& \lesssim\left\{\sum_{j=-\infty}^{\infty} \int_{x}\left|D_{j}(b)(x)\right|^{2}\left|S_{j}(f)(x)\right|^{2} d \mu(x)\right\}^{1 / 2}\left\{\sum_{j=-\infty}^{\infty} \int_{x}\left|\tilde{D}_{j}^{t}(g)(x)\right|^{2} d \mu(x)\right\}^{1 / 2} \\
& \lesssim\|b\|_{\mathrm{BMO}(x)}\|f\|_{L^{2}(x)}\|g\|_{L^{2}(x)},
\end{aligned}
$$

which together with a duality argument yields that

$$
\left\|P_{b}(f)\right\|_{L^{2}(x)} \lesssim\|b\|_{\mathrm{BMO}(x)}\|f\|_{L^{2}(x)} .
$$

(3) $P_{b}(1)=b$ and $P_{b}^{*}(1)=0$ in $\left(\mathcal{C}_{b}^{\beta}(x)\right)^{\prime}$. Since $\int_{x} \widetilde{D}_{j}(x, z) d \mu(x)=0$, from this, it follows that $P_{b}^{*}(1)=0$ in $\left(\mathcal{C}_{b}^{\beta}(\mathcal{X})\right)^{\prime}$. Also, since $\int_{\chi} S_{j}(y, z) d \mu(z)=1$, from this and Theorem 3.29 , it follows that $P_{b}(1)=b$.

We can now finish the proof of the sufficiency. For any given operator $T$ which satisfies (i), (ii), and (iii), let $b_{1}=T(1)$ and $b_{2}=T^{*}(1)$. Then, there exist paraproducts $P_{b_{i}}$ such that $P_{b_{i}}(1)=b_{i}$ and $P_{b_{i}}^{*}(1)=0$ for $i=1,2$. Then the operator $\tilde{T}=T-P_{b_{1}}-P_{b_{2}}^{*}$ lies in $\operatorname{WBP}(\beta)$ and $\widetilde{T}(1)=\widetilde{T}^{*}(1)=0$. Thus, by Theorem 5.23 , we know that $\widetilde{T}$ is bounded on $L^{2}(\mathcal{X})$, which together with the boundedness of $P_{b_{1}}$ and $P_{b_{2}}^{*}$ on $L^{2}(\mathcal{X})$ also yields the boundedness of $T$ on $L^{2}(X)$. This completes the proof of the sufficiency.

We now check the necessity. By Remark 2.14(iii), we know $T \in \operatorname{WBP}(\beta)$. To verify $T(1), T^{*}(1) \in \mathrm{BMO}(\mathcal{X})$, we first claim that if $T$ is as in Proposition 2.12 and $T$ is bounded on $L^{2}(\mathcal{X})$, then $T$ is also bounded from $L_{b}^{\infty}(\mathcal{X})$ to $\operatorname{BMO}(\mathcal{X})$, namely, for all $f \in L_{b}^{\infty}(\mathcal{X})$,

$$
\|T(f)\|_{\mathrm{BMO}(x)} \lesssim\|f\|_{L^{\infty}(x)}
$$

The proof of this claim is standard; see, for example, [75, pages 156-157] or [81, pages 118119]. For any ball $B=B\left(x_{0}, r\right)$ with some $x_{0} \in \mathcal{X}$ and $r>0$, let $C_{B}=T\left(f_{X X X B\left(x_{0}, 2 r\right)}\right)\left(x_{0}\right)$. Since 
$f \in L_{b}^{\infty}(\mathcal{X})$ and $K$ is locally integrable away from the diagonal of $\mathcal{X} \times \mathcal{X}$, it is easy to see that $\left|C_{B}\right|<\infty$. By the boundedness of $T$ on $L^{2}(\mathcal{X})$ and (2.49) together with Lemma 2.1(i), we have

$$
\begin{aligned}
& \frac{1}{\mu(B)} \int_{B}\left|T(f)(x)-C_{B}\right| d \mu(x) \\
& \quad \leq \frac{1}{\mu(B)} \int_{B}\left|T\left(f X_{B\left(x_{0}, 2 r\right)}\right)(x)\right| d \mu(x)+\frac{1}{\mu(B)} \int_{B}\left|T\left(f X x \backslash B\left(x_{0}, 2 r\right)\right)(x)-C_{B}\right| d \mu(x) \\
& \quad \lesssim\|T\|_{L^{2}(x) \rightarrow L^{2}(x)}\|f\|_{L^{\infty}(x)}+\frac{1}{\mu(B)} \int_{B} \int_{\chi \backslash B\left(x_{0}, 2 r\right)}\left|K(x, y)-K\left(x_{0}, y\right)\right||f(y)| d \mu(y) d \mu(x) \\
& \quad \lesssim\left(\|T\|_{\left.L^{2}(x) \rightarrow L^{2}(x)+C_{T}\right)\|f\|_{L^{\infty}(x)}}\right.
\end{aligned}
$$

which proves (5.243).

Using (5.243), we then can verify that if $T$ is bounded on $L^{2}(\mathcal{X})$, then $T(1) \in \mathrm{BMO}(\mathcal{X})$. To see this, for any $g \in \mathcal{C}_{b}^{\beta}(\mathcal{X})$ with supp $g \subset B\left(x_{0}, r\right)$ for some $x_{0} \in \mathcal{X}$ and $r>0$, let $\psi \in$ $C_{b}^{\beta}(\mathcal{X})$ be as in the proof of Proposition 2.12. By (5.243), $T(\psi) \in \mathrm{BMO}(\mathcal{X})$, which together with Theorem 5.19(i) below yields that

$$
|\langle T(\psi), g\rangle| \leq\|T(\psi)\|_{\mathrm{BMO}(\mathcal{X})}\|g\|_{H^{1}(\mathcal{X})} \lesssim\left(\|T\|_{L^{2}(\mathcal{X}) \rightarrow L^{2}(\mathcal{X})}+C_{T}\right)\|g\|_{H^{1}(\mathcal{X})}
$$

On the other hand, by (2.49) and Lemma 2.1(i), we also have

$$
\begin{aligned}
|\langle T(1-\psi), g\rangle| & =\left|\int_{x} \int_{d\left(y, x_{0}\right) \geq 2 r}\left[K(x, y)-K\left(x_{0}, y\right)\right](1-\psi(y)) g(x) d \mu(y) d \mu(x)\right| \\
& \lesssim C_{T} \int_{x}\left\{\int_{d\left(y, x_{0}\right) \geq 2 r} \frac{r^{\epsilon}}{V\left(x_{0}, y\right) d\left(x_{0}, y\right)^{\epsilon}} d \mu(y)\right\}|g(x)| d \mu(x) \\
& \lesssim C_{T}\|g\|_{L^{1}(x)} \\
& \lesssim C_{T}\|g\|_{H^{1}(x)} .
\end{aligned}
$$

Thus, $|\langle T(1), g\rangle| \lesssim\left(\|T\|_{L^{2}(\mathcal{X}) \rightarrow L^{2}(\mathcal{x})}+C_{T}\right)\|g\|_{H^{1}(\mathcal{X})}$, which together with Corollary 2.11(i), Proposition 5.21, Theorems 6.11, and 5.19(i) implies that $T(1) \in \mathrm{BMO}(\mathcal{X})$ and $\|T(1)\|_{\mathrm{BMO}(\mathcal{X})} \lesssim$ $\|T\|_{L^{2}(\mathcal{X}) \rightarrow L^{2}(\mathcal{X})}+C_{T}$. An argument similar to this also proves that $T^{*}(1) \in \mathrm{BMO}(\mathcal{X})$ and $\left\|T^{*}(1)\right\|_{\mathrm{BMO}(\mathcal{X})} \lesssim\|T\|_{L^{2}(\mathcal{X}) \rightarrow L^{2}(x)}+C_{T}$, which completes the proof of Theorem 5.56.

We now state a variant of the T(1)-theorem in the sense of Stein [75, page 294]. Let $\epsilon \in$ $(0,1], \beta \in(0, \epsilon)$, and let $T$ be a continuous linear operator from $C_{b}^{\beta}(\mathcal{X})$ to $\left(C_{b}^{\beta}(\mathcal{X})\right)^{\prime}$. We assume that associated to $T$, there is a standard kernel of order $\epsilon$, in the sense that if $f \in C_{b}^{\beta}(x)$, then, outside the support of $f$, the distribution $T f$ agrees with the function

$$
T(f)(x)=\int_{X} K(x, y) f(y) d \mu(y) .
$$


Similarly to [75, page 294], we assume that $T$ and $T^{*}$ are restrictedly bounded. Whenever $\phi^{R, x_{0}}$ is a normalized bump function for the ball $B\left(x_{0}, R\right)$ with some $x_{0} \in \mathcal{X}$ and $R>0$, the distributions $T\left(\phi^{R, x_{0}}\right)$ and $T^{*}\left(\phi^{R, x_{0}}\right)$ belong to $L^{2}(\mathcal{X})$, and the estimates

$$
\begin{aligned}
\left\|T\left(\phi^{R, x_{0}}\right)\right\|_{L^{2}(\chi)} & \leq A\left[\mu\left(B\left(x_{0}, R\right)\right)\right]^{1 / 2}, \\
\left\|T^{*}\left(\phi^{R, x_{0}}\right)\right\|_{L^{2}(\mathcal{X})} & \leq A\left[\mu\left(B\left(x_{0}, R\right)\right)\right]^{1 / 2}
\end{aligned}
$$

hold with an $A>0$ that is independent of $R, x_{0}$ and $\phi^{R, x_{0}}$.

Theorem 5.57. Let $\epsilon \in(0,1], \beta \in(0, \epsilon)$, and let $T$ be a continuous linear operator from $C_{b}^{\beta}(\mathcal{X})$ to $\left(C_{b}^{\beta}(X)\right)^{\prime}$ associated with a standard kernel of order $\epsilon$ in the sense of (5.247). Then $T$ extends to a bounded linear operator on $L^{2}(\mathcal{X})$ if and only if both $T$ and $T^{*}$ are restrictedly bounded in the sense of (5.248) and (5.249).

Proof. The necessity is obvious. We only need to prove the sufficiency. We first make the following claim that if $f \in \mathcal{C}_{b}^{\beta}(x)$, then

$$
T f \in L^{1}(X) .
$$

In fact, assume that supp $f \subset B\left(x_{0}, r\right)$ for some $x_{0} \in \mathcal{X}$ and $r>0$. Since $f$ is a multiple of a bump function, $T f \in L^{2}(\mathcal{X})$ by (5.248), and hence $\int_{B\left(x_{0}, 2 r\right)}|T f(x)| d \mu(x)<\infty$. If $x \notin B\left(x_{0}, 2 r\right)$, then by (5.247), $\int_{\chi} f(x) d \mu(x)=0$, and the regularity on $K$, we have

$$
\begin{aligned}
|T f(x)| & =\left|\int_{x} K(x, y) f(y) d \mu(y)\right| \\
& =\left|\int_{x}\left[K(x, y)-K\left(x, x_{0}\right)\right] f(y) d \mu(y)\right| \\
& \lesssim C_{T} \int_{B\left(x_{0}, r\right)} \frac{d\left(y, x_{0}\right)^{\epsilon}}{V\left(x, x_{0}\right) d\left(x, x_{0}\right)^{\epsilon}}|f(y)| d \mu(y) \\
& \lesssim C_{T}\|f\|_{L^{\infty}(x)} \mu\left(B\left(x_{0}, r\right)\right) \frac{r^{\epsilon}}{V\left(x, x_{0}\right) d\left(x, x_{0}\right)^{\epsilon}}
\end{aligned}
$$

which implies that $\int_{\mathcal{X} \backslash B\left(x_{0}, 2 r\right)}|T f(x)| d \mu(x)<\infty$. Thus, the claim (5.250) holds.

We now verify that $T(1) \in \operatorname{BMO}(\mathcal{X})$. To this end, we first prove that there exists a constant $A>0$ such that whenever $\phi^{R, x_{0}}$ is a normalized bump function for the ball $B\left(x_{0}, R\right)$ with $x_{0} \in \mathcal{X}$ and $R>0$, then $T\left(\phi^{R, x_{0}}\right) \in \mathrm{BMO}(\mathcal{X})$ with

$$
\left\|T\left(\phi^{R, x_{0}}\right)\right\|_{\mathrm{BMO}(\mathcal{X})} \leq A .
$$


Let $\widetilde{B}_{1}=B\left(\widetilde{x}_{0}, \widetilde{R}\right)$ with some $\tilde{x}_{0} \in \mathcal{X}$ and $\widetilde{R}>0$ be any ball, and let $\widetilde{B}_{2}=B\left(\tilde{x}_{0}, 2 \widetilde{R}\right)$ and $\widetilde{B}_{3}=B\left(\tilde{x}_{0}, 3 \widetilde{R}\right)$. Fix a function $\theta \in C_{b}^{\beta}(x)$ with $\theta(x)=1$ for $d\left(x, \tilde{x}_{0}\right) \leq 2 \widetilde{R}$ and $\theta(x)=0$ for $d\left(x, \tilde{x}_{0}\right) \geq 3 \tilde{R}$. Write

$$
\phi^{R, x_{0}}(x)=\phi^{R, x_{0}}(x) \theta(x)+\phi^{R, x_{0}}(x)(1-\theta(x))=f_{1}(x)+f_{2}(x)
$$

Observe that $f_{1}$ is, up to a bounded multiplicative constant, a normalized bump function for either the ball $B\left(x_{0}, R\right)$ or the ball $\widetilde{B}_{3}$, whichever has the smaller radius. Thus, by (5.248), we have

$$
\begin{aligned}
\int_{\tilde{B}_{1}}\left|T f_{1}(x)\right|^{2} d \mu(x) & \leq\left\|T f_{1}\right\|_{L^{2}(x)}^{2} \\
& \leq A^{2} \min \left\{\mu\left(B\left(x_{0}, R\right)\right), \mu\left(B\left(\tilde{x}_{0}, 3 \tilde{R}\right)\right)\right\} \\
& \leq A^{\prime} \mu\left(B\left(\tilde{x}_{0}, \tilde{R}\right)\right) .
\end{aligned}
$$

Since supp $f_{2} \subset\left(\mathcal{X} \backslash \widetilde{B}_{2}\right)$, for $x \in \widetilde{B}_{1}$, by (5.247), we have

$$
T f_{2}(x)=\int_{x} K(x, y) f_{2}(y) d \mu(y)
$$

Let $C_{\widetilde{B}_{1}}=\int_{\chi} K\left(\tilde{x}_{0}, y\right) f_{2}(y) d \mu(y)$. Then $\left|C_{\widetilde{B}_{1}}\right|<\infty$, and for $x \in \widetilde{B}_{1}$, by the regularity on $K$ on the first variable and Lemma 2.1(i),

$$
\begin{aligned}
\left|T f_{2}(x)-C_{\tilde{B}_{1}}\right| & \lesssim \int_{d\left(\tilde{x}_{0}, y\right)>2 \widetilde{R}}\left|K(x, y)-K\left(\tilde{x}_{0}, y\right)\right| d \mu(y) \\
& \lesssim \int_{d\left(\tilde{x}_{0}, y\right)>2 \widetilde{R}} \frac{d\left(x, \tilde{x}_{0}\right)^{\epsilon}}{V\left(\tilde{x}_{0}, y\right) d\left(\tilde{x}_{0}, y\right)^{\epsilon}} d \mu(y) \\
& \lesssim 1
\end{aligned}
$$

Combining the estimates for $f_{1}$ and $f_{2}$ gives that for any ball $\widetilde{B}_{1}$,

$$
\int_{\widetilde{B}_{1}}\left|T\left(\phi^{R, x_{0}}\right)-C_{\widetilde{B}_{1}}\right|^{2} d \mu(x) \leq A \mu\left(\widetilde{B}_{1}\right)
$$

which shows (5.252).

Let $\theta \in C_{b}^{1}(\mathbb{R})$ and $\theta(0)=1$. For any $v>0$ and $x \in X$, set $\theta_{v}(x)=\theta\left(v d\left(x, x_{1}\right)\right)$. By (5.252), $\left\{T\left(\theta_{v}\right)\right\}_{v>0}$ is uniformly bounded in $\operatorname{BMO}(\mathcal{X})$. Since $\operatorname{BMO}(\mathcal{X})=\left(H^{1}(x)\right)^{\prime}$ (see Theorem 5.19(i)), as is well known, $\mathrm{BMO}(\mathcal{X})$ is weakly* compact in the dual topology. Thus, every sequence $\left\{T\left(\theta_{v_{k}}\right)\right\}_{k \in \mathbb{N}}$ has a subsequence which weakly* converges. Let $f \in C_{b}^{\beta}(\mathcal{X})$. By (5.250) and $\left\langle T\left(\theta_{v}\right), f\right\rangle=\left\langle\theta_{v}, T^{*}(f)\right\rangle$, we know that whatever limit (denote it by $a$ ), we extract 
from a subsequence of $\left\{T\left(\theta_{v}\right)\right\}_{v>0}$, then $\langle a, f\rangle=\left\langle 1, T^{*}(f)\right\rangle$. This shows that the limit $a$ is independent of the subsequence, and we are justified in setting $a=T(1)=\lim _{v \rightarrow 0} T\left(\theta_{v}\right)$ and

$$
\langle T(1), f\rangle=\int_{x} T^{*}(f)(x) d \mu(x),
$$

with a similar statement when the roles of $T$ and $T^{*}$ are reversed. Thus, $T(1), T^{*}(1) \in$ $\operatorname{BMO}(x)$. Moreover, if $T$ is restrictedly bounded, then $T \in \operatorname{WBP}(\beta)$. Thus, by Theorem 5.56, we know $T$ is bounded on $L^{2}(\mathcal{X})$, which completes the proof of Theorem 5.57 .

Remark 5.58. From (5.258), we see that if $T$ is bounded on $L^{2}(\mathcal{X})$, then $T(1) \in\left(\mathscr{C}_{b}^{\beta}(\mathcal{X})\right)^{\prime}$ for a certain $\beta \in(0,1]$ is constant if and only if for any $f \in C_{b}^{\beta}(x)$,

$$
\int_{x} T^{*}(f)(x) d \mu(x)=0
$$

\section{Triebel-Lizorkin spaces with $p=\infty$}

In this section, we will develop a theory for Triebel-Lizorkin spaces with $p=\infty$ by using the Carleson characterizations. We again distinguish between the homogeneous and the inhomogeneous cases and examine the relations between these cases as well as with BMOtype spaces.

\subsection{Plancherel-Pôlya inequality and definition of $\dot{F}_{\infty, q}^{s}(\mathcal{X})$}

Throughout this and the next subsection, we will assume that $\mu(x)=\infty$. In this subsection, we introduce the norm in $\dot{F}_{\infty, q}^{s}(\mathcal{X})$ in a similar way as in [82] and, using the homogeneous discrete Calderon reproducing formulae, Theorem 4.13 , we will prove that the norm $\|\cdot\|_{\dot{F}_{\infty, q}^{s}}(\mathcal{x})$ is independent of the choices of ATIs and spaces of distributions via some Plancherel-Pôlya inequality; see also $[83,84]$.

Definition 6.1. Let $\epsilon_{1} \in(0,1], \epsilon_{2}>0, \epsilon_{3}>0, \epsilon \in\left(0, \epsilon_{1} \wedge \epsilon_{2}\right)$, and $\left\{S_{k}\right\}_{k \in \mathbb{Z}}$ be an $\left(\epsilon_{1}, \epsilon_{2}, \epsilon_{3}\right)$-ATI. For $k \in \mathbb{Z}$, set $D_{k}=S_{k}-S_{k-1}$. Let $|s|<\epsilon$ and $p(s, \epsilon)<q \leq \infty$. For any $f \in\left(\mathcal{G}_{0}^{\epsilon}(\beta, \gamma)\right)^{\prime}$ with $0<\beta, \gamma<\epsilon$, define

$$
\|f\|_{\dot{F}_{\infty, q}^{s}(x)}=\sup _{l \in \mathbb{Z}} \sup _{\alpha \in I_{l}}\left\{\frac{1}{\mu\left(Q_{\alpha}^{l}\right)} \int_{Q_{\alpha}^{l}} \sum_{k=l}^{\infty} 2^{k s q}\left|D_{k}(f)(x)\right|^{q} d \mu(x)\right\}^{1 / q},
$$

where the supremum is taken over all dyadic cubes as in Lemma 2.19 and the usual modification is made when $q=\infty$.

Remark 6.2. (i) From Lemma 2.19 and the doubling property (1.2), it is easy to see that an equivalent norm is obtained if the supremum in Definition 6.1 is taken with respect to all balls with positive radius instead of all dyadic cubes as in Lemma 2.19. 
(ii) Let $l_{0} \in \mathbb{Z}$. It is easy to see that there exists a constant $C_{l_{0}}>0$ such that for all $f \in\left(\mathcal{G}_{0}^{\epsilon}(\beta, \gamma)\right)^{\prime}$,

$$
\sup _{l \in \mathbb{Z}} \sup _{\alpha \in I_{l}}\left\{\frac{1}{\mu\left(Q_{\alpha}^{l}\right)} \int_{Q_{\alpha}^{l}} \sum_{k=l+l_{0}}^{\infty} 2^{k s q}\left|D_{k}(f)(x)\right|^{q} d \mu(x)\right\}^{1 / q} \lesssim C_{l_{0}}\|f\|_{\dot{F}_{\infty, q}^{s}(x)} .
$$

Thus,

$$
\|f\|_{\dot{F}_{\infty, q}^{s}(x)} \sim \sup _{l \in \mathbb{Z}} \sup _{\alpha \in I_{l}}\left\{\frac{1}{\mu\left(Q_{\alpha}^{l}\right)} \int_{Q_{\alpha}^{l}} \sum_{k=l+l_{0}}^{\infty} 2^{k s q}\left|D_{k}(f)(x)\right|^{q} d \mu(x)\right\}^{1 / q} .
$$

We now establish the following useful Plancherel-Pôlya inequality, which complements Proposition 5.4(ii) for the case $p=\infty$.

Proposition 6.3. Let $\epsilon_{1} \in(0,1], \epsilon_{2}>0, \epsilon_{3}>0, \epsilon \in\left(0, \epsilon_{1} \wedge \epsilon_{2}\right)$, and let $\left\{S_{k}\right\}_{k \in \mathbb{Z}}$ and $\left\{P_{k}\right\}_{k \in \mathbb{Z}}$ be two $\left(\epsilon_{1}, \epsilon_{2}, \epsilon_{3}\right)$-ATIs. For $k \in \mathbb{Z}$, set $D_{k}=S_{k}-S_{k-1}$ and $Q_{k}=P_{k}-P_{k-1}$. Let $|s|<\epsilon$ and $p(s, \epsilon)<q \leq \infty$. Then for all $f \in\left(\mathcal{G}_{0}^{\epsilon}(\beta, \gamma)\right)^{\prime}$ with $0<\beta, \gamma<\epsilon$,

$$
\begin{aligned}
& \sup _{l \in \mathbb{Z}} \sup _{\alpha \in I_{l}}\left\{\frac{1}{\mu\left(Q_{\alpha}^{l}\right)} \sum_{k=l}^{\infty} \sum_{\tau \in I_{k}} \sum_{v=1}^{N(k, \tau)} 2^{k s q} \mu\left(Q_{\tau}^{k, v}\right) X_{\left\{(\tau, v): Q_{\tau}^{k, v} \subset Q_{\alpha}^{l}\right\}}(\tau, v)\left[\sup _{x \in Q_{\tau}^{k, v}}\left|D_{k}(f)(x)\right|\right]^{q}\right\}^{1 / q} \\
& \sim \sup _{l \in \mathbb{Z}} \sup _{\alpha \in I_{l}}\left\{\frac{1}{\mu\left(Q_{\alpha}^{l}\right)} \sum_{k=l}^{\infty} \sum_{\tau \in I_{k}} \sum_{\nu=1}^{N(k, \tau)} 2^{k s q} \mu\left(Q_{\tau}^{k, v}\right) X_{\left\{(\tau, v): Q_{\tau}^{k, v} \subset Q_{\alpha}^{l}\right\}}(\tau, v)\left[\inf _{x \in Q_{\tau}^{k, v}}\left|Q_{k}(f)(x)\right|\right]^{q}\right\}^{1 / q} .
\end{aligned}
$$

Proof. To prove Proposition 6.3, it suffices to show that for all $f \in\left(\mathcal{G}_{0}^{\epsilon}(\beta, \gamma)\right)^{\prime}$ with $0<\beta, \gamma<\epsilon$, the left-hand side of (6.4) is controlled by its right-hand side.

Let all the notation be as in the proof of Proposition 5.4. Then, by (5.9) and (5.10), we have

$$
\begin{aligned}
& \frac{1}{\mu\left(Q_{\alpha}^{l}\right)} \sum_{k=l}^{\infty} \sum_{\tau \in I_{k}} \sum_{\nu=1}^{N(k, \tau)} 2^{k s q} \mu\left(Q_{\tau}^{k, v}\right) X_{\left\{(\tau, v): Q_{\tau}^{k, v} \subset Q_{\alpha}^{l}\right\}}(\tau, v)\left[\sup _{x \in Q_{\tau}^{k, v}}\left|D_{k}(f)(x)\right|\right]^{q} \\
& \lesssim \frac{1}{\mu\left(Q_{\alpha}^{l}\right)} \sum_{k=l}^{\infty} \sum_{\tau \in I_{k}} \sum_{\nu=1}^{N(k, \tau)} 2^{k s q} \mu\left(Q_{\tau}^{k, v}\right) X_{\left\{(\tau, v): Q_{\tau}^{k, v} \subset Q_{\alpha}^{l}\right\}}(\tau, v) \\
& \times\left[\sum_{k^{\prime}=l}^{\infty} \sum_{\tau^{\prime} \in I_{k^{\prime}}} \sum_{v^{\prime}=1}^{N\left(k^{\prime}, \tau^{\prime}\right)} 2^{-\left|k-k^{\prime}\right| \epsilon^{\prime}} \mu\left(Q_{\tau^{\prime}}^{k^{\prime}, v^{\prime}}\right)\left|Q_{k^{\prime}}(f)\left(y_{\tau^{\prime}}^{k^{\prime}, v^{\prime}}\right)\right|\right. \\
& \left.\times \frac{1}{V_{2^{-\left(k \wedge k^{\prime}\right)}}\left(y_{\tau}^{k, v}\right)+V_{2^{-\left(k \wedge k^{\prime}\right)}}\left(y_{\tau^{\prime}}^{k^{\prime}, v^{\prime}}\right)+V\left(y_{\tau}^{k, v}, y_{\tau^{\prime}}^{k^{\prime}, v^{\prime}}\right)}\left(\frac{2^{-\left(k \wedge k^{\prime}\right)}}{2^{-\left(k \wedge k^{\prime}\right)}+d\left(y_{\tau}^{k, v}, y_{\tau^{\prime}}^{k^{\prime}, v^{\prime}}\right)}\right)^{\epsilon}\right]^{q}
\end{aligned}
$$


Yongsheng Han et al.

$$
\begin{aligned}
& +\frac{1}{\mu\left(Q_{\alpha}^{l}\right)} \sum_{k=l}^{\infty} \sum_{\tau \in I_{k}} \sum_{v=1}^{N(k, \tau)} 2^{k s q} \mu\left(Q_{\tau}^{k, v}\right) X_{\left\{(\tau, v): Q_{\tau}^{k, v} \subset Q_{\alpha}^{l}\right\}}(\tau, v) \\
& \times\left[\sum_{k^{\prime}=-\infty}^{l-1} \sum_{\tau^{\prime} \in I_{k^{\prime}}} \sum_{v^{\prime}=1}^{N\left(k^{\prime}, \tau^{\prime}\right)} 2^{-\left|k-k^{\prime}\right| \epsilon^{\prime}} \mu\left(Q_{\tau^{\prime}}^{k^{\prime}, v^{\prime}}\right)\left|Q_{k^{\prime}}(f)\left(y_{\tau^{\prime}}^{k^{\prime}, v^{\prime}}\right)\right| \cdots\right]^{q} \\
& \equiv Y_{1}+\Upsilon_{2} .
\end{aligned}
$$

Then by Lemma 2.19, if $x \notin B\left(z_{\alpha}^{l}, 4 C_{6} 2^{-l}\right)$ and $y \in Q_{\alpha}^{l}$, then $d(x, y) \geq 3 C_{6} 2^{-l}$, where and in what follows, $z_{\alpha}^{l}$ is the "center" of $Q_{\alpha}^{l}$ as in Lemma 2.19. By Lemma 2.19 again, we can find $m_{1} \in \mathbb{N}$ such that $B\left(z_{\alpha}^{l}, 4 C_{6} 2^{-l}\right) \subset \bigcup_{i=1}^{m_{1}} Q_{\tau^{i}}^{l}, \tau^{i} \in I_{l}, B\left(z_{\alpha}^{l}, 4 C_{6} 2^{-l}\right) \cap Q_{\tau^{i}}^{l} \neq \varnothing$ and $m_{1}$ is no more than a constant which is independent of $\alpha$ and $l$; see the details for a proof of the last fact in [85, pages 1385-1386]. Moreover,

$$
\mu\left(Q_{\tau^{i}}^{l}\right) \sim \mu\left(Q_{\alpha}^{l}\right)
$$

With these choices, we further control $Y_{1}$ by

$$
\begin{aligned}
& Y_{1} \lesssim \frac{1}{\mu\left(Q_{\alpha}^{l}\right)} \sum_{k=l}^{\infty} \sum_{\tau \in I_{k}} \sum_{v=1}^{N(k, \tau)} 2^{k s q} \mu\left(Q_{\tau}^{k, v}\right) X_{\left\{(\tau, v): Q_{\tau}^{k, v} \subset Q_{\alpha}^{l}\right\}}(\tau, v) \\
& \times\left[\sum_{k^{\prime}=l}^{\infty} \sum_{\tau^{\prime} \in I_{k^{\prime}}} \sum_{v^{\prime}=1}^{N\left(k^{\prime}, \tau^{\prime}\right)} 2^{-\left|k-k^{\prime}\right| \epsilon^{\prime}} \mu\left(Q_{\tau^{\prime}}^{k^{\prime}, v^{\prime}}\right) X_{\left\{\left(\tau^{\prime}, v^{\prime}\right): Q_{\tau^{\prime}}^{k^{\prime}, v^{\prime}} \subset \cup_{i=1}^{m_{1} 1} Q_{\tau^{i}}^{l}\right\}}\left(\tau^{\prime}, v^{\prime}\right)\right. \\
& \times\left|Q_{k^{\prime}}(f)\left(y_{\tau^{\prime}}^{k^{\prime}, v^{\prime}}\right)\right| \frac{1}{V_{2^{-\left(k \wedge k^{\prime}\right)}}\left(y_{\tau}^{k, v}\right)+V_{2^{-\left(k \wedge k^{\prime}\right)}}\left(y_{\tau^{\prime}}^{k^{\prime}, v^{\prime}}\right)+V\left(y_{\tau}^{k, v}, y_{\tau^{\prime}}^{k^{\prime}, v^{\prime}}\right)} \\
& \left.\times\left(\frac{2^{-\left(k \wedge k^{\prime}\right)}}{2^{-\left(k \wedge k^{\prime}\right)}+d\left(y_{\tau}^{k, v}, y_{\tau^{\prime}}^{k^{\prime}, v^{\prime}}\right)}\right)^{\epsilon}\right]^{q} \\
& +\frac{1}{\mu\left(Q_{\alpha}^{l}\right)} \sum_{k=l}^{\infty} \sum_{\tau \in I_{k}} \sum_{\nu=1}^{N(k, \tau)} 2^{k s q} \mu\left(Q_{\tau}^{k, v}\right) X_{\left\{(\tau, v): Q_{\tau}^{k, v} \subset Q_{\alpha}^{l}\right\}}(\tau, v) \\
& \times\left[\sum_{k^{\prime}=l}^{\infty} \sum_{\tau^{\prime} \in I_{k^{\prime}}} \sum_{\nu^{\prime}=1}^{N\left(k^{\prime}, \tau^{\prime}\right)} 2^{-\left|k-k^{\prime}\right| \epsilon^{\prime}} \mu\left(Q_{\tau^{\prime}}^{k^{\prime}, v^{\prime}}\right) X_{\left\{\left(\tau^{\prime}, v^{\prime}\right): Q_{\tau^{\prime}}^{k^{\prime}, v^{\prime}} \cap\left(\cup_{i=1}^{m} Q_{\tau^{i}}^{l}\right)=\varnothing\right\}}\left(\tau^{\prime}, v^{\prime}\right)\right. \\
& \times\left|Q_{k^{\prime}}(f)\left(y_{\tau^{\prime}}^{k^{\prime}, v^{\prime}}\right)\right| \frac{1}{V_{2^{-\left(k \wedge k^{\prime}\right)}}\left(y_{\tau}^{k, v}\right)+V_{2^{-\left(k \wedge k^{\prime}\right)}}\left(y_{\tau^{\prime}}^{k^{\prime}, v^{\prime}}\right)+V\left(y_{\tau}^{k, v}, y_{\tau^{\prime}}^{k^{\prime}, v^{\prime}}\right)} \\
& \left.\times\left(\frac{2^{-\left(k \wedge k^{\prime}\right)}}{2^{-\left(k \wedge k^{\prime}\right)}+d\left(y_{\tau}^{k, v}, y_{\tau^{\prime}}^{k^{\prime}, v^{\prime}}\right)}\right)^{\epsilon}\right]^{q} \\
& \equiv Y_{1,1}+Y_{1,2} .
\end{aligned}
$$


We first estimate $Y_{1,1}$. If $q \leq 1$, by (5.5), Lemma 5.2, (5.12), (6.6), and choosing $\epsilon^{\prime} \in(0, \epsilon)$ such that $\epsilon^{\prime}>s$ and $q>p\left(s, \epsilon^{\prime}\right)$,

$$
\begin{aligned}
& Y_{1,1} \lesssim \frac{1}{\mu\left(Q_{\alpha}^{l}\right)} \sum_{i=1}^{m_{1}} \sum_{k^{\prime}=l}^{\infty} \sum_{\tau^{\prime} \in I_{k^{\prime}}} \sum_{v^{\prime}=1}^{N\left(k^{\prime}, \tau^{\prime}\right)} \sum_{k=l}^{\infty} 2^{k s q} X_{\left\{\left(\tau^{\prime}, v^{\prime}\right): Q_{\tau^{\prime}}^{k^{\prime}, v^{\prime}} \subset Q_{\tau^{i}}^{l}\right\}}\left(\tau^{\prime}, v^{\prime}\right) \\
& \times\left[\mu\left(Q_{\tau^{\prime}}^{k^{\prime}, \nu^{\prime}}\right)\left|Q_{k^{\prime}}(f)\left(y_{\tau^{\prime}}^{k^{\prime}, \nu^{\prime}}\right)\right|\right]^{q} 2^{-\left|k-k^{\prime}\right| \epsilon^{\prime} q} \\
& \times \sum_{\tau \in I_{k}} \sum_{v=1}^{N(k, \tau)} \mu\left(Q_{\tau}^{k, v}\right) X_{\left\{(\tau, v): Q_{\tau}^{k, v} \subset Q_{\alpha}^{l}\right\}}(\tau, v) \\
& \times\left[\frac{1}{V_{2^{-\left(k \wedge k^{\prime}\right)}}\left(y_{\tau}^{k, v}\right)+V_{2^{-\left(k \wedge k^{\prime}\right)}}\left(y_{\tau^{\prime}}^{k^{\prime}, \nu^{\prime}}\right)+V\left(y_{\tau}^{k, v}, y_{\tau^{\prime}}^{k^{\prime}, \nu^{\prime}}\right)}\left(\frac{2^{-\left(k \wedge k^{\prime}\right)}}{2^{-\left(k \wedge k^{\prime}\right)}+d\left(y_{\tau}^{k, v}, y_{\tau^{\prime}}^{k^{\prime}, \nu^{\prime}}\right)}\right)^{\epsilon}\right]^{q} \\
& \lesssim \sum_{i=1}^{m_{1}} \frac{1}{\mu\left(Q_{\tau^{i}}^{l}\right)} \sum_{k^{\prime}=l}^{\infty} \sum_{\tau^{\prime} \in I_{k^{\prime}}} \sum_{v^{\prime}=1}^{N\left(k^{\prime}, \tau^{\prime}\right)} 2^{k^{\prime} s q} \mu\left(Q_{\tau^{\prime}}^{k^{\prime}, v^{\prime}}\right)\left|Q_{k^{\prime}}(f)\left(y_{\tau^{\prime}}^{k^{\prime}, v^{\prime}}\right)\right|^{q} X_{\left\{\left(\tau^{\prime}, v^{\prime}\right): Q_{\tau^{\prime}}^{k^{\prime}, v^{\prime}} \subset Q_{\tau^{i}}^{l}\right\}}\left(\tau^{\prime}, v^{\prime}\right),
\end{aligned}
$$

which together with the arbitrary choice of $y_{\tau^{\prime}}^{k^{\prime}, v^{\prime}} \in Q_{\tau^{\prime}}^{k^{\prime}, v^{\prime}}$ shows that $\left(Y_{1,1}\right)^{1 / q}$ is controlled by the right-hand side of (6.4) in this case.

If $1<q \leq \infty$, choosing $\epsilon^{\prime}>|s|$ together with Hölder's inequality and Lemma 5.2 yields

$$
\begin{aligned}
& \sum_{k^{\prime}=l}^{\infty} \sum_{\tau^{\prime} \in I_{k^{\prime}}} \sum_{v^{\prime}=1}^{N\left(k^{\prime}, \tau^{\prime}\right)} 2^{k^{\prime} s} 2^{-\left|k-k^{\prime}\right| \epsilon^{\prime}} 2^{\left(k-k^{\prime}\right) s} \mu\left(Q_{\tau^{\prime}}^{k^{\prime}, v^{\prime}}\right) X_{\left\{\left(\tau^{\prime}, v^{\prime}\right): Q_{\tau^{\prime}}^{k^{\prime}, v^{\prime}} \subset Q_{\tau^{\prime}}^{l}\right\}}\left(\tau^{\prime}, v^{\prime}\right) \\
& \times\left|Q_{k^{\prime}}(f)\left(y_{\tau^{\prime}}^{k^{\prime}, v^{\prime}}\right)\right| \frac{1}{V_{2^{-\left(k \wedge k^{\prime}\right)}}\left(y_{\tau}^{k, v}\right)+V_{2^{-\left(k \wedge k^{\prime}\right)}}\left(y_{\tau^{\prime}}^{k^{\prime}, v^{\prime}}\right)+V\left(y_{\tau}^{k, v}, y_{\tau^{\prime}}^{k^{\prime}, v^{\prime}}\right)} \\
& \times\left(\frac{2^{-\left(k \wedge k^{\prime}\right)}}{2^{-\left(k \wedge k^{\prime}\right)}+d\left(y_{\tau}^{k, v}, y_{\tau^{\prime}}^{k^{\prime}, v^{\prime}}\right)}\right)^{\epsilon} \\
& \lesssim\left\{\sum_{k^{\prime}=l}^{\infty} \sum_{\tau^{\prime} \in I_{k^{\prime}}}^{N} \sum_{v^{\prime}=1}^{N\left(k^{\prime}, \tau^{\prime}\right)} 2^{k^{\prime} s q} 2^{-\left|k-k^{\prime}\right| \epsilon^{\prime}} 2^{\left(k-k^{\prime}\right) s} \mu\left(Q_{\tau^{\prime}}^{k^{\prime}, v^{\prime}}\right) X_{\left\{\left(\tau^{\prime}, v^{\prime}\right): Q_{\tau^{\prime}}^{k^{\prime}, v^{\prime}} \subset Q_{\tau^{i}}^{l}\right\}}\left(\tau^{\prime}, v^{\prime}\right)\right. \\
& \times\left|Q_{k^{\prime}}(f)\left(y_{\tau^{\prime}}^{k^{\prime}, v^{\prime}}\right)\right|^{q} \frac{1}{V_{2^{-\left(k \kappa k^{\prime}\right)}}\left(y_{\tau}^{k, v}\right)+V_{2^{-\left(k k k^{\prime}\right)}}\left(y_{\tau^{\prime}}^{k^{\prime}, \nu^{\prime}}\right)+V\left(y_{\tau}^{k, v}, y_{\tau^{\prime}}^{k^{\prime}, v^{\prime}}\right)} \\
& \left.\times\left(\frac{2^{-\left(k \wedge k^{\prime}\right)}}{2^{-\left(k \wedge k^{\prime}\right)}+d\left(y_{\tau}^{k, v}, y_{\tau^{\prime}}^{k^{\prime}, v^{\prime}}\right)}\right)^{\epsilon}\right\}^{1 / q}
\end{aligned}
$$


which together with Minkowski's inequality, (6.6), and Lemma 5.2 yields that

$\left(Y_{1,1}\right)^{1 / q} \lesssim \sum_{i=1}^{m_{1}}\left\{\frac{1}{\mu\left(Q_{\tau^{i}}^{l}\right)} \sum_{k^{\prime}=l}^{\infty} \sum_{\tau^{\prime} \in I_{k^{\prime}}} \sum_{v^{\prime}=1}^{N\left(k^{\prime}, \tau^{\prime}\right)} 2^{k^{\prime} s q} \mu\left(Q_{\tau^{\prime}}^{k^{\prime}, v^{\prime}}\right) X_{\left\{\left(\tau^{\prime}, v^{\prime}\right): Q_{\tau^{\prime}}^{k^{\prime}, \prime^{\prime}} \subset Q_{\tau^{i}}^{l}\right\}}\left(\tau^{\prime}, v^{\prime}\right)\left|Q_{k^{\prime}}(f)\left(y_{\tau^{\prime}}^{k^{\prime}, v^{\prime}}\right)\right|^{q}\right\}^{1 / q}$,

which completes the estimate for $Y_{1,1}$.

We now estimate $Y_{1,2}$. To this end, for $j \in \mathbb{Z}_{+}$, let

$$
I_{l}^{j}=\left\{\alpha^{\prime} \in I_{l}: \alpha^{\prime} \neq \tau^{1}, \ldots, \tau^{m_{1}}, 3 C_{6} 2^{j-l} \leq d\left(z_{\alpha^{\prime}}^{l}, z_{\alpha}^{l}\right)<3 C_{6} 2^{j-l+1}\right\}
$$

We first claim that there exists $m_{2} \in \mathbb{N}$ which is no more than a constant independent of $l$ and $j$ such that

$$
\bigcup_{\alpha^{\prime} \in I_{l}^{j}} Q_{\alpha^{\prime}}^{l} \subset \bigcup_{\substack{i=1, \ldots, m_{2} \\ \alpha^{i} \in I_{l-j}}} Q_{\alpha^{i}}^{l-j}, \quad Q_{\alpha^{i}}^{l-j} \cap\left(\bigcup_{\alpha^{\prime} \in I_{l}^{j}} Q_{\alpha^{\prime}}^{l}\right) \neq \varnothing
$$

for $i=1, \ldots, m_{2}$, and moreover, if $Q_{\tau^{\prime}}^{k^{\prime}, v^{\prime}} \subset \cup_{\alpha^{\prime} \in I_{l}^{j}} Q_{\alpha^{\prime}}^{l}$, then

$$
\mu\left(\bigcup_{\substack{i=1, \ldots, m_{2} \\ \alpha^{i} \in I_{l-j}}} Q_{\alpha^{i}}^{l-j}\right) \lesssim V_{2^{j-l}}\left(y_{\tau^{\prime}}^{k^{\prime}, v^{\prime}}\right)
$$

In fact, by Lemma 2.19, there exists $m_{2} \in \mathbb{N}$ such that (6.12) holds. Notice that for any fixed $i_{0} \in\left\{1, \ldots, m_{2}\right\}, \cup_{\alpha^{\prime} \in I_{l}^{j}} Q_{\alpha^{\prime}}^{l} \subset B\left(z_{\alpha^{i_{0}}}^{j-l}, 14 C_{6} 2^{j-l}\right)$. By an argument in [85, pages 1385-1386], we know that the number of $\alpha \in I_{l-j}$ such that $Q_{\alpha}^{l-j} \cap B\left(z_{\alpha^{i 0}}^{j-l}, 14 C_{6} 2^{j-l}\right) \neq \varnothing$ is no more than a constant which is independent of $j$ and $l$. Thus, the claim (6.12) holds.

To see the claim (6.13), we only need to notice that

$$
\bigcup_{\substack{i=1, \ldots, m_{2} \\ \alpha^{i} \in I_{l-j}}} Q_{\alpha^{i}}^{l-j} \subset B\left(y_{\tau^{\prime}}^{k^{\prime}, v^{\prime}}, 9 C_{6} 2^{j-l}\right)
$$

which implies the claim (6.13).

We also notice that if $Q_{\tau^{\prime}}^{k^{\prime}, v^{\prime}} \subset Q_{\alpha^{\prime}}^{l}$ with $\alpha^{\prime} \in I_{l^{\prime}}^{i}$, then for all $y \in Q_{\alpha^{\prime}}^{l}$

$$
d\left(y, y_{\tau^{\prime}}^{k^{\prime}, v^{\prime}}\right) \geq C_{6} 2^{j-l}
$$


Using these properties, we now estimate $Y_{1,2}$ by first considering the case $q \leq 1$. In fact, in this case, from (5.5), (6.15), (6.12), and (6.13), it follows that

$$
\begin{aligned}
Y_{1,2} \lesssim \sum_{j=0}^{\infty} 2^{-j[e q-n(1-q)]} \sum_{i=1}^{m_{2}} & \frac{1}{\mu\left(Q_{\alpha^{i}}^{l-j}\right)} \sum_{k^{\prime}=l}^{\infty} \sum_{\tau^{\prime} \in I_{k^{\prime}}} \sum_{v^{\prime}=1}^{N\left(k^{\prime}, \tau^{\prime}\right)} 2^{k^{\prime} s q} \mu\left(Q_{\tau^{\prime}}^{k^{\prime}, \nu^{\prime}}\right) \\
& \times X_{\left\{\left(\tau^{\prime}, v^{\prime}\right): Q_{\tau^{\prime}}^{k^{\prime}, v^{\prime}} \subset Q_{\alpha^{i}}^{l-j}\right\}}\left(\tau^{\prime}, v^{\prime}\right)\left|Q_{k^{\prime}}(f)\left(y_{\tau^{\prime}}^{k^{\prime}, v^{\prime}}\right)\right|^{q} \\
\times & \left(\sum_{k=l}^{\infty} 2^{-\left|k-k^{\prime}\right| \epsilon^{\prime} q} 2^{\left(k-k^{\prime}\right) s q} 2^{-\left(k \wedge k^{\prime}\right) e q} 2^{k^{\prime} n(1-q)} 2^{-\ln (1-q)+l e q}\right)
\end{aligned}
$$

which together with $q>n /(n+\epsilon)$ and choosing $\epsilon^{\prime}>s$ further implies that

$$
\begin{aligned}
Y_{1,2} \lesssim \sum_{j=0}^{\infty} 2^{-j[\varepsilon q-n(1-q)]} \sum_{i=1}^{m_{2}} & \frac{1}{\mu\left(Q_{\alpha^{i}}^{l-j}\right)} \sum_{k^{\prime}=l}^{\infty} \sum_{\tau^{\prime} \in I_{k^{\prime}}} \sum_{v^{\prime}=1}^{N\left(k^{\prime}, \tau^{\prime}\right)} 2^{k^{\prime} s q} \mu\left(Q_{\tau^{\prime}}^{k^{\prime}, v^{\prime}}\right) \\
& \times X_{\left\{\left(\tau^{\prime}, v^{\prime}\right): Q_{\tau^{\prime}}^{k^{\prime}, v^{\prime}} \subset Q_{\alpha^{i}}^{l-j}\right\}}\left(\tau^{\prime}, v^{\prime}\right)\left|Q_{k^{\prime}}(f)\left(y_{\tau^{\prime}}^{k^{\prime}, v^{\prime}}\right)\right|^{q}
\end{aligned}
$$

From this, it is easy to deduce the desired estimate for $Y_{1,2}$ in this case.

If $1<q \leq \infty$, by Hölder's inequality and Lemma 5.2 , we have

$$
\begin{aligned}
& \sum_{k^{\prime}=l}^{\infty} \sum_{\tau^{\prime} \in I_{k^{\prime}}} \sum_{v^{\prime}=1}^{N\left(k^{\prime}, \tau^{\prime}\right)} 2^{k^{\prime} s} 2^{-\left|k-k^{\prime}\right| \epsilon^{\prime}} 2^{\left(k-k^{\prime}\right) s} \mu\left(Q_{\tau^{\prime}}^{k^{\prime}, v^{\prime}}\right) X_{\left\{\left(\tau^{\prime}, v^{\prime}\right): Q_{\tau^{\prime}}^{k^{\prime}, v^{\prime}} \cap\left(\cup_{i=1}^{m_{1}} Q_{\tau^{i}}^{l}\right)=\varnothing\right\}}\left(\tau^{\prime}, v^{\prime}\right) \\
& \times\left|Q_{k^{\prime}}(f)\left(y_{\tau^{\prime}}^{k^{\prime}, v^{\prime}}\right)\right| \frac{1}{V_{2^{-\left(k \wedge k^{\prime}\right)}}\left(y_{\tau}^{k, v}\right)+V_{2^{-\left(k \wedge k^{\prime}\right)}}\left(y_{\tau^{\prime}}^{k^{\prime}, v^{\prime}}\right)+V\left(y_{\tau}^{k, v}, y_{\tau^{\prime}}^{k^{\prime}, v^{\prime}}\right)} \\
& \times\left(\frac{2^{-\left(k \wedge k^{\prime}\right)}}{2^{-\left(k \wedge k^{\prime}\right)}+d\left(y_{\tau}^{k, v}, y_{\tau^{\prime}}^{k^{\prime}, v^{\prime}}\right)}\right)^{\epsilon} \\
& \lesssim\left\{\sum_{k^{\prime}=l}^{\infty} \sum_{\tau^{\prime} \in I_{k^{\prime}}} \sum_{v^{\prime}=1}^{N\left(k^{\prime}, \tau^{\prime}\right)} 2^{k^{\prime} s q} 2^{-\left|k-k^{\prime}\right| \epsilon^{\prime}} 2^{\left(k-k^{\prime}\right) s} \mu\left(Q_{\tau^{\prime}}^{k^{\prime}, v^{\prime}}\right)\left|Q_{k^{\prime}}(f)\left(y_{\tau^{\prime}}^{k^{\prime}, v^{\prime}}\right)\right|^{q}\right. \\
& \times \frac{1}{V_{2^{-\left(k \wedge k^{\prime}\right)}}\left(y_{\tau}^{k, v}\right)+V_{2^{-\left(k \wedge k^{\prime}\right)}}\left(y_{\tau^{\prime}}^{k^{\prime}, \nu^{\prime}}\right)+V\left(y_{\tau}^{k, v}, y_{\tau^{\prime}}^{k^{\prime}, v^{\prime}}\right)} \\
& \left.\times X_{\left\{\left(\tau^{\prime}, v^{\prime}\right): Q_{\tau^{\prime}}^{k^{\prime}, v^{\prime}} \cap\left(\cup_{i=1}^{m_{1}} Q_{\tau^{i}}^{l}\right)=\varnothing\right\}}\left(\tau^{\prime}, v^{\prime}\right)\left(\frac{2^{-\left(k \wedge k^{\prime}\right)}}{2^{-\left(k \wedge k^{\prime}\right)}+d\left(y_{\tau}^{k, v}, y_{\tau^{\prime}}^{k^{\prime}, v^{\prime}}\right)}\right)^{\epsilon}\right\}^{1 / q},
\end{aligned}
$$

which together with (6.15), (6.12), and (6.13) yields that

$$
\begin{aligned}
Y_{1,2} \lesssim \sum_{j=0}^{\infty} 2^{-j e} \sum_{i=1}^{m_{2}} & \frac{1}{\mu\left(Q_{\alpha^{i}}^{l-j}\right)} \sum_{k^{\prime}=l}^{\infty} \sum_{\tau^{\prime} \in I_{k^{\prime}}} \sum_{v^{\prime}=1}^{N\left(k^{\prime}, \tau^{\prime}\right)} 2^{k^{\prime} s q} \mu\left(Q_{\tau^{\prime}}^{k^{\prime}, v^{\prime}}\right) \\
& \times X_{\left\{\left(\tau^{\prime}, v^{\prime}\right): Q_{\tau^{\prime}}^{k^{\prime}, v^{\prime}} \subset Q_{\alpha^{i}}^{l-j}\right\}}\left(\tau^{\prime}, v^{\prime}\right)\left|Q_{k^{\prime}}(f)\left(y_{\tau^{\prime}}^{k^{\prime}, v^{\prime}}\right)\right|^{q},
\end{aligned}
$$


where we choose $\epsilon^{\prime}>s$. From this, we deduce the desired estimate for $Y_{1,2}$ when $1<q \leq \infty$, which completes the estimate for $Y_{1}$.

We now estimate $Y_{2}$ by using the following trivial estimate that

$$
\left\{\sum_{\tau^{\prime} \in I_{k^{\prime}}} \sum_{v^{\prime}=1}^{N\left(k^{\prime}, \tau^{\prime}\right)} 2^{k^{\prime} s q}\left|Q_{k^{\prime}}(f)\left(y_{\tau^{\prime}}^{k^{\prime}, v^{\prime}}\right)\right|^{q}\right\}^{1 / q} \lesssim \text { RHS of }(6.4),
$$

where and in the sequel, RHS stands for "right-hand side." From this, it follows that if $q \leq 1$, by choosing $\epsilon^{\prime}>s$, we then have

$$
\begin{aligned}
& Y_{2} \lesssim \frac{1}{\mu\left(Q_{\alpha}^{l}\right)} \sum_{k=l}^{\infty} \sum_{\tau \in I_{k}} \sum_{v=1}^{N(k, \tau)} 2^{k s q} \mu\left(Q_{\tau}^{k, v}\right) X_{\left\{(\tau, v): Q_{\tau}^{k, v} \subset Q_{\alpha}^{l}\right\}}(\tau, v) \\
& \times\left(\sum_{k^{\prime}=-\infty}^{l-1} \sum_{\tau^{\prime} \in I_{k^{\prime}}} \sum_{v^{\prime}=1}^{N\left(k^{\prime}, \tau^{\prime}\right)} 2^{-\left(k-k^{\prime}\right) \epsilon^{\prime} q}\left|Q_{k^{\prime}}(f)\left(y_{\tau^{\prime}}^{k^{\prime}, v^{\prime}}\right)\right|^{q}\right) \\
& \lesssim(\text { RHS of }(6.4))^{q}
\end{aligned}
$$

if $1<q \leq \infty$, by Hölder's inequality, Lemma 5.2, and (6.20),

$$
\begin{aligned}
& Y_{2} \lesssim \frac{1}{\mu\left(Q_{\alpha}^{l}\right)} \sum_{k=l}^{\infty} \sum_{\tau \in I_{k}} \sum_{\nu=1}^{N(k, \tau)} 2^{k s q} \mu\left(Q_{\tau}^{k, v}\right) X_{\left\{(\tau, v): Q_{\tau}^{k, v} \subset Q_{\alpha}^{l}\right\}}(\tau, v) \\
& \times\left\{\sum_{k^{\prime}=-\infty}^{l-1} \sum_{\tau^{\prime} \in I_{k^{\prime}}} \sum_{v^{\prime}=1}^{N\left(k^{\prime}, \tau^{\prime}\right)} 2^{-\left(k-k^{\prime}\right) \epsilon^{\prime}} 2^{\left(k-k^{\prime}\right) s} \mu\left(Q_{\tau^{\prime}}^{k^{\prime}, v^{\prime}}\right)\left|Q_{k^{\prime}}(f)\left(y_{\tau^{\prime}}^{k^{\prime}, v^{\prime}}\right)\right|^{q}\right. \\
& \times \frac{1}{V_{2^{-\left(k \wedge k^{\prime}\right)}}\left(y_{\tau}^{k, v}\right)+V_{2^{-\left(k \wedge k^{\prime}\right)}}\left(y_{\tau^{\prime}}^{k^{\prime}, v^{\prime}}\right)+V\left(y_{\tau}^{k, v}, y_{\tau^{\prime}}^{k^{\prime}, v^{\prime}}\right)} \\
& \left.\times\left(\frac{2^{-\left(k \wedge k^{\prime}\right)}}{2^{-\left(k \wedge k^{\prime}\right)}+d\left(y_{\tau}^{k, v}, y_{\tau^{\prime}}^{k^{\prime}, v^{\prime}}\right)}\right)^{\epsilon}\right\} \\
& \lesssim(\text { RHS of }(6.4))^{q},
\end{aligned}
$$

which completes the proof of Proposition 6.3.

Remark 6.4. We point out that Remark 5.5 applies in a similar way to Proposition 6.3.

From Proposition 6.3, it is easy to deduce that the definition of the norm $\|\cdot\|_{\dot{F}_{\infty, q}^{s}(\mathcal{x})}$ is independent of the choice of ATIs. We omit the details. 
Proposition 6.5. Adopting the notation from Proposition 6.3, one has for all $f \in\left(\mathcal{G}_{0}^{\epsilon}(\beta, \gamma)\right)^{\prime}$ with $0<\beta, \gamma<\epsilon$,

$$
\begin{aligned}
& \sup _{l \in \mathbb{Z}} \sup _{\alpha \in I_{l}}\left\{\frac{1}{\mu\left(Q_{\alpha}^{l}\right)} \int_{Q_{\alpha}^{l}} \sum_{k=l}^{\infty} 2^{k s q}\left|D_{k}(f)(x)\right|^{q} d \mu(x)\right\}^{1 / q} \\
& \sim \sup _{l \in \mathbb{Z}} \sup _{\alpha \in I_{l}}\left\{\frac{1}{\mu\left(Q_{\alpha}^{l}\right)} \int_{Q_{\alpha}^{l}} \sum_{k=l}^{\infty} 2^{k s q}\left|Q_{k}(f)(x)\right|^{q} d \mu(x)\right\}^{1 / q} .
\end{aligned}
$$

The following theorem will show that the definition of the norm $\|\cdot\|_{\dot{F}_{\infty, q}^{s}(\mathcal{x})}$ is independent of the choice of the space of distributions.

Proposition 6.6. Let all the notation be as in Definition 6.1. Let $|s|<\epsilon$ and $p(s, \epsilon)<q \leq \infty$. If $f \in\left(\mathcal{G}_{0}^{\epsilon}\left(\beta_{1}, \gamma_{1}\right)\right)^{\prime}$ with $\max \{0,-s\}<\beta_{1}<\epsilon$ and $\max \{0, s\}<\gamma_{1}<\epsilon$, and if $\|f\|_{\dot{F}_{\infty, q}^{s}(\mathcal{x})}<\infty$, then $f \in\left(\dot{\mathcal{G}}_{0}^{\epsilon}\left(\beta_{2}, \gamma_{2}\right)\right)^{\prime}$ for every $\max \{0,-s\}<\beta_{2}<\epsilon$ and $\max \{0, s\}<\gamma_{2}<\epsilon$.

Proof. We use the same notation as in the proof of Proposition 5.7. Let $\psi \in \mathcal{G}(\epsilon, \epsilon)$. For any $f \in$ $\left(\mathcal{C}_{0}^{\epsilon}\left(\beta_{1}, \gamma_{1}\right)\right)^{\prime}$ with $\max \{0,-s\}<\beta_{1}<\epsilon$ and $\max \{0, s\}<\gamma_{1}<\epsilon$, when $q \leq 1$, by Theorem 4.13 together with (5.24), (5.25), and (5.5), we have

$$
\begin{aligned}
|\langle f, \psi\rangle|=\left|\sum_{k=-\infty}^{\infty} \sum_{\tau \in I_{k}} \sum_{\nu=1}^{N(k, \tau)} \mu\left(Q_{\tau}^{k, v}\right) D_{k}(f)\left(y_{\tau}^{k, v}\right)\left\langle\widetilde{D}_{k}\left(\cdot, y_{\tau}^{k, v}\right), \psi\right\rangle\right| \\
\lesssim\|\psi\|_{\mathcal{G}\left(\beta_{2}, \gamma_{2}\right)}\left\{\sum_{k=0}^{\infty} \sum_{\tau \in I_{k}} \sum_{\nu=1}^{N(k, \tau)} 2^{-k \beta_{2} q}\left[\mu\left(Q_{\tau}^{k, v}\right)\left|D_{k}(f)\left(y_{\tau}^{k, v}\right)\right|\right]^{q}\right. \\
\quad \times\left[\frac{1}{V_{1}\left(x_{1}\right)+V\left(x_{1}, y_{\tau}^{k, v}\right)} \frac{1}{\left(1+d\left(x_{1}, y_{\tau}^{k, v}\right)\right)^{\gamma_{2}}}\right]^{q} \\
+\sum_{k=-\infty}^{-1} \sum_{\tau \in I_{k}} \sum_{\nu=1}^{N(k, \tau)} 2^{k \gamma_{2}^{\prime} q}\left[\mu\left(Q_{\tau}^{k, v}\right)\left|D_{k}(f)\left(y_{\tau}^{k, v}\right)\right|\right]^{q} \\
\left.\times\left[\frac{1}{V_{2^{-k}}\left(x_{1}\right)+V\left(x_{1}, y_{\tau}^{k, v}\right)} \frac{2^{-k \gamma_{2}}}{\left(2^{-k}+d\left(x_{1}, y_{\tau}^{k, \nu}\right)\right)^{\gamma_{2}}}\right]^{q}\right\}^{1 / q} .
\end{aligned}
$$

Notice that for $k \in \mathbb{Z}_{+}$, when $d\left(x_{1}, y_{\tau}^{k, \nu}\right)<1$, then $V_{1}\left(x_{1}\right) \sim V_{1}\left(y_{\tau}^{k, \nu}\right) \gtrsim 2^{k \kappa} V_{2^{-k}}\left(y_{\tau}^{k, v}\right) \gtrsim$ $\mu\left(Q_{\tau}^{k, v}\right)$, while when $2^{l} \leq d\left(x_{1}, y_{\tau}^{k, v}\right)<2^{l+1}$ for some $l \in \mathbb{Z}_{+}$, then $V\left(x_{1}, y_{\tau}^{k, v}\right) \gtrsim V_{2^{l}}\left(y_{\tau}^{k, v}\right) \gtrsim$ $V_{2^{-k}}\left(y_{\tau}^{k, v}\right) \sim \mu\left(Q_{\tau}^{k, v}\right)$. Therefore, for $k \in \mathbb{Z}_{+}$,

$$
\frac{\mu\left(Q_{\tau}^{k, v}\right)}{V_{1}\left(x_{1}\right)+V\left(x_{1}, y_{\tau}^{k, v}\right)} \lesssim 1 .
$$

When $k \in \mathbb{Z} \backslash \mathbb{Z}_{+}$, noticing that if $d\left(x_{1}, y_{\tau}^{k, v}\right)<2^{-k}$, then

$$
V_{2^{-k}}\left(x_{1}\right) \gtrsim V_{2^{-k}}\left(y_{\tau}^{k, v}\right) \gtrsim \mu\left(Q_{\tau}^{k, v}\right) ;
$$


if $2^{l} 2^{-k} \leq d\left(x_{1}, y_{\tau}^{k, v}\right)<2^{l+1} 2^{-k}$ for some $l \in \mathbb{Z}_{+}$, then

$$
V\left(x_{1}, y_{\tau}^{k, v}\right) \gtrsim V_{2^{2-k}}\left(y_{\tau}^{k, v}\right) \gtrsim 2^{l \kappa} V_{2^{-k}}\left(y_{\tau}^{k, v}\right) \gtrsim \mu\left(Q_{\tau}^{k, v}\right),
$$

we also have

$$
\frac{\mu\left(Q_{\tau}^{k, v}\right)}{V_{2-k}\left(x_{1}\right)+V\left(x_{1}, y_{\tau}^{k, v}\right)} \lesssim 1
$$

We also need the following trivial estimate that

$$
\sum_{\tau \in I_{k}} \sum_{v=1}^{N(k, \tau)} 2^{k s q}\left|D_{k}(f)\left(y_{\tau}^{k, v}\right)\right|^{q} \lesssim\|f\|_{\dot{F}_{\infty, q}^{s}(x)}^{q}
$$

Using (6.25), (6.28), and (6.29) yields that

$$
\begin{aligned}
|\langle f, \psi\rangle| & \lesssim\|\psi\|_{\mathcal{G}\left(\beta_{2}, \gamma_{2}\right)}\|f\|_{\dot{F}_{\infty, q}^{s}(\mathcal{X})}\left\{\sum_{k=0}^{\infty} 2^{-k\left(\beta_{2}+s\right) q}+\sum_{k=-\infty}^{-1} 2^{k\left(\gamma_{2}^{\prime}-s\right) q}\right\}^{1 / q} \\
& \lesssim\|\psi\|_{\mathcal{G}\left(\beta_{2}, \gamma_{2}\right)}\|f\|_{\dot{F}_{\infty, q}^{s}(\mathcal{X})}
\end{aligned}
$$

where we chose $s<\gamma_{2}^{\prime}<\gamma_{2}$.

If $1<q \leq \infty$, Hölder's inequality, (6.25), (6.28), (6.29), and Lemma 2.1(ii) prove that

$$
\begin{aligned}
|\langle f, \psi\rangle| \lesssim\|\psi\|_{\mathcal{G}_{(}\left(\beta_{2}, \gamma_{2}\right)} & \left\{\sum _ { k = 0 } ^ { \infty } 2 ^ { - k \beta _ { 2 } } 2 ^ { - k s } \left[\sum_{\tau \in I_{k}} \sum_{v=1}^{N(k, \tau)} 2^{k s q} \mu\left(Q_{\tau}^{k, v}\right)\left|D_{k}(f)\left(y_{\tau}^{k, v}\right)\right|^{q}\right.\right. \\
& \times\left[\int_{x} \frac{1}{V_{1}\left(x_{1}\right)+V\left(x_{1}, y_{\tau}^{k, v}\right)} \frac{1}{\left(1+d\left(x_{1}, y_{\tau}^{k, v}\right)\right)^{\gamma_{2}}}\right]^{1 / q} \\
& +\sum_{k=-\infty}^{-1} 2^{k \gamma_{2}^{\prime}} 2^{-k s}\left[\sum_{\tau \in I_{k}} \sum_{v=1}^{N(k, \tau)} 2^{k s q} \mu\left(Q_{\tau}^{k, v}\right)\left|D_{k}(f)\left(y_{\tau}^{k, v}\right)\right|^{q}\right. \\
& \times\left[\frac{1}{\left(1+d\left(x_{1}, y\right)\right)^{\gamma_{2}}} d \mu(y)\right]^{1 / q^{\prime}} \\
& {\left.\left[\frac{1}{V_{2^{-k}}\left(x_{1}\right)+V\left(x_{1}, y\right)} \frac{2^{-k \gamma_{2}}}{\left(2^{-k}+d\left(x_{1}, y\right)\right)^{\gamma_{2}}} d \mu(y)\right]^{1 / q^{\prime}}\right\} }
\end{aligned}
$$

$\lesssim\|\psi\|_{\mathcal{G}\left(\beta_{2}, \gamma_{2}\right)}\|f\|_{\dot{F}_{\infty, q}^{s}(x)}$. 
Combining (6.30) and (6.31) with an argument similar to the proof of Proposition 5.7 then completes the proof of Proposition 6.6.

Now we can introduce the homogeneous Triebel-Lizorkin spaces $\dot{F}_{\infty, q}^{s}(\mathcal{X})$.

Definition 6.7. Let $\epsilon_{1} \in(0,1], \epsilon_{2}>0, \epsilon_{3}>0, \epsilon \in\left(0, \epsilon_{1} \wedge \epsilon_{2}\right)$ and let $\left\{S_{k}\right\}_{k \in \mathbb{Z}}$ be an $\left(\epsilon_{1}, \epsilon_{2}, \epsilon_{3}\right)$-ATI. For $k \in \mathbb{Z}$, set $D_{k}=S_{k}-S_{k-1}$. Let $|s|<\epsilon$ and $p(s, \epsilon)<q \leq \infty$. The space $\dot{F}_{\infty, q}^{s}(\mathcal{X})$ is defined to be the set of all $f \in\left(\mathcal{G}_{0}^{\epsilon}(\beta, \gamma)\right)^{\prime}$, for some $\beta, \gamma$ satisfying

$$
|s|<\beta<\epsilon, \quad \max \{s, 0,-s-\kappa\}<\gamma<\epsilon
$$

such that

$$
\|f\|_{F_{\infty, q}^{s}(x)}=\sup _{l \in \mathbb{Z}} \sup _{\alpha \in I_{l}}\left\{\frac{1}{\mu\left(Q_{\alpha}^{l}\right)} \int_{Q_{\alpha}^{l}} \sum_{k=l}^{\infty} 2^{k s q}\left|D_{k}(f)(x)\right|^{q} d \mu(x)\right\}^{1 / q}<\infty,
$$

where the supremum is taken over all dyadic cubes as in Lemma 2.19 and the usual modification is made when $q=\infty$.

Propositions 6.5 and 6.6 show that the definition of the spaces $\dot{F}_{\infty, q}^{s}(\mathcal{X})$ is independent of the choice of the ATI and the distribution space $(\mathcal{G}(\beta, \gamma))^{\prime}$, with $\beta$ and $\gamma$ satisfying (6.32).

Remark 6.8. To guarantee that the definition of the space $\dot{F}_{\infty, q}^{s}(\mathcal{X})$ is independent of the choice of the distribution space $(\mathcal{G}(\beta, \gamma))^{\prime}$, we only need the restriction that $\max \{0,-s\}<\beta<\epsilon$ and $\max \{0, s\}<\gamma<\epsilon$. Moreover, if we assume that $\max \{0, s\}<\beta<\epsilon$ and $\max \{0, s-\mathcal{\kappa}\}<\gamma<\epsilon$, we can then verify that $\mathcal{G}(\beta, \gamma) \subset \dot{F}_{\infty, q}^{s}(\mathcal{X})$; see Proposition 6.9 below.

\subsection{Properties of $\dot{F}_{\infty, q}^{s}(X)$ and boundedness of singular integrals}

In this subsection, we first present some basic properties of $\dot{F}_{\infty, q}^{s}(\mathcal{X})$. By establishing a maximal function characterization of $\dot{F}_{\infty, q}^{s}(\mathcal{X})$, we then establish some relations between the spaces $\dot{F}_{\infty, q}^{s}(\mathcal{X})$ and the spaces $\dot{C}^{s}(\mathcal{X})$ and between the spaces $\dot{F}_{\infty, q}^{s}(\mathcal{X})$ and the space $\operatorname{BMO}(\mathcal{X})$. Finally, we obtain the boundedness on $\dot{F}_{\infty, q}^{s}(\mathcal{X})$ of the singular integrals considered by Nagel and Stein in [44].

Proposition 6.9. Let $\epsilon$ be as in Definition 6.7, $|s|<\epsilon$, and $p(s, \epsilon)<q \leq \infty$. Then,

(i) $\dot{F}_{\infty, q_{0}}^{s}(\mathcal{X}) \subset \dot{F}_{\infty, q_{1}}^{s}(\mathcal{X})$ if $p(s, \epsilon)<q_{0} \leq q_{1} \leq \infty$;

(ii) $\dot{B}_{\infty, q}^{s}(\mathcal{X}) \subset \dot{F}_{\infty, q}^{s}(\mathcal{X}) \subset \dot{B}_{\infty, \infty}^{s}(\mathcal{X})$;

(iii) if $\max \{0,-s\}<\beta<\epsilon$ and $\max \{0, s\}<\gamma<\epsilon$, then $\dot{F}_{\infty, q}^{s}(\mathcal{X}) \subset\left(\mathcal{G}_{0}^{\epsilon}(\beta, \gamma)\right)^{\prime}$;

(iv) if $\max \{s, 0\}<\beta<\epsilon$ and $\max \{0,-s-\kappa\}<\gamma<\epsilon$, then $\mathcal{G}(\beta, \gamma) \subset \dot{F}_{\infty, q}^{s}(\mathcal{X})$;

(v) the spaces $\dot{F}_{\infty, q}^{s}(\mathcal{X}) / \mathcal{N}$ are complete. 
Proof. Property (i) is a simple corollary of (5.5).

To see (ii), $\dot{B}_{\infty, q}^{s}(\mathcal{X}) \subset \dot{F}_{\infty, q}^{s}(\mathcal{X})$ is obvious by the definitions of the both spaces $\dot{B}_{\infty, q}^{s}(\mathcal{X})$ and $\dot{F}_{\infty, q}^{s}(\mathcal{X})$, while $\dot{F}_{\infty, q}^{s}(\mathcal{X}) \subset \dot{B}_{\infty, \infty}^{s}(\mathcal{X})$ can be obtained by their definitions together with the Lebesgue differential theorem; we omit the details.

Property (iii) is a consequence of the second inclusion in Property (ii) of this proposition and Proposition 5.10(iii) on $\dot{B}_{\infty, \infty}^{s}(\mathcal{X})$, while Property (v) can be easily deduced from Property (iii), Property (iv) is a conclusion of the first inclusion in Property (ii) of this proposition and Proposition 5.10(iv) on $\dot{B}_{\infty, q}^{s}(\mathcal{X})$, which completes the proof of this proposition.

To obtain some relations between the spaces $\dot{F}_{\infty, q}^{s}(\mathcal{X})$ and the space $\operatorname{BMO}(\mathcal{X})$, we need the following technical result which in fact gives a new characterization of Triebel-Lizorkin space $\dot{F}_{\infty, q}^{s}(\mathcal{X})$. We first introduce a maximal function.

For any $x \in \mathcal{X}$ and $l \in \mathbb{Z}$, using Lemma 2.19, it is easy to prove that there exists a finite number of $\alpha \in I_{l}$ such that

$$
Q_{\alpha}^{l} \cap B\left(x, 2^{-l+1}\right) \neq \varnothing
$$

which will denote by $Q_{\alpha_{l}^{i}}^{l}$ with $i=1, \ldots, m_{l}(x)$, and moreover, $m_{l}(x)$ is no more than a positive integer $m \in \mathbb{N}$ which is independent of $l$ and $x$; see [85, pages 1385-1386] for a detailed proof. In what follows, for convenience sake, we will always assume that $m_{l}(x)=m$ by letting $Q_{\alpha_{1}^{i}}^{l}=\varnothing$ for $i=m_{l}(x)+1, \ldots, m$ when $m_{l}(x)<m$. Let $\left\{D_{k}\right\}_{k \in \mathbb{Z}}$ be as in Definition 6.7. Now for any $s \in \mathbb{R}, q \in(0, \infty], f \in(\mathcal{G}(\beta, \gamma))^{\prime}$ with $0<\beta, \gamma<\epsilon$ and $x \in \mathcal{X}$, we define the maximal function $\dot{\mathfrak{C}}_{q}^{s}(f)(x)$ by

$$
\dot{\mathfrak{C}}_{q}^{s}(f)(x)=\sup _{l \in \mathbb{Z}}\left[\frac{1}{\mu\left(\cup_{i=1}^{m} Q_{\alpha_{l}^{i}}^{l}\right)} \int_{\cup_{i=1}^{m} Q_{\alpha_{l}^{l}}^{l}} \sum_{k=l}^{\infty} 2^{k s q}\left|D_{k}(f)(y)\right|^{q} d \mu(y)\right]^{1 / q},
$$

where the usual modification is made when $q=\infty$.

Proposition 6.10. Let $\epsilon, s$, and $q$ be as in Definition 6.7. Then $f \in \dot{F}_{\infty, q}^{s}(x)$ if and only if $f \in$ $(\mathcal{G}(\beta, \gamma))^{\prime}$ with $\beta, \gamma$ as in $(6.32)$ and $\dot{\mathfrak{C}}_{q}^{s}(f) \in L^{\infty}(\mathcal{X})$. Moreover, in this case,

$$
\|f\|_{\dot{F}_{\infty, q}^{s}(x)} \sim\left\|\dot{\mathfrak{C}}_{q}^{s}(f)\right\|_{L^{\infty}(x)} .
$$

Proof. For any $s \in \mathbb{R}, q \in(0, \infty], f \in(\mathcal{G}(\beta, \gamma))^{\prime}$ with $\beta, \gamma$ as in (6.32) and $x \in \mathcal{X}$, let

$$
\dot{\mathfrak{C}}_{q, 1}^{s}(f)(x)=\sup _{l \in \mathbb{Z}} \sup _{\substack{Q_{\alpha}^{l} \ni x \\ \alpha \in I_{l}}}\left[\frac{1}{\mu\left(Q_{\alpha}^{l}\right)} \int_{Q_{\alpha}^{l}} \sum_{k=l}^{\infty} 2^{k s q}\left|D_{k}(f)(y)\right|^{q} d \mu(y)\right]^{1 / q},
$$

where the usual modification is made when $q=\infty$. Obviously,

$$
\|f\|_{\dot{F}_{\infty, q}^{s}(x)} \sim\left\|\dot{\mathfrak{C}}_{q, 1}^{s}(f)\right\|_{L^{\infty}(x)} .
$$


To prove the conclusion of this proposition, it suffices to verify that when

$$
\bigcup_{i=1}^{m_{l}(x)} Q_{\alpha_{l}^{l}}^{l} \supset B\left(x, 2^{-l+1}\right)
$$

for any $i_{1}, i_{2} \in\left\{1, \ldots, m_{l}(x)\right\}$,

$$
\mu\left(Q_{\alpha_{l}^{i_{1}}}^{l}\right) \sim \mu\left(Q_{\alpha_{l}^{i_{2}}}^{l}\right)
$$

To verify (6.40), by symmetry, it suffices to verify that

$$
\mu\left(Q_{\alpha_{l}^{i_{1}}}^{l}\right) \lesssim \mu\left(Q_{\alpha_{l}^{i_{2}}}^{l}\right)
$$

To this end, suppose $z_{k} \in B\left(x, 2^{-l+1}\right) \cap Q_{\alpha_{l}^{i_{k}}}^{l}$ with $k=1$, 2. Then for any $w \in B\left(z_{\alpha_{l}^{i_{1}}}^{l}, C_{6} 2^{-l}\right)$, we have

$$
\begin{aligned}
d\left(w, z_{\alpha_{l}^{i_{2}}}^{l}\right) & \leq d\left(w, z_{\alpha_{l}^{i_{1}}}^{l}\right)+d\left(z_{\alpha_{l}^{i_{1}}}^{l}, z_{1}\right)+d\left(z_{1}, x\right)+d\left(x, z_{2}\right)+d\left(z_{2}, z_{\alpha_{l}^{i_{2}}}^{l}\right) \\
& \leq 3 C_{6} 2^{-l}+2^{-l+2} .
\end{aligned}
$$

Thus, $Q_{\alpha_{l}^{i_{1}}}^{l} \subset B\left(z_{\alpha_{l}^{i_{1}}}^{l}, C_{6} 2^{-l}\right) \subset B\left(z_{\alpha_{l}^{i_{2}}}^{l}\left(3 C_{6}+4\right) 2^{-l}\right)$, which together with Lemma 2.19 and the double property of $\mu$ gives (6.41), and hence, completes the proof of Proposition 6.10.

Using Theorem 5.19(i) and Proposition 6.10, and an argument similar to that in [86], we can establish the connections between $\dot{F}_{\infty, q}^{s}(\mathcal{X})$ with $\mathrm{BMO}(\mathcal{X})$ and $\dot{C}^{s}(\mathcal{X})$ with $s>0$ as follows.

Theorem 6.11. Let $\epsilon$ be as in Definition 6.7. Then,

(i) if $0<s<\epsilon$, then $\dot{C}^{s}(\mathcal{X})=\dot{F}_{\infty, \infty}^{s}(\mathcal{X})=\dot{B}_{\infty, \infty}^{s}(\mathcal{X})$ with equivalent norms;

(ii) $\operatorname{BMO}(\mathcal{X})=\dot{F}_{\infty, 2}^{0}(x)$ with equivalent norm.

Proof. We first verify (i). Let $f \in \dot{C}^{s}(\mathcal{X})$ and let $\left\{D_{k}\right\}_{k \in \mathbb{Z}}$ be as in Definition 6.7. We first claim that $f \in\left(\dot{\mathcal{C}}_{0}^{\epsilon}(\beta, \gamma)\right)^{\prime}$ when $0<\beta<\epsilon$ and $s<\gamma<\epsilon$. In fact, from $f \in \dot{C}^{s}(\mathcal{X})$, it follows that for all $x \in \mathcal{X}$,

$$
\left|f(x)-f\left(x_{1}\right)\right| \lesssim\|f\|_{C^{s}(x)} d\left(x, x_{1}\right)^{s},
$$


which implies that for any $g \in \mathcal{G}(\beta, \gamma)$ with $0<\beta<\epsilon$ and $s<\gamma<\epsilon$,

$$
\begin{aligned}
|\langle f, g\rangle| & =\left|\int_{\mathcal{X}}\left[f(x)-f\left(x_{1}\right)\right] g(x) d \mu(x)\right| \\
& \lesssim\|f\|_{\dot{C}^{s}(\mathcal{X})}\|g\|_{\mathcal{G}(\beta, r)} \int_{\mathcal{X}} d\left(x, x_{1}\right)^{s} \frac{1}{V_{1}\left(x_{1}\right)+V\left(x_{1}, x\right)}\left(\frac{1}{1+d\left(x_{1}, x\right)}\right)^{\gamma} d \mu(x) \\
& \lesssim\|f\|_{\dot{C}^{s}(\mathcal{X})}\|g\|_{\mathcal{G}(\beta, \gamma)}
\end{aligned}
$$

where in the last step, we used Lemma 2.1(ii).

Moreover, for all $k \in \mathbb{Z}$ and $x \in \mathcal{X}$, by Lemma 2.1(ii) and $0<s<\epsilon<\epsilon_{2}$, we then have

$$
\begin{aligned}
\left|D_{k}(f)(x)\right| & =\left|\int_{\chi} D_{k}(x, y) f(y) d \mu(y)\right| \\
& =\left|\int_{\chi} D_{k}(x, y)[f(y)-f(x)] d \mu(y)\right| \\
& \lesssim\|f\|_{C^{s}(x)} \int_{x} \frac{1}{V_{2^{-k}}(x)+V_{2^{-k}}(y)+V(x, y)}\left(\frac{2^{-k}}{2^{-k}+d(x, y)}\right)^{\epsilon_{2}} d(x, y)^{s} d \mu(y) \\
& \lesssim 2^{-k s}\|f\|_{\dot{C}^{s}(\mathcal{X})}
\end{aligned}
$$

which proves that

$$
\|f\|_{\dot{F}_{\infty, \infty}^{s}(\mathcal{X})}=\sup _{k \in \mathbb{Z}} \sup _{x \in \mathcal{X}} 2^{k s}\left|D_{k}(f)(x)\right| \lesssim\|f\|_{\dot{C}^{s}(\mathcal{X})} .
$$

Thus, $\dot{C}^{s}(\mathcal{X}) \subset \dot{F}_{\infty, \infty}^{s}(\mathcal{X})$.

Conversely, let $f \in \dot{F}_{\infty, \infty}^{s}(\mathcal{X})$. By Proposition 6.6, without loss of generality, we may assume that $f \in(\mathcal{G}(\beta, \gamma))^{\prime}$ with $\beta, \gamma$ as in (6.32). Let all the notation as in Theorem 3.10. Then, by Theorem 3.13, for any $g \in \mathcal{G}(\beta, \gamma)$ with $\beta, \gamma$ as in (6.32), since $\int_{x} g(x) d \mu(x)=0$, we then have

$$
\langle f, g\rangle=\sum_{k=-\infty}^{\infty}\left\langle\widetilde{D}_{k} D_{k}(f)(\cdot), g(\cdot)\right\rangle=\sum_{k=-\infty}^{\infty}\left\langle\widetilde{D}_{k} D_{k}(f)(\cdot)-\widetilde{D}_{k} D_{k}(f)\left(x_{1}\right), g(\cdot)\right\rangle
$$

which means that in $(\mathcal{G}(\beta, \gamma))^{\prime}, f(x)=\sum_{k=-\infty}^{\infty}\left[\tilde{D}_{k} D_{k}(f)(\cdot)-\tilde{D}_{k} D_{k}(f)\left(x_{1}\right)\right]$. For $x \in \mathcal{X}$, we now let $h(x) \equiv \sum_{k=-\infty}^{\infty}\left[\tilde{D}_{k} D_{k}(f)(x)-\tilde{D}_{k} D_{k}(f)\left(x_{1}\right)\right]$ and we first verify that $h$ is a function satisfying the following growth condition that

$$
|h(x)| \lesssim\|f\|_{\dot{F}_{\infty, \infty}^{s}(x)} d\left(x, x_{1}\right)^{s}
$$


In fact, for any $x \in \mathcal{X}$, assume that $2^{-l_{0}-1}<d\left(x, x_{1}\right) \leq 2^{-l_{0}}$ with some $l_{0} \in \mathbb{Z}$ and write that

$$
\begin{aligned}
h(x) & =\sum_{k=-\infty}^{\infty} \int_{\chi}\left[\widetilde{D}_{k}(x, z)-\widetilde{D}_{k}\left(x_{1}, z\right)\right] D_{k}(f)(z) d \mu(z) \\
& =\sum_{k=-\infty}^{l_{0}-1} \int_{x}\left[\widetilde{D}_{k}(x, z)-\widetilde{D}_{k}\left(x_{1}, z\right)\right] D_{k}(f)(z) d \mu(z)+\sum_{k=l_{0}}^{\infty} \cdots \\
& \equiv J_{1}+J_{2} .
\end{aligned}
$$

For $J_{1}$, by the regularity of $\widetilde{D}_{k}$, Lemma 2.1(ii) and $s<\epsilon<\epsilon_{1}$, we have

$$
\begin{aligned}
\left|J_{1}\right| & \lesssim \sum_{k=-\infty}^{l_{0}-1} \int_{x}\left(\frac{d\left(x, x_{1}\right)}{2^{-k}+d(x, z)}\right)^{\epsilon_{1}} \frac{1}{V_{2^{-k}}(x)+V_{2^{-k}}(z)+V(x, z)}\left(\frac{2^{-k}}{2^{-k}+d(x, z)}\right)^{\epsilon_{2}}\left|D_{k}(f)(z)\right| d \mu(z) \\
& \lesssim\|f\|_{\dot{F}_{\infty, \infty}^{s}(x)} d\left(x, x_{1}\right)^{s} .
\end{aligned}
$$

To estimate $J_{2}$, by $s>0$ and Proposition 2.7(i), we obtain

$$
\begin{aligned}
J_{2} & \lesssim\|f\|_{\dot{F}_{\infty, \infty}^{s}(x)} \sum_{k=l_{0}}^{\infty} 2^{-k s} \int_{\chi}\left[\left|\widetilde{D}_{k}(x, z)\right|+\left|\widetilde{D}_{k}\left(x_{1}, z\right)\right|\right] d \mu(z) \\
& \lesssim\|f\|_{\dot{F}_{\infty, \infty}^{s}(x)} d\left(x, x_{1}\right)^{s} .
\end{aligned}
$$

Thus, our claim is true.

Notice that for any $x, y \in \mathcal{X}$,

$$
h(x)-h(y)=\sum_{k=-\infty}^{\infty}\left[\widetilde{D}_{k} D_{k}(f)(x)-\widetilde{D}_{k} D_{k}(f)(y)\right]
$$

Repeating the above proof yields that for all $x, y \in \mathcal{X}$,

$$
|h(x)-h(y)| \lesssim\|f\|_{F_{\infty, \infty}^{s}(x)} d(x, y)^{s}
$$

Thus, $h \in \dot{C}^{s}(\mathcal{X})$ and

$$
\|h\|_{\dot{C}^{s}(\mathcal{X})} \lesssim\|f\|_{\dot{F}_{\infty, \infty}^{s}(\mathcal{X})}
$$

In this sense, we say that $\dot{F}_{\infty, \infty}^{s}(\mathcal{X}) \subset \dot{C}^{s}(\mathcal{X})$, which finishes the proof of (i).

To see (ii), let $f \in \mathrm{BMO}(\mathcal{X})$ and $\left\{D_{k}\right\}_{k \in \mathbb{Z}}$ be as in Definition 6.7. Proposition 5.10(iv) and Theorem 5.19(i) immediately imply that $f \in\left(\mathcal{G}_{0}^{\epsilon}(\beta, \gamma)\right)^{\prime}$ with $0<\beta, \gamma<\epsilon$. Let now $Q_{\alpha}^{l}$ for $l \in \mathbb{Z}$ and $\alpha \in I_{l}$ be a dyadic cube as in Lemma 2.19. Set $B_{\alpha}^{l}=B\left(z_{\alpha}^{l}, 2 C_{6} 2^{-l}\right)$ and write

$$
f=\left(f-m_{B_{\alpha}^{l}}(f)\right) X_{B_{\alpha}^{l}}+\left(f-m_{B_{\alpha}^{l}}(f)\right) X_{\left\{x \backslash B_{\alpha}^{l}\right\}}+m_{B_{\alpha}^{l}}(f) \equiv f_{1}+f_{2}+f_{3} .
$$


Obviously, $\mu\left(Q_{\alpha}^{l}\right) \sim \mu\left(B_{\alpha}^{l}\right)$ and $D_{k}\left(f_{3}\right)=0$; in combination with Proposition 3.15, this shows that

$$
\begin{aligned}
\left\{\frac{1}{\mu\left(Q_{\alpha}^{l}\right)} \int_{Q_{\alpha}^{l}} \sum_{k=l}^{\infty}\left|D_{k}\left(f_{1}\right)(x)\right|^{2} d \mu(x)\right\}^{1 / 2} & \lesssim\left\{\frac{1}{\mu\left(Q_{\alpha}^{l}\right)} \int_{x}\left|f_{1}(x)\right|^{2} d \mu(x)\right\}^{1 / 2} \\
& \lesssim\left\{\frac{1}{\mu\left(B_{\alpha}^{l}\right)} \int_{B_{\alpha}^{l}}\left|f-m_{B_{\alpha}^{l}}(f)\right|^{2} d \mu(x)\right\}^{1 / 2} \\
& \lesssim\|f\|_{\mathrm{BMO}(x) .}
\end{aligned}
$$

To estimate $f_{2}$, notice that for $x \in Q_{\alpha}^{l}$ and $y \in \mathcal{X} \backslash B_{\alpha}^{l}$, then $d(x, y) \gtrsim 2^{-l}+d\left(y, z_{\alpha}^{l}\right)$, which together with the size condition of $D_{k}$ yields that for $x \in Q_{\alpha}^{l}$ and $k \geq l$,

$$
\begin{aligned}
\left|D_{k}\left(f_{2}\right)(x)\right| & =\left|\int_{x \backslash B_{\alpha}^{l}} D_{k}(x, y)\left[f(y)-m_{B_{\alpha}^{l}}(f)\right] d \mu(y)\right| \\
& \lesssim 2^{(l-k) \epsilon_{2}} \sum_{j=0}^{\infty} \frac{1}{2^{j \epsilon_{2}}} \frac{1}{V\left(z_{\alpha}^{l}, 2 C_{6} 2^{j-l+1}\right)} \int_{d\left(y, z_{\alpha}^{l}\right)<2 C_{6} 2^{j-l+1}}\left|f(y)-m_{B_{\alpha}^{l}}(f)\right| d \mu(y) .
\end{aligned}
$$

The definition of $\mathrm{BMO}(x)$ together with the double property of $\mu$ gives that

$$
\left|m_{B\left(z_{\alpha}^{l}, 2 C_{6} 2^{j-l+1}\right)}(f)-m_{B_{\alpha}^{l}}(f)\right| \lesssim(j+1)\|f\|_{\mathrm{BMO}(x)},
$$

which further implies that for $x \in Q_{\alpha}^{l}$ and $k \geq l$,

$$
\begin{aligned}
& \left|D_{k}\left(f_{2}\right)(x)\right| \\
& \lesssim 2^{(l-k) \epsilon_{2}} \sum_{j=0}^{\infty} \frac{1}{2^{j \epsilon_{2}}}\left\{\frac{1}{V\left(z_{\alpha}^{l}, 2 C_{6} 2^{j-l+1}\right)} \int_{d\left(y, z_{\alpha}^{l}\right)<2 C_{6} 2^{j-l+1}}\left|f(y)-m_{B\left(z_{\alpha}^{l}, 2 C_{6} 2^{j-l+1}\right)}(f)\right| d \mu(y)\right. \\
& \left.+\left|m_{B\left(z_{\alpha}^{l}, 2 C_{6} 2^{j-l+1}\right)}(f)-m_{B_{\alpha}^{l}}(f)\right|\right\} \\
& \lesssim 2^{(l-k) \epsilon_{2}}\|f\|_{\mathrm{BMO}(x)} .
\end{aligned}
$$

From this, it follows that

$$
\begin{aligned}
\left\{\frac{1}{\mu\left(Q_{\alpha}^{l}\right)} \int_{Q_{\alpha}^{l}} \sum_{k=l}^{\infty}\left|D_{k}\left(f_{2}\right)(x)\right|^{2} d \mu(x)\right\}^{1 / 2} & \lesssim\|f\|_{\mathrm{BMO}(x)}\left\{\sum_{k=l}^{\infty} 2^{2(l-k) \epsilon_{2}}\right\}^{1 / 2} \\
& \lesssim\|f\|_{\mathrm{BMO}(x)} .
\end{aligned}
$$

Combining the above estimates, we know that $f \in \dot{F}_{\infty, 2}^{0}(\mathcal{X})$ and

$$
\|f\|_{\dot{F}_{\infty, 2}^{0}(x)} \lesssim\|f\|_{\mathrm{BMO}(x)}
$$


We now prove the converse. Let $f \in \dot{F}_{\infty, 2}^{0}(\mathcal{X})$. By Proposition 6.6, without loss of generality, we may assume that $f \in(\mathcal{G}(\beta, \gamma))^{\prime}$ with $\beta, \gamma$ as in (6.32). In the rest of the proof of this theorem, we denote $\dot{\mathfrak{C}}_{2}^{0}$ and $\dot{S}_{2,1}^{0}$ simply by $\dot{\mathfrak{C}}$ and $\dot{S}$. Moreover, for $j \in \mathbb{Z}, f$ as above and $x \in \mathcal{X}$, we define

$$
\dot{S}^{j}(f)(x)=\left\{\sum_{k=j}^{\infty} \int_{d(x, y)<2^{-k}}\left|D_{k}(f)(y)\right|^{2} \frac{d \mu(y)}{V_{2^{-k}}(x)}\right\}^{1 / 2} .
$$

Obviously, $\dot{S}^{\infty}(f)(x)=\dot{S}(f)(x)$. Let the notation as in Theorem 3.11. For any $f$ as above and $x \in \mathcal{X}$, we also set

$$
\dot{\bar{S}}(f)(x)=\left\{\sum_{k=-\infty}^{\infty} \int_{d(x, y)<2^{-k}}\left|\bar{D}_{k}(f)(y)\right|^{2} \frac{d \mu(y)}{V_{2^{-k}}(x)}\right\}^{1 / 2} .
$$

Theorem 5.13 together with Remark 5.5 shows that for all $f \in H^{1}(\mathcal{X})$,

$$
\|\dot{\bar{S}}(f)\|_{L^{1}(\mathcal{X})} \lesssim\|f\|_{H^{1}(\mathcal{X})} .
$$

For any fixed $f$ as above and $x \in \mathcal{X}$, we define the "stopping-time" $j(x)$ by

$$
j(x)=\inf \left\{j \in \mathbb{Z}: \dot{S}^{j}(f)(x) \leq A \dot{\mathfrak{C}}(f)(x)\right\},
$$

where $A>0$ is a large constant to be determined later. We first claim that for any $y \in \mathcal{X}$ and $l \in \mathbb{Z}$, if we choose $A$ to be large enough, then there exists a constant $C_{12}>0$ such that

$$
\mu\left(\left\{x \in X: d(x, y)<2^{-l}, l \geq j(x)\right\}\right) \geq C_{12} \mu\left(B\left(y, 2^{-l}\right)\right) .
$$

In fact, let $B_{0}=B\left(y, 2^{-l}\right)$. Then $\bigcup_{x \in B_{0}} B\left(x, 2^{-l}\right) \subset B\left(y, 2^{-l+1}\right)$. Let

$$
B\left(y, 2^{-l+1}\right) \subset \bigcup_{i=1}^{m} Q_{\alpha_{l}^{i}}^{l} \equiv P
$$

as in the definition of $\dot{\mathfrak{C}}$. Let $w \in B\left(y, 2^{-l+1}\right) \cap Q_{\alpha_{l}^{i}}^{l}$. Then for any $x \in Q_{\alpha_{l}^{i}}^{l}, d(x, y) \leq d(x, w)+$ $d(w, y) \leq C_{6} 2^{-l}+2^{-l+1}$, which shows that

$$
P \subset B\left(y,\left(C_{6}+2\right) 2^{-l}\right) .
$$


Thus, $\mu\left(B_{0}\right) \sim \mu(P)$. From this, it follows that

$$
\begin{aligned}
\frac{1}{\mu\left(B_{0}\right)} \int_{B_{0}}\left[\dot{S}^{l}(f)(x)\right]^{2} d \mu(x) & =\sum_{k=l}^{\infty} \frac{1}{\mu\left(B_{0}\right)} \int_{B_{0}} \int_{d(x, z)<2^{-k}}\left|D_{k}(f)(z)\right|^{2} \frac{d \mu(z)}{V_{2^{-k}}(x)} d \mu(x) \\
& \leq C \frac{1}{\mu(P)} \sum_{k=l}^{\infty} \int_{P} \int_{B_{0}}\left|D_{k}(f)(z)\right|^{2} X_{B\left(z, 2^{-k}\right)}(x) \frac{d \mu(x)}{V_{2^{-k}}(x)} d \mu(z) \\
& \leq C_{13} \frac{1}{\mu(P)} \int_{P} \sum_{k=l}^{\infty}\left|D_{k}(f)(z)\right|^{2} d \mu(z) \\
& \leq C_{13}\left[\inf _{x \in B_{0}} \dot{C}(f)(x)\right]^{2},
\end{aligned}
$$

where $C, C_{13}>0$ are constants independent of $l$ and $x$. Thus, if $A^{2}>C_{13}$, then

$$
\mu\left(\left\{x \in B_{0}: S^{l}(f)(x)>A \dot{\mathfrak{C}}(f)(x)\right\}\right) \leq \frac{C_{13}}{A^{2}} \mu\left(B_{0}\right),
$$

which in turn shows (6.66) with $C_{12}=1-C_{13} / A^{2}>0$ if $A^{2}>C_{13}$.

Let $g \in \stackrel{\mathcal{G}}{b}_{b}\left(\epsilon_{1}, \epsilon_{2}\right)$ and $\|g\|_{H^{1}(x)} \leq 1$. By Theorem 3.11, (6.66), the Fubini theorem, Hölder's inequality, (6.64), and Proposition 6.10, we then have

$$
\begin{aligned}
|\langle f, g\rangle| & =\left|\left\langle f, \sum_{k=-\infty}^{\infty} D_{k} \bar{D}_{k}(g)\right\rangle\right| \\
& =\left|\sum_{k=-\infty}^{\infty}\left\langle D_{k}^{t}(f), \bar{D}_{k}(g)\right\rangle\right| \\
& \leq \sum_{k=-\infty}^{\infty} \int_{x}\left|D_{k}^{t}(f)(y) \bar{D}_{k}(g)(y)\right| d \mu(y) \\
& \lesssim \int_{x}\left[\sum_{k=l(x)}^{\infty} \int_{d(x, y)<2^{-k}}\left|D_{k}^{t}(f)(y) \bar{D}_{k}(g)(y)\right| \frac{d \mu(y)}{V_{2-k}(x)}\right] d \mu(x) \\
& \lesssim \int_{x} \dot{S}^{l(x)}(f)(x) \dot{\bar{S}}(g)(x) d \mu(x) \\
& \lesssim \int_{x} \dot{\mathfrak{C}}(f)(x) \dot{\bar{S}}(g)(x) d \mu(x) \\
& \lesssim\|\dot{\mathfrak{C}}(f)\|_{L^{\infty}(x)}\|g\|_{H^{1}(x)} \\
& \lesssim\|f\|_{\dot{F}_{\infty, 2}^{0}(x),}
\end{aligned}
$$


which together with Theorem 5.19(i) further implies that $f \in \operatorname{BMO}(\mathcal{X})$ and

$$
\|f\|_{\mathrm{BMO}(x)} \lesssim\|f\|_{\dot{F}_{\infty, 2}^{0}(x)}
$$

This finishes the proof of Theorem 6.11 .

We end this subsection by the boundedness on $\dot{F}_{\infty, q}^{s}(\mathcal{X})$ of singular integral operators of order $\epsilon$, which satisfy (I-1) through (I-4) in Subsection 5.2.

Theorem 6.12. Let $\epsilon$ and $q$ be as in Definition 6.7. If $T$ is a singular integral operator of order $\epsilon$, then $T$ is bounded from $\dot{\mathcal{G}}_{b}(\beta, \gamma)$ (with $0<\beta \leq \epsilon$ and $\gamma>0$ ) to $\dot{F}_{\infty, q}^{s}(\mathcal{X})$. Moreover, there exists a constant $C>0$ such that for all $f \in \dot{\mathcal{G}}_{b}(\beta, \gamma)$ with $0<\beta \leq \epsilon$ and $\gamma>0$,

$$
\|T f\|_{\hat{F}_{\infty, q}^{s}(\mathcal{X})} \leq C\|f\|_{\hat{F}_{\infty, q}^{s}(\mathcal{X})}
$$

Proof. Combining some estimates and technics used in the proof of Proposition 5.25 with those used in the proof of Proposition 6.3 gives the desired conclusions. The details are left to the reader.

Remark 6.13. By Theorem 8.15 below, if $p(s, \epsilon)<q \leq \infty$, then $\dot{F}_{\infty, q}^{s}(\mathcal{X})$ is the dual space of $\dot{F}_{1, q^{\prime}}^{-s}(\mathcal{X})$. In this case, Theorem 6.12 can be deduced from Theorem 5.23 together with a duality argument. This provides another proof of Theorem 6.12 .

\subsection{Inhomogeneous Plancherel-Pôlya inequality and definition of $F_{\infty, q}^{s}(X)$}

In this and the next subsection, $\mu(\mathcal{X})$ can be finite or infinite. We first introduce the norm in $\|\cdot\|_{F_{\infty, q}^{s}(\mathcal{X})}$ via some IATI and then verify that this norm is independent of the choices of IATIs and the distribution spaces; see also [84]. Similarly to the case of the space $\dot{F}_{\infty, q}^{s}(\mathcal{X})$, we need also first to establish an inhomogeneous Plancherel-Pôlya inequality related to the norm $\|\cdot\|_{F_{\infty, q}^{s}(x)}$.

Definition 6.14. Let $\epsilon_{1} \in(0,1], \epsilon_{2}>0, \epsilon_{3}>0, \epsilon \in\left(0, \epsilon_{1} \wedge \epsilon_{2}\right)$ and let $\left\{S_{k}\right\}_{k \in \mathbb{Z}_{+}}$be an $\left(\epsilon_{1}, \epsilon_{2}, \epsilon_{3}\right)$ IATI. Set $D_{k}=S_{k}-S_{k-1}$ for $k \in \mathbb{N}$, and $D_{0}=S_{0}$. Let $\left\{Q_{\tau}^{0, v}: \tau \in I_{0}, v=1, \ldots, N(0, \tau)\right\}$ with a fixed large $j \in \mathbb{N}$ be dyadic cubes as in Section 4. Let $|s|<\epsilon$ and $p(s, \epsilon)<q \leq \infty$. For any $f \in\left(\mathcal{G}_{0}^{\epsilon}(\beta, \gamma)\right)^{\prime}$ with $0<\beta, \gamma<\epsilon$, define

$$
\begin{aligned}
& \|f\|_{F_{\infty, q}^{s}(x)} \\
& \quad=\max \left\{\sup _{\substack{\tau \in I_{0} \\
\nu=1, \ldots, N(0, \tau)}} m_{Q_{\tau}^{0, v}}\left(\left|D_{0}(f)\right|\right), \sup _{l \in \mathbb{N}} \sup _{\alpha \in I_{l}}\left[\frac{1}{\mu\left(Q_{\alpha}^{l}\right)} \int_{Q_{\alpha}^{l}} \sum_{k=l}^{\infty} 2^{k s q}\left|D_{k}(f)(x)\right|^{q} d \mu(x)\right]^{1 / q}\right\},
\end{aligned}
$$

where the supremum is taken over all dyadic cubes as in Lemma 2.19 and Section 4, and the usual modification is made when $q=\infty$. 
To verify that the definition of $\|\cdot\|_{F_{\infty, q}^{s}(x)}$ is independent of the choice of IATIs, we need the following inequality of Plancherel-Pôlya type.

Proposition 6.15. Let $\epsilon_{1} \in(0,1], \epsilon_{2}>0, \epsilon_{3}>0, \epsilon \in\left(0, \epsilon_{1} \wedge \epsilon_{2}\right)$ and let $\left\{S_{k}\right\}_{k \in \mathbb{Z}}$ and $\left\{P_{k}\right\}_{k \in \mathbb{Z}}$ be two $\left(\epsilon_{1}, \epsilon_{2}, \epsilon_{3}\right)$-ATIs. Set $D_{k}=S_{k}-S_{k-1}$ and $Q_{k}=P_{k}-P_{k-1}$ for $k \in \mathbb{N}, D_{0}=S_{0}$, and $Q_{0}=S_{0}$. Let $\left\{Q_{\tau}^{0, v}: \tau \in I_{0}, v=1, \ldots, N(0, \tau)\right\}$ with a fixed large $j \in \mathbb{N}$ be dyadic cubes as in Section 4. Let $|s|<\epsilon$ and $p(s, \epsilon)<q \leq \infty$. Then for all $f \in\left(\mathcal{G}_{0}^{\epsilon}(\beta, \gamma)\right)^{\prime}$ with $0<\beta, \gamma<\epsilon$,

$$
\begin{aligned}
& \max \left\{\sup _{\substack{\tau \in I_{0} \\
v=1, \ldots, N(0, \tau)}} m_{Q_{\tau}^{0, v}}\left(\left|D_{0}(f)\right|\right),\right. \\
& \left.\quad \sup _{l \in \mathbb{N}} \sup _{\alpha \in I_{l}}\left[\frac{1}{\mu\left(Q_{\alpha}^{l}\right)} \sum_{k=l}^{\infty} \sum_{\tau \in I_{k}} \sum_{v=1}^{N(k, \tau)} 2^{k s q} \mu\left(Q_{\tau}^{k, v}\right) X_{\left\{(\tau, v): Q_{\tau}^{k, v} \subset Q_{\alpha}^{l}\right\}}(\tau, v)\left(\sup _{x \in Q_{\tau}^{k_{\nu}, v}}\left|D_{k}(f)(x)\right|\right)^{q}\right]^{1 / q}\right\} \\
& \sim \max \left\{\sup _{\substack{\tau \in I_{0} \\
v=1, \ldots, N(0, \tau)}} m_{Q_{\tau}^{0_{\tau}, v}}\left(\left|Q_{0}(f)\right|\right),\right. \\
& \left.\quad \sup _{l \in \mathbb{N}} \sup _{\alpha \in I_{l}}\left[\frac{1}{\mu\left(Q_{\alpha}^{l}\right)} \sum_{k=l}^{\infty} \sum_{\tau \in I_{k}} \sum_{v=1}^{N(k, \tau)} 2^{k s q} \mu\left(Q_{\tau}^{k, v}\right) X_{\left\{(\tau, v): Q_{\tau}^{k, v} \subset Q_{\alpha}^{l}\right\}}(\tau, v)\left(\inf _{x \in Q_{\tau}^{k_{\tau}, \nu}}\left|Q_{k}(f)(x)\right|\right)^{q}\right]^{1 / q}\right\} .
\end{aligned}
$$

Proof. To prove Proposition 6.15, it suffices to verify that for all $f \in\left(\mathcal{G}_{0}^{\epsilon}(\beta, \gamma)\right)^{\prime}$ with $0<\beta, \gamma<$ $\epsilon$, the left-hand side of (6.75) is controlled by its right side.

Let all the notation be as in Proposition 5.25. Then, as in the proof of Proposition 5.25, by (5.78) and (5.80), we still control $m_{Q_{\tau}^{0, v}}\left(\left|D_{0}(f)\right|\right)$ by $Z_{1}+Z_{2}$. Moreover, by (5.82) and Lemma 2.1(ii), we have

$$
Z_{1} \lesssim \sup _{\substack{\tau^{\prime} \in I_{0} \\ v^{\prime}=1, \ldots, N\left(0, \tau^{\prime}\right)}} m_{Q_{\tau^{\prime}}^{0, v^{\prime}}}\left(\left|Q_{0}(f)\right|\right)
$$

which is the desired estimate.

To estimate $Z_{2}$, we need the following trivial estimate that for $k^{\prime} \in \mathbb{N}$,

$$
\begin{aligned}
& {\left[\sum_{\tau^{\prime} \in I_{k^{\prime}}} \sum_{v^{\prime}=1}^{N\left(k^{\prime}, \tau^{\prime}\right)} 2^{k^{\prime} s q}\left|Q_{k^{\prime}}(f)\left(y_{\tau^{\prime}}^{k^{\prime}, v^{\prime}}\right)\right|^{q}\right]^{1 / q}} \\
& \quad \lesssim \sup _{l \in \mathbb{N}} \sup _{\alpha \in I_{l}}\left[\frac{1}{\mu\left(Q_{\alpha}^{l}\right)} \sum_{k=l}^{\infty} \sum_{\tau \in I_{k}} \sum_{v=1}^{N(k, \tau)} 2^{k s q} \mu\left(Q_{\tau}^{k, v}\right) X_{\left\{(\tau, v): Q_{\tau}^{k, v} \subset Q_{\alpha}^{l}\right\}}(\tau, v)\left(\inf _{x \in Q_{\tau}^{k, v}}\left|Q_{k}(f)(x)\right|\right)^{q}\right]^{1 / q} .
\end{aligned}
$$


By this and (5.82) together with $\mu\left(Q_{\tau^{\prime}}^{k^{\prime}, v^{\prime}}\right) \lesssim V_{1}\left(y_{\tau^{\prime}}^{k^{\prime}, v^{\prime}}\right)$ and (5.5), when $q \leq 1$, we have

$$
\begin{aligned}
Z_{2} & \lesssim \sum_{k^{\prime}=1}^{\infty} 2^{-k^{\prime}(\epsilon+s)}\left\{\sum_{\tau^{\prime} \in I_{k^{\prime}}} \sum_{v^{\prime}=1}^{N\left(k^{\prime}, \tau^{\prime}\right)} 2^{k^{\prime} s q}\left|Q_{k^{\prime}}(f)\left(y_{\tau^{\prime}}^{k^{\prime}, \nu^{\prime}}\right)\right|^{q}\right\}^{1 / q} \\
& \lesssim \text { RHS of (6.75), }
\end{aligned}
$$

where we used the assumption that $|s|<\epsilon$.

Similarly, when $1<q \leq \infty$, by Hölder's inequality and Lemma 2.1(ii),

$$
\begin{aligned}
Z_{2} \lesssim & \sum_{k^{\prime}=1}^{\infty} 2^{-k^{\prime}(\epsilon+s)}\left\{\sum_{\tau^{\prime} \in I_{k^{\prime}}} \sum_{v^{\prime}=1}^{N\left(k^{\prime}, \tau^{\prime}\right)} 2^{k^{\prime} s q}\left|Q_{k^{\prime}}(f)\left(y_{\tau^{\prime}}^{k^{\prime}, v^{\prime}}\right)\right|^{q}\right\}^{1 / q} \\
& \times\left\{\int_{x} \frac{1}{V_{1}\left(y_{\tau}^{0, v}\right)+V_{1}(y)+V\left(y_{\tau}^{0, v}, y\right)} \frac{1}{\left(1+d\left(y_{\tau}^{0, v}, y\right)\right)^{\epsilon}} d \mu(y)\right\}^{1 / q^{\prime}}
\end{aligned}
$$

$\lesssim$ RHS of (6.75).

Thus, $m_{Q_{\tau}^{0, v}}\left(\left|D_{0}(f)\right|\right)$ for $\tau \in I_{0}$ and $v=1, \ldots, N(0, \tau)$ is controlled by the right-hand side of (6.75).

We now verify that the second term of the left-hand side of (6.75) is also controlled by its right-hand side. To this end, for any $k \in \mathbb{N}$ and $z \in Q_{\tau}^{k, v}$, we also control $\left|D_{k}(f)(z)\right|$ by $Y_{1}+Y_{2}$. The estimate for $Y_{2}$ is similar to the estimates for $Y_{1}+Y_{2}$ in the proof of Proposition 6.3 and we omit the details. To estimate $\Upsilon_{1}$, by (5.95) and Lemma 2.1(ii), we have

$$
\begin{aligned}
& \sup _{x \in Q_{\tau}^{k, \nu}} Y_{1} \lesssim 2^{-k \epsilon}\left[\sup _{\substack{\tau^{\prime} \in I_{0} \\
\nu^{\prime}=1, \ldots, N\left(0, \tau^{\prime}\right)}} m_{Q_{\tau^{\prime}}^{0, v^{\prime}}}\left(\left|Q_{0}(f)\right|\right)\right] \\
& \times \sum_{\tau^{\prime} \in I_{0}} \sum_{v^{\prime}=1}^{N\left(0, \tau^{\prime}\right)} \mu\left(Q_{\tau^{\prime}}^{0, v^{\prime}}\right) \frac{1}{V_{1}\left(y_{\tau}^{k, v}\right)+V_{1}\left(y_{\tau^{\prime}}^{0, v^{\prime}}\right)+V\left(y_{\tau}^{k, v}, y_{\tau^{\prime}}^{0, v^{\prime}}\right)} \frac{1}{\left(1+d\left(y_{\tau}^{k, v}, y_{\tau^{\prime}}^{0, v^{\prime}}\right)\right)^{\epsilon}} \\
& \lesssim 2^{-k \epsilon}\left[\sup _{\substack{\tau^{\prime}=1, \ldots, N\left(0, I_{0}\right) \\
\nu^{\prime}\left(0, \tau^{\prime}\right)}} m_{Q_{\tau^{\prime}}^{0, v^{\prime}}}\left(\left|Q_{0}(f)\right|\right)\right] \text {, }
\end{aligned}
$$

which together with Lemma 2.19 and $|s|<\epsilon$ gives that for $l \in \mathbb{N}$ and $\alpha \in I_{l}$,

$$
\begin{aligned}
& {\left[\frac{1}{\mu\left(Q_{\alpha}^{l}\right)} \sum_{k=l}^{\infty} \sum_{\tau \in I_{k}} \sum_{v=1}^{N(k, \tau)} 2^{k s q} \mu\left(Q_{\tau}^{k, v}\right) X_{\left\{(\tau, v): Q_{\tau}^{k, v} \subset Q_{\alpha}^{l}\right\}}(\tau, v) \sup _{x \in Q_{\tau}^{k, v}}\left|Y_{1}\right|^{q}\right]^{1 / q}} \\
& \lesssim \sup _{\substack{\tau^{\prime} \in I_{0} \\
v^{\prime}=1, \ldots, N\left(0, \tau^{\prime}\right)}} m_{Q_{\tau^{\prime}}^{0, v^{\prime}}}\left(\left|Q_{0}(f)\right|\right) .
\end{aligned}
$$

This is the desired estimate for $Y_{1}$ and hence, we complete the proof of Proposition 6.15. 
Remark 6.16. We point out that Remark 5.5 applies in a similar way to Proposition 6.15 .

From Proposition 6.15 and Lemma 2.19, it is easy to deduce the following proposition. We omit the details.

Proposition 6.17. Adopting the notation from Proposition 6.15, one has for all for $f \in\left(\mathcal{G}_{0}^{\epsilon}(\beta, \gamma)\right)^{\prime}$ with $0<\beta, \gamma<\epsilon$,

$$
\begin{aligned}
\max & \left\{\sup _{\substack{\tau \in I_{0} \\
v=1, \ldots, N(0, \tau)}} m_{Q_{\tau}^{0, v}}\left(\left|D_{0}(f)\right|\right), \sup _{l \in \mathbb{N}} \sup _{\alpha \in I_{l}}\left[\frac{1}{\mu\left(Q_{\alpha}^{l}\right)} \int_{Q_{\alpha}^{l}} \sum_{k=l}^{\infty} 2^{k s q}\left|D_{k}(f)(x)\right|^{q} d \mu(x)\right]^{1 / q}\right\} \\
& \sim \max \left\{\sup _{\substack{\tau \in I_{0} \\
\nu=1, \ldots, N(0, \tau)}} m_{Q_{\tau}^{Q_{\nu}, v}}\left(\left|Q_{0}(f)\right|\right), \sup _{l \in \mathbb{N}} \sup _{\alpha \in I_{l}}\left[\frac{1}{\mu\left(Q_{\alpha}^{l}\right)} \int_{Q_{\alpha}^{l}} \sum_{k=l}^{\infty} 2^{k s q}\left|Q_{k}(f)(x)\right|^{q} d \mu(x)\right]^{1 / q}\right\} .
\end{aligned}
$$

Proposition 6.17 shows that the definition of the norm $\|\cdot\|_{F_{\infty, q}^{s}(x)}$ is independent of the choice of IATIs. We now verify that under some restrictions on $\beta$ and $\gamma$, it is also independent of the choice of distribution spaces.

Proposition 6.18. Let all the notation be as in Definition 6.14. Let $|s|<\epsilon$ and $p(s, \epsilon)<q \leq \infty$. If $f \in\left(\mathcal{G}_{0}^{\epsilon}\left(\beta_{1}, \gamma_{1}\right)\right)^{\prime}$ with $\max \{0,-s\}<\beta_{1}<\epsilon$ and $0<\gamma_{1}<\epsilon$, and if $\|f\|_{F_{\infty, q}^{s}(x)}<\infty$, then $f \in\left(\mathcal{G}_{0}^{\epsilon}\left(\beta_{2}, \gamma_{2}\right)\right)^{\prime}$ for every $\max \{0,-s\}<\beta_{2}<\epsilon$ and $0<\gamma_{2}<\epsilon$.

Proof. We use the notation from the proof of Proposition 5.28. Let $\psi \in \mathcal{G}(\epsilon, \epsilon)$, and $f \in$ $\left(\mathcal{G}_{0}^{\epsilon}\left(\beta_{1}, \gamma_{1}\right)\right)^{\prime}$ with $\max \left\{0, d(1-1 / q)_{+}-s-d\right\}<\beta_{1}<\epsilon$ and $0<\gamma_{1}<\epsilon$, and $\|f\|_{F_{\infty, q}^{s}(x)}<\infty$. To verify that $f \in\left(\mathcal{G}_{0}^{\epsilon}\left(\beta_{2}, \gamma_{2}\right)\right)^{\prime}$ with $\max \{0,-s\}<\beta_{2}<\epsilon$ and $0<\gamma_{2}<\epsilon$, we need the following trivial estimates that for $\tau \in I_{0}$ and $v=1, \ldots, N(0, \tau)$,

$$
m_{Q_{\tau}^{0, v}}\left(\left|D_{0}(f)\right|\right) \lesssim\|f\|_{F_{\infty, q}^{s}(x)}
$$

and that for $k \in \mathbb{N}$,

$$
\left[\sum_{\tau \in I_{k}} \sum_{v=1}^{N(k, \tau)} 2^{k s q}\left|D_{k}(f)\left(y_{\tau}^{k, v}\right)\right|^{q}\right]^{1 / q} \lesssim\|f\|_{F_{\infty, q}^{s}(\mathcal{X})} .
$$

When $q \leq 1$, by Theorem 4.16, (5.80), (5.105), (5.5), (6.25), (6.83), and (6.84), we have

$$
\begin{aligned}
|\langle f, \psi\rangle|= & \mid \sum_{\tau \in I_{0}} \sum_{\nu=1}^{N(0, \tau)} \int_{Q_{\tau}^{0, v}}\left\langle\tilde{D}_{0}(\cdot, y), \psi\right\rangle d \mu(y) D_{\tau, 1}^{0, v}(f) \\
& \quad+\sum_{k=1}^{\infty} \sum_{\tau \in I_{k}} \sum_{\nu=1}^{N(k, \tau)} \mu\left(Q_{\tau}^{k, v}\right)\left\langle\tilde{D}_{k}\left(\cdot, y_{\tau}^{k, v}\right), \psi\right\rangle D_{k}(f)\left(y_{\tau}^{k, \nu}\right) \mid \\
\lesssim & \|\psi\|_{\mathcal{G}\left(\beta_{2}, \gamma_{2}\right)}\|f\|_{F_{\infty, q}^{s}(\mathcal{x}),}
\end{aligned}
$$

where we used the assumption that $\gamma_{2}>0$ and $\beta_{2}>-s$. 
If $1<q \leq \infty$, by (6.83), Hölder's inequality, (6.25), and (6.84), we obtain

$$
\begin{aligned}
|\langle f, \psi\rangle| \lesssim\|\psi\|_{\mathcal{G}\left(\beta_{\left.2, \gamma_{2}\right)}\right)}\left\{f \|_{F_{\infty, q}^{s}(x)}+\sum_{k=1}^{\infty} 2^{-k \beta_{2}}\left[\sum_{\tau \in I_{k}} \sum_{v=1}^{N(k, \tau)} \mu\left(Q_{\tau}^{k, v}\right)\left|D_{k}(f)\left(y_{\tau}^{k, v}\right)\right|^{q}\right.\right. \\
\left.\times \frac{1}{V_{1}\left(x_{1}\right)+V\left(x_{1}, y_{\tau}^{k, v}\right)} \frac{1}{\left(1+d\left(x_{1}, y_{\tau}^{k, v}\right)\right)^{\gamma_{2}}}\right]^{1 / q} \\
\left.\quad \times\left[\int_{x} \frac{1}{V_{1}\left(x_{1}\right)+V\left(x_{1}, y\right)} \frac{1}{\left(1+d\left(x_{1}, y\right)\right)^{\gamma_{2}}} d \mu(y)\right]^{1 / q^{\prime}}\right\} \\
\lesssim\|\psi\|_{\mathcal{G}\left(\beta_{2}, \gamma_{2}\right)}\|f\|_{F_{\infty, q}^{s}(x),}
\end{aligned}
$$

where we used the assumption $\beta_{2}>-s$ and $\gamma_{2}>0$ again. This finishes the proof of Proposition 6.18.

Based on Propositions 6.17 and 6.18, we can now introduce the inhomogeneous Triebel-Lizorkin spaces $F_{\infty, q}^{s}(x)$.

Definition 6.19. Let $\epsilon_{1} \in(0,1], \epsilon_{2}>0, \epsilon_{3}>0, \epsilon \in\left(0, \epsilon_{1} \wedge \epsilon_{2}\right)$ and let $\left\{S_{k}\right\}_{k \in \mathbb{Z}}$ be an $\left(\epsilon_{1}, \epsilon_{2}, \epsilon_{3}\right)$ IATI. Set $D_{k}=S_{k}-S_{k-1}$ for $k \in \mathbb{N}$ and $D_{0}=S_{0}$. Let $\left\{Q_{\tau}^{0, v}: \tau \in I_{0}, v=1, \ldots, N(0, \tau)\right\}$ with a fixed large $j \in \mathbb{N}$ be dyadic cubes as in Section 4. Let $|s|<\epsilon$ and $p(s, \epsilon)<q \leq \infty$. The space $F_{\infty, q}^{s}(\mathcal{X})$ is defined to be the set of all $f \in\left(\mathcal{G}_{0}^{\epsilon}(\beta, \gamma)\right)^{\prime}$, for some $|s|<\beta<\epsilon$ and $0<\gamma<\epsilon$, such that

$$
\begin{aligned}
& \|f\|_{F_{\infty, q}^{s}(\mathcal{x})} \\
& \quad=\max \left\{\sup _{\substack{\tau \in I_{0} \\
\nu=1, \ldots, N(0, \tau)}} m_{Q_{\tau}^{0, v}}\left(\left|D_{0}(f)\right|\right), \sup _{l \in \mathbb{N}} \sup _{\alpha \in I_{l}}\left[\frac{1}{\mu\left(Q_{\alpha}^{l}\right)} \int_{Q_{\alpha}^{l}} \sum_{k=l}^{\infty} 2^{k s q}\left|D_{k}(f)(x)\right|^{q} d \mu(x)\right]^{1 / q}\right\}<\infty,
\end{aligned}
$$

where the supremum is taken over all dyadic cubes as in Lemma 2.19 and Section 4, and the usual modification is made when $q=\infty$.

Propositions 6.17 and 6.18 show that the definition of the space $F_{\infty, q}^{s}(x)$ is independent of the choices of IATIs and the distribution spaces, $(\mathcal{G}(\beta, \gamma))^{\prime}$ with $\beta$ and $\gamma$ with $|s|<\beta<\epsilon$ and $0<\gamma<\epsilon$.

Remark 6.20. To guarantee that the definition of the space $F_{\infty, q}^{s}(x)$ is independent of the choice of the distribution space $(\mathcal{G}(\beta, \gamma))^{\prime}$, we only need to restrict $\max \{0,-s\}<\beta<\epsilon$ and $0<\gamma<\epsilon$. However, if $\max \{0, s\}<\beta<\epsilon$ and $0<\gamma<\epsilon$, we can then verify that $\mathcal{G}(\beta, \gamma) \subset F_{\infty, q}^{s}(\mathcal{X})$; see Proposition 6.21 below. 


\subsection{Properties of $F_{\infty, q}^{s}(X)$ and boundedness of singular integrals}

In this subsection, we first present some basic properties of $F_{\infty, q}^{s}(\mathcal{X})$ and the relation between $F_{\infty, q}^{s}(\mathcal{X})$ and $\dot{F}_{\infty, q}^{s}(\mathcal{X})$. By establishing a maximal function characterization of $F_{\infty, q}^{s}(\mathcal{X})$, we then derive some relations between the spaces $F_{\infty, q}^{s}(\mathcal{X})$ and $C^{s}(\mathcal{X})$ and between the spaces $F_{\infty, q}^{s}(\mathcal{X})$ and $\operatorname{bmo}(\mathcal{X})$. Finally, we prove boundedness results on the spaces $F_{\infty, q}^{s}(\mathcal{X})$ for the classes of singular integral operators considered in Subsection 5.4.

Proposition 6.21. Let $\epsilon_{1} \in(0,1], \epsilon_{2}>0, \epsilon \in\left(0, \epsilon_{1} \wedge \epsilon_{2}\right),|s|<\epsilon$, and $p(s, \epsilon)<q \leq \infty$. Then, the following hold.

(i) $F_{\infty, q_{0}}^{s}(\mathcal{X}) \subset F_{\infty, q_{1}}^{s}(\mathcal{X})$ if $p(s, \epsilon)<q_{0} \leq q_{1} \leq \infty$.

(ii) Let $-\epsilon<s+\theta<\epsilon$ and $\theta>0$. Then for $p(s, \epsilon)<q_{0}, q_{1} \leq \infty$,

$$
F_{\infty, q_{0}}^{s+\theta}(x) \subset F_{\infty, q_{1}}^{s}(\mathcal{X}) .
$$

(iii) $B_{\infty, q}^{s}(\mathcal{X}) \subset F_{\infty, q}^{s}(x) \subset B_{\infty, \infty}^{s}(\mathcal{X})$.

(iv) If $\max \{0,-s\}<\beta<\epsilon$ and $0<\gamma<\epsilon$, then $F_{\infty, q}^{s}(\mathcal{X}) \subset\left(\mathcal{G}_{0}^{\epsilon}(\beta, \gamma)\right)^{\prime}$.

(v) If $\max \{s, 0\}<\beta<\epsilon$ and $0<\gamma<\epsilon$, then $\mathcal{G}(\beta, \gamma) \subset F_{\infty, q}^{s}(\mathcal{X})$.

(vi) The spaces $F_{\infty, q}^{s}(\mathcal{X})$ are complete.

Proof. Property (ii) can be established by an argument similar to that used for property (ii) of Proposition 5.31, while property (i) and property (iii) through property (vi) can be proved by an argument similar to those used for Proposition 6.9 via Proposition 5.31, which completes the proof of Proposition 6.21.

The following proposition gives a new characterization of the spaces $F_{\infty, q}^{s}(\mathcal{X})$ when $1 \leq q \leq \infty$.

Proposition 6.22. Let $\epsilon$ and $\left\{D_{k}\right\}_{k \in \mathbb{Z}_{+}}$be as in Definition 6.19. If $1 \leq q \leq \infty$, then $f \in F_{\infty, q}^{s}(\mathcal{X})$ if and only if $f \in\left(\mathcal{G}_{0}^{\epsilon}(\beta, \gamma)\right)^{\prime}$, for some $|s|<\beta<\epsilon$ and $0<\gamma<\epsilon$, and

$$
\sup _{l \in \mathbb{Z}_{+}} \sup _{\alpha \in I_{l}}\left[\frac{1}{\mu\left(Q_{\alpha}^{l}\right)} \int_{Q_{\alpha}^{l}} \sum_{k=l}^{\infty} 2^{k s q}\left|D_{k}(f)(x)\right|^{q} d \mu(x)\right]^{1 / q}<\infty .
$$

Moreover, in this case,

$$
\|f\|_{F_{\infty, q}^{s}(x)} \sim \sup _{l \in \mathbb{Z}_{+}} \sup _{\alpha \in I_{l}}\left[\frac{1}{\mu\left(Q_{\alpha}^{l}\right)} \int_{Q_{\alpha}^{l}} \sum_{k=l}^{\infty} 2^{k s q}\left|D_{k}(f)(x)\right|^{q} d \mu(x)\right]^{1 / q} .
$$

Proof. Fix $\tau \in I_{0}$ and $v=1, \ldots, N(0, \tau)$. Since $Q_{\tau}^{0, v} \subset Q_{\tau}^{0}$, then it is easy to verify that $Q_{\tau}^{0} \subset$ $B\left(z_{\tau}^{0, v}, 2 C_{6}\right)$, and hence $\mu\left(Q_{\tau}^{0, v}\right) \lesssim \mu\left(Q_{\tau}^{0}\right) \lesssim \mu\left(B\left(z_{\tau}^{0, v}, 2 C_{6}\right)\right) \lesssim \mu\left(Q_{\tau}^{0, v}\right)$. From this and $1 \leq q \leq \infty$ 
together with Hölder's inequality, it follows that

$$
\begin{aligned}
m_{Q_{\tau}^{0, v}}\left(\left|D_{0}(f)\right|\right) & \leq\left[\frac{1}{\mu\left(Q_{\tau}^{0, v}\right)} \int_{Q_{\tau}^{0, v}}\left|D_{0}(f)(x)\right|^{q} d \mu(x)\right]^{1 / q} \\
& \lesssim\left[\frac{1}{\mu\left(Q_{\tau}^{0}\right)} \int_{Q_{\tau}^{0}}\left|D_{0}(f)(x)\right|^{q} d \mu(x)\right]^{1 / q} .
\end{aligned}
$$

Thus,

$$
\|f\|_{F_{\infty, q}^{s}(x)} \lesssim \sup _{l \in \mathbb{Z}_{+}} \sup _{\alpha \in I_{l}}\left[\frac{1}{\mu\left(Q_{\alpha}^{l}\right)} \int_{Q_{\alpha}^{l}} \sum_{k=l}^{\infty} 2^{k s q}\left|D_{k}(f)(x)\right|^{q} d \mu(x)\right]^{1 / q} .
$$

To see the converse, it suffices to verify that for all $f \in F_{\infty, q}^{s}(\mathcal{X})$ and $\tau \in I_{0}$,

$$
\left[\frac{1}{\mu\left(Q_{\tau}^{0}\right)} \int_{Q_{\tau}^{0}}\left|D_{0}(f)(x)\right|^{q} d \mu(x)\right]^{1 / q} \lesssim\|f\|_{F_{\infty, q}^{s}(x)} .
$$

To see this, by the construction of $\left\{Q_{\tau}^{0, v}: \tau \in I_{0}, v=1, \ldots, N(0, \tau)\right\}$, we have

$$
\begin{aligned}
{\left[\frac{1}{\mu\left(Q_{\tau}^{0}\right)} \int_{Q_{\tau}^{0}}\left|D_{0}(f)(x)\right|^{q} d \mu(x)\right]^{1 / q} } & \lesssim\left\{\frac{1}{\mu\left(Q_{\tau}^{0}\right)} \sum_{v=1}^{N(0, \tau)} \mu\left(Q_{\tau}^{0, v}\right)\left[\sup _{x \in Q_{\tau}^{0, v}}\left|D_{0}(f)(x)\right|\right]^{q}\right\}^{1 / q} \\
& \lesssim \sup _{v=1, \ldots, N(0, \tau)} \sup _{x \in Q_{\tau}^{0, v}}\left|D_{0}(f)(x)\right| .
\end{aligned}
$$

Using Theorem 4.16 together with some estimates similar to those for $Z_{1}+Z_{2}$ in the proof of Proposition 6.15, we can then verify that

$$
\sup _{\nu=1, \ldots, N(0, \tau)} \sup _{x \in Q_{\tau}^{0, v}}\left|D_{0}(f)(x)\right| \lesssim\|f\|_{F_{\infty, q}^{s}(x)}
$$

which gives the desired estimate and hence, we complete the proof of Proposition 6.22. follows.

Using Proposition 6.22, we now complement Proposition 5.39(ii) for the case $p=\infty$ as

Proposition 6.23. Let $\epsilon>0$ be as in Definition 6.19, $0<s<\epsilon, 1 \leq q \leq \infty$ and $\mu(\mathcal{X})=\infty$. Then $F_{\infty, q}^{s}(\mathcal{X})=\dot{F}_{\infty, q}^{s}(\mathcal{X}) \cap L^{\infty}(\mathcal{X})$, and moreover, for any $f \in F_{\infty, q}^{s}(\mathcal{X})$,

$$
\|f\|_{F_{\infty, q}^{s}(\mathcal{Q})} \sim\|f\|_{\dot{F}_{\infty, q}^{s}(\mathcal{})}+\|f\|_{L^{\infty}(\mathcal{X})}
$$


Proof. Take $f \in F_{\infty, q}^{s}(\mathcal{X})$. By Proposition 6.18, without loss of generality, we may assume that $f \in(\mathcal{G}(\beta, \gamma))^{\prime}$ with $s<\beta, \gamma<\epsilon$ as in (6.32). Thus, we also have $f \in(\mathcal{G}(\beta, \gamma))^{\prime}$ with $s<\beta, \gamma<\epsilon$ as in (6.32); see the proof of Proposition 5.37. By Theorem 3.29, we have that

$$
f=\widetilde{D}_{0} S_{0}(f)+\sum_{k=1}^{\infty} \widetilde{D}_{k} D_{k}(f)
$$

holds in $\in(\mathcal{G}(\beta, \gamma))^{\prime}$ with $s<\beta<\epsilon$ and $0<\gamma<\epsilon$, where $\widetilde{D}_{k}$ with $k \in \mathbb{Z}_{+}$is as in Theorem 3.26. From this and Lemma 2.19, it follows that for any $x \in \mathcal{X}$,

$$
\begin{aligned}
& |f(x)| \lesssim \sum_{\tau \in I_{0}} \sum_{\nu=1}^{N(0, \tau)} \int_{Q_{\tau}^{0, \nu}}\left|\widetilde{D}_{0}(x, y)\right|\left|S_{0}(f)(y)\right| d \mu(y)+\sum_{k=1}^{\infty} \sum_{\tau \in I_{k}} \sum_{\nu=1}^{N(k, \tau)} \int_{Q_{\tau}^{k, \nu}}\left|\widetilde{D}_{k}(x, y)\right|\left|D_{k}(f)(y)\right| d \mu(y) \\
& \lesssim \sum_{\tau \in I_{0}} \sum_{\nu=1}^{N(0, \tau)} \mu\left(Q_{\tau}^{0, \nu}\right) \frac{1}{V_{1}(x)+V_{1}\left(y_{\tau}^{0, \nu}\right)+V\left(x, y_{\tau}^{0, \nu}\right)}\left(\frac{1}{1+d\left(x, y_{\tau}^{0, v}\right)}\right)^{\epsilon} m_{Q_{\tau}^{0, v}}\left(\left|S_{0}(f)\right|\right) \\
& +\sum_{k=1}^{\infty} \sum_{\tau \in I_{k}} \sum_{\nu=1}^{N(k, \tau)} \mu\left(Q_{\tau}^{k, v}\right) \frac{1}{V_{2^{-k}}(x)+V_{2^{-k}}\left(y_{\tau}^{k, v}\right)+V\left(x, y_{\tau}^{k, v}\right)} \\
& \times\left(\frac{2^{-k}}{2^{-k}+d\left(x, y_{\tau}^{k, v}\right)}\right)^{\epsilon}\left[\sup _{y \in Q_{\tau}^{k, v}}\left|D_{k}(f)(y)\right|\right] .
\end{aligned}
$$

Since $f \in F_{\infty, q}^{s}(\mathcal{X})$, by its definition, we have that for $\tau \in I_{0}$ and $v=1, \ldots, N(0, \tau)$,

$$
\left.m_{Q_{\tau}^{0, v}}|| S_{0}(f) \mid\right) \lesssim\|f\|_{F_{\infty, q}^{s}(x)}
$$

and the definition of $F_{\infty, q}^{s}(x)$ together with Proposition 6.15 also implies that for any $k \in \mathbb{N}$, $\tau \in I_{k}$, and $v=1, \ldots, N(k, \tau)$,

$$
\left\{\sum_{\tau \in I_{k}} \sum_{\nu=1}^{N(k, \tau)} 2^{k s q}\left[\sup _{y \in Q_{\tau}^{k, v}}\left|D_{k}(f)(y)\right|\right]^{q}\right\}^{1 / q} \lesssim\|f\|_{F_{\infty, q}^{s}(x)} .
$$

Notice that $1 \leq q \leq \infty$. Both estimates via Hölder's inequality, the fact that $\mu\left(Q_{\tau}^{k, v}\right) \sim$ $V_{2^{-k}}\left(y_{\tau}^{k, v}\right)$, Lemma 5.2, and the assumption that $s>0$ further yield that

$$
\begin{aligned}
& |f(x)| \lesssim\|f\|_{F_{\infty, q}^{s}(x)}\left\{1+\sum_{k=1}^{\infty} 2^{-k s}\left(\sum_{\tau \in I_{k}} \sum_{\nu=1}^{N(k, \tau)} \mu\left(Q_{\tau}^{k, \nu}\right)\left(\frac{2^{-k}}{2^{-k}+d\left(x, y_{\tau}^{k, v}\right)}\right)^{\epsilon q^{\prime}}\right.\right. \\
& \left.\left.\times \frac{1}{V_{2^{-k}}(x)+V_{2^{-k}}\left(y_{\tau}^{k, v}\right)+V\left(x, y_{\tau}^{k, v}\right)}\right)^{1 / q^{\prime}}\right\}
\end{aligned}
$$

$\lesssim\|f\|_{F_{\infty, q}^{s}(x)}$, 
namely, $f \in L^{\infty}(\mathcal{X})$ and

$$
\|f\|_{L^{\infty}(x)} \lesssim\|f\|_{F_{\infty, q}^{s}(x)}
$$

Moreover,

$$
\begin{aligned}
&\|f\|_{\dot{F}_{\infty, q}^{s}(x) \lesssim} \sup _{l \in \mathbb{N}} \sup _{\alpha \in I_{l}}\left[\frac{1}{\mu\left(Q_{\alpha}^{l}\right)} \int_{Q_{\alpha}^{l}} \sum_{k=l}^{\infty} 2^{k s q}\left|D_{k}(f)(x)\right|^{q} d \mu(x)\right]^{1 / q} \\
&+\sup _{l \in \mathbb{Z} \backslash \mathbb{N}} \sup _{\alpha \in I_{l}}\left[\frac{1}{\mu\left(Q_{\alpha}^{l}\right)} \int_{Q_{\alpha}^{l}} \sum_{k=l}^{\infty} 2^{k s q}\left|D_{k}(f)(x)\right|^{q} d \mu(x)\right]^{1 / q} \\
& \lesssim\|f\|_{F_{\infty, q}^{s}(x)}+\sup _{l \in \mathbb{Z} \backslash \mathbb{N}} \sup _{\alpha \in I_{l}}\left[\frac{1}{\mu\left(Q_{\alpha}^{l}\right)} \int_{Q_{\alpha}^{l}} \sum_{k=l}^{0} 2^{k s q}\left|D_{k}(f)(x)\right|^{q} d \mu(x)\right]^{1 / q} \\
&+\sup _{l \in \mathbb{Z} \backslash \mathbb{N}} \sup _{\alpha \in I_{l}}\left[\frac{1}{\mu\left(Q_{\alpha}^{l}\right)} \int_{Q_{\alpha}^{l}} \sum_{k=1}^{\infty} 2^{k s q}\left|D_{k}(f)(x)\right|^{q} d \mu(x)\right]^{1 / q}
\end{aligned}
$$

To estimate the second term, by Proposition 2.7(i), we have

$$
\left|D_{k}(f)(x)\right| \lesssim\|f\|_{L^{\infty}(x)} \lesssim\|f\|_{F_{\infty, q}^{s}(x)},
$$

which together with $s>0$ shows

$$
\begin{aligned}
\sup _{l \in \mathbb{Z} \backslash \mathbb{N}} \sup _{\alpha \in I_{l}}\left[\frac{1}{\mu\left(Q_{\alpha}^{l}\right)} \int_{Q_{\alpha}^{l}} \sum_{k=l}^{0} 2^{k s q}\left|D_{k}(f)(x)\right|^{q} d \mu(x)\right]^{1 / q} & \lesssim\|f\|_{F_{\infty, q}^{s}(x)} \sup _{l \in \mathbb{Z} \backslash \mathbb{N}}\left[\sum_{k=l}^{0} 2^{k s q}\right]^{1 / q} \\
& \lesssim\|f\|_{F_{\infty, q}^{s}(x) .}
\end{aligned}
$$

To estimate the third term, for any $l \in \mathbb{Z} \backslash \mathbb{N}$ and $\alpha \in I_{l}$, set

$$
I_{l}^{\alpha}=\left\{\beta \in I_{1}: Q_{\beta}^{1} \subset Q_{\alpha}^{l}\right\}
$$

Lemma 2.19 proves that

$$
\sum_{\beta \in I_{l}^{\alpha}} \mu\left(Q_{\beta}^{1}\right)=\mu\left(Q_{\alpha}^{l}\right)
$$


which together with Lemma 2.19 further yields that

$$
\begin{aligned}
\sup _{l \in \mathbb{Z} \backslash \mathbb{N}} & \sup _{\alpha \in I_{l}}\left[\frac{1}{\mu\left(Q_{\alpha}^{l}\right)} \int_{Q_{\alpha}^{l}} \sum_{k=1}^{\infty} 2^{k s q}\left|D_{k}(f)(x)\right|^{q} d \mu(x)\right]^{1 / q} \\
& \lesssim \sup _{l \in \mathbb{Z} \backslash \mathbb{N}} \sup _{\alpha \in I_{l}}\left[\frac{1}{\mu\left(Q_{\alpha}^{l}\right)} \sum_{\beta \in I_{l}^{\alpha}} \mu\left(Q_{\beta}^{1}\right) \frac{1}{\mu\left(Q_{\beta}^{1}\right)} \int_{Q_{\beta}^{1}} \sum_{k=1}^{\infty} 2^{k s q}\left|D_{k}(f)(x)\right|^{q} d \mu(x)\right]^{1 / q} \\
& \lesssim\|f\|_{F_{\infty, q}^{s}(\mathcal{x}) .}
\end{aligned}
$$

Thus, $f \in \dot{F}_{\infty, q}^{s}(\mathcal{X}) \cap L^{\infty}(\mathcal{X})$ and

$$
\|f\|_{L^{\infty}(\mathcal{X})}+\|f\|_{\dot{F}_{\infty, q}^{s}(\mathcal{}} \lesssim\|f\|_{F_{\infty, q}^{s}(x)}
$$

Conversely, let $f \in \dot{F}_{\infty, q}^{s}(\mathcal{X}) \cap L^{\infty}(\mathcal{X})$. Obviously, $f \in(\mathcal{G}(\beta, \gamma))^{\prime}$ with $s<\beta<\epsilon$ and $0<\gamma<\epsilon$. On the other hand, Proposition 2.7(i) shows that for any $x \in \mathcal{X}$,

$$
\left|S_{0}(f)(x)\right| \lesssim\|f\|_{L^{\infty}(x)}
$$

Since $1 \leq q \leq \infty$, by Proposition 6.22, (5.5) together with (6.110), and Lemma 2.19 together with (6.107), we obtain

$$
\begin{aligned}
\|f\|_{F_{\infty, q}^{s}(x)} \sim & \sup _{l \in \mathbb{N}} \sup _{\alpha \in I_{l}}\left[\frac{1}{\mu\left(Q_{\alpha}^{l}\right)} \int_{Q_{\alpha}^{l}} \sum_{k=l}^{\infty} 2^{k s q}\left|D_{k}(f)(x)\right|^{q} d \mu(x)\right]^{1 / q} \\
& +\sup _{\alpha \in I_{0}}\left[\frac{1}{\mu\left(Q_{\alpha}^{0}\right)} \int_{Q_{\alpha}^{0}}\left(\left|S_{0}(f)(x)\right|^{q}+\sum_{k=1}^{\infty} 2^{k s q}\left|D_{k}(f)(x)\right|^{q}\right) d \mu(x)\right]^{1 / q} \\
\lesssim & \|f\|_{\dot{F}_{\infty, q}^{s}(x)}+\sup _{\alpha \in I_{0}}\left\{\frac{1}{\mu\left(Q_{\alpha}^{0}\right)} \sum_{\beta \in I_{0}^{\alpha}} \mu\left(Q_{\beta}^{0}\right)\left[\frac{1}{\mu\left(Q_{\beta}^{0}\right)} \int_{Q_{\beta}^{0} k=1}^{\infty} 2^{k s q}\left|D_{k}(f)(x)\right|^{q} d \mu(x)\right]\right\}^{1 / q} \\
\lesssim & \|f\|_{\dot{F}_{\infty, q}^{s}(x),}
\end{aligned}
$$

which means that $f \in F_{\infty, q}^{s}(\mathcal{X})$. Thus,

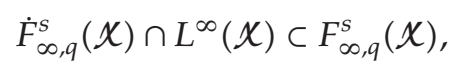

which completes the proof of Proposition 6.23. 
From Proposition 6.23 and Theorem 6.11(i), we easily deduce the following results.

Corollary 6.24. Let $\epsilon>0$ be as in Definition 6.19. Then,

(i) for any $s \in(0,1], C^{s}(\mathcal{X})=\dot{C}^{s}(\mathcal{X}) \cap L^{\infty}(\mathcal{X})$, and moreover, for all $f \in C^{s}(\mathcal{X})$,

$$
\|f\|_{C^{s}(\mathcal{X})}=\|f\|_{L^{\infty}(\mathcal{X})}+\|f\|_{\dot{C}^{s}(\mathcal{X})}
$$

(ii) for any $s \in(0, \epsilon), C^{s}(\mathcal{X})=B_{\infty, \infty}^{s}(\mathcal{X})=F_{\infty, \infty}^{s}(\mathcal{X})$ with equivalent norms.

Proof. Property (i) is a simple consequence of the definitions of both $C^{s}(\mathcal{X})$ and $\dot{C}^{s}(\mathcal{X})$.

When $\mu(\mathcal{X})=\infty$, then Property (ii) can be deduced from Property (i), Theorem 6.11(i), and Proposition 6.23, which completes the proof of Corollary 6.24 in this case.

An alternative way to prove Property (ii), which works when $\mu(\mathcal{X})=\infty$ and also when $\mu(x)<\infty$, follows the line of reasoning in the proof of Theorem 6.11(i). In fact, let $f \in C^{s}(\mathcal{X})$ and $\left\{D_{k}\right\}_{k \in \mathbb{Z}_{+}}$be as in Definition 6.19. Then from $f \in L^{\infty}(\mathcal{X})$, it follows that $f \in(\mathcal{G}(\beta, \gamma))^{\prime}$ with $s<\beta<\epsilon$ and $0<\gamma<\epsilon$. Moreover, by Proposition 2.7(i), we have that for all $x \in \mathcal{X}$,

$$
\left|D_{0}(f)(x)\right|=\left|\int_{\chi} D_{0}(x, y) f(y) d \mu(y)\right| \lesssim\|f\|_{L^{\infty}(x)}
$$

which together with (6.45) shows that $f \in F_{\infty, \infty}^{s}(\mathcal{X})$ and

$$
\|f\|_{F_{\infty, \infty}^{s}(x)} \lesssim\|f\|_{L^{\infty}(x)}
$$

Conversely, suppose $f \in F_{\infty, \infty}^{s}(\mathcal{X})$. By Proposition 6.18, we can assume that $f \in$ $(\mathcal{G}(\beta, \gamma))^{\prime}$ with $s<\beta<\epsilon$ and $0<\gamma<\epsilon$. Using the same notation as in Theorem 3.26, by Theorem 3.29 and the definition of $\|\cdot\|_{F_{\infty, \infty}^{s}(x)}$ together with $s>0$, we further obtain that for all $x \in x$,

$$
|f(x)|=\left|\sum_{k=0}^{\infty} \widetilde{D}_{k} D_{k}(f)(x)\right| \lesssim\|f\|_{F_{\infty, \infty}^{s}(x)} \sum_{k=0}^{\infty} 2^{-k s} \lesssim\|f\|_{F_{\infty, \infty}^{s}(x)} .
$$

Thus, $f \in L^{\infty}(\mathcal{X})$ and

$$
\|f\|_{L^{\infty}(x)} \lesssim\|f\|_{F_{\infty, \infty}^{s}(x)}
$$

Moreover, if $d(x, y) \geq 1 / 2$, then

$$
|f(x)-f(y)| \lesssim\|f\|_{L^{\infty}(x)} \lesssim\|f\|_{F_{\infty, \infty}^{s}(x)} d(x, y)^{s} .
$$


Assume that $2^{-l_{0}-1} \leq d(x, y)<2^{-l_{0}}$ with $l_{0} \in \mathbb{N}$. By the regularity of $\widetilde{D}_{k}$, Lemma 2.1(ii) and Proposition 2.7(i) together with $0<s<\epsilon<\epsilon_{1}$, we then obtain

$$
\begin{aligned}
|f(x)-f(y)| \lesssim & \left|\sum_{k=0}^{l_{0}-1} \int_{x}\left[\widetilde{D}_{k}(x, z)-\tilde{D}_{k}(y, z)\right] D_{k}(f)(z) d \mu(z)\right| \\
& +\sum_{k=l_{0}}^{\infty}\left\{\int_{x}\left|\widetilde{D}_{k}(x, z)\right|\left|D_{k}(f)(z)\right| d \mu(z)+\int_{x}\left|\widetilde{D}_{k}(y, z)\right|\left|D_{k}(f)(z)\right| d \mu(z)\right\} \\
\lesssim & \sum_{k=0}^{l_{0}-1} \int_{x}\left(\frac{d(x, y)}{2^{-k}+d(x, z)}\right)^{\epsilon_{1}} \frac{1}{V_{2^{-k}}(x)+V_{2^{-k}}(y)+V(x, y)} \\
& \times\left(\frac{2^{-k}}{2^{-k}+d(x, z)}\right)^{\epsilon_{2}}\left|D_{k}(f)(z)\right| d \mu(z)+\|f\|_{F_{\infty, \infty}^{s}(x)} \sum_{k=l_{0}}^{\infty} 2^{-k s} \\
\lesssim & \|f\|_{F_{\infty, \infty}^{s}(x)} d(x, y)^{s} .
\end{aligned}
$$

Thus, $f \in C^{s}(\mathcal{X})$ and

$$
\|f\|_{C^{s}(\chi)} \lesssim\|f\|_{F_{\infty, \infty}^{s}(x)},
$$

which completes the proof of Corollary 6.24(ii). and $q$.

We now establish the connection between $\dot{F}_{\infty, q}^{s}(\mathcal{X})$ and $F_{\infty, q}^{s}(\mathcal{X})$ for all admissible $s$

Proposition 6.25. Let $\epsilon, s, q$, and $S_{0}$ be as in Definition 6.19 and let $\mu(\mathcal{X})=\infty$. Then there exists a constant $C>0$ such that for all $f \in \dot{F}_{\infty, q}^{s}(\mathcal{X}), f-S_{0}(f) \in F_{\infty, q}^{s}(\mathcal{X})$ and

$$
\left\|f-S_{0}(f)\right\|_{F_{\infty, q}^{s}(x)} \leq C\|f\|_{\dot{F}_{\infty, q}^{s}(x)} .
$$

Proof. Let $f \in \dot{F}_{\infty, q}^{s}(\mathcal{X})$. By Proposition 6.6, we may assume that $f \in\left(\dot{\mathcal{G}}_{0}^{\epsilon}(\beta, \gamma)\right)^{\prime}$ with $\beta, \gamma$ as in (6.32). On the other hand, for any $g \in \mathcal{G}_{0}^{\epsilon}(\beta, \gamma)$ with $\beta, \gamma$ as in (6.32), from Lemma 5.36 and $\int_{x} S_{0}(x, y) d \mu(x)=1$, it is easy to deduce that $g-S_{0}^{t}(g) \in \mathcal{G}(\beta, \gamma)$ with $\beta, \gamma$ as in (6.32). Thus, from

$$
\left\langle f-S_{0}(f), g\right\rangle=\left\langle f, g-S_{0}^{t}(g)\right\rangle,
$$

we deduce that $f-S_{0}(f) \in\left(\mathcal{G}_{0}^{e}(\beta, \gamma)\right)^{\prime}$ with $\beta, \gamma$ as in (6.32). 
We now verify (6.121). In what follows, let $I$ be the identity operator, let $\left\{D_{k^{\prime}}\right\}_{k^{\prime} \in \mathbb{Z}}$ be as in Definition 6.7, and let $\left\{\widetilde{D}_{k^{\prime}}\right\}_{k^{\prime} \in \mathbb{Z}}$ be as in Theorem 4.11. We first claim that for all $k^{\prime} \in \mathbb{Z}$ and $x, y \in \mathcal{X}$,

$$
\begin{aligned}
& \left|\left(S_{0}\left(I-S_{0}\right) \tilde{D}_{k^{\prime}}\right)(x, y)\right| \\
& \quad \lesssim 2^{-\left|k^{\prime}\right| \epsilon} \frac{1}{V_{2^{-\left(0 \wedge k^{\prime}\right)}(x)+V_{2^{-}\left(0 \wedge k^{\prime}\right)}(y)+V(x, y)}}\left(\frac{2^{-\left(0 \wedge k^{\prime}\right)}}{2^{-\left(0 \wedge k^{\prime}\right)}+d(x, y)}\right)^{\epsilon}
\end{aligned}
$$

and that for all $k \in \mathbb{N}, k^{\prime} \in \mathbb{Z}$, and $x, y \in \mathbb{Z}$,

$$
\begin{aligned}
& \left|\left(D_{k}\left(I-S_{0}\right) \tilde{D}_{k^{\prime}}\right)(x, y)\right| \\
& \quad \lesssim 2^{-\left|k-k^{\prime}\right| \epsilon} \frac{1}{V_{2^{-\left(k \wedge k^{\prime}\right)}}(x)+V_{2^{-\left(k \wedge k^{\prime}\right)}}(y)+V(x, y)}\left(\frac{2^{-\left(k \wedge k^{\prime}\right)}}{2^{-\left(k \wedge k^{\prime}\right)}+d(x, y)}\right)^{\epsilon} .
\end{aligned}
$$

The estimate (6.124) is essentially the same as the estimate (5.130) by symmetry, while the estimate (6.123) can essentially be obtained by an argument similar to Cases 1 and 2 of the proof of the estimate (5.130).

Using the estimate (6.123) and Theorem 4.13, we have that for any $x \in \mathcal{X}$,

$$
\begin{aligned}
& \left|S_{0}\left(I-S_{0}\right)(f)(x)\right| \\
& =\left|\sum_{k^{\prime} \in \mathbb{Z}} \sum_{\tau^{\prime} \in I_{k^{\prime}}} \sum_{v^{\prime}=1}^{N\left(k^{\prime}, \tau^{\prime}\right)} \mu\left(Q_{\tau^{\prime}}^{k^{\prime}, v^{\prime}}\right)\left(S_{0}\left(I-S_{0}\right) \widetilde{D}_{k^{\prime}}\right)\left(x, y_{\tau^{\prime}}^{k^{\prime}, v^{\prime}}\right) D_{k^{\prime}}(f)\left(y_{\tau^{\prime}}^{k^{\prime}, v^{\prime}}\right)\right| \\
& \lesssim \sum_{k^{\prime} \in \mathbb{Z}} \sum_{\tau^{\prime} \in I_{k^{\prime}}} \sum_{v^{\prime}=1}^{N\left(k^{\prime}, \tau^{\prime}\right)} 2^{-\left|k^{\prime}\right| \epsilon} \mu\left(Q_{\tau^{\prime}}^{k^{\prime}, v^{\prime}}\right) \frac{1}{V_{2^{-\left(0 \wedge k^{\prime}\right)}}(x)+V_{2^{-\left(0 \wedge k^{\prime}\right)}}\left(y_{\tau^{\prime}}^{k^{\prime}, v^{\prime}}\right)+V\left(x, y_{\tau^{\prime}}^{k^{\prime}, v^{\prime}}\right)} \\
& \times\left(\frac{2^{-\left(0 \wedge k^{\prime}\right)}}{2^{-\left(0 \wedge k^{\prime}\right)}+d\left(x, y_{\tau^{\prime}}^{k^{\prime}, v^{\prime}}\right)}\right)^{e}\left|D_{k^{\prime}}(f)\left(y_{\tau^{\prime}}^{k^{\prime}, v^{\prime}}\right)\right| .
\end{aligned}
$$

From Proposition 6.3, we deduce the following trivial estimate that for all $k^{\prime} \in \mathbb{Z}$,

$$
\left\{\sum_{\tau^{\prime} \in I_{k^{\prime}}} \sum_{\nu^{\prime}=1}^{N\left(k^{\prime}, \tau^{\prime}\right)} 2^{k^{\prime} s q}\left|D_{k^{\prime}}(f)\left(y_{\tau^{\prime}}^{k^{\prime}, v^{\prime}}\right)\right|^{q}\right\}^{1 / q} \lesssim\|f\|_{\dot{F}_{\infty, q}^{s}}(x),
$$

and from Lemma 2.19, it follows that

$$
\frac{\mu\left(Q_{\tau^{\prime}}^{k^{\prime}, v^{\prime}}\right)}{V_{2^{-\left(0 \wedge k^{\prime}\right)}}(x)+V_{2^{-\left(0 \wedge k^{\prime}\right)}}\left(y_{\tau^{\prime}}^{k^{\prime}, v^{\prime}}\right)+V\left(x, y_{\tau^{\prime}}^{k^{\prime}, v^{\prime}}\right)} \lesssim 1 .
$$


Both estimates together with (5.5) show that when $q \leq 1$, for any $x \in \mathcal{X}$,

$$
\begin{aligned}
\left|S_{0}\left(I-S_{0}\right)(f)(x)\right| & \lesssim \sum_{k^{\prime} \in \mathbb{Z}} 2^{-\left|k^{\prime}\right| \epsilon} 2^{-k^{\prime} s}\left\{\sum_{\tau^{\prime} \in I_{k^{\prime}}} \sum_{v^{\prime}=1}^{N\left(k^{\prime}, \tau^{\prime}\right)} 2^{k^{\prime} s q}\left|D_{k^{\prime}}(f)\left(y_{\tau^{\prime}}^{k^{\prime}, v^{\prime}}\right)\right|^{q}\right\}^{1 / q} \\
& \lesssim\|f\|_{\dot{F}_{\infty, q}^{s}(x),}
\end{aligned}
$$

while when $1<q \leq \infty$, both estimates together with Hölder's inequality, Lemma 5.2, and Lemma 2.1(ii) still yield that for all $x \in \mathcal{X}$,

$$
\begin{aligned}
& \left|S_{0}\left(I-S_{0}\right)(f)(x)\right| \\
& \lesssim \sum_{k^{\prime} \in \mathbb{Z}} 2^{-\left|k^{\prime}\right| \epsilon} 2^{-k^{\prime} s}\left[\sum_{\tau^{\prime} \in I_{k^{\prime}}} \sum_{v^{\prime}=1}^{N\left(k^{\prime}, \tau^{\prime}\right)} \mu\left(Q_{\tau^{\prime}}^{k^{\prime}, v^{\prime}}\right) \frac{1}{V_{2^{-\left(0 \wedge k^{\prime}\right)}}(x)+V_{2^{-\left(0 \wedge k^{\prime}\right)}}\left(y_{\tau^{\prime}}^{k^{\prime}, v^{\prime}}\right)+V\left(x, y_{\tau^{\prime}}^{k^{\prime}, v^{\prime}}\right)}\right. \\
& \left.\times\left(\frac{2^{-\left(0 \wedge k^{\prime}\right)}}{2^{-\left(0 \wedge k^{\prime}\right)}+d\left(x, y_{\tau^{\prime}}^{k^{\prime}, v^{\prime}}\right)}\right)^{\epsilon}\right]^{1 / q^{\prime}}\left[\sum_{\tau^{\prime} \in I_{k^{\prime}}} \sum_{v^{\prime}=1}^{N\left(k^{\prime}, \tau^{\prime}\right)} 2^{k^{\prime} s q}\left|D_{k^{\prime}}(f)\left(y_{\tau^{\prime}}^{k^{\prime}, v^{\prime}}\right)\right|^{q}\right]^{1 / q} \\
& \lesssim\|f\|_{\dot{F}_{\infty, q}^{s}(x)} .
\end{aligned}
$$

Thus, for $\tau \in I_{0}$ and $v=1, \ldots, N(0, \tau)$,

$$
m_{Q_{\tau}^{0, v}}\left(\left|S_{0}\left(I-S_{0}\right)(f)\right|\right)=\frac{1}{\mu\left(Q_{\tau}^{0, v}\right)} \int_{Q_{\tau}^{0, v}}\left|S_{0}\left(I-S_{0}\right)(f)(x)\right| d \mu(x) \lesssim\|f\|_{\dot{F}_{\infty, q}^{s}(x)},
$$

which is the desired estimate.

For $l \in \mathbb{N}$ and $\alpha \in I_{l}$, from Theorem 5.16 together with (6.124), it follows that

$$
\begin{aligned}
& \frac{1}{\mu\left(Q_{\alpha}^{l}\right)} \int_{Q_{\alpha}^{l}} \sum_{k=l}^{\infty} 2^{k s q}\left|D_{k}\left(I-S_{0}\right)(f)(x)\right|^{q} d \mu(x) \\
& \lesssim \frac{1}{\mu\left(Q_{\alpha}^{l}\right)} \int_{Q_{\alpha}^{l}} \sum_{k=l}^{\infty} 2^{k s q}\left[\sum_{k^{\prime} \in \mathbb{Z}} \sum_{\tau^{\prime} \in I_{k^{\prime}}} \sum_{v^{\prime}=1}^{N\left(k^{\prime}, \tau^{\prime}\right)} 2^{-\left|k-k^{\prime}\right| e} \mu\left(Q_{\tau^{\prime}}^{k^{\prime}, v^{\prime}}\right) \frac{1}{V_{2^{-\left(k \wedge k^{\prime}\right)}}(x)+V_{2^{-\left(k \wedge k^{\prime}\right)}}\left(y_{\tau^{\prime}}^{k^{\prime}, v^{\prime}}\right)+V\left(x, y_{\tau^{\prime}}^{k^{\prime}, v^{\prime}}\right)}\right. \\
& \left.\times\left(\frac{2^{-\left(k \wedge k^{\prime}\right)}}{2^{-\left(k \wedge k^{\prime}\right)}+d\left(x, y_{\tau^{\prime}}^{k^{\prime}, v^{\prime}}\right)}\right)^{\epsilon}\left|D_{k^{\prime}}(f)\left(y_{\tau^{\prime}}^{k^{\prime}, v^{\prime}}\right)\right|\right]^{q} d \mu(x) .
\end{aligned}
$$

Then, an argument similar to that used to estimate $Y_{1}+Y_{2}$ in the proof of Proposition 6.3 together with Proposition 6.3 yields that

$$
\frac{1}{\mu\left(Q_{\alpha}^{l}\right)} \int_{Q_{\alpha}^{l}} \sum_{k=l}^{\infty} 2^{k s q}\left|D_{k}\left(I-S_{0}\right)(f)(x)\right|^{q} d \mu(x) \lesssim\|f\|_{\dot{F}_{\infty, q}^{s}(x)}^{q}
$$


Thus, $\left(I-S_{0}\right)(f) \in F_{\infty, q}^{s}(\mathcal{X})$ and

$$
\left\|\left(I-S_{0}\right)(f)\right\|_{F_{\infty, q}^{s}(\mathcal{X})} \lesssim\|f\|_{\dot{F}_{\infty, q}^{s}(x)}
$$

which completes the proof of Proposition 6.25.

Remark 6.26. We point out that Remark 5.38 applies in a similar way to Proposition 6.25.

To establish the equivalence between $\operatorname{bmo}(\mathcal{X})$ and $F_{\infty, 2}^{0}(\mathcal{X})$, we need the following technical result which in fact gives a new characterization of Triebel-Lizorkin space $F_{\infty, q}^{s}(x)$ when $1 \leq q \leq \infty$. We first introduce an inhomogeneous maximal function.

For any $x \in \mathcal{X}$ and $l \in \mathbb{Z}_{+}$, we choose $m \in \mathbb{N}$ by a way similar to that in the definition of $\dot{\mathfrak{C}}_{q}^{s}$. Let $\left\{D_{k}\right\}_{k \in \mathbb{Z}_{+}}$be as in Definition 6.19. Then for any $s \in \mathbb{R}, q \in(0, \infty], f \in(\mathcal{G}(\beta, \gamma))^{\prime}$ with $0<\beta, \gamma<\epsilon$ and $x \in \mathcal{X}$, we define the inhomogeneous maximal function $\mathfrak{C}_{q}^{s}(f)(x)$ by

$$
\mathfrak{C}_{q}^{s}(f)(x)=\sup _{l \in \mathbb{Z}_{+}}\left[\frac{1}{\mu\left(\cup_{i=1}^{m} Q_{\alpha_{l}^{i}}^{l}\right)} \int_{\cup_{i=1}^{m} Q_{\alpha_{l}^{i}}^{l}} \sum_{k=l}^{\infty} 2^{k s q}\left|D_{k}(f)(y)\right|^{q} d \mu(y)\right]^{1 / q},
$$

where the usual modification is made when $q=\infty$.

Using Proposition 6.22 and an argument similar to the proof of Proposition 6.10 yields the following characterization of $F_{\infty, q}^{s}(x)$ with $1 \leq q \leq \infty$; we omit the details.

Proposition 6.27. Let $\epsilon$ and $s$ be as in Definition 6.7 and let $1 \leq q \leq \infty$. Then $f \in F_{\infty, q}^{s}(x)$ if and only if $f \in(\mathcal{G}(\beta, \gamma))^{\prime}$ with $|s|<\beta<\epsilon$ and $0<\gamma<\epsilon$, and $\mathfrak{C}_{q}^{s}(f) \in L^{\infty}(\mathcal{X})$. Moreover, in this case,

$$
\|f\|_{F_{\infty, q}^{s}(x)} \sim\left\|\mathfrak{C}_{q}^{s}(f)\right\|_{L^{\infty}(x)}
$$

Now, from Theorem 5.44(i), Proposition 6.22, and Proposition 6.27, we can deduce the following relation between $\mathrm{bmo}(\mathcal{X})$ and $F_{\infty, 2}^{0}(\mathcal{X})$.

Theorem 6.28. $b m o(\mathcal{X})=F_{\infty, 2}^{0}(\mathcal{X})$, with equivalent norms.

Proof. Let $f \in \operatorname{bmo}(\mathcal{X})$ and $\left\{D_{k}\right\}_{k \in \mathbb{Z}_{+}}$be as in Definition 6.19. Proposition 6.21(v) and Theorem 5.44(i) immediately imply that $f \in\left(\mathcal{G}_{0}^{e}(\beta, \gamma)\right)^{\prime}$ with $0<\beta, \gamma<\epsilon$. Let now $Q_{\alpha}^{l}$ for $l \in \mathbb{Z}_{+}$and $\alpha \in I_{l}$ be a dyadic cube as in Lemma 2.19. Let $B_{\alpha}^{l}$ be as in the proof of Theorem 6.11(ii). We then decompose $f=f_{1}+f_{2}+f_{3}$ in the same way as in the proof of Theorem 6.11(ii). The estimations for $f_{1}$ and $f_{2}$ are as in the proof of Theorem 6.11(ii) by replacing Proposition 3.15 by Proposition 3.30. If $l \in \mathbb{N}$, then

$$
\left\{\frac{1}{\mu\left(Q_{\alpha}^{l}\right)} \int_{Q_{\alpha}^{l}} \sum_{k=l}^{\infty}\left|D_{k}\left(f_{3}\right)(x)\right| d \mu(x)\right\}^{1 / 2}=0
$$


while when $l=0$, we then have

$$
\left\{\frac{1}{\mu\left(Q_{\alpha}^{l}\right)} \int_{Q_{\alpha}^{l}} \sum_{k=l}^{\infty}\left|D_{k}\left(f_{3}\right)(x)\right| d \mu(x)\right\}^{1 / 2}=\left|f_{3}\right| \leq\|f\|_{\text {bmo }(x) .}
$$

Thus, $f \in F_{\infty, 2}^{0}(\mathcal{X})$ and $\|f\|_{F_{\infty, 2}^{0}(\mathcal{X})} \lesssim\|f\|_{\mathrm{bmo}(\mathcal{X})}$.

Conversely, using Theorem 5.44(i), Proposition 6.22, and Proposition 6.27 together with an argument similar to the proof of Theorem 6.11(ii), we can prove that if $f \in F_{\infty, 2}^{0}(X)$, then $f \in \operatorname{bmo}(x)$ and $\|f\|_{\text {bmo }(x)} \lesssim\|f\|_{F_{\infty, 2}^{0}(x)}$, which completes the proof of Theorem 6.28.

We end this subsection by considering the boundedness on $F_{\infty, q}^{s}(X)$ of singular integrals of order $(\epsilon, \sigma)$.

Theorem 6.29. Let $\epsilon, s$, and $q$ be as in Definition 6.19. Let $\sigma>0$ and let $T$ be a singular integral of $\operatorname{order}(\epsilon, \sigma)$. Then $T$ is bounded from $\mathcal{G}_{b}(\beta, \gamma)$ with $0<\beta \leq \epsilon$ and $\gamma>0$ to $F_{\infty, q}^{s}(\mathcal{X})$. Moreover, there exists a constant $C>0$ such that for all $f \in \mathcal{G}_{b}(\beta, \gamma)$ with $0<\beta \leq \epsilon$ and $\gamma>0$,

$$
\|T f\|_{F_{\infty, q}^{s}(x)} \leq C\|f\|_{F_{\infty, q}^{s}(x)} .
$$

Proof. Combining some estimates and technics used in the proof of Proposition 5.54 with those used in the proof of Proposition 6.15 gives the desired conclusions. The details are left to the reader.

Remark 6.30. By Theorem 8.18 below, if $p(s, \epsilon)<q \leq \infty$, then $F_{\infty, q}^{s}(x)$ is the dual space of $F_{1, q^{\prime}}^{-s}(\mathcal{X})$. In this case, Theorem 6.29 can be deduced from Theorem 5.48 together with a duality argument, which provides another proof of Theorem 6.29.

\section{Frame characterizations}

In this section, using the discrete Calderón reproducing formulae, we establish a frame characterization of Besov spaces and Triebel-Lizorkin spaces.

\subsection{Frame characterization of $\dot{B}_{p, q}^{s}(\mathcal{X})$ and $\dot{F}_{p, q}^{s}(\mathcal{X})$}

In this subsection, we assume that $\mu(\mathcal{X})=\infty$. We first introduce some spaces of sequences, $\dot{b}_{p, q}^{s}(\mathcal{X})$ and $\dot{f}_{p, q}^{s}(\mathcal{X})$.

Let

$$
\lambda=\left\{\lambda_{\tau}^{k, v}: k \in \mathbb{Z}, \tau \in I_{k}, v=1, \ldots, N(k, \tau)\right\}
$$

be a sequence of complex numbers. The space $\dot{b}_{p, q}^{s}(\mathcal{X})$ with $s \in \mathbb{R}$ and $0<p, q \leq \infty$ is the set of all $\lambda$ as in (7.1) such that

$$
\|\lambda\|_{\dot{b}_{p, q}^{s}(x)}=\left\{\sum_{k=-\infty}^{\infty} 2^{k s q}\left[\sum_{\tau \in I_{k}} \sum_{\nu=1}^{N(k, \tau)} \mu\left(Q_{\tau}^{k, v}\right)\left|\lambda_{\tau}^{k, v}\right|^{p}\right]^{q / p}\right\}^{1 / q}<\infty,
$$


and the space $\dot{f}_{p, q}^{s}(\mathcal{X})$ with $s \in \mathbb{R}, 0<p<\infty$, and $0<q \leq \infty$ is the set of all $\lambda$ as in (7.1) such that

$$
\|\lambda\|_{f_{p, q}^{s}(\mathcal{X})}=\left\|\left\{\sum_{k=-\infty}^{\infty} \sum_{\tau \in I_{k}} \sum_{\nu=1}^{N(k, \tau)} 2^{k s q}\left|\lambda_{\tau}^{k, \nu}\right|^{q} X_{Q_{\tau}^{k, \nu}}\right\}^{1 / q}\right\|_{L^{p}(\mathcal{X})}<\infty .
$$

Moreover, the space $\dot{f}_{\infty, q}^{s}(\mathcal{X})$ with $s \in \mathbb{R}$ and $0<q \leq \infty$ is the set of all $\lambda$ as in (7.1) such that

$$
\begin{aligned}
& \|\mathcal{\lambda}\|_{f_{s, q}^{s}(\mathcal{x})} \\
& \quad=\sup _{l \in \mathbb{Z}} \sup _{\alpha \in I_{l}}\left\{\frac{1}{\mu\left(Q_{\alpha}^{l}\right)}\left[\sum_{k=l}^{\infty} \sum_{\tau \in I_{k}} \sum_{v=1}^{N(k, \tau)} 2^{k s q} \mu\left(Q_{\tau}^{k, v}\right)\left|\lambda_{\tau}^{k, v}\right|{ }^{q} X_{\left\{(\tau, v): Q_{\tau}^{k, v} \subset Q_{\alpha}^{l}\right\}}(\tau, v)\right]\right\}^{1 / q}<\infty,
\end{aligned}
$$

where $\left\{Q_{\alpha}^{l}\right\}_{l \in \mathbb{Z}, \alpha \in I_{l}}$ are dyadic cubes as in Lemma 2.19 .

Proposition 7.1. Let $\epsilon$ be as in Definition 5.8, let $|s|<\epsilon$, and let $p(s, \epsilon)<p \leq \infty$. Let $\lambda$ be a sequence of numbers as in (7.1) and all the other notation as in Theorem 4.11. Then, the following hold.

(i) If $0<q \leq \infty$ and $\|\lambda\|_{\dot{b}_{p, q}^{s}(x)}<\infty$, then the series

$$
\sum_{k=-\infty}^{\infty} \sum_{\tau \in I_{k}} \sum_{v=1}^{N(k, \tau)} \lambda_{\tau}^{k, v} \mu\left(Q_{\tau}^{k, v}\right) \widetilde{D}_{k}\left(x, y_{\tau}^{k, v}\right)
$$

converges to some $f \in \dot{B}_{p, q}^{s}(\mathcal{X})$ both in the norm of $\dot{B}_{p, q}^{s}(\mathcal{X})$ and in $\left(\mathcal{G}_{0}^{\epsilon}(\beta, \gamma)\right)^{\prime}$ with

$$
\max \left\{0,-s+n\left(\frac{1}{p}-1\right)_{+}\right\}<\beta<\epsilon, \quad \max \left\{n\left(\frac{1}{p}-1\right)_{+}, s-\frac{\kappa}{p}\right\}<\gamma<\epsilon
$$

when $p, q<\infty$ and only in $\left(\mathcal{G}_{0}^{\epsilon}(\beta, \gamma)\right)^{\prime}$ with $\beta$ and $\gamma$ as in $(7.6)$ when $\max (p, q)=\infty$. Moreover, in all cases,

$$
\|f\|_{\dot{B}_{p, q}^{s}(x)} \leq C\|\lambda\|_{\dot{b}_{p, q}^{s}(x)}
$$

(ii) If $p(s, \epsilon)<q \leq \infty$ and $\|\lambda\|_{f_{s, q}^{s}(x)}<\infty$, then the series in (7.5) converges to some $f \in$ $\dot{F}_{p, q}^{s}(\mathcal{X})$ both in the norm of $\dot{F}_{p, q}^{s}(\mathcal{X})$ and in $\left(\dot{\mathcal{G}}_{0}^{\epsilon}(\beta, \gamma)\right)^{\prime}$ with $\beta$, $\gamma$ as in (7.6) when $p, q<\infty$ and only in $\left(\mathcal{G}_{0}^{\epsilon}(\beta, \gamma)\right)^{\prime}$ with $\beta$ and $\gamma$ as in $(7.6)$ when $\max (p, q)=\infty$. Moreover, in all cases,

$$
\|f\|_{\dot{P}_{p, q}^{s}(x)} \leq C\|\lambda\|_{\dot{f}_{p, q}^{s}(x)} .
$$


Proof. We first verify that the series in (7.5) converges in $\left(\mathcal{G}_{0}^{\epsilon}(\beta, \gamma)\right)^{\prime}$ with $\beta$ and $\gamma$ as in (7.6) if $\lambda \in \dot{b}_{p, q}^{s}(\mathcal{X})$ with $s, p, q$ as in (i). By Lemma 2.19 and the definition of $N(k, \tau)$ together with (2.59) in [85, page 1385], we know that for all $k \in \mathbb{Z}$ and $\tau \in I_{k}, N(k, \tau)$ is a finite set. Without loss of generality, since $\mu(\mathcal{X})=\infty$, we may assume that $I_{k}=\mathbb{N}$ for all $k \in \mathbb{Z}$. With this assumption, for $L \in \mathbb{N}$, we define

$$
f_{L}(x)=\sum_{k=-L}^{L} \sum_{\tau=1}^{L} \sum_{\nu=1}^{N(k, \tau)} \mu\left(Q_{\tau}^{k, \nu}\right) \lambda_{\tau}^{k, v} \widetilde{D}_{k}\left(x, y_{\tau}^{k, v}\right) .
$$

Then $f_{L} \in \mathcal{G}_{1}\left(\epsilon^{\prime}, \epsilon^{\prime}\right)$ with $\epsilon^{\prime} \in\left(0, \epsilon_{1} \wedge \epsilon_{2}\right)$, and $f_{L} \in\left(\mathcal{G}_{0}^{\epsilon}(\beta, \gamma)\right)^{\prime}$ with any $\beta, \gamma \in(0, \epsilon)$. For any $\psi \in \mathcal{G}(\beta, \gamma)$ with $\beta, \gamma$ as in (7.6), $L_{1}, L_{2} \in \mathbb{N}$ and $L_{1}<L_{2}$, we have

$$
\begin{aligned}
\left|\left\langle f_{L_{2}}-f_{L_{1}}, \psi\right\rangle\right| \leq & \sum_{k=-L_{2}}^{-L_{1}-1} \sum_{\tau=1}^{L_{1}} \sum_{v=1}^{N(k, \tau)} \mu\left(Q_{\tau}^{k, v}\right)\left|\lambda_{\tau}^{k, v}\right|\left|\left\langle\widetilde{D}_{k}\left(\cdot, y_{\tau}^{k, v}\right), \psi\right\rangle\right| \\
& +\sum_{k=L_{1}+1}^{L_{2}} \sum_{\tau=1}^{L_{1}} \sum_{\nu=1}^{N(k, \tau)} \mu\left(Q_{\tau}^{k, v}\right)\left|\lambda_{\tau}^{k, v}\right|\left|\left\langle\widetilde{D}_{k}\left(\cdot, y_{\tau}^{k, v}\right), \psi\right\rangle\right| \\
& +\sum_{k=-L_{2}}^{L_{2}} \sum_{\tau=L_{1}+1}^{L_{2}} \sum_{v=1}^{N(k, \tau)} \mu\left(Q_{\tau}^{k, v}\right)\left|\lambda_{\tau}^{k, v}\right|\left|\left\langle\widetilde{D}_{k}\left(\cdot, y_{\tau}^{k, v}\right), \psi\right\rangle\right| \\
\equiv & Z_{1}+Z_{2}+Z_{3} .
\end{aligned}
$$

Let us now consider two cases, respectively. We first consider the case $p \leq 1$. In this case, letting $\gamma^{\prime} \in(\max \{0, s-\kappa / p\}, \gamma)$, by (5.25) and $\gamma>n(1 / p-1)$ together with (5.31) and Hölder's inequality, we have

$$
\begin{aligned}
Z_{1} & \lesssim\|\psi\|_{\mathcal{G}(\beta, \gamma)} \sum_{k=-L_{2}}^{-L_{1}-1} \sum_{\tau=1}^{L_{1}} \sum_{\nu=1}^{N(k, \tau)} 2^{k \gamma^{\prime}} \mu\left(Q_{\tau}^{k, v}\right)\left|\lambda_{\tau}^{k, v}\right| \frac{1}{V_{2^{-k}}\left(x_{1}\right)+V\left(x_{1}, y_{\tau}^{k, v}\right)} \frac{2^{-k \gamma}}{\left(2^{-k}+d\left(x_{1}, y_{\tau}^{k, v}\right)\right)^{\gamma}} \\
& \lesssim\|\psi\|_{\mathcal{G}(\beta, \gamma)} \sum_{k=-L_{2}}^{-L_{1}-1} 2^{k\left(\gamma^{\prime}-s+\kappa / p\right)}\left[\sum_{\tau=1}^{L_{1}} \sum_{v=1}^{N(k, \tau)} 2^{k s p} \mu\left(Q_{\tau}^{k, v}\right)\left|\lambda_{\tau}^{k, v}\right|^{p}\right]^{1 / p} .
\end{aligned}
$$

If $q \leq 1$, by (5.5), we further obtain

$$
\begin{aligned}
Z_{1} & \lesssim\|\psi\|_{\mathcal{G}(\beta, \gamma)}\left\{\sum_{k=-L_{2}}^{-L_{1}-1} 2^{k\left(\gamma^{\prime}-s+\kappa / p\right) q}\left[\sum_{\tau=1}^{L_{1}} \sum_{v=1}^{N(k, \tau)} 2^{k s p} \mu\left(Q_{\tau}^{k, v}\right)\left|\lambda_{\tau}^{k, v}\right|^{p}\right]^{q / p}\right\}^{1 / q} \\
& \lesssim\|\psi\|_{\mathcal{G}(\beta, \gamma)}\left\{\sum_{k=-L_{2}}^{-L_{1}-1}\left[\sum_{\tau=1}^{L_{1}} \sum_{v=1}^{N(k, \tau)} 2^{k s p} \mu\left(Q_{\tau}^{k, v}\right)\left|\lambda_{\tau}^{k, v}\right|^{p}\right]^{q / p}\right\}^{1 / q}
\end{aligned}
$$


while if $1<q \leq \infty$, by Hölder's inequality, we have

$$
\begin{aligned}
Z_{1} & \lesssim\|\psi\|_{\mathcal{G}(\beta, \gamma)}\left\{\sum_{k=-L_{2}}^{-L_{1}-1}\left[\sum_{\tau=1}^{L_{1}} \sum_{v=1}^{N(k, \tau)} 2^{k s p} \mu\left(Q_{\tau}^{k, \nu}\right)\left|\lambda_{\tau}^{k, v}\right|^{p}\right]^{q / p}\right\}^{1 / q}\left\{\sum_{k=-L_{2}}^{-L_{1}-1} 2^{k\left(\gamma^{\prime}-s+\kappa / p\right) q^{\prime}}\right\}^{1 / q^{\prime}} \\
& \lesssim\|\psi\|_{\mathcal{G}(\beta, \gamma)} 2^{-L_{1}\left(\gamma^{\prime}-s+\kappa / p\right)}\left\{\sum_{k=-L_{2}}^{-L_{1}-1}\left[\sum_{\tau=1}^{L_{1}} \sum_{v=1}^{N(k, \tau)} 2^{k s p} \mu\left(Q_{\tau}^{k, v}\right)\left|\lambda_{\tau}^{k, v}\right|^{p}\right]^{q / p}\right\}^{1 / q}
\end{aligned}
$$

Thus,

$$
\lim _{L_{1}, L_{2} \rightarrow \infty} Z_{1}=0
$$

Replacing the estimates (5.25) and (5.31) respectively by the estimates (5.24) and (5.30), and using some similar computations to the estimate for $Z_{1}$, we obtain

$$
\begin{aligned}
Z_{2} & \lesssim\|\psi\|_{\mathcal{G}(\beta, \gamma)} \sum_{k=L_{1}+1}^{L_{2}} \sum_{\tau=1}^{L_{1}} \sum_{\nu=1}^{N(k, \tau)} 2^{-k \beta} \mu\left(Q_{\tau}^{k, v}\right)\left|\lambda_{\tau}^{k, v}\right| \frac{1}{V_{1}\left(x_{1}\right)+V\left(x_{1}, y_{\tau}^{k, v}\right)} \frac{1}{\left(1+d\left(x_{1}, y_{\tau}^{k, v}\right)\right)^{\gamma}} \\
& \lesssim\|\psi\|_{\mathcal{G}(\beta, \gamma)} \sum_{k=L_{1}+1}^{L_{2}} 2^{-k[s+\beta-d(1 / p-1)]}\left[\sum_{\tau=1}^{L_{1}} \sum_{\nu=1}^{N(k, \tau)} 2^{k s p} \mu\left(Q_{\tau}^{k, v}\right)\left|\lambda_{\tau}^{k, v}\right|^{p}\right]^{1 / p} \\
& \lesssim\|\psi\|_{\mathcal{G}(\beta, \gamma)} \begin{cases}\left\{\sum_{k=L_{1}+1}^{L_{2}}\left[\sum_{\tau=1}^{L_{1}} \sum_{v=1}^{N(k, \tau)} 2^{k s p} \mu\left(Q_{\tau}^{k, v}\right)\left|\lambda_{\tau}^{k, v}\right|^{p}\right]^{q / p}\right\}^{1 / q}, & q \leq 1 \\
2^{-L_{1}[s+\beta-d(1 / p-1)]}\left\{\sum_{k=L_{1}+1}^{L_{2}}\left[\sum_{\tau=1}^{L_{1}} \sum_{v=1}^{N(k, \tau)} 2^{k s p} \mu\left(Q_{\tau}^{k, v}\right)\left|\lambda_{\tau}^{k, v}\right|^{p}\right]^{q / p}\right\}^{1 / q}, & 1<q \leq \infty .\end{cases}
\end{aligned}
$$

where we used the assumption that $\beta>-s+d(1 / p-1)$. Thus,

$$
\lim _{L_{1}, L_{2} \rightarrow \infty} Z_{2}=0 .
$$


The estimates (5.24) and (5.25) together with (5.5), (5.30), and (5.31) further yield that

$$
\begin{aligned}
Z_{3} \lesssim\|\psi\|_{\mathcal{G}(\beta, \gamma)} & \left\{\sum_{k=0}^{L_{2}} \sum_{\tau=L_{1}+1}^{L_{2}} \sum_{\nu=1}^{N(k, \tau)} 2^{-k \beta} \mu\left(Q_{\tau}^{k, v}\right)\left|\lambda_{\tau}^{k, v}\right| \frac{1}{V_{1}\left(x_{1}\right)+V\left(x_{1}, y_{\tau}^{k, v}\right)} \frac{1}{\left(1+d\left(x_{1}, y_{\tau}^{k, v}\right)\right)^{\gamma}}\right. \\
& \left.+\sum_{k=-L_{2}}^{-1} \sum_{\tau=L_{1}+1}^{L_{2}} \sum_{v=1}^{N(k, \tau)} 2^{k \gamma^{\prime}} \mu\left(Q_{\tau}^{k, v}\right)\left|\lambda_{\tau}^{k, v}\right| \frac{1}{V_{2^{-k}}\left(x_{1}\right)+V\left(x_{1}, y_{\tau}^{k, v}\right)} \frac{2^{-k \gamma}}{\left(2^{-k}+d\left(x_{1}, y_{\tau}^{k, v}\right)\right)^{\gamma}}\right\} \\
\lesssim\|\psi\|_{\mathcal{G}(\beta, \gamma)} & \left\{\sum_{k=0}^{L_{2}} 2^{-k[\beta+s-d(1 / p-1)]}+\sum_{k=-L_{2}}^{-1} 2^{k\left(\gamma^{\prime}-s+\kappa / p\right)}\right\}\left[\sum_{\tau=L_{1}+1}^{L_{2}} \sum_{v=1}^{N(k, \tau)} 2^{k s p} \mu\left(Q_{\tau}^{k, v}\right)\left|\lambda_{\tau}^{k, v}\right|^{p}\right]^{1 / p},
\end{aligned}
$$

where we chose $\gamma^{\prime}>0$ as in the estimate for $Z_{1}$. For any given $\delta>0$, since $\gamma^{\prime}>s-\kappa / p$ and $\beta>d(1 / p-1)-s$, we can fix $L_{2}^{0} \in \mathbb{N}$ such that

$$
\sum_{k=L_{2}^{0}+1}^{\infty} 2^{-k[\beta+s-d(1 / p-1)]}+\sum_{k=-\infty}^{-L_{2}^{0}-1} 2^{k\left(\gamma^{\prime}-s+\kappa / p\right)}<\delta
$$

Since for all $k \in \mathbb{Z}$,

$$
\left[\sum_{\tau=L_{1}+1}^{L_{2}} \sum_{v=1}^{N(k, \tau)} 2^{k s p} \mu\left(Q_{\tau}^{k, v}\right)\left|\lambda_{\tau}^{k, v}\right|^{p}\right]^{1 / p} \leq\left[\sum_{\tau \in I_{k}} \sum_{v=1}^{N(k, \tau)} 2^{k s p} \mu\left(Q_{\tau}^{k, v}\right)\left|\lambda_{\tau}^{k, v}\right|^{p}\right]^{1 / p}<\|\lambda\|_{b_{p, q}^{s}(x)},
$$

we can choose $N \in \mathbb{N}$ such that if $L_{1}>N$, then

$$
\left[\sum_{\tau=L_{1}+1}^{L_{2}} \sum_{\nu=1}^{N(k, \tau)} 2^{k s p} \mu\left(Q_{\tau}^{k, v}\right)\left|\lambda_{\tau}^{k, v}\right|^{p}\right]^{1 / p}<\delta
$$

for all $k=-L_{2}^{0},-L_{2}^{0}+1, \ldots, L_{2}^{0}$. From (7.18), (7.19), and (7.20), it follows that if $L_{2}>L_{1}>N$, then

$$
\begin{aligned}
Z_{3} \lesssim & \|\psi\|_{\mathcal{G}(\beta, \gamma)}\left\{\sum_{k=0}^{L_{2}^{0}} 2^{-k[\beta+s-d(1 / p-1)]}+\sum_{k=-L_{2}^{0}}^{-1} 2^{k\left(\gamma^{\prime}-s+\kappa / p\right)}\right\} \delta \\
& +C\|\psi\|_{\mathcal{G}(\beta, \gamma)}\|\lambda\|_{\dot{b}_{p, q}^{s}(\mathcal{X})}\left\{\sum_{k=L_{2}^{0}+1}^{\infty} 2^{-k[\beta+s-d(1 / p-1)]}+\sum_{k=-\infty}^{-L_{2}^{0}-1} 2^{k\left(\gamma^{\prime}-s+\kappa / p\right)}\right\} \\
& \lesssim\|\psi\|_{\mathcal{G}(\beta, \gamma)} \delta,
\end{aligned}
$$

which just means that

$$
\lim _{L_{1}, L_{2} \rightarrow \infty} Z_{3}=0
$$


We now consider the case $1<p \leq \infty$. Replacing (5.5) by Hölder's inequality and using Lemma 5.2, similarly to the estimate for the case $p \leq 1$, we obtain

$$
\begin{aligned}
Z_{1} \lesssim & \|\psi\|_{\mathcal{G}(\beta, \gamma)} \sum_{k=-L_{2}}^{-L_{1}-1} 2^{k\left(\gamma^{\prime}-s\right)}\left[\sum_{\tau=1}^{L_{1}} \sum_{\nu=1}^{N(k, \tau)} 2^{k s p} \mu\left(Q_{\tau}^{k, v}\right)\left|\lambda_{\tau}^{k, v}\right|^{p}\right. \\
& \left.\times \frac{1}{V_{2^{-k}}\left(x_{1}\right)+V\left(x_{1}, y_{\tau}^{k, \nu}\right)} \frac{2^{-k \gamma}}{\left(2^{-k}+d\left(x_{1}, y_{\tau}^{k, v}\right)\right)^{\gamma}}\right]^{1 / p} \\
& \times\left[\int_{X} \frac{1}{V_{2^{-k}}\left(x_{1}\right)+V\left(x_{1}, y\right)} \frac{2^{-k \gamma}}{\left(2^{-k}+d\left(x_{1}, y\right)\right)^{\gamma}} d \mu(y)\right]^{1 / p^{\prime}} \\
\lesssim & \|\psi\|_{\mathcal{G}(\beta, \gamma)} \sum_{k=-L_{2}}^{-L_{1}-1} 2^{k\left(\gamma^{\prime}-s+\kappa / p\right)}\left[\sum_{\tau=1}^{L_{1}} \sum_{v=1}^{N(k, \tau)} 2^{k s p} \mu\left(Q_{\tau}^{k, v}\right)\left|\lambda_{\tau}^{k, v}\right|^{p}\right]^{1 / p}
\end{aligned}
$$

Then repeating the proof of the case $p \leq 1$ yields that

$$
\lim _{L_{1}, L_{2} \rightarrow \infty} Z_{1}=0
$$

Similarly, for $Z_{2}$, Hölder's inequality and Lemma 5.2 imply that

$$
\begin{aligned}
& Z_{2} \lesssim\|\psi\|_{\mathcal{G}(\beta, \gamma)} 2^{-k(s+\beta)}\left[\sum_{k=L_{1}+1}^{L_{2}} \sum_{\tau=1}^{L_{1}} \sum_{\nu=1}^{N(k, \tau)} 2^{k s p} \mu\left(Q_{\tau}^{k, v}\right)\left|\lambda_{\tau}^{k, v}\right|^{p}\right]^{1 / p} \\
& \times\left[\int_{X} \frac{1}{V_{1}\left(x_{1}\right)+V\left(x_{1}, y\right)} \frac{1}{\left(1+d\left(x_{1}, y\right)\right)^{\gamma}} d \mu(y)\right]^{1 / p^{\prime}} \\
& \lesssim\|\psi\|_{\mathcal{G}(\beta, \gamma)} \sum_{k=L_{1}+1}^{L_{2}} 2^{-k(s+\beta)}\left[\sum_{\tau=1}^{L_{1}} \sum_{\nu=1}^{N(k, \tau)} 2^{k s p} \mu\left(Q_{\tau}^{k, v}\right)\left|\lambda_{\tau}^{k, v}\right|^{p}\right]^{1 / p} .
\end{aligned}
$$

Using the fact that $\beta>-s$ and repeating the proof of the case $p \leq 1$ show that

$$
\lim _{L_{1}, L_{2} \rightarrow \infty} Z_{2}=0
$$

To estimate $Z_{3}$, let

$$
X_{L_{1}}^{L_{2}, k}=\bigcup_{\tau=L_{1}+1}^{L_{2}} \bigcup_{\nu=1}^{N(k, \tau)} Q_{\tau}^{k, v} .
$$


The Hölder inequality shows that

$$
\begin{aligned}
Z_{3} \lesssim & \|\psi\|_{\mathcal{G}(\beta, \gamma)}\left\{\sum_{k=0}^{L_{2}} 2^{-k(\beta+s)}\left[\int_{x_{L_{1}}^{L_{2}, k}} \frac{1}{V_{1}\left(x_{1}\right)+V\left(x_{1}, y\right)} \frac{1}{\left(1+d\left(x_{1}, y\right)\right)^{\gamma}} d \mu(y)\right]^{1 / p^{\prime}}\right. \\
& \left.+\sum_{k=-L_{2}}^{-1} 2^{k\left(\gamma^{\prime}-s+\kappa / p\right)}\left[\int_{\chi_{L_{1}}^{L_{2}, k}} \frac{1}{V_{2^{-k}}\left(x_{1}\right)+V\left(x_{1}, y\right)} \frac{2^{-k \gamma}}{\left(2^{-k \gamma}+d\left(x_{1}, y\right)\right)^{\gamma}} d \mu(y)\right]^{1 / p^{\prime}}\right\} \\
\times & {\left[\sum_{\tau=L_{1}+1}^{L_{2}} \sum_{v=1}^{N(k, \tau)} 2^{k s p} \mu\left(Q_{\tau}^{k, v}\right)\left|\lambda_{\tau}^{k, v}\right|^{p}\right]^{1 / p}, }
\end{aligned}
$$

where we chose $\gamma^{\prime} \in(\max \{0, s-\kappa / p\}, \gamma)$. If $p \in(1, \infty)$, using the facts that $\gamma^{\prime}>s-\kappa / p$ and $\beta>-s,(7.19),(7.20)$,

$$
\begin{gathered}
\int_{x} \frac{1}{V_{1}\left(x_{1}\right)+V\left(x_{1}, y\right)} \frac{1}{\left(1+d\left(x_{1}, y\right)\right)^{\gamma}} d \mu(y) \lesssim 1, \\
\int_{x} \frac{1}{V_{2^{-k}}\left(x_{1}\right)+V\left(x_{1}, y\right)} \frac{2^{-k \gamma}}{\left(2^{-k \gamma}+d\left(x_{1}, y\right)\right)^{\gamma}} d \mu(y) \lesssim 1,
\end{gathered}
$$

and repeating the argument for the case $p \leq 1$, we can verify that

$$
\lim _{L_{1}, L_{2} \rightarrow \infty} Z_{3}=0
$$

If $p=\infty$, replacing $(7.20)$ by

$$
\begin{gathered}
\lim _{L_{1}, L_{2} \rightarrow \infty} \int_{x_{L_{1}}^{L_{2}, k}} \frac{1}{V_{1}\left(x_{1}\right)+V\left(x_{1}, y\right)} \frac{1}{\left(1+d\left(x_{1}, y\right)\right)^{\gamma}} d \mu(y)=0, \\
\lim _{L_{1}, L_{2} \rightarrow \infty} \int_{X_{L_{1}}^{L_{2}, k}} \frac{1}{V_{2^{-k}}\left(x_{1}\right)+V\left(x_{1}, y\right)} \frac{2^{-k \gamma}}{\left(2^{-k \gamma}+d\left(x_{1}, y\right)\right)^{\gamma}} d \mu(y)=0
\end{gathered}
$$

for any given $k \in \mathbb{Z}$, by an argument similar to the case $p \leq 1$, we still obtain that

$$
\lim _{L_{1}, L_{2} \rightarrow \infty} Z_{3}=0
$$

Thus, for any give $\psi \in \mathcal{G}(\beta, \gamma),\left\{\left\langle f_{L}, \psi\right\rangle\right\}_{L \in \mathbb{N}}$ is a Cauchy sequence, which means that the series in (7.5) converges to some $f \in\left(\dot{\mathcal{G}}_{0}^{\epsilon}(\beta, \gamma)\right)^{\prime}$ with $\beta, \gamma$ as in (7.6) if $\lambda \in \dot{b}_{p, q}^{s}(\mathcal{X})$ with $s, p, q$ as in the theorem.

If $\lambda \in \dot{f}_{p, q}^{s}(\mathcal{X})$, by the proved fact on $\dot{b}_{p, q}^{s}(\mathcal{X})$ and

$$
\dot{b}_{p, \min (p, q)}^{s}(\mathcal{X}) \subset \dot{f}_{p, q}^{s}(\mathcal{X}) \subset \dot{b}_{p, \max (p, q)}^{s}(\mathcal{X})
$$


(see [87, Proposition 2.3]), we also obtain that the series in (7.5) converges in $\left(\mathcal{G}_{0}^{\epsilon}(\beta, \gamma)\right)^{\prime}$ with $\beta$ and $\gamma$ as in (7.6).

Let us now verify that the series in (7.5) converges in the norm of $\dot{B}_{p, q}^{s}(\mathcal{X})$ or $\dot{F}_{p, q}^{s}(\mathcal{X})$ when $p, q<\infty$ if $\lambda \in \dot{b}_{p, q}^{s}(\mathcal{X})$ or $\lambda \in \dot{f}_{p, q}^{s}(\mathcal{X})$, respectively. To this end, let $f$ be the series in (7.5). For $L \in \mathbb{N}$, in $\left(\mathcal{C}_{0}^{\epsilon}(\beta, \gamma)\right)^{\prime}$ with $\beta$ and $\gamma$ as in (7.6), we then have

$$
\begin{aligned}
f-f_{L}= & \sum_{k=L+1}^{\infty} \sum_{\tau \in I_{k}} \sum_{v=1}^{N(k, \tau)} \mu\left(Q_{\tau}^{k, v}\right) \lambda_{\tau}^{k, v} \widetilde{D}_{k}\left(x, y_{\tau}^{k, v}\right) \\
& +\sum_{k=-\infty}^{-L-1} \sum_{\tau \in I_{k}} \sum_{v=1}^{N(k, \tau)} \mu\left(Q_{\tau}^{k, v}\right) \lambda_{\tau}^{k, v} \widetilde{D}_{k}\left(x, y_{\tau}^{k, v}\right) \\
& +\sum_{k=-L}^{L} \sum_{\tau=L+1}^{\infty} \sum_{v=1}^{N(k, \tau)} \mu\left(Q_{\tau}^{k, v}\right) \lambda_{\tau}^{k, v} \widetilde{D}_{k}\left(x, y_{\tau}^{k, v}\right) .
\end{aligned}
$$

Replacing $Q_{k^{\prime}}(f)\left(y_{\tau^{\prime}}^{k^{\prime}, v^{\prime}}\right)$ in the proof of Proposition 5.4 by $\lambda_{\tau}^{k, v}$ here and repeating the proof of Proposition 5.4, we can verify that

$$
\begin{aligned}
& \left\|f-f_{L}\right\|_{\dot{B}_{p, q}^{s}(x)} \lesssim\left\{\sum_{k=L+1}^{\infty} 2^{k s q}\left[\sum_{\tau \in I_{k}} \sum_{v=1}^{N(k, \tau)} \mu\left(Q_{\tau}^{k, v}\right)\left|\lambda_{\tau}^{k, v}\right|^{p}\right]^{q / p}\right\}^{1 / q} \\
& +\left\{\sum_{k=-\infty}^{-L-1} 2^{k s q}\left[\sum_{\tau \in I_{k}} \sum_{\nu=1}^{N(k, \tau)} \mu\left(Q_{\tau}^{k, v}\right)\left|\lambda_{\tau}^{k, v}\right|^{p}\right]^{q / p}\right\}^{1 / q} \\
& +\left\{\sum_{k=-L}^{L} 2^{k s q}\left[\sum_{\tau=L+1}^{\infty} \sum_{\nu=1}^{N(k, \tau)} \mu\left(Q_{\tau}^{k, v}\right)\left|\lambda_{\tau}^{k, v}\right|^{p}\right]^{q / p}\right\}^{1 / q}, \\
& \left\|f-f_{L}\right\|_{\dot{F}_{p, q}^{s}(x)} \lesssim\left\|\left\{\sum_{k=L+1}^{\infty} \sum_{\tau \in I_{k}} \sum_{\nu=1}^{N(k, \tau)} 2^{k s q}\left|\lambda_{\tau}^{k, v}\right|^{q} X_{Q_{\tau}^{k, v}}\right\}^{1 / q}\right\|_{L^{p}(x)} \\
& +\left\|\left\{\sum_{k=-\infty}^{-L-1} \sum_{\tau \in I_{k}} \sum_{\nu=1}^{N(k, \tau)} 2^{k s q}\left|\lambda_{\tau}^{k, v}\right|^{q} \chi_{Q_{\tau}^{k, \nu}}\right\}^{1 / q}\right\|_{L^{p}(\mathcal{X})} \\
& +\left\|\left\{\sum_{k=-L}^{L} \sum_{\tau=L+1}^{\infty} \sum_{\nu=1}^{N(k, \tau)} 2^{k s q}\left|\lambda_{\tau}^{k, v}\right|^{q} X_{Q_{\tau}^{k, v}}\right\}^{1 / q}\right\|_{L^{p}(\mathcal{X})} .
\end{aligned}
$$

Thus, from Lebesgue's dominated convergence theorems on the integral and the series, it is easy to deduce that

$$
\begin{aligned}
& \left\|f-f_{L}\right\|_{\dot{B}_{p, q}^{s}(x)} \longrightarrow 0, \\
& \left\|f-f_{L}\right\|_{\dot{F}_{p, q}^{s}(x)} \longrightarrow 0
\end{aligned}
$$


as $L \rightarrow \infty$. Moreover, by Proposition 5.10(iv), we know that $f_{L} \in \dot{B}_{p, q}^{s}(\mathcal{X})$ and $f_{L} \in \dot{F}_{p, q}^{s}(\mathcal{X})$ if we choose $\epsilon^{\prime}$ large enough. Thus, $f \in \dot{B}_{p, q}^{s}(\mathcal{X})$ if $\lambda \in \dot{b}_{p, q}^{s}(\mathcal{X})$ and $f \in \dot{F}_{p, q}^{s}(\mathcal{X})$ if $\lambda \in \dot{f}_{p, q}^{s}(\mathcal{X})$ when $p, q<\infty$.

The same arguments as in the proof of Propositions 5.4 and 6.3 for the space $\dot{B}_{p, q}^{s}(\mathcal{X})$ and the space $\dot{F}_{p, q}^{s}(\mathcal{X})$ with all $p, q$ as in the assumption of the theorem yield (7.7) and (7.8), respectively, which completes the proof of Proposition 7.1.

From Theorem 4.13, Proposition 7.1, and the Plancherel-Pôlya inequalities, Propositions 5.4 and 6.3, we obtain the following frame characterizations of the spaces $\dot{B}_{p, q}^{s}(\mathcal{X})$ and $\dot{F}_{p, q}^{s}(X)$.

Theorem 7.2. Let $\epsilon$ be as in Definition 5.8, let $|s|<\epsilon$, and let $p(s, \epsilon)<p \leq \infty$. Let all the other notation be as in Theorem 4.11 and $\lambda_{\tau}^{k, v}=D_{k}(f)\left(y_{\tau}^{k, v}\right)$ for $k \in \mathbb{Z}, \tau \in I_{k}$ and $v=1, \ldots, N(k, \tau)$, where $y_{\tau}^{k, v}$ is any fixed element in $Q_{\tau}^{k, v}$. Then, the following hold.

(i) If $0<q \leq \infty$, then $f \in \dot{B}_{p, q}^{s}(x)$, if and only if $f \in\left(\mathcal{G}_{0}^{\epsilon}(\beta, \gamma)\right)^{\prime}$ for some $\beta, \gamma$ as in (5.35),

$$
f(x)=\sum_{k=-\infty}^{\infty} \sum_{\tau \in I_{k}} \sum_{v=1}^{N(k, \tau)} \mu\left(Q_{\tau}^{k, v}\right) D_{k}(f)\left(y_{\tau}^{k, v}\right) \widetilde{D}_{k}\left(x, y_{\tau}^{k, v}\right),
$$

holds in $\left(\dot{\mathcal{G}}_{0}^{\epsilon}(\beta, \gamma)\right)^{\prime}$ and $\lambda \in \dot{b}_{p, q}^{s}(\mathcal{X})$. Moreover, in this case,

$$
\|f\|_{\dot{B}_{p, q}^{s}(x)} \sim\|\lambda\|_{\dot{b}_{p, q}^{s}(x)} .
$$

(ii) If $p(s, \epsilon)<q \leq \infty$, then $f \in \dot{F}_{p, q}^{s}(X)$ if and only if $f \in\left(\mathcal{G}_{0}^{\epsilon}(\beta, \gamma)\right)^{\prime}$ for some $\beta, \gamma$ as in (5.35), (7.37), holds in $\left(\mathcal{G}_{0}^{\epsilon}(\beta, \gamma)\right)^{\prime}$ and $\lambda \in \dot{f}_{p, q}^{s}(\mathcal{X})$. Moreover, in this case,

$$
\|f\|_{\dot{F}_{p, q}^{s}(x)} \sim\|\lambda\|_{\dot{f}_{p, q}^{s}(x)} .
$$

\subsection{Frame characterization of $B_{p, q}^{s}(X)$ and $F_{p, q}^{s}(X)$}

Again, in this subsection, $\mu(x)$ can be finite or infinite. We also first introduce some spaces of sequences, $b_{p, q}^{s}(\mathcal{X})$ and $f_{p, q}^{s}(\mathcal{X})$.

Let

$$
\lambda=\left\{\lambda_{\tau}^{k, v}: k \in \mathbb{Z}_{+}, \tau \in I_{k}, v=1, \ldots, N(k, \tau)\right\}
$$

be a sequence of complex numbers. The space $b_{p, q}^{s}(\mathcal{X})$ with $s \in \mathbb{R}$ and $0<p, q \leq \infty$ is the set of all $\lambda$ as in (7.40) such that

$$
\|\lambda\|_{b_{p, q}^{s}(\mathcal{x})}=\left\{\sum_{k=0}^{\infty} 2^{k s q}\left[\sum_{\tau \in I_{k}} \sum_{\nu=1}^{N(k, \tau)} \mu\left(Q_{\tau}^{k, v}\right)\left|\lambda_{\tau}^{k, v}\right|^{p}\right]^{q / p}\right\}^{1 / q}<\infty,
$$


and the space $f_{p, q}^{s}(\mathcal{X})$ with $s \in \mathbb{R}, 0<p<\infty$, and $0<q \leq \infty$ is the set of all $\lambda$ as in (7.40) such that

$$
\|\lambda\|_{f_{p, q}^{s}(\mathcal{x})}=\left\|\left\{\sum_{k=0}^{\infty} \sum_{\tau \in I_{k}} \sum_{\nu=1}^{N(k, \tau)} 2^{k s q}\left|\lambda_{\tau}^{k, \nu}\right|^{q} X_{Q_{\tau}^{k, \nu}}\right\}^{1 / q}\right\|_{L^{p}(\mathcal{X})}<\infty .
$$

Moreover, the space $f_{\infty, q}^{s}(\mathcal{X})$ with $s \in \mathbb{R}$ and $0<q \leq \infty$ is the set of all $\lambda$ as in (7.40) such that

$$
\begin{aligned}
\|\lambda\|_{f_{\infty, q}^{s}(\mathcal{l})=\max }\left[\sup _{\substack{\tau \in I_{0} \\
v=1, \ldots, N(0, \tau)}}\left|\lambda_{\tau}^{0, v}\right|,\right. \\
\left.\quad \sup _{l \in \mathbb{N}} \sup _{\alpha \in I_{l}}\left\{\frac{1}{\mu\left(Q_{\alpha}^{l}\right)}\left[\sum_{k=l}^{\infty} \sum_{\tau \in I_{k}} \sum_{\nu=1}^{N(k, \tau)} 2^{k s q} \mu\left(Q_{\tau}^{k, v}\right)\left|\lambda_{\tau}^{k, v}\right|^{q} X_{\left\{(\tau, v): Q_{\tau}^{k, v} \subset Q_{\alpha}^{l}\right\}}(\tau, v)\right]\right\}^{1 / q}\right]<\infty,
\end{aligned}
$$

where $\left\{Q_{\alpha}^{l}\right\}_{l \in \mathbb{N}, \alpha \in I_{l}}$ are dyadic cubes as in Lemma 2.19.

Proposition 7.3. Let $\epsilon$ be as in Definition 5.29, let $|s|<\epsilon$, and let $p(s, \epsilon)<p \leq \infty$. Let $\lambda$ be a sequence of numbers as in (7.40) and all the other notation as in Theorem 4.14. Then, the following hold.

(i) If $0<q \leq \infty$ and $\|\lambda\|_{b_{p, q}^{s}(x)}<\infty$, then the series

$$
\sum_{\tau \in I_{0}} \sum_{\nu=1}^{N(0, \tau)} \lambda_{\tau}^{0, v} \int_{Q_{\tau}^{0, v}} \tilde{D}_{0}(x, y) d \mu(y)+\sum_{k=1}^{\infty} \sum_{\tau \in I_{k}} \sum_{v=1}^{N(k, \tau)} \lambda_{\tau}^{k, v} \mu\left(Q_{\tau}^{k, v}\right) \tilde{D}_{k}\left(x, y_{\tau}^{k, v}\right)
$$

converges to some $f \in B_{p, q}^{s}(\mathcal{X})$ both in the norm of $B_{p, q}^{s}(\mathcal{X})$ and in $\left(\mathcal{G}_{0}^{\epsilon}(\beta, \gamma)\right)^{\prime}$ with

$$
\max \left\{0,-s+n\left(\frac{1}{p}-1\right)_{+}\right\}<\beta<\epsilon, \quad n\left(\frac{1}{p}-1\right)_{+}<\gamma<\epsilon,
$$

when $p, q<\infty$ and only in $\left(\mathcal{G}_{0}^{\epsilon}(\beta, \gamma)\right)^{\prime}$ with $\beta$ and $\gamma$ as in $(7.45)$ when $\max (p, q)=\infty$. Moreover, in all cases,

$$
\|f\|_{B_{p, q}^{s}(x)} \leq C\|\lambda\|_{b_{p, q}^{s}(x)}
$$

(ii) If $p(s, \epsilon)<q \leq \infty$ and $\|\lambda\|_{f_{p, q}^{s}(x)}<\infty$, then the series in (7.44) converges to some $f \in$ $F_{p, q}^{s}(\mathcal{X})$ both in the norm of $F_{p, q}^{s}(\mathcal{X})$ and in $\left(\mathcal{G}_{0}^{e}(\beta, \gamma)\right)^{\prime}$ with $\beta, \gamma$ as in $(7.45)$ when $p, q<$ $\infty$ and only in $\left(\mathcal{G}_{0}^{\epsilon}(\beta, \gamma)\right)^{\prime}$ with $\beta$ and $\gamma$ as in $(7.45)$ when $\max (p, q)=\infty$. Moreover, in all cases,

$$
\|f\|_{F_{p, q}^{s}(x)} \leq C\|\lambda\|_{f_{p, q}^{s}(x)} .
$$


Proof. In what follows, for simplicity, we set

$$
\widetilde{D}_{Q_{\tau}^{0, v}}(x)=\frac{1}{\mu\left(Q_{\tau}^{0, v}\right)} \int_{Q_{\tau}^{0, v}} \tilde{D}_{0}(x, y) d \mu(y) .
$$

Let us first show that the series in (7.44) converges in $(\mathcal{G}(\beta, \gamma))^{\prime}$ with $\beta$ and $\gamma$ as in (7.45). As in the proof of Proposition 7.1, we know that for all $k \in \mathbb{Z}_{+}$and $\tau \in I_{k}, N(k, \tau)$ is a finite set. Let us suppose $I_{k}=\mathbb{N}$ for all $k \in \mathbb{Z}_{+}$; the other cases are easier. With this assumption, for $L \in \mathbb{N}$, we define

$$
f_{L}(x)=\sum_{\tau=1}^{L} \sum_{v=1}^{N(0, \tau)} \mu\left(Q_{\tau}^{0, v}\right) \lambda_{\tau}^{0, v} \widetilde{D}_{Q_{\tau}^{0, v}}(x)+\sum_{k=1}^{L} \sum_{\tau=1}^{L} \sum_{v=1}^{N(k, \tau)} \mu\left(Q_{\tau}^{k, v}\right) \lambda_{\tau}^{k, v} \widetilde{D}_{k}\left(x, y_{\tau}^{k, v}\right)
$$

Then $f_{L} \in \mathcal{G}_{1}(\epsilon, \epsilon)$ and $f_{L} \in\left(\mathcal{G}_{0}^{\epsilon}(\beta, \gamma)\right)^{\prime}$ with any $\beta, \gamma \in(0, \epsilon)$, where $\epsilon$ can be any positive number in $\left(0, \epsilon_{1} \wedge \epsilon_{2}\right)$. In what follows, we choose $\epsilon>\max (\beta, \gamma)$ such that $p>p(s, \epsilon)$ for the spaces $b_{p, q}^{s}(\mathcal{X})$, and $p, q>p(s, \epsilon)$ for the spaces $f_{p, q}^{s}(\mathcal{X})$.

For any $\psi \in \mathcal{G}(\beta, \gamma)$ with $(\beta, \gamma)$ as in (7.45), $L_{1}, L_{2} \in \mathbb{N}$ and $L_{1}<L_{2}$, we have

$$
\begin{aligned}
\left|\left\langle f_{L_{2}}-f_{L_{1}}, \psi\right\rangle\right| \leq & \sum_{\tau=L_{1}+1}^{L_{2}} \sum_{\nu=1}^{N(0, \tau)} \mu\left(Q_{\tau}^{0, v}\right)\left|\lambda_{\tau}^{0, v}\right|\left|\left\langle\widetilde{D}_{Q_{\tau}^{0, v}}, \psi\right\rangle\right| \\
& +\sum_{k=L_{1}+1}^{L_{2}} \sum_{\tau=1}^{L_{2}} \sum_{v=1}^{N(k, \tau)} \mu\left(Q_{\tau}^{k, v}\right)\left|\lambda_{\tau}^{k, v}\right|\left|\left\langle\widetilde{D}_{k}\left(\cdot, y_{\tau}^{k, v}\right), \psi\right\rangle\right| \\
& +\sum_{k=1}^{L_{1}} \sum_{\tau=L_{1}+1}^{L_{2}} \sum_{v=1}^{N(k, \tau)} \mu\left(Q_{\tau}^{k, v}\right)\left|\lambda_{\tau}^{k, v}\right|\left|\left\langle\widetilde{D}_{k}\left(\cdot, y_{\tau}^{k, v}\right), \psi\right\rangle\right| \\
\equiv & Y_{1}+Y_{2}+Y_{3} .
\end{aligned}
$$

From (5.105), (5.5) when $p \leq 1$ or Hölder's inequality when $1<p \leq \infty$, and $\gamma>$ $n(1 / p-1)_{+}$together with (5.30), it follows that when $p \leq 1$,

$$
\left|Y_{1}\right| \lesssim\|\psi\|_{\mathcal{G}(\beta, \gamma)}\left\{\sum_{\tau=L_{1}+1}^{L_{2}} \sum_{\nu=1}^{N(0, \tau)} \mu\left(Q_{\tau}^{0, v}\right)\left|\lambda_{\tau}^{0, \nu}\right|^{p}\right\}^{1 / p}
$$

while when $1<p \leq \infty$,

$$
\begin{aligned}
\left|Y_{1}\right| \lesssim & \|\psi\|_{\mathcal{G}(\beta, \gamma)}\left\{\sum_{\tau=L_{1}+1}^{L_{2}} \sum_{\nu=1}^{N(0, \tau)} \mu\left(Q_{\tau}^{0, v}\right)\left|\lambda_{\tau}^{0, v}\right|^{p}\right\}^{1 / p} \\
& \times\left\{\int_{\chi_{L_{1}}^{L_{2}, 0}} \frac{1}{V_{1}\left(x_{1}\right)+V\left(x_{1}, y\right)} \frac{1}{\left(1+d\left(x_{1}, y\right)\right)^{\gamma}} d \mu(y)\right\}^{1 / p^{\prime}},
\end{aligned}
$$

where $\mathcal{X}_{L_{1}}^{L_{2}, 0}$ is as in the proof of Proposition 7.1. 
For $Y_{2}$, by (5.105), (5.5) when $p \leq 1$ or Hölder's inequality when $1<p \leq \infty, \gamma>$ $n(1 / p-1)_{+}$together with (5.30), and Lemma 2.1(ii), we obtain

$$
\left|Y_{2}\right| \lesssim\|\psi\|_{\mathcal{G}(\beta, \gamma)} \begin{cases}\sum_{k=L_{1}+1}^{L_{2}} 2^{-k[\beta+s-n(1 / p-1)]} 2^{k s}\left[\sum_{\tau=1}^{L_{2}} \sum_{v=1}^{N(k, \tau)} \mu\left(Q_{\tau}^{k, v}\right)\left|\lambda_{\tau}^{k, v}\right|^{p}\right]^{1 / p}, \quad p \leq 1 \\ \sum_{k=L_{1}+1}^{L_{2}} 2^{-k(\beta+s)} 2^{k s}\left[\sum_{\tau=1}^{L_{2}} \sum_{v=1}^{N(k, \tau)} \mu\left(Q_{\tau}^{k, v}\right)\left|\lambda_{\tau}^{k, v}\right|^{p}\right]^{1 / p}, & 1<p \leq \infty .\end{cases}
$$

From this and (5.5) when $q \leq 1$ or Hölder's inequality when $1<q \leq \infty$ again, it further follows that when $p \leq 1$ and $q \leq 1$,

$$
\left|Y_{2}\right| \lesssim\|\psi\|_{\mathcal{G}(\beta, \gamma)}\left\{\sum_{k=L_{1}+1}^{L_{2}} 2^{k s q}\left[\sum_{\tau=1}^{L_{2}} \sum_{\nu=1}^{N(k, \tau)} \mu\left(Q_{\tau}^{k, v}\right)\left|\lambda_{\tau}^{k, v}\right|^{p}\right]^{q / p}\right\}^{1 / q},
$$

while when $1<q \leq \infty$,

$$
\left|Y_{2}\right| \lesssim\|\psi\|_{\mathcal{G}(\beta, \gamma)}\left\{\sum_{k=L_{1}+1}^{L_{2}} 2^{k s q}\left[\sum_{\tau=1}^{L_{2}} \sum_{\nu=1}^{N(k, \tau)} \mu\left(Q_{\tau}^{k, v}\right)\left|\lambda_{\tau}^{k, v}\right|^{p}\right]^{q / p}\right\}^{1 / q}\left\{\sum_{k=L_{1}+1}^{L_{2}} 2^{-k[\beta+s-n(1 / p-1)] q^{\prime}}\right\}^{1 / q^{\prime}},
$$

and that when $1<p \leq \infty$,

$$
\left|Y_{2}\right| \lesssim\|\psi\|_{\mathcal{G}(\beta, \gamma)} \begin{cases}\left\{\sum_{k=L_{1}+1}^{L_{2}} 2^{k s q}\left[\sum_{\tau=1}^{L_{2}} \sum_{v=1}^{N(k, \tau)} \mu\left(Q_{\tau}^{k, v}\right)\left|\lambda_{\tau}^{k, v}\right|^{p}\right]^{q / p}\right\}^{1 / q}, & q \leq 1 \\ \left\{\sum_{k=L_{1}+1}^{L_{2}} 2^{k s q}\left[\sum_{\tau=1}^{L_{2}} \sum_{v=1}^{N(k, \tau)} \mu\left(Q_{\tau}^{k, v}\right)\left|\lambda_{\tau}^{k, v}\right|^{p}\right]^{q / p}\right\}^{1 / q}\left\{\sum_{k=L_{1}+1}^{L_{2}} 2^{-k(\beta+s) q^{\prime}}\right\}^{1 / q^{\prime}}, & 1<q \leq \infty .\end{cases}
$$

where we used the fact that $\beta>\max \left(0, n(1 / p-1)_{+}-s\right)$.

Similarly, by (5.105), (5.5) when $p \leq 1$ or Hölder's inequality when $1<p \leq \infty$, and $r>n(1 / p-1)$, together with (5.30), we have

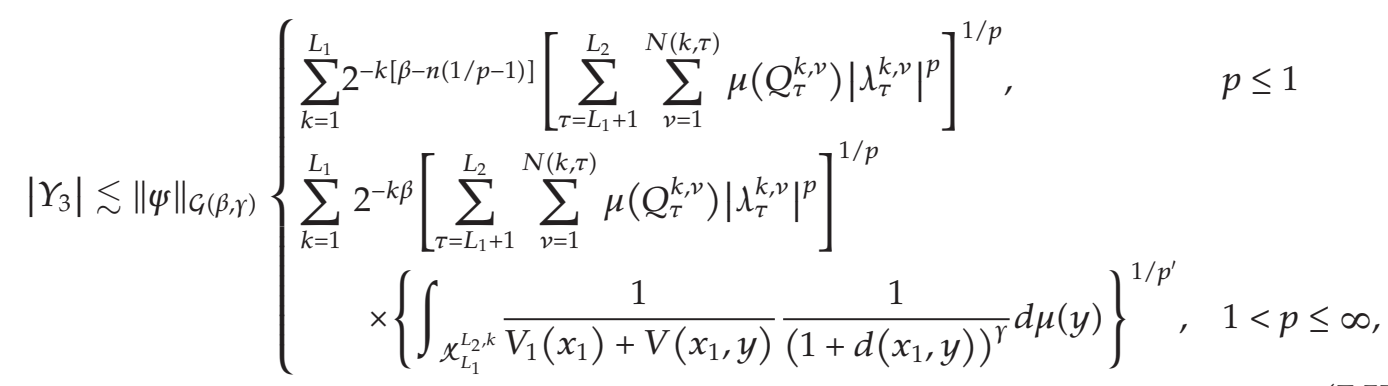

where $\mathcal{X}_{L_{1}}^{L_{2}, k}$ is as in the proof of Proposition 7.1. 
From this and (5.5) when $q \leq 1$ or Hölder's inequality when $1<q \leq \infty$, we further deduce that when $p \leq 1$,

$$
\left|Y_{3}\right| \lesssim\|\psi\|_{\mathcal{G}(\beta, \gamma)}\left\{\sum_{k=1}^{L_{1}} 2^{k s q}\left[\sum_{\tau=L_{1}+1}^{L_{2}} \sum_{\nu=1}^{N(k, \tau)} \mu\left(Q_{\tau}^{k, \nu}\right)\left|\lambda_{\tau}^{k, v}\right|^{p}\right]^{q / p}\right\}^{1 / q}
$$

and that when $1<p \leq \infty$ and $q \leq 1$,

$$
\left|Y_{3}\right| \lesssim\|\psi\|_{\mathcal{G}(\beta, \gamma)}\left\{\sum_{k=1}^{L_{1}} 2^{k s q}\left[\sum_{\tau=L_{1}+1}^{L_{2}} \sum_{\nu=1}^{N(k, \tau)} \mu\left(Q_{\tau}^{k, v}\right)\left|\lambda_{\tau}^{k, v}\right|^{p}\right]^{q / p}\right\}^{1 / q},
$$

or that when $1<p \leq \infty$ and $1<q \leq \infty$,

$$
\begin{aligned}
\left|Y_{3}\right| \lesssim & \|\psi\|_{\mathcal{G}(\beta, \gamma)}\left\{\sum_{k=1}^{L_{1}} 2^{k s q}\left[\sum_{\tau=L_{1}+1}^{L_{2}} \sum_{v=1}^{N(k, \tau)} \mu\left(Q_{\tau}^{k, v}\right)\left|\lambda_{\tau}^{k, v}\right|^{p}\right]^{q / p}\right\}^{1 / q} \\
& \times\left\{\sum_{k=1}^{L_{2}} 2^{-k(\beta+s) q^{\prime}}\left[\int_{x_{L_{1}}^{L_{2}, k}} \frac{1}{V_{1}\left(x_{1}\right)+V\left(x_{1}, y\right)} \frac{1}{\left(1+d\left(x_{1}, y\right)\right)^{\gamma}} d \mu(y)\right]^{q^{\prime} / p^{\prime}}\right\}^{1 / q^{\prime}},
\end{aligned}
$$

where we used the fact that $\beta>\max \left(0, n(1 / p-1)_{+}-s\right)$.

Combining (7.51) through (7.60), by $\lambda \in b_{p, q}^{s}(x)$,

$$
\int_{x} \frac{1}{V_{1}\left(x_{1}\right)+V\left(x_{1}, y\right)} \frac{1}{\left(1+d\left(x_{1}, y\right)\right)^{r}} d \mu(y)<\infty
$$

when $p=\infty$, and

$$
\sum_{k=1}^{\infty} 2^{-k[\beta+s-n(1 / p-1)]}<\infty
$$

when $p \leq 1$ and $q=\infty$, or

$$
\sum_{k=1}^{\infty} 2^{-k(\beta+s)}<\infty
$$

when $1<p \leq \infty$ and $q=\infty$, it is easy to see that $\left\{\left\langle f_{L}, \psi\right\rangle\right\}_{L \in \mathbb{N}}$ is a Cauchy sequence. This just means that the series in (7.44) converges to some $f \in\left(\mathcal{G}_{0}^{\epsilon}(\beta, \gamma)\right)^{\prime}$ with $\beta, \gamma$ satisfying (7.45) if $\lambda \in b_{p, q}^{s}(\mathcal{X})$ with $s, p, q$ as in the theorem. If $\lambda \in f_{p, q}^{s}(\mathcal{X})$ with $s, p, q$ as in the theorem, by this fact and

$$
b_{p, \min (p, q)}^{s}(\mathcal{X}) \subset f_{p, q}^{s}(\mathcal{X}) \subset b_{p, \max (p, q)}^{s}(\mathcal{X})
$$


(see [87, Proposition 2.3]), we also obtain that the series in $(7.44)$ converges in $\left(\mathcal{G}_{0}^{\epsilon}(\beta, \gamma)\right)^{\prime}$ with $\beta$ and $\gamma$ as in (7.45).

Let us now verify that the series in (7.44) converges in the norm of $B_{p, q}^{s}(\mathcal{X})$ when $p, q<\infty$, if $\lambda \in b_{p, q}^{s}(\mathcal{X})$. Let $f$ be the series in (7.44). We estimate the norm in $B_{p, q}^{s}(\mathcal{X})$ of $f-f_{L}$ by writing

$$
\begin{aligned}
f-f_{L}= & \sum_{\tau=L+1}^{\infty} \sum_{v=1}^{N(0, \tau)} \mu\left(Q_{\tau}^{0, v}\right) \lambda_{\tau}^{0, v} \widetilde{D}_{Q_{\tau}^{0, v}}(x) \\
& +\sum_{k=1}^{\infty} \sum_{\tau=L+1}^{\infty} \sum_{v=1}^{N(k, \tau)} \mu\left(Q_{\tau}^{k, v}\right) \lambda_{\tau}^{k, v} \widetilde{D}_{k}\left(x, y_{\tau}^{k, v}\right) \\
& +\sum_{k=L+1}^{\infty} \sum_{\tau=1}^{L} \sum_{v=1}^{N(k, \tau)} \mu\left(Q_{\tau}^{k, v}\right) \lambda_{\tau}^{k, v} \widetilde{D}_{k}\left(x, y_{\tau}^{k, v}\right) .
\end{aligned}
$$

Replacing $Q_{\tau^{\prime}, 1}^{0, v^{\prime}}(f)$ and $Q_{k^{\prime}}(f)\left(y_{\tau^{\prime}}^{k^{\prime}, v^{\prime}}\right)$ in the proof of Proposition 5.25 respectively by $\lambda_{\tau}^{0, v}$ and $\lambda_{\tau}^{k, v}$ here, and repeating the proof of Proposition 5.25, we then obtain

$$
\begin{aligned}
& \left\|f-f_{L}\right\|_{B_{p, q}^{s}(x)} \lesssim\left\{\sum_{\tau=L+1}^{\infty} \sum_{\nu=1}^{N(0, \tau)} \mu\left(Q_{\tau}^{0, v}\right)\left|\lambda_{\tau}^{0, v}\right|^{p}\right\}^{1 / p} \\
& +\left\{\sum_{k=1}^{\infty} 2^{k s q}\left[\sum_{\tau=L+1}^{\infty} \sum_{\nu=1}^{N(k, \tau)} \mu\left(Q_{\tau}^{k, v}\right)\left|\lambda_{\tau}^{k, v}\right|^{p}\right]^{q / p}\right\}^{1 / q} \\
& +\left\{\sum_{k=L+1}^{\infty} 2^{k s q}\left[\sum_{\tau=1}^{L} \sum_{v=1}^{N(k, \tau)} \mu\left(Q_{\tau}^{k, v}\right)\left|\lambda_{\tau}^{k, v}\right|^{p}\right]^{q / p}\right\}^{1 / q}, \\
& \left\|f-f_{L}\right\|_{F_{p, q}^{s}(x)} \lesssim\left\{\sum_{\tau=L+1}^{\infty} \sum_{\nu=1}^{N(0, \tau)} \mu\left(Q_{\tau}^{0, v}\right)\left|\lambda_{\tau}^{0, v}\right|^{p}\right\}^{1 / p} \\
& +\left\|\left\{\sum_{k=1}^{\infty} \sum_{\tau=L+1}^{\infty} \sum_{\nu=1}^{N(k, \tau)} 2^{k s q}\left|\lambda_{\tau}^{k, \nu}\right|{ }^{q} X_{Q_{\tau}^{k, v}}\right\}^{1 / q}\right\|_{L^{p}(\mathcal{X})} \\
& +\left\|\left\{\sum_{k=L+1}^{\infty} \sum_{\tau=1}^{L} \sum_{\nu=1}^{N(k, \tau)} 2^{k s q}\left|\lambda_{\tau}^{k, v}\right|^{q} X_{Q_{\tau}^{k, v}}\right\}^{1 / q}\right\|_{L^{p}(\mathcal{X})} .
\end{aligned}
$$

Then Lebesgue's dominated convergence theorems on the integral and the series show that

$$
\begin{aligned}
& \left\|f-f_{L}\right\|_{B_{p, q}^{s}(x)} \longrightarrow 0, \\
& \left\|f-f_{L}\right\|_{F_{p, q}^{s}(x)} \longrightarrow 0
\end{aligned}
$$

as $L \rightarrow \infty$. Moreover, by Proposition 5.31(v), we know that $f_{L} \in B_{p, q}^{s}(\mathcal{X})$ and $f_{L} \in F_{p, q}^{s}(\mathcal{X})$ 
if we choose $\epsilon$ large enough. Thus, $f \in B_{p, q}^{s}(\mathcal{X})$ if $\lambda \in b_{p, q}^{s}(\mathcal{X})$ and $f \in F_{p, q}^{s}(\mathcal{X})$ if $\lambda \in f_{p, q}^{s}(\mathcal{X})$ when $p, q<\infty$.

The same arguments as in the proof of Propositions 5.25 and 6.15 for the space $B_{p, q}^{s}(\mathcal{X})$ and the space $F_{p, q}^{s}(\mathcal{X})$ with all $p, q$ as in the assumption of the theorem yield (7.46) and (7.47), respectively, which completes the proof of Proposition 7.3.

From Theorem 4.16, Proposition 7.3, and the Plancherel-Pôlya inequalities, Propositions 5.25 and 6.15, we obtain the following frame characterizations of the spaces $B_{p, q}^{s}(x)$ and $F_{p, q}^{s}(X)$.

Theorem 7.4. Let $\epsilon$ be as in Definition 5.29, let $|s|<\epsilon$, and let $p(s, \epsilon)<p \leq \infty$. Let all the other notation be as in Theorem 4.14, $\lambda_{\tau}^{0, v}=m_{Q_{\tau}^{0, v}}\left(D_{0}(f)\right)$ for $\tau \in I_{0}$ and $v=1, \ldots, N(0, \tau)$, and $\lambda_{\tau}^{k, v}=$ $D_{k}(f)\left(y_{\tau}^{k, v}\right)$ for $k \in \mathbb{N}, \tau \in I_{k}$ and $v=1, \ldots, N(k, \tau)$, where $y_{\tau}^{k, v}$ is any fixed element in $Q_{\tau}^{k, v}$. Then, the following hold.

(i) If $0<q \leq \infty$, then $f \in B_{p, q}^{s}(x)$ if and only if $f \in\left(\mathcal{G}_{0}^{\epsilon}(\beta, \gamma)\right)^{\prime}$ for some $\beta, \gamma$ as in (5.111),

$$
f(x)=\sum_{\tau \in I_{0}} \sum_{\nu=1}^{N(0, \tau)} \lambda_{\tau}^{0, v} \int_{Q_{\tau}^{k, v}} \widetilde{D}_{0}(x, y) d \mu(y)+\sum_{k=1}^{\infty} \sum_{\tau \in I_{k}} \sum_{v=1}^{N(k, \tau)} \mu\left(Q_{\tau}^{k, v}\right) D_{k}(f)\left(y_{\tau}^{k, v}\right) \widetilde{D}_{k}\left(x, y_{\tau}^{k, v}\right)
$$

holds in $\left(\mathcal{G}_{0}^{\epsilon}(\beta, \gamma)\right)^{\prime}$, and $\lambda \in b_{p, q}^{s}(\mathcal{X})$. Moreover, in this case,

$$
\|f\|_{B_{p, q}^{s}(x)} \sim\|\lambda\|_{b_{p, q}^{s}(x)} .
$$

(ii) If $p(s, \epsilon)<q \leq \infty$, then $f \in F_{p, q}^{s}(\mathcal{X})$ if and only if $f \in\left(\mathcal{G}_{0}^{\epsilon}(\beta, \gamma)\right)^{\prime}$ for some $\beta, \gamma$ as in (5.111), (7.68) holds in $\left(\mathcal{G}_{0}^{\epsilon}(\beta, \gamma)\right)^{\prime}$, and $\lambda \in f_{p, q}^{s}(\mathcal{X})$. Moreover, in this case,

$$
\|f\|_{F_{p, q}^{s}(x)} \sim\|\lambda\|_{f_{p, q}^{s}(x)} .
$$

\section{Real interpolation and dual spaces}

In this section, using the frame characterization of Besov spaces and Triebel-Lizorkin spaces in the last section, we characterize real interpolation spaces for our scales of Besov and Triebel-Lizorkin spaces and determine their dual spaces (when $p \geq 1$ ).

\subsection{Real interpolation spaces}

Let us first recall some general background on the real interpolation method; see [3, pages 62-64] or [88, 89].

Let $\mathscr{t}$ be a linear complex Hausdorff space, and let $\mathcal{A}_{0}$ and $\mathcal{A}_{1}$ be two complex quasiBanach spaces such that $\mathscr{A}_{0} \subset \mathscr{d}$ and $\mathscr{A}_{1} \subset \mathscr{d}$. Let $\mathscr{A}_{0}+\mathscr{A}_{1}$ be the set of all elements $a \in \mathscr{d}$ which can be represented as $a=a_{0}+a_{1}$ with $a_{0} \in \mathcal{A}_{0}$ and $a_{1} \in \mathcal{A}_{1}$. If $0<t<\infty$ and $a \in \mathscr{A}_{0}+\mathcal{A}_{1}$, then Peetre's $K$-functional is given by

$$
K(t, a)=K\left(t, a ; \mathscr{A}_{0}, \mathcal{A}_{1}\right)=\inf \left(\left\|a_{0}\right\|_{\mathcal{A}_{0}}+t\left\|a_{1}\right\|_{\mathcal{A}_{1}}\right),
$$


where the infimum is taken over all representations of $a$ of the form $a=a_{0}+a_{1}$ with $a_{0} \in \mathcal{A}_{0}$ and $a_{1} \in \mathscr{A}_{1}$.

Definition 8.1. Let $0<\sigma<1$. If $0<q<\infty$, then one defines the interpolation space

$$
\left(\mathcal{A}_{0}, \mathcal{A}_{1}\right)_{\sigma, q} \equiv\left\{a: a \in \mathscr{A}_{0}+\mathcal{A}_{1},\|a\|_{\left(\mathcal{A}_{0}, \mathcal{A}_{1}\right)_{\sigma, q}} \equiv\left\{\int_{0}^{\infty}\left[t^{-\sigma} K(t, a)\right]^{q} \frac{d t}{t}\right\}^{1 / q}<\infty\right\} \text {. }
$$

If $q=\infty$, then one defines

$$
\left(\mathcal{A}_{0}, \mathcal{A}_{1}\right)_{\sigma, \infty} \equiv\left\{a: a \in \mathcal{A}_{0}+\mathcal{A}_{1},\|a\|_{\left(\mathcal{A}_{0}, \mathscr{A}_{1}\right)_{\sigma, \infty}} \equiv \sup _{0<t<\infty} t^{-\sigma} K(t, a)<\infty\right\} .
$$
page 64].

The following basic properties of $\left(\mathcal{A}_{0}, \mathcal{A}_{1}\right)_{\sigma, q}$ are proved in [3, pages 63-64] and [88,

Proposition 8.2. Let $\mathcal{A}_{0}$ and $\mathcal{A}_{1}$ be two complex quasi-Banach spaces. Let $0<\sigma<1$ and $0<q \leq \infty$. Then,

(i) $\left(\mathscr{A}_{0}, \mathscr{A}_{1}\right)_{\sigma, q}$ is a quasi-Banach space;

(ii) $\left(\mathscr{A}_{0}, \mathcal{A}_{1}\right)_{\sigma, q}=\left(\mathcal{A}_{1}, \mathcal{A}_{0}\right)_{1-\sigma, q}$;

(iii) let th be a linear complex Hausdorff space, and let $\boldsymbol{B}_{0}$ and $\boldsymbol{B}_{1}$ be two complex quasi-Banach spaces such that $\mathcal{A}_{0} \subset \boldsymbol{B}_{0} \subset$ de and $\mathcal{A}_{1} \subset \boldsymbol{B}_{1} \subset \mathcal{H}$. Then $\left(\mathcal{A}_{0}, \mathcal{A}_{1}\right)_{\sigma, q} \subset\left(\mathbb{B}_{0}, \boldsymbol{B}_{1}\right)_{\sigma, q}$.

Using Theorems 7.2 and 7.4 together with the method of retraction and coretraction as in the proofs of Theorems 2.4.1 and 2.4.2 in [89], we can easily deduce the following interpolation theorems; see also [90].

Theorem 8.3. Let $\epsilon$ be as in Definition 5.8 and $\sigma \in(0,1)$.

(i) Let $-\epsilon<s_{0}, s_{1}<\epsilon, s_{0} \neq s_{1}, 1 \leq p \leq \infty$, and $1 \leq q_{0}, q_{1}, q \leq \infty$. Then

$$
\left(\dot{B}_{p, q_{0}}^{s_{0}}(\mathcal{X}), \dot{B}_{p, q_{1}}^{s_{1}}(\mathcal{X})\right)_{\sigma, q}=\dot{B}_{p, q}^{s}(\mathcal{X}),
$$

where $s=(1-\sigma) s_{0}+\sigma s_{1}$.

(ii) Let $-\epsilon<s<\epsilon, 1 \leq p \leq \infty, 1 \leq q_{0}, q_{1} \leq \infty$, and $q_{0} \neq q_{1}$. Then

$$
\left(\dot{B}_{p, q_{0}}^{s}(\mathcal{X}), \dot{B}_{p, q_{1}}^{s}(\mathcal{X})\right)_{\sigma, q}=\dot{B}_{p, q}^{s}(\mathcal{X}),
$$

where $1 / q=(1-\sigma) / q_{0}+\sigma / q_{1}$.

(iii) Let $-\epsilon<s_{0}, s_{1}<\epsilon, 1 \leq p_{0}, p_{1} \leq \infty$, and $p_{0} \neq p_{1}$. Then

$$
\left(\dot{B}_{p_{0}, p_{0}}^{S_{0}}(\mathcal{X}), \dot{B}_{p_{1}, p_{1}}^{S_{1}}(\mathcal{X})\right)_{\sigma, p}=\dot{B}_{p, p}^{s}(\mathcal{X})
$$

where $1 / p=(1-\sigma) / p_{0}+\sigma / p_{1}$ and $s=(1-\sigma) s_{0}+\sigma s_{1}$. 
Theorem 8.4. Let $\epsilon$ be as in Definition 5.8, $-\epsilon<s_{0}, s_{1}<\epsilon, 1 \leq p_{0}, p_{1}<\infty, 1 \leq q_{0}, q_{1} \leq \infty$, $\sigma \in(0,1), s=(1-\sigma) s_{0}+\sigma s_{1}, 1 / p=(1-\sigma) / p_{0}+\sigma / p_{1}$, and $1 / q=(1-\sigma) / q_{0}+\sigma / q_{1}$.

(i) If $s_{0} \neq s_{1}$, then

$$
\left(\dot{F}_{p_{0}, q_{0}}^{s_{0}}(\mathcal{X}), \dot{F}_{p_{1}, q_{1}}^{s_{1}}(\mathcal{X})\right)_{\sigma, p}=\dot{F}_{p, p}^{s}(\mathcal{X})
$$

(ii) If $s_{0}=s_{1}=s, p_{0}=q_{0}, p_{1}=q_{1}$, and $q_{0} \neq q_{1}$, then

$$
\left(\dot{F}_{p_{0}, p_{0}}^{s}(\mathcal{X}), \dot{F}_{p_{1}, p_{1}}^{s}(\mathcal{X})\right)_{\sigma, p}=\dot{F}_{p, p}^{s}(\mathcal{X})
$$

(iii) If $s_{0}=s_{1}=s, q_{0}=q_{1}=q$, and $p_{0} \neq p_{1}$, then

$$
\left(\dot{F}_{p_{0}, q}^{s}(\mathcal{X}), \dot{F}_{p_{1}, q}^{s}(\mathcal{X})\right)_{\sigma, p}=\dot{F}_{p, q}^{s}(\mathcal{X}) .
$$

Theorem 8.5. Let $\epsilon$ be as in Definition 5.29 and $\sigma \in(0,1)$.

(i) Let $-\epsilon<s_{0}, s_{1}<\epsilon, s_{0} \neq s_{1}, 1 \leq p \leq \infty$, and $1 \leq q_{0}, q_{1}, q \leq \infty$. Then

$$
\left(B_{p, q_{0}}^{s_{0}}(\mathcal{X}), B_{p, q_{1}}^{s_{1}}(\mathcal{X})\right)_{\sigma, q}=B_{p, q}^{s}(\mathcal{X}),
$$

where $s=(1-\sigma) s_{0}+\sigma s_{1}$.

(ii) Let $-\epsilon<s<\epsilon, 1 \leq p \leq \infty, 1 \leq q_{0}, q_{1} \leq \infty$, and $q_{0} \neq q_{1}$. Then

$$
\left(B_{p, q_{0}}^{s}(\mathcal{X}), B_{p, q_{1}}^{s}(\mathcal{X})\right)_{\sigma, q}=B_{p, q}^{s}(\mathcal{X})
$$

where $1 / q=(1-\sigma) / q_{0}+\sigma / q_{1}$.

(iii) Let $-\epsilon<s_{0}, s_{1}<\epsilon, 1 \leq p_{0}, p_{1} \leq \infty$, and $p_{0} \neq p_{1}$. Then

$$
\left(B_{p_{0}, p_{0}}^{S_{0}}(\mathcal{X}), B_{p_{1}, p_{1}}^{s_{1}}(\mathcal{X})\right)_{\sigma, p}=B_{p, p}^{s}(\mathcal{X})
$$

where $1 / p=(1-\sigma) / p_{0}+\sigma / p_{1}$ and $s=(1-\sigma) s_{0}+\sigma s_{1}$.

Theorem 8.6. Let $\epsilon$ be as in Definition 5.29, $-\epsilon<s_{0}, s_{1}<\epsilon, 1 \leq p_{0}, p_{1}<\infty, 1 \leq q_{0}, q_{1} \leq \infty$, $\sigma \in(0,1), s=(1-\sigma) s_{0}+\sigma s_{1}, 1 / p=(1-\sigma) / p_{0}+\sigma / p_{1}$, and $1 / q=(1-\sigma) / q_{0}+\sigma / q_{1}$.

(i) If $s_{0} \neq s_{1}$, then

$$
\left(F_{p_{0}, q_{0}}^{S_{0}}(X), F_{p_{1}, q_{1}}^{s_{1}}(X)\right)_{\sigma, p}=F_{p, p}^{s}(X)
$$

(ii) If $s_{0}=s_{1}=s, p_{0}=q_{0}, p_{1}=q_{1}$, and $q_{0} \neq q_{1}$, then

$$
\left(B_{p_{0}, p_{0}}^{S}(\mathcal{X}), B_{p_{1}, p_{1}}^{s}(\mathcal{X})\right)_{\sigma, p}=B_{p, p}^{S}(\mathcal{X}) .
$$


(iii) If $s_{0}=s_{1}=s, q_{0}=q_{1}=q$, and $p_{0} \neq p_{1}$, then

$$
\left(F_{p_{0}, q}^{s}(\mathcal{X}), F_{p_{1}, q}^{s}(\mathcal{X})\right)_{\sigma, p}=F_{p, q}^{s}(\mathcal{X}) .
$$

Proofs of Theorem 8.3 through Theorem 8.6. The proofs of Theorem 8.3 through Theorem 8.6 are similar by using [89, Theorem 1.2.4]. We only give an outline here; see also [90, the proofs of Proposition 3.3 and Theorem 3.1].

To prove Theorems 8.3 and 8.4, by Proposition 5.10(iii), we know that

$$
\dot{B}_{p_{i}, q_{i}}^{s_{i}}(\mathcal{X}), \dot{F}_{p_{i}, q_{i}}^{s_{i}}(\mathcal{X}) \subset\left(\dot{\mathcal{G}}_{0}^{\epsilon}\left(\beta_{i}, \gamma_{i}\right)\right)^{\prime}
$$

where $\max \left\{0,-s_{i}+n\left(1 / p_{i}-1\right)_{+}\right\}<\beta_{i}<\epsilon$ and $\max \left\{n\left(1 / p_{i}-1\right)_{+}, s_{i}-\kappa / p_{i}\right\}<\gamma_{i}<\epsilon$ with $i=0,1$. We then let $\beta=\max \left(\beta_{0}, \beta_{1}\right)$ and $\gamma=\left(\gamma_{0}, \gamma_{1}\right)$. Then

$$
\dot{B}_{p_{i}, q_{i}}^{s_{i}}(\mathcal{X}), \dot{F}_{p_{i}, q_{i}}^{s_{i}}(\mathcal{X}) \subset\left(\mathcal{G}_{0}^{\epsilon}(\beta, \gamma)\right)^{\prime} .
$$

In this sense, $\left\{\dot{B}_{p_{0}, q_{0}}^{s_{0}}(\mathcal{X}), \dot{B}_{p_{1}, q_{1}}^{s_{1}}(\mathcal{X})\right\}$ and $\left\{\dot{F}_{p_{0}, q_{0}}^{s_{0}}(\mathcal{X}), \dot{F}_{p_{1}, q_{1}}^{s_{1}}(\mathcal{X})\right\}$ are interpolation couples in the sense of [89, Section 1.2.1]. Now, for $f \in\left(\mathcal{G}_{0}^{\epsilon}(\beta, \gamma)\right)^{\prime}$, with the notation of Theorem 4.11, we can define the coretraction operator $\dot{S}$ by

$$
\dot{S}(f)(x)=\left\{\dot{S}(f)_{k}(x)\right\}_{k=-\infty}^{\infty}
$$

where for $k \in \mathbb{Z}$,

$$
\dot{S}(f)_{k}(x)=\sum_{\tau \in I_{k}} \sum_{v=1}^{N(k, \tau)} D_{k}(f)\left(y_{\tau}^{k, v}\right) X_{Q_{\tau}^{k, v}}(x)
$$

and the corresponding retraction operator $\dot{R}$ by

$$
\dot{R}\left(\left\{f_{k}\right\}\right)(x)=\sum_{k=-\infty}^{\infty} \sum_{\tau \in I_{k}} \sum_{\nu=1}^{N(k, \tau)}\left[\int_{Q_{\tau}^{k, \nu}} f_{k}(y) d \mu(y)\right] \tilde{D}_{k}\left(x, y_{\tau}^{k, \nu}\right) .
$$

By Theorem 4.13, for any $f \in\left(\mathcal{G}_{0}^{\epsilon}(\beta, \gamma)\right)^{\prime}$, we have $\dot{R} \dot{S}(f)(x)=f(x)$. In what follows, for $s \in \mathbb{R}$, $0<q \leq \infty$, and $0<p \leq \infty$, we say $\left\{f_{k}\right\}_{k=-\infty}^{\infty} \in \dot{\ell}^{s, q}\left(L^{p}\right)(x)$, if

$$
\left\|\left\{f_{k}\right\}_{k=-\infty}^{\infty}\right\|_{\dot{\ell}^{s, q}\left(L^{p}\right)(x)}=\left\{\sum_{k=-\infty}^{\infty} 2^{k s q}\left\|f_{k}\right\|_{L^{p}(\mathcal{X})}^{q}\right\}^{1 / q}<\infty ;
$$

and we say $\left\{f_{k}\right\}_{k=-\infty}^{\infty} \in L^{p}\left(\dot{\ell}^{s, q}\right)(\mathcal{X})$, if

$$
\left\|\left\{f_{k}\right\}_{k=-\infty}^{\infty}\right\|_{L^{p}(\dot{e}, s)(x)}=\left\|\left\{\sum_{k=-\infty}^{\infty} 2^{k s q}\left|f_{k}(x)\right|^{q}\right\}^{1 / q}\right\|_{L^{p}(x)}<\infty,
$$


where the usual modifications are made when $p=\infty$ or $q=\infty$. If $F$ is an interpolation functor, then one obtains by [89, Theorem 1.2.4] that

$$
\begin{aligned}
& \|f\|_{F\left(\left\{\dot{B}_{p_{0}, q_{0}}^{s_{0}}(x), \dot{B}_{p_{1}, q_{1}}^{\dot{s}_{1}}(x)\right\}\right)} \sim\|\dot{S}(f)\|_{F\left(\left\{\dot{\ell}^{s_{0}, q_{0}}\left(L^{p_{0}}\right)(x), \dot{\ell}^{s_{1}, q_{1}}\left(L^{p_{1}}\right)(x)\right\}\right)},
\end{aligned}
$$

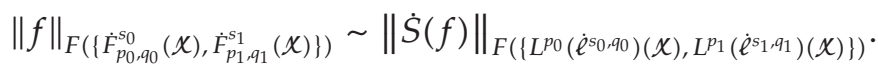

Using Proposition 7.1 and Theorem 7.2, we can then finish the proofs of Theorems 8.3 and 8.4 by the same procedures as those in [89, pages 182-183] and [89, pages 185-186].

To prove Theorems 8.5 and 8.6 , by Proposition 5.31(iv), we know that

$$
B_{p_{i}, q_{i}}^{S_{i}}(\mathcal{X}), F_{p_{i}, q_{i}}^{S_{i}}(\mathcal{X}) \subset\left(\mathcal{G}_{0}^{\epsilon}\left(\beta_{i}, \gamma_{i}\right)\right)^{\prime},
$$

where $\max \left\{0,-s_{i}+n\left(1 / p_{i}-1\right)_{+}\right\}<\beta_{i}<\epsilon$ and $n\left(1 / p_{i}-1\right)_{+}<\gamma_{i}<\epsilon$ with $i=0$, 1 . We then let $\beta=\max \left(\beta_{0}, \beta_{1}\right)$ and $\gamma=\left(\gamma_{0}, \gamma_{1}\right)$. Then

$$
B_{p_{i}, q_{i}}^{s_{i}}(\mathcal{X}), F_{p_{i}, q_{i}}^{S_{i}}(\mathcal{X}) \subset\left(\mathcal{G}_{0}^{\epsilon}(\beta, \gamma)\right)^{\prime}
$$

In this sense, $\left\{B_{p_{0}, q_{0}}^{s_{0}}(\mathcal{X}), B_{p_{1}, q_{1}}^{S_{1}}(\mathcal{X})\right\}$ and $\left\{F_{p_{0}, q_{0}}^{S_{0}}(\mathcal{X}), F_{p_{1}, q_{1}}^{S_{1}}(\mathcal{X})\right\}$ are interpolation couples in the sense of [89, Section 1.2.1]. Now, for $f \in\left(\mathcal{G}_{0}^{\epsilon}(\beta, \gamma)\right)^{\prime}$, with the notation of Theorem 4.14, we can define the coretraction operator $S$ by

$$
S(f)(x)=\left\{S(f)_{k}(x)\right\}_{k=0}^{\infty}
$$

where

$$
S(f)_{0}(x)=\sum_{\tau \in I_{0}} \sum_{v=1}^{N(0, \tau)} m_{Q_{\tau}^{0, v}}\left(D_{0}(f)\right) \chi_{Q_{\tau}^{0, v}}(x)
$$

and for $k \in \mathbb{N}$,

$$
S(f)_{k}(x)=\sum_{\tau \in I_{k}} \sum_{\nu=1}^{N(k, \tau)} D_{k}(f)\left(y_{\tau}^{k, v}\right) X_{Q_{\tau}^{k, v}}(x)
$$

and the corresponding retraction operator $R$ by

$$
R\left(\left\{f_{k}\right\}\right)(x)=\sum_{\tau \in I_{0}} \sum_{\nu=1}^{N(0, \tau)}\left[\int_{Q_{\tau}^{0, \nu}} f_{0}(y) d \mu(y)\right] \widetilde{D}_{Q_{\tau}^{0, v}}(x)+\sum_{k=1}^{\infty} \sum_{\tau \in I_{k}} \sum_{\nu=1}^{N(k, \tau)}\left[\int_{Q_{\tau}^{k, \nu}} f_{k}(y) d \mu(y)\right] \widetilde{D}_{k}\left(x, y_{\tau}^{k, \nu}\right),
$$

where $\widetilde{D}_{Q_{\tau}^{0, v}}(x)$ is as in the proof of Proposition 7.3. By Theorem 4.16, for any $f \in\left(\mathcal{G}_{0}^{\epsilon}(\beta, \gamma)\right)^{\prime}$, 
we have $R S(f)(x)=f(x)$. In what follows, for $s \in \mathbb{R}, 0<q \leq \infty$, and $0<p \leq \infty$, we say $\left\{f_{k}\right\}_{k=0}^{\infty} \in \ell^{s, q}\left(L^{p}\right)(x)$, if

$$
\left\|\left\{f_{k}\right\}_{k=0}^{\infty}\right\|_{\mathcal{\ell}^{s, q}\left(L^{p}\right)(\mathcal{X})}=\left\{\sum_{k=0}^{\infty} 2^{k s q}\left\|f_{k}\right\|_{L^{p}(\mathcal{X})}^{q}\right\}^{1 / q}<\infty ;
$$

and we say $\left\{f_{k}\right\}_{k=0}^{\infty} \in L^{p}\left(\ell^{s, q}\right)(\mathcal{X})$, if

$$
\left\|\left\{f_{k}\right\}_{k=0}^{\infty}\right\|_{L^{p}\left(\mathcal{\ell}^{s, q)}(\mathcal{X})\right.}=\left\|\left\{\sum_{k=0}^{\infty} 2^{k s q}\left|f_{k}(x)\right|^{q}\right\}^{1 / q}\right\|_{L^{p}(\mathcal{X})}<\infty,
$$

where the usual modifications are made when $p=\infty$ or $q=\infty$. If $F$ is an interpolation functor, then one obtains by [89, Theorem 1.2.4] that

$$
\begin{aligned}
& \|f\|_{F\left(\left\{B_{p_{0}, q_{0}}^{s_{0}}(x), B_{p_{1}, q_{1}}^{s_{1}}(x)\right\}\right)} \sim\|S(f)\|_{F\left(\left\{\ell^{s_{0}, q_{0}}\left(L^{p_{0}}\right)(x), \ell^{s_{1}, q_{1}}\left(L^{p_{1}}\right)(x)\right\}\right)^{\prime}} \\
& \|f\|_{F\left(\left\{F_{p_{0}, q_{0}}^{s_{0}}(\mathcal{X}), F_{p_{1}, q_{1}}^{s_{1}}(x)\right\}\right)} \sim\|S(f)\|_{F\left(\left\{L^{p_{0}}\left(\ell^{s_{0}, q_{0}}\right), L^{p_{1}}\left(\ell^{s_{1}, q_{1}}\right)\right\}\right)^{\prime}} .
\end{aligned}
$$

Using Proposition 7.3 and Theorem 7.4, we then finish the proofs of Theorems 8.5 and 8.6 by the same procedures as those in [89, pages 182-183] and [89, pages 185-186], which completes the proofs of Theorem 8.3 through Theorem 8.6.

We remark that Theorem 8.3 through Theorem 8.6 only deal with Besov spaces and Triebel-Lizorkin spaces which are Banach spaces, since $p, q \geq 1$. Using Theorems 7.2 and 7.4 together with the following fact that for $0<p_{0} \neq p_{1} \leq \infty$ and $\sigma \in(0,1)$, if $1 / p=(1-\sigma) / p_{0}+$ $\sigma / p_{1}$

$$
\begin{aligned}
& \left(\dot{\ell}^{p_{0}}, \dot{\ell}^{p_{1}}\right)_{\sigma, p}=\dot{\ell}^{p} \\
& \left(\ell^{p_{0}}, \ell^{p_{1}}\right)_{\sigma, p}=\ell^{p}
\end{aligned}
$$

(see [88, Theorem 5.2.1] and also [89, Remark 1.18.6/5]), by a method similar to the proofs of Theorem 8.3 through Theorem 8.6 (see also [82, Corollary 6.6]), we can easily establish the following interpolation theorem which covers also cases when $p<1$. We omit the details.

Theorem 8.7. Let $\epsilon$ be as in Definition 5.8, $s_{0}, s_{1} \in(-\epsilon, \epsilon), p\left(s_{0}, \epsilon\right)<p_{0} \leq \infty, p\left(s_{1}, \epsilon\right)<p_{1} \leq \infty$, and $p_{0} \neq p_{1}$. Let $\sigma \in(0,1), 1 / p=(1-\sigma) / p_{0}+\sigma / p_{1}$, and $s=(1-\sigma) s_{0}+\sigma s_{1}$. Then,

(i) $\left(\dot{B}_{p_{0}, p_{0}}^{s_{0}}(\mathcal{X}), \dot{B}_{p_{1}, p_{1}}^{s_{1}}(\mathcal{X})\right)_{\sigma, p}=\dot{B}_{p, p}^{s}(\mathcal{X})$;

(ii) $\left(B_{p_{0}, p_{0}}^{s_{0}}(\mathcal{X}), B_{p_{1}, p_{1}}^{s_{1}}(\mathcal{X})\right)_{\sigma, p}=B_{p, p}^{s}(\mathcal{X})$.

Making use of Calderón reproducing formulae in place of frame characterizations, we can also directly establish the following real interpolation theorems for Besov and TriebelLizorkin spaces which are only quasi-Banach spaces; see also [3, Theorem 2.4.2] and [91]. 
Theorem 8.8. Let $\epsilon$ be as in Definition 5.8, $\sigma \in(0,1), s_{0}, s_{1} \in(-\epsilon, \epsilon), s_{0} \neq s_{1}$, and $s=(1-\sigma) s_{0}+$ $\sigma s_{1}$. Then

(i) if $\max \left\{p\left(s_{0}, \epsilon\right), p\left(s_{1}, \epsilon\right)\right\}<p \leq \infty$ and $0<q_{0}, q_{1}, q \leq \infty$, then

$$
\left(\dot{B}_{p, q_{0}}^{s_{0}}(\mathcal{X}), \dot{B}_{p, q_{1}}^{s_{1}}(\mathcal{X})\right)_{\sigma, q}=\dot{B}_{p, q}^{s}(\mathcal{X})
$$

(ii) if $\max \left\{p\left(s_{0}, \epsilon\right), p\left(s_{1}, \epsilon\right)\right\}<p \leq \infty, p\left(s_{i}, \epsilon\right)<q_{i} \leq \infty$ for $i=0,1$ and $0<q \leq \infty$, then

$$
\left(\dot{F}_{p, q_{0}}^{s_{0}}(\mathcal{X}), \dot{F}_{p, q_{1}}^{s_{1}}(\mathcal{X})\right)_{\sigma, q}=\dot{B}_{p, q}^{s}(\mathcal{X}) .
$$

Proof. We first verify (i). By Proposition 5.10(iii), we know that

$$
\dot{B}_{p, q_{0}}^{s_{0}}(\mathcal{X}), \dot{B}_{p, q_{1}}^{s_{1}}(\mathcal{X}) \subset\left(\dot{\mathcal{G}}_{0}^{\epsilon}(\beta, \gamma)\right)^{\prime}
$$

with $\max \left\{0,-s_{0}+n(1 / p-1)_{+},-s_{1}+n(1 / p-1)_{+}\right\}<\beta<\epsilon$ and $\max \left\{n(1 / p-1)_{+}, s_{0}-\kappa / p, s_{1}-\right.$ $\kappa / p\}<\gamma<\epsilon$. Thus, we can take $\mathscr{\ell}=\left(\mathcal{G}_{0}^{\epsilon}(\beta, \gamma)\right)^{\prime}$ with $\beta$ and $\gamma$ as above.

We now verify that

$$
\left(\dot{B}_{p, \infty}^{s_{0}}(\mathcal{X}), \dot{B}_{p, \infty}^{s_{1}}(\mathcal{X})\right)_{\sigma, q} \subset \dot{B}_{p, q}^{s}(\mathcal{X}) .
$$

By Proposition 8.2(ii), without loss of generality, we may assume that $s_{0}>s_{1}$.

Assume that $f \in\left(\dot{B}_{p, \infty}^{s_{0}}(\mathcal{X}), \dot{B}_{p, \infty}^{s_{1}}(\mathcal{X})\right)_{\sigma, q}$ and $f=f_{0}+f_{1}$ with $f_{0} \in \dot{B}_{p, \infty}^{s_{0}}(\mathcal{X})$ and $f_{1} \in$ $\dot{B}_{p, \infty}^{s_{1}}(\mathcal{X})$. Let $\left\{D_{k}\right\}_{k \in \mathbb{Z}}$ be as in Definition 5.8. Then,

$$
\begin{aligned}
2^{k s_{0}}\left\|D_{k}(f)\right\|_{L^{p}(\mathcal{X})} & \lesssim 2^{k s_{0}}\left\|D_{k}\left(f_{0}\right)\right\|_{L^{p}(\mathcal{X})}+2^{k\left(s_{0}-s_{1}\right)} 2^{k s_{1}}\left\|D_{k}\left(f_{1}\right)\right\|_{L^{p}(\mathcal{X})} \\
& \lesssim\left\|f_{0}\right\|_{\dot{B}_{p, \infty}^{s_{0}}(\mathcal{X})}+2^{k\left(s_{0}-s_{1}\right)}\left\|f_{1}\right\|_{\dot{B}_{p, o}^{s_{1}}(\mathcal{X})} .
\end{aligned}
$$

Taking the infimum on all representations $f=f_{0}+f_{1}$ yields that

$$
2^{k s_{0}}\left\|D_{k}(f)\right\|_{L^{p}(\mathcal{X})} \lesssim K\left(2^{k\left(s_{0}-s_{1}\right)}, f ; \dot{B}_{p, \infty}^{s_{0}}(\mathcal{X}), \dot{B}_{p, \infty}^{s_{1}}(\mathcal{X})\right)
$$


If $0<q<\infty$, from (8.39), it follows that

$$
\begin{aligned}
\int_{0}^{\infty} t^{-\sigma q} & {\left[K\left(t, f ; \dot{B}_{p, \infty}^{s_{0}}(x), \dot{B}_{p, \infty}^{s_{1}}(x)\right)\right]^{q} \frac{d t}{t} } \\
& =\sum_{k=-\infty}^{\infty} \int_{2^{(k-1)\left(s_{0}-s_{1}\right)}}^{2^{k\left(s_{0}-s_{1}\right)}} t^{-\sigma q}\left[K\left(t, f ; \dot{B}_{p, \infty}^{s_{0}}(x), \dot{B}_{p, \infty}^{s_{1}}(x)\right)\right]^{q} \frac{d t}{t} \\
& \gtrsim \sum_{k=-\infty}^{\infty} 2^{-\sigma q k\left(s_{0}-s_{1}\right)}\left[K\left(2^{k\left(s_{0}-s_{1}\right)}, f ; \dot{B}_{p, \infty}^{s_{0}}(x), \dot{B}_{p, \infty}^{s_{1}}(x)\right)\right]^{q} \\
& \gtrsim \sum_{k=-\infty}^{\infty} 2^{k s q}\left\|D_{k}(f)\right\|_{L^{p}(x)}^{q} \\
& \gtrsim\|f\|_{\dot{B}_{p, q}^{s}(x)^{\prime}}^{q}
\end{aligned}
$$

and if $q=\infty$, by (8.39), we then have

$$
\begin{aligned}
\|f\|_{\dot{B}_{p, \infty}^{s}(x)} & =\sup _{k \in \mathbb{Z}} 2^{k s}\left\|D_{k}(f)\right\|_{L^{p}(x)} \\
& \lesssim \sup _{k \in \mathbb{Z}} 2^{k\left(s-s_{0}\right)} K\left(2^{k\left(s_{0}-s_{1}\right)}, f ; \dot{B}_{p, \infty}^{s_{0}}(x), \dot{B}_{p, \infty}^{s_{1}}(x)\right) \\
& \lesssim \sup _{0<t<\infty} t^{-\sigma} K\left(t, f ; \dot{B}_{p, \infty}^{s_{0}}(\mathcal{X}), \dot{B}_{p, \infty}^{s_{1}}(\mathcal{X})\right) .
\end{aligned}
$$

Thus, (8.37) holds.

We now prove that if $0<r<q$, then

$$
\dot{B}_{p, q}^{s}(\mathcal{X}) \subset\left(\dot{B}_{p, r}^{s_{0}}(\mathcal{X}), \dot{B}_{p, r}^{s_{1}}(\mathcal{X})\right)_{\sigma, q}
$$

Without loss of generality, we may assume that $s_{0}>s_{1}$ again. Then we also have $s_{0}>s>s_{1}$. In what follows, we only consider the case $q<\infty$ and we omit the details for the case $q=\infty$ by similarity and simplicity. Let now $f \in \dot{B}_{p, q}^{s}(\mathcal{X})$. We then write

$$
\begin{aligned}
\|f\|_{\left(\dot{B}_{p, r}^{s_{0}}(\mathcal{X}), \dot{B}_{p, r}^{s_{1}}(\mathcal{X})\right)_{\sigma, q}}^{q} & =\int_{0}^{\infty} t^{-\sigma q}\left[K\left(t, f ; \dot{B}_{p, r}^{s_{0}}(\mathcal{X}), \dot{B}_{p, r}^{s_{1}}(\mathcal{X})\right)\right]^{q} \frac{d t}{t} \\
& \lesssim \sum_{j=-\infty}^{\infty} 2^{-j \sigma q\left(s_{0}-s_{1}\right)}\left[K\left(2^{j\left(s_{0}-s_{1}\right)}, f ; \dot{B}_{p, r}^{s_{0}}(\mathcal{X}), \dot{B}_{p, r}^{s_{1}}(\mathcal{X})\right)\right]^{q} .
\end{aligned}
$$

Let all the notation be as in Theorem 4.11. For any $j \in \mathbb{Z}$, we write

$$
\begin{aligned}
f(x) & =\sum_{k=-\infty}^{j} \sum_{\tau \in I_{k}} \sum_{\nu=1}^{N(k, \tau)} \mu\left(Q_{\tau}^{k, v}\right) D_{k}(f)\left(y_{\tau}^{k, v}\right) \widetilde{D}_{k}\left(x, y_{\tau}^{k, v}\right)+\sum_{k=j+1}^{\infty} \sum_{\tau \in I_{k}} \sum_{\nu=1}^{N(k, \tau)} \cdots \\
& \equiv f_{0}^{j}+f_{1}^{j}
\end{aligned}
$$


From this and the definition of $K$-functional, it follows that

$$
\begin{aligned}
& \|f\|_{\left(\dot{B}_{p, r}^{s_{0}}(\chi), \dot{B}_{p, r}^{s_{1}}(\chi)\right)_{\sigma, q}}^{q} \\
& \lesssim \sum_{j=-\infty}^{\infty} 2^{-j \sigma q\left(s_{0}-s_{1}\right)}\left(\left\|f_{0}^{j}\right\|_{B_{p, r}^{s_{0}}(x)}^{q}+2^{j\left(s_{0}-s_{1}\right)}\left\|f_{1}^{j}\right\|_{B_{p, r}^{s_{1}}(x)}^{q}\right) \\
& \lesssim \sum_{j=-\infty}^{\infty} 2^{j q\left(s-s_{0}\right)}\left\{\sum_{k^{\prime}=-\infty}^{\infty} 2^{k^{\prime} s_{0} r}\left[\sum_{\tau^{\prime} \in I_{k^{\prime}}} \sum_{v^{\prime}=1}^{N\left(k^{\prime}, \tau^{\prime}\right)} \mu\left(Q_{\tau^{\prime}}^{k^{\prime}, v^{\prime}}\right)\left|D_{k^{\prime}}\left(f_{0}^{j}\right)\left(y_{\tau^{\prime}}^{k^{\prime}, \nu^{\prime}}\right)\right|^{p}\right]^{r / p}\right\}^{q / r} \\
& +\sum_{j=-\infty}^{\infty} 2^{j q\left(s-s_{1}\right)}\left\{\sum_{k^{\prime}=-\infty}^{\infty} 2^{k^{\prime} s_{1} r}\left[\sum_{\tau^{\prime} \in I_{k^{\prime}}} \sum_{v^{\prime}=1}^{N\left(k^{\prime}, \tau^{\prime}\right)} \mu\left(Q_{\tau^{\prime}}^{k^{\prime}, v^{\prime}}\right)\left|D_{k^{\prime}}\left(f_{1}^{j}\right)\left(y_{\tau^{\prime}}^{k^{\prime}, v^{\prime}}\right)\right|^{p}\right]^{r / p}\right\}^{q / r} \\
& \equiv J_{1}+J_{2} \text {. }
\end{aligned}
$$

We first estimate $J_{1}$ in the case $p \leq 1$. In this case, by (5.10), (5.5), Lemma 5.2, and (5.12), we have

$$
\begin{aligned}
J_{1} \lesssim \sum_{j=-\infty}^{\infty} 2^{j q\left(s-s_{0}\right)}\left\{\sum_{k^{\prime}=-\infty}^{\infty} 2^{k^{\prime} s_{0} r}\right. & \left(\sum_{k=-\infty}^{j} 2^{-\left|k^{\prime}-k\right| \epsilon p} 2^{n\left[k-\left(k \wedge k^{\prime}\right)\right](1-p)}\right. \\
& \left.\left.\quad \times \sum_{\tau \in I_{k}} \sum_{\nu=1}^{N(k, \tau)} \mu\left(Q_{\tau}^{k, v}\right)\left|D_{k}(f)\left(y_{\tau}^{k, v}\right)\right|^{p}\right)^{r / p}\right\}^{q / r}
\end{aligned}
$$

Now, we choose $\epsilon^{\prime} \in\left(\left|s_{0}\right|, \epsilon\right)$ such that $p>n /\left(n+s_{0}+\epsilon^{\prime}\right)$. Using (5.5) when $r / p \leq 1$ or Hölder's inequality when $1<r / p<\infty$ then further shows that

$$
\begin{gathered}
J_{1} \lesssim \sum_{j=-\infty}^{\infty} 2^{j q\left(s-s_{0}\right)}\left\{\sum _ { k ^ { \prime } = - \infty } ^ { \infty } 2 ^ { k ^ { \prime } s _ { 0 } r } \left[\sum_{k=-\infty}^{j} 2^{-\left|k^{\prime}-k\right| \epsilon^{\prime} r} 2^{n\left[k-\left(k \wedge k^{\prime}\right)\right](1 / p-1) r}\right.\right. \\
\left.\left.\quad \times\left(\sum_{\tau \in I_{k}} \sum_{v=1}^{N(k, \tau)} \mu\left(Q_{\tau}^{k, v}\right)\left|D_{k}(f)\left(y_{\tau}^{k, v}\right)\right|^{p}\right)^{r / p}\right]\right\}^{q / r} \\
\lesssim \sum_{j=-\infty}^{\infty} 2^{j q\left(s-s_{0}\right)}\left\{\sum_{k=-\infty}^{j} 2^{k s_{0} r}\left(\sum_{\tau \in I_{k}} \sum_{v=1}^{N(k, \tau)} \mu\left(Q_{\tau}^{k, v}\right)\left|D_{k}(f)\left(y_{\tau}^{k, v}\right)\right|^{p}\right)^{r / p}\right\}^{q / r} .
\end{gathered}
$$

Since $q / r>1$, by Hölder's inequality and $s_{0}>s$, we have

$$
\begin{aligned}
J_{1} & \lesssim \sum_{j=-\infty}^{\infty} 2^{j q\left(s-s_{0}\right) / 2}\left\{\sum_{k=-\infty}^{j} 2^{k\left(s_{0}-s\right) q / 2} 2^{k s q}\left(\sum_{\tau \in I_{k}} \sum_{\nu=1}^{N(k, \tau)} \mu\left(Q_{\tau}^{k, v}\right)\left|D_{k}(f)\left(y_{\tau}^{k, v}\right)\right|^{p}\right)^{q / p}\right\} \\
& \lesssim\|f\|_{B_{p, q}^{s}(x)^{\prime}}^{q}
\end{aligned}
$$

which is the desired estimate in the case $p \leq 1$. 
While when $1<p \leq \infty$, letting $\epsilon^{\prime} \in\left(\left|s_{0}\right|, \epsilon\right)$, by Hölder's inequality and Lemma 5.2, we have

$$
\begin{aligned}
& J_{1} \lesssim \sum_{j=-\infty}^{\infty} 2^{j q\left(s-s_{0}\right)}\left\{\sum _ { k ^ { \prime } = - \infty } ^ { \infty } 2 ^ { k ^ { \prime } s _ { 0 } r } \left(\sum_{\tau^{\prime} \in I_{k^{\prime}}} \sum_{v^{\prime}=1}^{N\left(k^{\prime}, \tau^{\prime}\right)} \mu\left(Q_{\tau^{\prime}}^{k^{\prime}, v^{\prime}}\right)\right.\right. \\
& \times\left[\sum_{k=-\infty}^{j} 2^{-\left|k^{\prime}-k\right| \epsilon^{\prime} p} \sum_{\tau \in I_{k}} \sum_{\nu=1}^{N(k, \tau)} \mu\left(Q_{\tau}^{k, v}\right)\left|D_{k}(f)\left(y_{\tau}^{k, v}\right)\right|^{p}\right. \\
& \times \frac{1}{V_{2^{-\left(k \wedge k^{\prime}\right)}}\left(y_{\tau^{\prime}}^{k^{\prime}, v^{\prime}}\right)+V_{2^{-\left(k \wedge k^{\prime}\right)}}\left(y_{\tau}^{k, v}\right)+V\left(y_{\tau^{\prime}}^{k^{\prime}, v^{\prime}}, y_{\tau}^{k, v}\right)} \\
& \left.\left.\left.\times\left(\frac{2^{-\left(k \wedge k^{\prime}\right)}}{2^{-\left(k \wedge k^{\prime}\right)}+d\left(y_{\tau^{\prime}}^{k^{\prime}, \nu^{\prime}}, y_{\tau}^{k, \nu}\right)}\right)^{\epsilon}\right]\right)^{r / p}\right\}^{q / r} \\
& \lesssim \sum_{j=-\infty}^{\infty} 2^{j q\left(s-s_{0}\right)}\left\{\sum_{k^{\prime}=-\infty}^{\infty} 2^{k^{\prime} s_{0} r}\left[\sum_{k=-\infty}^{j} 2^{-\left|k^{\prime}-k\right| \epsilon^{\prime} p}\left(\sum_{\tau \in I_{k}} \sum_{\nu=1}^{N(k, \tau)} \mu\left(Q_{\tau}^{k, v}\right)\left|D_{k}(f)\left(y_{\tau}^{k, v}\right)\right|^{p}\right)\right]^{r / p}\right\}^{q / r} .
\end{aligned}
$$

Let $\epsilon^{\prime \prime}=\epsilon^{\prime}$ when $r / p \leq 1$ and $\epsilon^{\prime \prime} \in\left(\left|s_{0}\right|, \epsilon^{\prime}\right)$ when $r / p>1$. By (5.5) when $r / p \leq 1$ or Hölder's inequality when $r / p>1$, we further obtain

$$
\begin{aligned}
J_{1} & \lesssim \sum_{j=-\infty}^{\infty} 2^{j q\left(s-s_{0}\right)}\left\{\sum_{k^{\prime}=-\infty}^{\infty} 2^{k^{\prime} s_{0} r}\left[\sum_{k=-\infty}^{j} 2^{-\left|k^{\prime}-k\right| \epsilon^{\prime \prime} r}\left(\sum_{\tau \in I_{k}} \sum_{\nu=1}^{N(k, \tau)} \mu\left(Q_{\tau}^{k, \nu}\right)\left|D_{k}(f)\left(y_{\tau}^{k, v}\right)\right|^{p}\right)^{r / p}\right]\right\}^{q / r} \\
& \lesssim\|f\|_{\dot{B}_{p, q}^{s}(x)^{\prime}}^{q}
\end{aligned}
$$

where in the last step, we omit some estimates similar to the case $p \leq 1$. This completes the estimate for $J_{1}$.

We now turn to the estimate for $J_{2}$. We also need to consider two cases. We first assume that $p \leq 1$. In this case, the estimate (5.10) and Lemma 5.2 prove that

$$
\begin{aligned}
J_{2} \lesssim \sum_{j=-\infty}^{\infty} 2^{j q\left(s-s_{1}\right)}\left\{\sum_{k^{\prime}=-\infty}^{\infty} 2^{k^{\prime} s_{1} r}(\right. & \sum_{k=j+1}^{\infty} 2^{-\left|k^{\prime}-k\right| \epsilon p} 2^{n\left[k-\left(k \wedge k^{\prime}\right)\right](1-p)} \\
& \left.\times \sum_{\tau \in I_{k}} \sum_{\nu=1}^{N(k, \tau)} \mu\left(Q_{\tau}^{k, \nu}\right)\left|D_{k}(f)\left(y_{\tau}^{k, \nu}\right)\right|^{p}\right)^{r / p}
\end{aligned}
$$


Now let $\epsilon^{\prime}=0$ when $r / p \leq 1$ or $\epsilon^{\prime} \in\left(0, s-s_{1}\right)$ when $r / p>1$. Using (5.5) when $r / p \leq 1$ or Hölder's inequality when $r / p>1$ gives that

$$
\begin{gathered}
J_{2} \lesssim \sum_{j=-\infty}^{\infty} 2^{j q\left(s-s_{1}-\epsilon^{\prime}\right)}\left\{\sum _ { k ^ { \prime } = - \infty } ^ { \infty } 2 ^ { k ^ { \prime } s _ { 1 } r } \left[\sum_{k=j+1}^{\infty} 2^{-\left|k^{\prime}-k\right| \epsilon r} 2^{n\left[k-\left(k \wedge k^{\prime}\right)\right](1 / p-1) r} 2^{k \epsilon^{\prime} r}\right.\right. \\
\left.\left.\times\left(\sum_{\tau \in I_{k}} \sum_{v=1}^{N(k, \tau)} \mu\left(Q_{\tau}^{k, v}\right)\left|D_{k}(f)\left(y_{\tau}^{k, v}\right)\right|^{p}\right)^{r / p}\right]\right\}^{q / r} \\
\lesssim \sum_{j=-\infty}^{\infty} 2^{j q\left(s-s_{1}-\epsilon^{\prime}\right)}\left\{\sum_{k=j+1}^{\infty} 2^{k\left(s_{1}+\epsilon^{\prime}\right) r}\left(\sum_{\tau \in I_{k}} \sum_{\nu=1}^{N(k, \tau)} \mu\left(Q_{\tau}^{k, v}\right)\left|D_{k}(f)\left(y_{\tau}^{k, v}\right)\right|^{p}\right)^{r / p}\right\}^{q / r} .
\end{gathered}
$$

Using $q / r>1$ together with Hölder's inequality then further yields that

$$
\begin{aligned}
J_{2} & \lesssim \sum_{j=-\infty}^{\infty} 2^{j q\left(s-s_{1}-\epsilon^{\prime}\right) / 2}\left\{\sum_{k=j+1}^{\infty} 2^{k\left(s_{1}+\epsilon^{\prime}-s\right) q / 2} 2^{k s q}\left(\sum_{\tau \in I_{k}} \sum_{\nu=1}^{N(k, \tau)} \mu\left(Q_{\tau}^{k, \nu}\right)\left|D_{k}(f)\left(y_{\tau}^{k, \nu}\right)\right|^{p}\right)^{q / p}\right\} \\
& \lesssim\|f\|_{\dot{B}_{p, q}^{s}(\mathcal{q})}^{q} .
\end{aligned}
$$

If $1<p \leq \infty$, letting $\epsilon^{\prime \prime} \in\left(0, s-s_{1}\right)$, by Hölder's inequality and Lemma 5.2, we obtain

$$
J_{2} \lesssim \sum_{j=-\infty}^{\infty} 2^{j q\left(s-s_{1}-\epsilon^{\prime \prime}\right)}\left\{\sum_{k^{\prime}=-\infty}^{\infty} 2^{k^{\prime} s_{1} r}\left(\sum_{k=j+1}^{\infty} 2^{-\left|k^{\prime}-k\right| \epsilon p} 2^{k \epsilon^{\prime \prime} p} \sum_{\tau \in I_{k}} \sum_{\nu=1}^{N(k, \tau)} \mu\left(Q_{\tau}^{k, \nu}\right)\left|D_{k}(f)\left(y_{\tau}^{k, \nu}\right)\right|^{p}\right)^{r / p}\right\}^{q / r} .
$$

Now, if $r / p \leq 1$, we then take $\epsilon^{\prime}=0$, and if $r / p>1$, we take $\epsilon^{\prime} \in\left(0, s-\epsilon^{\prime \prime}-s_{1}\right)$; by (5.5) or Hölder's inequality, we obtain

$$
\begin{aligned}
J_{2} \lesssim \sum_{j=-\infty}^{\infty} 2^{j q\left(s-s_{1}-\epsilon^{\prime \prime}-\epsilon^{\prime}\right)}\left\{\sum _ { k ^ { \prime } = - \infty } ^ { \infty } 2 ^ { k ^ { \prime } s _ { 1 } r } \left[\sum_{k=j+1}^{\infty} 2^{-\left|k^{\prime}-k\right| e r} 2^{k\left(\epsilon^{\prime \prime}+\epsilon^{\prime}\right) r}\right.\right. \\
\\
\left.\left.\quad \times\left(\sum_{\tau \in I_{k}} \sum_{\nu=1}^{N(k, \tau)} \mu\left(Q_{\tau}^{k, v}\right)\left|D_{k}(f)\left(y_{\tau}^{k, v}\right)\right|^{p}\right)^{r / p}\right]\right\}^{q / r} \\
\lesssim\|f\|_{\dot{B}_{p, q}^{s}(x)}^{q}
\end{aligned}
$$

Thus, (8.42) holds. 
From (8.37), (8.42), and Proposition 8.2(iii) together with Proposition 5.10(i), by taking $0<r<q_{0}$ and $0<r<q_{1}$, we deduce that

$$
\begin{aligned}
\dot{B}_{p, q}^{s}(\mathcal{X}) & \subset\left(\dot{B}_{p, r}^{s_{0}}(\mathcal{X}), \dot{B}_{p, r}^{s_{1}}(\mathcal{X})\right)_{\sigma, q} \\
& \subset\left(\dot{B}_{p, q_{0}}^{s_{0}}(\mathcal{X}), \dot{B}_{p, q_{1}}^{s_{1}}(\mathcal{X})\right)_{\sigma, q} \\
& \subset\left(\dot{B}_{p, \infty}^{s_{0}}(\mathcal{X}), \dot{B}_{p, \infty}^{s_{1}}(\mathcal{X})\right)_{\sigma, q} \\
& \subset \dot{B}_{p, q}^{s}(\mathcal{X}) .
\end{aligned}
$$

Thus, (i) holds.

To see (ii), by (i) and Proposition 8.2(iii) together with Propositions 5.10(ii) and 6.9(ii), we have

$$
\begin{aligned}
\dot{B}_{p, q}^{s}(\mathcal{X}) & =\left(\dot{B}_{p, \min \left\{p, q_{0}\right\}}^{s_{0}}(\mathcal{X}), \dot{B}_{p, \min \left\{p, q_{1}\right\}}^{s_{1}}(\mathcal{X})\right)_{\sigma, q} \\
& \subset\left(\dot{F}_{p, q_{0}}^{s_{0}}(\mathcal{X}), \dot{F}_{p, q_{1}}^{s_{1}}(\mathcal{X})\right)_{\sigma, q} \\
& \subset\left(\dot{B}_{p, \max \left\{p, q_{0}\right\}}^{s_{0}}(\mathcal{X}), \dot{B}_{p, \max \left\{p, q_{1}\right\}}^{s_{1}}(\mathcal{X})\right)_{\sigma, q} \\
& =\dot{B}_{p, q}^{s}(\mathcal{X}) .
\end{aligned}
$$

Thus, (ii) holds, which completes the proof of Theorem 8.8 .

Theorem 8.9. Let $\epsilon$ be as in Definition 5.29, $\sigma \in(0,1), s_{0}, s_{1} \in(-\epsilon, \epsilon), s_{0} \neq s_{1}$, and $s=(1-\sigma) s_{0}+$ $\sigma s_{1}$. Then

(i) if $\max \left\{p\left(s_{0}, \epsilon\right), p\left(s_{1}, \epsilon\right)\right\}<p \leq \infty$ and $0<q_{0}, q_{1}, q \leq \infty$, then

$$
\left(B_{p, q_{0}}^{S_{0}}(\mathcal{X}), B_{p, q_{1}}^{S_{1}}(\mathcal{X})\right)_{\sigma, q}=B_{p, q}^{s}(\mathcal{X})
$$

(ii) if $\max \left\{p\left(s_{0}, \epsilon\right), p\left(s_{1}, \epsilon\right)\right\}<p \leq \infty, p\left(s_{i}, \epsilon\right)<q_{i} \leq \infty$ for $i=0,1$ and $0<q \leq \infty$, then

$$
\left(F_{p, q_{0}}^{s_{0}}(X), F_{p, q_{1}}^{s_{1}}(X)\right)_{\sigma, q}=B_{p, q}^{s}(x) .
$$

Proof. Similarly to the proof of Theorem 8.8, we only need to verify (i), while (ii) can be deduced from (i), Propositions 8.10, 5.31, and 6.21.

To prove (i), by Proposition 5.31(iv), we know that

$$
B_{p, q_{0}}^{s_{0}}(\mathcal{X}), B_{p, q_{1}}^{s_{1}}(\mathcal{X}) \subset\left(\mathcal{G}_{0}^{\epsilon}(\beta, \gamma)\right)^{\prime}
$$

with $\max \left\{0,-s_{0}+n(1 / p-1)_{+},-s_{1}+n(1 / p-1)_{+}\right\}<\beta<\epsilon$ and $n(1 / p-1)_{+}<\gamma<\epsilon$. Thus, in this case, we can take $\mathscr{H}=\left(\mathcal{C}_{0}^{\epsilon}(\beta, \gamma)\right)^{\prime}$ with $\beta$ and $\gamma$ as above. 
We now verify that

$$
\left(B_{p, \infty}^{s_{0}}(\mathcal{X}), B_{p, \infty}^{s_{1}}(\mathcal{X})\right)_{\sigma, q} \subset B_{p, q}^{s}(\mathcal{X}) .
$$

By Proposition 8.2(ii), without loss of generality, we may assume that $s_{0}>s_{1}$.

Assume that $f \in\left(B_{p, \infty}^{s_{0}}(\mathcal{X}), B_{p, \infty}^{s_{1}}(\mathcal{X})\right)_{\sigma, q}$ and $f=f_{0}+f_{1}$ with $f_{0} \in B_{p, \infty}^{s_{0}}(\mathcal{X})$ and $f_{1} \in$ $B_{p, \infty}^{s_{1}}(\mathcal{X})$. Let $\left\{D_{k}\right\}_{k \in \mathbb{Z}_{+}}$be as in Definition 5.29. Then,

$$
\begin{aligned}
2^{k s_{0}}\left\|D_{k}(f)\right\|_{L^{p}(\mathcal{X})} & \lesssim 2^{k s_{0}}\left\|D_{k}\left(f_{0}\right)\right\|_{L^{p}(\mathcal{X})}+2^{k\left(s_{0}-s_{1}\right)} 2^{k s_{1}}\left\|D_{k}\left(f_{1}\right)\right\|_{L^{p}(\mathcal{X})} \\
& \lesssim\left\|f_{0}\right\|_{B_{p, \infty}^{s_{0}}(\mathcal{X})}+2^{k\left(s_{0}-s_{1}\right)}\left\|f_{1}\right\|_{B_{p, \infty}^{s_{1}}(\mathcal{X})} .
\end{aligned}
$$

Taking the infimum on all representations $f=f_{0}+f_{1}$ yields that for all $k \in \mathbb{Z}_{+}$,

$$
2^{k s_{0}}\left\|D_{k}(f)\right\|_{L^{p}(\mathcal{X})} \lesssim K\left(2^{k\left(s_{0}-s_{1}\right)}, f ; B_{p, \infty}^{s_{0}}(\mathcal{X}), B_{p, \infty}^{s_{1}}(\mathcal{X})\right) .
$$

If $0<q<\infty$, from (8.63), it follows that

$$
\begin{aligned}
\int_{0}^{\infty} t^{-\sigma q} & {\left[K\left(t, f ; B_{p, \infty}^{s_{0}}(\mathcal{X}), B_{p, \infty}^{s_{1}}(\mathcal{X})\right)\right]^{q} \frac{d t}{t} } \\
\quad & \sum_{k=-\infty}^{\infty} \int_{2^{(k-1)\left(s_{0}-s_{1}\right)}}^{2^{k\left(s_{0}-s_{1}\right)}} t^{-\sigma q}\left[K\left(t, f ; B_{p, \infty}^{s_{0}}(\mathcal{X}), B_{p, \infty}^{s_{1}}(\mathcal{X})\right)\right]^{q} \frac{d t}{t} \\
& \gtrsim \sum_{k=0}^{\infty} 2^{-\sigma q k\left(s_{0}-s_{1}\right)}\left[K\left(2^{k\left(s_{0}-s_{1}\right)}, f ; B_{p, \infty}^{s_{0}}(\mathcal{X}), B_{p, \infty}^{s_{1}}(\mathcal{X})\right)\right]^{q} \\
& \gtrsim \sum_{k=0}^{\infty} 2^{k s q}\left\|D_{k}(f)\right\|_{L^{p}(\mathcal{X})}^{q} \\
& \gtrsim\|f\|_{B_{p, q}^{s}(\mathcal{X})^{\prime}}^{q}
\end{aligned}
$$

and if $q=\infty$, by (8.63), we then have

$$
\begin{aligned}
\|f\|_{B_{p, \infty}^{s}(x)} & =\sup _{k \in \mathbb{Z}_{+}} 2^{k s}\left\|D_{k}(f)\right\|_{L^{p}(x)} \\
& \lesssim \sup _{k \in \mathbb{Z}_{+}} 2^{k\left(s-s_{0}\right)} K\left(2^{k\left(s_{0}-s_{1}\right)}, f ; B_{p, \infty}^{s_{0}}(\mathcal{X}), B_{p, \infty}^{s_{1}}(\mathcal{X})\right) \\
& \lesssim \sup _{0<t<\infty} t^{-\sigma} K\left(t, f ; B_{p, \infty}^{s_{0}}(\mathcal{X}), B_{p, \infty}^{s_{1}}(\mathcal{X})\right) .
\end{aligned}
$$

Thus, (8.61) holds.

We now prove that if $0<r<q$, then

$$
B_{p, q}^{S}(\mathcal{X}) \subset\left(B_{p, r}^{S_{0}}(\mathcal{X}), B_{p, r}^{s_{1}}(\mathcal{X})\right)_{\sigma, q} .
$$


Without loss of generality, we may assume that $s_{0}>s_{1}$ again. Then we also have $s_{0}>s>s_{1}$. In what follows, we only consider the case $q<\infty$ and we omit the details for the case $q=\infty$ by similarity and simplicity. Let now $f \in B_{p, q}^{s}(x)$. We then write

$$
\begin{aligned}
\|f\|_{\left(B_{p, r}^{s_{0}}(\mathcal{X}), B_{p, r}^{s_{1}}(\mathcal{X})\right)_{\sigma, q}}^{q} & \int_{0}^{\infty} t^{-\sigma q}\left[K\left(t, f ; B_{p, r}^{s_{0}}(\mathcal{X}), B_{p, r}^{s_{1}}(\mathcal{X})\right)\right]^{q} \frac{d t}{t} \\
\lesssim & \int_{0}^{1} t^{-\sigma q}\left[K\left(t, f ; B_{p, r}^{s_{0}}(\mathcal{X}), B_{p, r}^{s_{1}}(\mathcal{X})\right)\right]^{q} \frac{d t}{t} \\
& +\sum_{j=0}^{\infty} 2^{-j \sigma q\left(s_{0}-s_{1}\right)}\left[K\left(2^{j\left(s_{0}-s_{1}\right)}, f ; B_{p, r}^{s_{0}}(\mathcal{X}), B_{p, r}^{s_{1}}(\mathcal{X})\right)\right]^{q} \\
\equiv & Y_{1}+Y_{2} .
\end{aligned}
$$

To estimate the first term, by Proposition 5.31(ii), we have

$$
K\left(t, f ; B_{p, r}^{s_{0}}(\mathcal{X}), B_{p, r}^{s_{1}}(\mathcal{X})\right) \leq t\|f\|_{B_{p, r}^{s_{1}}(\mathcal{X})} \lesssim t\|f\|_{B_{p, r}^{s_{1}}(\mathcal{X})^{\prime}}
$$

which shows that

$$
Y_{1}=\int_{0}^{1} t^{-\sigma q}\left[K\left(t, f ; B_{p, r}^{s_{0}}(\mathcal{X}), B_{p, r}^{s_{1}}(\mathcal{X})\right)\right]^{q} \frac{d t}{t} \lesssim\|f\|_{B_{p, r}^{s}(\mathcal{X})}^{q}
$$

To estimate the second term, let all the notation be as in Theorem 4.14. For any $j \in \mathbb{N}$, we write

$$
\begin{aligned}
f(x)= & \left\{\sum_{\tau \in I_{0}} \sum_{v=1}^{N(0, \tau)} \mu\left(Q_{\tau}^{0, v}\right) m_{Q_{\tau}^{0, v}}\left(D_{0}(f)\right) \widetilde{D}_{Q_{\tau}^{0, v}}(x)+\sum_{k=1}^{j} \sum_{\tau \in I_{k}} \sum_{\nu=1}^{N(k, \tau)} \mu\left(Q_{\tau}^{k, v}\right) D_{k}(f)\left(y_{\tau}^{k, v}\right) \widetilde{D}_{k}\left(x, y_{\tau}^{k, v}\right)\right\} \\
& +\sum_{k=j+1}^{\infty} \sum_{\tau \in I_{k}} \sum_{\nu=1}^{N(k, \tau)} \mu\left(Q_{\tau}^{k, v}\right) D_{k}(f)\left(y_{\tau}^{k, v}\right) \widetilde{D}_{k}\left(x, y_{\tau}^{k, v}\right) \\
\equiv & f_{0}^{j}+f_{1}^{j},
\end{aligned}
$$

where $\widetilde{D}_{Q_{\tau}^{0, v}}(x)$ is as in the proof of Proposition 7.3. From this and the definition of $K$ functional, it follows that

$$
\begin{aligned}
Y_{2} \lesssim & \sum_{j=0}^{\infty} 2^{-j \sigma q\left(s_{0}-s_{1}\right)}\left(\left\|f_{0}^{j}\right\|_{B_{p, r}^{s_{0}(x)}}^{q}+2^{j\left(s_{0}-s_{1}\right)}\left\|f_{1}^{j}\right\|_{B_{p, r}^{s_{1}}(x)}^{q}\right) \\
\lesssim & \sum_{j=0}^{\infty} 2^{j q\left(s-s_{0}\right)}\left\{\sum_{\tau^{\prime} \in I_{0}} \sum_{v^{\prime}=1}^{N\left(0, \tau^{\prime}\right)} \mu\left(Q_{\tau^{\prime}}^{0, v^{\prime}}\right)\left[m_{Q_{\tau^{\prime}}^{0, v^{\prime}}}\left(\left|D_{0}\left(f_{0}^{j}\right)\right|\right)\right]^{p}\right\}^{q / p} \\
& +\sum_{j=0}^{\infty} 2^{j q\left(s-s_{0}\right)}\left\{\sum_{k^{\prime}=1}^{\infty} 2^{k^{\prime} s_{0} r}\left[\sum_{\tau^{\prime} \in I_{k^{\prime}}} \sum_{v^{\prime}=1}^{N\left(k^{\prime}, \tau^{\prime}\right)} \mu\left(Q_{\tau^{\prime}}^{k^{\prime}, \nu^{\prime}}\right)\left|D_{k^{\prime}}\left(f_{0}^{j}\right)\left(y_{\tau^{\prime}}^{k^{\prime}, v^{\prime}}\right)\right|^{p}\right]^{r / p}\right\}^{q / r}
\end{aligned}
$$


Yongsheng Han et al.

$$
\begin{aligned}
& +\sum_{j=0}^{\infty} 2^{j q\left(s-s_{1}\right)}\left\{\sum_{\tau^{\prime} \in I_{0}} \sum_{v^{\prime}=1}^{N\left(0, \tau^{\prime}\right)} \mu\left(Q_{\tau^{\prime}}^{0, v^{\prime}}\right)\left[m_{Q_{\tau^{\prime}}^{0, v^{\prime}}}\left(\left|D_{0}\left(f_{1}^{j}\right)\right|\right)\right]^{p}\right\}^{q / p} \\
& +\sum_{j=0}^{\infty} 2^{j q\left(s-s_{1}\right)}\left\{\sum_{k^{\prime}=1}^{\infty} 2^{k^{\prime} s_{1} r}\left[\sum_{\tau^{\prime} \in I_{k^{\prime}}} \sum_{v^{\prime}=1}^{N\left(k^{\prime}, \tau^{\prime}\right)} \mu\left(Q_{\tau^{\prime}}^{k^{\prime}, v^{\prime}}\right)\left|D_{k^{\prime}}\left(f_{1}^{j}\right)\left(y_{\tau^{\prime}}^{k^{\prime}, v^{\prime}}\right)\right|^{p}\right]^{r / p}\right\}^{q / r} \\
\equiv & J_{1}+J_{2}+J_{3}+J_{4} .
\end{aligned}
$$

The estimate (5.82), (5.5), and Lemma 5.2 show that when $p \leq 1$,

$$
\begin{aligned}
J_{1} \lesssim & \sum_{j=0}^{\infty} 2^{j\left(s-s_{0}\right) q}\left\{\sum_{\tau \in I_{0}} \sum_{v=1}^{N(0, \tau)} \mu\left(Q_{\tau}^{0, v}\right)\left[m_{Q_{\tau}^{0, v}}\left(\left|D_{0}(f)\right|\right)\right]^{p}\right\}^{q / p} \\
& +\sum_{j=0}^{\infty} 2^{j\left(s-s_{0}\right) q}\left\{\sum_{k=1}^{j} 2^{-k \epsilon p} \sum_{\tau \in I_{k}} \sum_{v=1}^{N(k, \tau)}\left[\mu\left(Q_{\tau}^{k, v}\right)\left|D_{k}(f)\left(y_{\tau}^{k, v}\right)\right|\right]^{p}\left[V_{1}\left(y_{\tau}^{k, \nu}\right)\right]^{1-p}\right\}^{q / p} \\
& \lesssim\|f\|_{B_{p, q}^{s}\left(\mathcal{q}(x)^{\prime}\right.}
\end{aligned}
$$

where in the last inequality, we used (5.5) when $q / p \leq 1$ or Hölder's inequality when $1<$ $q / p<\infty$.

When $1<p \leq \infty$, from (5.82), Hölder's inequality, and Lemma 5.2, it follows that

$$
\begin{aligned}
& J_{1} \lesssim \sum_{j=0}^{\infty} 2^{j\left(s-s_{0}\right) q}\left\{\sum_{\tau^{\prime} \in I_{0}} \sum_{v^{\prime}=1}^{N\left(0, \tau^{\prime}\right)} \mu\left(Q_{\tau^{\prime}}^{0, v^{\prime}}\right)\right. \\
& \times\left(\sum_{\tau \in I_{0}} \sum_{\nu=1}^{N(0, \tau)} \mu\left(Q_{\tau}^{0, \nu}\right)\left[m_{Q_{\tau}^{0, v}}\left(\left|D_{0}(f)\right|\right)\right]^{p}\right. \\
& \left.\left.\times \frac{1}{V_{1}\left(y_{\tau^{\prime}}^{0, v^{\prime}}\right)+V_{1}\left(y_{\tau}^{0, v}\right)+V\left(y_{\tau^{\prime}}^{0, v^{\prime}}, y_{\tau}^{0, v}\right)} \frac{1}{\left(1+d\left(y_{\tau^{\prime}}^{0, v^{\prime}}, y_{\tau}^{0, v}\right)\right)^{\epsilon}}\right)\right\}^{q / p} \\
& +\sum_{j=0}^{\infty} 2^{j\left(s-s_{0}\right) q}\left\{\sum_{\tau^{\prime} \in I_{0}} \sum_{v^{\prime}=1}^{N\left(0, \tau^{\prime}\right)} \mu\left(Q_{\tau^{\prime}}^{0, v^{\prime}}\right)\right. \\
& \times\left[\sum_{k=1}^{j} 2^{-k \epsilon^{\prime} p} \sum_{\tau \in I_{k}} \sum_{v=1}^{N(k, \tau)} \mu\left(Q_{\tau}^{k, v}\right)\left|D_{k}(f)\left(y_{\tau}^{k, v}\right)\right|^{p}\right. \\
& \left.\left.\times \frac{1}{V_{1}\left(y_{\tau^{\prime}}^{0, v^{\prime}}\right)+V_{1}\left(y_{\tau}^{k, v}\right)+V\left(y_{\tau^{\prime}}^{0, v^{\prime}}, y_{\tau}^{k, v}\right)} \frac{1}{\left(1+d\left(y_{\tau^{\prime}}^{0, v^{\prime}}, y_{\tau}^{k, v}\right)\right)^{\epsilon}}\right]\right\}^{q / p} \\
& \lesssim\|f\|_{B_{p, q}^{s}(x)^{\prime}}^{q}
\end{aligned}
$$

where we chose $\epsilon^{\prime} \in(|s|, \epsilon)$. 
By (5.82), we write

$$
\begin{aligned}
& J_{2} \lesssim \sum_{j=0}^{\infty} 2^{j\left(s-s_{0}\right) q}\left\{\sum _ { k ^ { \prime } = 1 } ^ { \infty } 2 ^ { k ^ { \prime } s _ { 0 } r } \left(\sum_{\tau^{\prime} \in I_{k^{\prime}}} \sum_{v^{\prime}=1}^{N\left(k^{\prime}, \tau^{\prime}\right)} \mu\left(Q_{\tau^{\prime}}^{k^{\prime}, v^{\prime}}\right)\right.\right. \\
& \times\left[\sum_{\tau \in I_{0}} \sum_{v=1}^{N(0, \tau)} 2^{-k^{\prime} \epsilon} \mu\left(Q_{\tau}^{0, v}\right) m_{Q_{\tau}^{0, v}}\left(\left|D_{0}(f)\right|\right)\right. \\
& \times \frac{1}{V_{1}\left(y_{\tau^{\prime}}^{k^{\prime}, \nu^{\prime}}\right)+V_{1}\left(y_{\tau}^{0, v}\right)+V\left(y_{\tau^{\prime}}^{k^{\prime}, \nu^{\prime}}, y_{\tau}^{0, v}\right)} \\
& \left.\left.\left.\times \frac{1}{\left(1+d\left(y_{\tau^{\prime}}^{k^{\prime}, v^{\prime}}, y_{\tau}^{0, v}\right)\right)^{\epsilon}}\right]^{p}\right)^{r / p}\right\}^{q / r} \\
& +\sum_{j=0}^{\infty} 2^{j\left(s-s_{0}\right) q}\left\{\sum _ { k ^ { \prime } = 1 } ^ { \infty } 2 ^ { k ^ { \prime } s _ { 0 } r } \left(\sum _ { \tau ^ { \prime } \in I _ { k ^ { \prime } } } \sum _ { v ^ { \prime } = 1 } ^ { N ( k ^ { \prime } , \tau ^ { \prime } ) } \mu ( Q _ { \tau ^ { \prime } } ^ { k ^ { \prime } , v ^ { \prime } } ) \left[\sum_{k=1}^{j} 2^{-\left|k^{\prime}-k\right| \epsilon} \sum_{\tau \in I_{k}} \sum_{v=1}^{N(k, \tau)} \mu\left(Q_{\tau}^{k, v}\right)\left|D_{k}(f)\left(y_{\tau}^{k, v}\right)\right|\right.\right.\right. \\
& \left.\left.\left.\times\left|\left(\widetilde{D}_{k}^{\prime} D_{k}\right)\left(y_{\tau^{\prime}}^{k^{\prime}, v^{\prime}}, y_{\tau}^{k, v}\right)\right|\right]^{p}\right)^{r / p}\right\}^{q / r} \\
& \equiv J_{2,1}+J_{2,2} \text {. }
\end{aligned}
$$

The estimate $J_{2,2}$ is completely similar to that for $J_{1}$. Thus, we only need to estimate $J_{2,1}$. When $p \leq 1$, by (5.5) and Lemma 5.2, we have

$$
J_{2,1} \lesssim \sum_{j=0}^{\infty} 2^{j\left(s-s_{0}\right) q}\left\{\sum_{k^{\prime}=1}^{\infty} 2^{k^{\prime}\left(s_{0}-\epsilon\right) r}\right\}^{q / r}\left(\sum_{\tau \in I_{0}} \sum_{\nu=1}^{N(0, \tau)} \mu\left(Q_{\tau}^{0, v}\right)\left[m_{Q_{\tau}^{0, v}}\left(\left|D_{0}(f)\right|\right)\right]^{p}\right)^{q / p} \lesssim\|f\|_{B_{p, q}^{s}(x)^{\prime}}^{q}
$$

while when $1<p \leq \infty$, Hölder's inequality and Lemma 5.2 give that

$$
\begin{aligned}
& J_{2,1} \lesssim \sum_{j=0}^{\infty} 2^{j\left(s-s_{0}\right) q}\left\{\sum _ { k ^ { \prime } = 1 } ^ { \infty } 2 ^ { k ^ { \prime } s _ { 0 } r } \left(\sum_{\tau^{\prime} \in I_{k^{\prime}}} \sum_{v^{\prime}=1}^{N\left(k^{\prime}, \tau^{\prime}\right)} \mu\left(Q_{\tau^{\prime}}^{k^{\prime}, v^{\prime}}\right)\right.\right. \\
& \times\left[\sum_{\tau \in I_{0}} \sum_{\nu=1}^{N(0, \tau)} 2^{-k^{\prime} \epsilon p} \mu\left(Q_{\tau}^{0, v}\right)\right. \\
& \times\left[m_{Q_{\tau}^{0, v}}\left(\left|D_{0}(f)\right|\right)\right]^{p} \frac{1}{V_{1}\left(y_{\tau^{\prime}}^{k^{\prime}, v^{\prime}}\right)+V_{1}\left(y_{\tau}^{0, v}\right)+V\left(y_{\tau^{\prime}}^{k^{\prime}, v^{\prime}}, y_{\tau}^{0, v}\right)} \\
& \left.\left.\left.\times \frac{1}{\left(1+d\left(y_{\tau^{\prime}}^{k^{\prime}, v^{\prime}}, y_{\tau}^{0, v}\right)\right)^{\epsilon}}\right]\right)^{r / p}\right\}^{q / r} \\
& \lesssim\|f\|_{B_{p, q}^{s}(x)^{\prime}}^{q}
\end{aligned}
$$

which is also the desired estimate. 
Similarly, from (5.82), (5.5), Hölder's inequality, and Lemma 5.2, it follows that if $p \leq 1$,

$$
\begin{aligned}
& J_{3} \lesssim \sum_{j=0}^{\infty} 2^{j\left(s-s_{1}\right) q}\left\{\sum_{\tau^{\prime} \in I_{0}} \sum_{\nu^{\prime}=1}^{N\left(0, \tau^{\prime}\right)} \mu\left(Q_{\tau^{\prime}}^{0, v^{\prime}}\right)\right. \\
& \quad \times\left[\sum_{k=j+1}^{\infty} \sum_{\tau \in I_{k}} \sum_{v=1}^{N(k, \tau)} \mu\left(Q_{\tau}^{k, v}\right)\left|D_{k}(f)\left(y_{\tau}^{k, v}\right)\right|\right. \\
& \left.\left.\quad \times 2^{-k \epsilon} \frac{1}{V_{1}\left(y_{\tau^{\prime}}^{0, v^{\prime}}\right)+V_{1}\left(y_{\tau}^{k, v}\right)+V\left(y_{\tau^{\prime}}^{0, v^{\prime}}, y_{\tau}^{k, v}\right)} \frac{1}{\left(1+d\left(y_{\tau^{\prime}}^{0, v^{\prime}}, y_{\tau}^{k, v}\right)\right)^{d+\epsilon}}\right]^{p}\right\}^{q / p} \\
& \lesssim \sum_{j=0}^{\infty} 2^{j\left(s-s_{1}\right) q}\left[\sum_{k=j+1}^{\infty} 2^{-k(\epsilon+n-n / p) p} \sum_{\tau \in I_{k}} \sum_{v=1}^{N(k, \tau)} \mu\left(Q_{\tau}^{k, v}\right)\left|D_{k}(f)\left(y_{\tau}^{k, v}\right)\right|^{p}\right]^{q / p} \\
& \lesssim\|f\|_{B_{p, q}^{s}(\mathcal{q})^{\prime}}^{q / p}
\end{aligned}
$$

where we choose $a_{1}=1$ if $q / p \leq 1$ and $a_{1} \in(0,1)$ if $q / p>1$ such that $a_{1} s>(1-$ $\left.a_{1}\right)(\epsilon+n-n / p)+s_{1}$, while when $1<p \leq \infty$, by Hölder's inequality and Lemma 5.2, we have

$$
\begin{aligned}
& J_{3} \lesssim \sum_{j=0}^{\infty} 2^{j\left(s-s_{1}\right) q}\left\{\sum_{\tau^{\prime} \in I_{0}} \sum_{v^{\prime}=1}^{N\left(0, \tau^{\prime}\right)} \mu\left(Q_{\tau^{\prime}}^{0, v^{\prime}}\right)\right. \\
& \quad \times\left[\sum_{k=j+1}^{\infty} \sum_{\tau \in I_{k}} \sum_{v=1}^{N(k, \tau)} \mu\left(Q_{\tau}^{k, v}\right)\left|D_{k}(f)\left(y_{\tau}^{k, v}\right)\right|^{p}\right. \\
& \left.\left.\quad \times 2^{-k \epsilon^{\prime} p} \frac{1}{V_{1}\left(y_{\tau^{\prime}}^{0, v^{\prime}}\right)+V_{1}\left(y_{\tau}^{k, v}\right)+V\left(y_{\tau^{\prime}}^{0, v^{\prime}}, y_{\tau}^{k, v}\right)} \frac{1}{\left(1+d\left(y_{\tau^{\prime}}^{0, v^{\prime}}, y_{\tau}^{k, v}\right)\right)^{\epsilon}}\right]\right\}^{q / p} \\
& \lesssim\|f\|_{B_{p, q}^{s}(x)^{\prime}}^{q}
\end{aligned}
$$

where we chose $\epsilon^{\prime} \in\left(\left|s_{1}\right|, \epsilon\right)$ and $a_{2} \in(0,1)$ such that $a_{2} \epsilon^{\prime}>\left(1-a_{2}\right) s-s_{1}$.

The estimate $J_{4}$ is similar to that for $J_{2}$ and we omit the details, which completes the proof of Theorem 8.9.

\subsection{Dual spaces}

In this subsection, we are going to identify the dual spaces of some classes of Besov spaces and Triebel-Lizorkin spaces. To this end, we first recall the definitions of some auxiliary function spaces. Let $0<p, q \leq \infty$. The spaces $L^{p}\left(\dot{\ell}^{q}\right)(\mathcal{X})$ and $\dot{\ell}^{q}\left(L^{p}\right)(\mathcal{X})$ are respectively defined to be 
the set of all sequences $f=\left\{f_{k}\right\}_{k \in \mathbb{Z}}$ of $\mu$-measurable functions on $\mathcal{X}$ such that

$$
\begin{aligned}
& \|f\|_{L^{p}\left(\dot{\ell}^{q}\right)(\mathcal{X})}=\left\|\left\{\sum_{k=-\infty}^{\infty}\left|f_{k}\right|^{q}\right\}^{1 / q}\right\|_{L^{p}(\mathcal{X})}<\infty, \\
& \|f\|_{\dot{\ell}^{q}\left(L^{p}\right)(\mathcal{X})}=\left\{\sum_{k=-\infty}^{\infty}\left\|f_{k}\right\|_{L^{p}(\mathcal{X})}^{q}\right\}^{1 / q}<\infty ;
\end{aligned}
$$

and the spaces $L^{p}\left(\ell^{q}\right)(\mathcal{X})$ and $\ell^{q}\left(L^{p}\right)(\mathcal{X})$ are respectively defined to be the set of all sequences $f=\left\{f_{k}\right\}_{k \in \mathbb{Z}_{+}}$of $\mu$-measurable functions on $\mathcal{X}$ such that

$$
\begin{aligned}
& \|f\|_{L^{p}\left(\ell^{q}\right)(\mathcal{X})}=\left\|\left\{\sum_{k=0}^{\infty}\left|f_{k}\right|^{q}\right\}^{1 / q}\right\|_{L^{p}(\mathcal{X})}<\infty, \\
& \|f\|_{\ell^{q}\left(L^{p}\right)(x)}=\left\{\sum_{k=0}^{\infty}\left\|f_{k}\right\|_{L^{p}(\mathcal{X})}^{q}\right\}^{1 / q}<\infty .
\end{aligned}
$$

The following result is well known (cf., e.g., [3, Proposition 2.11.1, pages 177-178]).

Proposition 8.10. Let $1 \leq p<\infty$ and $0<q<\infty$. Then the following hold.

(i) $g \in\left(L^{p}\left(\dot{\ell^{q}}\right)(x)\right)^{\prime}$ if and only if there exists a sequence $\left\{g_{j}\right\}_{j \in \mathbb{Z}} \in L^{p^{\prime}}\left(\dot{\ell}^{q^{\prime}}\right)(X)$ such that

$$
g(f)=\sum_{j=-\infty}^{\infty} \int_{x} g_{j}(x) f_{j}(x) d \mu(x)
$$

$$
\text { for every } f=\left\{f_{j}\right\}_{j \in \mathbb{Z}} \in L^{p}(\dot{\ell} q)(\mathcal{X}) \text {, and }\|g\| \sim\left\|\left\{g_{j}\right\}_{j \in \mathbb{Z}}\right\|_{L^{p^{\prime}}\left(\dot{\ell} q^{\prime}\right)(x)} \text {. }
$$

(ii) $g \in\left(\dot{\ell}^{q}\left(L^{p}\right)(X)\right)^{\prime}$ if and only if there is a sequence $\left\{g_{j}\right\}_{j \in \mathbb{Z}} \in \dot{\ell}^{q^{\prime}}\left(L^{p^{\prime}}\right)(\mathcal{X})$ such that

$$
\begin{gathered}
\qquad g(f)=\sum_{j=-\infty}^{\infty} \int_{x} g_{j}(x) f_{j}(x) d \mu(x) \\
\text { for every } f=\left\{f_{j}\right\}_{j \in \mathbb{Z}} \in \dot{\ell}^{q}\left(L^{p}\right)(x) \text {, and }\|g\| \sim\left\|\left\{g_{j}\right\}_{j \in \mathbb{Z}}\right\|_{\dot{\ell}^{\prime}\left(L^{p^{\prime}}\right)(x)} .
\end{gathered}
$$

Using Proposition 8.10, by a procedure similar to the proof of Theorem 2.11.2 in [3], we can establish the following duality theorem for homogeneous Besov spaces and TriebelLizorkin spaces. In what follows, when $0<q<1$, we also let $q^{\prime} \equiv \infty$. 
Theorem 8.11. Let $\epsilon$ be as in Definition 5.8 and $|s|<\epsilon$. Then, the following hold.

(i) If $1 \leq p<\infty$ and $0<q<\infty$, then

$$
\left(\dot{B}_{p, q}^{s}(\mathcal{X})\right)^{\prime}=\dot{B}_{p^{\prime}, q^{\prime}}^{-s}(\mathcal{X})
$$

More precisely, given $g \in \dot{B}_{p^{\prime}, q^{\prime}}^{-s}(\mathcal{X})$, then $\mathcal{L}_{g}(f) \equiv\langle f, g\rangle$ defines a linear functional on $\dot{\mathcal{G}}(\epsilon, \epsilon) \cap$ $\dot{B}_{p, q}^{s}(X)$ such that

$$
\left|\mathcal{L}_{g}(f)\right| \leq C\|f\|_{B_{p, q}^{s}(x)}\|g\|_{B_{p^{\prime}, q^{\prime}}^{-s}(x)}
$$

where $C>0$ is independent of $f$, and this linear functional naturally extends to $\dot{B}_{p, q}^{s}(x)$ by continuity with norm at most $C\|g\|_{\dot{B}_{p^{\prime}, q^{\prime}}^{-s}(x)}$.

Conversely, if $\mathcal{\perp}$ is a linear functional on $\dot{B}_{p, q}^{s}(\mathcal{X})$, then there exists a unique $g \in \dot{B}_{p^{\prime}, q^{\prime}}^{-s}(\mathcal{X})$ such that $\mathcal{L}$ is the natural extension of $\mathcal{L}_{g^{\prime}}$, with $\|g\|_{\dot{B}_{p^{\prime}, q^{\prime}}^{-s}(x)} \leq C\|\mathcal{L}\|$.

(ii) If $1<p, q<\infty$, then

$$
\left(\dot{F}_{p, q}^{s}(X)\right)^{\prime}=\dot{F}_{p^{\prime}, q^{\prime}}^{-s}(\mathcal{X})
$$

More precisely, given $g \in \dot{F}_{p^{\prime}, q^{\prime}}^{-s}(\mathcal{X})$, then $\mathcal{L}_{g}(f) \equiv\langle f, g\rangle$ defines a linear functional on $\mathcal{G}(\epsilon, \epsilon) \cap$ $\dot{F}_{p, q}^{s}(X)$ such that

$$
\left|\mathcal{L}_{g}(f)\right| \leq C\|f\|_{\dot{F}_{p, q}^{s}(x)}\|g\|_{\dot{F}_{p^{\prime}, q^{\prime}}^{-s}(x)^{\prime}}
$$

where $C>0$ is independent of $f$, and this linear functional naturally extends to $\dot{F}_{p, q}^{s}(\mathcal{X})$ by continuity with norm at most $C\|g\|_{p_{p^{\prime}, q^{\prime}}(\boldsymbol{x})}$.

Conversely, if $\mathcal{\perp}$ is a linear functional on $\dot{F}_{p, q}^{s}(\mathcal{X})$, then there exists a unique $g \in \dot{F}_{p^{\prime}, q^{\prime}}^{-s}(\mathcal{X})$ such that $\mathcal{L}$ is the natural extension of $\mathcal{L}_{g}$, with $\|g\|_{\hat{F}_{p^{\prime}, q^{\prime}}^{-s}(x)} \leq C\|\mathcal{L}\|$.

Proof. Let $s \in(-\epsilon, \epsilon)$. We first claim that if $1<p<\infty$ and $p(s, \epsilon)<q<\infty$, then

$$
\dot{F}_{p^{\prime}, q^{\prime}}^{-s}(X) \subset\left(\dot{F}_{p, q}^{s}(X)\right)^{\prime}
$$

and that if $1 \leq p<\infty$ and $0<q<\infty$,

$$
\dot{B}_{p^{\prime}, q^{\prime}}^{-s}(\mathcal{X}) \subset\left(\dot{B}_{p, q}^{s}(\mathcal{X})\right)^{\prime} .
$$


We first verify (8.87). Observe first that $1<p^{\prime}<\infty$ and $1<q^{\prime} \leq \infty$. Let $f \in \dot{F}_{p^{\prime}, q^{\prime}}^{-s}(\mathcal{X})$ and all the notation be as in Theorem 3.10. For any $\varphi \in \mathcal{G}_{(}\left(\epsilon_{1}, \epsilon_{2}\right)$, by the definition of $\dot{F}_{p^{\prime}, q^{\prime}}^{-s}(\mathcal{X})$ and Theorem 3.13 together with Hölder's inequality and Remark 5.5, we have

$$
\begin{aligned}
|f(\varphi)| & =\left|\sum_{k=-\infty}^{\infty}\left\langle D_{k}(f), \tilde{D}_{k}^{t}(\varphi)\right\rangle\right| \\
& \leq \int_{\chi} \sum_{k=-\infty}^{\infty}\left|D_{k}(f)(x) \| \tilde{D}_{k}^{t}(\varphi)(x)\right| d \mu(x) \\
& \lesssim\|f\|_{F_{p^{\prime}, q^{\prime}}^{-s}(x)}\|\varphi\|_{\dot{F}_{p, q}^{s}(x)^{\prime}}
\end{aligned}
$$

which together with the Hahn-Banach theorem and Proposition 5.21 shows (8.87).

Similarly, to see (8.88), for any $f \in \dot{B}_{p^{\prime}, q^{\prime}}^{-s}(\mathcal{X})$ with $1<p^{\prime}, q^{\prime} \leq \infty$ and $\varphi \in \mathcal{G}_{1}\left(\epsilon_{1}, \epsilon_{2}\right)$, we have

$$
\begin{aligned}
|f(\varphi)| & =\left|\sum_{k=-\infty}^{\infty}\left\langle D_{k}(f), \tilde{D}_{k}^{t}(\varphi)\right\rangle\right| \\
& \leq \sum_{k=-\infty}^{\infty}\left\|D_{k}(f)\right\|_{L^{p^{\prime}}(x)}\left\|\tilde{D}_{k}^{t}(\varphi)\right\|_{L^{p}(x)} \\
& \leq\|f\|_{\dot{B}_{p^{\prime}, q^{\prime}}^{-s}(x)}\|\varphi\|_{\dot{B}_{p, q}^{s}(x)^{\prime}}
\end{aligned}
$$

which together with the Hahn-Banach theorem and Proposition 5.21 again proves (8.88).

We now complete the proof of (ii). Let $s \in(-\epsilon, \epsilon)$ and let $1<p, q<\infty$. Let $\left\{D_{k}\right\}_{k \in \mathbb{Z}}$ be as in Definition 5.8. Then

$$
f \in \dot{F}_{p, q}^{s}(x) \longrightarrow\left\{2^{k s} D_{k}(f)\right\}_{k=-\infty}^{\infty}
$$

is a one-to-one mapping from $\dot{F}_{p, q}^{s}(\mathcal{X})$ onto a subspace of $L^{p}\left(\dot{\ell}^{q}\right)(\mathcal{X})$, and every functional $g \in$ $\left(\dot{F}_{p, q}^{s}(\mathcal{X})\right)^{\prime}$ can be interpreted as a functional on that subspace. By the Hahn-Banach theorem, $g$ can be extended to a continuous linear functional on $L^{p}\left(\dot{\ell}^{q}\right)(\mathcal{X})$, where the norm of $g$ is preserved. If $\varphi \in \mathcal{G}_{0}^{\epsilon}(\beta, \gamma)$ with some fixed $|s|<\beta<\epsilon$ and $\max \{s-\mathcal{\kappa} / p, 0,-s-\kappa(1-1 / p)\}<$ $r<\epsilon$, then Proposition 8.10(ii) yields

$$
\begin{gathered}
g(\varphi)=\sum_{k=-\infty}^{\infty} \int_{x} g_{k}(x) D_{k}(\varphi)(x) d \mu(x), \\
\left\|\left\{2^{-s k} g_{k}\right\}_{k=-\infty}^{\infty}\right\|_{L^{p^{\prime}}\left(\dot{(}^{\prime}\right)(x)} \sim\|g\| .
\end{gathered}
$$

The formula (8.92) can be written as

$$
g(\varphi)=\sum_{k=-\infty}^{\infty} \int_{x} D_{k}^{t}\left(g_{k}\right)(x) \varphi(x) d \mu(x)
$$


which means that in $\left(\mathcal{G}_{0}^{\epsilon}(\beta, \gamma)\right)^{\prime}$ with $|s|<\beta<\epsilon$ and $\max \{s-\kappa / p, 0,-s-\kappa(1-1 / p)\}<\gamma<\epsilon$, we have

$$
g(x)=\sum_{k=-\infty}^{\infty} D_{k}^{t}\left(g_{k}\right)(x)
$$

Repeating the proof of Proposition 5.4(ii), we find that

$$
\|g\|_{\dot{F}_{p^{\prime}, q^{\prime}}^{-s}(x)} \lesssim\left\|\left\{2^{-s k} g_{k}\right\}_{k=-\infty}^{\infty}\right\|_{L^{p^{\prime}\left(\ell^{\prime} q^{\prime}\right)(x)}}
$$

Thus, $g \in \dot{F}_{p^{\prime}, q^{\prime}}^{-s}(\mathcal{X})$, which shows (ii).

To finish the proof of the theorem, we still need to establish the converse of (8.88). To this end, we first let $s \in(-\epsilon, \epsilon), 1 \leq p, q<\infty$, and $g \in\left(\dot{B}_{p, q}^{s}(\mathcal{X})\right)^{\prime}$. Repeating the above proof with $\dot{\ell}^{q^{\prime}}\left(L^{p^{\prime}}\right)(\mathcal{X})$ instead of $L^{p^{\prime}}\left(\dot{\ell}^{q^{\prime}}\right)(\mathcal{X})$, for any $\varphi \in \dot{\mathcal{G}}_{0}^{\epsilon}(\beta, \gamma)$ with some fixed $|s|<\beta<\epsilon$ and $\max \{s-\kappa / p, 0,-s-\mathcal{\kappa}(1-1 / p)\}<\gamma<\epsilon$, we see that (8.92) holds, and that

$$
\left\|\left\{2^{-s k} g_{k}\right\}_{k=-\infty}^{\infty}\right\|_{\ell^{q^{\prime}}\left(L^{p^{\prime}}\right)(x)} \sim\|g\| .
$$

Similarly, (8.92) means that (8.95) holds in $\left(\mathcal{G}_{0}^{\epsilon}(\beta, \gamma)\right)^{\prime}$ with $|s|<\beta<\epsilon$ and $\max \{s-\kappa / p, 0,-s-$ $\kappa(1-1 / p)\}<\gamma<\epsilon$. Repeating the proof of Proposition 5.6(ii), we obtain

$$
\|g\|_{\dot{B}_{p^{\prime}, g^{\prime}}^{-s}(x)} \lesssim\left\|\left\{2^{-s k} g_{k}\right\}_{k=-\infty}^{\infty}\right\|_{\dot{\ell}^{q^{\prime}}\left(L^{p^{\prime}}\right)(x)}
$$

Thus, $g \in \dot{B}_{p^{\prime}, q^{\prime}}^{-s}(\mathcal{X})$, which proves (i) when $s \in(-\epsilon, \epsilon)$ and $1 \leq p, q<\infty$.

Finally, let $s \in(-\epsilon, \epsilon), 1 \leq p<\infty$, and $0<q<1$. Then $\dot{B}_{p, q}^{s}(\mathcal{X}) \subset \dot{B}_{p, 1}^{s}(\mathcal{X})$ by Proposition 5.10(i), which gives

$$
\dot{B}_{p^{\prime}, \infty}^{-s}(\mathcal{X})=\left(\dot{B}_{p, 1}^{s}(\mathcal{X})\right)^{\prime} \subset\left(\dot{B}_{p, q}^{s}(\mathcal{X})\right)^{\prime} .
$$

On the other hand, if $g \in\left(\dot{B}_{p, q}^{s}(\mathcal{X})\right)^{\prime}$ with $s \in(-\epsilon, \epsilon), 1 \leq p<\infty$, and $0<q<1$ and letting $\left\{D_{k}\right\}_{k \in \mathbb{Z}}$ be as in Definition 5.8, we then have that for all $l \in \mathbb{Z}$,

$$
\left|\left(D_{l} g\right)(\varphi)\right|=\left|g\left(D_{l}^{t}(\varphi)\right)\right| \lesssim\|g\|\left\|D_{l}^{t}(\varphi)\right\|_{\dot{B}_{p, q}^{s}(x)} .
$$

We now estimate $\left\|D_{l}^{t}(\varphi)\right\|_{\dot{B}_{p, q}^{s}(x)}$. For any $k, l \in \mathbb{Z}$, by Theorem 3.10, we have

$$
D_{k} D_{l}^{t}(\varphi)=\sum_{k^{\prime}=-\infty}^{\infty} D_{k} D_{l}^{t} \tilde{D}_{k^{\prime}} D_{k^{\prime}}(\varphi)
$$


in $\mathcal{G}_{0}^{\epsilon}(\beta, \gamma)$ with $\beta$ and $\gamma$ as above. Using (3.2) of Lemma 3.2 and (iii) of Proposition 2.7 together with Hölder's inequality and the Fubini theorem gives

$$
\begin{aligned}
& \left\|D_{k} D_{l}^{t} \widetilde{D}_{k^{\prime}} D_{k^{\prime}}(\varphi)\right\|_{L^{p}(\mathcal{X})} \lesssim 2^{-|k-l| \epsilon_{1}^{\prime}}\left\|\widetilde{D}_{k^{\prime}} D_{k^{\prime}}(\varphi)\right\|_{L^{p}(\mathcal{X})} \lesssim 2^{-|k-l| \epsilon_{1}^{\prime}}\left\|D_{k^{\prime}}(\varphi)\right\|_{L^{p}(\mathcal{X})^{\prime}} \\
& \left\|D_{k} D_{l}^{t} \widetilde{D}_{k^{\prime}} D_{k^{\prime}}(\varphi)\right\|_{L^{p}(\mathcal{X})} \lesssim\left\|D_{l}^{t} \tilde{D}_{k^{\prime}} D_{k^{\prime}}(\varphi)\right\|_{L^{p}(\mathcal{X})} \lesssim 2^{-\left|l-k^{\prime}\right| \epsilon_{1}^{\prime \prime}}\left\|D_{k^{\prime}}(\varphi)\right\|_{L^{p}(\mathcal{X})^{\prime}}
\end{aligned}
$$

where $\epsilon_{1}^{\prime}$ and $\epsilon_{1}^{\prime \prime}$ can be any positive number in $\left(0, \epsilon_{1}\right)$. These estimates together with the geometric means and Proposition 2.7(iii) again yield that for any $\sigma \in(0,1)$,

$$
\begin{aligned}
\left\|D_{k} D_{l}^{t} \widetilde{D}_{k^{\prime}} D_{k^{\prime}}(\varphi)\right\|_{L^{p}(\mathcal{X})} & \lesssim 2^{-|k-l| \epsilon_{1}^{\prime} \sigma} 2^{-\left|l-k^{\prime}\right| \epsilon_{1}^{\prime \prime}(1-\sigma)}\left\|D_{k^{\prime}}(\varphi)\right\|_{L^{p}(\mathcal{X})} \\
& \lesssim 2^{-|k-l| \epsilon_{1}^{\prime} \sigma} 2^{-\left|l-k^{\prime}\right| \epsilon_{1}^{\prime \prime}(1-\sigma)}\|\varphi\|_{L^{p}(\mathcal{X})^{.}}
\end{aligned}
$$

If we choose $\sigma \in(0,1)$ and $\epsilon_{1}^{\prime} \in\left(0, \epsilon_{1}\right)$ such that $\epsilon_{1}^{\prime} \sigma>|s|$, then from the above estimate and (8.101) together with Definition 5.1 and (5.5), it follows that

$$
\begin{aligned}
\left\|D_{l}^{t}(\varphi)\right\|_{\dot{B}_{p, q}^{s}(\mathcal{x})} & \lesssim\left\{\sum_{k=-\infty}^{\infty} 2^{k s q}\left\|D_{k} D_{l}^{t}(\varphi)\right\|_{L^{p}(\mathcal{x})}^{q}\right\}^{1 / q} \\
& \lesssim\left\{\sum_{k=-\infty}^{\infty} 2^{k s q}\left[\sum_{k^{\prime}=-\infty}^{\infty} 2^{-|k-l| \epsilon_{1}^{\prime} \sigma} 2^{-\left|l-k^{\prime}\right| \epsilon_{1}^{\prime \prime}(1-\sigma)}\|\varphi\|_{L^{p}(\mathcal{X})}\right]^{q}\right\}^{1 / q} \\
& \lesssim 2^{l s}\|\varphi\|_{L^{p}(\mathcal{x})}
\end{aligned}
$$

Thus,

$$
\left|\left(D_{l} g\right)(\varphi)\right| \lesssim 2^{-l s}\|\varphi\|_{L^{p}(x)}
$$

which implies that

$$
\sup _{l \in \mathbb{Z}}\left\{2^{-l s}\left\|D_{l}(g)\right\|_{L^{p^{\prime}}(x)}\right\} \lesssim\|g\|
$$

That is, $g \in \dot{B}_{p^{\prime} \infty}^{-s}(\mathcal{X})$, which completes the proof of (i), and hence, Theorem 8.11.

To determine the dual space of $\dot{F}_{p, q}^{s}(x)$ when $p=1$, following [82], we first consider the corresponding spaces of sequences. Let $\lambda$ be a sequence as in (7.1). Then for $s \in \mathbb{R}, 0<q \leq \infty$, and $x \in \mathcal{X}$, we define

$$
\begin{aligned}
\dot{G}^{s, q}(\lambda)(x) & =\left\{\sum_{k=-\infty}^{\infty} \sum_{\tau \in I_{k}} \sum_{\nu=1}^{N(k, \tau)} 2^{k s q}\left|\lambda_{\tau}^{k, v}\right|^{q} \chi_{Q_{\tau}^{k, v}}(x)\right\}^{1 / q}, \\
\dot{\mathfrak{G}}_{\tau, k, v}^{s, q}(\lambda)(x) & =\left\{\sum_{k^{\prime}=k+1}^{\infty} \sum_{Q_{\tau^{\prime}}^{k^{\prime}, \nu^{\prime}} \subset Q_{\tau}^{k, v}} 2^{k^{\prime} s q}\left|\lambda_{\tau^{\prime}}^{k^{\prime}, \nu^{\prime}}\right|^{q} \chi_{Q_{\tau^{\prime}}^{k^{\prime}, \nu^{\prime}}}(x)\right\}^{1 / q} .
\end{aligned}
$$


Let $\dot{m}_{\tau, k, v}^{s, q}(\lambda)$ denote the "1/4-median" of $\dot{\mathfrak{G}}_{\tau, k, v}^{s, q}(\lambda)$ on $Q_{\tau}^{k, v}$, namely,

$$
\dot{m}_{\tau, k, v}^{s, q}=\inf \left\{\delta>0: \mu\left(\left\{x \in Q_{\tau}^{k, v}: \dot{\mathfrak{G}}_{\tau, k, v}^{s, q}(\lambda)(x)>\delta\right\}\right)<\frac{\mu\left(Q_{\tau}^{k, v}\right)}{4}\right\} .
$$

We also set

$$
\dot{m}^{s, q}(\lambda)(x)=\sup _{k, \tau, v} \dot{m}_{\tau, k, v}^{s, q}(\lambda) \chi_{Q_{\tau}^{k, v}}(x)
$$

The following conclusion is trivial by the definition of $\dot{f}_{\infty, q}^{s}(\mathcal{X})$.

Proposition 8.12. Let $\delta \in(0,1], s \in \mathbb{R}$, and $0<q \leq \infty$. Assume that for each dyadic cube $Q$, there exists a measurable set $E_{Q} \subset Q$ such that $\mu\left(E_{Q}\right) / \mu(Q) \geq \delta$. Then

$$
\|\lambda\|_{f_{\infty, q}^{s}(\mathcal{X})} \sim \sup _{l \in \mathbb{Z}} \sup _{\alpha \in I_{l}}\left\{\frac{1}{\mu\left(Q_{\alpha}^{l}\right)}\left[\sum_{k=l}^{\infty} \sum_{\tau \in I_{k}} \sum_{\nu=1}^{N(k, \tau)} 2^{k s q} \mu\left(E_{Q_{\tau}^{k, v}}\right)\left|\lambda_{\tau}^{k, v}\right|^{q} X_{\left\{(\tau, v): Q_{\tau}^{k, v} \subset Q_{\alpha}^{l}\right\}}(\tau, v)\right]\right\}^{1 / q}
$$

We now establish a characterization of $\dot{f}_{\infty, q}^{s}(\mathcal{X})$ by means of $\dot{m}^{s, q}$.

Proposition 8.13. Let $s \in \mathbb{R}$ and $0<q \leq \infty$. Then

$$
\|\lambda\|_{f_{s, q}^{s}(x)} \sim\left\|\dot{m}^{s, q}(\lambda)\right\|_{L^{\infty}(x)} .
$$

Proof. By Chebyshev's inequality, we see that

$$
\begin{aligned}
\mu\left(\left\{x \in Q_{\tau}^{k, v}: \dot{\mathfrak{G}}_{\tau, k, v}^{s, q}(\lambda)(x)>\delta\right\}\right) & \leq \frac{1}{\delta^{q}} \int_{Q_{\tau}^{k, \nu}}\left[\dot{\mathfrak{G}}_{\tau, k, v}^{s, q}(\lambda)(x)\right]^{q} d \mu(x) \\
& \leq \frac{\mu\left(Q_{\tau}^{k, \nu}\right)}{\delta^{q}}\|\lambda\|_{\dot{f}_{\infty, q}^{s}(x)}^{q}(x) \\
& <\frac{1}{4} \mu\left(Q_{\tau}^{k, \nu}\right),
\end{aligned}
$$

if $\delta>4^{1 / \delta}\|\lambda\|_{f_{\infty, q}^{s}(x)}$. Hence,

$$
\left\|\dot{m}^{s, q}(\lambda)\right\|_{L^{\infty}(\chi)} \lesssim\|\lambda\|_{f_{\infty, q}^{s}(\chi)} .
$$

To establish the converse, we introduce the extended integer-valued stopping time $k(x)$ for $x \in X$ by

$$
k(x)=\inf \left\{k \in \mathbb{Z}:\left(\sum_{k^{\prime}=k}^{\infty} \sum_{\tau^{\prime} \in I_{k^{\prime}}} \sum_{\nu^{\prime}=1}^{N\left(k^{\prime}, \tau^{\prime}\right)} 2^{k^{\prime} s q}\left|\lambda_{\tau^{\prime}}^{k^{\prime}, v^{\prime}}\right|^{q} \chi_{Q_{\tau^{\prime}}^{k^{\prime}, \nu^{\prime}}}\right)^{1 / q} \leq \dot{m}^{s, q}(\lambda)(x)\right\} .
$$


Also, for any $Q_{\tau}^{k, v}$, set

$$
E_{Q_{\tau}^{k, v}}=\left\{x \in Q_{\tau}^{k, v}: k(x) \leq k\right\} .
$$

Then, obviously,

$$
E_{Q_{\tau}^{k, v}}=\left\{x \in Q_{\tau}^{k, v}: \dot{\mathfrak{G}}_{\tau, k, v}^{s, q}(\lambda)(x) \leq \dot{m}^{s, q}(\lambda)(x)\right\} .
$$

From (8.108), it follows that

$$
\begin{aligned}
\mu(\{x & \left.\left.\in Q_{\tau}^{k, v}: \dot{\mathfrak{G}}_{\tau, k, v}^{s, q}(\lambda)(x)>\dot{m}^{s, q}(\lambda)(x)\right\}\right) \\
& \leq \mu\left(\left\{x \in Q_{\tau}^{k, v}: \dot{\mathfrak{G}}_{\tau, k, v}^{s, q}(\lambda)(x)>\dot{m}_{\tau, k, v}^{s, q}(\lambda)\right\}\right) \leq \frac{1}{4} \mu\left(Q_{\tau}^{k, v}\right),
\end{aligned}
$$

and hence, $\mu\left(E_{Q_{\tau}^{k, v}}\right) \geq(3 / 4) \mu\left(Q_{\tau}^{k, v}\right)$, and

$$
\left(\sum_{k=-\infty}^{\infty} \sum_{\tau \in I_{k}} \sum_{\nu=1}^{N(k, \tau)} 2^{k s q}\left|\lambda_{\tau}^{k, v}\right|^{q} X_{E_{Q_{\tau}}^{k, v}}(x)\right)^{1 / q} \lesssim \dot{m}^{s, q}(\lambda)(x)
$$

which together with Proposition 8.12 yields

$$
\|\lambda\|_{\dot{f}_{\infty, q}^{s}(\mathcal{})} \lesssim\left\|\dot{m}^{s, q}(\lambda)\right\|_{L^{\infty}(\mathcal{X})} .
$$

This finishes the proof of Proposition 8.13.

We next prove the following duality for $\dot{f}_{1, q}^{s}(\mathcal{X})$.

Proposition 8.14. Assume that $s \in \mathbb{R}$ and $0<q<\infty$. Then $\left(\dot{f}_{1, q}^{s}(\mathcal{X})\right)^{\prime}=\dot{f}_{\infty, q^{\prime}}^{-s}(\mathcal{X})$. In particular, if $\lambda=\left\{\lambda_{\tau}^{k, v}: k \in \mathbb{Z}, \tau \in I_{k}, v=1, \ldots, N(k, \tau)\right\} \in \dot{f}_{\infty, q^{\prime}}^{-s}(\mathcal{X})$, then the map

$$
t=\left\{t_{Q_{\tau}^{k, v}}: k \in \mathbb{Z}, \tau \in I_{k}, v=1, \ldots, N(k, \tau)\right\} \longmapsto\langle t, \lambda\rangle,
$$

where

$$
\langle t, \lambda\rangle=\sum_{k=-\infty}^{\infty} \sum_{\tau \in I_{k}} \sum_{\nu=1}^{N(k, \tau)} t_{Q_{\tau}^{k, v}} \overline{\lambda_{\tau}^{k, v}} \mu\left(Q_{\tau}^{k, v}\right),
$$

defines a continuous linear functional on $\dot{f}_{1, q}^{s}(\mathcal{X})$ with operator norm $\|\lambda\|_{\left(f_{1, q}^{s}(x)\right)^{\prime}}$ equivalent to $\|\lambda\|_{\dot{f}_{1, q}^{s}(\mathcal{X})}$, and every $\ell \in\left(\dot{f}_{1, q}^{s}(\mathcal{X})\right)^{\prime}$ is of this form for some $\lambda \in \dot{f}_{1, q}^{s}(\mathcal{X})$. 
Proof. We first assume that $1 \leq q<\infty$. Similarly to the proof of Proposition 8.13 , let

$$
E_{Q_{\tau}^{k, v}}=\left\{x \in Q_{\tau}^{k, v}: \dot{\mathfrak{G}}_{\tau, k, v}^{-s, q^{\prime}}(\lambda)(x) \leq \dot{m}^{-s, q^{\prime}}(\lambda)(x)\right\} .
$$

Then $\mu\left(E_{Q_{\tau}^{k, v}}\right) \geq(3 / 4) \mu\left(Q_{\tau}^{k, v}\right)$ and

$$
\left(\sum_{k=-\infty}^{\infty} \sum_{\tau \in I_{k}} \sum_{\nu=1}^{N(k, \tau)} 2^{-k s q^{\prime}}\left|\lambda_{\tau}^{k, v}\right|^{q^{\prime}} X_{E_{Q_{\tau}^{k, \nu}}}(x)\right)^{1 / q^{\prime}} \lesssim \dot{m}^{-s, q^{\prime}}(\lambda)(x) .
$$

From this, Hölder's inequality, and Proposition 8.13, it follows that

$$
\begin{aligned}
& \left|\sum_{k=-\infty}^{\infty} \sum_{\tau \in I_{k}} \sum_{v=1}^{N(k, \tau)} \mu\left(Q_{\tau}^{k, v}\right) t_{Q_{\tau}^{k, v}} \overline{\lambda_{\tau}^{k, v}}\right| \\
& \lesssim \int_{\chi} \sum_{k=-\infty}^{\infty} \sum_{\tau \in I_{k}} \sum_{\nu=1}^{N(k, \tau)}\left(2^{k s}\left|t_{Q_{\tau}^{k, \nu}}\right| X_{Q_{\tau}^{k, v}}(x)\right)\left(2^{-k s}\left|\lambda_{\tau}^{k, v}\right| X_{Q_{Q_{\tau}^{k}}^{k, v}}(x)\right) d \mu(x) \\
& \lesssim \int_{\chi}\left\{\sum_{k=-\infty}^{\infty} \sum_{\tau \in I_{k}} \sum_{\nu=1}^{N(k, \tau)} 2^{k s q}\left|t_{Q_{\tau}^{k, v}}\right|^{q} X_{Q_{\tau}^{k, v}}(x)\right\}^{1 / q}\left\{\sum_{k=-\infty}^{\infty} \sum_{\tau \in I_{k}} \sum_{\nu=1}^{N(k, \tau)} 2^{-k s q^{\prime}}\left|\lambda_{\tau}^{k, v}\right|^{q^{\prime}} X_{Q_{Q_{\tau}}^{k, v}}(x)\right\}^{1 / q^{\prime}} d \mu(x) \\
& \lesssim\|t\|_{\dot{f}_{1, q}^{s}(x)}\left\|\dot{m}^{-s, q^{\prime}}(\lambda)\right\|_{L^{\infty}(x)} \\
& \lesssim\|t\|_{\dot{f}_{1, q}^{s}(x)}\|\lambda\|_{\dot{f}_{\infty, q^{\prime}}^{-s}(x)}
\end{aligned}
$$

which yields

$$
\|\lambda\|_{\left(\dot{f}_{1, q}^{s}(x)\right)^{\prime}} \lesssim\|\lambda\|_{\dot{f}_{\infty, q^{\prime}}^{-s}(x)}
$$

if $1 \leq q<\infty$. The case $0<q<1$ then follows from the trivial imbedding $\dot{f}_{1, q}^{s}(\mathcal{X}) \subset \dot{f}_{1,1}^{-s}(\mathcal{X})$. form:

Conversely, by Proposition 8.10(i), it is easy to see that every $\ell \in\left(\dot{f}_{1, q}^{s}(\mathcal{X})\right)^{\prime}$ is of the

$$
t \longmapsto \sum_{k=-\infty}^{\infty} \sum_{\tau \in I_{k}} \sum_{\nu=1}^{N(k, \tau)} \mu\left(Q_{\tau}^{k, v}\right) t_{Q_{\tau}^{k, v}} \lambda_{\tau}^{k, v}
$$

for some $\lambda$ as in (7.1). Assume first again that $1 \leq q<\infty$. Fix a dyadic cube $Q_{\alpha}^{l}$. Let $y$ be the sequence space of all dyadic cubes $Q_{\tau}^{k, v}$ such that $Q_{\tau}^{k, v} \subset Q_{\alpha}^{l}$, and let $\sigma$ be a measure on $y$ such 
that the $\sigma$-measure of the point $Q_{\tau}^{k, v}$ is $\mu\left(Q_{\tau}^{k, v}\right) / \mu\left(Q_{\alpha}^{l}\right)$. Then

$$
\begin{aligned}
& \left\{\frac{1}{\mu\left(Q_{\alpha}^{l}\right)} \sum_{k=l}^{\infty} \sum_{\tau \in I_{k}} \sum_{\nu=1}^{N(k, \tau)} 2^{-k s q} \mu\left(Q_{\tau}^{k, v}\right)\left|\lambda_{\tau}^{k, v}\right|^{q^{\prime}} X_{\left\{(\tau, v): Q_{\tau}^{k, v} \subset Q_{\alpha}^{l}\right\}}(\tau, v)\right\}^{1 / q^{\prime}} \\
& =\left\|\left\{2^{-k s} \lambda_{\tau}^{k, v}\right\}_{Q_{\tau}^{k, v} \subset Q_{\alpha}^{l}}\right\|_{\ell^{q^{\prime}}(y, d \sigma)} \\
& =\sup _{\|t\|_{\ell^{q}(, d, d) \leq 1}}\left|\frac{1}{\mu\left(Q_{\alpha}^{l}\right)} \sum_{k=l}^{\infty} \sum_{\tau \in I_{k}} \sum_{\nu=1}^{N(k, \tau)} t_{Q_{\tau}^{k, \nu}} 2^{-k s} \overline{\lambda_{\tau}^{k, v}} \mu\left(Q_{\tau}^{k, v}\right)\right| \\
& \leq\|\lambda\|_{\left(\dot{f}_{1, q}^{s}(x)\right)^{\prime}} \sup _{\|t\|_{l^{q}(y, d \sigma)} \leq 1}\left\|\left\{2^{-k s} t_{Q_{\tau}^{k, \nu}} \frac{1}{\mu\left(Q_{\alpha}^{l}\right)}\right\}_{Q_{\tau}^{k, v} \subset Q_{\alpha}^{l}}\right\|_{\dot{f}_{1, q}^{s}(\mathcal{X})} .
\end{aligned}
$$

However, by Hölder's inequality, we have

$$
\begin{aligned}
\left\|\left\{2^{-k s} t_{Q_{\tau}^{k, v}} \frac{1}{\mu\left(Q_{\alpha}^{l}\right)}\right\}_{Q_{\tau}^{k, v} \subset Q_{\alpha}^{l}}\right\|_{f_{1, q}^{s}(\mathcal{X})} \\
\quad=\frac{1}{\mu\left(Q_{\alpha}^{l}\right)} \int_{Q_{\alpha}^{l}}\left\{\sum_{k=l}^{\infty} \sum_{\tau \in I_{k}} \sum_{v=1}^{N(k, \tau)}\left|t_{Q_{\tau}^{k, v}}\right|^{q} X_{\left\{(\tau, v): Q_{\tau}^{k, v} \subset Q_{\alpha}^{l}\right\}}(\tau, v) X_{Q_{\tau}^{k, v}}(x)\right\}^{1 / q} d \mu(x) \\
\quad \lesssim\left\{\frac{1}{\mu\left(Q_{\alpha}^{l}\right)} \sum_{k=l}^{\infty} \sum_{\tau \in I_{k}} \sum_{v=1}^{N(k, \tau)}\left|t_{Q_{\tau}^{k, v}}\right|^{q} X_{\left\{(\tau, v): Q_{\tau}^{k, v} \subset Q_{\alpha}^{l}\right\}}(\tau, v) \mu\left(Q_{\tau}^{k, v}\right)\right\}^{1 / q} \\
\sim\|t\|_{\ell(y, d \sigma)} .
\end{aligned}
$$

Thus, if $1 \leq q<\infty$, then

$$
\|\lambda\|_{f_{\infty, q^{\prime}}^{-s}(x)} \lesssim\|\lambda\|_{\left(f_{1, q}^{s}(x)\right)^{\prime}}
$$

For $0<q<1$, we have $q^{\prime}=\infty$. In this case, for any $Q_{\tau}^{k, v}$, we set $\left(t^{\mathrm{Q}_{\tau}^{k, v}}\right)_{\mathrm{Q}_{\tau^{\prime}}^{k^{\prime}, v^{\prime}}}=2^{-k s} / \mu\left(Q_{\tau}^{k, v}\right)$ for $Q_{\tau^{\prime}}^{k^{\prime}, v^{\prime}}=Q_{\tau}^{k, v}$ and 0 otherwise. Clearly, $\left\|t^{Q_{\tau}^{k, v}}\right\|_{f_{1, q}^{s}(x)}=1$ and hence,

$$
\|\lambda\|_{\dot{f}_{\infty, s, \infty}^{-s}(x)} \leq \sup _{k, \tau, v}\left|\left\langle t^{Q_{\tau}^{k, v}}, \lambda\right\rangle\right| \lesssim\|\lambda\|_{\left(f_{1, q}^{s}(x)\right)^{\prime}}
$$

which completes the proof of Proposition 8.14.

From Proposition 7.1, Theorem 7.2, and Proposition 8.14, by a standard method as in [82, pages 79-80], we can obtain the dual space of $\dot{F}_{p, q}^{s}(\mathcal{X})$ when $p=1$ as follows. We omit the details. 
Theorem 8.15. Let $\epsilon$ be as in Definition 6.1, $|s|<\epsilon$, and $p(s, \epsilon)<q<\infty$. Then $\left(\dot{F}_{1, q}^{s}(\mathcal{X})\right)^{\prime}=$ $\dot{F}_{\infty, q^{\prime}}^{-s}(\mathcal{X})$ in the sense of that in Theorem 8.11(ii).

A slight modification of the proof of Proposition 2.11.1 in [3, pages 177-178] again gives the following inhomogeneous version of Propositions 8.10 and 8.14. We omit the details.

Proposition 8.16. Let $1 \leq p<\infty$ and $0<q<\infty$. Then the following hold.

(i) $g \in\left(L^{p}\left(\ell^{q}\right)(X)\right)^{\prime}$ if and only if there is a sequence $\left\{g_{j}\right\}_{j \in \mathbb{Z}_{+}} \in L^{p^{\prime}}\left(\ell^{q^{\prime}}\right)(X)$ such that

$$
g(f)=\sum_{j=0}^{\infty} \int_{x} g_{j}(x) f_{j}(x) d \mu(x)
$$

$$
\text { for every } f=\left\{f_{j}\right\}_{j \in \mathbb{Z}_{+}} \in L^{p}\left(\ell^{q}\right)(x) \text {, and }\|g\| \sim\left\|\left\{g_{j}\right\}_{j \in \mathbb{Z}_{+}}\right\|_{L^{p^{\prime}}\left(\ell^{q^{\prime}}\right)(x)} .
$$

(ii) $g \in\left(\ell^{q}\left(L^{p}\right)(X)\right)^{\prime}$ if and only if there is a sequence $\left\{g_{j}\right\}_{j \in \mathbb{Z}_{+}} \in \ell^{q^{\prime}}\left(L^{p^{\prime}}\right)(X)$

$$
g(f)=\sum_{j=0}^{\infty} \int_{\chi} g_{j}(x) f_{j}(x) d \mu(x)
$$

$$
\text { for every } f=\left\{f_{j}\right\}_{j \in \mathbb{Z}_{+}} \in \ell^{q}\left(L^{p}\right)(\mathcal{X}) \text {, and }\|g\| \sim\left\|\left\{g_{j}\right\}_{j \in \mathbb{Z}_{+}}\right\|_{\ell_{q^{\prime}\left(L^{p^{\prime}}\right)(x)}} \text {. }
$$

Theorem 8.17. Assume that $s \in \mathbb{R}$ and $0<q<\infty$. Then $\left(f_{1, q}^{s}(\mathcal{X})\right)^{\prime}=f_{\infty, q^{\prime}}^{-s}(\mathcal{X})$. In particular, if $\lambda=\left\{\lambda_{\tau}^{k, v}: k \in \mathbb{Z}_{+}, \tau \in I_{k}, v=1, \ldots, N(k, \tau)\right\} \in f_{\infty, q^{\prime}}^{-s}(\mathcal{X})$, then the map

$$
t=\left\{t_{Q_{\tau}^{k, v}}: k \in \mathbb{Z}_{+}, \tau \in I_{k}, v=1, \ldots, N(k, \tau)\right\} \longrightarrow\langle t, \lambda\rangle,
$$

where

$$
\langle t, \lambda\rangle=\sum_{k=0}^{\infty} \sum_{\tau \in I_{k}} \sum_{v=1}^{N(k, \tau)} t_{Q_{\tau}^{k, v}} \overline{\lambda_{\tau}^{k, v}} \mu\left(Q_{\tau}^{k, v}\right),
$$

defines a continuous linear functional on $f_{1, q}^{s}(x)$ with operator norm $\|\lambda\|_{\left(f_{1, q}^{s}(x)\right)^{\prime}}$ equivalent to $\|\lambda\|_{f_{1, q}^{s}(x)}$, and every $\ell \in\left(f_{1, q}^{s}(\mathcal{X})\right)^{\prime}$ is of this form for some $\lambda \in f_{1, q}^{s}(\mathcal{X})$.

With Proposition 8.16 and Theorem 8.17, respectively, in place of Propositions 8.10 and 8.14 , by a procedure similar to the proof of Theorem 8.11 (see also [3, the proof of Theorem 2.11.2]) and Theorem 5.6 in [82, pages 79-80], we can establish the following inhomogeneous version of Theorems 8.11 and 8.15 , which describes the dual spaces of some inhomogeneous Besov and Triebel-Lizorkin spaces. We omit the details. 
Theorem 8.18. Let $\epsilon$ be as in Definition 5.8 and $|s|<\epsilon$. Then, the following hold.

(i) If $1 \leq p<\infty$ and $0<q<\infty$, then

$$
\left(B_{p, q}^{s}(\mathcal{X})\right)^{\prime}=B_{p^{\prime}, q^{\prime}}^{-s}(X)
$$

More precisely, given $g \in B_{p^{\prime}, q^{\prime}}^{-s}(\mathcal{X})$, then $\mathcal{L}_{g}(f) \equiv\langle f, g\rangle$ defines a linear functional on $\mathcal{G}(\epsilon, \epsilon) \cap$ $B_{p, q}^{s}(X)$ such that

$$
\left|\mathcal{L}_{g}(f)\right| \leq C\|f\|_{B_{p, q}^{s}(x)}\|g\|_{B_{p^{\prime}, q^{\prime}}^{-s}(x)}
$$

where $C>0$ is independent of $f$, and this linear functional naturally extends to $B_{p, q}^{s}(\mathcal{X})$ by continuity with norm at most $C\|g\|_{B_{p^{\prime}, q^{\prime}}^{-s}(x)}$.

Conversely, if $\mathcal{\perp}$ is a linear functional on $B_{p, q}^{s}(\mathcal{X})$, then there exists a unique $g \in B_{p^{\prime}, q^{\prime}}^{-s}(X)$ such that $\mathcal{L}$ is the natural extension of $\mathcal{L}_{g}$, with $\|g\|_{B_{p^{\prime}, q^{\prime}}^{-s}(x)} \leq C\|\mathcal{L}\|$.

(ii) If $1<p, q<\infty$, or $p=1$ and $p(s, \epsilon)<q<\infty$, then

$$
\left(F_{p, q}^{s}(x)\right)^{\prime}=F_{p^{\prime}, q^{\prime}}^{-s}(x) .
$$

More precisely, given $g \in F_{p^{\prime}, q^{\prime}}^{-s}(\mathcal{X})$, then $\mathcal{L}_{g}(f) \equiv\langle f, g\rangle$ defines a linear functional on $\mathcal{G}(\epsilon, \epsilon) \cap$ $F_{p, q}^{s}(X)$ such that

$$
\left|\mathcal{L}_{g}(f)\right| \leq C\|f\|_{F_{p, q}^{s}(x)}\|g\|_{F_{p^{\prime}, q^{\prime}}^{-s}(x)^{\prime}}
$$

where $C>0$ is independent of $f$, and this linear functional naturally extends to $F_{p, q}^{s}(\mathcal{X})$ by continuity with norm at most $C\|g\|_{F_{p^{\prime}, g^{\prime}}^{-s}(\boldsymbol{x})}$.

Conversely, if $\mathcal{L}$ is a linear functional on $F_{p, q}^{s}(\mathcal{X})$, then there exists a unique $g \in F_{p^{\prime}, q^{\prime}}^{-s}(x)$ such that $\mathcal{L}$ is the natural extension of $\mathcal{L}_{g}$, with $\|g\|_{F_{p^{\prime}, q^{\prime}}^{-s}(x)} \leq C\|\mathcal{L}\|$.

\section{Acknowledgments}

The authors would like to thank the referees for their many helpful suggestions and corrections, which improve the presentation of this paper. Dachun Yang also wishes to express his sincerely thanks to Professor Hans Triebel for several helpful suggestions on this paper. Dachun Yang is supported by National Science Foundation for Distinguished Young Scholars (no. 10425106) and NCET (no. 04-0142) of Ministry of Education of China.

\section{References}

[1] H. Triebel, Theory of Function Spaces. II, vol. 84 of Monographs in Mathematics, Birkhäuser, Basel, Switzerland, 1992.

[2] H. Triebel, Theory of Function Spaces. III, vol. 100 of Monographs in Mathematics, Birkhäuser, Basel, Switzerland, 2006.

[3] H. Triebel, Theory of Function Spaces, vol. 78 of Monographs in Mathematics, Birkhäuser, Basel, Switzerland, 1983. 
[4] A. Jonsson and H. Wallin, "Function spaces on subsets of $\mathbb{R}^{n}$," Mathematical Reports, vol. 2, no. 1, pp. $1-221,1984$.

[5] H. Triebel, Fractals and Spectra, vol. 91 of Monographs in Mathematics, Birkhäuser, Basel, Switzerland, 1997.

[6] H. Triebel, The Structure of Functions, vol. 97 of Monographs in Mathematics, Birkhäuser, Basel, Switzerland, 2001.

[7] K.-T. Sturm, "On the geometry of metric measure spaces. I," Acta Mathematica, vol. 196, no. 1, pp. 65-131, 2006.

[8] K.-T. Sturm, "On the geometry of metric measure spaces. II," Acta Mathematica, vol. 196, no. 1, pp. 133-177, 2006.

[9] J. Lott and C. Villani, "Ricci curvature for metric-measure spaces via optimal transport," to appear in Annals of Mathematics. Second Series.

[10] S. Semmes, "An introduction to analysis on metric spaces," Notices of the American Mathematical Society, vol. 50, no. 4, pp. 438-443, 2003.

[11] N. J. Korevaar and R. M. Schoen, "Sobolev spaces and harmonic maps for metric space targets," Communications in Analysis and Geometry, vol. 1, no. 3-4, pp. 561-659, 1993.

[12] P. Hajłasz and P. Koskela, "Sobolev met Poincaré," Memoirs of the American Mathematical Society, vol. 145 , no. 688 , pp. 1-101, 2000.

[13] J. Heinonen, Lectures on Analysis on Metric Spaces, Universitext, Springer, New York, NY, USA, 2001.

[14] A. Grigor'yan, J. Hu, and K.-S. Lau, "Heat kernels on metric measure spaces and an application to semilinear elliptic equations," Transactions of the American Mathematical Society, vol. 355, no. 5, pp. 2065-2095, 2003.

[15] A. Grigor'yan, "Heat kernels and function theory on metric measure spaces," in Heat Kernels and Analysis on Manifolds, Graphs, and Metric Spaces (Paris, 2002), vol. 338 of Contemporary Mathematics, pp. 143-172, American Mathematical Society, Providence, RI, USA, 2003.

[16] S. Keith and X. Zhong, "The Poincaré inequality is an open ended condition," Annals of Mathematics. Second Series, vol. 167, no. 2, pp. 575-599, 2008.

[17] P. Hajłasz, "Sobolev spaces on an arbitrary metric space," Potential Analysis, vol. 5, no. 4, pp. 403-415, 1996.

[18] P. Koskela and P. MacManus, "Quasiconformal mappings and Sobolev spaces," Studia Mathematica, vol. 131, no. 1, pp. 1-17, 1998.

[19] B. Franchi, P. Hajłasz, and P. Koskela, "Definitions of Sobolev classes on metric spaces," Annales de l'Institut Fourier, vol. 49, no. 6, pp. 1903-1924, 1999.

[20] A. E. Gatto and S. Vági, "On Sobolev spaces of fractional order and $\epsilon$-families of operators on spaces of homogeneous type," Studia Mathematica, vol. 133, no. 1, pp. 19-27, 1999.

[21] Y. Liu, G. Lu, and R. L. Wheeden, "Some equivalent definitions of high order Sobolev spaces on stratified groups and generalizations to metric spaces," Mathematische Annalen, vol. 323, no. 1, pp. 157-174, 2002.

[22] N. Shanmugalingam, "Newtonian spaces: an extension of Sobolev spaces to metric measure spaces," Revista Matemática Iberoamericana, vol. 16, no. 2, pp. 243-279, 2000.

[23] J. Heinonen, P. Koskela, N. Shanmugalingam, and J. T. Tyson, "Sobolev classes of Banach spacevalued functions and quasiconformal mappings," Journal d'Analyse Mathématique, vol. 85, pp. 87-139, 2001.

[24] S. Klainerman and I. Rodnianski, "A geometric approach to the Littlewood-Paley theory," Geometric and Functional Analysis, vol. 16, no. 1, pp. 126-163, 2006.

[25] D. Danielli, N. Garofalo, and D.-M. Nhieu, "Non-doubling Ahlfors measures, perimeter measures, and the characterization of the trace spaces of Sobolev functions in Carnot-Carathéodory spaces," Memoirs of the American Mathematical Society, vol. 182, no. 857, pp. 1-119, 2006.

[26] P. Hajłasz, T. Iwaniec, J. Malý, and J. Onninen, "Weakly differentiable mappings between manifolds," Memoirs of the American Mathematical Society, vol. 192, no. 899, pp. 1-72, 2008.

[27] G. Furioli, C. Melzi, and A. Veneruso, "Littlewood-Paley decompositions and Besov spaces on Lie groups of polynomial growth," Mathematische Nachrichten, vol. 279, no. 9-10, pp. 1028-1040, 2006.

[28] R. R. Coifman and G. Weiss, "Extensions of Hardy spaces and their use in analysis," Bulletin of the American Mathematical Society, vol. 83, no. 4, pp. 569-645, 1977.

[29] R. R. Coifman and G. Weiss, Analyse Harmonique Non-Commutative sur Certains Espaces Homogènes, vol. 242 of Lecture Notes in Mathematics, Springer, Berlin, Germany, 1971.

[30] R. A. Macías and C. Segovia, "A decomposition into atoms of distributions on spaces of homogeneous type," Advances in Mathematics, vol. 33, no. 3, pp. 271-309, 1979. 
[31] Y. Han, "Calderon-type reproducing formula and the Tb theorem," Revista Matemática Iberoamericana, vol. 10, no. 1, pp. 51-91, 1994.

[32] X. T. Duong and L. Yan, "Hardy spaces of spaces of homogeneous type," Proceedings of the American Mathematical Society, vol. 131, no. 10, pp. 3181-3189, 2003.

[33] R. A. Macías and C. Segovia, "Lipschitz functions on spaces of homogeneous type," Advances in Mathematics, vol. 33, no. 3, pp. 257-270, 1979.

[34] Y. Han and E. T. Sawyer, "Littlewood-Paley theory on spaces of homogeneous type and the classical function spaces," Memoirs of the American Mathematical Society, vol. 110, no. 530, pp. 1-126, 1994.

[35] Y. Han, "Inhomogeneous Calderón reproducing formula on spaces of homogeneous type," The Journal of Geometric Analysis, vol. 7, no. 2, pp. 259-284, 1997.

[36] Y. Han and D. Yang, "New characterizations and applications of inhomogeneous Besov and TriebelLizorkin spaces on homogeneous type spaces and fractals," Dissertationes Mathematicae, vol. 403, pp. 1-102, 2002.

[37] Y. Han and D. Yang, "Some new spaces of Besov and Triebel-Lizorkin type on homogeneous spaces," Studia Mathematica, vol. 156, no. 1, pp. 67-97, 2003.

[38] A. Bellaïche and J.-J. Risler, Eds., Sub-Riemannian Geometry, vol. 144 of Progress in Mathematics, Birkhäuser, Basel, Switzerland, 1996.

[39] R. S. Strichartz, “Sub-Riemannian geometry," Journal of Differential Geometry, vol. 24, no. 2, pp. 221263, 1986.

[40] E. M. Stein, "Some geometrical concepts arising in harmonic analysis," Geometric and Functional Analysis, pp. 434-453, 2000, Special Volume, Part I.

[41] A. Nagel and E. M. Stein, "The $\square_{b}$-heat equation on pseudoconvex manifolds of finite type in $\mathbb{C}^{2}$," Mathematische Zeitschrift, vol. 238, no. 1, pp. 37-88, 2001.

[42] A. Nagel and E. M. Stein, "Differentiable control metrics and scaled bump functions," Journal of Differential Geometry, vol. 57, no. 3, pp. 465-492, 2001.

[43] A. Nagel and E. M. Stein, "The $\bar{\partial}_{b}$-complex on decoupled boundaries in $\mathbb{C}^{n}$," Annals of Mathematics. Second Series, vol. 164, no. 2, pp. 649-713, 2006.

[44] A. Nagel and E. M. Stein, "On the product theory of singular integrals," Revista Matemática Iberoamericana, vol. 20, no. 2, pp. 531-561, 2004.

[45] A. Nagel, J.-P. Rosay, E. M. Stein, and S. Wainger, "Estimates for the Bergman and Szegö kernels in $\mathbb{C}^{2}, "$ Annals of Mathematics. Second Series, vol. 129, no. 1, pp. 113-149, 1989.

[46] D.-C. Chang, A. Nagel, and E. M. Stein, "Estimates for the $\bar{\partial}$-Neumann problem in pseudoconvex domains of finite type in $\mathbb{C}^{2}$," Acta Mathematica, vol. 169, no. 1, pp. 153-228, 1992.

[47] K. D. Koenig, "On maximal Sobolev and Hölder estimates for the tangential Cauchy-Riemann operator and boundary Laplacian," American Journal of Mathematics, vol. 124, no. 1, pp. 129-197, 2002.

[48] Y. Han, D. Müller, and D. Yang, "Littlewood-Paley characterizations for Hardy spaces on spaces of homogeneous type," Mathematische Nachrichten, vol. 279, no. 13-14, pp. 1505-1537, 2006.

[49] D. Müller and D. Yang, "A difference characterization of Besov and Triebel-Lizorkin spaces on RDspaces," to appear in Forum Mathematicum.

[50] J.-O. Strömberg and A. Torchinsky, Weighted Hardy Spaces, vol. 1381 of Lecture Notes in Mathematics, Springer, Berlin, Germany, 1989.

[51] R. L. Wheeden, "A characterization of some weighted norm inequalities for the fractional maximal function," Studia Mathematica, vol. 107, no. 3, pp. 257-272, 1993.

[52] A. Jonsson, "Besov spaces on closed subsets of $\mathbb{R}^{n}$," Transactions of the American Mathematical Society, vol. 341, no. 1, pp. 355-370, 1994.

[53] A. Jonsson, "Besov spaces on closed sets by means of atomic decompositions," Research Reports 7, Department of Mathematics, University of Umeå, Umeå, Sweden, 1993.

[54] P. Bylund and J. Gudayol, "On the existence of doubling measures with certain regularity properties," Proceedings of the American Mathematical Society, vol. 128, no. 11, pp. 3317-3327, 2000.

[55] H. Federer, Geometric Measure Theory, vol. 153 of Die Grundlehren der mathematischen Wissenschaften, Springer, New York, NY, USA, 1969.

[56] A. Nagel, E. M. Stein, and S. Wainger, "Balls and metrics defined by vector fields. I. Basic properties," Acta Mathematica, vol. 155, no. 1-2, pp. 103-147, 1985.

[57] N. Th. Varopoulos, "Analysis on Lie groups," Journal of Functional Analysis, vol. 76, no. 2, pp. 346-410, 1988.

[58] N. Th. Varopoulos, L. Saloff-Coste, and T. Coulhon, Analysis and Geometry on Groups, vol. 100 of Cambridge Tracts in Mathematics, Cambridge University Press, Cambridge, UK, 1992. 
[59] A. Nagel, F. Ricci, and E. M. Stein, "Harmonic analysis and fundamental solutions on nilpotent Lie groups," in Analysis and Partial Differential Equations, vol. 122 of Lecture Notes in Pure and Applied Mathematics, pp. 249-275, Dekker, New York, NY, USA, 1990.

[60] G. Alexopoulos, "Spectral multipliers on Lie groups of polynomial growth," Proceedings of the American Mathematical Society, vol. 120, no. 3, pp. 973-979, 1994.

[61] Y. Guivarc'h, "Croissance polynomiale et périodes des fonctions harmoniques," Bulletin de la Société Mathématique de France, vol. 101, pp. 333-379, 1973.

[62] J. W. Jenkins, "Growth of connected locally compact groups," Journal of Functional Analysis, vol. 12, pp. 113-127, 1973.

[63] A. Nagel and E. M. Stein, "Corrigenda: "On the product theory of singular integrals"," Revista Matemática Iberoamericana, vol. 21, no. 2, pp. 693-694, 2005.

[64] G. David, J.-L. Journé, and S. Semmes, "Opérateurs de Calderón-Zygmund, fonctions para-accrétives et interpolation," Revista Matemática Iberoamericana, vol. 1, no. 4, pp. 1-56, 1985.

[65] R. A. Macías, C. Segovia, and J. L. Torrea, "Singular integral operators with non-necessarily bounded kernels on spaces of homogeneous type," Advances in Mathematics, vol. 93, no. 1, pp. 25-60, 1992.

[66] E. M. Stein, Singular Integrals and Differentiability Properties of Functions, Princeton Mathematical Series, no. 30, Princeton University Press, Princeton, NJ, USA, 1970.

[67] K. Yosida, Functional Analysis, Classics in Mathematics, Springer, Berlin, Germany, 1995.

[68] G. David and J.-L. Journé, "A boundedness criterion for generalized Calderon-Zygmund operators," Annals of Mathematics. Second Series, vol. 120, no. 2, pp. 371-397, 1984.

[69] Y. Meyer, "Les nouveaux opérateurs de Calderón-Zygmund," Astérisque, no. 131, pp. 237-254, 1985.

[70] F. John and L. Nirenberg, "On functions of bounded mean oscillation," Communications on Pure and Applied Mathematics, vol. 14, no. 3, pp. 415-426, 1961.

[71] Y. Meyer and R. Coifman, Wavelets, vol. 48 of Cambridge Studies in Advanced Mathematics, Cambridge University Press, Cambridge, UK, 1997.

[72] M. Christ, "A $T(b)$ theorem with remarks on analytic capacity and the Cauchy integral," Colloquium Mathematicum, vol. 60/61, no. 2, pp. 601-628, 1990.

[73] D. Goldberg, "A local version of real Hardy spaces," Duke Mathematical Journal, vol. 46, no. 1, pp. 27-42, 1979.

[74] Da. Yang, Do. Yang, and Y. Zhou, "Localized Campanato spaces on RD-spaces and their applications to Schrödinger operators," submitted.

[75] E. M. Stein, Harmonic Analysis: Real-Variable Methods, Orthogonality, and Oscillatory Integrals, vol. 43 of Princeton Mathematical Series, Princeton University Press, Princeton, NJ, USA, 1993.

[76] Y. Meyer, Wavelets and Operators, vol. 37 of Cambridge Studies in Advanced Mathematics, Cambridge University Press, Cambridge, UK, 1992.

[77] C. Fefferman and E. M. Stein, "Some maximal inequalities," American Journal of Mathematics, vol. 93, no. 1, pp. 107-115, 1971.

[78] M. Frazier, B. Jawerth, and G. Weiss, Littlewood-Paley Theory and the Study of Function Spaces, vol. 79 of CBMS Regional Conference Series in Mathematics, American Mathematical Society, Providence, RI, USA, 1991.

[79] Y. Han, "Plancherel-Pôlya type inequality on spaces of homogeneous type and its applications," Proceedings of the American Mathematical Society, vol. 126, no. 11, pp. 3315-3327, 1998.

[80] D. Deng, Y. Han, and D. Yang, "Inhomogeneous Plancherel-Pôlya inequalities on spaces of homogeneous type and their applications," Communications in Contemporary Mathematics, vol. 6, no. 2, pp. 221-243, 2004.

[81] J. Duoandikoetxea, Fourier Analysis, vol. 29 of Graduate Studies in Mathematics, American Mathematical Society, Providence, RI, USA, 2001.

[82] M. Frazier and B. Jawerth, "A discrete transform and decompositions of distribution spaces," Journal of Functional Analysis, vol. 93, no. 1, pp. 34-170, 1990.

[83] D. Yang, "Some new Triebel-Lizorkin spaces on spaces of homogeneous type and their frame characterizations," Science in China. Series A, vol. 48, no. 1, pp. 12-39, 2005.

[84] D. Yang, "Some new inhomogeneous Triebel-Lizorkin spaces on metric measure spaces and their various characterizations," Studia Mathematica, vol. 167, no. 1, pp. 63-98, 2005.

[85] X. T. Duong and L. Yan, "New function spaces of BMO type, the John-Nirenberg inequality, interpolation, and applications," Communications on Pure and Applied Mathematics, vol. 58, no. 10, pp. 1375-1420, 2005.

[86] Y. Han and D. Yang, "New characterization of BMO $\left(\mathbb{R}^{n}\right)$ space," Boletín de la Sociedad Matemática Mexicanae. Tercera Serie, vol. 10, no. 1, pp. 95-103, 2004. 
[87] H. Triebel, "Function spaces in Lipschitz domains and on Lipschitz manifolds. Characteristic functions as pointwise multipliers," Revista Matemática Complutense, vol. 15, no. 2, pp. 475-524, 2002.

[88] J. Bergh and J. Löfström, Interpolation Spaces. An Introduction, Springer, Berlin, Germany, 1976.

[89] H. Triebel, Interpolation Theory, Function Spaces, Differential Operators, Johann Ambrosius Barth, Heidelberg, Germany, 2nd edition, 1995.

[90] D. Yang, "Frame characterizations of Besov and Triebel-Lizorkin spaces on spaces of homogeneous type and their applications," Georgian Mathematical Journal, vol. 9, no. 3, pp. 567-590, 2002.

[91] D. Yang, "Real interpolations for Besov and Triebel-Lizorkin spaces on spaces of homogeneous type," Mathematische Nachrichten, vol. 273, no. 1, pp. 96-113, 2004. 


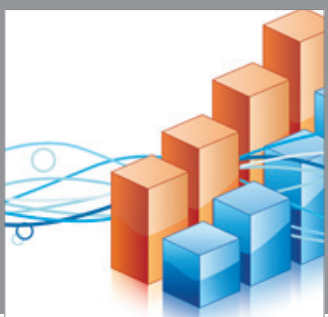

Advances in

Operations Research

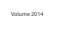

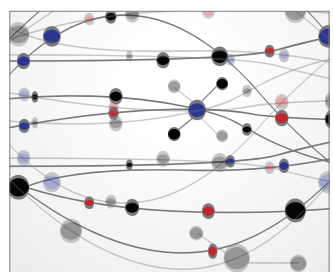

\section{The Scientific} World Journal
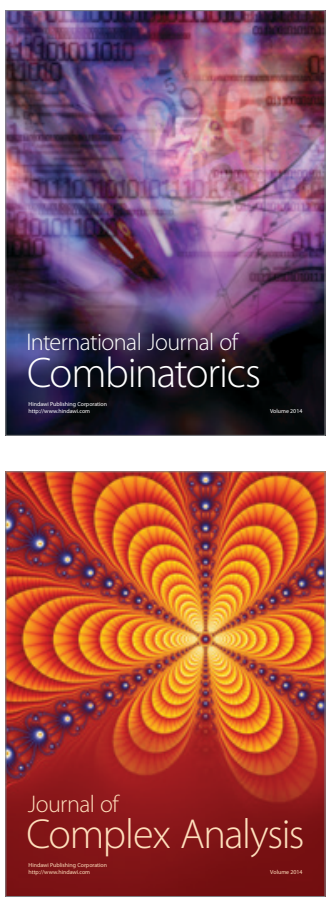

International Journal of

Mathematics and

Mathematical

Sciences
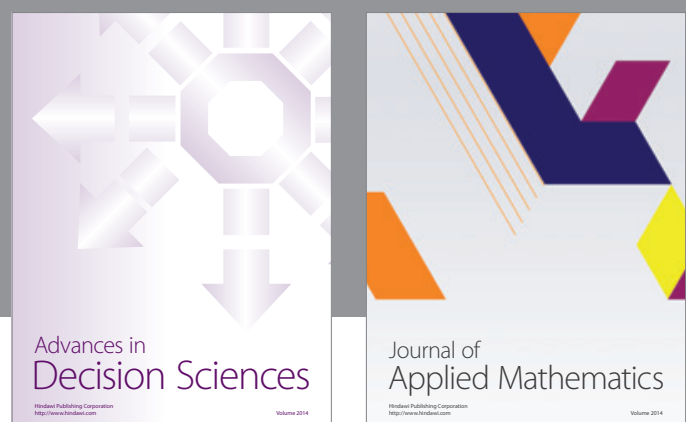

Journal of

Applied Mathematics
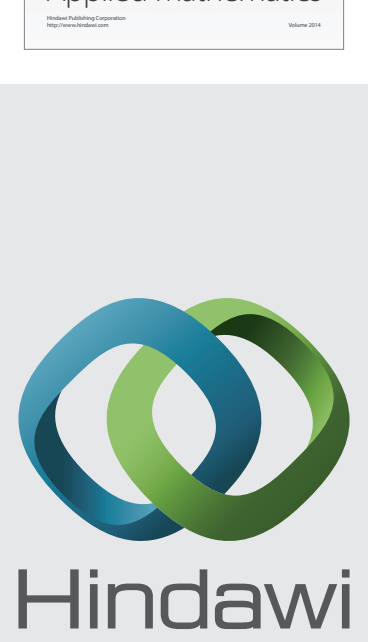

Submit your manuscripts at http://www.hindawi.com
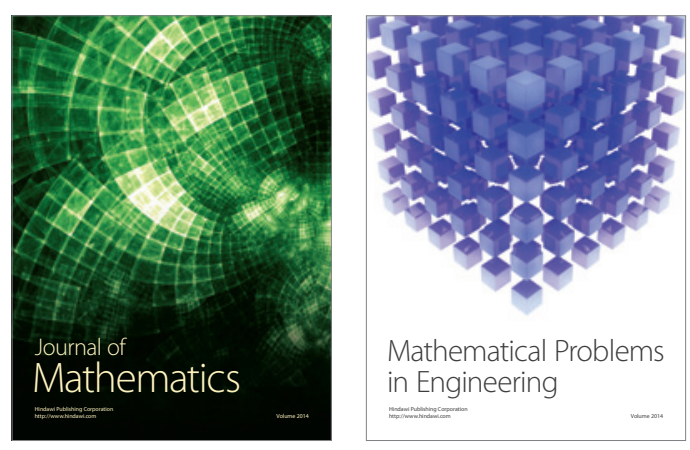

Mathematical Problems in Engineering
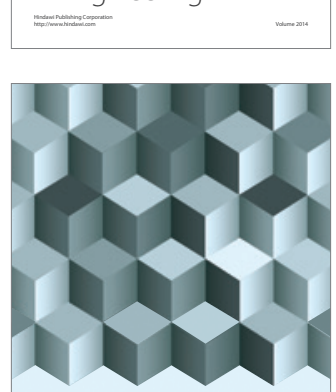

Journal of

Function Spaces
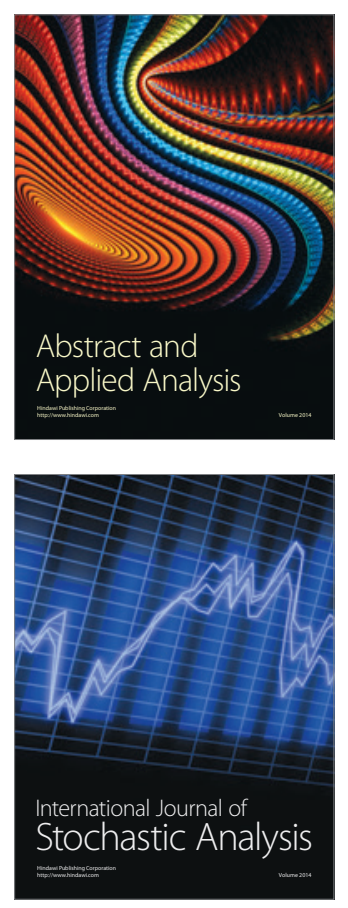

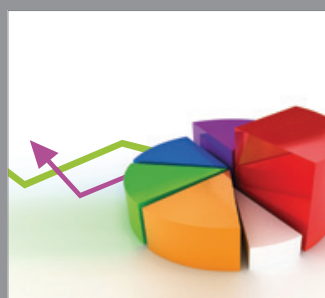

ournal of

Probability and Statistics

Promensencen
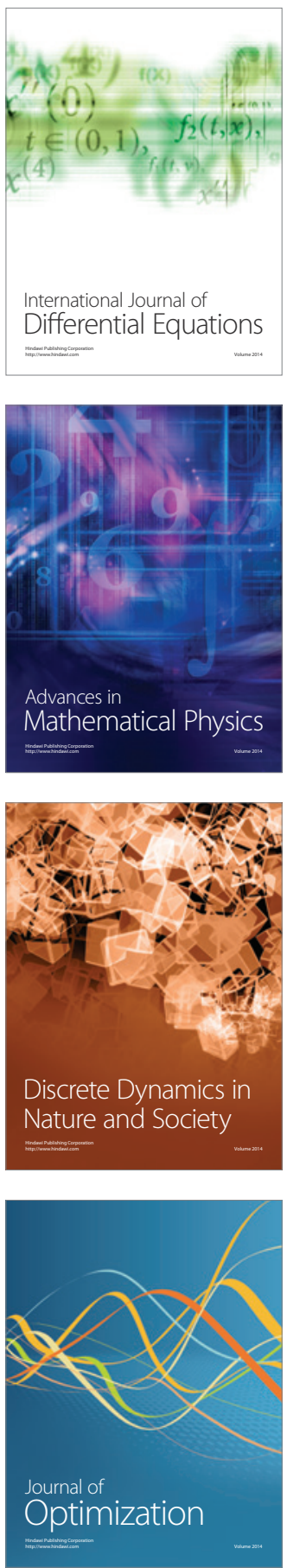